Supporting Information

\title{
Amino Acid Schiff Base Bearing Benzophenone Imine as a Platform for Highly Congested Unnatural $\alpha$-Amino Acid Synthesis
}

Yohei Matsumoto, Jun Sawamura, Yumi Murata, Takashi Nishikata,* Ryo Yazaki* and Takashi Ohshima*

Graduate School of Pharmaceutical Sciences, Kyushu University, Fukuoka, Japan

Graduate School of Science and Engineering, Yamaguchi University, Ube, Yamaguchi 755-8611, Japan

nisikata@yamaguchi-u.ac.jp

yazaki@phar.kyushu-u.ac.jp, ohshima@phar.kyushu-u.ac.jp

1. General

2. Instrumentation

3. Materials

4. Substrate Syntheses and Characterization

5. General Procedure and Characterization of Products

5-1. $\alpha$-Alkylation

5-2. $\beta$-Alkylation

6. Three-Components Cross-Coupling

7. Diastereoselective Reaction using Chiral Auxiliary

8. Transformation of the Products

9. Control Experiment

10. Full Data of Optimization Study

11. References

12. NMR Spectra of Products 
Supporting information Amino Acid Schiff Base Bearing Benzophenone Imine as a Platform for Highly Congested Unnatural $\alpha$-Amino Acid Synthesis

\section{General}

All reactions were carried out using heat gun dried glassware under a positive pressure of dry argon unless otherwise noted. The test tubes were fitted with a 3-way glass stopcock and catalytic reactions were run under argon atmosphere. Air- and moisture-sensitive liquids were transferred via a syringe and a stainless-steel needle. Reactions were magnetically stirred and monitored by thin layer chromatography using Merck Silica Gel 60 F254 plates. All work-up and purification procedures were carried out with reagent-grade solvents under ambient atmosphere. Flash chromatography was performed using silica gel $60 \mathrm{~N}$ (spherical neutral, particle size $40-50 \mu \mathrm{m}$ ) purchased from Kanto Chemical Co. Ltd.

\section{Instrumentation}

NMR was recorded on $500 \mathrm{MHz}$ Bruker Advanced III. Chemical shifts for proton are reported in parts per million downfield from tetramethylsilane and are referenced to residual protium in the NMR solvent $\left(\mathrm{CDCl}_{3}: \delta 7.26 \mathrm{ppm}\right)$. For ${ }^{13} \mathrm{C} \mathrm{NMR}$, chemical shifts were reported in the scale relative to NMR solvent $\left(\mathrm{CDCl}_{3}: 77.0 \mathrm{ppm}\right)$ as an internal reference. NMR data are reported as follows: chemical shifts, multiplicity (s: singlet, d: doublet, dd: doublet of doublets, t: triplet, q: quartet, sep: septet, m: multiplet, br: broad signal), coupling constant $(\mathrm{Hz})$, and integration. Infrared (IR) spectra were recorded on with PerkinElmer Spectrum One FT-IR Spectrometer. High-resolution mass spectroscopy (HRMS) was obtained with Bruker MicrOTOF II. Optical rotation was measured with JASCO DIP-370 polarimeter.

\section{Materials}

Deoxidized toluene was purchased from Wako Pure Chemical Industries (Wako) and used as received. Deoxidized THF was purchased from Kanto Chemical Co., Inc. (Kanto) and used as received. $\mathrm{CH}_{2} \mathrm{Cl}_{2}$ and tert-butylbenzene were dried over MS4A. Copper(II) acetate was purchased from Wako Pure Chemical Industries (Wako) and stored in an inert atmosphere glove box. Alkyl bromides $\mathbf{2}$ were prepared according to the previous report. ${ }^{1}$

\section{Substrate Syntheses and Characterization}

\section{General Procedure $A^{2}\left(1 a, 1 a^{\prime}, 1 j, 11-10,1 \delta, 13\right)$}

A flame-dried $25 \mathrm{~mL}$ round-bottom flask equipped with a magnetic stirring bar was filled with argon, and charged with the corresponding amino acid methyl ester hydrochloride (1.0 equiv.), benzophenone imine (1.0 equiv.) and $\mathrm{CH}_{2} \mathrm{Cl}_{2}(1.0 \mathrm{M})$. The mixture was stirred overnight at ambient temperature. After 
the reaction mixture was filtered, the solution was poured into sat. $\mathrm{NaHCO}_{3}$ aq and extracted with $\mathrm{CH}_{2} \mathrm{Cl}_{2}$. The combined organic layers were washed with brine, dried over sodium sulfate, and filtrated then purified by flash column chromatography.

\section{General Procedure $B^{3}$ (1b-1i)}

A flame-dried $25 \mathrm{~mL}$ round-bottom flask equipped with a magnetic stirring bar was filled with argon, and charged with methyl $\mathrm{N}$-(diphenylmethylene)glycinate (1.1 equiv.), $\left.\mathrm{Pd}^{(}{ }^{t} \mathrm{Bu}_{3} \mathrm{P}\right)_{2}(1.0 \mathrm{~mol} \%), \mathrm{K}_{3} \mathrm{PO}_{4}(3.0$ equiv.), toluene $(0.33 \mathrm{M})$ and aryl chloride (1.0 equiv.). The mixture was stirred at $100{ }^{\circ} \mathrm{C}$ for $20 \mathrm{~h}$, and the reaction mixture was filtered through plug of Celite. After evaporation of the organic solvent under reduced pressure, the residue was purified by silica gel flash chromatography.

\section{Characterization of Substrates and Products}

\section{5-1. $\alpha$-Amino acid Schiff base}<smiles>COC(=O)C(N=C(c1ccccc1)c1ccccc1)c1ccccc1</smiles>

Methyl 2-((diphenylmethylene)amino)-2-phenylacetate (1a); CAS Registry Number 171181-53-6

Prepared according to General Method A and purified by flash column chromatography (Hexane/Et $t_{2} \mathrm{O}=$ 5/1 (1\% Et $3 \mathrm{~N}), 67 \%$ yield, white solid); ${ }^{1} \mathrm{H} \mathrm{NMR}\left(500 \mathrm{MHz}, \mathrm{CDCl}_{3}, 27^{\circ} \mathrm{C}\right) \delta 7.72(\mathrm{~d}, J=7.0 \mathrm{~Hz}, 2 \mathrm{H}, \mathrm{ArH})$, 7.45-7.38 (m, 6H, ArH), 7.35-7.28 (m, 5H, ArH), $7.09(\mathrm{~m}, 2 \mathrm{H}, \mathrm{ArH}), 5.16(\mathrm{~s}, 1 \mathrm{H}, \mathrm{ArCH}), 3.68(\mathrm{~s}, 3 \mathrm{H}, \mathrm{OCH})$; ${ }^{13} \mathrm{C}$ NMR $\left(125 \mathrm{MHz}, \mathrm{CDCl}_{3}, 27{ }^{\circ} \mathrm{C}\right) \delta 172.0,170.3,139.4,139.1,136.1,130.5,129.0,128.8,128.6,128.5$, $128.0,127.9,127.8,127.7,69.6,52.4$.<smiles>CCC(C)OC(=O)C(N=C(c1ccccc1)c1ccccc1)c1ccccc1</smiles>

Tert-butyl 2-((diphenylmethylene)amino)-2-phenylacetate (1a'); CAS Registry Number 366018-76-0 
Supporting information Amino Acid Schiff Base Bearing Benzophenone Imine as a Platform for Highly Congested Unnatural $\alpha$-Amino Acid Synthesis

Prepared according to General Procedure A and purified by flash column chromatography (Hexane/AcOEt $=15 / 1\left(0.5 \% \mathrm{Et}_{3} \mathrm{~N}\right), 43 \%$ yield, colorless oil); ${ }^{1} \mathrm{H} \mathrm{NMR}\left(500 \mathrm{MHz}, \mathrm{CDCl}_{3}, 2{ }^{\circ} \mathrm{C}\right) \delta 7.72(\mathrm{~d}, J$ $=7.0 \mathrm{~Hz}, 2 \mathrm{H}, \mathrm{ArH}), 7.43(\mathrm{~m}, 5 \mathrm{H}, \operatorname{ArH}), 7.38(\mathrm{t}, J=7.3,1.7 \mathrm{~Hz}, 1 \mathrm{H}, \operatorname{ArH}), 7.32(\mathrm{q}, J=8.3 \mathrm{~Hz}, 4 \mathrm{H}, \operatorname{ArH}), 7.26(\mathrm{t}$, $J=8.0 \mathrm{~Hz}, 1 \mathrm{H}, \mathrm{ArH}), 7.11(\mathrm{~m}, 2 \mathrm{H}, \mathrm{ArH}), 5.01(\mathrm{~s}, 1 \mathrm{H}, \mathrm{ArCH}), 1.37\left(\mathrm{~s}, 9 \mathrm{H}, \mathrm{C}\left(\mathrm{CH}_{3}\right)_{3}\right) ;{ }^{13} \mathrm{C} \mathrm{NMR}\left(125 \mathrm{MHz}, \mathrm{CDCl}_{3}\right.$, $\left.27^{\circ} \mathrm{C}\right) \delta 170.5,169.6,139.7,139.6,136.4,130.3,128.9,128.6,128.5,128.3,128.0,127.9,127.8,127.4$, $81.2,70.2,27.9$.<smiles>COC(=O)C(N=C(c1ccccc1)c1ccc(C)cc1)c1ccccc1</smiles>

Methyl 2-((diphenylmethylene)amino)-2-(p-tolyl)acetate (1b); CAS Registry Number 137321-01-8 Prepared according to General Method B and purified by flash column chromatography (Hexane/Et $2 \mathrm{O}=$ $5 / 1\left(1 \% \mathrm{Et}_{3} \mathrm{~N}\right), 61 \%$ yield, white solid); ${ }^{1} \mathrm{H}$ NMR $\left(500 \mathrm{MHz}, \mathrm{CDCl}_{3}, 27^{\circ} \mathrm{C}\right) \delta 7.71(\mathrm{~d}, J=11.5 \mathrm{~Hz}, 2 \mathrm{H}, \mathrm{ArH})$, 7.45-7.44 (m, 3H, ArH), 7.43-7.37 (m, 1H, ArH), 7.35-7.30 (m, 4H, ArH), 7.13 (d, J = 8.0 Hz, 2H, ArH), 7.09$7.07(\mathrm{~m}, 2 \mathrm{H}, \mathrm{ArH}), 5.12(\mathrm{~s}, 1 \mathrm{H}, \mathrm{ArCH}), 3.67\left(\mathrm{~s}, 3 \mathrm{H}, \mathrm{OCH}_{3}\right), 2.33\left(\mathrm{~s}, 3 \mathrm{H}, \mathrm{ArCH}_{3}\right) ;{ }^{13} \mathrm{C} \mathrm{NMR}\left(125 \mathrm{MHz}, \mathrm{CDCl}_{3}\right.$, $\left.27^{\circ} \mathrm{C}\right) \delta 172.2,170.1,139.5,137.5,136.2,136.2,130.4,129.2,129.0,128.8,128.5,128.0,127.8,127.7$, $69.4,52.4,21.2$.<smiles>COC(=O)C(N=C(c1ccccc1)c1ccc(OC)cc1)c1ccccc1</smiles>

Methyl 2-((diphenylmethylene)amino)-2-(4-methoxyphenyl)acetate (1c); CAS Registry Number 191401-39-5

Prepared according to General Method B and purified by flash column chromatography (Hexane/Et $2 \mathrm{O}=$ $5 / 1\left(1 \% \mathrm{Et}_{3} \mathrm{~N}\right), 60 \%$ yield, yellow solid); ${ }^{1} \mathrm{H}$ NMR $\left(500 \mathrm{MHz}, \mathrm{CDCl}_{3}, 27^{\circ} \mathrm{C}\right) \delta 7.71(\mathrm{~d}, J=7.0 \mathrm{~Hz}, 2 \mathrm{H}, \mathrm{ArH})$, 7.45-7.43 (m, 3H, ArH), 7.39-7.37 (m, 1H, ArH), 7.35-7.31 (m, 4H, ArH), 7.09-7.07 (m, 2H, ArH), $6.86(\mathrm{~d}, J$ $=9.0 \mathrm{~Hz}, 2 \mathrm{H}, \mathrm{ArH}), 5.10(\mathrm{~s}, 1 \mathrm{H}, \mathrm{ArCH}), 3.79\left(\mathrm{~s}, 3 \mathrm{H}, \mathrm{ArOCH}_{3}\right), 3.68\left(\mathrm{~s}, 3 \mathrm{H}, \mathrm{OCH}_{3}\right) ;{ }^{13} \mathrm{CNMR}\left(125 \mathrm{MHz}, \mathrm{CDCl}_{3}\right.$, 
$\left.27^{\circ} \mathrm{C}\right) \delta 172.3,170.0,159.2,139.4,136,2,131.4,130.4,129.0,129.0,128.8,128.5,128.0,127.7,113.9$, $69.0,55.3,52.3$.<smiles>COC(=O)C(N=C(c1ccccc1)c1ccc(Cl)cc1)c1ccccc1</smiles>

Methyl 2-(4-chlorophenyl)-2-((diphenyImethylene)amino)acetate (1d); CAS Registry Number 2027496$87-1$

Prepared according to General Method B and purified by flash column chromatography (Hexane/Et $2 \mathrm{O}=$ $5 / 1\left(1 \% \mathrm{Et}_{3} \mathrm{~N}\right), 32 \%$ yield, yellow solid); ${ }^{1} \mathrm{H}$ NMR $\left(500 \mathrm{MHz}, \mathrm{CDCl}_{3}, 27^{\circ} \mathrm{C}\right) \delta 7.71(\mathrm{~d}, J=7.0 \mathrm{~Hz}, 2 \mathrm{H}, \mathrm{ArH})$, 7.46-7.42 (m, 3H, ArH), 7.41-7.39 (m, 1H, ArH), 7.37-7.34 (m, 4H, ArH), 7.33-7.29 (m, 2H, ArH), 7.08-7.06 $(\mathrm{m}, 2 \mathrm{H}, \mathrm{ArH}), 5.12(\mathrm{~s}, 1 \mathrm{H}, \mathrm{ArCH}), 3.68\left(\mathrm{~s}, 3 \mathrm{H}, \mathrm{OCH}_{3}\right) ;{ }^{13} \mathrm{C} \mathrm{NMR}\left(125 \mathrm{MHz}, \mathrm{CDCl}_{3}, 27^{\circ} \mathrm{C}\right) \delta 171.6,170.8$, $139.2,137.6,136.0,133.7,130.7,129.3,129.0,128.9,128.7,128.6,128.1,127.6,68.8,52.5$.<smiles>COC(=O)C(N=C(c1ccccc1)c1ccccc1)c1ccc(-c2ccccc2)cc1</smiles>

\section{Methyl 2-([1,1'-biphenyl]-4-yl)-2-((diphenylmethylene)amino)acetate (1e)}

Prepared according to General Method B and purified by flash column chromatography (Hexane/Et $\mathrm{t}_{2} \mathrm{O}=$ 5/1 (1\% Et 3 N), 70\% yield, yellow solid); IR (neat) 1736, 1620, 1485, 1248, $1157 \mathrm{~cm}^{-1} ;{ }^{1} \mathrm{H}$ NMR (500 MHz, $\left.\mathrm{CDCl}_{3}, 27^{\circ} \mathrm{C}\right) \delta 7.71(\mathrm{~d}, J=7.0 \mathrm{~Hz}, 2 \mathrm{H}, \mathrm{ArH}), 7.56-7.50(\mathrm{~m}, 4 \mathrm{H}, \mathrm{ArH}), 7.49-7.38(\mathrm{~m}, 8 \mathrm{H}, \mathrm{ArH})$, 7.37-7.32 (m, $3 \mathrm{H}, \mathrm{ArH}), 7.12-7.11(\mathrm{~m}, 2 \mathrm{H}, \mathrm{ArH}), 5.21(\mathrm{~s}, 1 \mathrm{H}, \mathrm{ArCH}), 3.71\left(\mathrm{~s}, 3 \mathrm{H}, \mathrm{OCH}_{3}\right) ;{ }^{13} \mathrm{C} \mathrm{NMR}\left(125 \mathrm{MHz}, \mathrm{CDCl}_{3}, 27^{\circ} \mathrm{C}\right)$ ठ 172.0, 170.4, 140.8, 140.7, 139.4, 138.2, 136.1, 130.5, 129.0, 128.9, 128.7, 128.6, 128.3, 128.1, 128.1, 127.7, 127.3, 127.1, 69.4, 52.5; HRMS (ESI) m/z calcd. for $\mathrm{C}_{28} \mathrm{H}_{23} \mathrm{NNaO}_{2}{ }^{+} 428.1621$ found 428.1617 . 
Supporting information Amino Acid Schiff Base Bearing Benzophenone Imine as a Platform for Highly Congested Unnatural $\alpha$-Amino Acid Synthesis<smiles>COC(=O)C(N=C(c1ccccc1)c1ccccc1)c1cccc(C)c1</smiles>

Methyl 2-((diphenylmethylene)amino)-2-(m-tolyl)acetate (1f); CAS Registry Number 2027496-82-6 Prepared according to General Method B and purified by flash column chromatography (Hexane/Et ${ }_{2} \mathrm{O}=$ $5 / 1\left(1 \% \mathrm{Et}_{3} \mathrm{~N}\right), 69 \%$ yield, white solid); ${ }^{1} \mathrm{H}$ NMR $\left(500 \mathrm{MHz}, \mathrm{CDCl}_{3}, 27^{\circ} \mathrm{C}\right) \delta 7.72(\mathrm{~d}, J=10.0 \mathrm{~Hz}, 2 \mathrm{H}, \mathrm{ArH})$, 7.45-7.43 (m, 3H, ArH), 7.41-7.38 (m, 1H, ArH), 7.35-7.32 (m, 2H, ArH), 7.25 (s, 1H, ArH), 7.21-7.20 (m, $2 \mathrm{H}, \mathrm{ArH}), 7.10-7.07(\mathrm{~m}, 3 \mathrm{H}, \mathrm{ArH}), 5.12(\mathrm{~s}, 1 \mathrm{H}, \mathrm{ArCH}), 3.68(\mathrm{~s}, 3 \mathrm{H}, \mathrm{OCH}), 2.33\left(\mathrm{~s}, 3 \mathrm{H}, \mathrm{CH}_{3}\right) ;{ }^{13} \mathrm{C}$ NMR $(125$ $\left.\mathrm{MHz}, \mathrm{CDCl}_{3}, 27^{\circ} \mathrm{C}\right) \delta 172.1,170.2,139.4,139.0,138.2,136.2,130.5,129.0,128.8,128.6,128.5,128.5$, $128.4,128.0,127.7,125.0,69.6,52.4,21.5$.<smiles>COC(=O)C(N=C(c1ccccc1)c1ccccc1)c1cccc(OC)c1</smiles>

Methyl 2-((diphenylmethylene)amino)-2-(3-methoxyphenyl)acetate (1g); CAS Registry Number 2027496-84-8

Prepared according to General Method B and purified by flash column chromatography (Hexane/Et $\mathrm{E}_{2} \mathrm{O}=$ $5 / 1\left(1 \% \mathrm{Et}_{3} \mathrm{~N}\right), 61 \%$ yield, yellow solid); ${ }^{1} \mathrm{H}$ NMR $\left(500 \mathrm{MHz}, \mathrm{CDCl}_{3}, 27^{\circ} \mathrm{C}\right) \delta 7.71(\mathrm{~d}, J=8.5 \mathrm{~Hz}, 2 \mathrm{H}, \mathrm{ArH})$, 7.45-7.43 (m, 3H, ArH), 7.41-7.38 (m, 1H, ArH), $7.34(\mathrm{t}, J=6.0 \mathrm{~Hz}, 2 \mathrm{H}, \operatorname{ArH}), 7.23(\mathrm{t}, J=7.5 \mathrm{~Hz}, 1 \mathrm{H}, \operatorname{ArH})$, 7.10-7.08 (m, 2H, ArH), 7.03-7.02 (m, 1H, ArH), $6.98(\mathrm{~d}, J=7.5 \mathrm{~Hz}, 1 \mathrm{H}, \operatorname{ArH}), 6.83(\mathrm{dd}, J=8.0,2.5 \mathrm{~Hz}, 1 \mathrm{H}$, $\operatorname{ArH}), 5.13(\mathrm{~s}, 1 \mathrm{H}, \operatorname{ArCH}), 3.80\left(\mathrm{~s}, 3 \mathrm{H}, \mathrm{ArOCH}_{3}\right), 3.68\left(\mathrm{~s}, 3 \mathrm{H}, \mathrm{OCH}_{3}\right) ;{ }^{13} \mathrm{C} \mathrm{NMR}\left(125 \mathrm{MHz}, \mathrm{CDCl}_{3}, 27{ }^{\circ} \mathrm{C}\right) \delta$ $171.9,170.4,159.7,140.6,139.4,136.1,130.5,129.4,129.0,128.8,128.6,128.0,127.7,120.3,113.5$, $113.4,69.5,55.3,52.4$. 
Supporting information Amino Acid Schiff Base Bearing Benzophenone Imine as a Platform for Highly Congested Unnatural $\alpha$-Amino Acid Synthesis<smiles>COC(=O)C(N=C(c1ccccc1)c1ccccc1)c1ccccc1C</smiles>

Methyl 2-((diphenylmethylene)amino)-2-(o-tolyl)acetate (1h); CAS Registry Number 191401-38-4

Prepared according to General Method B and purified by flash column chromatography $\left(\right.$ Hexane $/ \mathrm{Et}_{2} \mathrm{O}=$ 5/1 (1\% Et $3 \mathrm{~N}), 75 \%$ yield, white solid); $\left.{ }^{1} \mathrm{H} \mathrm{NMR} \mathrm{(500} \mathrm{MHz,} \mathrm{CDCl}_{3}, 27{ }^{\circ} \mathrm{C}\right) \delta 7.71(\mathrm{~d}, J=7.0 \mathrm{~Hz}, 2 \mathrm{H}, \mathrm{ArH})$, $7.58(\mathrm{~d}, J=9.0 \mathrm{~Hz}, 1 \mathrm{H}, \operatorname{ArH}), 7.45-7.42(\mathrm{~m}, 3 \mathrm{H}, \operatorname{ArH}), 7.40-7.37(\mathrm{~m}, 1 \mathrm{H}, \operatorname{ArH}), 7.33(\mathrm{t}, J=7.5 \mathrm{~Hz}, 2 \mathrm{H}, \operatorname{ArH})$, 7.20-7.14 (m, 2H, ArH), $7.08(\mathrm{~d}, J=7.0 \mathrm{~Hz}, 1 \mathrm{H}, \operatorname{ArH}), 7.05-7.04(\mathrm{~m}, 2 \mathrm{H}, \operatorname{ArH}), 5.33(\mathrm{~s}, 1 \mathrm{H}, \operatorname{ArCH}), 3.68(\mathrm{~s}$, $\left.3 \mathrm{H}, \mathrm{OCH}_{3}\right), 2.05\left(\mathrm{~s}, 3 \mathrm{H}, \mathrm{CH}_{3}\right) ;{ }^{13} \mathrm{C} \mathrm{NMR}\left(125 \mathrm{MHz}, \mathrm{CDCl}_{3}, 27^{\circ} \mathrm{C}\right) \delta 172.2,170.2,139.4,138.0,136.5,136.0$, $130.4,130.4,128.9,128.9,128.7,128.6,128.0,127.6,127.5,126.2,66.6,52.3,19.2$.<smiles>COC(=O)C(N=C(c1ccccc1)c1ccccc1)c1ccc2ccccc2c1</smiles>

Methyl 2-((diphenylmethylene)amino)-2-(naphthalen-2-yl)acetate (1i); CAS Registry Number 183614539-1

Prepared according to General Method B and purified by flash column chromatography (Hexane/Et $t_{2} \mathrm{O}=$ 5/1 (1\% Et $\left.{ }_{3} \mathrm{~N}\right), 66 \%$ yield, white solid); ${ }^{1} \mathrm{H} \mathrm{NMR}\left(500 \mathrm{MHz}, \mathrm{CDCl}_{3}, 27^{\circ} \mathrm{C}\right) \delta 7.83-7.79(\mathrm{~m}, 3 \mathrm{H}, \operatorname{ArH}), 7.75(\mathrm{~d}$, $J=7.0 \mathrm{~Hz}, 2 \mathrm{H}, \operatorname{ArH}), 7.61(\mathrm{~d}, J=8.5 \mathrm{~Hz}, 1 \mathrm{H}, \operatorname{ArH}), 7.47-7.41(\mathrm{~m}, 4 \mathrm{H}, \operatorname{ArH}), 7.40-7.39(\mathrm{~m}, 1 \mathrm{H}, \operatorname{ArH}), 7.35(\mathrm{t}, J$ $=8.0 \mathrm{~Hz}, 2 \mathrm{H}, \mathrm{ArH}), 7.10(\mathrm{~d}, J=7.0 \mathrm{~Hz}, 2 \mathrm{H}, \mathrm{ArH}), 5.32(\mathrm{~s}, 1 \mathrm{H}, \mathrm{ArCH}), 3.69\left(\mathrm{~s}, 3 \mathrm{H}, \mathrm{OCH}_{3}\right) ;{ }^{13} \mathrm{C} \mathrm{NMR}(125 \mathrm{MHz}$, $\left.\mathrm{CDCl}_{3}, 27^{\circ} \mathrm{C}\right) \delta 172.0,171.33,139.4,136.6,136.1,133.3,133.0,130.5,129.0,128.9,128.6,128.2,128.1$, $128.1,127.7,127.7,126.8,126.0,126.0,125.9,69.8,52.4$. 
Supporting information Amino Acid Schiff Base Bearing Benzophenone Imine as a Platform for Highly Congested Unnatural $\alpha$-Amino Acid Synthesis<smiles>COC(=O)C(N=C(c1ccccc1)c1ccccc1)c1cccs1</smiles>

Methyl 2-((diphenylmethylene)amino)-2-(thiophen-2-yl)acetate (1j); CAS Registry Number 2027496$92-8$

Prepared according to General Method $\mathrm{A}$ and purified by flash column chromatography (Hexane/Et ${ }_{2} \mathrm{O}=$ $5 / 1\left(1 \% \mathrm{Et}_{3} \mathrm{~N}\right), 46 \%$ yield, white solid); ${ }^{1} \mathrm{H} \mathrm{NMR}\left(500 \mathrm{MHz}, \mathrm{CDCl}_{3}, 27^{\circ} \mathrm{C}\right) \delta 7.73(\mathrm{~d}, J=7.0 \mathrm{~Hz}, 2 \mathrm{H}, \mathrm{ArH})$, 7.47-7.45 (m, 3H, ArH), 7.43-7.40 (m, 1H, ArH), $7.35(\mathrm{t}, J=7.0 \mathrm{~Hz}, 2 \mathrm{H}, \operatorname{ArH}), 7.28-7.27(\mathrm{~m}, 1 \mathrm{H}, \operatorname{ArH}), 7.15-$ $7.13(\mathrm{~m}, 2 \mathrm{H}, \mathrm{ArH}), 6.96(\mathrm{~d}, \mathrm{~J}=4.0 \mathrm{~Hz}, 2 \mathrm{H}, \operatorname{ArH}), 5.44(\mathrm{~s}, 1 \mathrm{H}, \operatorname{ArCH}), 3.72\left(\mathrm{~s}, 3 \mathrm{H}, \mathrm{OCH}_{3}\right) ;{ }^{13} \mathrm{C} \mathrm{NMR}(125 \mathrm{MHz}$, $\left.\mathrm{CDCl}_{3}, 27^{\circ} \mathrm{C}\right) \delta 171.1,170.9,141.7,139.1,135.7,130.7,129.1,129.0,128.7,128.1,127.7,126.5,125.4$, $124.8,65.4,52.7$.<smiles>COc1ccc(C2=NC(c3ccccc3)C(=O)O2)cc1</smiles>

2-(4-Methoxyphenyl)-4-phenyloxazol-5(4H)-one (1k)33; CAS Registry Number 28172-58-9

To a solution of DL-2-(4-methoxybenzamido)-2-phenylacetic acid (2.8 mmol, 1.0 equiv.) in $\mathrm{CH}_{2} \mathrm{Cl}_{2}(39 \mathrm{~mL}$, 0.072 M) under argon atmosphere was added 1-(3-dimethylaminopropyl)-3-ethylcarbodiimide hydrochloride ( $3.6 \mathrm{mmol}, 1.3$ equiv.) at $0{ }^{\circ} \mathrm{C}$. After stirring at ambient temperature for $1 \mathrm{~h}$, the reaction mixture was poured into $\mathrm{H}_{2} \mathrm{O}$ and extracted with $\mathrm{CH}_{2} \mathrm{Cl}_{2}$. The combined organic layers were extracted with sat. $\mathrm{NaHCO}_{3}$ aq and dried over sodium sulfate and filtered. After removal of solvent under reduced pressure, the product was obtained as a solid which was used after recrystallization (62\% yield, white solid); ${ }^{1} \mathrm{H}$ NMR ( $\left.500 \mathrm{MHz}, \mathrm{CDCl}_{3}, 27^{\circ} \mathrm{C}\right) \delta 8.04(\mathrm{~d}, J=8.0 \mathrm{~Hz}, 2 \mathrm{H}, \operatorname{ArH}), 7.45-7.36(\mathrm{~m}, 5 \mathrm{H}, \mathrm{ArH}), 7.01(\mathrm{~d}, J=$ $9.0 \mathrm{~Hz}, 2 \mathrm{H}, \mathrm{ArH}), 5.45(\mathrm{~s}, 1 \mathrm{H}, \mathrm{ArCH}), 3.90\left(\mathrm{~s}, 3 \mathrm{H}, \mathrm{OCH}_{3}\right) ;{ }^{13} \mathrm{C} \mathrm{NMR}\left(125 \mathrm{MHz}, \mathrm{CDCl}_{3}, 2{ }^{\circ} \mathrm{C}\right) \delta 176.5,163.5$, $162.3,133.8,130.0,129.0,128.7,126.7,118.0,114.3,68.1,55.5$. 
Supporting information Amino Acid Schiff Base Bearing Benzophenone Imine as a Platform for Highly Congested Unnatural $\alpha$-Amino Acid Synthesis<smiles>COC(=O)C(C)N=C(c1ccccc1)c1ccccc1</smiles>

Methyl 2-((diphenylmethylene)amino)propanoate (1I); CAS Registry Number 195600-97-6

Prepared according to General Method A and purified by flash column chromatography $($ Hexane/Et $2 \mathrm{O}=$ 5/1 (1\% Et $3 \mathrm{~N}), 92 \%$ yield, white solid); ${ }^{1} \mathrm{H} \mathrm{NMR}\left(500 \mathrm{MHz}, \mathrm{CDCl}_{3}, 27^{\circ} \mathrm{C}\right) \delta 7.64(\mathrm{~d}, J=7.0 \mathrm{~Hz}, 2 \mathrm{H}, \mathrm{ArH})$, 7.48-7.44 (m, 3H, ArH), 7.37 (t, J=7.5 Hz, 1H, ArH), 7.33 (t, J=8.5 Hz, 2H, ArH), $6.19(\mathrm{~d}, J=8.0 \mathrm{~Hz}, 2 \mathrm{H}$, $\operatorname{ArH}), 4.18(\mathrm{q}, J=7.0 \mathrm{~Hz}, 1 \mathrm{H}, \mathrm{CH}), 3.72\left(\mathrm{~s}, 3 \mathrm{H}, \mathrm{OCH}_{3}\right), 1.42\left(\mathrm{~d}, J=6.5 \mathrm{~Hz}, 3 \mathrm{H}, \mathrm{CH}_{3}\right) ;{ }^{13} \mathrm{C} \mathrm{NMR}(125 \mathrm{MHz}$, $\left.\mathrm{CDCl}_{3}, 27^{\circ} \mathrm{C}\right) \delta 173.5,169.7,139.5,136.2,130.3,128.8,128.7,128.6,128.1,127.7,60.6,52.1,19.2$.<smiles>CCC(N=C(c1ccccc1)c1ccccc1)C(=O)OC</smiles>

Methyl 2-((diphenylmethylene)amino)butanoate (1m); CAS Registry Number 553645-47-9

Prepared according to General Method A and purified by flash column chromatography (Hexane/Et $t_{2} \mathrm{O}=$ 5/1 (1\% Et $3 \mathrm{~N}), 50 \%$ yield, colorless oil); ${ }^{1} \mathrm{H}$ NMR $\left(500 \mathrm{MHz}, \mathrm{CDCl}_{3}, 27^{\circ} \mathrm{C}\right) \delta 7.65(\mathrm{~d}, \mathrm{~J}=7.0 \mathrm{~Hz}, 2 \mathrm{H}, \mathrm{ArH})$, 7.47-7.45 (m, 3H, ArH), 7.44-7.40 (m, 1H, ArH), $7.33(\mathrm{t}, J=7.0 \mathrm{~Hz}, 2 \mathrm{H}, \mathrm{ArH})$, 7.18-7.16 (m, 2H, ArH), 4.01 $(\mathrm{dd}, J=8.0,5.0 \mathrm{~Hz}, 1 \mathrm{H}, \mathrm{CH}), 3.72\left(\mathrm{~s}, 3 \mathrm{H}, \mathrm{OCH}_{3}\right), 2.00-1.85\left(\mathrm{~m}, 2 \mathrm{H}, \mathrm{CH}_{2}\right), 0.85\left(\mathrm{t}, J=7.5 \mathrm{~Hz}, 3 \mathrm{H}, \mathrm{CH}_{3}\right) ;{ }^{13} \mathrm{C}$ $\operatorname{NMR}\left(125 \mathrm{MHz}, \mathrm{CDCl}_{3}, 27^{\circ} \mathrm{C}\right) \delta 172.9,170.4,139.6,136.5,130.3,128.8,128.6,128.5,128.1,127.9,66.8$, $52.0,27.0,10.5$<smiles>COC(=O)C(Cc1ccccc1)N=C(c1ccccc1)c1ccccc1</smiles>

Methyl 2-((diphenylmethylene)amino)-3-phenylpropanoate (1n); CAS Registry Number 120238-42-8

Prepared according to General Method A and purified by flash column chromatography (Hexane:Et ${ }_{2} \mathrm{O}=$ 5:1 (1\% Et $\left.{ }_{3} \mathrm{~N}\right), 46 \%$ yield, white solid); ${ }^{1} \mathrm{H} \mathrm{NMR}\left(500 \mathrm{MHz}, \mathrm{CDCl}_{3}, 27{ }^{\circ} \mathrm{C}\right) \delta 7.58(\mathrm{~d}, J=7.0 \mathrm{~Hz}, 2 \mathrm{H}, \mathrm{ArH})$, 
7.39-7.27 (m, 6H, ArH), 7.20-7.14 (m, 3H, ArH), $7.02(\mathrm{dd}, J=7.5,2.0 \mathrm{~Hz}, 2 \mathrm{H}, \operatorname{ArH}), 7.03-7.01(\mathrm{~m}, 2 \mathrm{H}, \operatorname{ArH})$, $4.26\left(\mathrm{dd}, J=9.5,4.5 \mathrm{~Hz}, 1 \mathrm{H}, \mathrm{CH}\right.$ ), $3.73\left(\mathrm{~s}, 3 \mathrm{H}, \mathrm{OCH}_{3}\right), 3.27$ (dd, $J=13.5,4.0 \mathrm{~Hz}, 1 \mathrm{H}, \mathrm{ArCH}_{2}$ ), 3.17 (dd, $J=$ 13.0, $\left.9.5 \mathrm{~Hz}, 1 \mathrm{H}, \mathrm{ArCH}_{2}\right) ;{ }^{13} \mathrm{C}$ NMR $\left(125 \mathrm{MHz}, \mathrm{CDCl}_{3}, 27^{\circ} \mathrm{C}\right) \delta 172.3,170.9,139.4,137.9,136.1,130.3$, $129.9,128.8,128.3,128.2,128.2,128.0,127.6,126.3,67.3,52.2,39.8$.<smiles>COC(=O)CCC(N=C(c1ccccc1)c1ccccc1)C(=O)OC</smiles>

Dimethyl 2-((diphenylmethylene)amino)pentanedioate (10); CAS Registry Number 221035-75-2 Prepared according to General Method A and purified by flash column chromatography (Hexane/Et $2 \mathrm{O}=$ $5 / 1\left(1 \% \mathrm{Et}_{3} \mathrm{~N}\right), 46 \%$ yield, colorless oil); ${ }^{1} \mathrm{H}$ NMR $\left(500 \mathrm{MHz}, \mathrm{CDCl}_{3}, 27^{\circ} \mathrm{C}\right) \delta 7.63(\mathrm{~d}, J=7.5 \mathrm{~Hz}, 2 \mathrm{H}, \mathrm{ArH})$, 7.46-7.43 (m, 3H, ArH), $7.40(\mathrm{t}, J=7.5 \mathrm{~Hz}, 1 \mathrm{H}, \operatorname{ArH}), 7.33(\mathrm{t}, J=7.5 \mathrm{~Hz}, 2 \mathrm{H}, \operatorname{ArH}), 7.19-7.17(\mathrm{~m}, 2 \mathrm{H}, \operatorname{ArH})$, $4.13(\mathrm{t}, J=7.0 \mathrm{~Hz}, 1 \mathrm{H}, \mathrm{CH}), 3.71\left(\mathrm{~s}, 3 \mathrm{H}, \mathrm{OCH}_{3}\right), 3.58\left(\mathrm{~s}, 3 \mathrm{H}, \mathrm{OCH}_{3}\right), 2.36\left(\mathrm{t}, J=7.5 \mathrm{~Hz}, 2 \mathrm{H}, \mathrm{CH}_{2} \mathrm{COOCH}_{3}\right)$, $2.24\left(q, J=7.5 \mathrm{~Hz}, 2 \mathrm{H}, \mathrm{CH}_{2}\right) ;{ }^{13} \mathrm{C}$ NMR $\left(125 \mathrm{MHz}, \mathrm{CDCl}_{3}, 27^{\circ} \mathrm{C}\right) \delta 173.4,172.2,171.2,139.3,136.1,130.5$, $128.9,128.7,128.6,128.1,127.8,64.1,52.2,51.5,30.4,28.6$.<smiles>C[C@H]1CC[C@H](OC(=O)C(N=C(c2ccccc2)c2ccccc2)c2ccccc2)[C@H](C(C)(C)c2cccc3ccccc23)C1</smiles>

$(1 R, 2 S, 5 R)-5-M e t h y l-2-(2-($ naphthalen-1-yl)propan-2-yl)cyclohexyl 2-((diphenylmethylene)amino)-2phenylacetate (1ס)

Prepared according to General Method A and purified by flash column chromatography (Hexane/Et $2 \mathrm{O}=$ 19/1 (1\% Et $\left.{ }_{3} \mathrm{~N}\right)$, diastereomixture, $72 \%$ yield, $d r=1.2 / 1$, white solid); IR (neat) $2359,2343,1732 \mathrm{~cm}^{-1} ;{ }^{1} \mathrm{H}$ $\operatorname{NMR}\left(500 \mathrm{MHz}, \mathrm{CDCl}_{3}, 27^{\circ} \mathrm{C}\right) \delta 8.37-8.35(\mathrm{~m}, 1 \mathrm{H}, \mathrm{ArH}), 8.27(\mathrm{~d}, J=8.5 \mathrm{~Hz}, 1 \mathrm{H}, \operatorname{ArH}), 7.83(\mathrm{t}, J=8.0 \mathrm{~Hz}, 2 \mathrm{H}$, $\operatorname{ArH}), 7.73(\mathrm{t}, J=7.0 \mathrm{~Hz}, 4 \mathrm{H}, \operatorname{ArH}), 7.62(\mathrm{t}, J=4.5 \mathrm{~Hz}, 1 \mathrm{H}, \operatorname{ArH}), 7.54-7.50(\mathrm{~m}, 4 \mathrm{H}, \operatorname{ArH}), 7.47(\mathrm{~d}, J=7.5 \mathrm{~Hz}$, $2 \mathrm{H}, \operatorname{ArH})$, 7.45-7.42 (m, 6H, ArH), 7.40-7.36 (m, 3H, ArH), 7.34-7.28 (m, 9H, ArH), 7.25-7.23 (m, $1 \mathrm{H}, \operatorname{ArH})$, 7.13-7.08 (m, 10H, ArH), $5.09(\mathrm{~s}, 1 \mathrm{H}, \mathrm{ArCH}), 5.06(\mathrm{td}, J=10.5,4.5 \mathrm{~Hz}, 1 \mathrm{H}, \mathrm{OCH}), 4.10(\mathrm{br}, 1 \mathrm{H}, \mathrm{OCH}), 4.73$ (br, 1H, ArCH), 2.71-2.67 (m, 2H, CH), $1.98\left(\mathrm{~d}, J=12.5 \mathrm{~Hz}, 1 \mathrm{H}, \mathrm{CH}_{2}\right), 1.80\left(\mathrm{~d}, J=10.5 \mathrm{~Hz}, 1 \mathrm{H}, \mathrm{CH}_{2}\right), 1.49(\mathrm{~m}$, 
Supporting information Amino Acid Schiff Base Bearing Benzophenone Imine as a Platform for Highly Congested Unnatural $\alpha$-Amino Acid Synthesis

$\left.2 \mathrm{H}, \mathrm{CH}_{2}\right), 1.42\left(\mathrm{~s}, 3 \mathrm{H}, \mathrm{CH}_{3}\right), 1.41\left(\mathrm{~s}, 3 \mathrm{H}, \mathrm{CH}_{3}\right), 1.40-1.35\left(\mathrm{~m}, 2 \mathrm{H}, \mathrm{CH}_{2}\right), 1.33\left(\mathrm{~s}, 3 \mathrm{H}, \mathrm{CH}_{3}\right), 1.29\left(\mathrm{~s}, 3 \mathrm{H}, \mathrm{CH}_{3}\right)$, 1.13-1.07 (m, $\left.1 \mathrm{H}, \mathrm{CH}_{2}\right), 1.01-0.91\left(\mathrm{~m}, 3 \mathrm{H}, \mathrm{CH}_{2}\right), 0.79\left(\mathrm{~d}, J=6.5 \mathrm{~Hz}, 3 \mathrm{H}, \mathrm{CHCH}_{3}\right), 0.74(\mathrm{~d}, J=6.5 \mathrm{~Hz}, 3 \mathrm{H}$, $\left.\mathrm{CHCH}_{3}\right)$ 0.68-0.66 (m, 2H, CH 2$), 0.61-0.53\left(\mathrm{~m}, 2 \mathrm{H}, \mathrm{CH}_{2}\right) ;{ }^{13} \mathrm{C} \mathrm{NMR}\left(125 \mathrm{MHz}, \mathrm{CDCl}_{3}, 27{ }^{\circ} \mathrm{C}\right) \delta 170.8,170.5$, 169.9, 169.8, 146.3, 139.6, 139.5, 139.4, 139.0, 136.6, 136.2, 135.2, 135.1, 131.6, 131.4, 130.4, 130.4, 129.7, 129.7, 129.0, 129.0, 128.8, 128.8, 128.7, 128.5, 128.5, 128.3, 128.1, 128.0, 127.9, 127.8, 127.3, 127.2, 126.6, 126.6, 125.0, 125.0, 124.6, 124.6, 124.5, 123.8, 123.4, 76.4, 76.1, 70.5, 47.6, 47.3, 42.2, 41.6, 41.5, 40.7, 34.6, 31.3, 31.1, 30.0, 27.9, 27.1, 25.6, 21.8, 21.7; HRMS (ESI) m/z calcd. for $\mathrm{C}_{41} \mathrm{H}_{42} \mathrm{NO}_{2}{ }^{+}$ 580.3210 found 580.3197 .

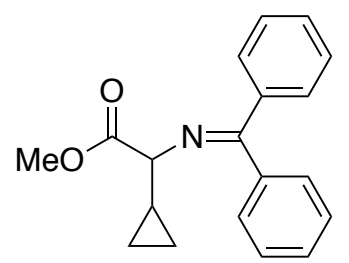

Methyl 2-cyclopropyl-2-((diphenylmethylene)amino)acetate (13)

Prepared according to General Method A and purified by flash column chromatography (Hexane/Et ${ }_{2} \mathrm{O}=$ $5 / 1\left(1 \% \mathrm{Et}_{3} \mathrm{~N}\right), 83 \%$ yield, colorless oil); IR (neat) 2360, $2341 \mathrm{~cm}^{-1} ;{ }^{1} \mathrm{H} \mathrm{NMR}\left(500 \mathrm{MHz}, \mathrm{CDCl}_{3}, 27^{\circ} \mathrm{C}\right) \delta 7.64$ (d, J = 7.0 Hz, 2H, ArH), $7.43(\mathrm{~d}, J=6.5 \mathrm{~Hz}, 3 \mathrm{H}, \operatorname{ArH}), 7.39(\mathrm{t}, J=7.5 \mathrm{~Hz}, 1 \mathrm{H}, \operatorname{ArH}), 7.33(\mathrm{t}, J=7.5 \mathrm{~Hz}, 2 \mathrm{H}$, $\operatorname{ArH}), 7.16(\mathrm{~d}, J=7.0 \mathrm{~Hz}, 2 \mathrm{H}, A r), 3.73\left(\mathrm{~s}, 3 \mathrm{H}, \mathrm{OCH}_{3}\right), 3.56(\mathrm{~d}, J=7.5 \mathrm{~Hz}, 1 \mathrm{H}, \mathrm{NCH}), 1.52-1.47(\mathrm{~m}, 1 \mathrm{H}, \mathrm{CH})$, 0.54-0.46 (m, 2H, CH $), 0.28-0.23\left(\mathrm{~m}, 1 \mathrm{H}, \mathrm{CH}_{2}\right), 0.20-0.16\left(\mathrm{~m}, 1 \mathrm{H}, \mathrm{CH}_{2}\right) ;{ }^{13} \mathrm{C} \mathrm{NMR}\left(125 \mathrm{MHz}, \mathrm{CDCl}_{3}, 27^{\circ} \mathrm{C}\right) \delta$ 172.7, 170.2, 139.6, 136.3, 130.3, 128.9, 128.6, 128.5, 128.0, 127.9, 68.8, 52.5, 14.8, 2.7, 2.4; HRMS (ESI) $\mathrm{m} / \mathrm{z}$ calcd. for $\mathrm{C}_{19} \mathrm{H}_{20} \mathrm{NO}_{2}{ }^{+} 294.1489$ found 294.1499.

\section{General Procedure and Characterization of Products}

\section{5-1. $\alpha$-Alkylation}

\section{General Procedure $C$ ( $\alpha-A$ lkylation of aryl substrates)}

In an inert atmosphere glove box, a $4 \mathrm{~mL}$ vial was charged with copper(II) acetate $(0.010 \mathrm{mmol}, 10$ mol\%) and $\mathbf{L} 5(0.010 \mathrm{mmol}, 10 \mathrm{~mol} \%)$, followed by tert-butylbenzene $(0.50 \mathrm{~mL})$. After the solution was stirred at ambient temperature for 10 minutes, $\alpha$-amino acid Schiff base 1 ( $0.10 \mathrm{mmol}, 1.0$ equiv.), alkyl bromide 2 ( $0.20 \mathrm{mmol}, 2.0$ equiv.) and $\mathrm{K}_{2} \mathrm{CO}_{3}(0.10 \mathrm{mmol}, 1.0$ equiv.) were added and removed from the glove box. The reaction solution was stirred at $100{ }^{\circ} \mathrm{C}$ for 24 hours. Copper salt was removed by silica gel short pad column chromatography and washed with excess ethyl acetate. Evaporation of the solvent gave the crude product, which was purified by flash column chromatography. 
Supporting information Amino Acid Schiff Base Bearing Benzophenone Imine as a Platform for Highly Congested Unnatural $\alpha$-Amino Acid Synthesis

\section{General Procedure D ( $\alpha$-Alkylation of aliphatic substrates)}

In an inert atmosphere glove box, a $4 \mathrm{~mL}$ light resident vial was charged with copper(II) acetate (0.010 mmol, $10 \mathrm{~mol} \%)$ and $\mathbf{L 5}(0.010 \mathrm{mmol}, 10 \mathrm{~mol} \%)$, followed by tert-butylbenzene $(0.50 \mathrm{~mL})$. After the solution was stirred at ambient temperature for 10 minutes, $\alpha$-amino acid Schiff base 1 (0.10 mmol, 1.0 equiv.), alkyl bromide 2 (0.20 mmol, 2.0 equiv.), $\mathrm{K}_{2} \mathrm{CO}_{3}(0.10 \mathrm{mmol}, 1.0$ equiv.) and AgOAc (0.12 mmol, 1.2 equiv.) were added and removed from the glovebox. The reaction solution was stirred at $100{ }^{\circ} \mathrm{C}$ for 24 hours. Copper salt was removed by silica gel short pad column chromatography and washed with excess ethyl acetate. Evaporation of the solvent gave the crude product, which was purified by flash column chromatography.

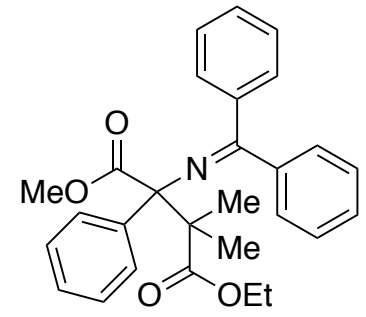

\section{4-Ethyl 1-methyl 2-((diphenylmethylene)amino)-3,3-dimethyl-2-phenylsuccinate (3aa)}

Prepared according to General Method C and purified by flash column chromatography (Hexane/Et ${ }_{2} \mathrm{O}=$ 5/1 (1\% Et $3 \mathrm{~N}), 81 \%$ yield, white solid); IR (neat) 2359, 2341, 1730, 1234,1134 $\mathrm{cm}^{-1} ;{ }^{1} \mathrm{H} \mathrm{NMR}(500 \mathrm{MHz}$, $\left.\mathrm{CDCl}_{3}, 27^{\circ} \mathrm{C}\right) \delta 7.73(\mathrm{~d}, J=7.5 \mathrm{~Hz}, 2 \mathrm{H}, \mathrm{ArH}), 7.46-7.42(\mathrm{~m}, 3 \mathrm{H}, \mathrm{ArH}), 7.38(\mathrm{t}, J=9.5 \mathrm{~Hz}, 2 \mathrm{H}, \operatorname{ArH}), 7.25-7.21$ $(\mathrm{m}, 4 \mathrm{H}, \operatorname{ArH}), 7.13(\mathrm{t}, J=7.5 \mathrm{~Hz}, 2 \mathrm{H}, \mathrm{ArH}), 6.77$ (d, J = $7.5 \mathrm{~Hz}, 2 \mathrm{H}, \operatorname{ArH}), 4.09\left(\mathrm{q}, J=7.0 \mathrm{~Hz}, 2 \mathrm{H}, \mathrm{OCH}_{2} \mathrm{CH}_{3}\right)$, $3.12\left(\mathrm{~s}, 3 \mathrm{H}, \mathrm{OCH}_{3}\right), 1.58\left(\mathrm{~s}, 3 \mathrm{H}, \mathrm{CH}_{3}\right), 1.21\left(\mathrm{t}, \mathrm{J}=7.5 \mathrm{~Hz}, 3 \mathrm{H}, \mathrm{OCH}_{2} \mathrm{CH}_{3}\right), 1.17\left(\mathrm{~s}, 3 \mathrm{H}, \mathrm{CH}_{3}\right) ;{ }^{13} \mathrm{C}$ NMR (125 $\left.\mathrm{MHz}, \mathrm{CDCl}_{3}, 2^{\circ} \mathrm{C}\right) \delta 176.0,170.9,167.8,141.6,141.1,136.4,130.2,129.4,129.1,128.8,128.4,128.0$, 127.1, 127.1, 127.0, 75.3, 60.6, 54.0, 50.9, 24.3, 22.5, 14.0; HRMS (ESI) m/z calcd. for $\mathrm{C}_{28} \mathrm{H}_{30} \mathrm{NO}_{4}{ }^{+}$ 444.2169 found 444.2159 .<smiles>CCOC(=O)C(C)(C)C(N=C(c1ccccc1)c1ccccc1)(C(=O)OCC)c1ccccc1</smiles>

1-(Tert-butyl) 4-ethyl 2-((diphenylmethylene)amino)-3,3-dimethyl-2-phenylsuccinate (3aa') 
Prepared according to General Procedure $\mathrm{C}$ and purified by flash column chromatography (Hexane/Et $\mathrm{t}_{2} \mathrm{O}$ $=15 / 1\left(0.5 \% \mathrm{Et}_{3} \mathrm{~N}\right), 78 \%$ yield, colorless oil); IR (neat) 1720, 1626, 1445, 1246, $1150 \mathrm{~cm}^{-1} ;{ }^{1} \mathrm{H}$ NMR (500 $\left.\mathrm{MHz}, \mathrm{CDCl}_{3}, 27^{\circ} \mathrm{C}\right) \delta 7.67(\mathrm{~d}, J=7.5 \mathrm{~Hz}, 2 \mathrm{H}, \operatorname{ArH}), 7.51(\mathrm{~d}, J=7.0 \mathrm{~Hz}, 2 \mathrm{H}, \operatorname{ArH}), 7.40(\mathrm{t}, J=7.5 \mathrm{~Hz}, 1 \mathrm{H}, \operatorname{ArH})$, $7.34(\mathrm{t}, J=7.5 \mathrm{~Hz}, 2 \mathrm{H}, \operatorname{ArH}), 7.20-7.12(\mathrm{~m}, 4 \mathrm{H}, \operatorname{ArH}), 7.03(\mathrm{t}, J=8.0 \mathrm{~Hz}, 2 \mathrm{H}, \operatorname{ArH}), 6.75(\mathrm{~d}, J=8.0 \mathrm{~Hz}, 2 \mathrm{H}$, $\operatorname{ArH})$, 4.04-3.97 (m, 2H, OCH $\left.\mathrm{CH}_{3}\right), 1.50\left(\mathrm{~s}, 3 \mathrm{H}, \mathrm{CH}_{3}\right), 1.38\left(\mathrm{~s}, 3 \mathrm{H}, \mathrm{CH}_{3}\right), 1.20\left(\mathrm{~s}, 9 \mathrm{H}, \mathrm{C}\left(\mathrm{CH}_{3}\right)_{3}\right), 1.10(\mathrm{t}, \mathrm{J}=7.0$ $\left.\mathrm{Hz}, 3 \mathrm{H}, \mathrm{OCH}_{2} \mathrm{CH}_{3}\right) ;{ }^{13} \mathrm{C}$ NMR $\left(125 \mathrm{MHz}, \mathrm{CDCl}_{3}, 2{ }^{\circ} \mathrm{C}\right) \delta 175.7,171.1,168.4,167.5,143.0,141.4,137.5$, $129.8,129.7,129.4,129.2,128.1,127.7,127.1,126.7,126.7,82.5,76.1,60.4,60.2,27.7,23.5,23.1,21.0$, 14.2, 13.9; HRMS (ESI) $\mathrm{m} / \mathrm{z}$ calcd. for $\mathrm{C}_{31} \mathrm{H}_{36} \mathrm{NO}_{4}{ }^{+} 486.2639$ found 486.2647 .<smiles>CCOC(=O)C(C)(N=C(c1ccccc1)c1ccccc1)C(C)(C)C(=O)OC</smiles>

\section{4-Ethyl 1-methyl 2-((diphenylmethylene)amino)-3,3-dimethyl-2-( $p$-tolyl)succinate (3ba)}

Prepared according to General Method $\mathrm{C}$ and purified by flash column chromatography (Hexane/Et ${ }_{2} \mathrm{O}=$ $5 / 1\left(1 \% \mathrm{Et}_{3} \mathrm{~N}\right), 72 \%$ yield, white solid); IR (neat) $1728,1625,1469,1444,1431,1265,1134 \mathrm{~cm}^{-1} ;{ }^{1} \mathrm{H} \mathrm{NMR}$ $\left(500 \mathrm{MHz}, \mathrm{CDCl}_{3}, 27^{\circ} \mathrm{C}\right) \delta 7.72(\mathrm{~d}, J=7.0 \mathrm{~Hz}, 2 \mathrm{H}, \mathrm{ArH}), 7.43(\mathrm{~d}, J=7.5 \mathrm{~Hz}, 1 \mathrm{H}, \operatorname{ArH}), 7.37(\mathrm{t}, J=9.0 \mathrm{~Hz}, 2 \mathrm{H}$, $\operatorname{ArH}), 7.32(\mathrm{~d}, J=10.0 \mathrm{~Hz}, 2 \mathrm{H}, \operatorname{ArH}), 7.25-7.21(\mathrm{~m}, 1 \mathrm{H}, \operatorname{ArH}), 7.14(\mathrm{t}, J=8.0 \mathrm{~Hz}, 2 \mathrm{H}, \operatorname{ArH}), 7.05(\mathrm{~d}, J=8.0 \mathrm{~Hz}$, $2 \mathrm{H}, \mathrm{ArH}), 6.79(\mathrm{~d}, J=7.5 \mathrm{~Hz}, 2 \mathrm{H}, \mathrm{ArH}), 4.09\left(\mathrm{q}, J=7.0 \mathrm{~Hz}, 2 \mathrm{H}, \mathrm{OCH}_{2} \mathrm{CH}_{3}\right), 3.10(\mathrm{~s}, 3 \mathrm{H}, \mathrm{OCH}), 2.33(\mathrm{~s}, 3 \mathrm{H}$, $\left.\operatorname{ArCH}_{3}\right), 1.57\left(\mathrm{~s}, 3 \mathrm{H}, \mathrm{CH}_{3}\right), 1.21\left(\mathrm{t}, J=7.5 \mathrm{~Hz}, 3 \mathrm{H}, \mathrm{OCH}_{2} \mathrm{CH}_{3}\right), 1.16\left(\mathrm{~s}, 3 \mathrm{H}, \mathrm{CH}_{3}\right) ;{ }^{13} \mathrm{C} \mathrm{NMR}\left(125 \mathrm{MHz}, \mathrm{CDCl}_{3}\right.$, $\left.27^{\circ} \mathrm{C}\right) \delta 176.1,170.0,167.3,141.7,138.0,136.6,130.1,129.2,129.2,129.1,128.8,128.4,127.9,127.7$, 127.1, 75.2, 60.6, 54.0, 50.8, 24.3, 22.5, 21.0, 14.0; HRMS (ESI) m/z calcd. for $\mathrm{C}_{29} \mathrm{H}_{32} \mathrm{NO}_{4}{ }^{+} 458.2326$ found 458.2279 .<smiles>CCOC(=O)C(C)(C)C(N=C(c1ccccc1)c1ccccc1)(C(=O)OC)c1ccc(OC)cc1</smiles>

4-Ethyl 1-methyl 2-((diphenylmethylene)amino)-2-(4-methoxyphenyl)-3,3-dimethylsuccinate (3ca) 
Prepared according to General Method $\mathrm{C}$ and purified by flash column chromatography (Hexane/Et ${ }_{2} \mathrm{O}=$ $5 / 1\left(1 \% \mathrm{Et}_{3} \mathrm{~N}\right), 85 \%$ yield, white solid); IR (neat) $2358,1728,1508,1252,1180,1136 \mathrm{~cm}^{-1} ;{ }^{1} \mathrm{H}$ NMR (500 $\left.\mathrm{MHz}, \mathrm{CDCl}_{3}, 27^{\circ} \mathrm{C}\right) \delta 7.72(\mathrm{~d}, J=7.5 \mathrm{~Hz}, 2 \mathrm{H}, \operatorname{ArH}), 7.45-7.35(\mathrm{~m}, 5 \mathrm{H}, \operatorname{ArH}), 7.23(\mathrm{~d}, J=7.5 \mathrm{~Hz}, 1 \mathrm{H}, \operatorname{ArH})$, $7.14(\mathrm{t}, J=7.5 \mathrm{~Hz}, 2 \mathrm{H}, \mathrm{ArH}), 6.78(\mathrm{t}, J=9.5 \mathrm{~Hz}, 4 \mathrm{H}, \mathrm{ArH}), 4.08\left(\mathrm{q}, J=7.0 \mathrm{~Hz}, 2 \mathrm{H}, \mathrm{OCH}_{2} \mathrm{CH}_{3}\right), 3.81(\mathrm{~s}, 3 \mathrm{H}$, $\left.\operatorname{ArOCH}_{3}\right), 3.11\left(\mathrm{~s}, 3 \mathrm{H}, \mathrm{OCH}_{3}\right), 1.55\left(\mathrm{~s}, 3 \mathrm{H}, \mathrm{CH}_{3}\right), 1.21\left(\mathrm{t}, J=7.5 \mathrm{~Hz}, 3 \mathrm{H}, \mathrm{OCH}_{2} \mathrm{CH}_{3}\right), 1.17\left(\mathrm{~s}, 3 \mathrm{H}, \mathrm{CH}_{3}\right) ;{ }^{13} \mathrm{CNMR}$ (125 MHz, CDCl, $\left.2{ }^{\circ} \mathrm{C}\right) \delta 176.1,171.1,167.4,158.3,141.7,136.4,133.3,130.5,130.1,129.1,128.8$, 128.4, 127.9, 127.1, 112.2, 74.9, 60.6, 55.1, 54.0, 50.8, 24.2, 22.5, 14.0; HRMS (ESI) m/z calcd. for $\mathrm{C}_{29} \mathrm{H}_{32} \mathrm{NO}_{5}{ }^{+} 474.2275$ found 474.2259 .<smiles>CCOC(=O)C(C)(N=C(c1ccccc1)c1ccccc1)C(C)(C)C(=O)OC</smiles>

\section{4-Ethyl 1-methyl 2-(4-chlorophenyl)-2-((diphenylmethylene)amino)-3,3-dimethylsuccinate (3da)}

Prepared according to General Method $\mathrm{C}$ and purified by flash column chromatography (Hexane/Et ${ }_{2} \mathrm{O}=$ 5/1 (1\% Et $\left.{ }_{3} \mathrm{~N}\right), 93 \%$ yield, white solid); IR (neat) $1730,1626,1491,1265,1236 \mathrm{~cm}^{-1} ;{ }^{1} \mathrm{H} \mathrm{NMR}(500 \mathrm{MHz}$, $\left.\mathrm{CDCl}_{3}, 27^{\circ} \mathrm{C}\right) \delta 7.71(\mathrm{~d}, J=7.5 \mathrm{~Hz}, 2 \mathrm{H}, \operatorname{ArH}), 7.47-7.42(\mathrm{~m}, 3 \mathrm{H}, \mathrm{ArH}), 7.38(\mathrm{t}, J=8.0 \mathrm{~Hz}, 2 \mathrm{H}, \operatorname{ArH}), 7.24-7.22$ $(\mathrm{m}, 3 \mathrm{H}, \operatorname{ArH}), 7.16(\mathrm{t}, J=7.5 \mathrm{~Hz}, 2 \mathrm{H}, \mathrm{ArH}), 6.75(\mathrm{~d}, J=7.5 \mathrm{~Hz}, 2 \mathrm{H}, \mathrm{ArH}), 4.08\left(\mathrm{q}, J=7.0 \mathrm{~Hz}, 2 \mathrm{H}, \mathrm{OCH}_{2} \mathrm{CH}_{3}\right)$, $3.12(\mathrm{~s}, 3 \mathrm{H}, \mathrm{OCH}), 1.56\left(\mathrm{~s}, 3 \mathrm{H}, \mathrm{CH}_{3}\right), 1.21\left(\mathrm{t}, J=7.0 \mathrm{~Hz}, 3 \mathrm{H}, \mathrm{OCH}_{2} \mathrm{CH}_{3}\right), 1.17\left(\mathrm{~s}, 3 \mathrm{H}, \mathrm{CH}_{3}\right) ;{ }^{13} \mathrm{CNMR}(125$ $\left.\mathrm{MHz}, \mathrm{CDCl}_{3}, 27^{\circ} \mathrm{C}\right) \delta 175.8,170.7,168.1,141.4,139.7,136.2,133.1,130.8,130.4,129.1,128.7,128.6$, 128.0, 127.2, 127.1, 74.8, 60.7, 54.0, 51.0, 24.2, 22.5, 14.0; HRMS (ESI) m/z calcd. for $\mathrm{C}_{28} \mathrm{H}_{29} \mathrm{CINO}_{4}{ }^{+}$ 478.1780 found 478.1756 .

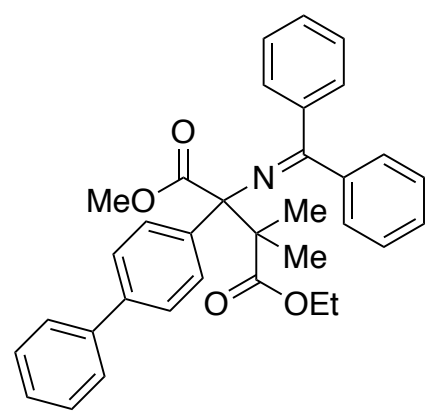

4-Ethyl 1-methyl 2-([1,1'-biphenyl]-4-yl)-2-((diphenylmethylene)amino)-3,3-dimethylsuccinate (3ea) 

for Highly Congested Unnatural $\alpha$-Amino Acid Synthesis

Prepared according to General Method $\mathrm{C}$ and purified by flash column chromatography (Hexane/Et $\mathrm{t}_{2} \mathrm{O}=$ 5/1 (1\% Et $\left.t_{3} \mathrm{~N}\right), 75 \%$ yield, white solid); IR (neat) $1728,1626,1265,1136 \mathrm{~cm}^{-1} ;{ }^{1} \mathrm{H}$ NMR (500 MHz, $\mathrm{CDCl}_{3}$, $\left.27^{\circ} \mathrm{C}\right) \delta 7.75(\mathrm{~d}, J=7.5 \mathrm{~Hz}, 2 \mathrm{H}, \mathrm{ArH}), 7.63(\mathrm{~d}, J=8.5 \mathrm{~Hz}, 2 \mathrm{H}, \operatorname{ArH}), 7.53-7.49(\mathrm{~m}, 3 \mathrm{H}, \operatorname{ArH}), 7.45-7.37(\mathrm{~m}$, $5 \mathrm{H}, \operatorname{ArH}), 7.33(\mathrm{t}, J=7.5 \mathrm{~Hz}, 1 \mathrm{H}, \operatorname{ArH}), 7.24-7.22(\mathrm{~m}, 2 \mathrm{H}, \operatorname{ArH}), 7.15(\mathrm{t}, J=7.5 \mathrm{~Hz}, 2 \mathrm{H}, \operatorname{ArH}), 6.81(\mathrm{~d}, J=7.5$ $\mathrm{Hz}, 2 \mathrm{H}, \mathrm{ArH}), 4.10\left(\mathrm{q}, \mathrm{J}=7.0 \mathrm{~Hz}, 2 \mathrm{H}, \mathrm{OCH}_{2} \mathrm{CH}_{3}\right), 3.14\left(\mathrm{~s}, 3 \mathrm{H}, \mathrm{OCH}_{3}\right), 1.61\left(\mathrm{~s}, 3 \mathrm{H}, \mathrm{CH}_{3}\right), 1.22\left(\mathrm{~s}, 3 \mathrm{H}, \mathrm{CH}_{3}\right), 1.22$ (t, $\left.J=7.5 \mathrm{~Hz}, 3 \mathrm{H}, \mathrm{OCH}_{2} \mathrm{CH}_{3}\right) ;{ }^{13} \mathrm{C}$ NMR $\left(125 \mathrm{MHz}, \mathrm{CDCl}_{3}, 27^{\circ} \mathrm{C}\right) \delta 176.2,171.0,167.7,141.6,140.6,140.2$, 139.4, 136.4, 130.2, 129.8, 129.1, 128.8, 128.8, 128.5, 128.0, 127.3, 127.2, 127.0, 125.5, 75.2, 60.7, 54.1, 50.9, 24.3, 22.6, 14.0; HRMS (ESI) m/z calcd. for $\mathrm{C}_{34} \mathrm{H}_{34} \mathrm{NO}_{4}{ }^{+} 520.2482$ found 520.2456 .<smiles>CCOC(=O)C(C)(N=C(c1ccccc1)c1ccccc1)C(C(C)=O)(C(C)=O)c1cccc(C)c1</smiles>

\section{4-Ethyl 1-methyl 2-((diphenylmethylene)amino)-3,3-dimethyl-2-(m-tolyl)succinate (3fa)}

Prepared according to General Method $\mathrm{C}$ and purified by flash column chromatography (Hexane/Et $\mathrm{E}_{2} \mathrm{O}=$ 5/1 (1\% Et $\left.{ }_{3} \mathrm{~N}\right), 94 \%$ yield, white solid); IR (neat) $2361,1626,1626,1223,1134 \mathrm{~cm}^{-1} ;{ }^{1} \mathrm{H} \mathrm{NMR}(500 \mathrm{MHz}$, $\left.\mathrm{CDCl}_{3}, 27^{\circ} \mathrm{C}\right) \delta 7.72(\mathrm{~d}, J=7.0 \mathrm{~Hz}, 2 \mathrm{H}, \mathrm{ArH}), 7.44(\mathrm{t}, J=7.5 \mathrm{~Hz}, 1 \mathrm{H}, \operatorname{ArH}), 7.23(\mathrm{t}, J=7.5 \mathrm{~Hz}, 2 \mathrm{H}, \operatorname{ArH}), 7.25-$ $7.22(\mathrm{~m}, 2 \mathrm{H}, \operatorname{ArH}), 7.13(\mathrm{q}, J=8.0 \mathrm{~Hz}, 3 \mathrm{H}, \operatorname{ArH}), 7.06(\mathrm{~d}, J=7.5 \mathrm{~Hz}, 2 \mathrm{H}, \operatorname{ArH}), 6.80(\mathrm{~d}, J=8.5 \mathrm{~Hz}, 2 \mathrm{H}, \operatorname{ArH})$, 4.09 (q, J = 7.0 Hz, 2H, OCH $\left.\mathrm{CH}_{3}\right), 3.12\left(\mathrm{~s}, 3 \mathrm{H}, \mathrm{OCH}_{3}\right), 2.03\left(\mathrm{~s}, 3 \mathrm{H}, \mathrm{ArCH}_{3}\right), 1.57\left(\mathrm{~s}, 3 \mathrm{H}, \mathrm{CH}_{3}\right), 1.21(\mathrm{t}, J=7.5$ $\left.\mathrm{Hz}, 3 \mathrm{H}, \mathrm{OCH}_{2} \mathrm{CH}_{3}\right), 1.16\left(\mathrm{~s}, 3 \mathrm{H}, \mathrm{CH}_{3}\right) ;{ }^{13} \mathrm{C}$ NMR $\left(125 \mathrm{MHz}, \mathrm{CDCl}_{3}, 27^{\circ} \mathrm{C}\right) \delta 176.1,170.9,167.5,141.7,140.9$, $136.5,136.2$, 130.1, 129.9, 129.1, 128.8, 128.4, 127.9, 127.7, 127.1, 126.8, 126.4, 75.3, 60.6, 54.1, 50.8, 24.4, 22.5, 21.9, 14.0; HRMS (ESI) m/z calcd. for $\mathrm{C}_{29} \mathrm{H}_{32} \mathrm{NO}_{4}{ }^{+} 458.2326$ found 458.2311 .<smiles>CCOC(=O)C(C)(N=C(c1ccccc1)c1ccccc1)C(C(C)=O)(C(C)=O)c1cccc(OC)c1</smiles>

4-Ethyl 1-methyl 2-((diphenylmethylene)amino)-2-(3-methoxyphenyl)-3,3-dimethylsuccinate (3ga) 
Prepared according to General Method $\mathrm{C}$ and purified by flash column chromatography (Hexane/Et ${ }_{2} \mathrm{O}=$ $5 / 1\left(1 \% \mathrm{Et}_{3} \mathrm{~N}\right), 83 \%$ yield, white solid); IR (neat) $1730,1251,1229 \mathrm{~cm}^{-1} ;{ }^{1} \mathrm{H} \mathrm{NMR}\left(500 \mathrm{MHz}, \mathrm{CDCl}_{3}, 27^{\circ} \mathrm{C}\right) \delta$ $7.71(\mathrm{~d}, J=7.5 \mathrm{~Hz}, 2 \mathrm{H}, \operatorname{ArH}), 7.43(\mathrm{t}, J=6.5 \mathrm{~Hz}, 1 \mathrm{H}, \operatorname{ArH}), 7.36(\mathrm{t}, J=7.5 \mathrm{~Hz}, 2 \mathrm{H}, \operatorname{ArH}), 7.23(\mathrm{~d}, J=7.5 \mathrm{~Hz}$, $1 \mathrm{H}, \operatorname{ArH}), 7.18-7.14(\mathrm{~m}, 3 \mathrm{H}, \mathrm{ArH}), 7.09-7.03(\mathrm{~m}, 2 \mathrm{H}, \mathrm{ArH}), 6.83-6.80(\mathrm{~m}, 3 \mathrm{H}, \mathrm{ArH}), 4.09(\mathrm{q}, J=7.0 \mathrm{~Hz}, 2 \mathrm{H}$, $\left.\mathrm{OCH}_{2} \mathrm{CH}_{3}\right), 3.74\left(\mathrm{~s}, 3 \mathrm{H}, \mathrm{ArOCH}_{3}\right), 3.11\left(\mathrm{~s}, 3 \mathrm{H}, \mathrm{OCH}_{3}\right), 1.58\left(\mathrm{~s}, 3 \mathrm{H}, \mathrm{CH}_{3}\right), 1.21\left(\mathrm{t}, J=7.0 \mathrm{~Hz}, 3 \mathrm{H}, \mathrm{OCH}_{2} \mathrm{CH}_{3}\right)$, $1.18\left(\mathrm{~s}, 3 \mathrm{H}, \mathrm{CH}_{3}\right) ;{ }^{13} \mathrm{C}$ NMR $\left(125 \mathrm{MHz}, \mathrm{CDCl}_{3}, 2{ }^{\circ} \mathrm{C}\right) \delta 176.1,170.8,167.7,158.3,142.6,141.6,136.4$, 130.2, 129.1, 128.8, 128.4, 127.9, 127.7, 127.1, 122.0, 115.8, 112.2, 75.2, 60.6, 55.1, 54.1, 50.9, 24.4, 22.5, 14.0; HRMS (ESI) $\mathrm{m} / \mathrm{z}$ calcd. for $\mathrm{C}_{29} \mathrm{H}_{32} \mathrm{NO}_{5}{ }^{+} 474.2275$ found 474.2268 .

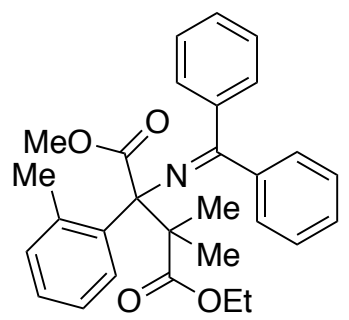

\section{4-Ethyl 1-methyl 2-((diphenylmethylene)amino)-3,3-dimethyl-2-(o-tolyl)succinate (3ha)}

Prepared according to General Method $\mathrm{C}$ and purified by flash column chromatography (Hexane/Et $\mathrm{E}_{2} \mathrm{O}=$ 5/1 (1\% Et ${ }_{3} \mathrm{~N}$ ), 50\% yield, white solid); IR (neat) $2361,1726,1445,1223,1132 \mathrm{~cm}^{-1} ;{ }^{1} \mathrm{H} \mathrm{NMR}(500 \mathrm{MHz}$, $\left.\mathrm{CDCl}_{3}, 27^{\circ} \mathrm{C}\right) \delta 7.67(\mathrm{~d}, J=8.0 \mathrm{~Hz}, 2 \mathrm{H}, \mathrm{ArH}), 7.42(\mathrm{t}, J=7.5 \mathrm{~Hz}, 1 \mathrm{H}, \operatorname{ArH}), 7.36(\mathrm{t}, J=7.5 \mathrm{~Hz}, 2 \mathrm{H}, \operatorname{ArH}), 7.24$ $(\mathrm{m}, 2 \mathrm{H}, \mathrm{ArH}), 7.17-7.06(\mathrm{~m}, 5 \mathrm{H}, \mathrm{ArH}), 6.82(\mathrm{~s}, 2 \mathrm{H}, \mathrm{ArH}), 4.17-4.02\left(\mathrm{~m}, 2 \mathrm{H}, \mathrm{OCH}_{2} \mathrm{CH}_{3}\right), 3.09\left(\mathrm{~s}, 3 \mathrm{H}, \mathrm{OCH}_{3}\right)$, $2.28\left(\mathrm{~s}, 3 \mathrm{H}, \mathrm{ArCH}_{3}\right), 1.60\left(\mathrm{~s}, 3 \mathrm{H}, \mathrm{CH}_{3}\right), 1.30\left(\mathrm{~s}, 3 \mathrm{H}, \mathrm{CH}_{3}\right), 1.19\left(\mathrm{t}, \mathrm{J}=7.5 \mathrm{~Hz}, 3 \mathrm{H}, \mathrm{OCH}_{2} \mathrm{CH}_{3}\right) ;{ }^{13} \mathrm{C} \mathrm{NMR}^{(125}$ $\left.\mathrm{MHz}, \mathrm{CDCl}_{3}, 27^{\circ} \mathrm{C}\right) \delta 176.2,171.3,141.9,139.6,137.6,136.1,132.7,130.8,130.1,129.2,128.9,128.7$, $127.9,127.2,127.1,124.4,65.9,60.6,55.6,50.9,29.7,24.5,22.3,13.9$; HRMS (ESI) m/z calcd. for $\mathrm{C}_{29} \mathrm{H}_{32} \mathrm{NO}_{4}{ }^{+} 458.2326$ found 458.2336 .<smiles>CCOC(=O)C(C)(C)C(N=C(c1ccccc1)c1ccccc1)(C(=O)OC)c1ccc2ccccc2c1</smiles>

4-Ethyl 1-methyl 2-((diphenylmethylene)amino)-3,3-dimethyl-2-(naphthalen-2-yl)succinate (3ia) 
Prepared according to General Method $\mathrm{C}$ and purified by flash column chromatography (Hexane/Et ${ }_{2} \mathrm{O}=$ $5 / 1\left(1 \% \mathrm{Et}_{3} \mathrm{~N}\right), 86 \%$ yield, white solid); IR (neat) $1726,1626,1445,1231,1121 \mathrm{~cm}^{-1} ;{ }^{1} \mathrm{H} \mathrm{NMR}(500 \mathrm{MHz}$, $\left.\mathrm{CDCl}_{3}, 27^{\circ} \mathrm{C}\right) \delta 7.93(\mathrm{~s}, 1 \mathrm{H}, \mathrm{ArH}), 7.81(\mathrm{~d}, J=8.0 \mathrm{~Hz}, 1 \mathrm{H}, \mathrm{ArH}), 7.77(\mathrm{~d}, J=7.5 \mathrm{~Hz}, 3 \mathrm{H}, \operatorname{ArH}), 7.72(\mathrm{~d}, J=8.5$ $\mathrm{Hz}, 1 \mathrm{H}, \operatorname{ArH}), 7.60(\mathrm{~d}, J=8.0 \mathrm{~Hz}, 1 \mathrm{H}, \operatorname{ArH}), 7.49-7.39(\mathrm{~m}, 5 \mathrm{H}, \operatorname{ArH}), 7.19(\mathrm{t}, J=7.5 \mathrm{~Hz}, 1 \mathrm{H}, \operatorname{ArH}), 7.07(\mathrm{t}, J=$ $7.5 \mathrm{~Hz}, 2 \mathrm{H}, \mathrm{ArH}), 6.78(\mathrm{~d}, J=7.5 \mathrm{~Hz}, 2 \mathrm{H}, \mathrm{ArH}), 4.11\left(\mathrm{q}, J=7.0 \mathrm{~Hz}, 2 \mathrm{H}, \mathrm{OCH}_{2} \mathrm{CH}_{3}\right), 3.14\left(\mathrm{~s}, 3 \mathrm{H}, \mathrm{OCH}_{3}\right), 1.63(\mathrm{~s}$, $\left.3 \mathrm{H}, \mathrm{CH}_{3}\right), 1.20\left(\mathrm{t}, J=7.5 \mathrm{~Hz}, 3 \mathrm{H}, \mathrm{OCH}_{2} \mathrm{CH}_{3}\right), 1.18\left(\mathrm{~s}, 3 \mathrm{H}, \mathrm{CH}_{3}\right) ;{ }^{13} \mathrm{C} \mathrm{NMR}\left(125 \mathrm{MHz}, \mathrm{CDCl}_{3}, 2{ }^{\circ} \mathrm{C}\right) \delta 176.2$, $171.0,167.9,141.7,138.7,136.4,132.4,132.3,130.3,129.2,128.8,128.8,128.5,128.5,128.0,127.5$, 127.2, 127.1, 126.2, 126.1, 125.6, 75.4, 60.7, 54.3, 51.0, 24.5, 22.6, 14.0; HRMS (ESI) m/z calcd. for $\mathrm{C}_{32} \mathrm{H}_{32} \mathrm{NO}_{4}{ }^{+} 494.2326$ found 494.2323 .<smiles>CCOC(=O)C(C)(C)C(N=C(c1ccccc1)c1ccccc1)(C(C)=O)c1cccs1</smiles>

\section{4-Ethyl 1-methyl 2-((diphenylmethylene)amino)-3,3-dimethyl-2-(thiophen-2-yl)succinate (3ja)}

Prepared according to General Method $\mathrm{C}$ and purified by flash column chromatography (Hexane/Et $\mathrm{E}_{2} \mathrm{O}=$ $5 / 1\left(1 \% \mathrm{Et}_{3} \mathrm{~N}\right), 35 \%$ yield, white solid); IR (neat) $1728,1622,1445,1248,1229,1136 \mathrm{~cm}^{-1} ;{ }^{1} \mathrm{H}$ NMR (500 $\left.\mathrm{MHz}, \mathrm{CDCl}_{3}, 27^{\circ} \mathrm{C}\right) \delta 7.72(\mathrm{~d}, J=8.0 \mathrm{~Hz}, 2 \mathrm{H}, \mathrm{ArH}), 7.42(\mathrm{t}, J=7.0 \mathrm{~Hz}, 1 \mathrm{H}, \operatorname{ArH}), 7.36(\mathrm{t}, J=7.0 \mathrm{~Hz}, 2 \mathrm{H}, \operatorname{ArH})$, 7.25-7.23 (m, 2H, ArH), $7.17(\mathrm{t}, J=8.0 \mathrm{~Hz}, 2 \mathrm{H}, \operatorname{ArH}), 7.09(\mathrm{~d}, J=3.5 \mathrm{~Hz}, 1 \mathrm{H}, \operatorname{ArH}), 6.94(\mathrm{t}, J=4.5 \mathrm{~Hz}, 1 \mathrm{H}$, $\operatorname{ArH}), 6.72(\mathrm{~d}, J=8.0 \mathrm{~Hz}, 2 \mathrm{H}, \mathrm{ArH}), 4.11-4.04\left(\mathrm{~m}, 2 \mathrm{H}, \mathrm{OCH}_{2} \mathrm{CH}_{3}\right), 3.17(\mathrm{~s}, 3 \mathrm{H}, \mathrm{OCH}), 1.54\left(\mathrm{~s}, 3 \mathrm{H}, \mathrm{CH}_{3}\right), 1.27$ $\left(\mathrm{s}, 3 \mathrm{H}, \mathrm{CH}_{3}\right), 1.21\left(\mathrm{t}, \mathrm{J}=9.0 \mathrm{~Hz}, 3 \mathrm{H}, \mathrm{OCH}_{2} \mathrm{CH}_{3}\right) ;{ }^{13} \mathrm{C} \mathrm{NMR}\left(125 \mathrm{MHz}, \mathrm{CDCl}_{3}, 27^{\circ} \mathrm{C}\right) \delta 175.5,170.5,168.3$, 143.2, 141.4, 136.5, 130.3, 129.2, 128.6, 128.4, 128.2, 127.9, 127.2, 126.3, 125.5, 74.5, 60.7, 53.6, 51.2, 23.2, 22.3, 14.1; HRMS (ESI) $\mathrm{m} / \mathrm{z}$ calcd. for $\mathrm{C}_{26} \mathrm{H}_{28} \mathrm{NO}_{4} \mathrm{~S}^{+} 450.1734$ found 450.1705 .<smiles>CCOC(=O)C(C)(C)C1(c2ccccc2)N=C(c2ccc(OC)cc2)OC1=O</smiles>

Ethyl 2-(2-(4-methoxyphenyl)-5-oxo-4-phenyl-4,5-dihydrooxazol-4-yl)-2-methylpropanoate (3ka) 
Supporting information Amino Acid Schiff Base Bearing Benzophenone Imine as a Platform for Highly Congested Unnatural $\alpha$-Amino Acid Synthesis

Prepared according to General Method $\mathrm{C}$ and purified by flash column chromatography (Hexane/Et ${ }_{2} \mathrm{O}=$ $10 / 1,81 \%$ yield, regioisomers $=4.2 / 1$, white solid); IR (neat) $1805,1722,1651,1512,1257,1142 \mathrm{~cm}^{-1} ;{ }^{1} \mathrm{H}$ NMR $\left(500 \mathrm{MHz}, \mathrm{CDCl}_{3}, 27^{\circ} \mathrm{C}\right) \delta 7.99(\mathrm{~d}, J=9.0 \mathrm{~Hz}, 2 \mathrm{H}, \mathrm{ArH}), 7.82(\mathrm{~d}, J=8.0 \mathrm{~Hz}, 2 \mathrm{H}, \mathrm{ArH}), 7.37-7.30(\mathrm{~m}, 3 \mathrm{H}$, $\operatorname{ArH}), 6.90(\mathrm{~d}, J=9.0 \mathrm{~Hz}, 2 \mathrm{H}, \mathrm{ArH}), 4.06-4.00\left(\mathrm{~m}, 2 \mathrm{H}, \mathrm{OCH}_{2} \mathrm{CH}_{3}\right), 3.86\left(\mathrm{~s}, 3 \mathrm{H}, \mathrm{OCH}_{3}\right), 1.48\left(\mathrm{~s}, 3 \mathrm{H}, \mathrm{CH}_{3}\right), 1.25$ $\left(\mathrm{s}, 3 \mathrm{H}, \mathrm{CH}_{3}\right), 1.12\left(\mathrm{t}, J=7.0 \mathrm{~Hz}, 3 \mathrm{H}, \mathrm{OCH}_{2} \mathrm{CH}_{3}\right) ;{ }^{13} \mathrm{C} \mathrm{NMR}\left(125 \mathrm{MHz}, \mathrm{CDCl}_{3}, 27^{\circ} \mathrm{C}\right) \delta 177.7,174.9,163.1$, $135.3,131.0,129.9,128.3,127.8,118.6,114.1,113.6,75.8,61.3,55.5,51.2,24.0,21.0,13.8$; HRMS (ESI) $\mathrm{m} / \mathrm{z}$ calcd. for $\mathrm{C}_{22} \mathrm{H}_{24} \mathrm{NO}_{5}{ }^{+} 382.1649$ found 382.1650 .

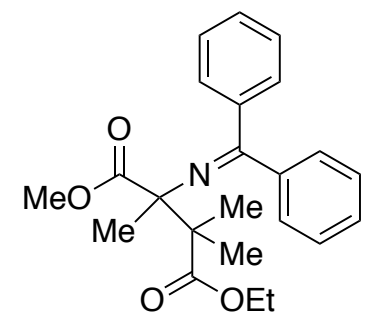

\section{4-Ethyl 1-methyl 2-((diphenylmethylene)amino)-2,3,3-trimethylsuccinate (3la)}

Prepared according to General Method D and purified by flash column chromatography (Hexane/Acetone = 9/1 (1\% Et $\left.\mathrm{E}_{3} \mathrm{~N}\right), 44 \%$ yield, colorless oil); IR (neat) 2361, 1722, 1445, 1248, $1103 \mathrm{~cm}^{-1}$; ${ }^{1} \mathrm{H} \mathrm{NMR}\left(500 \mathrm{MHz}, \mathrm{CDCl}_{3}, 27^{\circ} \mathrm{C}\right) \delta 7.59(\mathrm{~d}, J=7.5 \mathrm{~Hz}, 2 \mathrm{H}, \mathrm{ArH}), 7.38-7.28(\mathrm{~m}, 4 \mathrm{H}, \mathrm{ArH}), 7.30(\mathrm{t}, J=7.5 \mathrm{~Hz}$, $2 \mathrm{H}, \mathrm{ArH}), 7.13-7.11(\mathrm{~m}, 2 \mathrm{H}, \mathrm{ArH}), 4.13-4.06\left(\mathrm{~m}, 2 \mathrm{H}, \mathrm{OCH}_{2} \mathrm{CH}_{3}\right), 3.31(\mathrm{~s}, 3 \mathrm{H}, \mathrm{OCH}), 1.47\left(\mathrm{~s}, 3 \mathrm{H}, \mathrm{CH}_{3}\right), 1.45$ $\left(\mathrm{s}, 3 \mathrm{H}, \mathrm{CH}_{3}\right), 1.33\left(\mathrm{~s}, 3 \mathrm{H}, \mathrm{CH}_{3}\right), 1.22\left(\mathrm{t}, J=7.0 \mathrm{~Hz}, 3 \mathrm{H}, \mathrm{OCH}_{2} \mathrm{CH}_{3}\right) ;{ }^{13} \mathrm{C} \mathrm{NMR}\left(125 \mathrm{MHz}, \mathrm{CDCl}_{3}, 27^{\circ} \mathrm{C}\right) \delta 176.0$, 173.1, 166.3, 141.4, 137.3, 130.0, 128.6, 128.5, 128.3, 127.9, 127.7, 69.8, 60.5, 52.0, 51.2, 22.3, 21.6, 21.5, 14.1; HRMS (ESI) m/z calcd. for $\mathrm{C}_{23} \mathrm{H}_{28} \mathrm{NO}_{4}{ }^{+} 382.2013$ found 382.2039.

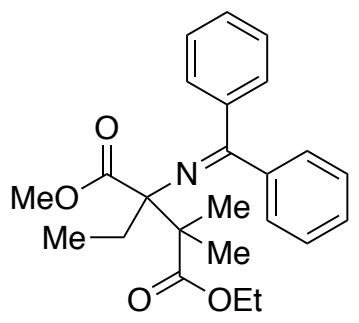

\section{4-Ethyl 1-methyl 2-((diphenylmethylene)amino)-2-ethyl-3,3-dimethylsuccinate (3ma)}

Prepared according to General Method D and purified by flash column chromatography (Hexane/Acetone = 9/1 $\left(1 \% \mathrm{Et}_{3} \mathrm{~N}\right), 40 \%$ yield, colorless oil); IR (neat) 1728, 1267, 1233, $1142 \mathrm{~cm}^{-1} ;{ }^{1} \mathrm{H}$ NMR $\left(500 \mathrm{MHz}, \mathrm{CDCl}_{3}, 27^{\circ} \mathrm{C}\right) \delta 7.58(\mathrm{~d}, J=7.5 \mathrm{~Hz}, 2 \mathrm{H}, \operatorname{ArH}), 7.38-7.34(\mathrm{~m}, 4 \mathrm{H}, \operatorname{ArH}), 7.30(\mathrm{t}, J=7.0 \mathrm{~Hz}, 2 \mathrm{H}$, $\operatorname{ArH}), 7.12(\mathrm{~d}, J=7.5 \mathrm{~Hz}, 2 \mathrm{H}, \mathrm{ArH}), 4.12-4.03\left(\mathrm{~m}, 2 \mathrm{H}, \mathrm{OCH}_{2} \mathrm{CH}_{3}\right), 3.14\left(\mathrm{~s}, 3 \mathrm{H}, \mathrm{OCH}_{3}\right), 2.17-2.09(\mathrm{~m}, 1 \mathrm{H}$, 
$\left.\mathrm{CCH}_{2} \mathrm{CH}_{3}\right), 1.91-1.84\left(\mathrm{~m}, 1 \mathrm{H}, \mathrm{CCH}_{2} \mathrm{CH}_{3}\right), 1.52\left(\mathrm{~s}, 3 \mathrm{H}, \mathrm{CH}_{3}\right), 1.51\left(\mathrm{~s}, 3 \mathrm{H}, \mathrm{CH}_{3}\right), 1.21(\mathrm{t}, J=7.5 \mathrm{~Hz}, 3 \mathrm{H}$, $\left.\mathrm{OCH}_{2} \mathrm{CH}_{3}\right), 0.93\left(\mathrm{t}, J=7.5 \mathrm{~Hz}, 3 \mathrm{H}, \mathrm{CCH}_{2} \mathrm{CH}_{3}\right) ;{ }^{13} \mathrm{C} \mathrm{NMR}\left(125 \mathrm{MHz}, \mathrm{CDCl}_{3}, 27^{\circ} \mathrm{C}\right) \delta 176.7,173.0,164.9,141.6$, $136.7,129.8,128.6,128.5,128.2,127.9,127.5,72.3,60.5,51.2,50.8,28.5,24.2,21.7,14.0,10.3$; HRMS (ESI) $\mathrm{m} / \mathrm{z}$ calcd. for $\mathrm{C}_{24} \mathrm{H}_{30} \mathrm{NO}_{4}{ }^{+} 396.2169$ found 396.2195 .

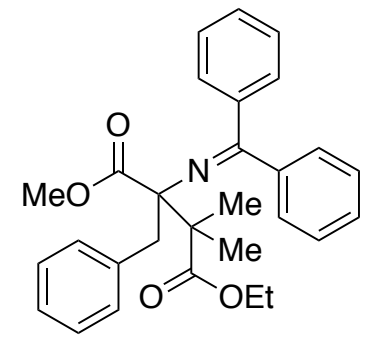

\section{4-Ethyl 1-methyl 2-benzyl-2-((diphenylmethylene)amino)-3,3-dimethylsuccinate (3na)}

Prepared according to General Method D and purified by flash column chromatography (Hexane/Acetone = 9/1 (1\% Et $3 \mathrm{~N}), 42 \%$ yield, colorless oil); IR (neat) 1728, 1260, 1219, $1142 \mathrm{~cm}^{-1} ;{ }^{1} \mathrm{H}$ NMR (500 MHz, CDCl, $\left.27^{\circ} \mathrm{C}\right) \delta 7.49$ (d, J = 7.5 Hz, 2H, ArH), $7.37(\mathrm{t}, J=7.5 \mathrm{~Hz}, 1 \mathrm{H}, \mathrm{ArH}), 7.33-7.28(\mathrm{~m}, 5 \mathrm{H}$, $\operatorname{ArH}), 7.22-7.12(\mathrm{~m}, 5 \mathrm{H}, \mathrm{ArH}), 7.05(\mathrm{t}, J=7.5 \mathrm{~Hz}, 2 \mathrm{H}, \mathrm{ArH}), 4.13-4.07\left(\mathrm{~m}, 1 \mathrm{H}, \mathrm{OCH}_{2} \mathrm{CH}_{3}\right), 4.05-3.99(\mathrm{~m}, 1 \mathrm{H}$, $\left.\mathrm{OCH}_{2} \mathrm{CH}_{3}\right), 3.49\left(\mathrm{~d}, J=13.5 \mathrm{~Hz}, 1 \mathrm{H}, \mathrm{ArCH}_{2}\right), 3.28\left(\mathrm{~d}, J=13.0 \mathrm{~Hz}, 1 \mathrm{H}, \mathrm{ArCH}_{2}\right), 2.77\left(\mathrm{~s}, 3 \mathrm{H}, \mathrm{OCH}_{3}\right), 1.62(\mathrm{~s}, 3 \mathrm{H}$, $\left.\mathrm{CH}_{3}\right), 1.45\left(\mathrm{~s}, 3 \mathrm{H}, \mathrm{CH}_{3}\right), 1.20\left(\mathrm{t}, J=7.5 \mathrm{~Hz}, 3 \mathrm{H}, \mathrm{OCH}_{2} \mathrm{CH}_{3}\right) ;{ }^{13} \mathrm{C} \mathrm{NMR}\left(125 \mathrm{MHz}, \mathrm{CDCl}_{3}, 27^{\circ} \mathrm{C}\right) \delta 176.2,171.9$, 164.2, 142.7, 137.6, 136.3, 133.1, 129.5, 128.8, 128.0, 127.7, 127.7, 127.4, 127.1, 126.3, 75.2, 60.6, 51.7, 50.4, 39.2, 23.1, 22.1, 14.2; HRMS (ESI) m/z calcd. for $\mathrm{C}_{29} \mathrm{H}_{32} \mathrm{NO}_{4}{ }^{+} 458.2326$ found 458.2344 .

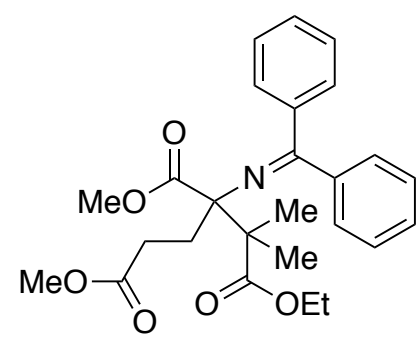

\section{4-Ethyl 1,3-dimethyl 3-((diphenylmethylene)amino)-4-methylpentane-1,3,4-tricarboxylate (3oa)}

Prepared according to General Method D and purified by flash column chromatography (Hexane/Acetone = 9/1 (1\% Et $\left.\mathrm{Et}_{3} \mathrm{~N}\right), 33 \%$ yield, colorless oil); IR (neat) 2359, 1732, 1435, 1198, $1165 \mathrm{~cm}^{-1}$; ${ }^{1} \mathrm{H}$ NMR $\left(500 \mathrm{MHz}, \mathrm{CDCl}_{3}, 27^{\circ} \mathrm{C}\right) \delta 7.54(\mathrm{~d}, J=7.0 \mathrm{~Hz}, 2 \mathrm{H}, \mathrm{ArH}), 7.40-7.36(\mathrm{~m}, 3 \mathrm{H}, \mathrm{ArH}), 7.35-7.28(\mathrm{~m}, 3 \mathrm{H}$, $\mathrm{ArH})$, 7.16-7.14 (m, 2H, ArH), 4.14-4.05 (m, 2H, OCH $\left.\mathrm{CH}_{3}\right), 3.55\left(\mathrm{~s}, 3 \mathrm{H}, \mathrm{OCH}_{3}\right), 3.04\left(\mathrm{~s}, 3 \mathrm{H}, \mathrm{OCH}_{3}\right), 2.53-$ $2.41\left(\mathrm{~m}, 3 \mathrm{H}, \mathrm{CH}_{2}\right), 2.32-2.29\left(\mathrm{~m}, 1 \mathrm{H}, \mathrm{CH}_{2}\right), 1.51\left(\mathrm{~s}, 3 \mathrm{H}, \mathrm{CH}_{3}\right), 1.48\left(\mathrm{~s}, 3 \mathrm{H}, \mathrm{CH}_{3}\right), 1.22(\mathrm{t}, J=7.5 \mathrm{~Hz}, 3 \mathrm{H}$, 
Supporting information Amino Acid Schiff Base Bearing Benzophenone Imine as a Platform for Highly Congested Unnatural $\alpha$-Amino Acid Synthesis

$\left.\mathrm{OCH}_{2} \mathrm{CH}_{3}\right) ;{ }^{13} \mathrm{C}$ NMR $\left(125 \mathrm{MHz}, \mathrm{CDCl}_{3}, 27^{\circ} \mathrm{C}\right) \delta 176.4,174.0,172.6,165.3,141.3,136.1,130.0,128.6$, 128.3, 127.9, 127.6, 71.8, 60.7, 51.4, 51.3, 50.9, 30.4, 30.4, 23.8, 22.0, 14.0; HRMS (ESI) m/z calcd. for $\mathrm{C}_{26} \mathrm{H}_{32} \mathrm{NO}_{6}{ }^{+} 454.2224$ found 454.2236 .<smiles>COC(=O)C(N=C(c1ccccc1)c1ccccc1)(C(=O)OC)c1ccccc1</smiles>

Methyl 1-(1-((diphenylmethylene)amino)-2-methoxy-2-oxo-1-phenylethyl)cyclohexane-1-carboxylate (3ab)

Prepared according to General Method C and purified by flash column chromatography (Hexane/Et ${ }_{2} \mathrm{O}=$ 5/1 (1\% Et $\left.{ }_{3} \mathrm{~N}\right), 73 \%$ yield, white solid); IR (neat) $1730,1217,1132 \mathrm{~cm}^{-1} ;{ }^{1} \mathrm{H} \mathrm{NMR}\left(500 \mathrm{MHz}, \mathrm{CDCl}_{3}, 27^{\circ} \mathrm{C}\right) \delta$ $7.74(\mathrm{~d}, J=7.5 \mathrm{~Hz}, 2 \mathrm{H}, \operatorname{ArH}), 7.45(\mathrm{t}, J=7.5 \mathrm{~Hz}, 1 \mathrm{H}, \operatorname{ArH}), 7.39(\mathrm{t}, J=7.5 \mathrm{~Hz}, 2 \mathrm{H}, \operatorname{ArH}), 7.20(\mathrm{t}, J=7.5 \mathrm{~Hz}$, 1H, ArH), 7.09 (t, J= $7.5 \mathrm{~Hz}, 2 \mathrm{H}, \operatorname{ArH}), 6.66(\mathrm{~d}, J=7.5 \mathrm{~Hz}, 2 \mathrm{H}, \operatorname{ArH}), 3.61\left(\mathrm{~s}, 3 \mathrm{H}, \mathrm{OCH}_{3}\right), 3.14(\mathrm{~s}, 3 \mathrm{H}, \mathrm{OCH})_{3}$, $2.61\left(\mathrm{~d}, J=7.0 \mathrm{~Hz}, 1 \mathrm{H}, \mathrm{CH}_{2}\right), 1.86\left(\mathrm{td}, J=13.0,4.0 \mathrm{~Hz}, 1 \mathrm{H}, \mathrm{CH}_{2}\right), 1.76-1.71\left(\mathrm{~m}, 2 \mathrm{H}, \mathrm{CH}_{2}\right), 1.62-1.59(\mathrm{~m}, 3 \mathrm{H}$, $\left.\mathrm{CH}_{2}\right), 1.14\left(\mathrm{~m}, 1 \mathrm{H}, \mathrm{CH}_{2}\right), 1.16-1.12\left(\mathrm{~m}, 2 \mathrm{H}, \mathrm{CH}_{2}\right) ;{ }^{13} \mathrm{C} \mathrm{NMR}\left(125 \mathrm{MHz} \mathrm{CDCl}_{3}, 2{ }^{\circ} \mathrm{C}\right) \delta 174.3,170.5,167.7$, 141.7, 140.7, 136.7, 130.2, 129.8, 129.1, 128.8, 128.4, 128.0, 127.1, 127.0, 126.7, 76.0, 59.1, 51.4, 50.8, 31.3, 29.3, 25.6, 24.4, 23.9; HRMS (ESI) m/z calcd. for $\mathrm{C}_{30} \mathrm{H}_{32} \mathrm{NO}_{4}{ }^{+} 470.2326$ found 470.2310 .

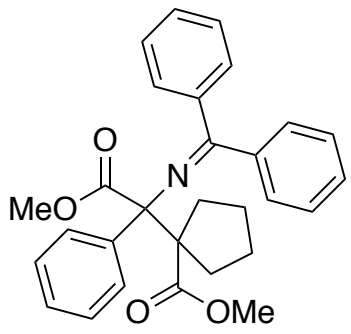

Methyl 1-(1-((diphenylmethylene)amino)-2-methoxy-2-oxo-1-phenylethyl)cyclopentane-1-carboxylate (3ac)

Prepared according to General Method C and purified by flash column chromatography (Hexane/Et $t_{2} \mathrm{O}=$ 5/1 (1\% Et $\left.{ }_{3} \mathrm{~N}\right), 78 \%$ yield, white solid); IR (neat) 1732, 1626, 1445, 1231, $1161 \mathrm{~cm}^{-1} ;{ }^{1} \mathrm{H} \mathrm{NMR}(500 \mathrm{MHz}$, $\left.\mathrm{CDCl}_{3}, 27^{\circ} \mathrm{C}\right) \delta 7.68(\mathrm{~d}, J=7.0 \mathrm{~Hz}, 2 \mathrm{H}, \mathrm{ArH}), 7.44(\mathrm{t}, J=7.0 \mathrm{~Hz}, 1 \mathrm{H}, \mathrm{ArH}), 7.37(\mathrm{t}, J=7.5 \mathrm{~Hz}, 2 \mathrm{H}, \operatorname{ArH}), 7.33-$ $7.31(\mathrm{~m}, 2 \mathrm{H}, \mathrm{ArH}), 7.25-7.22(\mathrm{~m}, 4 \mathrm{H}, \operatorname{ArH}), 7.13(\mathrm{t}, J=7.5 \mathrm{~Hz}, 2 \mathrm{H}, \operatorname{ArH}), 6.79(\mathrm{~d}, J=7.0 \mathrm{~Hz}, 2 \mathrm{H}, \operatorname{ArH}), 3.62$ 
$\left(\mathrm{s}, 3 \mathrm{H}, \mathrm{OCH}_{3}\right), 3.17\left(\mathrm{~s}, 3 \mathrm{H}, \mathrm{OCH}_{3}\right), 2.56-2.49\left(\mathrm{~m}, 1 \mathrm{H}, \mathrm{CH}_{2}\right), 2.30-2.24\left(\mathrm{~m}, 1 \mathrm{H}, \mathrm{CH}_{2}\right), 2.16-2.11\left(\mathrm{~m}, 1 \mathrm{H}, \mathrm{CH}_{2}\right)$, 1.87-1.80 (m, $\left.1 \mathrm{H}, \mathrm{CH}_{2}\right), 1.77-1.69\left(\mathrm{~m}, 1 \mathrm{H}, \mathrm{CH}_{2}\right), 1.64\left(\mathrm{~m}, 3 \mathrm{H}, \mathrm{CH}_{2}\right) ;{ }^{13} \mathrm{C} \mathrm{NMR}\left(125 \mathrm{MHz}, \mathrm{CDCl}_{3}, 27^{\circ} \mathrm{C}\right) \delta$ 176.3, 171.5, 168.7, 141.7, 141.4, 136.4, 130.3, 129.0, 128.7, 128.6, 128.6, 128.1, 127.1, 127.0, 127.0, 74.1, 67.3, 51.8, 51.0, 36.0, 32.0, 25.4, 24.3; HRMS (ESI) $\mathrm{m} / \mathrm{z}$ calcd. for $\mathrm{C}_{29} \mathrm{H}_{30} \mathrm{NO}_{4}{ }^{+} 456.2169$ found 456.2168 .

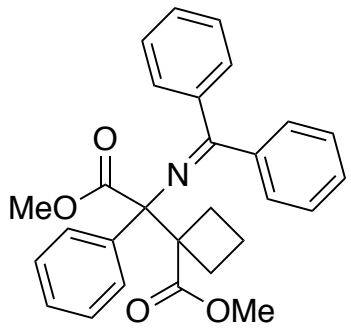

Methyl 1-(1-((diphenylmethylene)amino)-2-methoxy-2-oxo-1-phenylethyl)cyclobutane-1-carboxylate (3ad)

Prepared according to General Method C and purified by flash column chromatography (Hexane/Et ${ }_{2} \mathrm{O}=$ $5 / 1\left(1 \% \mathrm{Et}_{3} \mathrm{~N}\right), 52 \%$ yield, white solid); IR (neat) 1722, 1626, 1445, 1204, $1117 \mathrm{~cm}^{-1} ;{ }^{1} \mathrm{H} \mathrm{NMR}(500 \mathrm{MHz}$, $\left.\mathrm{CDCl}_{3}, 27^{\circ} \mathrm{C}\right) \delta 7.79(\mathrm{~d}, J=7.5 \mathrm{~Hz}, 2 \mathrm{H}, \mathrm{ArH}), 7.47(\mathrm{t}, J=7.5 \mathrm{~Hz}, 1 \mathrm{H}, \mathrm{ArH}), 7.40(\mathrm{t}, J=7.5 \mathrm{~Hz}, 2 \mathrm{H}, \operatorname{ArH}), 7.36-$ $7.32(\mathrm{~m}, 2 \mathrm{H}, \operatorname{ArH}), 7.27-7.22(\mathrm{~m}, 4 \mathrm{H}, \operatorname{ArH}), 7.14(\mathrm{t}, J=7.5 \mathrm{~Hz}, 2 \mathrm{H}, \operatorname{ArH}), 6.75(\mathrm{~d}, J=7.5 \mathrm{~Hz}, 2 \mathrm{H}, \operatorname{ArH}), 3.65$ (s, 3H, OCH $\mathrm{OCH}_{3}, 3.21\left(\mathrm{~s}, 3 \mathrm{H}, \mathrm{CH}_{3}\right), 3.14-3.07\left(\mathrm{~m}, 1 \mathrm{H}, \mathrm{CH}_{2}\right), 3.01-2.96\left(\mathrm{~m}, 1 \mathrm{H}, \mathrm{CH}_{2}\right), 2.17-2.12\left(\mathrm{~m}, 1 \mathrm{H}, \mathrm{CH}_{2}\right)$, 2.01-1.90 (m, 3H, $\left.\mathrm{CH}_{2}\right) ;{ }^{13} \mathrm{C}$ NMR $\left(125 \mathrm{MHz}, \mathrm{CDCl}_{3}, 27^{\circ} \mathrm{C}\right) \delta 175.7,171.2,168.6,141.2,139.9,136.4,130.4$, 128.9, 128.7, 128.5, 128.3, 128.1, 127.2, 127.0, 126.9, 72.6, 60.5, 51.9, 51.0, 30.9, 25.0, 15.8; HRMS (ESI) $\mathrm{m} / \mathrm{z}$ calcd. for $\mathrm{C}_{28} \mathrm{H}_{28} \mathrm{NO}_{4}{ }^{+} 442.2013$ found 442.1983 .<smiles>COC(=O)C(N=C(c1ccccc1)c1ccccc1)(c1ccccc1)C1(C(=O)OCc2ccccc2)CCCC1</smiles>

Benzyl 1-(1-((diphenylmethylene)amino)-2-methoxy-2-oxo-1-phenylethyl)cyclopentane-1-carboxylate (3ae) 
Supporting information Amino Acid Schiff Base Bearing Benzophenone Imine as a Platform for Highly Congested Unnatural $\alpha$-Amino Acid Synthesis

Prepared according to General Method $\mathrm{C}$ and purified by flash column chromatography (Hexane/Et ${ }_{2} \mathrm{O}=$ 5/1 (1\% Et $\left.\mathrm{t}_{3} \mathrm{~N}\right), 80 \%$ yield, white solid); IR (neat) $1728,1626,1445,1231,1157 \mathrm{~cm}^{-1} ;{ }^{1} \mathrm{H} \mathrm{NMR}(500 \mathrm{MHz}$, $\left.\mathrm{CDCl}_{3}, 27^{\circ} \mathrm{C}\right) \delta 7.66(\mathrm{~d}, J=7.0 \mathrm{~Hz}, 2 \mathrm{H}, \operatorname{ArH}), 7.43(\mathrm{t}, J=7.5 \mathrm{~Hz}, 1 \mathrm{H}, \operatorname{ArH}), 7.36(\mathrm{t}, J=7.0 \mathrm{~Hz}, 2 \mathrm{H}, \operatorname{ArH}), 7.33-$ $7.28(\mathrm{~m}, 7 \mathrm{H}, \operatorname{ArH}), 7.24-7.17(\mathrm{~m}, 4 \mathrm{H}, \operatorname{ArH}), 7.12(\mathrm{t}, J=7.5 \mathrm{~Hz}, 2 \mathrm{H}, \operatorname{ArH}), 6.78(\mathrm{~d}, J=7.5 \mathrm{~Hz}, 2 \mathrm{H}, \operatorname{ArH}), 5.11$ (d, J = 7.5 Hz, 1H, ArCH $)_{2}, 4.97\left(\mathrm{~d}, J=7.0 \mathrm{~Hz}, 1 \mathrm{H}, \mathrm{ArCH}_{2}\right), 2.94\left(\mathrm{~s}, 3 \mathrm{H}, \mathrm{OCH}_{3}\right), 2.54-2.48\left(\mathrm{~m}, 1 \mathrm{H}, \mathrm{CH}_{2}\right), 2.30-$ $2.28\left(\mathrm{~m}, 1 \mathrm{H}, \mathrm{CH}_{2}\right), 2.19-2.13\left(\mathrm{~m}, 1 \mathrm{H}, \mathrm{CH}_{2}\right), 1.85-1.80\left(\mathrm{~m}, 1 \mathrm{H}, \mathrm{CH}_{2}\right), 1.75-1.70\left(\mathrm{~m}, 1 \mathrm{H}, \mathrm{CH}_{2}\right), 1.63-1.58(\mathrm{~m}$, $\left.3 \mathrm{H}, \mathrm{CH}_{2}\right) ;{ }^{13} \mathrm{C}$ NMR $\left(125 \mathrm{MHz}, \mathrm{CDCl}_{3}, 27^{\circ} \mathrm{C}\right) \delta 175.5,171.3,168.6,141.6,141.4,136.3,135.6,130.3,129.0$, $128.7,128.6,128.5,128.4,128.2,128.0,127.1,127.1,127.0,74.1,67.2,66.8,50.8,36.0,32.0,25.4$, 24.3; HRMS (ESI) m/z calcd. for $\mathrm{C}_{35} \mathrm{H}_{34} \mathrm{NO}_{4}{ }^{+} 532.2482$ found 532.2484 .

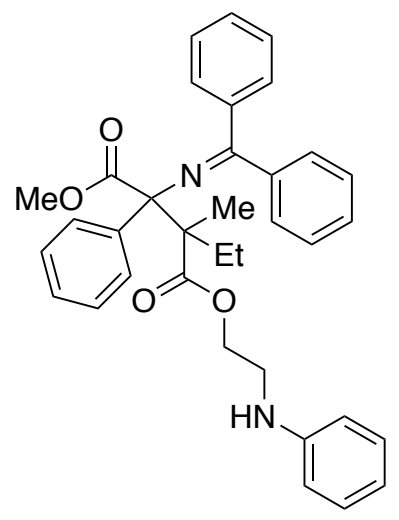

1-Methyl 4-(2-(phenylamino)ethyl) 2-((diphenylmethylene)amino)-3-ethyl-3-methyl-2-

\section{phenylsuccinate (3af)}

Prepared according to General Method $\mathrm{C}$ and purified by flash column chromatography (Hexane/Et ${ }_{2} \mathrm{O}=$ 1/1 (1\% Et $\left.{ }_{3} \mathrm{~N}\right)$, diastereomixture, 76\% yield, $\mathrm{dr}=1.2 / 1$, white solid); IR (neat) $1722,1603,1506,1227 \mathrm{~cm}$ 1; ${ }^{1} \mathrm{H}$ NMR (500 MHz, CDCl, $\left.27^{\circ} \mathrm{C}\right) \delta 7.74(\mathrm{~d}, J=7.5 \mathrm{~Hz}, 4 \mathrm{H}, \mathrm{ArH}$, major diastereomer), 7.59 (m, 2H, $\mathrm{ArH}$, minor diastereomer), $7.52(\mathrm{~m}, 2 \mathrm{H}, \mathrm{ArH}$, major diastereomer $), 7.45-7.42(\mathrm{~m}, 4 \mathrm{H}, \mathrm{ArH}$, minor diastereomer), 7.39-7.35 (m, 4H, ArH, major diastereomer), 7.27-7.06 (m, 6H, $\mathrm{ArH}$, major diastereomer), 7.27-7.06 (m, 10H, ArH, minor diastereomer), 6.67 (d, J = 7.5 Hz, 2H, ArH, major diastereomer), 6.61 (d, J $=7.0 \mathrm{~Hz}, 2 \mathrm{H}, \mathrm{ArH}$, minor diastereomer), $6.46(\mathrm{~d}, J=8.0 \mathrm{~Hz}, 2 \mathrm{H}, \mathrm{ArH}$, major diastereomer), 6.41 (d, $J=7.5$ $\mathrm{Hz}, 2 \mathrm{H}, \mathrm{ArH}$, minor diastereomer), 4.36-4.33 (m, $1 \mathrm{H}, \mathrm{OCH}_{2}$, major diastereomer), 4.24-4.22 $\left(\mathrm{m}, 1 \mathrm{H}, \mathrm{OCH}_{2}\right.$, major diastereomer), 4.21-4.15 (m, $1 \mathrm{H}, \mathrm{OCH}_{2}$, minor diastereomer), 4.11-4.08 $\left(\mathrm{m}, 1 \mathrm{H}, \mathrm{OCH}_{2}\right.$, minor diastereomer), 3.98 (br, $1 \mathrm{H}, \mathrm{NH}$, major diastereomer), 3.90 (br, $1 \mathrm{H}, \mathrm{NH}$, minor diastereomer), 3.37-3.35 (m, $2 \mathrm{H}, \mathrm{NHCH}_{2}$, major diastereomer), 3,31-3.29 (m, 2H, $\mathrm{NHCH}_{2}$, minor diastereomer), $3.17\left(\mathrm{~s}, 3 \mathrm{H}, \mathrm{OCH}_{3}\right.$, minor diastereomer), $3.05\left(\mathrm{~s}, 3 \mathrm{H}, \mathrm{OCH}_{3}\right.$, major diastereomer), 2.76-2.73 $\left(\mathrm{m}, 1 \mathrm{H}, \mathrm{CH}_{2} \mathrm{CH}_{3}\right.$, minor 
diastereomer), 2.13-2.10 (m, $1 \mathrm{H}, \mathrm{CH}_{2} \mathrm{CH}_{3}$, major diastereomer), 1.77-1.73 (m, $1 \mathrm{H}, \mathrm{CH}_{2} \mathrm{CH}_{3}$, minor diastereomer), 1.56-1.52 (m, $1 \mathrm{H}, \mathrm{CH}_{2} \mathrm{CH}_{3}$, major diastereomer), 1.40 (s, $3 \mathrm{H}, \mathrm{CH}_{3}$, minor diastereomer), $1.13\left(\mathrm{~s}, 3 \mathrm{H}, \mathrm{CH}_{3}\right.$, major diastereomer), $0.84\left(\mathrm{t}, J=7.5 \mathrm{~Hz}, 3 \mathrm{H}, \mathrm{CH}_{2} \mathrm{CH}_{3}\right.$, major diastereomer), $0.84(\mathrm{t}, J=7.5$ $\mathrm{Hz}, 3 \mathrm{H}, \mathrm{CH}_{2} \mathrm{CH}_{3}$, minor diastereomer); ${ }^{13} \mathrm{C}$ NMR $\left(125 \mathrm{MHz}, \mathrm{CDCl}_{3}, 2{ }^{\circ} \mathrm{C}\right) \delta 175.1,174.3,171.1,170.7$, $167.8,167.1,147.6,141.7,141.5,141.0,140.9,136.7,136.3,130.3,129.7,129.7,129.3,129.2,129.1$, $129.0,128.7,128.6,128.5,128.3,128.1,128.1,127.2,127.2,127.1,127.0,117.6,117.5,112.9,112.8$, $76.2,75.8,63.4,63.2,58.3,58.1,51.1,51.0,42.6,42.5,28.0,27.1,18.0,17.7,9.9,9.7 ;$ HRMS (ESI) m/z calcd. for $\mathrm{C}_{35} \mathrm{H}_{37} \mathrm{~N}_{2} \mathrm{O}_{4}{ }^{+} 549.2748$ found 549.2747 .

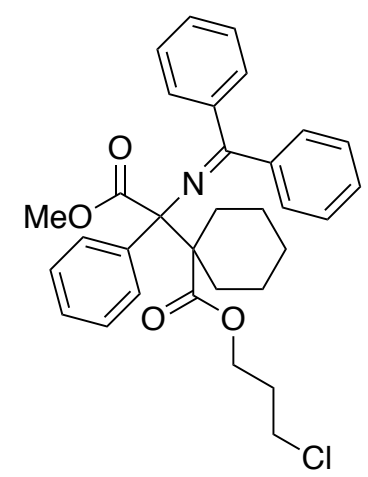

\section{3-Chloropropyl 1-(1-((diphenylmethylene)amino)-2-methoxy-2-oxo-1-phenylethyl)cyclohexane-1-}

\section{carboxylate (3ag)}

Prepared according to General Method $\mathrm{C}$ and purified by flash column chromatography (Hexane/Et ${ }_{2} \mathrm{O}=$ 2/1 (1\% Et $\left.{ }_{3} \mathrm{~N}\right), 63 \%$ yield, white solid); IR (neat) $1732,1444,1201,1128 \mathrm{~cm}^{-1} ;{ }^{1} \mathrm{H} \mathrm{NMR}\left(500 \mathrm{MHz}, \mathrm{CDCl}_{3}\right.$, $\left.27^{\circ} \mathrm{C}\right) \delta 7.73(\mathrm{~d}, J=7.5 \mathrm{~Hz}, 2 \mathrm{H}, \mathrm{ArH}), 7.45(\mathrm{t}, J=7.5 \mathrm{~Hz}, 1 \mathrm{H}, \operatorname{ArH}), 7.39(\mathrm{t}, J=7.5 \mathrm{~Hz}, 2 \mathrm{H}, \mathrm{ArH}), 7.28-7.25$ $(\mathrm{m}, 5 \mathrm{H}, \operatorname{ArH}), 7.20(\mathrm{t}, J=7.5 \mathrm{~Hz}, 1 \mathrm{H}, \operatorname{ArH}), 7.10(\mathrm{t}, J=7.5 \mathrm{~Hz}, 2 \mathrm{H}, \operatorname{ArH}), 6.65(\mathrm{~d}, J=7.0 \mathrm{~Hz}, 2 \mathrm{H}, \operatorname{ArH}), 4.20-$ $4.10\left(\mathrm{~m}, 2 \mathrm{H}, \mathrm{OCH}_{2}\right), 3.52-3.46\left(\mathrm{~m}, 2 \mathrm{H}, \mathrm{CH}_{2} \mathrm{Cl}\right), 3.15\left(\mathrm{~s}, 3 \mathrm{H}, \mathrm{OCH}_{3}\right), 2.60\left(\mathrm{~d}, J=11.0 \mathrm{~Hz}, 1 \mathrm{H}, \mathrm{CH}_{2}\right), 2.04-1.99$ $\left(\mathrm{m}, 2 \mathrm{H}, \mathrm{CH}_{2}\right), 1.89\left(\mathrm{td}, J=13.0,4.0 \mathrm{~Hz}, 1 \mathrm{H}, \mathrm{CH}_{2}\right), 1.76-1.60\left(\mathrm{~m}, 4 \mathrm{H}, \mathrm{CH}_{3}\right), 1.37-1.31\left(\mathrm{~m}, 1 \mathrm{H}, \mathrm{CH}_{2}\right), 1.21(\mathrm{t}, J$ $\left.=7.0 \mathrm{~Hz}, 1 \mathrm{H}, \mathrm{CH}_{2}\right), 1.14\left(\mathrm{~m}, 2 \mathrm{H}, \mathrm{CH}_{2}\right) ;{ }^{13} \mathrm{C}$ NMR $\left(125 \mathrm{MHz}, \mathrm{CDCl}_{3}, 27^{\circ} \mathrm{C}\right) \delta 173.8,170.4,167.7,141.6,140.7$, 136.6, 130.2, 129.7, 129.0, 128.7, 128.4, 128.0, 127.1, 127.1, 126.8, 76.0, 61.1, 59.0, 50.9, 41.4, 31.5, 31.3, 29.3, 25.6, 24.3, 23.9; HRMS (ESI) m/z calcd. for $\mathrm{C}_{32} \mathrm{H}_{35} \mathrm{ClNO}_{4}{ }^{+} 532.2249$ found 532.2228. 
<smiles>CCC(CC)(C(=O)OCCOC(=O)c1ccccc1I)C(N=C(c1ccccc1)c1ccccc1)(C(=O)OC)c1ccccc1</smiles>

\section{4-(2-((2-lodobenzoyl)oxy)ethyl) 1-methyl 2-((diphenylmethylene)amino)-3,3-diethyl-2-}

\section{phenylsuccinate (3ah)}

Prepared according to General Method C and purified by flash column chromatography (Hexane/Et ${ }_{2} \mathrm{O}=$ $1 / 1\left(1 \% \mathrm{Et}_{3} \mathrm{~N}\right), 46 \%$ yield, white solid); IR (neat) $1728,1445,1287,1219,1105,1014 \mathrm{~cm}^{-1} ;{ }^{1} \mathrm{H}$ NMR (500 $\left.\mathrm{MHz}_{\mathrm{CDCl}}, 27^{\circ} \mathrm{C}\right) \delta 7.99(\mathrm{~d}, J=8.0 \mathrm{~Hz}, 1 \mathrm{H}, \mathrm{ArH}), 7.73(\mathrm{~d}, J=7.5 \mathrm{~Hz}, 1 \mathrm{H}, \operatorname{ArH}), 7.69(\mathrm{~d}, J=7.5 \mathrm{~Hz}, 2 \mathrm{H}, \operatorname{ArH})$, $7.52(\mathrm{~s}, 2 \mathrm{H}, \operatorname{ArH}), 7.42(\mathrm{t}, J=7.5 \mathrm{~Hz}, 1 \mathrm{H}, \operatorname{ArH}), 7.35(\mathrm{t}, J=7.5 \mathrm{~Hz}, 3 \mathrm{H}, \operatorname{ArH}), 7.23-7.18(\mathrm{~m}, 4 \mathrm{H}, \operatorname{ArH}), 7.14(\mathrm{t}$, $J=8.0 \mathrm{~Hz}, 1 \mathrm{H}, \mathrm{ArH}), 7.09(\mathrm{t}, J=7.5 \mathrm{~Hz}, 2 \mathrm{H}, \mathrm{ArH}), 6.64(\mathrm{~d}, J=7.5 \mathrm{~Hz}, 2 \mathrm{H}, \mathrm{ArH}), 4.48(\mathrm{t}, J=5.0 \mathrm{~Hz}, 2 \mathrm{H}, \mathrm{OCH})_{2}$, 4.41-4.36 (m, 1H, OCH $)_{2}$, 4.33-4.28 (m, 1H, OCH 2$), 3.10\left(\mathrm{~s}, 3 \mathrm{H}, \mathrm{OCH}_{3}\right), 2.56-2.49\left(\mathrm{~m}, 1 \mathrm{H}, \mathrm{CH}_{2}\right), 2.06-1.99$ $\left(\mathrm{m}, 1 \mathrm{H}, \mathrm{CH}_{2}\right), 1.80-1.76\left(\mathrm{~m}, 2 \mathrm{H}, \mathrm{CH}_{2}\right), 0.95\left(\mathrm{t}, \mathrm{J}=7.5 \mathrm{~Hz}, 3 \mathrm{H}, \mathrm{CH}_{3}\right), 0.88\left(\mathrm{t}, J=7.5 \mathrm{~Hz}, 3 \mathrm{H}, \mathrm{CH}_{3}\right) ;{ }^{13} \mathrm{C}$ NMR $\left(125 \mathrm{MHz} \mathrm{CDCl}_{3}, 2{ }^{\circ} \mathrm{C}\right) \delta 174.5,170.7,167.1,166.0,141.7,141.4,141.2,136.5,134.4,134.4,132.8$, 131.1, 130.2, 129.5, 129.1, 128.8, 128.4, 128.0, 127.9, 127.2, 127.1, 126.9, 94.3, 63.2, 62.0, 61.0, 51.1, 24.8, 24.4, 10.1, 10.0; HRMS (ESI) m/z calcd. for $\mathrm{C}_{37} \mathrm{H}_{37} \mathrm{INO}_{6}{ }^{+} 718.1660$ found 718.1661 .<smiles>COC(=O)C(N=C(c1ccccc1)c1ccccc1)(c1ccccc1)C(C)(C)C(=O)OCc1ccccc1OC</smiles>

1-(2-methoxybenzyl) 4-methyl 2-butyl-3-((diphenylmethylene)amino)-2-methyl-3-phenylsuccinate (3ai) 
Prepared according to General Method $\mathrm{C}$ and purified by flash column chromatography (Hexane/Et ${ }_{2} \mathrm{O}=$ 4/1 (1\% Et $\left.{ }_{3} \mathrm{~N}\right)$, diastereomixture, $\mathrm{dr}=1 / 1,50 \%$ yield, white solid); IR (neat) 1730, 1493, 1246, $1145 \mathrm{~cm}^{-1}$; ${ }^{1} \mathrm{H}$ NMR $\left(500 \mathrm{MHz}, \mathrm{CDCl}_{3}, 27^{\circ} \mathrm{C}\right) \delta 7.68(\mathrm{~d}, J=7.5 \mathrm{~Hz}, 2 \mathrm{H}, \operatorname{ArH}), 7.49(\mathrm{~s}, 2 \mathrm{H}, \mathrm{ArH}), 7.41(\mathrm{t}, J=7.5 \mathrm{~Hz}, 1 \mathrm{H}$, $\operatorname{ArH}), 7.31(\mathrm{t}, J=7.5 \mathrm{~Hz}, 3 \mathrm{H}, \operatorname{ArH}), 7.28-7.19(\mathrm{~m}, 5 \mathrm{H}, \operatorname{ArH}), 7.10(\mathrm{t}, J=7.5 \mathrm{~Hz}, 2 \mathrm{H}, \operatorname{ArH}), 6.87(\mathrm{t}, J=7.5 \mathrm{~Hz}$, $1 \mathrm{H}, \operatorname{ArH}), 6.84(\mathrm{~d}, J=8.0 \mathrm{~Hz}, 1 \mathrm{H}, \operatorname{ArH}), 6.73(\mathrm{~d}, J=7.5 \mathrm{~Hz}, 2 \mathrm{H}, \operatorname{ArH}), 5.22\left(\mathrm{~d}, J=7.5 \mathrm{~Hz}, 1 \mathrm{H}, \operatorname{ArCH}_{2}\right), 5.08(\mathrm{~d}$, $\left.J=7.5 \mathrm{~Hz}, 1 \mathrm{H}, \mathrm{ArCH}_{2}\right), 3.77\left(\mathrm{~s}, 3 \mathrm{H}, \mathrm{ArOCH}_{3}\right), 2.91\left(\mathrm{~s}, 3 \mathrm{H}, \mathrm{OCH}_{3}\right), 1.31-1.26\left(\mathrm{~m}, 5 \mathrm{H}, \mathrm{CH}_{2}\right), 1.13\left(\mathrm{~s}, 3 \mathrm{H}, \mathrm{CH}_{3}\right)$, 0.90-0.84 (m, 4H, $\left.\mathrm{CH}_{2} \mathrm{CH}_{3}\right) ;{ }^{13} \mathrm{C} \mathrm{NMR}\left(125 \mathrm{MHz}, \mathrm{CDCl}_{3}, 27^{\circ} \mathrm{C}\right) \delta 174.4,170.5,167.4,157.3,141.7,141.1$, $136.9,130.0,129.8,129.4,129.1,129.0,128.6,128.2,127.9,127.0,126.9,124.6,120.3,110.1,75.8$, 61.8, 57.6, 55.2, 50.8, 34.1, 27.8, 23.6, 18.8, 14.1; HRMS (ESI) m/z calcd. for $\mathrm{C}_{37} \mathrm{H}_{40} \mathrm{NO}_{5}{ }^{+} 578.2901$ found 578.2896.



\section{1-(2-Bromoethyl) 4-methyl 3-((diphenylmethylene)amino)-3-phenyl-2,2-dipropylsuccinate (3aj)}

Prepared according to General Method $\mathrm{C}$ and purified by flash column chromatography (Hexane/Et $\mathrm{E}_{2} \mathrm{O}=$ 2/1 (1\% Et $3 \mathrm{~N}), 25 \%$ yield, colorless oil); IR (neat) $2361,2340,1630 \mathrm{~cm}^{-1} ;{ }^{1} \mathrm{H} \mathrm{NMR} \mathrm{(500} \mathrm{MHz}, \mathrm{CDCl}_{3}, 27^{\circ} \mathrm{C}$ ) $\delta 7.81(\mathrm{~d}, J=7.0 \mathrm{~Hz}, 1 \mathrm{H}, \operatorname{ArH}), 7.70(\mathrm{~d}, J=7.0 \mathrm{~Hz}, 2 \mathrm{H}, \operatorname{ArH}), 7.59(\mathrm{t}, J=7.5 \mathrm{~Hz}, 1 \mathrm{H}, \operatorname{ArH}), 7.50-7.43(\mathrm{~m}, 4 \mathrm{H}$, $\operatorname{ArH}), 7.39(\mathrm{t}, J=7.0 \mathrm{~Hz}, 2 \mathrm{H}, \operatorname{ArH}), 7.21(\mathrm{t}, J=7.0 \mathrm{~Hz}, 1 \mathrm{H}, \operatorname{ArH}), 7.10(\mathrm{t}, J=7.5 \mathrm{~Hz}, 2 \mathrm{H}, \operatorname{ArH}), 6.66(\mathrm{~d}, J=7.0$ $\mathrm{Hz}, 2 \mathrm{H}, \mathrm{ArH}), 4.33-4.23(\mathrm{~m}, 2 \mathrm{H}, \mathrm{OCH}), 3.42\left(\mathrm{t}, J=7.0 \mathrm{~Hz}, 2 \mathrm{H}, \mathrm{CH}_{2} \mathrm{Br}\right), 3.14\left(\mathrm{~s}, 3 \mathrm{H}, \mathrm{OCH}_{3}\right), 2.40-2.34(\mathrm{~m}, 1 \mathrm{H}$, $\left.\mathrm{CH}_{2}\right), 2.08-2.05\left(\mathrm{~m}, 1 \mathrm{H}, \mathrm{CH}_{2}\right), 2.03-1.90\left(\mathrm{~m}, 2 \mathrm{H}, \mathrm{CH}_{2}\right), 1.43-1.30\left(\mathrm{~m}, 4 \mathrm{H}, \mathrm{CH}_{2}\right), 0.93\left(\mathrm{t}, \mathrm{J}=7.0 \mathrm{~Hz}, 3 \mathrm{H}, \mathrm{CH}_{3}\right)$, $0.78\left(\mathrm{t}, J=7.0 \mathrm{~Hz}, 3 \mathrm{H}, \mathrm{CH}_{3}\right) ;{ }^{13} \mathrm{C} \mathrm{NMR}\left(125 \mathrm{MHz}, \mathrm{CDCl}_{3}, 27^{\circ} \mathrm{C}\right) \delta 174.4,170.8,167.1,141.2,136.6,132.4$, 130.2 , 130.1, 129.5, 129.2, 128.8, 128.3, 128.0, 127.1, 126.9, 64.0, 60.8, 51.0, 34.8, 34.4, 28.5, 18.7, 18.2, 15.3, 15.2; HRMS (ESI) m/z calcd. for $\mathrm{C}_{32} \mathrm{H}_{37} \mathrm{BrNO}_{4}{ }^{+} 578.1900$ found 578.1950 . 
<smiles>COC(=O)C(N=C(c1ccccc1)c1ccccc1)(C(=O)OCCCCCCO)C1(C(=O)OCCCCCO)CCC1</smiles>

6-Hydroxyhexyl 1-(1-((diphenylmethylene)amino)-2-methoxy-2-oxo-1-phenylethyl)cyclobutane-1carboxylate (3ak)

Prepared according to General Method $\mathrm{C}$ and purified by flash column chromatography (Hexane/Et ${ }_{2} \mathrm{O} / \mathrm{MeOH}=10 / 1 / 1\left(1 \% \mathrm{Et}_{3} \mathrm{~N}\right), 36 \%$ yield, white solid); IR (neat) 1744, 1628, 1445, $1120 \mathrm{~cm}^{-1}$; ${ }^{1} \mathrm{H} \mathrm{NMR}\left(500 \mathrm{MHz}, \mathrm{CDCl}_{3}, 27^{\circ} \mathrm{C}\right) \delta 7.78(\mathrm{~d}, J=7.0 \mathrm{~Hz}, 2 \mathrm{H}, \mathrm{ArH}), 7.47-7.40(\mathrm{~m}, 1 \mathrm{H}, \mathrm{ArH}), 7.40(\mathrm{t}, J=7.5 \mathrm{~Hz}$, $2 \mathrm{H}, \operatorname{ArH}), 7.36-7.34(\mathrm{~m}, 2 \mathrm{H}, \mathrm{ArH}), 7.24-7.21(\mathrm{~m}, 4 \mathrm{H}, \mathrm{ArH}), 7.14(\mathrm{t}, J=7.5 \mathrm{~Hz}, 2 \mathrm{H}, \operatorname{ArH}), 6.75(\mathrm{~d}, J=7.5 \mathrm{~Hz}$, $2 \mathrm{H}, \mathrm{ArH}), 4.10-4.02(\mathrm{~m}, 2 \mathrm{H}, \mathrm{OCH}), 3.61\left(\mathrm{t}, J=6.5 \mathrm{~Hz}, 2 \mathrm{H}, \mathrm{CH}_{2} \mathrm{OH}\right), 3.21\left(\mathrm{~s}, 3 \mathrm{H}, \mathrm{OCH}_{3}\right), 3.10(\mathrm{dd}, J=9.5,2.5$ $\left.\mathrm{Hz}, 1 \mathrm{H}, \mathrm{CH}_{2}\right), 3.00\left(\mathrm{dd}, J=9.5,2.5 \mathrm{~Hz}, 1 \mathrm{H}, \mathrm{CH}_{2}\right), 2.14-2.11\left(\mathrm{~m}, 1 \mathrm{H}, \mathrm{CH}_{2}\right), 1.98-1.90\left(\mathrm{~m}, 3 \mathrm{H}, \mathrm{CH}_{2}\right), 1.61-1.51$ $\left(\mathrm{m}, 3 \mathrm{H}, \mathrm{CH}_{2}\right), 1.36-1.19\left(\mathrm{~m}, 5 \mathrm{H}, \mathrm{CH}_{2}\right) ;{ }^{13} \mathrm{C}$ NMR $\left(125 \mathrm{MHz}, \mathrm{CDCl}_{3}, 27^{\circ} \mathrm{C}\right) \delta 175.3,171.2,168.6,141.2,140.0$, 136.4, 130.4, 130.1, 128.9, 128.7, 128.5, 128.3, 128.1, 127.2, 127.0, 72.5, 64.8, 62.8, 60.4, 51.0, 32.6, 31.0, 28.4, 25.7, 25.3, 25.1, 15.9; HRMS (ESI) m/z calcd. for $\mathrm{C}_{33} \mathrm{H}_{37} \mathrm{NNaO}_{5}{ }^{+} 550.2564$ found 550.2546.

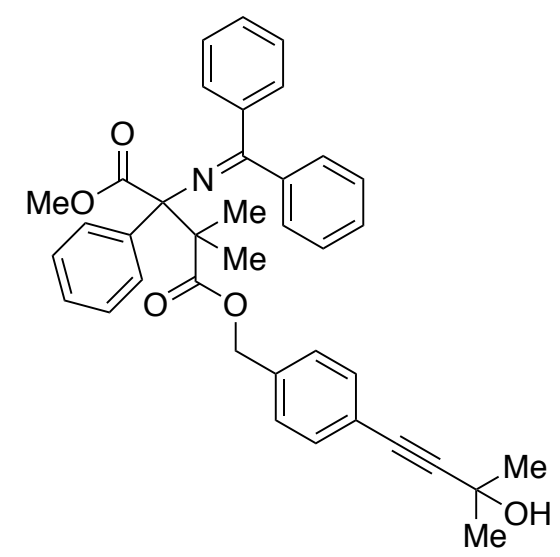

4-(4-(3-Hydroxy-3-methylbut-1-yn-1-yl)benzyl) 1-methyl 2-((diphenylmethylene)amino)-3,3-dimethyl2-phenylsuccinate (3al) 
Supporting information Amino Acid Schiff Base Bearing Benzophenone Imine as a Platform for Highly Congested Unnatural $\alpha$-Amino Acid Synthesis

Prepared according to General Method $\mathrm{C}$ and purified by flash column chromatography (Hexane/Et $\mathrm{t}_{2} \mathrm{O}=$ 2/1 (1\% Et $\left.{ }_{3} \mathrm{~N}\right), 86 \%$ yield, white solid); IR (neat) $2926,1728,1444,1234,1128 \mathrm{~cm}^{-1} ;{ }^{1} \mathrm{H}$ NMR (500 MHz, $\left.\mathrm{CDCl}_{3}, 27^{\circ} \mathrm{C}\right) \delta 7.69(\mathrm{~d}, J=7.5 \mathrm{~Hz}, 2 \mathrm{H}, \mathrm{ArH}), 7.47-7.41(\mathrm{~m}, 3 \mathrm{H}, \operatorname{ArH}), 7.35-7.32(\mathrm{~m}, 4 \mathrm{H}, \mathrm{ArH}), 7.25-7.19(\mathrm{~m}$, $6 \mathrm{H}, \operatorname{ArH}), 7.11(\mathrm{t}, J=7.5 \mathrm{~Hz}, 2 \mathrm{H}, \operatorname{ArH}), 6.72(\mathrm{~d}, J=7.5 \mathrm{~Hz}, 2 \mathrm{H}, \operatorname{ArH}), 5.03(\mathrm{q}, J=7.0 \mathrm{~Hz}, 2 \mathrm{H}, \operatorname{ArCH})^{2}, 2.96(\mathrm{~s}$, $\left.3 \mathrm{H}, \mathrm{OCH}_{3}\right), 1.62\left(\mathrm{~s}, 6 \mathrm{H}, \mathrm{C}(\mathrm{OH})\left(\mathrm{CH}_{3}\right)_{2}\right), 1.58\left(\mathrm{~s}, 3 \mathrm{H}, \mathrm{CH}_{3}\right), 1.21\left(\mathrm{~s}, 3 \mathrm{H}, \mathrm{CH}_{3}\right) ;{ }^{13} \mathrm{C} \mathrm{NMR}\left(125 \mathrm{MHz}, \mathrm{CDCl}_{3}, 27^{\circ} \mathrm{C}\right)$ $\delta 175.8,170.8,167.7,141.5,140.9,136.4,136.0,131.6,130.2,129.4,129.1,128.7,128.5,128.2,128.0$, 127.2, 127.1, 127.0, 122.5, 94.1, 81.8, 75.3, 66.1, 65.6, 54.0, 50.9, 31.5, 29.7, 24.2, 22.5; HRMS (ESI) m/z calcd. for $\mathrm{C}_{38} \mathrm{H}_{38} \mathrm{NO}_{5}{ }^{+} 588.2744$ found 588.2743 .

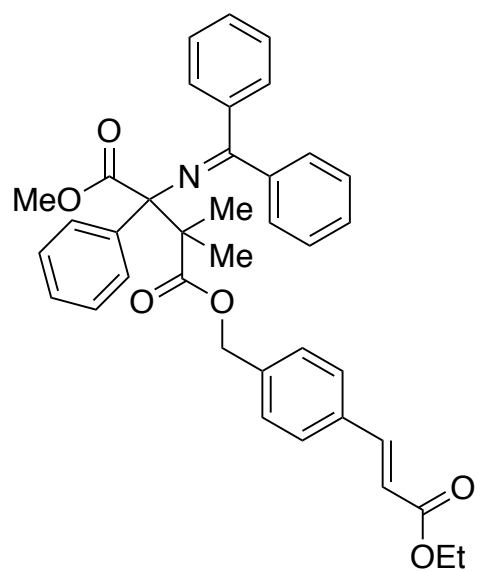

(E)-4-(4-(3-Ethoxy-3-oxoprop-1-en-1-yl)benzyl) 1-methyl 2-((diphenylmethylene)amino)-3,3-dimethyl-

\section{2-phenylsuccinate (3am)}

Prepared according to General Method $\mathrm{C}$ and purified by flash column chromatography (Hexane/Et ${ }_{2} \mathrm{O}=$ 2/1 (1\% Et $\left.{ }_{3} \mathrm{~N}\right), 69 \%$ yield, white solid); IR (neat) $2359,1713,1636,1445,1126 \mathrm{~cm}^{-1} ;{ }^{1} \mathrm{H} \mathrm{NMR}(500 \mathrm{MHz}$, $\left.\mathrm{CDCl}_{3}, 27^{\circ} \mathrm{C}\right) \delta 7.69(\mathrm{~d}, J=7.5 \mathrm{~Hz}, 2 \mathrm{H}, \mathrm{ArH}), 7.66(\mathrm{~d}, J=16.0 \mathrm{~Hz}, 1 \mathrm{H}, \operatorname{ArCH}), 7.48-7.41(\mathrm{~m}, 5 \mathrm{H}, \operatorname{ArH}), 7.34-$ $7.30(\mathrm{~m}, 4 \mathrm{H}, \operatorname{ArH}), 7.26-7.19(\mathrm{~m}, 4 \mathrm{H}, \operatorname{ArH}), 7.11(\mathrm{t}, J=7.5 \mathrm{~Hz}, 2 \mathrm{H}, \operatorname{ArH}), 6.71(\mathrm{~d}, J=7.5 \mathrm{~Hz}, 2 \mathrm{H}, \operatorname{ArH}), 6.42$ (d, J = 16.0 Hz, 1H, ArCHCH), 5.06 (q, J =13.0 Hz, 2H, ArCH $), 4.27\left(q, J=7.0 \mathrm{~Hz}, 2 \mathrm{H}, \mathrm{OCH}_{2} \mathrm{CH}_{3}\right), 2.98(\mathrm{~s}, 3 \mathrm{H}$, $\left.\mathrm{OCH}_{3}\right), 1.59\left(\mathrm{~s}, 3 \mathrm{H}, \mathrm{CH}_{3}\right), 1.34\left(\mathrm{t}, J=7.0 \mathrm{~Hz}, 3 \mathrm{H}, \mathrm{OCH}_{2} \mathrm{CH}_{3}\right), 1.23\left(\mathrm{~s}, 3 \mathrm{H}, \mathrm{CH}_{3}\right) ;{ }^{13} \mathrm{C} \mathrm{NMR}\left(125 \mathrm{MHz}, \mathrm{CDCl}_{3}\right.$, $\left.27^{\circ} \mathrm{C}\right) \delta 175.8,170.8,167.7,167.0,144.0,141.5,140.9,138.1,136.4,134.2,130.2,129.4,129.1,128.7$, $128.5,128.1,128.0,127.9,127.2,127.1,127.0,125.4,125.2,118.5,75.3,66.1,54.0,50.9,31.4,24.1$, 22.5, 14.3; HRMS (ESI) m/z calcd. for $\mathrm{C}_{38} \mathrm{H}_{38} \mathrm{NO}_{6}{ }^{+} 604.2694$ found 604.2651 . 
Supporting information Amino Acid Schiff Base Bearing Benzophenone Imine as a Platform for Highly Congested Unnatural $\alpha$-Amino Acid Synthesis<smiles>CCOC(=O)C(C)(C(=O)OCC)C(N)(C(=O)OC)C(=O)C(=NC(c1ccccc1)c1ccccc1)c1ccccc1</smiles>

\section{2,2-Diethyl 1-methyl 1-((diphenylmethylene)amino)-1-phenylpropane-1,2,2-tricarboxylate (3an)}

Prepared according to General Method C and purified by flash column chromatography (Hexane/Et $t_{2} \mathrm{O}=$ $5 / 1\left(1 \% \mathrm{Et}_{3} \mathrm{~N}\right), 37 \%$ yield, white solid); IR (neat) $1728,1447,1260,1105 \mathrm{~cm}^{-1} ;{ }^{1} \mathrm{H}^{\mathrm{NMR}}\left(500 \mathrm{MHz}, \mathrm{CDCl}_{3}\right.$, $\left.27^{\circ} \mathrm{C}\right) \delta 7.76(\mathrm{~d}, J=7.5 \mathrm{~Hz}, 2 \mathrm{H}, \operatorname{ArH}), 7.44-7.41(\mathrm{~m}, 3 \mathrm{H}, \operatorname{ArH}), 7.39-7.35(\mathrm{~m}, 2 \mathrm{H}, \operatorname{ArH}), 7.25-7.23(\mathrm{~m}, 4 \mathrm{H}$, $\operatorname{ArH}), 7.15(t, J=7.5 \mathrm{~Hz}, 2 \mathrm{H}, \operatorname{ArH}), 6.77(\mathrm{~d}, J=7.5 \mathrm{~Hz}, 2 \mathrm{H}, \mathrm{ArH}), 4.25-4.19\left(\mathrm{~m}, 2 \mathrm{H}, \mathrm{OCH}_{2} \mathrm{CH}_{3}\right), 4.18-4.11(\mathrm{~m}$, $\left.1 \mathrm{H}, \mathrm{OCH}_{2} \mathrm{CH}_{3}\right), 4.09-3.95\left(\mathrm{~m}, 1 \mathrm{H}, \mathrm{OCH}_{2} \mathrm{CH}_{3}\right), 3.15\left(\mathrm{~s}, 3 \mathrm{H}, \mathrm{OCH}_{3}\right), 1.69\left(\mathrm{~s}, 3 \mathrm{H}, \mathrm{CH}_{3}\right), 1.23(\mathrm{t}, J=7.0 \mathrm{~Hz}, 3 \mathrm{H}$, $\left.\mathrm{OCH}_{2} \mathrm{CH}_{3}\right), 1.17\left(\mathrm{t}, J=7.0 \mathrm{~Hz}, 3 \mathrm{H}, \mathrm{OCH}_{2} \mathrm{CH}_{3}\right) ;{ }^{13} \mathrm{C} \mathrm{NMR}\left(125 \mathrm{MHz}, \mathrm{CDCl}_{3}, 27^{\circ} \mathrm{C}\right) \delta 171.6,170.4,170.4,166.0$, 141.6, 138.9, 136.0, 130.2, 129.7, 129.3, 128.6, 128.5, 127.9, 127.6, 127.2, 127.1, 75.5, 65.2, 61.2, 61.2, 51.4, 19.3, 14.1, 13.7; HRMS (ESI) m/z calcd. for $\mathrm{C}_{30} \mathrm{H}_{32} \mathrm{NO}_{6}{ }^{+} 502.2224$ found 502.2211.

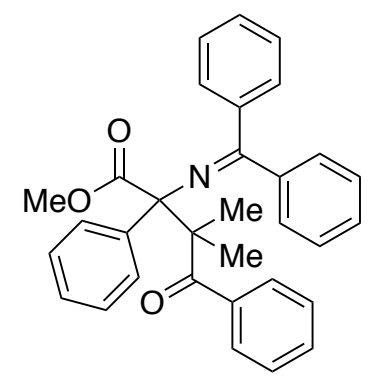

\section{Methyl 2-((diphenylmethylene)amino)-3,3-dimethyl-4-oxo-2,4-diphenylbutanoate (3ao)}

Prepared according to General Method C and purified by flash column chromatography (Hexane/Et ${ }_{2} \mathrm{O}=$ 5/1 (1\% Et 3 N), 69\% yield, white solid); IR (neat) 1674, 1618, 1232, $961 \mathrm{~cm}^{-1} ;{ }^{1} \mathrm{H}$ NMR (500 MHz, $\mathrm{CDCl}_{3}$, $\left.27^{\circ} \mathrm{C}\right) \delta 7.57(\mathrm{~d}, J=7.0 \mathrm{~Hz}, 4 \mathrm{H}, \mathrm{ArH}), 7.43-7.40(\mathrm{~m}, 3 \mathrm{H}, \mathrm{ArH}), 7.37-7.32(\mathrm{~m}, 3 \mathrm{H}, \mathrm{ArH}), 7.30-7.22(\mathrm{~m}, 6 \mathrm{H}$, $\operatorname{ArH}), 7.14(\mathrm{t}, J=7.5 \mathrm{~Hz}, 2 \mathrm{H}, \mathrm{ArH}), 6.75(\mathrm{~d}, J=7.5 \mathrm{~Hz}, 2 \mathrm{H}, \mathrm{ArH}), 3.17\left(\mathrm{~s}, 3 \mathrm{H}, \mathrm{OCH}_{3}\right), 1.72\left(\mathrm{~s}, 3 \mathrm{H}, \mathrm{CH}_{3}\right), 1.25(\mathrm{~s}$, $\left.3 \mathrm{H}, \mathrm{CH}_{3}\right) ;{ }^{13} \mathrm{C}$ NMR $\left(125 \mathrm{MHz}, \mathrm{CDCl}_{3}, 27^{\circ} \mathrm{C}\right) \delta 210.9,171.2,168.1,141.9,141.5,141.4,136.4,130.2,129.8$, 129.8, 129.0, 128.6, 128.4, 127.9, 127.7, 127.2, 127.1, 127.1, 75.7, 59.9, 51.0, 25.5, 24.2; HRMS (ESI) m/z calcd. for $\mathrm{C}_{32} \mathrm{H}_{30} \mathrm{NO}_{3}{ }^{+} 476.2220$ found 476.2195. 
Supporting information Amino Acid Schiff Base Bearing Benzophenone Imine as a Platform for Highly Congested Unnatural $\alpha$-Amino Acid Synthesis<smiles>COC(=O)C(N=C(c1ccccc1)c1ccccc1)(c1ccccc1)C(C)(C)C(=O)N1CCOCC1</smiles>

Methyl 2-((diphenylmethylene)amino)-3,3-dimethyl-4-morpholino-4-oxo-2-phenylbutanoate (3ap)

Prepared according to General Method C and purified by flash column chromatography

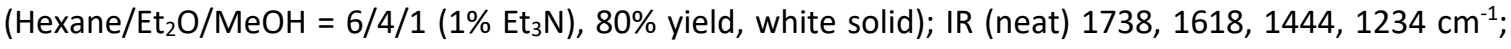
${ }^{1} \mathrm{H} \mathrm{NMR}\left(500 \mathrm{MHz}, \mathrm{CDCl}_{3}, 27^{\circ} \mathrm{C}\right) \delta 7.59(\mathrm{~d}, J=7.0 \mathrm{~Hz}, 2 \mathrm{H}, \mathrm{ArH}), 7.43(\mathrm{t}, J=7.5 \mathrm{~Hz}, 1 \mathrm{H}, \operatorname{Ar} H), 7.37(\mathrm{t}, J=7.5$ $\mathrm{Hz}, 2 \mathrm{H}, \operatorname{ArH}), 7.24-7.18(\mathrm{~m}, 6 \mathrm{H}, \mathrm{ArH}), 7.09(\mathrm{t}, J=7.5 \mathrm{~Hz}, 2 \mathrm{H}, \operatorname{ArH}), 6.59(\mathrm{~d}, J=7.0 \mathrm{~Hz}, 2 \mathrm{H}, \operatorname{ArH}), 3.78(\mathrm{br}$, $\left.4 \mathrm{H}, \mathrm{NCH}_{2}\right), 3.54-3.46\left(\mathrm{~m}, 4 \mathrm{H}, \mathrm{OCH}_{2}\right), 3.22\left(\mathrm{~s}, 3 \mathrm{H}, \mathrm{OCH}_{3}\right), 1.67\left(\mathrm{~s}, 3 \mathrm{H}, \mathrm{CH}_{3}\right), 1.29\left(\mathrm{t}, 3 \mathrm{H}, \mathrm{CH}_{3}\right) ;{ }^{13} \mathrm{C} \mathrm{NMR}(125$ $\left.\mathrm{MHz}_{1} \mathrm{CDCl}_{3}, 27^{\circ} \mathrm{C}\right) \delta 175.5,170.8,169.0,141.9,141.4,136.5,130.4,130.1,128.6,128.3,128.3,128.1$, 127.1, 127.1, 126.9, 75.6, 67.0, 55.3, 50.9, 46.0, 26.1, 24.7; HRMS (ESI) m/z calcd. for $\mathrm{C}_{30} \mathrm{H}_{33} \mathrm{~N}_{2} \mathrm{O}_{4}{ }^{+}$ 485.2435 found 485.2424 .<smiles>COC(=O)C(N=C(c1ccccc1)c1ccccc1)(c1ccccc1)C1(C)CC(=O)N(c2ccccc2)C1</smiles>

Methyl 2-((diphenylmethylene)amino)-2-(3-methyl-2-oxo-1-phenylazetidin-3-yl)-2-phenylacetate (3aq)

Prepared according to General Method C and purified by flash column chromatography (Hexane/Et ${ }_{2} \mathrm{O}=$ $2 / 1\left(1 \% \mathrm{Et}_{3} \mathrm{~N}\right), \mathrm{dr}=1.2 / 1,51 \%$ yield, white solid); IR (neat) 1746, 1599, 1500, $1389 \mathrm{~cm}^{-1} ;{ }^{1} \mathrm{H}$ NMR (500 $\left.\mathrm{MHz} \mathrm{CDCl}_{3}, 27^{\circ} \mathrm{C}\right) \delta 7.54(\mathrm{~d}, J=7.5 \mathrm{~Hz}, 2 \mathrm{H}, \mathrm{ArH}$, major diastereomer), 7.48-7.07 (m, 16H, ArH, major diastereomer), 7.48-7.07 (m, 18H, ArH, minor diastereomer), $6.82(\mathrm{~d}, J=7.5 \mathrm{~Hz}, 2 \mathrm{H}, \operatorname{ArH}$, major diastereomer), $6.77(\mathrm{~d}, J=7.0 \mathrm{~Hz}, 2 \mathrm{H}, \mathrm{ArH}), 4.16-4.14\left(\mathrm{~m}, 2 \mathrm{H}, \mathrm{NCH}_{2}\right.$, minor diastereomer), $3.46(\mathrm{~d}, J=6.0$ $\mathrm{Hz}, 1 \mathrm{H}, \mathrm{NCH}_{2}$, major diastereomer), 3.39 (s, 3H, $\mathrm{OCH}_{3}$, minor diastereomer), 3.33 (s, 3H, OCH $\mathrm{H}_{3}$ major 
Supporting information Amino Acid Schiff Base Bearing Benzophenone Imine as a Platform for Highly Congested Unnatural $\alpha$-Amino Acid Synthesis

diastereomer), $3.28\left(\mathrm{~d}, J=5.5 \mathrm{~Hz}, 1 \mathrm{H}, \mathrm{NCH}_{2}\right.$, major diastereomer), $1.49\left(\mathrm{~s}, 3 \mathrm{H}, \mathrm{CH}_{3}\right.$, major diastereomer), $1.44\left(\mathrm{~s}, 3 \mathrm{H}, \mathrm{CH}_{3}\right.$, minor diastereomer); ${ }^{13} \mathrm{C}$ NMR $\left(125 \mathrm{MHz}, \mathrm{CDCl}_{3}, 2{ }^{\circ} \mathrm{C}\right) \delta 171.0,170.7,169.1,169.0$, 168.1 , 168.0, 141.1, 140.7, 139.6, 138.7, 138.6, 138.5, 136.5, 136.4, 130.3, 130.3, 129.5, 129.1, 129.1, $129.1,129.0,128.8,128.4,128.4,128.3,128.2,127.9,127.9,127.7,127.3,127.3,127.2,123.6,123.5$, $116.4,116.4,75.3,74.5,63.1,62.8,51.8,51.7,51.2,50.4,18.5,17.8$; HRMS (ESI) $\mathrm{m} / \mathrm{z}$ calcd. for $\mathrm{C}_{32} \mathrm{H}_{29} \mathrm{~N}_{2} \mathrm{O}_{3}{ }^{+} 489.2173$ found 489.2163 .<smiles>CC(=O)C(N=C(c1ccccc1)c1ccccc1)(c1ccccc1)C(C)(C)C</smiles>

\section{Methyl 2-((diphenylmethylene)amino)-3,3-dimethyl-2-phenylbutanoate (3ar)}

Prepared according to modified General Method C and purified by flash column chromatography (Hexane/Et ${ }_{2} \mathrm{O}=19 / 1\left(1 \% \mathrm{Et}_{3} \mathrm{~N}\right), 47 \%$ yield, white solid); IR (neat) 1726, 1624, 1246, 1209, 1028, $1003 \mathrm{~cm}$ 1; ${ }^{1} \mathrm{H} \mathrm{NMR}\left(500 \mathrm{MHz}, \mathrm{CDCl}_{3}, 27^{\circ} \mathrm{C}\right) \delta 7.71(\mathrm{~d}, J=7.5 \mathrm{~Hz}, 2 \mathrm{H}, \operatorname{ArH}), 7.67(\mathrm{br}, 2 \mathrm{H}, \operatorname{ArH}), 7.42(\mathrm{t}, J=7.0 \mathrm{~Hz}, 1 \mathrm{H}$, $\operatorname{ArH}), 7.36(\mathrm{t}, J=8.0 \mathrm{~Hz}, 2 \mathrm{H}, \operatorname{ArH}), 7.24-7.22(\mathrm{~m}, 3 \mathrm{H}, \operatorname{ArH}), 7.19(\mathrm{t}, J=7.5 \mathrm{~Hz}, 1 \mathrm{H}, \operatorname{ArH}), 7.10(\mathrm{t}, J=7.5 \mathrm{~Hz}$, $\left.2 \mathrm{H}, \mathrm{ArH}), 6.68(\mathrm{~d}, J=7.5 \mathrm{~Hz}, 2 \mathrm{H}, \mathrm{ArH}), 3.15(\mathrm{~s}, 3 \mathrm{H}, \mathrm{OCH})_{3}\right), 1.07\left(\mathrm{~s}, 3 \mathrm{H}, \mathrm{C}\left(\mathrm{CH}_{3}\right)_{3}\right) ;{ }^{13} \mathrm{C} \mathrm{NMR}\left(125 \mathrm{MHz}, \mathrm{CDCl}_{3}\right.$, $\left.27^{\circ} \mathrm{C}\right) \delta 172.0,166.8,142.6,141.9,137.1,129.9,129.7,128.9,128.8,128.1,127.9,127.1,126.7,126.4$, 76.4, 50.4, 41.5, 27.1; HRMS (ESI) m/z calcd. for $\mathrm{C}_{26} \mathrm{H}_{28} \mathrm{NO}_{2}{ }^{+} 386.2115$ found 386.2114.

\section{5-2. $\beta$-Alkylation}

\section{General Procedure E ( $\beta$-Alkylation)}

In an inert atmosphere glove box, a $4 \mathrm{~mL}$ light resident vial was charged with copper(II) acetate $(0.010$ $\mathrm{mmol}, 10 \mathrm{~mol} \%)$ and $\mathbf{L} 5(0.010 \mathrm{mmol}, 10 \mathrm{~mol} \%)$, followed by tert-butylbenzene $(0.50 \mathrm{~mL})$. After the solution was stirred at ambient temperature for 10 minutes, $\alpha$-amino acid Schiff base $1(0.10 \mathrm{mmol}, 1.0$ equiv.), alkyl bromide 2 ( $0.20 \mathrm{mmol}, 2.0$ equiv.) and $\mathrm{AgOAc}(0.30 \mathrm{mmol}, 3.0$ equiv.) were added and removed from the glovebox. The reaction solution was stirred at $110{ }^{\circ} \mathrm{C}$ for 48 hours. Copper salt was removed by silica gel short pad column chromatography and washed with excess ethyl acetate. Evaporation of the solvent gave the crude product, which was purified by flash column chromatography. 
Supporting information Amino Acid Schiff Base Bearing Benzophenone Imine as a Platform for Highly Congested Unnatural $\alpha$-Amino Acid Synthesis<smiles>CCOC(=O)C(C)(C)/C=C(/N=C(c1ccccc1)c1ccccc1)C(=O)OC</smiles>

\section{5-Ethyl 1-methyl (E)-2-((diphenylmethylene)amino)-4,4-dimethylpent-2-enedioate (4a)}

Prepared according to General Method E and purified by flash column chromatography $\left(\right.$ Hexane/Et ${ }_{2} \mathrm{O}=$ 4/1 (1\% Et 3 N), 81\% yield, yellow solid); IR (neat) 1720, 1595, 1445, 1244, $1136 \mathrm{~cm}^{-1} ;{ }^{1} \mathrm{H} \mathrm{NMR}(500 \mathrm{MHz}$, $\left.\mathrm{CDCl}_{3}, 27^{\circ} \mathrm{C}\right) \delta 7.72(\mathrm{~d}, J=7.5 \mathrm{~Hz}, 2 \mathrm{H}, \mathrm{ArH}), 7.45(\mathrm{t}, J=7.5 \mathrm{~Hz}, 1 \mathrm{H}, \operatorname{ArH})$, 7.38-7.35 (m, 5H, ArH), 7.32-7.30 $(\mathrm{m}, 2 \mathrm{H}, \mathrm{ArH}), 6.18(\mathrm{~s}, 1 \mathrm{H}, \mathrm{CH}), 3.91\left(\mathrm{q}, J=7.5 \mathrm{~Hz}, 2 \mathrm{H}, \mathrm{OCH}_{2} \mathrm{CH}_{3}\right), 3.30\left(\mathrm{~s}, 3 \mathrm{H}, \mathrm{OCH}_{3}\right), 1.47\left(\mathrm{~s}, 6 \mathrm{H}, \mathrm{CH}_{3}\right), 1.07$ (t, $\left.J=7.0 \mathrm{~Hz}, 3 \mathrm{H}, \mathrm{OCH}_{2} \mathrm{CH}_{3}\right) ;{ }^{13} \mathrm{C}$ NMR $\left(125 \mathrm{MHz}, \mathrm{CDCl}_{3}, 27^{\circ} \mathrm{C}\right) \delta 175.9,169.4,164.7,138.7,138.4,137.0$, 133.4, 130.9, 129.6, 129.2, 128.7, 128.0, 127.9, 60.7, 51.4, 43.1, 25.7, 14.0; HRMS (ESI) m/z calcd. for $\mathrm{C}_{23} \mathrm{H}_{26} \mathrm{NO}_{4}{ }^{+} 380.1856$ found 380.1794 .<smiles>COC(=O)/C(=C\C1(C(=O)OC)CCCCC1)N=C(c1ccccc1)c1ccccc1</smiles>

Methyl (E)-1-(2-((diphenylmethylene)amino)-3-methoxy-3-oxoprop-1-en-1-yl)cyclohexane-1carboxylate (4b)

Prepared according to General Method E and purified by flash column chromatography (Hexane/Et ${ }_{2} \mathrm{O}=$ $5 / 1\left(1 \% \mathrm{Et}_{3} \mathrm{~N}\right), 93 \%$ yield, yellow solid); IR (neat) 1721, 1595, 1445, $1242 \mathrm{~cm}^{-1} ;{ }^{1} \mathrm{H} \mathrm{NMR}\left(500 \mathrm{MHz}, \mathrm{CDCl}_{3}\right.$, $\left.27^{\circ} \mathrm{C}\right) \delta 7.71(\mathrm{~d}, J=7.5 \mathrm{~Hz}, 2 \mathrm{H}, \operatorname{ArH}), 7.45(\mathrm{t}, J=7.5 \mathrm{~Hz}, 1 \mathrm{H}, \operatorname{ArH}), 7.41-7.36(\mathrm{~m}, 6 \mathrm{H}, \operatorname{ArH}), 7.27-7.25(\mathrm{~m}$, $1 \mathrm{H}, \operatorname{ArH}), 6.16(\mathrm{~s}, 1 \mathrm{H}, \mathrm{CH}), 3.47\left(\mathrm{~s}, 3 \mathrm{H}, \mathrm{OCH}_{3}\right), 3.30\left(\mathrm{~s}, 3 \mathrm{H}, \mathrm{OCH}_{3}\right), 2.01-1.96\left(\mathrm{~m}, 2 \mathrm{H}, \mathrm{CH}_{2}\right), 1.92-1.87(\mathrm{~m}, 2 \mathrm{H}$, $\left.\mathrm{CH}_{2}\right), 1.59-1.55\left(\mathrm{~m}, 3 \mathrm{H}, \mathrm{CH}_{2}\right), 1.49-1.47\left(\mathrm{~m}, 1 \mathrm{H}, \mathrm{CH}_{2}\right), 1.45-1.36\left(\mathrm{~m}, 1 \mathrm{H}, \mathrm{CH}_{2}\right), 1.33\left(\mathrm{~s}, 1 \mathrm{H}, \mathrm{CH}_{2}\right) ;{ }^{13} \mathrm{C} \mathrm{NMR}$ $\left(125 \mathrm{MHz}_{\mathrm{CDCl}}, 2 \mathrm{C}^{\circ} \mathrm{C}\right) \delta 175.4,169.7,164.7,138.7,138.5,137.0,131.2,130.9,129.6,129.2,128.6$, 128.0, 127.9, 51.8, 51.4, 47.4, 33.9, 25.5, 22.6; HRMS (ESI) m/z calcd. for $\mathrm{C}_{25} \mathrm{H}_{28} \mathrm{NO}_{4}{ }^{+} 406.2013$ found 406.1897. 
Supporting information Amino Acid Schiff Base Bearing Benzophenone Imine as a Platform for Highly Congested Unnatural $\alpha$-Amino Acid Synthesis<smiles>COC(=O)/C(=C\C1(C(=O)OC)CCCC1)N=C(c1ccccc1)c1ccccc1</smiles>

\section{Methyl (E)-1-(2-((diphenylmethylene)amino)-3-methoxy-3-oxoprop-1-en-1-yl)cyclopentane-1- carboxylate (4c)}

Prepared according to General Method E and purified by flash column chromatography $\left(\right.$ Hexane/Et ${ }_{2} \mathrm{O}=$ 5/1 (1\% Et $\left.{ }_{3} \mathrm{~N}\right), 73 \%$ yield, yellow solid); IR (neat) 1721, 1447, $1244 \mathrm{~cm}^{-1} ;{ }^{1} \mathrm{H} \mathrm{NMR}\left(500 \mathrm{MHz}, \mathrm{CDCl}_{3}, 27^{\circ} \mathrm{C}\right)$ $\delta 7.71(\mathrm{~d}, J=7.0 \mathrm{~Hz}, 2 \mathrm{H}, \operatorname{ArH}), 7.45(\mathrm{t}, J=7.5 \mathrm{~Hz}, 1 \mathrm{H}, \operatorname{ArH}), 7.38-7.36(\mathrm{~m}, 5 \mathrm{H}, \operatorname{ArH})$, 7.28-7.27 (m, 2H, $\operatorname{ArH})$, $6.23(\mathrm{~s}, 1 \mathrm{H}, \mathrm{CH}), 3.50\left(\mathrm{~s}, 3 \mathrm{H}, \mathrm{OCH}_{3}\right), 3.34\left(\mathrm{~s}, 3 \mathrm{H}, \mathrm{OCH}_{3}\right), 2.36-2.30\left(\mathrm{~m}, 2 \mathrm{H}, \mathrm{CH}_{2}\right), 1.93-1.87\left(\mathrm{~m}, 2 \mathrm{H}, \mathrm{CH}_{2}\right)$, 1.76-1.68 (m, 4H, $\left.\mathrm{CH}_{2}\right) ;{ }^{13} \mathrm{C}$ NMR $\left(125 \mathrm{MHz}, \mathrm{CDCl}_{3}, 27^{\circ} \mathrm{C}\right) \delta 175.6,169.6,164.7,138.7,138.5,137.0,132.6$, 130.9, 129.6, 129.2, 128.6, 128.1, 127.9, 54.2, 52.0, 51.5, 37.4, 24.6; HRMS (ESI) m/z calcd. for $\mathrm{C}_{24} \mathrm{H}_{26} \mathrm{NO}_{4}{ }^{+} 392.1856$ found 392.1708 .<smiles>COC(=O)/C(=C/C1(C(=O)OC)CCC1)N=C(c1ccccc1)c1ccccc1</smiles>

Methyl (E)-1-(2-((diphenylmethylene)amino)-3-methoxy-3-oxoprop-1-en-1-yl)cyclobutane-1-

\section{carboxylate (4d)}

Prepared according to General Method E and purified by flash column chromatography (Hexane/Et ${ }_{2} \mathrm{O}=$ $5 / 1\left(1 \% \mathrm{Et}_{3} \mathrm{~N}\right), 49 \%$ yield, yellow solid); IR (neat) $1718,1255,1090,1015 \mathrm{~cm}^{-1} ;{ }^{1} \mathrm{H} \mathrm{NMR}\left(500 \mathrm{MHz}, \mathrm{CDCl}_{3}\right.$, $\left.27^{\circ} \mathrm{C}\right) \delta 7.62(\mathrm{~d}, J=7.5 \mathrm{~Hz}, 2 \mathrm{H}, \operatorname{ArH}), 7.38(\mathrm{t}, J=7.5 \mathrm{~Hz}, 1 \mathrm{H}, \operatorname{ArH}), 7.31-7.28(\mathrm{~m}, 5 \mathrm{H}, \operatorname{ArH}), 7.14-7.12(\mathrm{~m}$, $2 \mathrm{H}, \mathrm{ArH}), 6.21(\mathrm{~s}, 1 \mathrm{H}, \mathrm{CH}), 3.46\left(\mathrm{~s}, 3 \mathrm{H}, \mathrm{OCH}_{3}\right), 3.42\left(\mathrm{~s}, 3 \mathrm{H}, \mathrm{OCH}_{3}\right), 2.52-2.47\left(\mathrm{~m}, 2 \mathrm{H}, \mathrm{CH}_{2}\right), 2.34-2.28(\mathrm{~m}, 2 \mathrm{H}$, $\left.\mathrm{CH}_{2}\right), 1.94-1.81\left(\mathrm{~m}, 2 \mathrm{H}, \mathrm{CH}_{2}\right) ;{ }^{13} \mathrm{C}$ NMR $\left(125 \mathrm{MHz}, \mathrm{CDCl}_{3}, 27^{\circ} \mathrm{C}\right) \delta 174.0,169.1,163.7,137.6,137.4,135.7$, 129.9, 128.5, 128.1, 127.5, 127.4, 127.0, 126.9, 51.0, 50.8, 46.9, 31.1, 15.3; HRMS (ESI) m/z calcd. for $\mathrm{C}_{23} \mathrm{H}_{24} \mathrm{NO}_{4}{ }^{+} 378.1700$ found 378.1674 . 
Supporting information Amino Acid Schiff Base Bearing Benzophenone Imine as a Platform for Highly Congested Unnatural $\alpha$-Amino Acid Synthesis<smiles>COC(=O)/C(=C\C1(C(=O)OCc2ccccc2)CCCC1)N=C(c1ccccc1)c1ccccc1</smiles>

Benzyl (E)-1-(2-((diphenylmethylene)amino)-3-methoxy-3-oxoprop-1-en-1-yl)cyclopentane-1carboxylate (4e)

Prepared according to General Method E and purified by flash column chromatography (Hexane/Et ${ }_{2} \mathrm{O}=$ 5/1 (1\% Et $3 \mathrm{~N}), 77 \%$ yield, yellow solid); IR (neat) $1721,1445,1242,1163 \mathrm{~cm}^{-1} ;{ }^{1} \mathrm{H} \mathrm{NMR}\left(500 \mathrm{MHz}, \mathrm{CDCl}_{3}\right.$, $\left.27^{\circ} \mathrm{C}\right) \delta 7.70(\mathrm{~d}, J=7.0 \mathrm{~Hz}, 2 \mathrm{H}, \operatorname{ArH}), 7.44(\mathrm{t}, J=7.0 \mathrm{~Hz}, 1 \mathrm{H}, \operatorname{ArH}), 7.37-7.31(\mathrm{~m}, 5 \mathrm{H}, \operatorname{ArH}), 7.29-7.24(\mathrm{~m}$, $5 \mathrm{H}, \mathrm{ArH}), 7.19-7.18(\mathrm{~m}, 2 \mathrm{H}, \mathrm{ArH}), 6.25(\mathrm{~s}, 1 \mathrm{H}, \mathrm{CH}), 4.93\left(\mathrm{~s}, 2 \mathrm{H}, \mathrm{OCH}_{2}\right), 3.33\left(\mathrm{~s}, 3 \mathrm{H}, \mathrm{OCH}_{3}\right), 2.86-2.33(\mathrm{~m}, 2 \mathrm{H}$, $\left.\mathrm{CH}_{2}\right)$, 1.94-1.89 (m, 2H, CH$), 1.74-1.65\left(\mathrm{~m}, 4 \mathrm{H}, \mathrm{CH}_{2}\right) ;{ }^{13} \mathrm{C} \mathrm{NMR}\left(125 \mathrm{MHz}, \mathrm{CDCl}_{3}, 27^{\circ} \mathrm{C}\right) \delta 174.9,169.6$, $164.8,138.7,138.7,137.0,136.3,132.8,130.9,129.6,129.2,128.7,128.4,128.1,127.8,66.5,54.4,51.5$, 37.4, 24.6; HRMS (ESI) m/z calcd. for $\mathrm{C}_{30} \mathrm{H}_{30} \mathrm{NO}_{4}{ }^{+} 468.2169$ found 468.2166.

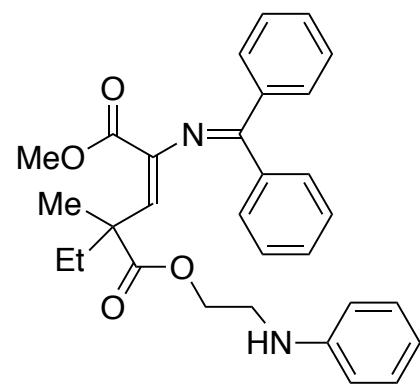

1-Methyl 5-(2-(phenylamino)ethyl) (E)-2-((diphenylmethylene)amino)-4-ethyl-4-methylpent-2enedioate (4f)

Prepared according to General Method E and purified by flash column chromatography (Hexane/Et ${ }_{2} \mathrm{O}=$ $5 / 1\left(1 \% \mathrm{Et}_{3} \mathrm{~N}\right), 57 \%$ yield, yellow solid); IR (neat) $1722,1602,1508,1250 \mathrm{~cm}^{-1} ;{ }^{1} \mathrm{H} \mathrm{NMR}\left(500 \mathrm{MHz}, \mathrm{CDCl}_{3}\right.$, $\left.27^{\circ} \mathrm{C}\right) \delta 7.69(\mathrm{~d}, J=7.5 \mathrm{~Hz}, 2 \mathrm{H}, \operatorname{ArH}), 7.43(\mathrm{t}, J=7.5 \mathrm{~Hz}, 1 \mathrm{H}, \operatorname{ArH}), 7.39-7.33(\mathrm{~m}, 5 \mathrm{H}, \operatorname{ArH}), 7.30-7.28(\mathrm{~m}$, $2 \mathrm{H}, \operatorname{ArH}), 7.13(\mathrm{t}, J=7.5 \mathrm{~Hz}, 2 \mathrm{H}, \operatorname{ArH}), 6.68(\mathrm{t}, J=7.5 \mathrm{~Hz}, 1 \mathrm{H}, \operatorname{ArH}), 6.54(\mathrm{~d}, J=8.5 \mathrm{~Hz}, \operatorname{ArH}), 6.19(\mathrm{~s}, 1 \mathrm{H}$, $\mathrm{CH}), 4.22-4.18\left(\mathrm{~m}, 1 \mathrm{H}, \mathrm{OCH}_{2}\right), 4.12-4.08\left(\mathrm{~m}, 1 \mathrm{H}, \mathrm{OCH}_{2}\right), 3.94(\mathrm{~s}, 1 \mathrm{H}, \mathrm{NH}), 3.31\left(\mathrm{~s}, 3 \mathrm{H}, \mathrm{OCH}_{3}\right), 3.28-3.25(\mathrm{~m}$, $2 \mathrm{H}, \mathrm{NCH}_{2}$ ), 1.97-1.89 (m, 1H, $\left.\mathrm{CH}_{2} \mathrm{CH}_{3}\right), 1.83-1.76\left(\mathrm{~m}, 1 \mathrm{H}, \mathrm{CH}_{2} \mathrm{CH}_{3}\right), 1.41\left(\mathrm{~s}, 3 \mathrm{H}, \mathrm{CH}_{3}\right), 0.92(\mathrm{t}, J=7.5 \mathrm{~Hz}, 3 \mathrm{H}$, $\left.\mathrm{CH}_{2} \mathrm{CH}_{3}\right) ;{ }^{13} \mathrm{C}$ NMR $\left(125 \mathrm{MHz}, \mathrm{CDCl}_{3}, 27^{\circ} \mathrm{C}\right) \delta 175.2,170.1,164.7,147.8,138.8,138.7,137.1,132.8,131.0$, 
129.6, 129.4, 129.2, 128.6, 128.1, 128.0, 117.5, 112.9, 63.3, 51.5, 47.1, 42.6, 32.5, 21.4, 8.9; HRMS (ESI) $\mathrm{m} / \mathrm{z}$ calcd. for $\mathrm{C}_{30} \mathrm{H}_{33} \mathrm{~N}_{2} \mathrm{O}_{4}{ }^{+} 485.2435$ found 485.2457 .

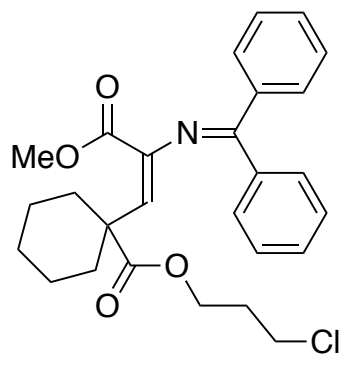

3-Chloropropyl (E)-1-(2-((diphenylmethylene)amino)-3-methoxy-3-oxoprop-1-en-1-yl)cyclohexane-1carboxylate (4g)

Prepared according to General Method E and purified by flash column chromatography $\left(\right.$ Hexane/Et $_{2} \mathrm{O}=$ $4 / 1\left(1 \% \mathrm{Et}_{3} \mathrm{~N}\right), 81 \%$ yield, yellow solid); IR (neat) $1720,1622,1447,1244 \mathrm{~cm}^{-1} ;{ }^{1} \mathrm{H} \mathrm{NMR}\left(500 \mathrm{MHz}, \mathrm{CDCl}_{3}\right.$ $\left.27{ }^{\circ} \mathrm{C}\right) \delta 7.69(\mathrm{~d}, J=7.5 \mathrm{~Hz}, 2 \mathrm{H}, \operatorname{ArH}), 7.46(\mathrm{t}, J=7.5 \mathrm{~Hz}, 1 \mathrm{H}, \operatorname{ArH}), 7.39-7.36(\mathrm{~m}, 5 \mathrm{H}, \operatorname{ArH}), 7.25-7.24(\mathrm{~m}$, $2 \mathrm{H}, \mathrm{ArH}), 6.16(\mathrm{~s}, 1 \mathrm{H}, \mathrm{CH}), 4.02\left(\mathrm{t}, J=6.0 \mathrm{~Hz}, 2 \mathrm{H}, \mathrm{OCH}_{2}\right), 3.42\left(\mathrm{t}, J=7.0 \mathrm{~Hz}, 2 \mathrm{H}, \mathrm{CH}_{2} \mathrm{Cl}\right), 3.32\left(\mathrm{~s}, 3 \mathrm{H}, \mathrm{OCH}_{3}\right)$, 2.00-1.95 (m, 2H, CH $)$, 1.92-1.86 (m, 4H, $\left.\mathrm{CH}_{2}\right), 1.62-1.48\left(\mathrm{~m}, 5 \mathrm{H}, \mathrm{CH}_{2}\right), 1.44-1.39\left(\mathrm{~m}, 1 \mathrm{H}, \mathrm{CH}_{2}\right) ;{ }^{13} \mathrm{C} \mathrm{NMR}$ $\left(125 \mathrm{MHz} \mathrm{CDCl}_{3}, 2{ }^{\circ} \mathrm{C}\right) \delta 174.8,169.8,164.7,138.8,138.8,137.0,131.3,131.0,129.6,129.3,128.6$, 128.1, 127.9, 61.3, 51.5, 47.5, 41.3, 34.0, 31.5, 25.5, 22.6; HRMS (ESI) m/z calcd. for $\mathrm{C}_{27} \mathrm{H}_{31} \mathrm{CINO}_{4}{ }^{+}$ 468.1936 found 468.1952 .

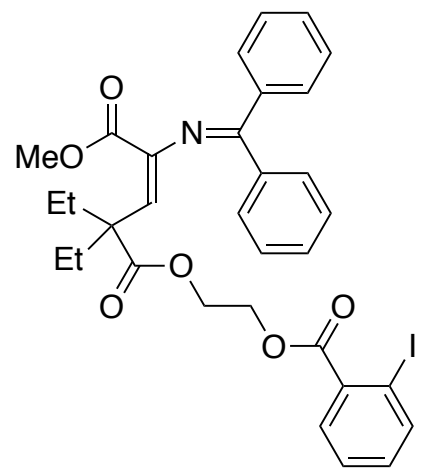

5-(2-((2-lodobenzoyl)oxy)ethyl) 1-methyl (E)-2-((diphenylmethylene)amino)-4,4-diethylpent-2-

\section{enedioate (4h)}

Prepared according to General Method E and purified by flash column chromatography (Hexane/Et $t_{2} \mathrm{O}=$ 2/1 (1\% Et $\left.{ }_{3} \mathrm{~N}\right), 45 \%$ yield, yellow solid); IR (neat) 1720, 1249, $1132 \mathrm{~cm}^{-1} ;{ }^{1} \mathrm{H} \mathrm{NMR}\left(500 \mathrm{MHz}, \mathrm{CDCl}_{3}, 27^{\circ} \mathrm{C}\right)$ $\delta 7.98(\mathrm{~d}, J=7.5 \mathrm{~Hz}, 1 \mathrm{H}, \operatorname{ArH}), 7.79-7.76(\mathrm{~m}, 1 \mathrm{H}, \operatorname{ArH}), 7.70(\mathrm{t}, J=7.5 \mathrm{~Hz}, 2 \mathrm{H}, \operatorname{ArH}), 7.45(\mathrm{t}, J=7.5 \mathrm{~Hz}, 1 \mathrm{H}$, 
$\operatorname{ArH})$, 7.39-7.34 (m, 6H, ArH), 7.33-7.27 (m, 2H, ArH), $7.15(\mathrm{t}, J=7.5 \mathrm{~Hz}, 1 \mathrm{H}, \operatorname{ArH}), 6.23(\mathrm{~s}, 1 \mathrm{H}, \mathrm{CH}), 4.40-$ $\left.\left.4.37(\mathrm{~m}, 2 \mathrm{H}, \mathrm{OCH})_{2}\right), 4.23-4.20(\mathrm{~m}, 2 \mathrm{H}, \mathrm{OCH})_{2}\right), 3.28\left(\mathrm{~s}, 3 \mathrm{H}, \mathrm{OCH}_{3}\right), 2.03-1.91\left(\mathrm{~m}, 4 \mathrm{H}, \mathrm{CH}_{2} \mathrm{CH}_{3}\right), 0.87(\mathrm{t}, J=$ $\left.7.5 \mathrm{~Hz}, 6 \mathrm{H}, \mathrm{CH}_{2} \mathrm{CH}_{3}\right) ;{ }^{13} \mathrm{C} \mathrm{NMR}\left(125 \mathrm{MHz}, \mathrm{CDCl}_{3}, 27^{\circ} \mathrm{C}\right) \delta 174.7,169.3,166.0,164.6,141.4,138.8,138.6$, 137.0, 134.4, 132.8, 132.7, 131.5, 131.3, 131.2, 131.0, 129.6, 129.3, 128.7, 128.1, 127.9, 63.2, 62.1, 51.8, 51.5, 28.0, 9.0; HRMS (ESI) m/z calcd. for $\mathrm{C}_{33} \mathrm{H}_{33} \mathrm{INO}_{6}{ }^{+} 654.1347$ found 654.1342 .

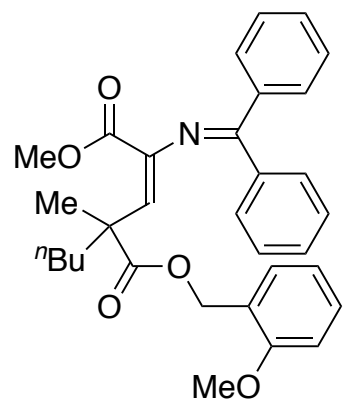

\section{5-(2-Methoxybenzyl) 1-methyl (E)-4-butyl-2-((diphenylmethylene)amino)-4-methylpent-2-enedioate}

\section{(4i)}

Prepared according to General Method $\mathrm{E}$ and purified by flash column chromatography (Hexane/Et $\mathrm{t}_{2} \mathrm{O}=$ $4 / 1\left(1 \% \mathrm{Et}_{3} \mathrm{~N}\right), 75 \%$ yield, yellow solid); IR (neat) $1721,1605,1495,1244,1136 \mathrm{~cm}^{-1} ;{ }^{1} \mathrm{H} \mathrm{NMR}(500 \mathrm{MHz}$, $\left.\mathrm{CDCl}_{3}, 27^{\circ} \mathrm{C}\right) \delta 7.72(\mathrm{~d}, J=7.5 \mathrm{~Hz}, 2 \mathrm{H}, \mathrm{ArH}), 7.42(\mathrm{t}, J=7.5 \mathrm{~Hz}, 1 \mathrm{H}, \mathrm{ArH}), 7.34(\mathrm{t}, J=7.5 \mathrm{~Hz}, 5 \mathrm{H}, \operatorname{ArH}), 7.27$ $(\mathrm{d}, J=8.0 \mathrm{~Hz}, 2 \mathrm{H}, \operatorname{ArH}), 7.24(\mathrm{dd}, J=8.0,1.5 \mathrm{~Hz}, 1 \mathrm{H}, \operatorname{ArH}), 7.16(\mathrm{~d}, J=7.5 \mathrm{~Hz}, 1 \mathrm{H}, \operatorname{ArH}), 6.85(\mathrm{t}, J=7.5 \mathrm{~Hz}$, $1 \mathrm{H}, \operatorname{ArH}), 6.82(\mathrm{~d}, J=8.0 \mathrm{~Hz}, 1 \mathrm{H}, \operatorname{ArH}), 6.24(\mathrm{~s}, 1 \mathrm{H}, \mathrm{CH}), 4.97(\mathrm{q}, J=13.0 \mathrm{~Hz}, 2 \mathrm{H}, \mathrm{OCH}), 3.75(\mathrm{~s}, 3 \mathrm{H}$, $\left.\operatorname{ArOCH}_{3}\right), 3.29\left(\mathrm{~s}, 3 \mathrm{H}, \mathrm{OCH}_{3}\right), 1.92\left(\mathrm{td}, J=13.5,4.5 \mathrm{~Hz}, 1 \mathrm{H}, \mathrm{CH}_{2}\right), 1.77\left(\mathrm{td}, J=13.0,4.0 \mathrm{~Hz}, 1 \mathrm{H}, \mathrm{CH}_{2}\right), 1.48(\mathrm{~s}$, $\left.3 \mathrm{H}, \mathrm{CH}_{3}\right), 1.36-1.19\left(\mathrm{~m}, 4 \mathrm{H}, \mathrm{CH}_{2}\right), 0.87\left(\mathrm{t}, J=7.0 \mathrm{~Hz}, 3 \mathrm{H}, \mathrm{CH}_{2} \mathrm{CH}_{3}\right) ;{ }^{13} \mathrm{C} \mathrm{NMR}\left(125 \mathrm{MHz}, \mathrm{CDCl}_{3}, 27{ }^{\circ} \mathrm{C}\right) \delta$ 175.2, 169.3, 164.9, 157.3, 138.7, 138.5, 137.0, 132.9, 130.8, 129.7, 129.4, 129.2, 129.1, 128.8, 128.0, $127.8,124.6,120.2,110.2,62.0,55.2,51.4,47.2,39.5,26.7,23.2,21.9,14.0 ;$ HRMS (ESI) m/z calcd. for $\mathrm{C}_{32} \mathrm{H}_{36} \mathrm{NO}_{5}{ }^{+} 514.2588$ found 514.2617 .<smiles>COC(=O)/C(=C\C(C(=O)OCCBr)(C(C)(F)F)C(C)(F)F)N=C(c1ccccc1)c1ccccc1</smiles>

5-(2-Bromoethyl) 1-methyl (E)-2-((diphenylmethylene)amino)-4,4-dipropylpent-2-enedioate (4j) 
Prepared according to General Method E and purified by flash column chromatography (Hexane/Et ${ }_{2} \mathrm{O}=$ 4/1 (1\% Et $\left.\mathrm{t}_{3} \mathrm{~N}\right), 46 \%$ yield, yellow solid); IR (neat) $1726,1236,1209,1136 \mathrm{~cm}^{-1} ;{ }^{1} \mathrm{H} \mathrm{NMR}\left(500 \mathrm{MHz}, \mathrm{CDCl}_{3}\right.$, $\left.27^{\circ} \mathrm{C}\right) \delta 7.70(\mathrm{~d}, J=7.5 \mathrm{~Hz}, 2 \mathrm{H}, \operatorname{ArH}), 7.46(\mathrm{t}, J=7.5 \mathrm{~Hz}, 1 \mathrm{H}, \operatorname{ArH}), 7.40-7.37(\mathrm{~m}, 5 \mathrm{H}, \mathrm{ArH}), 7.28-7.27(\mathrm{~m}$, $2 \mathrm{H}, \mathrm{ArH}), 6.26(\mathrm{~s}, 1 \mathrm{H}, \mathrm{CH}), 4.16\left(\mathrm{t}, J=6.5 \mathrm{~Hz}, 2 \mathrm{H}, \mathrm{OCH}\right.$ ), $3.33\left(\mathrm{t}, J=6.5 \mathrm{~Hz}, 2 \mathrm{H}, \mathrm{CH}_{2} \mathrm{Br}\right), 3.29\left(\mathrm{~s}, 3 \mathrm{H}, \mathrm{OCH}_{3}\right)$, 1.97-1.91 (m, 2H, $\left.\mathrm{CH}_{2}\right), 1.87-1.85\left(\mathrm{~m}, 2 \mathrm{H}, \mathrm{CH}_{2}\right), 1.36-1.26\left(\mathrm{~m}, 4 \mathrm{H}, \mathrm{CH}_{2}\right), 0.94-0.91\left(\mathrm{~m}, 6 \mathrm{H}, \mathrm{CH}_{3}\right) ;{ }^{13} \mathrm{C} \mathrm{NMR}$ $\left(125 \mathrm{MHz}, \mathrm{CDCl}_{3}, 27^{\circ} \mathrm{C}\right) \delta 174.7,169.3,164.6,138.8,138.3,137.0,131.8,131.0,129.6,129.3,128.7$, 128.1 127.9, 63.9, 51.5, 51.2, 38.2, 28.7, 18.0, 14.6; HRMS (ESI) m/z calcd. for $\mathrm{C}_{27} \mathrm{H}_{33} \mathrm{BrNO}_{4}{ }^{+} 514.1587$ found 514.1584 .

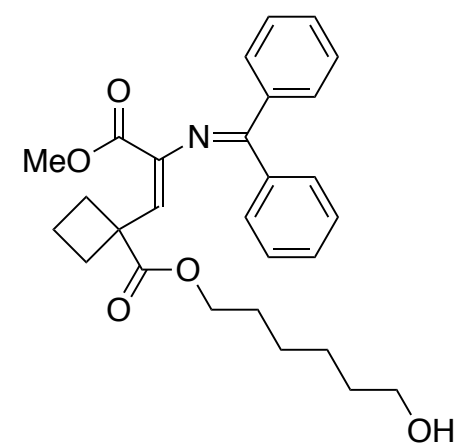

6-Hydroxyhexyl (E)-1-(2-((diphenylmethylene)amino)-3-methoxy-3-oxoprop-1-en-1-yl)cyclobutane-1carboxylate (4k)

Prepared according to General Method $\mathrm{E}$ and purified by flash column chromatography (Hexane/Et $2 \mathrm{O}=$ 2/1 (1\% Et $3 \mathrm{~N}), 24 \%$ yield, yellow solid); IR (neat) 2357, 1722, $1240 \mathrm{~cm}^{-1} ;{ }^{1} \mathrm{H} \mathrm{NMR}\left(500 \mathrm{MHz}, \mathrm{CDCl}_{3}, 27^{\circ} \mathrm{C}\right.$ ) $\delta 7.69(\mathrm{~d}, J=7.5 \mathrm{~Hz}, 2 \mathrm{H}, \mathrm{ArH}), 7.44(\mathrm{t}, J=7.5 \mathrm{~Hz}, 1 \mathrm{H}, \mathrm{ArH}), 7.38-7.35(\mathrm{~m}, 5 \mathrm{H}, \mathrm{ArH}), 7.21-7.19(\mathrm{~m}, 2 \mathrm{H}, \mathrm{ArH})$, $6.28(\mathrm{~s}, 1 \mathrm{H}, \mathrm{ArCH}), 3.93(\mathrm{t}, J=7.0 \mathrm{~Hz}, 2 \mathrm{H}, \mathrm{OCH}), 3.60\left(\mathrm{~m}, 2 \mathrm{H}, \mathrm{OCH}_{2}\right), 3.48\left(\mathrm{~s}, 3 \mathrm{H}, \mathrm{OCH}_{3}\right), 2.58-2.53(\mathrm{~m}, 2 \mathrm{H}$, $\left.\mathrm{CH}_{2}\right), 2.43-2.37\left(\mathrm{~m}, 2 \mathrm{H}, \mathrm{CH}_{2}\right), 1.98-1.89\left(\mathrm{~m}, 2 \mathrm{H}, \mathrm{CH}_{2}\right), 1.31-1.26\left(\mathrm{~m}, 8 \mathrm{H}, \mathrm{CH}_{2}\right) ;{ }^{13} \mathrm{C} \mathrm{NMR}\left(125 \mathrm{MHz}, \mathrm{CDCl}_{3}\right.$, $\left.27^{\circ} \mathrm{C}\right) \delta 174.6,169.9,164.8,138.6,138.4,136.7,130.9,129.5,129.1,128.9,128.4,128.1,127.9,64.8$, 62.8, 51.8, 48.2, 32.6, 32.1, 28.4, 25.6, 25.3, 16.4; HRMS (ESI) m/z calcd. for $\mathrm{C}_{28} \mathrm{H}_{34} \mathrm{NO}_{5}{ }^{+} 464.2431$ found 464.2430 . 
Supporting information Amino Acid Schiff Base Bearing Benzophenone Imine as a Platform for Highly Congested Unnatural $\alpha$-Amino Acid Synthesis

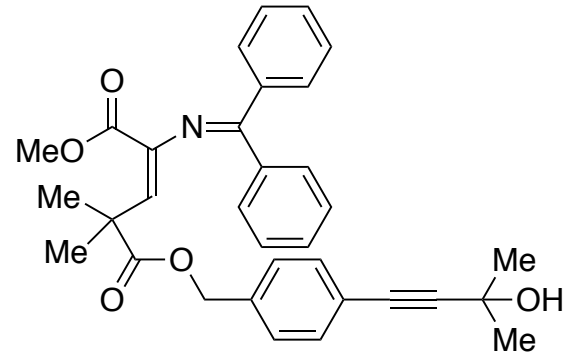

5-(4-(3-hydroxy-3-methylbut-1-yn-1-yl)benzyl) 1-methyl (E)-2-((diphenylmethylene)amino)-4,4dimethylpent-2-enedioate (4I)

Prepared according to General Method E and purified by flash column chromatography (Hexane/Et ${ }_{2} \mathrm{O}=$ $1 / 1\left(1 \% \mathrm{Et}_{3} \mathrm{~N}\right), 80 \%$ yield, yellow solid); IR (neat) $1721,1445,1246,1132 \mathrm{~cm}^{-1} ;{ }^{1} \mathrm{H} \mathrm{NMR}\left(500 \mathrm{MHz}, \mathrm{CDCl}_{3}\right.$, $\left.27^{\circ} \mathrm{C}\right) \delta 7.68(\mathrm{~d}, J=7.0 \mathrm{~Hz}, 2 \mathrm{H}, \operatorname{ArH}), 7.44(\mathrm{t}, J=7.5 \mathrm{~Hz}, 1 \mathrm{H}, \operatorname{ArH}), 7.37-7.30(\mathrm{~m}, 7 \mathrm{H}, \operatorname{ArH}), 7.27-7.25(\mathrm{~m}$, $2 \mathrm{H}, \mathrm{ArH}), 7.10(\mathrm{~d}, J=8.5 \mathrm{~Hz}, 2 \mathrm{H}, \mathrm{ArH}), 6.18(\mathrm{~s}, 1 \mathrm{H}, \mathrm{CH}), 4.87\left(\mathrm{~s}, 2 \mathrm{H}, \mathrm{OCH}_{2}\right), 3.29\left(\mathrm{~s}, 3 \mathrm{H}, \mathrm{OCH}_{3}\right), 1.61(\mathrm{~s}, 6 \mathrm{H}$, $\left.\mathrm{CH}_{3}\right), 1.48\left(\mathrm{~s}, 6 \mathrm{H}, \mathrm{CH}_{3}\right) ;{ }^{13} \mathrm{C} \mathrm{NMR}\left(125 \mathrm{MHz} \mathrm{CDCl}_{3}, 27^{\circ} \mathrm{C}\right) \delta 175.6,169.7,164.7$ 138.7, 138.6, 137.0, 136.4, 133.4, 131.6, 131.0, 129.6, 129.3, 128.7, 128.1 127.9, 127.6, 122.3, 94.0, 81.9, 66.0, 65.6, 51.5, 43.2, 31.5, 25.8; $\mathrm{HRMS}$ (ESI) m/z calcd. for $\mathrm{C}_{33} \mathrm{H}_{34} \mathrm{NO}_{5}{ }^{+} 524.2431$ found 524.2450.

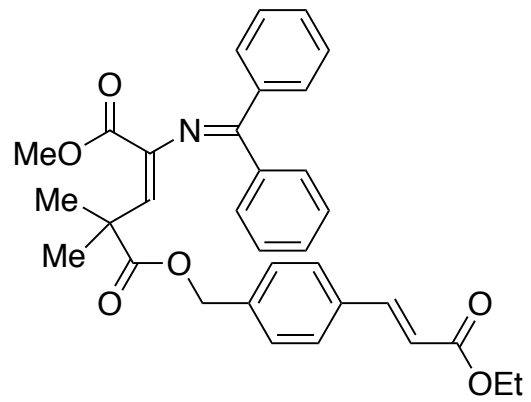

5-(4-((E)-3-Ethoxy-3-oxoprop-1-en-1-yl)benzyl) 1-methyl (E)-2-((diphenylmethylene)amino)-4,4dimethylpent-2-enedioate $(4 \mathrm{~m})$

Prepared according to General Method E and purified by flash column chromatography $\left(\right.$ Hexane/Et $_{2} \mathrm{O}=$ 2/1 (1\% Et $\left.{ }_{3} \mathrm{~N}\right), 56 \%$ yield, yellow solid); IR (neat) 1712, 1636, 1445, 1246, 1155, $1130 \mathrm{~cm}^{-1} ;{ }^{1} \mathrm{H}$ NMR (500 $\left.\mathrm{MHz}_{\mathrm{CDCl}}, 27^{\circ} \mathrm{C}\right) \delta 7.68(\mathrm{~d}, J=7.5 \mathrm{~Hz}, 2 \mathrm{H}, \mathrm{ArH}), 7.64(\mathrm{~d}, J=16.0 \mathrm{~Hz}, 1 \mathrm{H}, \operatorname{ArCH}), 7.45-7.41(\mathrm{~m}, 3 \mathrm{H}, \operatorname{ArH})$, 7.36-7.32 (m, 5H, ArH), 7.27-7.24 (m, 2H, ArH), $7.18(\mathrm{~d}, J=8.5 \mathrm{~Hz}, 2 \mathrm{H}, \operatorname{ArH}), 6.40(\mathrm{~d}, J=16.0 \mathrm{~Hz}, 1 \mathrm{H}$, $\operatorname{ArCHCH}), 6.19(\mathrm{~s}, 1 \mathrm{H}, \mathrm{CH}), 4.90\left(\mathrm{~s}, 2 \mathrm{H}, \mathrm{OCH}_{2}\right), 4.27\left(\mathrm{q}, J=7.0 \mathrm{~Hz}, 2 \mathrm{H}, \mathrm{OCH}_{2} \mathrm{CH}_{3}\right), 3.29\left(\mathrm{~s}, 3 \mathrm{H}, \mathrm{OCH}_{3}\right), 1.50(\mathrm{~s}$, $\left.6 \mathrm{H}, \mathrm{CH}_{3}\right), 1.34\left(\mathrm{t}, \mathrm{J}=7.0 \mathrm{~Hz}, 3 \mathrm{H}, \mathrm{OCH}_{2} \mathrm{CH}_{3}\right) ;{ }^{13} \mathrm{C} \mathrm{NMR}\left(125 \mathrm{MHz}, \mathrm{CDCl}_{3}, 27{ }^{\circ} \mathrm{C}\right) \delta 175.6,169.7,167.0,164.7$, 144.1, 138.7, 138.7, 138.5, 137.0, 134.0, 133.4, 131.0, 129.6, 129.3, 128.7, 128.1, 128.1, 128.1, 127.9, 118.4, 65.9, 60.6, 51.5, 43.2, 25.8, 14.4; HRMS (ESI) m/z calcd. for $\mathrm{C}_{33} \mathrm{H}_{34} \mathrm{NO}_{6}{ }^{+} 540.2381$ found 540.2383. 
<smiles>CCOC(=O)C(C)(C=C(N=C(c1ccccc1)c1ccccc1)C(=O)OC)C(=O)OC</smiles>

\section{3,3-Diethyl 1-methyl (E)-1-((diphenylmethylene)amino)but-1-ene-1,3,3-tricarboxylate (4n)}

Prepared according to General Method $\mathrm{E}$ and purified by flash column chromatography (Hexane/Et ${ }_{2} \mathrm{O}=$ 5/1 (1\% Et $3 \mathrm{~N}), 63 \%$ yield, yellow solid); IR (neat) 1724, 1595, 1445, 1215, $1119 \mathrm{~cm}^{-1} ;{ }^{1} \mathrm{H} \mathrm{NMR}(500 \mathrm{MHz}$, $\left.\mathrm{CDCl}_{3}, 27^{\circ} \mathrm{C}\right) \delta 7.71(\mathrm{~d}, J=7.0 \mathrm{~Hz}, 2 \mathrm{H}, \mathrm{ArH}), 7.46(\mathrm{t}, J=7.0 \mathrm{~Hz}, 1 \mathrm{H}, \operatorname{ArH}), 7.39-7.36(\mathrm{~m}, 5 \mathrm{H}, \mathrm{ArH}), 7.32-7.30$ $(\mathrm{m}, 2 \mathrm{H}, \mathrm{ArH}), 6.70(\mathrm{~s}, 1 \mathrm{H}, \mathrm{CH}), 4.12-4.05\left(\mathrm{~m}, 4 \mathrm{H}, \mathrm{OCH}_{2} \mathrm{CH}_{3}\right), 3.29\left(\mathrm{~s}, 3 \mathrm{H}, \mathrm{OCH}_{3}\right), 1.74\left(\mathrm{~s}, 3 \mathrm{H}, \mathrm{CH}_{3}\right), 1.16(\mathrm{t}, J$ $\left.=7.0 \mathrm{~Hz}, 6 \mathrm{H}, \mathrm{OCH}_{2} \mathrm{CH}_{3}\right) ;{ }^{13} \mathrm{C}$ NMR $\left(125 \mathrm{MHz}, \mathrm{CDCl}_{3}, 2{ }^{\circ} \mathrm{C}\right) \delta 170.6,170.0,164.2,139.4,138.5,136.9$, $131.1,129.8,129.4,128.9,128.0,127.9,127.3,61.7,54.8,51.5,20.9,13.9$; HRMS (ESI) m/z calcd. for $\mathrm{C}_{25} \mathrm{H}_{28} \mathrm{NO}_{6}{ }^{+} 438.1911$ found 438.1912 .

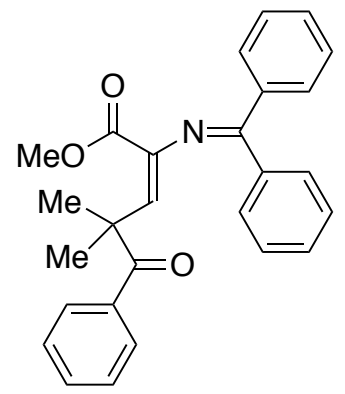

\section{Methyl (E)-2-((diphenylmethylene)amino)-4,4-dimethyl-5-oxo-5-phenylpent-2-enoate (40)}

Prepared according to General Method E and purified by flash column chromatography (Hexane/Et ${ }_{2} \mathrm{O}=$

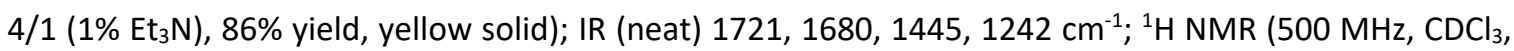
$\left.27^{\circ} \mathrm{C}\right) \delta 7.94(\mathrm{~d}, J=7.0 \mathrm{~Hz}, 2 \mathrm{H}, \operatorname{ArH}), 7.47(\mathrm{t}, J=7.5 \mathrm{~Hz}, 1 \mathrm{H}, \operatorname{ArH}), 7.43-7.40(\mathrm{~m}, 3 \mathrm{H}, \operatorname{ArH}), 7.36(\mathrm{t}, J=7.5$ $\mathrm{Hz}, 2 \mathrm{H}, \operatorname{ArH}), 7.30(\mathrm{t}, J=7.5 \mathrm{~Hz}, 3 \mathrm{H}, \operatorname{ArH}), 7.25(\mathrm{t}, J=7.5 \mathrm{~Hz}, 2 \mathrm{H}, \operatorname{ArH}), 6.89(\mathrm{~d}, J=7.5 \mathrm{~Hz}, 2 \mathrm{H}, \operatorname{ArH}), 6.56(\mathrm{~s}$, $\left.1 \mathrm{H}, \mathrm{CH}), 3.27\left(\mathrm{~s}, 3 \mathrm{H}, \mathrm{OCH}_{3}\right), 1.54\left(\mathrm{~s}, 6 \mathrm{H}, \mathrm{CH}_{3}\right) ;{ }^{13} \mathrm{C} \mathrm{NMR} \mathrm{(125} \mathrm{MHz}, \mathrm{CDCl}_{3}, 2{ }^{\circ} \mathrm{C}\right) \delta 201.4,169.4,164.9$, 138.4 , 138.2, 136.8, 135.9, 135.2, 131.9, 131.0, 129.7, 129.2, 128.9, 128.4, 127.9, 127.8, 51.5, 47.4, 26.9; HRMS (ESI) $\mathrm{m} / \mathrm{z}$ calcd. for $\mathrm{C}_{27} \mathrm{H}_{26} \mathrm{NO}_{3}{ }^{+} 412.1907$ found 412.1904 . 
Supporting information Amino Acid Schiff Base Bearing Benzophenone Imine as a Platform for Highly Congested Unnatural $\alpha$-Amino Acid Synthesis<smiles>COC(=O)/C(=C\C(C)(C)C(=O)N1CCOCC1)N=C(c1ccccc1)c1ccccc1</smiles>

\section{Methyl (E)-2-((diphenylmethylene)amino)-4,4-dimethyl-5-morpholino-5-oxopent-2-enoate (4p)}

Prepared according to General Method E and purified by flash column chromatography $\left(\right.$ Hexane/Acetone $=10 / 1\left(1 \% \mathrm{Et}_{3} \mathrm{~N}\right), 44 \%$ yield, yellow solid); IR (neat) 1719, 1636, 1240, 1113 $\mathrm{cm}^{-1} ;{ }^{1} \mathrm{H}$ $\operatorname{NMR}\left(500 \mathrm{MHz}, \mathrm{CDCl}_{3}, 27^{\circ} \mathrm{C}\right) \delta 7.69(\mathrm{~d}, J=7.5 \mathrm{~Hz}, 2 \mathrm{H}, \mathrm{ArH}), 7.45(\mathrm{t}, J=7.5 \mathrm{~Hz}, 1 \mathrm{H}, \operatorname{ArH}), 7.39-7.35(\mathrm{~m}, 5 \mathrm{H}$, $\operatorname{ArH}), 7.22(\mathrm{~m}, 2 \mathrm{H}, \mathrm{ArH}), 6.18(\mathrm{~s}, 1 \mathrm{H}, \mathrm{CH}), 3.67\left(\mathrm{br}, 8 \mathrm{H}, \mathrm{NCH}_{2} \mathrm{CH}_{2} \mathrm{O}\right), 3.38\left(\mathrm{~s}, 3 \mathrm{H}, \mathrm{OCH}_{3}\right), 1.39\left(\mathrm{~s}, 6 \mathrm{H}, \mathrm{CH}_{3}\right)$; ${ }^{13} \mathrm{C}$ NMR $\left(125 \mathrm{MHz}, \mathrm{CDCl}_{3}, 27{ }^{\circ} \mathrm{C}\right) \delta 173.5,169.9,165.0,138.4,138.0,137.0,132.1,131.1,129.6,129.3$, 128.5, 128.1, 128.0, 66.9, 65.9, 51.7, 42.5, 26.8; HRMS (ESI) m/z calcd. for $\mathrm{C}_{25} \mathrm{H}_{29} \mathrm{~N}_{2} \mathrm{O}_{4}{ }^{+} 421.2122$ found 421.2107.

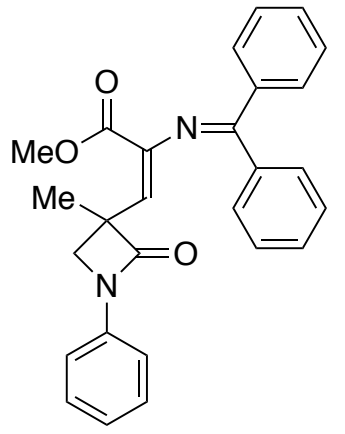

\section{Methyl (E)-2-((diphenylmethylene)amino)-3-(3-methyl-2-oxo-1-phenylazetidin-3-yl)acrylate (4q)}

Prepared according to General Method E and purified by flash column chromatography (Hexane/Acetone = 10/1 (1\% Et $3 \mathrm{~N}), 39 \%$ yield, yellow solid); IR (neat) 1745, 1722, $1256 \mathrm{~cm}^{-1} ;{ }^{1} \mathrm{H}$ NMR $\left(500 \mathrm{MHz}, \mathrm{CDCl}_{3}, 27^{\circ} \mathrm{C}\right) \delta 7.57(\mathrm{~d}, J=7.5 \mathrm{~Hz}, 2 \mathrm{H}, \mathrm{ArH}), 7.39-7.38(\mathrm{~m}, 3 \mathrm{H}, \operatorname{ArH}), 7.32-7.30(\mathrm{~m}, 3 \mathrm{H}, \operatorname{ArH})$, 7.18-7.12 (m, 6H, ArH), 6.98-6.94 (m, 1H, ArH), $6.17(\mathrm{~s}, 1 \mathrm{H}, \mathrm{CH}), 4.29\left(\mathrm{~d}, J=5.0 \mathrm{~Hz}, 1 \mathrm{H}, \mathrm{CH}_{2}\right), 3.48(\mathrm{~d}, J=$ $\left.5.0 \mathrm{~Hz}, 1 \mathrm{H}, \mathrm{CH}_{2}\right), 3.36\left(\mathrm{~s}, 3 \mathrm{H}, \mathrm{OCH}_{3}\right), 1.63$ (s, 3H, $\left.\mathrm{CH}_{3}\right) ;{ }^{13} \mathrm{C} \mathrm{NMR}\left(125 \mathrm{MHz}, \mathrm{CDCl}_{3}, 27{ }^{\circ} \mathrm{C}\right) \delta 170.5,168.0$, $164.0,140.9,138.5,138.2,136.8,130.9,129.5,129.3,128.8,128.7,127.9,127.8,125.3,123.5,116.2$, 53.0, 51.7, 51.4, 21.5; HRMS (ESI) $\mathrm{m} / \mathrm{z}$ calcd. for $\mathrm{C}_{27} \mathrm{H}_{25} \mathrm{~N}_{2} \mathrm{O}_{3}{ }^{+} 425.1860$ found 425.1857 . 
Supporting information Amino Acid Schiff Base Bearing Benzophenone Imine as a Platform for Highly Congested Unnatural $\alpha$-Amino Acid Synthesis<smiles>COC(=O)/C(=C\C1([N+](=O)[O-])COCOC1)N=C(c1ccccc1)c1ccccc1</smiles>

\section{Methyl (E)-2-((diphenylmethylene)amino)-3-(5-nitro-1,3-dioxan-5-yl)acrylate (4r)}

Prepared according to General Method E and purified by flash column chromatography $\left(\right.$ Hexane/Et ${ }_{2} \mathrm{O}=$ 2/1 (1\% Et 3 N), 15\% yield, yellow solid); IR (neat) 2359, 1724, 1445, 1263, $1121 \mathrm{~cm}^{-1}$; ${ }^{1} \mathrm{H} \mathrm{NMR}(500 \mathrm{MHz}$, $\left.\mathrm{CDCl}_{3}, 27^{\circ} \mathrm{C}\right) \delta$ 7.72-7.68 (m, 2H, $\left.\mathrm{ArH}\right), 7.50-7.47(\mathrm{~m}, 1 \mathrm{H}, \mathrm{ArH}), 7.41(\mathrm{~m}, 6 \mathrm{H}, \mathrm{ArH})$, 7.17-7.15 (m, 1H, $\left.\operatorname{ArH}\right)$, $5.77(\mathrm{~s}, 1 \mathrm{H}, \mathrm{CH}), 5.01\left(\mathrm{~d}, J=6.0 \mathrm{~Hz}, 1 \mathrm{H}, \mathrm{OCH}_{2}\right), 4.97\left(\mathrm{~d}, J=12.5 \mathrm{~Hz}, 2 \mathrm{H}, \mathrm{OCH}_{2}\right), 4.74(\mathrm{~d}, J=6.0 \mathrm{~Hz}, 1 \mathrm{H}$, $\left.\mathrm{OCH}_{2}\right), 4.19\left(\mathrm{~d}, \mathrm{~J}=12.5 \mathrm{~Hz}, 2 \mathrm{H}, \mathrm{OCH}_{2}\right), 3.38\left(\mathrm{~s}, 3 \mathrm{H}, \mathrm{OCH}_{3}\right) ;{ }^{13} \mathrm{C} \mathrm{NMR}\left(125 \mathrm{MHz}, \mathrm{CDCl}_{3}, 27^{\circ} \mathrm{C}\right) \delta 172.5,163.2$, 144.5, 129.8, 128.9, 128.3, 115.7, 93.8, 85.7, 69.9, 52.2; HRMS (ESI) m/z calcd. for $\mathrm{C}_{21} \mathrm{H}_{21} \mathrm{~N}_{2} \mathrm{O}_{6}{ }^{+} 397.1394$ found 397.1382 .<smiles>CCOC(=O)C(C)(C)/C(C)=C(/N=C(c1ccccc1)c1ccccc1)C(=O)OC</smiles>

\section{5-Ethyl 1-methyl (E)-2-((diphenylmethylene)amino)-3,4,4-trimethylpent-2-enedioate (4s)}

Prepared according to General Method E and purified by flash column chromatography (Hexane/Et $\mathrm{t}_{2} \mathrm{O}=$ $5 / 1\left(1 \% \mathrm{Et}_{3} \mathrm{~N}\right), 46 \%$ yield, yellow solid); IR (neat) 1718, 1605, 1445, 1225, 1130, $1053 \mathrm{~cm}^{-1} ;{ }^{1} \mathrm{H}$ NMR (500 $\left.\mathrm{MHz}, \mathrm{CDCl}_{3}, 27^{\circ} \mathrm{C}\right) \delta 7.64(\mathrm{~d}, J=7.0 \mathrm{~Hz}, 2 \mathrm{H}, \operatorname{ArH}), 7.42-7.39(\mathrm{~m}, 4 \mathrm{H}, \operatorname{ArH}), 7.35$ (t, $\left.J=7.5 \mathrm{~Hz}, 2 \mathrm{H}, \operatorname{ArH}\right)$, 7.31-7.29 (m, 2H, $\mathrm{ArH}), 3.67\left(\mathrm{q}, J=7.0 \mathrm{~Hz}, 1 \mathrm{H}, \mathrm{OCH}_{2} \mathrm{CH}_{3}\right), 3.10\left(\mathrm{~s}, 3 \mathrm{H}, \mathrm{OCH}_{3}\right), 1.99\left(\mathrm{~s}, 3 \mathrm{H}, \mathrm{CH}_{3}\right), 1.45(\mathrm{~s}, 6 \mathrm{H}$, $\left.\left(\mathrm{CH}_{3}\right)_{2}\right), 0.97\left(\mathrm{t}, \mathrm{J}=7.5 \mathrm{~Hz}, 3 \mathrm{H}, \mathrm{OCH}_{2} \mathrm{CH}_{3}\right) ;{ }^{13} \mathrm{C} \mathrm{NMR}\left(125 \mathrm{MHz}, \mathrm{CDCl}_{3}, 2{ }^{\circ} \mathrm{C}\right) \delta 176.6,166.6,166.3,141.6$, 139.0, 136.9, 135.2, 130.6, 129.7, 129.3, 127.9, 60.2, 50.7, 47.1, 24.1, 15.4, 13.9; HRMS (ESI) m/z calcd. for $\mathrm{C}_{24} \mathrm{H}_{28} \mathrm{NO}_{4}{ }^{+} 394.2013$ found 394.2004. 
Supporting information Amino Acid Schiff Base Bearing Benzophenone Imine as a Platform for Highly Congested Unnatural $\alpha$-Amino Acid Synthesis<smiles>CCOC(=O)C(C)(C)C(C(=O)OC)=C(N=C(c1ccccc1)c1ccccc1)c1ccccc1</smiles>

5-Ethyl 1-methyl (E)-2-((diphenylmethylene)amino)-4,4-dimethyl-3-phenylpent-2-enedioate (4t)

Prepared according to General Method $\mathrm{E}$ and purified by flash column chromatography (Hexane/Et ${ }_{2} \mathrm{O}=$ 5/1 (1\% Et $\left.{ }_{3} \mathrm{~N}\right), 39 \%$ yield, yellow solid); IR (neat) 1717, 1591, 1445, 1242, $1140 \mathrm{~cm}^{-1} ;{ }^{1} \mathrm{H}$ NMR (500 MHz, $\left.\mathrm{CDCl}_{3}, 27^{\circ} \mathrm{C}\right) \delta 7.72(\mathrm{~d}, J=7.0 \mathrm{~Hz}, 2 \mathrm{H}, \mathrm{ArH}), 7.45(\mathrm{td}, J=7.0,1.0 \mathrm{~Hz}, 1 \mathrm{H}, \mathrm{ArH}), 7.40-7.30(\mathrm{~m}, 7 \mathrm{H}, \mathrm{ArH})$, 7.29-7.22 (m, 3H, ArH), 7.17 (d, J=8.0 Hz, 2H, ArH), $3.86\left(\mathrm{q}, J=7.0 \mathrm{~Hz}, 2 \mathrm{H}, \mathrm{OCH}_{2} \mathrm{CH}_{3}\right), 2.73\left(\mathrm{~s}, 3 \mathrm{H}, \mathrm{OCH}_{3}\right)$, $1.35\left(\mathrm{~s}, 6 \mathrm{H}, \mathrm{CH}_{3}\right), 1.06\left(\mathrm{t}, J=7.0 \mathrm{~Hz}, 3 \mathrm{H}, \mathrm{OCH}_{2} \mathrm{CH}_{3}\right) ;{ }^{13} \mathrm{C} \mathrm{NMR}\left(125 \mathrm{MHz}, \mathrm{CDCl}_{3}, 27^{\circ} \mathrm{C}\right) \delta 176.8,168.4,165.7$, $143.9,138.8,138.4,137.3,136.7,130.9,129.8,129.3,129.2,128.8,128.0,127.9,127.5,126.9,60.5$, 50.5, 46.8, 25.5, 13.9; HRMS (ESI) m/z calcd. for $\mathrm{C}_{29} \mathrm{H}_{30} \mathrm{NO}_{4}{ }^{+} 456.2169$ found 456.2172 .

\section{Three-Components Cross-Coupling}

\section{General Procedure F}

In an inert atmosphere glove box, a $4 \mathrm{~mL}$ vial was charged with copper(II) acetate $(0.010 \mathrm{mmol}, 10$ mol\%) and $\mathbf{L} 5(0.010 \mathrm{mmol}, 10 \mathrm{~mol} \%)$, followed by tert-butylbenzene $(0.50 \mathrm{~mL})$. After the solution was stirred at ambient temperature for 10 minutes, $\alpha$-amino acid Schiff base $(0.10 \mathrm{mmol}, 1.0$ equiv.), alkyl bromide $2\left(0.12 \mathrm{mmol}, 1.2\right.$ equiv.) and $\mathrm{K}_{2} \mathrm{CO}_{3}(0.10 \mathrm{mmol}, 1.0$ equiv.) were added and removed from the glovebox. Styrene $(0.20 \mathrm{mmol}, 2.0$ equiv.) was added in an inert atmosphere and the reaction solution was stirred at $100{ }^{\circ} \mathrm{C}$ for 48 hours. Copper salt was removed by silica gel short pad column chromatography and washed with excess ethyl acetate. Evaporation of the solvent gave the crude product, which was purified by flash column chromatography. 
Supporting information Amino Acid Schiff Base Bearing Benzophenone Imine as a Platform for Highly Congested Unnatural $\alpha$-Amino Acid Synthesis<smiles>CCOC(=O)C(C)(C)CC(c1ccccc1)C(N=C(c1ccccc1)c1ccccc1)(C(=O)OC)c1ccccc1</smiles>

\section{6-Ethyl 1-methyl 2-((diphenylmethylene)amino)-5,5-dimethyl-2,3-diphenylhexanedioate (6a)}

Prepared according to General Procedure F and purified by flash column chromatography (Hexane/Et ${ }_{2} \mathrm{O}$ $=5 / 1\left(1 \% \mathrm{Et}_{3} \mathrm{~N}\right)$, diastereomixture, 71\% yield, $\mathrm{dr}=1 / 1$, colorless oil); IR (neat) 1722, 1624, 1445, 1219, 1134, 1117, $1028 \mathrm{~cm}^{-1} ;{ }^{1} \mathrm{H}$ NMR $\left(500 \mathrm{MHz} \mathrm{CDCl}_{3}, 27^{\circ} \mathrm{C}\right) \delta$ 7.69-7.66 (m, 4H, ArH), 7.65-7.40 (m, 2H, $\left.\mathrm{ArH}\right)$, 7.38-7.34 (m, 6H, ArH), 7.30-7.28 (m, 2H, ArH), 7.25-7.19 (m, 6H, ArH), 7.18-7.15 (m, 4H, ArH), 7.12-7.09 (m, 7H, ArH), 7.07-7.04 (m, 3H, ArH), $7.00(\mathrm{~m}, 2 \mathrm{H}, \operatorname{ArH}), 6.61-6.59(\mathrm{~m}, 4 \mathrm{H}, \mathrm{ArH}), 3.76(\mathrm{~d}, J=10.5 \mathrm{~Hz}, 1 \mathrm{H}$, $\mathrm{CH}_{2}$ ), $3.67\left(\mathrm{~d}, J=10.0 \mathrm{~Hz}, 1 \mathrm{H}, \mathrm{CH}_{2}\right), 3.50-3.46\left(\mathrm{~m}, 2 \mathrm{H}, \mathrm{OCH}_{2} \mathrm{CH}_{3}\right), 3.26-3.21\left(\mathrm{~m}, 2 \mathrm{H}, \mathrm{OCH}_{2} \mathrm{CH}_{3}\right), 3.18(\mathrm{~s}, 3 \mathrm{H}$, $\left.\mathrm{OCH}_{3}\right), 3.00\left(\mathrm{~s}, 3 \mathrm{H}, \mathrm{OCH}_{3}\right), 2.57\left(\mathrm{~d}, \mathrm{~J}=9.0 \mathrm{~Hz}, 1 \mathrm{H}, \mathrm{CH}_{2}\right), 2.32-2.29(\mathrm{~m}, 1 \mathrm{H}, \mathrm{CH}), 1.98\left(\mathrm{~d}, J=7.0 \mathrm{~Hz}, 1 \mathrm{H}, \mathrm{CH}_{2}\right)$, 1.97-1.94 (m, $1 \mathrm{H}, \mathrm{CH}), 1.15\left(\mathrm{~s}, 3 \mathrm{H}, \mathrm{CH}_{3}\right), 1.07\left(\mathrm{~s}, 3 \mathrm{H}, \mathrm{CH}_{3}\right), 1.00\left(\mathrm{~s}, 6 \mathrm{H}, \mathrm{CH}_{3}\right), 0.97(\mathrm{t}, J=7.0 \mathrm{~Hz}, 3 \mathrm{H}$, $\left.\mathrm{OCH}_{2} \mathrm{CH}_{3}\right), 0.96\left(\mathrm{t}, J=7.0 \mathrm{~Hz}, 3 \mathrm{H}, \mathrm{OCH}_{2} \mathrm{CH}_{3}\right) ;{ }^{13} \mathrm{C} \mathrm{NMR}\left(125 \mathrm{MHz}, \mathrm{CDCl}_{3}, 27^{\circ} \mathrm{C}\right) \delta 177.4,177.1,172.2,171.5$, 168.0, 167.2, 142.1, 141.5, 141.4, 141.0, 139.7, 138.7, 137.0, 136.7, 130.2, 130.1, 129.5, 129.0, 128.7, 128.4, 128.3, 128.2, 127.9, 127.9, 127.2, 127.1, 126.9, 126.8, 126.6, 126.5, 76.3, 75.7, 59.8, 59.8, 55.1, 54.5, 50.9, 50.8, 41.6, 41.5, 41.4, 40.3, 27.7, 27.6, 24.3, 24.0, 13.8, 13.8; HRMS (ESI) m/z calcd. for $\mathrm{C}_{36} \mathrm{H}_{38} \mathrm{NO}_{4}{ }^{+} 548.2795$ found 548.2797.

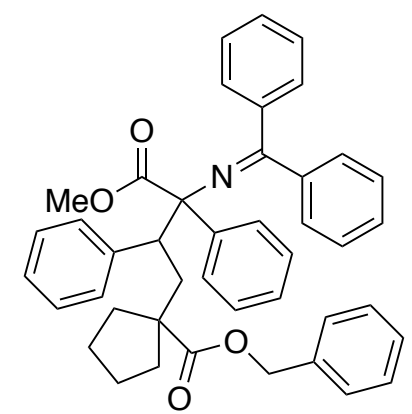

Benzyl 1-(3-((diphenylmethylene)amino)-4-methoxy-4-oxo-2,3-diphenylbutyl)cyclopentane -1carboxylate (6b)

Prepared according to General Procedure $F$ and purified by flash column chromatography (Hexane/toluene/Et ${ }_{2} \mathrm{O}=5 / 5 / 1\left(1 \% \mathrm{Et}_{3} \mathrm{~N}\right)$, diastereomixture, $58 \%$ yield, $\mathrm{dr}=1.1 / 1$, colorless oil); IR (neat) 
$1722,1622,1445,1161 \mathrm{~cm}^{-1} ;{ }^{1} \mathrm{H}$ NMR $\left(500 \mathrm{MHz}, \mathrm{CDCl}_{3}, 27^{\circ} \mathrm{C}\right) \delta$ 7.68-7.64 (m, 4H, ArH) , 7.44-7.40 (m, 2H, $\operatorname{ArH})$, 7.36-7.33 (m, 6H, ArH), 7.30-7.26 (m, 6H, ArH), 7.25-7.18 (m, 7H, ArH), 7.18-7.09 (m, 15H, ArH), 7.07-7.04 (m, 4H, ArH), 7.00 (br, 2H, ArH), 6.62-6.58 (m, 4H, $\operatorname{ArH}), 4.43\left(\mathrm{dd}, J=16.5,12.5 \mathrm{~Hz}, 2 \mathrm{H}, \operatorname{ArCH}_{2}\right.$ ), $4.08\left(\mathrm{dd}, J=16.0,13.0 \mathrm{~Hz}, 2 \mathrm{H}, \mathrm{ArCH}_{2}\right), 3.73\left(\mathrm{~d}, J=10.5 \mathrm{~Hz}, 1 \mathrm{H}, \mathrm{CHCH}_{2}\right), 3.62\left(\mathrm{~d}, J=10.0 \mathrm{~Hz}, 1 \mathrm{H}, \mathrm{CHCH}_{2}\right.$ ), $3.17\left(\mathrm{~s}, 3 \mathrm{H}, \mathrm{OCH}_{3}\right), 2.95\left(\mathrm{~s}, 3 \mathrm{H}, \mathrm{OCH}_{3}\right), 2.68\left(\mathrm{~d}, J=13.0 \mathrm{~Hz}, 1 \mathrm{H}, \mathrm{CHCH}_{2}\right), 2.46(\mathrm{dd}, J=14.0,11.0 \mathrm{~Hz}, 1 \mathrm{H}, \mathrm{CH})$, 2.33-2.28 (m, $\left.1 \mathrm{H}, \mathrm{CH}_{2}\right), 2.22-2.18\left(\mathrm{~m}, 1 \mathrm{H}, \mathrm{CH}_{2}\right), 2.14\left(\mathrm{~d}, J=13.0 \mathrm{~Hz}, 1 \mathrm{H}, \mathrm{CHCH}_{2}\right), 2.06(\mathrm{dd}, J=13.5,11.0 \mathrm{~Hz}$, $1 \mathrm{H}, \mathrm{CH}), 1.85-1.76\left(\mathrm{~m}, 2 \mathrm{H}, \mathrm{CH}_{2}\right), 1.72-1.68\left(\mathrm{~m}, 1 \mathrm{H}, \mathrm{CH}_{2}\right), 1.64-1.61\left(\mathrm{~m}, 1 \mathrm{H}, \mathrm{CH}_{2}\right), 1.57-1.51\left(\mathrm{~m}, 1 \mathrm{H}, \mathrm{CH}_{2}\right)$, 1.48-1.38 (m, 8H, CH $), 1.31\left(\mathrm{q}, J=5.0 \mathrm{~Hz}, 1 \mathrm{H}, \mathrm{CH}_{2}\right) ;{ }^{13} \mathrm{C} \mathrm{NMR}\left(125 \mathrm{MHz}, \mathrm{CDCl}_{3}, 27{ }^{\circ} \mathrm{C}\right) \delta 176.8,176.6$, $172.2,171.5,168.0,167.3,142.2,141.4,141.0,139.5,138.5,137.0,126.7,136.6,130.2,130.1,129.4$, $129.0,128.7,128.4,128.3,128.3,128.2,128.1,127.9,127.6,127.6,127.5,127.2,127.2,127.1,126.9$, $126.8,126.7,126.6,76.3,75.7,65.4,65.3,56.4,55.6,53.9,50.9,40.0,38.7,38.5,38.4,33.7,33.7,24.1$, 23.9; HRMS (ESI) m/z calcd. for $\mathrm{C}_{43} \mathrm{H}_{42} \mathrm{NO}_{4}{ }^{+} 636.3108$ found 636.3092 .

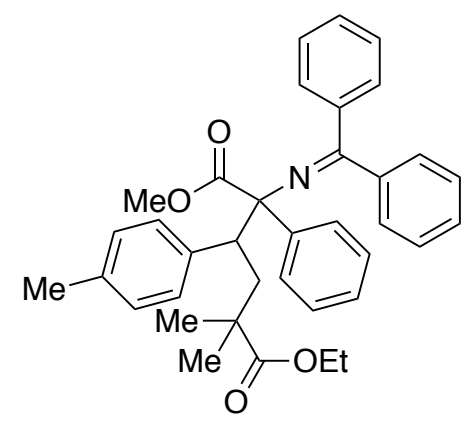

6-Ethyl 1-methyl 2-((diphenylmethylene)amino)-5,5-dimethyl-2-phenyl-3-(p-tolyl) hexanedioate (6c) Prepared according to General Procedure $F$ and purified by flash column chromatography (Hexane/toluene/Et ${ }_{2} \mathrm{O}=5 / 5 / 1\left(1 \% \mathrm{Et}_{3} \mathrm{~N}\right)$, diastereomixture, $57 \%$ yield, $\mathrm{dr}=1.1 / 1$, colorless oil); IR (neat) $1719,1445,1265 \mathrm{~cm}^{-1} ;{ }^{1} \mathrm{H} \mathrm{NMR}\left(500 \mathrm{MHz}, \mathrm{CDCl}_{3}, 27^{\circ} \mathrm{C}\right) \delta 7.68(\mathrm{~d}, J=7.0 \mathrm{~Hz}, 2 \mathrm{H}, \mathrm{ArH}), 7.66(\mathrm{~d}, J=7.0 \mathrm{~Hz}$, $2 \mathrm{H}, \operatorname{ArH}), 7.41(\mathrm{td}, J=6.0,1.0 \mathrm{~Hz}, 2 \mathrm{H}, \mathrm{ArH}), 7.37-7.33(\mathrm{~m}, 7 \mathrm{H}, \operatorname{ArH}), 7.29(\mathrm{~d}, J=7.5 \mathrm{~Hz}, 2 \mathrm{H}, \mathrm{ArH}), 7.25-7.14$ $(\mathrm{m}, 9 \mathrm{H}, \mathrm{ArH}), 7.11(\mathrm{t}, J=7.5 \mathrm{~Hz}, 2 \mathrm{H}, \mathrm{ArH}), 7.05(\mathrm{t}, J=7.5 \mathrm{~Hz}, 2 \mathrm{H}, \mathrm{ArH}), 6.94-6.88(\mathrm{~m}, 6 \mathrm{H}, \mathrm{ArH}), 6.61-6.58$ $(\mathrm{m}, 4 \mathrm{H}, \mathrm{ArH}), 3.73\left(\mathrm{~d}, J=10.0 \mathrm{~Hz}, 1 \mathrm{H}, \mathrm{CH}_{2}\right), 3.64\left(\mathrm{~d}, J=10.0 \mathrm{~Hz}, 1 \mathrm{H}, \mathrm{CH}_{2}\right), 3.53-3.48\left(\mathrm{~m}, 2 \mathrm{H}, \mathrm{OCH}_{2} \mathrm{CH}_{3}\right)$, 3.32-3.24 (m, $\left.2 \mathrm{H}, \mathrm{OCH}_{2} \mathrm{CH}_{3}\right), 3.18\left(\mathrm{~s}, 3 \mathrm{H}, \mathrm{OCH}_{3}\right), 3.02\left(\mathrm{~s}, 3 \mathrm{H}, \mathrm{OCH}_{3}\right), 2.53\left(\mathrm{~d}, J=13.0 \mathrm{~Hz}, 1 \mathrm{H}, \mathrm{CH}_{2}\right), 2.30-$ $2.27(\mathrm{~m}, 1 \mathrm{H}, \mathrm{CH}), 2.25\left(\mathrm{~s}, 6 \mathrm{H}, \mathrm{ArCH}_{3}\right), 1.95\left(\mathrm{~d}, \mathrm{~J}=13.0,1 \mathrm{H}, \mathrm{CH}_{2}\right), 1.90-1,85(\mathrm{~m}, 1 \mathrm{H}, \mathrm{CH}) 1.14\left(\mathrm{~s}, 3 \mathrm{H}, \mathrm{CH}_{3}\right)$, $1.06\left(\mathrm{~s}, 3 \mathrm{H}, \mathrm{CH}_{3}\right), 1.00\left(\mathrm{~s}, 3 \mathrm{H}, \mathrm{CH}_{3}\right), 0.99\left(\mathrm{~s}, 3 \mathrm{H}, \mathrm{CH}_{3}\right), 0.98\left(\mathrm{t}, J=7.0 \mathrm{~Hz}, 3 \mathrm{H}, \mathrm{OCH}_{2} \mathrm{CH}_{3}\right), 0.98(\mathrm{t}, J=7.5 \mathrm{~Hz}$, $\left.3 \mathrm{H}, \mathrm{OCH}_{2} \mathrm{CH}_{3}\right) ;{ }^{13} \mathrm{C} \mathrm{NMR}\left(125 \mathrm{MHz}, \mathrm{CDCl}_{3}, 2{ }^{\circ} \mathrm{C}\right) \delta 177.4,177.2,172.3,171.5,167.9,167.1,141.5,140.9$, $137.1,136.8,136.5,136.3,135.8,135.5,130.1,130.0,129.6,129.0,128.8,128.4,128.3,128.3,128.1$, 
127.9, 127.6, 127.3, 127.2, 127.1, 127.1, 126.8, 126.7, 76.3, 75.8, 59.8, 59.8, 54.8, 54.1, 50.9, 50.8, 41.6, $41.5,41.5,40.2,27.6,27.5,24.4,24.1,21.0,13.8,13.8$; HRMS (ESI) m/z calcd. for $\mathrm{C}_{37} \mathrm{H}_{40} \mathrm{NO}_{4}{ }^{+} 562.2952$ found 562.2957 .

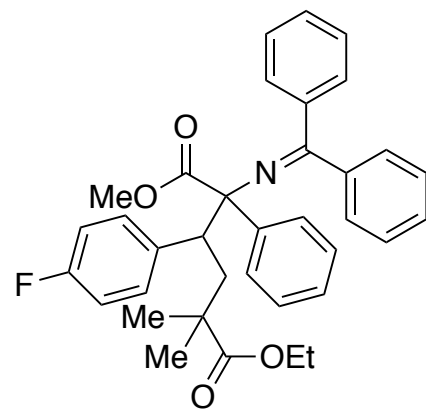

6-Ethyl 1-methyl 2-((diphenylmethylene)amino)-3-(4-fluorophenyl)-5,5-dimethyl-2-

phenylhexanedioate (6d)

Prepared according to General Procedure F and purified by flash column chromatography (Hexane/toluene/AcOEt = 5/5/1 (1\% Et $\left.\mathrm{E}_{3} \mathrm{~N}\right)$, diastereomixture, $63 \%$ yield, $\mathrm{dr}=1.1 / 1$, colorless oil); IR (neat) $1721,1508,1221,1134 \mathrm{~cm}^{-1} ;{ }^{1} \mathrm{H}$ NMR $\left(500 \mathrm{MHz}, \mathrm{CDCl}_{3}, 27^{\circ} \mathrm{C}\right) \delta 7.67(\mathrm{~d}, J=7.0 \mathrm{~Hz}, 2 \mathrm{H}, \mathrm{ArH}), 7.65$ $(\mathrm{d}, J=8.5 \mathrm{~Hz}, 2 \mathrm{H}, \mathrm{ArH}), 7.42(\mathrm{t}, J=7.0 \mathrm{~Hz}, 2 \mathrm{H}, \operatorname{ArH}), 7.37(\mathrm{t}, J=8.0 \mathrm{~Hz}, 4 \mathrm{H}, \operatorname{ArH}), 7.34-7.27(\mathrm{~m}, 4 \mathrm{H}, \mathrm{ArH})$, 7.24-7.15 (m, 10H, ArH), $7.12(\mathrm{t}, J=7.5 \mathrm{~Hz}, 2 \mathrm{H}, \operatorname{ArH}), 7.07(\mathrm{t}, J=7.5 \mathrm{~Hz}, 2 \mathrm{H}, \operatorname{ArH}), 6.96(\mathrm{br}, 2 \mathrm{H}, \operatorname{ArH}), 6.82$ $(\mathrm{t}, J=8.5 \mathrm{~Hz}, 3 \mathrm{H}, \operatorname{ArH}), 6.77(\mathrm{br}, 1 \mathrm{H}, \mathrm{ArH}), 6.63-6.61(\mathrm{~m}, 4 \mathrm{H}, \mathrm{ArH}), 3.74\left(\mathrm{~d}, J=10.5 \mathrm{~Hz}, 1 \mathrm{H}, \mathrm{CH}_{2}\right), 3.69(\mathrm{~d}, J$ $\left.=10.5 \mathrm{~Hz}, 1 \mathrm{H}, \mathrm{CH}_{2}\right), 3.55-3.51\left(\mathrm{~m}, 2 \mathrm{H}, \mathrm{OCH}_{2} \mathrm{CH}_{3}\right), 3.33-3.30\left(\mathrm{~m}, 2 \mathrm{H}, \mathrm{OCH}_{2} \mathrm{CH}_{3}\right), 3.17(\mathrm{~s}, 3 \mathrm{H}, \mathrm{OCH})_{3}, 3.05(\mathrm{~s}$, $3 \mathrm{H}, \mathrm{OCH}_{3}$ ), $2.55\left(\mathrm{~d}, J=13.5 \mathrm{~Hz}, 1 \mathrm{H}, \mathrm{CH}_{2}\right.$ ), 2.24 (dd, J = 14.0, $11.0 \mathrm{~Hz}, 1 \mathrm{H}, \mathrm{CH}$ ), $1.93\left(\mathrm{~d}, J=14.0 \mathrm{~Hz}, 1 \mathrm{H}, \mathrm{CH}_{2}\right.$ ), $1.90\left(\mathrm{dd}, J=14.0,11.5 \mathrm{~Hz}, 1 \mathrm{H}, \mathrm{C}_{2}\right), 1.15\left(\mathrm{~s}, 3 \mathrm{H}, \mathrm{CH}_{3}\right), 1.07\left(\mathrm{~s}, 3 \mathrm{H}, \mathrm{CH}_{3}\right), 1.01\left(\mathrm{~s}, 3 \mathrm{H}, \mathrm{CH}_{3}\right), 1.01\left(\mathrm{~s}, 3 \mathrm{H}, \mathrm{CH}_{3}\right)$, $1.00\left(\mathrm{t}, J=7.0 \mathrm{~Hz}, 3 \mathrm{H}, \mathrm{OCH}_{2} \mathrm{CH}_{3}\right), 0.99\left(\mathrm{t}, J=7.0 \mathrm{~Hz}, 3 \mathrm{H}, \mathrm{OCH}_{2} \mathrm{CH}_{3}\right) ;{ }^{13} \mathrm{C} \mathrm{NMR}\left(125 \mathrm{MHz}, \mathrm{CDCl}_{3}, 27^{\circ} \mathrm{C}\right) \delta$ 177.2 , 177.0, 172.2, 171.6, 168.2, 167.4, 141.7, 141.4, 141.2, 140.9, 136.9, 136.6, 135.3, 134.6, 130.2, $130.2,129.3,129.0,128.9,128.8,128.4,128.3,128.3,128.2,128.0,127.3,127.2,127.0,126.9,126.9$, $113.7,113.6,113.3,113.2,76.1,75.7,59.9,59.9,54.3,53.7,51.0,50.9,41.7,41.4,41.4,40.5,27.8,27.7$, 24.3, 24.0, 13.8; HRMS (ESI) m/z calcd. for $\mathrm{C}_{36} \mathrm{H}_{37} \mathrm{FNO}_{4}{ }^{+} 566.2701$ found 566.2709 .

\section{Diastereoselective Reaction using Chiral Auxiliary}

\section{General Procedure G}

In an inert atmosphere glove box, a $4 \mathrm{~mL}$ vial was charged with copper(II) acetate $(0.030 \mathrm{mmol}, 10$ mol\%) and $\mathbf{L} 5(0.030 \mathrm{mmol}, 10 \mathrm{~mol} \%)$, followed by THF $(3.0 \mathrm{~mL})$. After the solution was stirred at 
ambient temperature for 10 minutes, $\alpha$-amino acid Schiff base ( $0.30 \mathrm{mmol}, 1.0$ equiv.), alkyl bromide 2 $\left(0.20 \mathrm{mmol}, 2.0\right.$ equiv.) and $\mathrm{KO}^{t} \mathrm{Bu}(0.20 \mathrm{mmol}, 2.0$ equiv.) were added. After the solution was stirred at ambient temperature for 3 hours, alkyl bromide $2\left(0.20 \mathrm{mmol}, 2.0\right.$ equiv.) and $\mathrm{KO} \mathrm{B}^{t} \mathrm{Bu}(0.20 \mathrm{mmol}, 2.0$ equiv.) were added and stirred for 3 hours. Copper salt was removed by silica gel short pad column chromatography and washed with excess ethyl acetate. Evaporation of the solvent gave the crude product, which was purified by flash column chromatography.

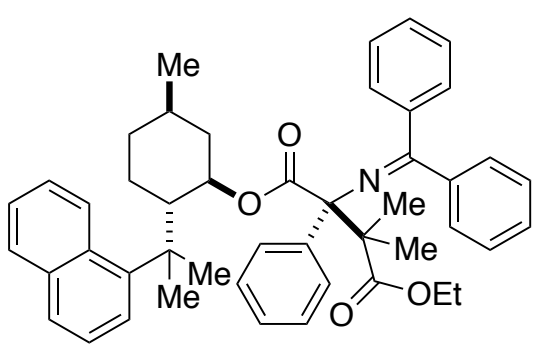

4-Ethyl 1-((1R,2S,5R)-5-methyl-2-(2-(naphthalen-1-yl)propan-2-yl)cyclohexyl) (S)-2-

((diphenylmethylene)amino)-3,3-dimethyl-2-phenylsuccinate (38a major)

Prepared according to General Procedure $\mathrm{G}$ and purified by flash column chromatography (Hexane/Et $\mathrm{t}_{2} \mathrm{O}$ $=19 / 1\left(1 \% \mathrm{Et}_{3} \mathrm{~N}\right), 61 \%$ yield, colorless oil); IR (neat) 1711, 1624, 1445, $1140 \mathrm{~cm}^{-1} ;{ }^{1} \mathrm{H} \mathrm{NMR}(500 \mathrm{MHz}$, $\left.\mathrm{CDCl}_{3}, 27^{\circ} \mathrm{C}\right) \delta 8.18(\mathrm{~d}, J=8.5 \mathrm{~Hz}, 1 \mathrm{H}, \mathrm{ArH}), 7.78(\mathrm{~d}, J=9.0 \mathrm{~Hz}, 1 \mathrm{H}, \mathrm{ArH}), 7.61(\mathrm{t}, J=4.5 \mathrm{~Hz}, 3 \mathrm{H}, \mathrm{ArH}), 7.36$ $(\mathrm{t}, J=7.0 \mathrm{~Hz}, 1 \mathrm{H}, \operatorname{ArH}), 7.32-7.24(\mathrm{~m}, 8 \mathrm{H}, \operatorname{ArH}), 7.11(\mathrm{t}, J=7.5 \mathrm{~Hz}, 1 \mathrm{H}, \operatorname{ArH}), 7.06-7.01(\mathrm{~m}, 5 \mathrm{H}, \operatorname{ArH}), 6.95$ (d, J = 7.0 Hz, 2H, ArH), $5.07(\mathrm{td}, J=10.5,4.0 \mathrm{~Hz}, 1 \mathrm{H}, \mathrm{OCH}), 4.10-4.01\left(\mathrm{~m}, 2 \mathrm{H}, \mathrm{OCH}_{2} \mathrm{CH}_{3}\right), 2.68(\mathrm{td}, J=12.0$, $4.0 \mathrm{~Hz}, 1 \mathrm{H}, \mathrm{CH}), 2.26\left(\mathrm{~d}, \mathrm{~J}=10.0 \mathrm{~Hz}, 1 \mathrm{H}, \mathrm{CH}_{2}\right), 1.58\left(\mathrm{~s}, 3 \mathrm{H}, \mathrm{CH}_{3}\right), 1.52\left(\mathrm{~s}, 3 \mathrm{H}, \mathrm{CH}_{3}\right), 1.37\left(\mathrm{~s}, 3 \mathrm{H}, \mathrm{CH}_{3}\right), 1.26$ $\left(\mathrm{m}, 2 \mathrm{H}, \mathrm{CH}_{2}\right), 1.24\left(\mathrm{~s}, 3 \mathrm{H}, \mathrm{CH}_{3}\right), 1.09\left(\mathrm{t}, J=7.0 \mathrm{~Hz}, 3 \mathrm{H}, \mathrm{OCH}_{2} \mathrm{CH}_{3}\right), 0.90-0.82\left(\mathrm{~m}, 1 \mathrm{H}, \mathrm{CH}_{2}\right), 0.80(\mathrm{~d}, J=6.5 \mathrm{~Hz}$, $\left.3 \mathrm{H}, \mathrm{CHCH}_{3}\right), 0.77-0.72\left(\mathrm{~m}, 2 \mathrm{H}, \mathrm{CH}_{2}\right), 0.49\left(\mathrm{qd}, J=12.5,4.0 \mathrm{~Hz}, 1 \mathrm{H}, \mathrm{CH}_{2}\right) ;{ }^{13} \mathrm{C} \mathrm{NMR}\left(125 \mathrm{MHz}, \mathrm{CDCl}_{3}, 27^{\circ} \mathrm{C}\right)$ б 175.9, 169.3, 167.9, 146.4, 143.7, 138.4, 137.5, 135.0, 131.5, 129.7, 129.6, 129.3, 129.2, 127.8, 127.6, 127.2, 127.0, 126.8, 126.7, 126.3, 125.0, 124.5, 124.4, 123.9, 78.8, 78.5, 60.3, 55.1, 47.7, 42.6, 40.9, 34.7, 31.4, 30.3, 28.3, 25.0, 23.6, 23.4, 21.8, 13.9; HRMS (ESI) $\mathrm{m} / \mathrm{z}$ calcd. for $\mathrm{C}_{47} \mathrm{H}_{52} \mathrm{NO}_{4}{ }^{+} 694.3891$ found 694.3827. 
Supporting information Amino Acid Schiff Base Bearing Benzophenone Imine as a Platform for Highly Congested Unnatural $\alpha$-Amino Acid Synthesis<smiles>CCOC(=O)C(C)(C)[C@](N=C(c1ccccc1)c1ccccc1)(C(=O)O[C@H]1C[C@@H](C)CC[C@H]1C(C)(C)c1cccc2ccccc12)c1ccccc1</smiles>

4-Ethyl 1-((1R,2S,5R)-5-methyl-2-(2-(naphthalen-1-yl)propan-2-yl)cyclohexyl) (R)-2-

((diphenylmethylene)amino)-3,3-dimethyl-2-phenylsuccinate (38a minor)

Prepared according to General Procedure $\mathrm{G}$ and purified by flash column chromatography (Hexane/Et $\mathrm{t}_{2} \mathrm{O}$ $=19 / 1\left(1 \% \mathrm{Et}_{3} \mathrm{~N}\right), 5 \%$ yield, colorless oil); IR (neat) $1711,1624,1445,1140 \mathrm{~cm}^{-1} ;{ }^{1} \mathrm{H} \mathrm{NMR}\left(500 \mathrm{MHz}, \mathrm{CDCl}_{3}\right.$, $\left.27^{\circ} \mathrm{C}\right) \delta 8.51(\mathrm{~d}, J=8.5 \mathrm{~Hz}, 1 \mathrm{H}, \mathrm{ArH}), 7.84(\mathrm{dd}, J=8.0,2.0 \mathrm{~Hz}, 1 \mathrm{H}, \operatorname{ArH}), 7.67-7.63(\mathrm{~m}, 3 \mathrm{H}, \operatorname{ArH}), 7.48-7.43$ $(\mathrm{m}, 5 \mathrm{H}, \operatorname{ArH}), 7.33-7.27(\mathrm{~m}, 3 \mathrm{H}, \mathrm{ArH}), 7.17-7.05(\mathrm{~m}, 7 \mathrm{H}, \mathrm{ArH}), 6.85(\mathrm{~d}, J=7.5 \mathrm{~Hz}, 2 \mathrm{H}, \operatorname{ArH}), 4.81(\mathrm{td}, J=$ 10.0, 4.0 Hz, 1H, OCH), 4.17-4.05 (m, 2H, OCH $\left.\mathrm{CH}_{3}\right), 2.93-2.88(\mathrm{~m}, 1 \mathrm{H}, \mathrm{CH}), 2.33(\mathrm{~d}, J=11.5 \mathrm{~Hz}, 1 \mathrm{H}, \mathrm{CH})$, $1.74\left(\mathrm{~s}, 3 \mathrm{H}, \mathrm{CH}_{3}\right), 1.57\left(\mathrm{~s}, 3 \mathrm{H}, \mathrm{CH}_{3}\right), 1.47\left(\mathrm{~s}, 3 \mathrm{H}, \mathrm{CH}_{3}\right), 1.38\left(\mathrm{~s}, 3 \mathrm{H}, \mathrm{CH}_{3}\right), 1.30-1.21\left(\mathrm{~m}, 2 \mathrm{H}, \mathrm{CH}_{2}\right), 1.22(\mathrm{t}, J=$ $\left.7.0 \mathrm{~Hz}, 3 \mathrm{H}, \mathrm{OCH}_{2} \mathrm{CH}_{3}\right), 0.92-0.84\left(\mathrm{~m}, 3 \mathrm{H}, \mathrm{CH}_{2}\right), 0.79\left(\mathrm{~d}, J=6.5 \mathrm{~Hz}, 3 \mathrm{H}, \mathrm{CHCH}_{3}\right), 0.66-0.58\left(\mathrm{~m}, 1 \mathrm{H}, \mathrm{CH}_{2}\right) ;{ }^{13} \mathrm{C}$ NMR (125 MHz, CDCl $\left.27^{\circ} \mathrm{C}\right) \delta 176.0,169.2,168.5,146.1,139.6,135.1,131.5,130.6,129.9,129.7$, 129.4, 129.3, 128.5, 127.6, 127.3, 126.8, 126.5, 126.3, 125.0, 124.7., 124.6, 123.9, 78.7, 76.0, 60.5, 53.8, 47.6, 42.8, 40.3, 34.3, 31.1, 30.2, 28.1, 24.6, 23.8, 21.9, 14.1; HRMS (ESI) m/z calcd. for $\mathrm{C}_{47} \mathrm{H}_{52} \mathrm{NO}_{4}{ }^{+}$ 694.3891 found 694.3838 .

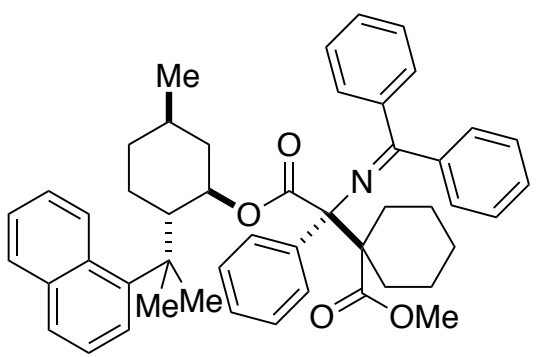

Methyl 1-((S)-1-((diphenylmethylene)amino)-2-(((1R,2S,5R)-5-methyl-2-(2-(naphthalen-1-yl)propan-2yl)cyclohexyl)oxy)-2-oxo-1-phenylethyl)cyclohexane-1-carboxylate (38b)

Prepared according to General Procedure $\mathrm{G}$ and purified by flash column chromatography (Hexane/Et ${ }_{2} \mathrm{O}$ $=19 / 1\left(1 \% \mathrm{Et}_{3} \mathrm{~N}\right)$, diastereomixture, 65\% yield, $\mathrm{dr}=94 / 6$, white solid); IR (neat) 2943, 1715, 1206, 1132 $\mathrm{cm}^{-1} ;{ }^{1} \mathrm{H}$ NMR $\left(500 \mathrm{MHz}^{\mathrm{CDCl}} 3,27^{\circ} \mathrm{C}\right) \delta 8.23(\mathrm{~d}, J=9.0 \mathrm{~Hz}, 1 \mathrm{H}, \mathrm{ArH}), 7.80(\mathrm{dd}, J=8.0,1.0 \mathrm{~Hz}, 1 \mathrm{H}, \operatorname{ArH})$, $7.63(\mathrm{t}, J=7.5 \mathrm{~Hz}, 3 \mathrm{H}, \operatorname{ArH}), 7.38(\mathrm{t}, J=8.0 \mathrm{~Hz}, 1 \mathrm{H}, \operatorname{ArH}), 7.36-7.26(\mathrm{~m}, 7 \mathrm{H}, \operatorname{ArH}), 7.13-7.10(\mathrm{~m}, 5 \mathrm{H}, \operatorname{ArH})$, $7.03(\mathrm{t}, J=7.5,2 \mathrm{H}, \operatorname{ArH}), 6.83(\mathrm{~d}, J=6.5 \mathrm{~Hz}, 2 \mathrm{H}, \operatorname{ArH}), 4.96(\mathrm{td}, J=10.5,4.0 \mathrm{~Hz}, 1 \mathrm{H}, \mathrm{OCH}), 3.54(\mathrm{~s}, 3 \mathrm{H}$, 
Supporting information Amino Acid Schiff Base Bearing Benzophenone Imine as a Platform for Highly Congested Unnatural $\alpha$-Amino Acid Synthesis

$\left.\mathrm{OCH}_{3}\right), 2.66(\mathrm{td}, J=11.5,4.0 \mathrm{~Hz}, 1 \mathrm{H}, \mathrm{CH}), 2.43\left(\mathrm{~d}, J=12.5 \mathrm{~Hz}, 1 \mathrm{H}, \mathrm{CH}_{2}\right), 2.28\left(\mathrm{~d}, J=14.0 \mathrm{~Hz}, 1 \mathrm{H}, \mathrm{CH}_{2}\right), 2.13$ (qd, $J=10.0,4.5 \mathrm{~Hz}, 2 \mathrm{H}, \mathrm{CH}_{2}$ ), $1.89\left(\mathrm{td}, J=12.5,4.0 \mathrm{~Hz}, 1 \mathrm{H}, \mathrm{CH}_{2}\right.$ ), $1.76\left(\mathrm{q}, J=8.0 \mathrm{~Hz}, 2 \mathrm{H}, \mathrm{CH}_{2}\right.$ ), 1.66-1.63 $\left(\mathrm{m}, 1 \mathrm{H}, \mathrm{CH}_{2}\right), 1.40-1.34\left(\mathrm{~m}, 1 \mathrm{H}, \mathrm{CH}_{2}\right), 1.31\left(\mathrm{~s}, 3 \mathrm{H}, \mathrm{CH}_{3}\right), 1.26\left(\mathrm{~s}, 3 \mathrm{H}, \mathrm{CH}_{3}\right), 1.21-1.16\left(\mathrm{~m}, 2 \mathrm{H}, \mathrm{CH}_{2}\right), 0.84$ (qd, $J=10.0,2.5 \mathrm{~Hz}, 2 \mathrm{H}, \mathrm{CH}_{2}$ ), $0.76\left(\mathrm{~d}, J=6.5 \mathrm{~Hz}, 3 \mathrm{H}, \mathrm{CHCH}_{3}\right), 055$ (q, J=11.5 Hz, 1H, $\mathrm{CH}_{2}$ ), 0.46 (qd, $J=13.0$, $\left.4.0 \mathrm{~Hz}, 1 \mathrm{H}, \mathrm{CH}_{2}\right) ;{ }^{13} \mathrm{C}$ NMR $\left(125 \mathrm{MHz} \mathrm{CDCl}_{3}, 2{ }^{\circ} \mathrm{C}\right) \delta 174.0,168.8,167.9,146.3,143.9,138.7,138.0$, 135.0, 131.5, 129.7, 129.6, 129.4, 129.2, 128.0, 127.7, 127.2, 127.1, 127.0, 126.7, 126.2, 125.0, 124.6, 124.4, 123.9, 78.9, 78.6, 60.4, 51.1, 47.4, 42.5, 40.6, 34.6, 31.3, 30.9, 30.3, 30.2, 28.3, 25.7, 25.1, 24.0, 21.7; HRMS (ESI) m/z calcd. for $\mathrm{C}_{49} \mathrm{H}_{53} \mathrm{NNaO}_{4}{ }^{+} 742.3867$ found 742.3823 .

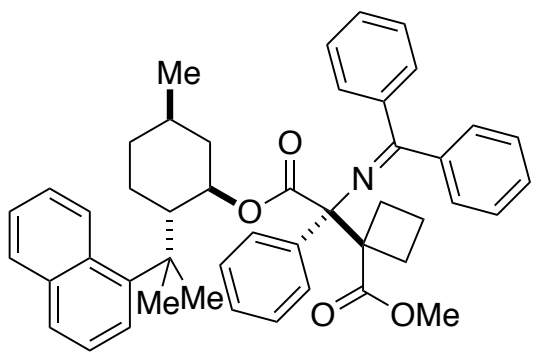

methyl 1-((S)-1-((diphenylmethylene)amino)-2-(((1R,2S,5R)-5-methyl-2-(2-(naphthalen-1-yl)propan-2yl)cyclohexyl)oxy)-2-oxo-1-phenylethyl)cyclobutane-1-carboxylate (38d)

Prepared according to General Procedure $\mathrm{G}$ and purified by flash column chromatography (Hexane/Et ${ }_{2} \mathrm{O}$ = 19/1 (1\% Et $\left.{ }_{3} \mathrm{~N}\right)$, diastereomixture, 23\% yield, $\mathrm{dr}=96 / 4$, colorless oil); IR (neat) 2359, 1719, 1263, cm ${ }^{-1}$; ${ }^{1} \mathrm{H} \mathrm{NMR}\left(500 \mathrm{MHz}, \mathrm{CDCl}_{3}, 27^{\circ} \mathrm{C}\right) \delta 8.20(\mathrm{~d}, J=8.5 \mathrm{~Hz}, 1 \mathrm{H}, \mathrm{ArH}), 7.79(\mathrm{dd}, J=8.0,1.0 \mathrm{~Hz}, 1 \mathrm{H}, \operatorname{ArH}), 7.67(\mathrm{~d}$, $J=7.0 \mathrm{~Hz}, 2 \mathrm{H}, \operatorname{ArH}), 7.63(\mathrm{dd}, J=6.0,3.0 \mathrm{~Hz}, 1 \mathrm{H}, \operatorname{ArH}), 7.36-7.28(\mathrm{~m}, 3 \mathrm{H}, \operatorname{ArH}), 7.24-7.21(\mathrm{~m}, 4 \mathrm{H}, \operatorname{Ar} H)$, $7.09(\mathrm{t}, J=6.5 \mathrm{~Hz}, 1 \mathrm{H}, \mathrm{ArH}), 7.02-6.97(\mathrm{~m}, 3 \mathrm{H}, \mathrm{ArH}), 6.95-6.90(\mathrm{~m}, 6 \mathrm{H}, \mathrm{ArH}), 5.10(\mathrm{td}, J=10.0,4.0 \mathrm{~Hz}, 1 \mathrm{H}$, $\mathrm{OCH}$ ), $3.50\left(\mathrm{~s}, 3 \mathrm{H}, \mathrm{OCH}_{3}\right.$ ), $3.24\left(\mathrm{q}, J=10.0 \mathrm{~Hz}, 1 \mathrm{H}, \mathrm{CH}_{2}\right.$ ), $3.16\left(\mathrm{q}, J=10.0 \mathrm{~Hz}, 1 \mathrm{H}, \mathrm{CH}_{2}\right.$ ), $2.76(\mathrm{qd}, J=11.0$, $3.5 \mathrm{~Hz}, 2 \mathrm{H}, \mathrm{CH}), 2.28-2.26\left(\mathrm{~m}, 2 \mathrm{H}, \mathrm{CH}_{2}\right), 2.14\left(\mathrm{q}, J=10.0 \mathrm{~Hz}, 1 \mathrm{H}, \mathrm{CH}_{2}\right), 2.01\left(\mathrm{q}, J=10.0 \mathrm{~Hz}, 1 \mathrm{H}, \mathrm{CH}_{2}\right), 1.43$ $\left(\mathrm{s}, 6 \mathrm{H}, \mathrm{CAr}\left(\mathrm{CH}_{3}\right)_{2}\right), 1.33-1.19\left(\mathrm{~m}, 3 \mathrm{H}, \mathrm{CH}_{2}\right), 0.92-0.86\left(\mathrm{~m}, 2 \mathrm{H}, \mathrm{CH}_{2}\right), 0.83$ (d, J = 6.5 Hz, 3H, $\left.\mathrm{CHCH}_{3}\right), 0.59$ (qd, $\left.J=12.5,4.0 \mathrm{~Hz}, 1 \mathrm{H}, \mathrm{CH}_{2}\right) ;{ }^{13} \mathrm{C} \mathrm{NMR}\left(125 \mathrm{MHz}, \mathrm{CDCl}_{3}, 2{ }^{\circ} \mathrm{C}\right) \delta 175.5,170.0,169.1,146.2,142.5,140.6$, 138.2, 134.9, 131.6, 130.0, 129.6, 128.7, 128.4, 128.1, 127.8, 127.3, 127.0, 126.8, 126.5, 126.2, 125.0, 124.6, 14.6, 123.9, 79.2, 60.2, 51.7, 48.1, 42.5, 41.1, 34.6, 31.4, 30.7, 30.0, 28.3, 27.5, 25.1, 21.8, 16.9; HRMS (ESI) m/z calcd. for $\mathrm{C}_{47} \mathrm{H}_{50} \mathrm{NO}_{4}{ }^{+} 692.3734$ found 692.3714 . 
Supporting information Amino Acid Schiff Base Bearing Benzophenone Imine as a Platform for Highly Congested Unnatural $\alpha$-Amino Acid Synthesis<smiles>C[C@H]1CC[C@@H]([C@@H](OC(=O)[C@](N=C(c2ccccc2)c2ccccc2)(c2ccccc2)C2(C(=O)OCc3ccccc3)CCCC2)C(C)(C)C)[C@H](C(C)(C)C)C1</smiles>

Benzyl 1-((S)-1-((diphenylmethylene)amino)-2-(((1R,2S,5R)-5-methyl-2-(2-(naphthalen-1-yl)propan-2yl)cyclohexyl)oxy)-2-oxo-1-phenylethyl)cyclopentane-1-carboxylate (3ठe)

Prepared according to General Procedure $\mathrm{G}$ and purified by flash column chromatography (Hexane/Et $\mathrm{t}_{2} \mathrm{O}$ = 19/1 (1\% Et $\left.{ }_{3} \mathrm{~N}\right)$, diastereomixture, 55\% yield, $d r=92 / 8$, colorless oil); IR (neat) 2357, 1717, 1263, 1161 $\mathrm{cm}^{-1} ;{ }^{1} \mathrm{H}$ NMR $\left(500 \mathrm{MHz}, \mathrm{CDCl}_{3}, 27^{\circ} \mathrm{C}\right) \delta 8.19(\mathrm{~d}, J=9.0 \mathrm{~Hz}, 1 \mathrm{H}, \operatorname{ArH}), 7.78(\mathrm{~d}, J=7.0 \mathrm{~Hz}, 1 \mathrm{H}, \operatorname{ArH}), 7.61$ (dd, $J=7.5,2.5 \mathrm{~Hz}, 1 \mathrm{H}, \operatorname{ArH}), 7.54(\mathrm{~d}, J=7.5 \mathrm{~Hz}, 2 \mathrm{H}, \operatorname{ArH}), 7.36-7.28(\mathrm{~m}, 4 \mathrm{H}, \mathrm{ArH})$, 7.25-7.16 (m, 8H, ArH), 7.09 (t, J = 7.5 Hz, 1H, ArH), 7.04-6.98 (m, 4H, ArH), 6.96-6.91 (m, 3H, ArH), $6.88(\mathrm{~d}, J=7.5 \mathrm{~Hz}, 2 \mathrm{H}, \operatorname{ArH}), 5.08$ $(\mathrm{td}, J=10.5,4.0 \mathrm{~Hz}, 1 \mathrm{H}, \mathrm{OCH}), 5.07\left(\mathrm{~d}, J=11.5 \mathrm{~Hz}, 1 \mathrm{H}, \mathrm{OCH}_{2}\right), 4.81$ (d, J=12.5 Hz, 1H, OCH $), 2.74(\mathrm{td}, J=$ 12.5, $4.0 \mathrm{~Hz}, 1 \mathrm{H}, \mathrm{CH}), 2.65-2.60\left(\mathrm{~m}, 1 \mathrm{H}, \mathrm{CH}_{2}\right), 2.57-2.52\left(\mathrm{~m}, 1 \mathrm{H}, \mathrm{CH}_{2}\right), 2.47\left(\mathrm{~m}, 1 \mathrm{H}, \mathrm{CH}_{2}\right), 2.28\left(\mathrm{~m}, 2 \mathrm{H}, \mathrm{CH}_{2}\right)$, $1.90\left(\mathrm{~m}, 3 \mathrm{H}, \mathrm{CH}_{2}\right), 1.43\left(\mathrm{~s}, 3 \mathrm{H}, \mathrm{CH}_{3}\right), 1.36\left(\mathrm{~s}, 3 \mathrm{H}, \mathrm{CH}_{3}\right), 1.30-1.26\left(\mathrm{~m}, 2 \mathrm{H}, \mathrm{CH}_{2}\right), 0.87\left(\mathrm{~m}, 2 \mathrm{H}, \mathrm{CH}_{2}\right), 0.82(\mathrm{~d}, J$ $\left.=5.5 \mathrm{~Hz}, 3 \mathrm{H}, \mathrm{CHCH}_{3}\right), 0.53\left(\mathrm{qd}, J=13.0,4.0 \mathrm{~Hz}, 1 \mathrm{H}, \mathrm{CH}_{2}\right) ;{ }^{13} \mathrm{C} \mathrm{NMR}\left(125 \mathrm{MHz}, \mathrm{CDCl}_{3}, 27^{\circ} \mathrm{C}\right) \delta 175.1,170.0$, 168.5, 146.3, 143.2, 140.2, 138.4, 136.3, 135.0, 131.6, 129.8, 129.6, 128.9, 128.1, 127.7, 127.5, 127.4, 127.2, 127.1, 126.8, 126.7, 126.2, 125.0, 124.6, 124.5, 123.9, 79.3, 77.5, 68.1, 66.1, 47.9, 42.6, 40.8, 35.1, 34.7, 32.3, 31.4, 30.5, 28.3, 25.1, 24.7, 24.3, 21.8; HRMS (ESI) m/z calcd. for $\mathrm{C}_{54} \mathrm{H}_{56} \mathrm{NO}_{4}{ }^{+} 782.4204$ found 782.4202.<smiles>CC1CC[C@@H](C(C)(C)c2cccc3ccccc23)C(OC(=O)[C@](N=C(c2ccccc2)c2ccccc2)(c2ccccc2)C(C)(C)C(=O)c2ccccc2)C1</smiles>

(1R,2S,5R)-5-Methyl-2-(2-(naphthalen-1-yl)propan-2-yl)cyclohexyl (S)-2-((diphenylmethylene)amino)3,3-dimethyl-4-oxo-2,4-diphenylbutanoate (380) 
Supporting information Amino Acid Schiff Base Bearing Benzophenone Imine as a Platform for Highly Congested Unnatural $\alpha$-Amino Acid Synthesis

Prepared according to General Procedure $\mathrm{G}$ and purified by flash column chromatography (Hexane/Et ${ }_{2} \mathrm{O}$ $=19 / 1\left(1 \% \mathrm{Et}_{3} \mathrm{~N}\right)$, diastereomixture, 62\% yield, $\mathrm{dr}=89 / 11$, white solid); IR (neat) 1713, 1445, $1217 \mathrm{~cm}^{-1}$; ${ }^{1} \mathrm{H} \mathrm{NMR}\left(500 \mathrm{MHz}, \mathrm{CDCl}_{3}, 27^{\circ} \mathrm{C}\right) \delta 8.26(\mathrm{~d}, J=9.0 \mathrm{~Hz}, 1 \mathrm{H}, \mathrm{ArH}), 7.78(\mathrm{dd}, J=13.5,1.5 \mathrm{~Hz}, 1 \mathrm{H}, \mathrm{ArH}), 7.62-$ $7.60(\mathrm{~m}, 1 \mathrm{H}, \operatorname{ArH}), 7.51(\mathrm{~m}, 2 \mathrm{H}, \operatorname{ArH}), 7.45(\mathrm{~m}, 2 \mathrm{H}, \operatorname{ArH}), 7.39-7.32(\mathrm{~m}, 10 \mathrm{H}, \operatorname{ArH})$, 7.30-7.27 (m, 2H, ArH), 7.16-7.08 (m, 1H, ArH), 7.07-7.01 (m, 7H, ArH), $5.14(\mathrm{td}, J=10.5,4.0 \mathrm{~Hz}, 1 \mathrm{H}, \mathrm{OCH}), 2.73(\mathrm{td}, J=10.5,4.0$ $\mathrm{Hz}, 1 \mathrm{H}, \mathrm{CH}), 2.34\left(\mathrm{~d}, \mathrm{~J}=10.5 \mathrm{~Hz}, 1 \mathrm{H}, \mathrm{CH}_{2}\right), 1.65\left(\mathrm{~s}, 3 \mathrm{H}, \mathrm{CH}_{3}\right), 1.55\left(\mathrm{~s}, 3 \mathrm{H}, \mathrm{CH}_{3}\right), 1.43\left(\mathrm{~s}, 3 \mathrm{H}, \mathrm{CH}_{3}\right), 1.26(\mathrm{~m}$, $\left.2 \mathrm{H}, \mathrm{CH}_{2}\right), 1.26\left(\mathrm{~s}, 3 \mathrm{H}, \mathrm{CH}_{3}\right), 0.93-0.86\left(\mathrm{~m}, 1 \mathrm{H}, \mathrm{CH}_{2}\right), 0.82\left(\mathrm{~d}, \mathrm{~J}=6.5 \mathrm{~Hz}, 3 \mathrm{H}, \mathrm{CHCH}_{3}\right), 0.80-0.77\left(\mathrm{~m}, 2 \mathrm{H}, \mathrm{CH}_{2}\right)$, $0.51\left(\mathrm{qd}, J=12.5,4.0 \mathrm{~Hz}, 1 \mathrm{H}, \mathrm{CH}_{2}\right) ;{ }^{13} \mathrm{C} \mathrm{NMR}\left(125 \mathrm{MHz}, \mathrm{CDCl}_{3}, 27^{\circ} \mathrm{C}\right) \delta 209.6,170.2,168.2,146.6,142.4$, 137.2, 135.0, 131.6, 129.9, 129.6, 129.5, 129.2, 129.1, 128.0, 127.9, 127.7, 127.6, 127.4, 127.1, 127.0, 126.9, 126.4, 125.1, 124.6, 124.4, 123.9, 79.4, 78.8, 59.9, 47.7, 42.7, 41.0, 34.7, 31.4, 30.3, 28.3, 24.8, 24.7, 21.9; HRMS (ESI) m/z calcd. for $\mathrm{C}_{51} \mathrm{H}_{52} \mathrm{NO}_{3}{ }^{+} 726.3942$ found 726.3923 .

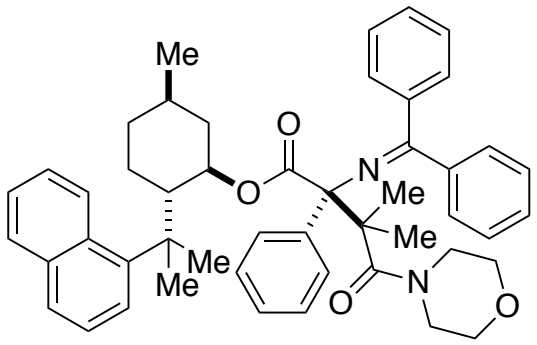

$(1 R, 2 S, 5 R)-5-M e t h y l-2-(2-($ naphthalen-1-yl)propan-2-yl)cyclohexyl (S)-2-((diphenylmethylene)amino)-

\section{3,3-dimethyl-4-morpholino-4-oxo-2-phenylbutanoate (38p)}

Prepared according to General Procedure $G$ and purified by flash column chromatography (Hexane/AcOEt = 4/1 (1\% Et $\left.{ }_{3} \mathrm{~N}\right)$, diastereomixture, 60\% yield, $\mathrm{dr}=89 / 11$, white solid); IR (neat) 1711, 1445, 1215, $1115 \mathrm{~cm}^{-1} ;{ }^{1} \mathrm{H}$ NMR $\left(500 \mathrm{MHz}, \mathrm{CDCl}_{3}, 27^{\circ} \mathrm{C}\right) \delta 8.37-8.35(\mathrm{~m}, 1 \mathrm{H}, \mathrm{ArH}), 7.83-7.81(\mathrm{~m}, 1 \mathrm{H}, \mathrm{ArH})$, $7.64(\mathrm{dd}, J=7.5,1.5 \mathrm{~Hz}, 2 \mathrm{H}, \operatorname{ArH})$, 7.51-7.46 (m, 2H, ArH), 7.42-7.41 (m, 2H, ArH), 7.33-7.28 (m, 5H, ArH), 7.19-7.10 (m, 5H, ArH), 7.05 (br, 2H, ArH), 6.90 (br, 2H, ArH), $4.95(\mathrm{td}, J=10.0,4.0 \mathrm{~Hz}, 1 \mathrm{H}, \mathrm{OCH}), 3.69-$ $3.60\left(\mathrm{~m}, 4 \mathrm{H}, \mathrm{NCH}_{2}\right), 3.52-3.46\left(\mathrm{~m}, 4 \mathrm{H}, \mathrm{OCH}_{2}\right), 2.75(\mathrm{td}, J=11.5,3.5 \mathrm{~Hz}, 1 \mathrm{H}, \mathrm{CH}), 2.37(\mathrm{~d}, J=10.5 \mathrm{~Hz}, 1 \mathrm{H}$, $\left.\mathrm{CH}_{2}\right), 1.66\left(\mathrm{~s}, 3 \mathrm{H}, \mathrm{CH}_{3}\right), 1.58\left(\mathrm{~s}, 3 \mathrm{H}, \mathrm{CH}_{3}\right), 1.42-1.34\left(\mathrm{~m}, 1 \mathrm{H}, \mathrm{CH}_{2}\right), 1.26\left(\mathrm{~s}, 3 \mathrm{H}, \mathrm{CH}_{3}\right), 1.26\left(\mathrm{~m}, 1 \mathrm{H}, \mathrm{CH}_{2}\right), 1.15$ $\left(\mathrm{s}, 3 \mathrm{H}, \mathrm{CH}_{3}\right), 0.91-0.82\left(\mathrm{~m}, 2 \mathrm{H}, \mathrm{CH}_{2}\right), 0.80\left(\mathrm{~d}, J=6.5 \mathrm{~Hz}, 3 \mathrm{H}, \mathrm{CHCH}_{3}\right), 0.78\left(\mathrm{~m}, 1 \mathrm{H}, \mathrm{CH}_{2}\right), 0.51(\mathrm{qd}, J=12.5$, $\left.3.5 \mathrm{~Hz}, 1 \mathrm{H}, \mathrm{CH}_{2}\right) ;{ }^{13} \mathrm{C}$ NMR $\left(125 \mathrm{MHz}, \mathrm{CDCl}_{3}, 27^{\circ} \mathrm{C}\right) \delta 175.0,169.6,168.9,146.4,138.5,135.0,131.5,130.1$, 129.7, 129.2, 128.7, 127.8, 127.3, 127.2, 127.0, 126.3, 125.0, 124.6, 124.4, 123.9, 78.9, 78.1, 67.0, 56.7, 47.4, 46.4, 42.7, 40.8, 34.5, 31.3, 30.3, 28.3, 26.1, 25.8, 25.1, 21.9; HRMS (ESI) m/z calcd. for $\mathrm{C}_{49} \mathrm{H}_{55} \mathrm{~N}_{2} \mathrm{O}_{4}{ }^{+}$ 735.4156 found 735.4154 . 
Supporting information Amino Acid Schiff Base Bearing Benzophenone Imine as a Platform for Highly Congested Unnatural $\alpha$-Amino Acid Synthesis

\section{Determination of Absolute Configuration of the Product}

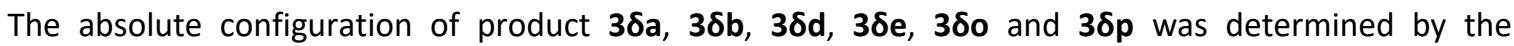
transformation of the analog $\mathbf{3} \delta \mathrm{r}$ to the known product $\mathbf{S 1}$ in the literature ${ }^{4}$ as described below.

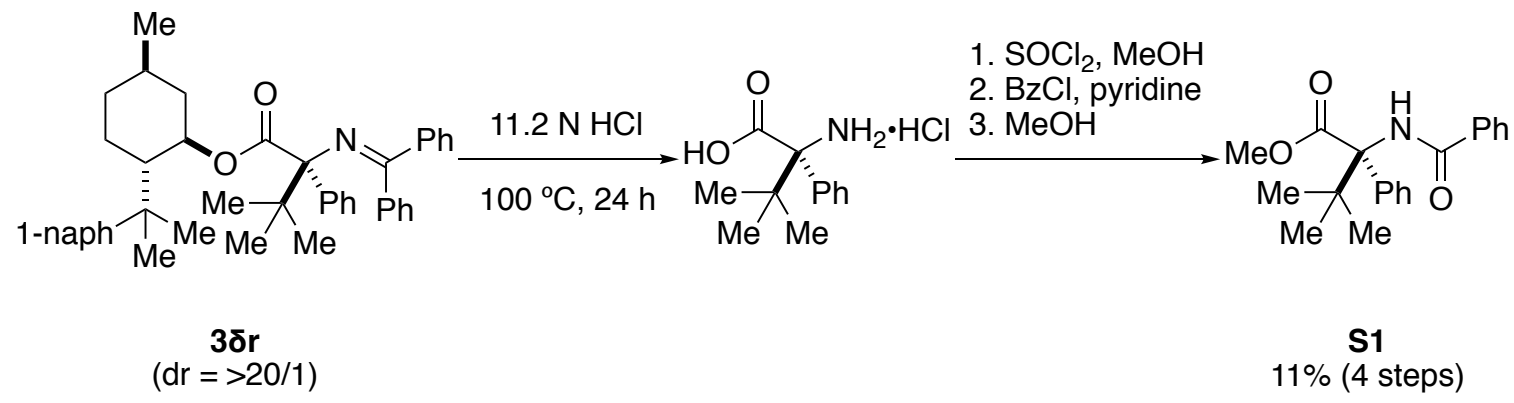

Methyl (R)-2-benzamido-3,3-dimethyl-2-phenylbutanoate (S1); CAS Registry Number 1429442-53-4

A $4 \mathrm{~mL}$ vial was charged with $3 \delta \mathrm{r}(0.15 \mathrm{mmol}, 1.0$ equiv.) and $11.2 \mathrm{M} \mathrm{HCl}(5.0 \mathrm{~mL})$ and the solution was stirred overnight at $100{ }^{\circ} \mathrm{C}$. After consumption of starting material was confirmed by TLC analysis, the solution was poured into $\mathrm{H}_{2} \mathrm{O}(5.0 \mathrm{~mL})$ and extracted with diethyl ether $(3 \times 10.0 \mathrm{~mL})$. The water layer was combined and water was removed in vacuo, and the white solid was obtained. Then, a $4 \mathrm{~mL}$ vial was charged with the obtained solid and $\mathrm{MeOH}(2.0 \mathrm{~mL}) . \mathrm{SOCl}_{2}\left(0.17 \mathrm{mmol}, 1.1\right.$ equiv.) was added at $0{ }^{\circ} \mathrm{C}$ and the solution was stirred overnight at ambient temperature. After evaporation of the solvent, pyridine $(2.0 \mathrm{~mL})$ and benzoyl chloride $(0.45 \mathrm{mmol}, 3.0$ equiv.) was added and the reaction mixture was heated at $50{ }^{\circ} \mathrm{C}$ for 24 hours. After consumption of starting material was confirmed by TLC analysis, the solvent was removed in vacuo. $\mathrm{MeOH}(2.0 \mathrm{~mL})$ was poured into the reaction mixture and stirred for 24 hours. The solution was poured into sat. $\mathrm{NaHCO}_{3}$ aq and extracted with $\mathrm{CH}_{2} \mathrm{Cl}_{2}$. The combined organic layers were washed with $1 \mathrm{~N} \mathrm{HCl}$ and brine, dried over sodium sulfate, and evaporation of the solvent gave the crude product, which was purified by flash column chromatography. (Hexane/EtOAc $=4 / 1(1 \%$ $\mathrm{Et}_{3} \mathrm{~N}, 11 \%$ yield, colorless oil); ${ }^{1} \mathrm{H} \mathrm{NMR}\left(500 \mathrm{MHz}, \mathrm{CDCl}_{3}, 27^{\circ} \mathrm{C}\right) \delta 7.83(\mathrm{t}, \mathrm{J}=7.0 \mathrm{~Hz}, 4 \mathrm{H}, \mathrm{ArH}), 7.55(\mathrm{t}, \mathrm{J}=$ $7.5 \mathrm{~Hz}, 1 \mathrm{H}, \operatorname{ArH}), 7.49(\mathrm{t}, J=8.0 \mathrm{~Hz}, 2 \mathrm{H}, \operatorname{ArH}), 7.30(\mathrm{t}, J=8.0 \mathrm{~Hz}, 2 \mathrm{H}, \operatorname{ArH}), 7.27-7.25(\mathrm{~m}, 1 \mathrm{H}, \operatorname{ArH}), 6.80(\mathrm{~s}$,

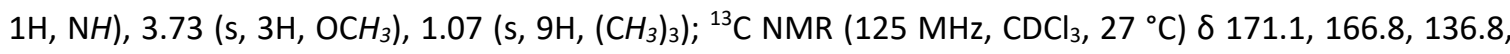
134.8, 131.7, 128.8, 128.3, 127.2, 127.0, 127.0, 70.0, 52.1, 38.6, 26.6; $[\alpha]_{\mathrm{D}}{ }^{24}=-43.1$ (c 0.55, $\mathrm{CHCl}_{3}$ ). Lit. $[\alpha]_{\mathrm{D}}^{20}=-57.8\left(\mathrm{c} 1.12, \mathrm{CHCl}_{3}\right){ }^{4}$

\section{Transformation of the Product}


Supporting information Amino Acid Schiff Base Bearing Benzophenone Imine as a Platform for Highly Congested Unnatural $\alpha$-Amino Acid Synthesis<smiles>CCOC(=O)C(C)(N)C(N)(C(=O)OC)c1ccccc1</smiles>

\section{4-Ethyl 1-methyl 2-amino-3,3-dimethyl-2-phenylsuccinate (7)}

A $4 \mathrm{~mL}$ vial was charged with $3 a a(0.10 \mathrm{mmol}, 1.0$ equiv.), $\mathrm{MeOH}(1.0 \mathrm{~mL})$ and $1 \mathrm{~N} \mathrm{HCl}(1.0 \mathrm{~mL})$ and the solution was stirred overnight at ambient temperature. After consumption of starting material was confirmed by TLC analysis, the solution was poured into water $(5.0 \mathrm{~mL})$ and extracted with diethyl ether $(5.0 \mathrm{~mL})$. The water layer was poured into sat. $\mathrm{NaHCO}_{3}$ aq $(5.0 \mathrm{~mL})$ and extracted with diethyl ether $(3 \mathrm{x}$ $5.0 \mathrm{~mL}$ ). The combined organic layers were washed with brine, dried over sodium sulfate, and filtrated then purified by flash column chromatography (Hexane/Et ${ }_{2} \mathrm{O} / \mathrm{MeOH}=10 / 1 / 1,86 \%$ yield, white solid); IR (neat) 1730, 1712, 1226, 1132, $1030 \mathrm{~cm}^{-1} ;{ }^{1} \mathrm{H} \mathrm{NMR}\left(500 \mathrm{MHz}, \mathrm{CDCl}_{3}, 2{ }^{\circ} \mathrm{C}\right) \delta 7.51$ (d, $\mathrm{J}=13.0 \mathrm{~Hz}, 2 \mathrm{H}$, $\operatorname{ArH})$, 7.34-7.30 (m, 3H, ArH), $4.17\left(\mathrm{q}, J=7.0 \mathrm{~Hz}, 2 \mathrm{H}, \mathrm{OCH}_{2} \mathrm{CH}_{3}\right), 3.72\left(\mathrm{~s}, 3 \mathrm{H}, \mathrm{OCH}_{3}\right), 2.51\left(\mathrm{~s}, 2 \mathrm{H}, \mathrm{NH}_{2}\right), 1.28$ $\left(\mathrm{t}, J=7.5 \mathrm{~Hz}, 3 \mathrm{H}, \mathrm{OCH}_{2} \mathrm{CH}_{3}\right), 1.24\left(\mathrm{~s}, 3 \mathrm{H}, \mathrm{CH}_{3}\right), 1.19\left(\mathrm{~s}, 3 \mathrm{H}, \mathrm{CH}_{3}\right) ;{ }^{13} \mathrm{C} \mathrm{NMR}\left(125 \mathrm{MHz}, \mathrm{CDCl}_{3}, 27^{\circ} \mathrm{C}\right) \delta 178.3$, 176.4, 139.6, 127.9, 127.8, 127.6, 69.2, 60.8, 52.2, 48.8, 23.9, 22.7, 14.1; HRMS (ESI) m/z calcd. for $\mathrm{C}_{15} \mathrm{H}_{22} \mathrm{NO}_{4}{ }^{+} 280.1543$ found 280.1532 .<smiles>CC(N)(C(=O)O)C(N)(C(=O)O)c1ccccc1</smiles>

\section{2-Amino-3,3-dimethyl-2-phenylsuccinic acid hydrochloride (8)}

A $4 \mathrm{~mL}$ vial was charged with $3 a a(0.10 \mathrm{mmol}, 1.0$ equiv.) and $6 \mathrm{M} \mathrm{HCl}(1.0 \mathrm{~mL})$ and the solution was stirred overnight at $100{ }^{\circ} \mathrm{C}$. After consumption of starting material was confirmed by TLC analysis, the solution was poured into $1 \mathrm{~N} \mathrm{HCl}(5.0 \mathrm{~mL})$ and extracted with diethyl ether $(3 \times 5.0 \mathrm{~mL})$. The water layer was combined and water was removed in vacuo, and the product was obtained (89\% yield, white solid). The combined organic layers were washed with brine, dried over sodium sulfate, and evaporation of the solvent gave benzophenone (89\% yield, white solid); IR (neat) 2361, 1749, 1481, 1292, $1107 \mathrm{~cm}^{-1} ;{ }^{1} \mathrm{H}$ NMR (500 MHz, $\left.\mathrm{D}_{2} \mathrm{O}, 27^{\circ} \mathrm{C}\right) \delta 7.43(\mathrm{~m}, 5 \mathrm{H}, \mathrm{ArH}), 1.32\left(\mathrm{~s}, 3 \mathrm{H}, \mathrm{CH}_{3}\right), 1.13\left(\mathrm{~s}, 3 \mathrm{H}, \mathrm{CH}_{3}\right) ;{ }^{13} \mathrm{C} \mathrm{NMR}(125 \mathrm{MHz}$, $\left.\mathrm{D}_{2} \mathrm{O}, 2{ }^{\circ} \mathrm{C}\right) \delta 180.1,171.8,132.0,129.8,129.0,126.5,47.8,22.8,21.6$; HRMS (ESI) $\mathrm{m} / \mathrm{z}$ calcd. for $\mathrm{C}_{12} \mathrm{H}_{16} \mathrm{NO}_{4}{ }^{+} 238.1074$ found 238.1039 . 
Supporting information Amino Acid Schiff Base Bearing Benzophenone Imine as a Platform for Highly Congested Unnatural $\alpha$-Amino Acid Synthesis<smiles>CCOC(=O)C(C)(C)C(NC(=O)CNC(=O)OCC1c2ccccc2-c2ccccc21)(C(=O)OC)c1ccccc1</smiles>

\section{4-Ethyl 1-methyl 2-(2-((((9H-fluoren-9-yl)methoxy)carbonyl)amino)acetamido)-3,3-dimethyl-2- phenylsuccinate (9)}

A $4 \mathrm{~mL}$ vial was charged with 7aa $\left(0.10 \mathrm{mmol}, 1.0\right.$ equiv.) and $\mathrm{CH}_{2} \mathrm{Cl}_{2}(0.25 \mathrm{~mL})$, followed by pyridine (0.20 mmol, 2.0 equiv.). The Fmoc- $\mathrm{Gly}-\mathrm{Cl}$ solution in $\mathrm{CH}_{2} \mathrm{Cl}_{2}(0.25 \mathrm{~mL})$ was added at $0{ }^{\circ} \mathrm{C}$ and the solution was stirred for 24 hours at ambient temperature. After consumption of starting material was confirmed by TLC analysis, the solution was poured into sat. $\mathrm{NaHCO}_{3}$ aq and extracted with $\mathrm{CH}_{2} \mathrm{Cl}_{2}$. The combined organic layers were washed with $1 \mathrm{~N} \mathrm{HCl}$ and brine, dried over sodium sulfate, and evaporation of the solvent gave the product (72\% yield, colorless oil); IR (neat) 1726, 1705, 1514, 1240, $1143 \mathrm{~cm}^{-1} ;{ }^{1} \mathrm{H} \mathrm{NMR}$ $\left(500 \mathrm{MHz} \mathrm{CDCl}_{3}, 27^{\circ} \mathrm{C}\right) \delta 8.37(\mathrm{~s}, 1 \mathrm{H}, \mathrm{ArH}), 7.76-7.72(\mathrm{~m}, 4 \mathrm{H}, \mathrm{ArH}, \mathrm{NH}), 7.63-7.61(\mathrm{~m}, 2 \mathrm{H}, \operatorname{ArH}), 7.40-7.37$ $(\mathrm{m}, 2 \mathrm{H}, \operatorname{ArH}), 7.30-7.27(\mathrm{~m}, 5 \mathrm{H}, \mathrm{ArH}), 5.55(\mathrm{t}, J=5.5 \mathrm{~Hz}, 1 \mathrm{H}, \mathrm{NH}), 4.44-4.39\left(\mathrm{~m}, 2 \mathrm{H}, \mathrm{CH}_{2}\right), 4.27-4.24(\mathrm{~m}, 1 \mathrm{H}$, $\left.\mathrm{ArCH}_{2}\right)$, 4.09-3.95 (m, 3H, $\left.\mathrm{ArCH}_{2}, \mathrm{OCH}_{2} \mathrm{CH}_{3}\right), 3.71\left(\mathrm{~s}, 3 \mathrm{H}, \mathrm{OCH}_{3}\right), 1.25\left(\mathrm{~s}, 3 \mathrm{H}, \mathrm{CH}_{3}\right), 1.16(\mathrm{t}, J=7.5 \mathrm{~Hz}$, $\left.\mathrm{OCH}_{2} \mathrm{CH}_{3}\right), 1.07\left(\mathrm{~s}, 3 \mathrm{H}, \mathrm{CH}_{3}\right) ;{ }^{13} \mathrm{C}$ NMR $\left(125 \mathrm{MHz}_{2} \mathrm{CDCl}_{3}, 27^{\circ} \mathrm{C}\right) \delta 176.2,169.9,168.6,156.6,143.9,143.8$, 141.3, 134.8, 128.7, 127.8, 127.7, 127.4, 127.1, 125.2, 120.0, 68.5, 67.4, 61.8, 52.6, 48.4, 47.1, 44.7, 22.3, 21.8, 13.8; HRMS (ESI) m/z calcd. for $\mathrm{C}_{32} \mathrm{H}_{34} \mathrm{~N}_{2} \mathrm{NaO}_{7}{ }^{+} 581.2258$ found 581.2256.<smiles>CCOC(=O)C(C)(C)CC(N)C(=O)OC</smiles>

\section{1-Ethyl 5-methyl 4-amino-2,2-dimethylpentanedioate (10)}

$25 \mathrm{~mL}$ round-bottom flask was charged with 4 a $(0.20 \mathrm{mmol}, 1.0$ equiv.) and EtOH (3.5 mL), followed by $10 \% \mathrm{Pd} / \mathrm{C}(0.020 \mathrm{mmol}, 10 \mathrm{~mol} \%)$. After filling the flask with hydrogen, the solution was stirred for 24 hours at ambient temperature. After consumption of starting material was confirmed by TLC analysis, the reaction mixture was filtered and purified by flash column chromatography (Hexane/Et ${ }_{2} \mathrm{O}=1 / 1,65 \%$ yield, colorless oil); IR (neat) 1738, 1699, 1263, 1207, $1024 \mathrm{~cm}^{-1} ;{ }^{1} \mathrm{H} \mathrm{NMR}\left(500 \mathrm{MHz}, \mathrm{CDCl}_{3}, 27^{\circ} \mathrm{C}\right) \delta 4.15-$ $4.08\left(\mathrm{~m}, 2 \mathrm{H}, \mathrm{OCH}_{2}\right), 3.01\left(\mathrm{~s}, 3 \mathrm{H}, \mathrm{OCH}_{3}\right), 3.51(\mathrm{q}, J=5.0 \mathrm{~Hz}, 1 \mathrm{H}, \mathrm{CH}), 1.97\left(\mathrm{dd}, J=14.0,9.5 \mathrm{~Hz}, 1 \mathrm{H}, \mathrm{CH}_{2}\right)$, $1.88\left(\mathrm{dd}, J=14.0,4.5 \mathrm{~Hz}, 1 \mathrm{H}, \mathrm{CH}_{2}\right), 1.26\left(\mathrm{t}, J=7.0 \mathrm{~Hz}, 3 \mathrm{H}, \mathrm{OCH}_{2} \mathrm{CH}_{3}\right), 1.23\left(\mathrm{~s}, 3 \mathrm{H}, \mathrm{CH}_{3}\right), 1.23\left(\mathrm{~s}, 3 \mathrm{H}, \mathrm{CH}_{3}\right)$; 
${ }^{13} \mathrm{C} \mathrm{NMR}\left(125 \mathrm{MHz}, \mathrm{CDCl}_{3}, 27^{\circ} \mathrm{C}\right) \delta 177.8,176.8,60.4,52.1,52.0,44.1,40.7,27.2,23.5,14.1$; HRMS (ESI) $\mathrm{m} / \mathrm{z}$ calcd. for $\mathrm{C}_{10} \mathrm{H}_{20} \mathrm{NO}_{4}{ }^{+} 218.1387$ found 218.1385 .

\section{Control Experiment}<smiles>COC(=O)C(C)N=C(c1ccccc1)c1ccccc1</smiles>

11

11

In an inert atmosphere glove box, a $4 \mathrm{~mL}$ vial was charged with copper salt $(0.10 \mathrm{mmol}, 100 \mathrm{~mol} \%)$ and L5 $(0.10 \mathrm{mmol}, 100 \mathrm{~mol} \%)$, followed by tert-butylbenzene $(0.50 \mathrm{~mL})$. After the solution was stirred at ambient temperature for 10 minutes, 1 l (0.10 mmol, 1.0 equiv.) and $\mathrm{K}_{2} \mathrm{CO}_{3}(0.10 \mathrm{mmol}, 1.0$ equiv.) were added and removed from the glovebox. The reaction solution was stirred at $100{ }^{\circ} \mathrm{C}$ for 24 hours. Copper salt was removed by silica gel short pad column chromatography and washed with excess ethyl acetate. Evaporation of the solvent gave the crude product $(\mathrm{dr}=1.2 / 1)$, which was purified by flash column chromatography.

\section{Dimethyl 2,3-bis((diphenylmethylene)amino)-2,3-dimethylsuccinate (11)}

Major isomer: IR (neat) 2359, 2342, $1636 \mathrm{~cm}^{-1} ;{ }^{1} \mathrm{H}$ NMR $\left(500 \mathrm{MHz}, \mathrm{CDCl}_{3}, 27^{\circ} \mathrm{C}\right) \delta 7.56(\mathrm{~d}, J=7.0 \mathrm{~Hz}, 4 \mathrm{H}$, $\operatorname{ArH}), 7.41-7.38(\mathrm{~m}, 6 \mathrm{H}, \operatorname{ArH}), 7.32(\mathrm{t}, J=7.0 \mathrm{~Hz}, 2 \mathrm{H}, \operatorname{ArH}), 7.25-7.23(\mathrm{~m}, 4 \mathrm{H}, \mathrm{ArH}), 7.17(\mathrm{~m}, 4 \mathrm{H}, \operatorname{ArH}), 3.36$ (s, 3H, OCH $\left.\mathrm{OCH}_{3}\right), 1.63\left(\mathrm{~s}, 6 \mathrm{H}, \mathrm{CH}_{3}\right) ;{ }^{13} \mathrm{C} \mathrm{NMR}\left(125 \mathrm{MHz}, \mathrm{CDCl}_{3}, 27{ }^{\circ} \mathrm{C}\right) \delta 172.5,166.5,141.1,137.3,130.0$, 128.8, 128.6, 128.3, 127.8, 127.6, 73.6, 51.2, 22.5; HRMS (ESI) m/z calcd. for $\mathrm{C}_{34} \mathrm{H}_{33} \mathrm{~N}_{2} \mathrm{O}_{4}{ }^{+} 533.2435$ found 533.2418.

Minor isomer: IR (neat) 2361, 1732, 1634, $1248 \mathrm{~cm}^{-1} ;{ }^{1} \mathrm{H}$ NMR $\left(500 \mathrm{MHz}, \mathrm{CDCl}_{3}, 27^{\circ} \mathrm{C}\right) \delta 7.60(\mathrm{~d}, \mathrm{~J}=7.0$ $\mathrm{Hz}, 4 \mathrm{H}, \operatorname{ArH}), 7.40-7.39(\mathrm{~m}, 6 \mathrm{H}, \mathrm{ArH}), 7.33(\mathrm{t}, J=7.5 \mathrm{~Hz}, 2 \mathrm{H}, \operatorname{ArH}), 7.26(\mathrm{t}, J=7.5 \mathrm{~Hz}, 4 \mathrm{H}, \operatorname{ArH}), 7.20-7.18$ $(\mathrm{m}, 4 \mathrm{H}, \mathrm{ArH}), 3.42\left(\mathrm{~s}, 6 \mathrm{H}, \mathrm{OCH}_{3}\right), 1.46\left(\mathrm{~s}, 6 \mathrm{H}, \mathrm{CH}_{3}\right) ;{ }^{13} \mathrm{C} \mathrm{NMR}\left(125 \mathrm{MHz}, \mathrm{CDCl}_{3}, 27^{\circ} \mathrm{C}\right) \delta 173.2,165.9,141.5$, 138.0, 129.9, 128.8, 128.5, 128.3, 127.8, 127.7, 74.1, 51.4, 20.8; HRMS (ESI) m/z calcd. for $\mathrm{C}_{34} \mathrm{H}_{33} \mathrm{~N}_{2} \mathrm{O}_{4}{ }^{+}$ 533.2435 found 533.2418 . 
Supporting information Amino Acid Schiff Base Bearing Benzophenone Imine as a Platform for Highly Congested Unnatural $\alpha$-Amino Acid Synthesis

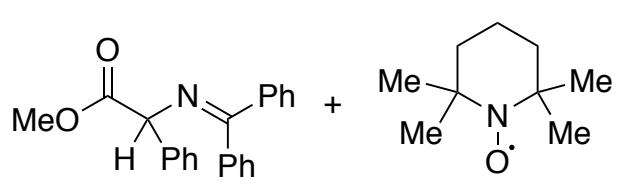

1a

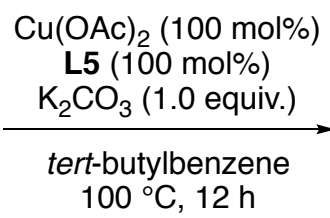

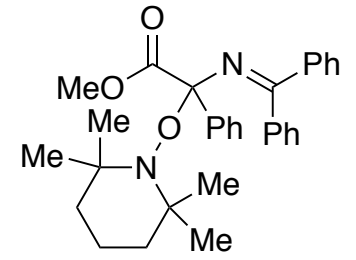

12

In an inert atmosphere glove box, a $4 \mathrm{~mL}$ vial was charged with copper(II) acetate $(0.10 \mathrm{mmol}, 100$ mol\%) and $\mathbf{L 5}(0.10 \mathrm{mmol}, 100 \mathrm{~mol} \%)$, followed by tert-butylbenzene $(0.50 \mathrm{~mL})$. After the solution was stirred at ambient temperature for 10 minutes, $1 \mathrm{a}\left(0.10 \mathrm{mmol}, 1.0\right.$ equiv.), $\mathrm{K}_{2} \mathrm{CO}_{3}(0.10 \mathrm{mmol}, 1.0$ equiv.) and TEMPO ( $0.20 \mathrm{mmol}, 2.0$ equiv.) were added and removed from the glovebox. The reaction solution was stirred at $100{ }^{\circ} \mathrm{C}$ for 24 hours. Copper salt was removed by silica gel short pad column chromatography and washed with excess ethyl acetate. Evaporation of the solvent gave the crude product and TEMPO adduct 12 was detected by HRMS (ESI). (m/z calcd. for $\mathrm{C}_{31} \mathrm{H}_{37} \mathrm{~N}_{2} \mathrm{O}_{3}{ }^{+} 485.2799$ found 485.2798)<smiles>CCOC(=O)C(C)(C)CC/C=C(/N=C(/Pc1ccccc1)c1ccccc1)C(=O)OC</smiles>

13 2a

14

$$
15
$$

In an inert atmosphere glove box, a $4 \mathrm{~mL}$ vial was charged with copper(II) acetate $(0.010 \mathrm{mmol}, 10$ mol\%) and $\mathbf{L} 5(0.010 \mathrm{mmol}, 10 \mathrm{~mol} \%)$, followed by tert-butylbenzene $(0.50 \mathrm{~mL})$. After the solution was stirred at ambient temperature for 10 minutes, 13 (0.10 mmol, 1.0 equiv.), 2a (0.20 mmol, 2.0 equiv.) and $\mathrm{K}_{2} \mathrm{CO}_{3}$ ( $0.10 \mathrm{mmol}, 1.0$ equiv.) were added and removed from the glove box. The reaction solution was stirred at $100{ }^{\circ} \mathrm{C}$ for 24 hours. Copper salt was removed by silica gel short pad column chromatography and washed with excess ethyl acetate. Evaporation of the solvent gave the crude product.

\section{7-Ethyl 1-methyl (E)-2-((diphenylmethylene)amino)-6,6-dimethylhept-2-enedioate (14)}

IR (neat) 1722, 1244, $1144 \mathrm{~cm}^{-1} ;{ }^{1} \mathrm{H}$ NMR $\left(500 \mathrm{MHz}, \mathrm{CDCl}_{3}, 27^{\circ} \mathrm{C}\right) \delta 7.71$ (d, J = $7.0 \mathrm{~Hz}, 2 \mathrm{H}, \operatorname{ArH}$, minor isomer), 7.52 (d, $J=7.0 \mathrm{~Hz}, 2 \mathrm{H}, \mathrm{ArH}$, major isomer), 7.47-7.12 (m, 6H, ArH, major isomer), 7.47-7.12 (m, $8 \mathrm{H}, \mathrm{ArH}$, minor isomer), $7.04(\mathrm{~d}, J=7.0 \mathrm{~Hz}, 2 \mathrm{H}, \mathrm{ArH}$, major isomer), $6.12(\mathrm{~d}, J=10.5 \mathrm{~Hz}, 1 \mathrm{H}, \mathrm{CH}$, major 
Supporting information Amino Acid Schiff Base Bearing Benzophenone Imine as a Platform for Highly Congested Unnatural $\alpha$-Amino Acid Synthesis

isomer), $6.01\left(\mathrm{~d}, \mathrm{~J}=10.0 \mathrm{~Hz}, 1 \mathrm{H}, \mathrm{CH}\right.$, minor isomer), 4.12-4.08 (m, $2 \mathrm{H}, \mathrm{OCH}_{2} \mathrm{CH}_{3}$, minor isomer), 4.02-3.93 (m, $2 \mathrm{H}, \mathrm{OCH}_{2} \mathrm{CH}_{3}$, major isomer), $3.27\left(\mathrm{~s}, 3 \mathrm{H}, \mathrm{OCH}_{3}\right), 3.22\left(\mathrm{~s}, 3 \mathrm{H}, \mathrm{OCH}_{3}\right.$, minor isomer), $3.07(\mathrm{t}, J=10.0 \mathrm{~Hz}$, $\mathrm{CH}_{2}$, major isomer), 2.95 (t, $\mathrm{J}=10.0 \mathrm{~Hz}, \mathrm{CH}_{2}$, minor isomer), 2.09-2.04 (m, 2H, $\mathrm{CH}_{2}$, minor isomer), 2.01$1.92\left(\mathrm{~m}, 2 \mathrm{H}, \mathrm{CH}_{2}\right.$, major isomer), 1.78 (d, J = $13.0 \mathrm{~Hz}, 1 \mathrm{H}, \mathrm{CH}_{2}$, major isomer), 1.72 (d, J = 11.5 Hz, $1 \mathrm{H}, \mathrm{CH}_{2}$, minor isomer), 1.29 (s, 3H, $\mathrm{CH}_{3}$, major isomer), 1.25 (s, 3H, $\mathrm{CH}_{3}$, minor isomer), 1.22 (s, 3H, $\mathrm{CH}_{3}$, major isomer), $1.20\left(\mathrm{~s}, 3 \mathrm{H}, \mathrm{CH}_{3}\right.$, minor isomer), $1.17\left(\mathrm{t}, J=7.0 \mathrm{~Hz}, 3 \mathrm{H}, \mathrm{OCH}_{2} \mathrm{CH}_{3}\right.$, major isomer), $1.16(\mathrm{t}, J=7.0$

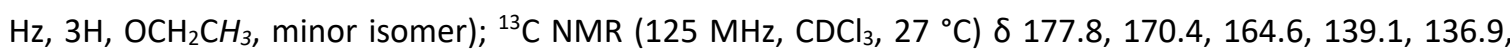
132.8, 130.6, 129.8, 129.6, 129.0, 128.8, 128.7, 127.9, 127.8, 127.6, 60.2, 51.2, 42.0, 42.0, 27.3, 27.2, 24.1, 14.0; HRMS (ESI) m/z calcd. for $\mathrm{C}_{25} \mathrm{H}_{30} \mathrm{NO}_{4}{ }^{+} 408.2169$ found 408.1976 .

\section{2,7-Diethyl 3-methyl (E)-3-((diphenylmethylene)amino)-2,7-dimethyloct-4-ene-2,3,7-tricarboxylate} (15)

IR (neat) 2360, 1724, 1238, 1136, $1028 \mathrm{~cm}^{-1} ;{ }^{1} \mathrm{H}$ NMR $\left(500 \mathrm{MHz}, \mathrm{CDCl}_{3}, 27^{\circ} \mathrm{C}\right) \delta 7.62(\mathrm{~d}, J=7.0 \mathrm{~Hz}, 2 \mathrm{H}$, $\operatorname{ArH}), 7.38(\mathrm{t}, J=7.5 \mathrm{~Hz}, 1 \mathrm{H}, \operatorname{ArH}), 7.33-7.30(\mathrm{~m}, 5 \mathrm{H}, \operatorname{ArH}), 7.13-7.11(\mathrm{~m}, 2 \mathrm{H}, \operatorname{ArH}), 6.03(\mathrm{~d}, J=15.5 \mathrm{~Hz}, 1 \mathrm{H}$, $\mathrm{CH}), 5.37\left(\mathrm{~m}, 1 \mathrm{H}, \mathrm{CHCH}_{2}\right), 4.08\left(\mathrm{q}, J=7.0 \mathrm{~Hz}, 1 \mathrm{H}, \mathrm{OCH}_{2} \mathrm{CH}_{3}\right), 4.01\left(\mathrm{q}, J=7.0 \mathrm{~Hz}, 1 \mathrm{H}, \mathrm{OCH}_{2} \mathrm{CH}_{3}\right), 3.04(\mathrm{~s}, 3 \mathrm{H}$, $\mathrm{OCH}_{3}$ ), 2.33 (q, J = 7.5 Hz, 1H, $\mathrm{CH}_{2}$ ), 2.27 (q, J = 7.5 Hz, 1H, CH $\left.J=7.5 \mathrm{~Hz}, 3 \mathrm{H}, \mathrm{OCH}_{2} \mathrm{CH}_{3}\right), 1.18\left(\mathrm{~s}, 3 \mathrm{H}, \mathrm{CH}_{3}\right), 1.16\left(\mathrm{~s}, 3 \mathrm{H}, \mathrm{CH}_{3}\right), 1.09\left(\mathrm{t}, J=7.5 \mathrm{~Hz}, 3 \mathrm{H}, \mathrm{OCH}_{2} \mathrm{CH}_{3}\right) ;{ }^{13} \mathrm{C} \mathrm{NMR}^{\mathrm{N}}$ $\left(125 \mathrm{MHz}, \mathrm{CDCl}_{3}, 2{ }^{\circ} \mathrm{C}\right) \delta 177.5,175.9,171.1,166.6,141.7,136.9,133.4,129.9,129.0,128.9,128.4$, 128.2, 127.8, 127.3, 74.3, 60.5, 60.3, 52.0, 50.9, 43.8, 42.6, 25.3, 25.0, 22.3, 14.1, 14.0; HRMS (ESI) m/z calcd. for $\mathrm{C}_{31} \mathrm{H}_{40} \mathrm{NO}_{6}{ }^{+} 522.2850$ found 522.2849.

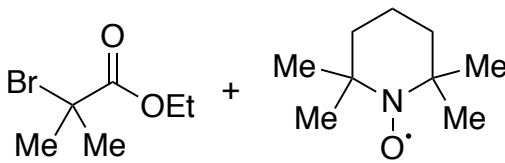

$2 a$

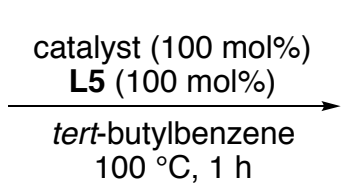

$100^{\circ} \mathrm{C}, 1 \mathrm{~h}$

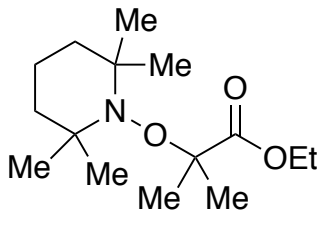

16

In an inert atmosphere glove box, a $4 \mathrm{~mL}$ vial was charged with copper(II) acetate $(0.10 \mathrm{mmol}, 100$ mol\%) and L5 $(0.10 \mathrm{mmol}, 100 \mathrm{~mol} \%)$, followed by tert-butylbenzene $(0.50 \mathrm{~mL})$. After the solution was stirred at ambient temperature for 10 minutes, $2 \mathrm{a}\left(0.10 \mathrm{mmol}, 1.0\right.$ equiv.), $\mathrm{K}_{2} \mathrm{CO}_{3}(0.10 \mathrm{mmol}, 1.0$ equiv.) and TEMPO ( $0.20 \mathrm{mmol}, 2.0$ equiv.) were added and removed from the glovebox. The reaction solution was stirred at $100{ }^{\circ} \mathrm{C}$ for 1 hours. Copper salt was removed by silica gel short pad column 
chromatography and washed with excess ethyl acetate. Evaporation of the solvent gave the crude product.

Ethyl 2-methyl-2-((2,2,6,6-tetramethylpiperidin-1-yl)oxy)propanoate (16); CAS Registry Number 212128-91-1

${ }^{1} \mathrm{H}$ NMR $\left(500 \mathrm{MHz}, \mathrm{CDCl}_{3}, 27^{\circ} \mathrm{C}\right) \delta 4.17\left(\mathrm{q}, J=7.5 \mathrm{~Hz}, 2 \mathrm{H}, \mathrm{OCH}_{2} \mathrm{CH}_{3}\right), 1.58-1.49\left(\mathrm{~m}, 2 \mathrm{H}, \mathrm{CH}_{2}\right), 1.47(\mathrm{~s}, 6 \mathrm{H}$, $\left.\mathrm{OC}\left(\mathrm{CH}_{3}\right)_{2}\right), 1.45-1.39\left(\mathrm{~m}, 2 \mathrm{H}, \mathrm{CH}_{2}\right), 1.30\left(\mathrm{~m}, 2 \mathrm{H}, \mathrm{CH}_{2}\right), 1.30\left(\mathrm{t}, 3 \mathrm{H}, \mathrm{OCH}_{2} \mathrm{CH}_{3}\right), 1.15\left(\mathrm{~s}, 6 \mathrm{H}, \mathrm{CH}_{3}\right), 1.00(\mathrm{~s}, 6 \mathrm{H}$, $\left.\mathrm{CH}_{3}\right) ;{ }^{13} \mathrm{C}$ NMR $\left(125 \mathrm{MHz}, \mathrm{CDCl}_{3}, 27^{\circ} \mathrm{C}\right) \delta 176.1,81.1,60.6,59.5,40.6,33.5,24.5,20.5,17.1,14.2$.

\section{Full Data of Optimization Study}

\section{Table S1. Optimization of Solvents}

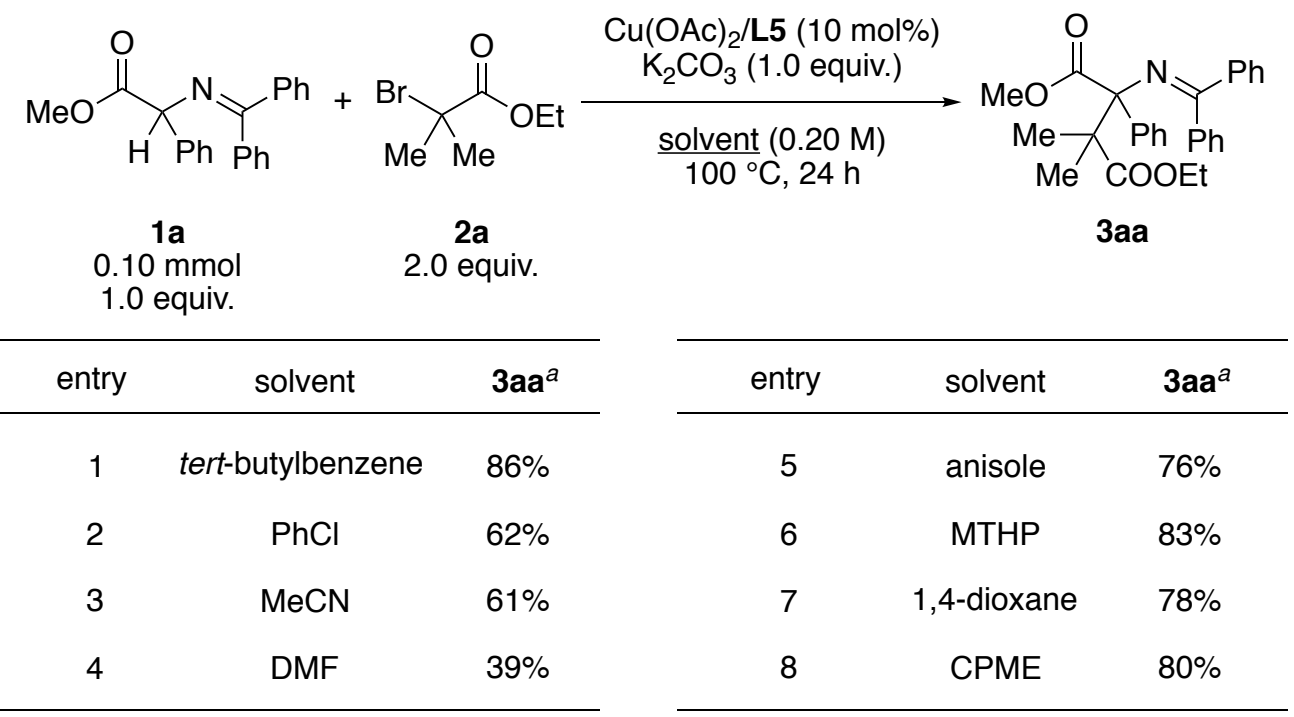

aYields were determined by ${ }^{1} \mathrm{H}-\mathrm{NMR}$ analysis. 
Table S2. Optimization of Bases

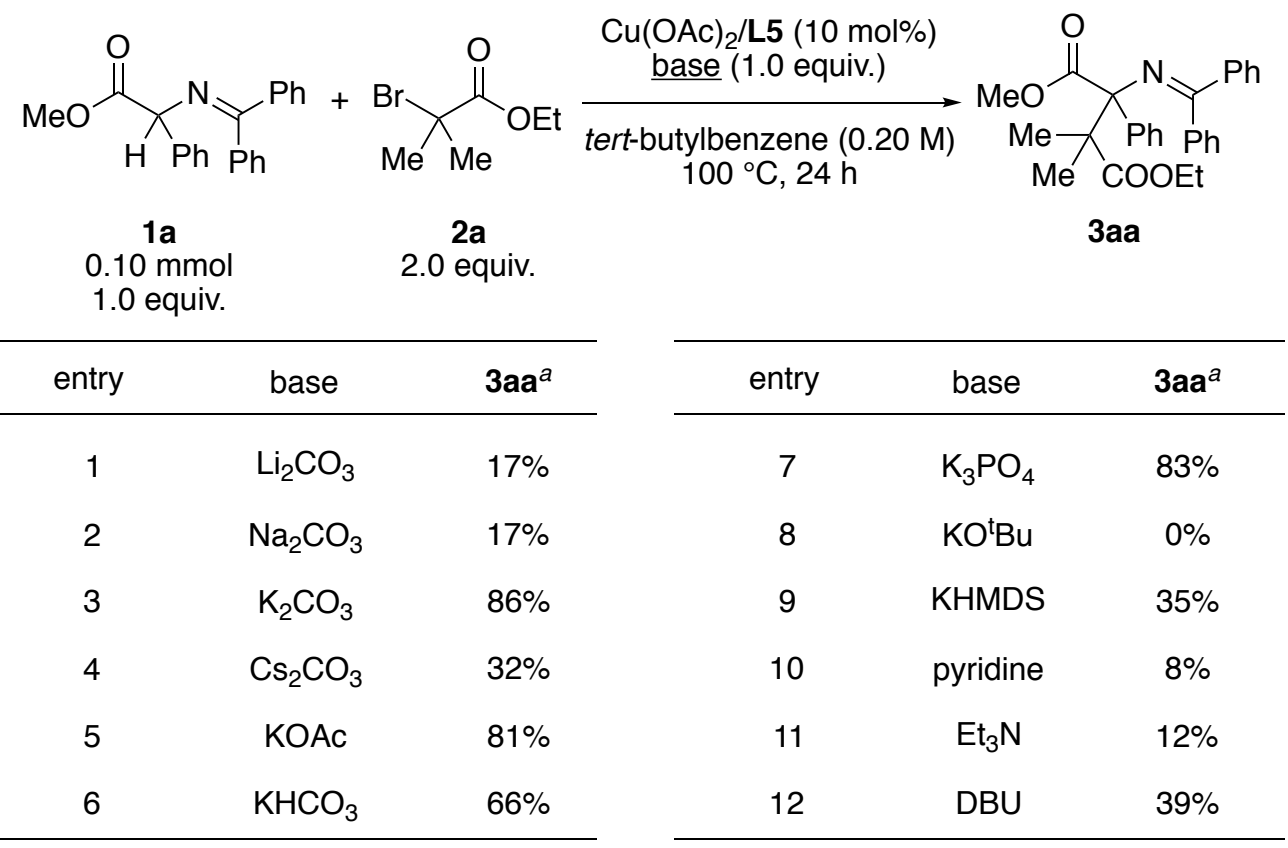

aYields were determined by ${ }^{1} \mathrm{H}-\mathrm{NMR}$ analysis.

Table S3. Optimization of Catalysts

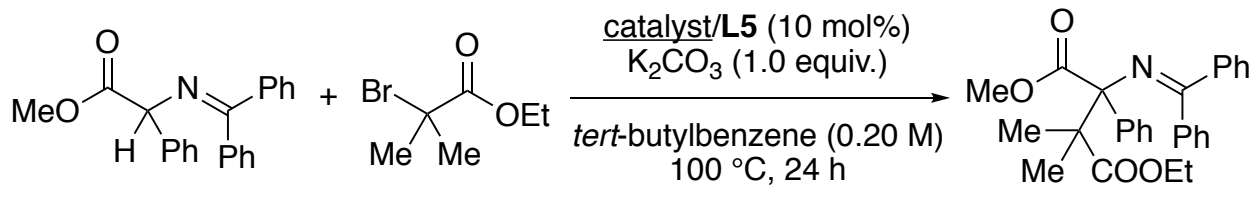

$1 \mathrm{a}$

$0.10 \mathrm{mmol}$

1.0 equiv.

\begin{tabular}{ccc}
\hline entry & catalyst & 3aa $^{a}$ \\
\hline 1 & $\mathrm{Cu}(\mathrm{OAc})_{2}$ & $86 \%$ \\
2 & $\mathrm{Cu}(\mathrm{OPiv})_{2}$ & $84 \%$ \\
3 & $\mathrm{Cu}(\mathrm{OTf})_{2}$ & $11 \%$ \\
4 & $\mathrm{CuCl}_{2}$ & $9 \%$ \\
5 & $\mathrm{CuBr}_{2}$ & $42 \%$ \\
6 & $\mathrm{CuOAc}^{2}$ & $22 \%$
\end{tabular}

$2 a$

2.0 equiv.

3aa

\begin{tabular}{ccc}
\hline entry & catalyst & 3aa $^{a}$ \\
\hline 7 & $(\text { CuOTf })_{2} \cdot$ benzene $^{b}$ & $7 \%$ \\
8 & $\mathrm{CuCl}$ & $18 \%$ \\
9 & $\mathrm{CuBr}$ & $9 \%$ \\
10 & $\mathrm{Cul}$ & $72 \%$ \\
11 & $\mathrm{Fe}(\mathrm{OAc})_{2}$ & $0 \%$ \\
12 & $\mathrm{NiBr}_{2} \cdot$ diglyme & $0 \%$ \\
\hline
\end{tabular}

aYields were determined by ${ }^{1} \mathrm{H}-\mathrm{NMR}$ analysis. ${ }^{b_{5}} \mathrm{~mol} \%$ of $(\mathrm{CuOTf})_{2} \cdot$ benzene complex was used. 
Supporting information

Amino Acid Schiff Base Bearing Benzophenone Imine as a Platform for Highly Congested Unnatural $\alpha$-Amino Acid Synthesis

Table S4. Optimization of Ligands ${ }^{a}$

$$
\text { 2.0 equiv. }
$$

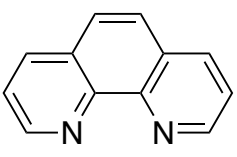

L1: $66 \%$<smiles>c1ccc(-c2ccnc3c2ccc2c(-c4ccccc4)ccnc23)cc1</smiles>

L5: $86 \%$<smiles>c1ccc(Oc2ccnc3c2ccc2c(Oc4ccccc4)ccnc23)cc1</smiles>

L9: $63 \%$<smiles>Cc1ccnc2c1ccc1c(C)ccnc12</smiles>

L2: $5 \%$<smiles>Cc1cc(-c2ccccc2)c2ccc3c(-c4ccccc4)cc(C)nc3c2n1</smiles>

L6: $15 \%$<smiles>CN(C)c1ccnc2c1ccc1c(N(C)C)ccnc12</smiles>

L10: $32 \%$<smiles>CN(C)CCCN(C)C</smiles>

L14: 9\%<smiles>COc1ccnc2c1ccc1c(OC)ccnc12</smiles>

L3: $30 \%$<smiles>Clc1ccnc2c1ccc1c(Cl)ccnc12</smiles>

L4: $17 \%$<smiles>c1ccc(-c2ccccn2)nc1</smiles>

L7: $35 \%$<smiles>CCC(C)c1ccnc(-c2cc(CC(C)C)ccn2)c1</smiles>

L8: $49 \%$<smiles>c1ccc(-c2ccc3ccc4ccc(-c5ccccc5)nc4c3n2)cc1</smiles>

L11: 3\%<smiles>COc1ccnc(-c2cc(OC)ccn2)c1</smiles>

L12: 4\%<smiles>CN(C)CCN(C)CCN(C)C</smiles>

L16: 39\%

L13: $12 \%$

L15: 22\% 


\section{References}

1. (a) Hama, T.; Culkin, D. A.; Hartwig, J. F. J. Am. Chem. Soc. 2006, 128, 4976-4985. (b) Fujishita, T.; Mikamiyama, M. Kawai, M.; Akiyama, T. WO2010/11023 A1 2010 (c) Zumbrägel, N.; Machui, P.;

Nonnhoff, J.; Gröger, H. J. Org. Chem. 2019, 84, 1440-1447. (d) Murata, Y.; Takeuchi, K.; Nishikata, T. Tetrahedron 2019, 75, 2726-2736.

2. O’Donnell, M. J.; Polt, R. L. J. Org. Chem. 1982, 47, 2663-2666.

3. Tanaka, T.; Tanaka, T.; Tsuji, T.; Yazaki, R.; Ohshima, T. Org. Lett. 2018, 20, 3541-3544.

4. Reeves, J. T.; Tan, Z.; Herbage, M. A.; Han, Z. S.; Marsini, M. A.; Li, Z.; Li, G.; Xu, Y.; Fandrick, K. R.; Gonnella, N. C.; et al. J. Am. Chem. Soc. 2013, 135, 5565-5568. 


\section{NMR Spectra of New Compounds}

吉
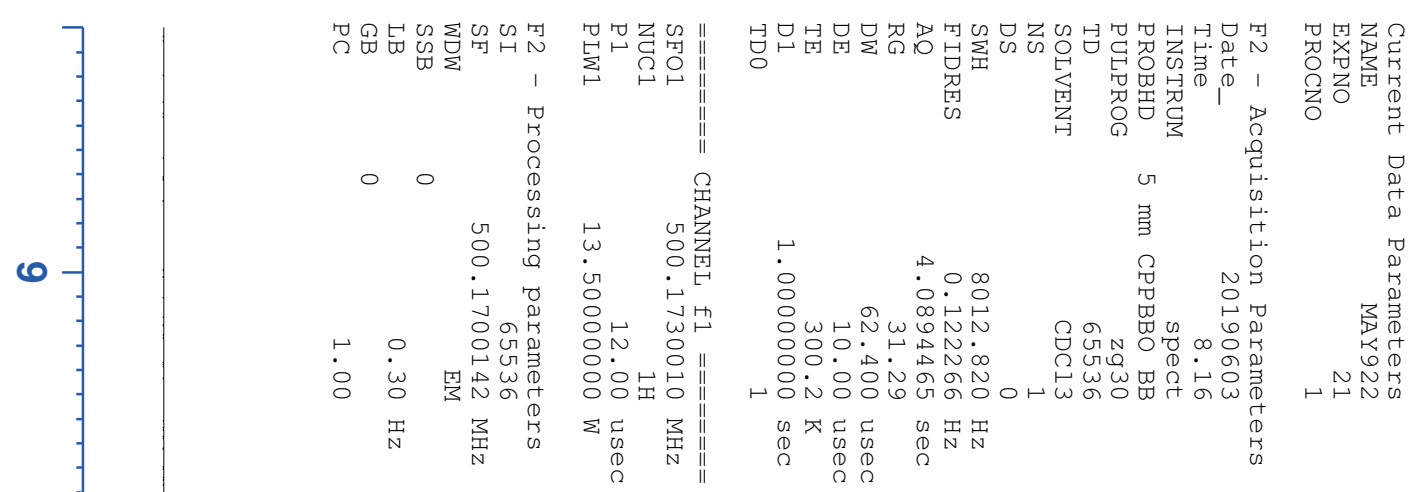

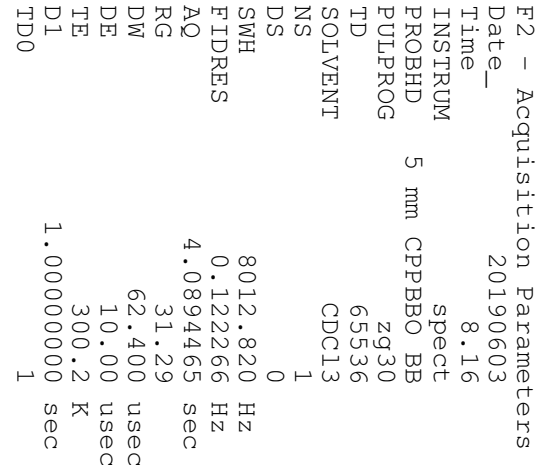
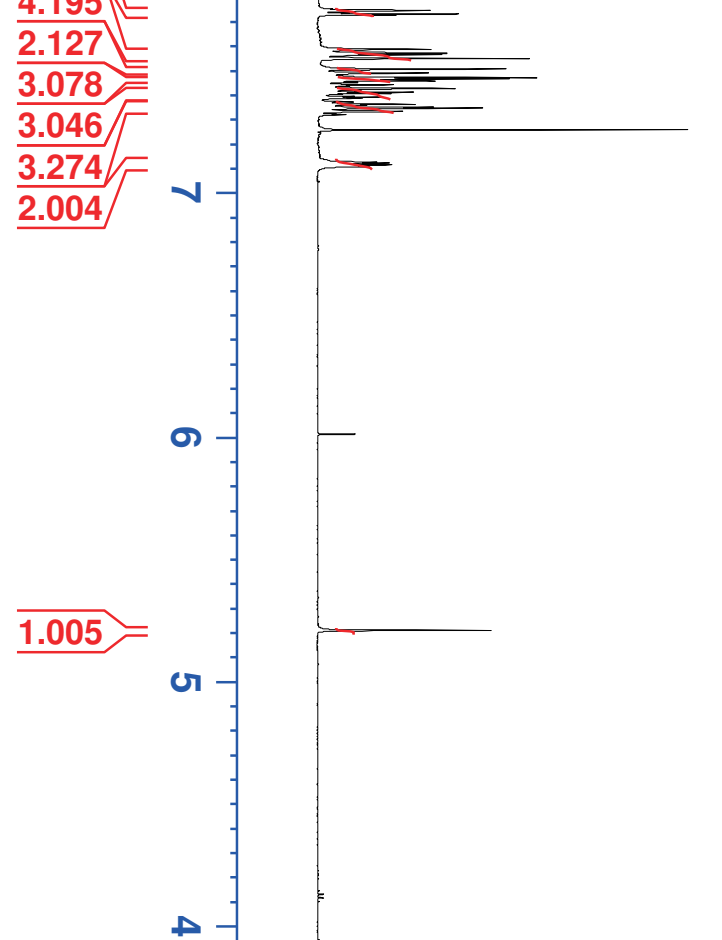

.705

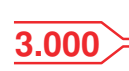

$+$

$\omega$
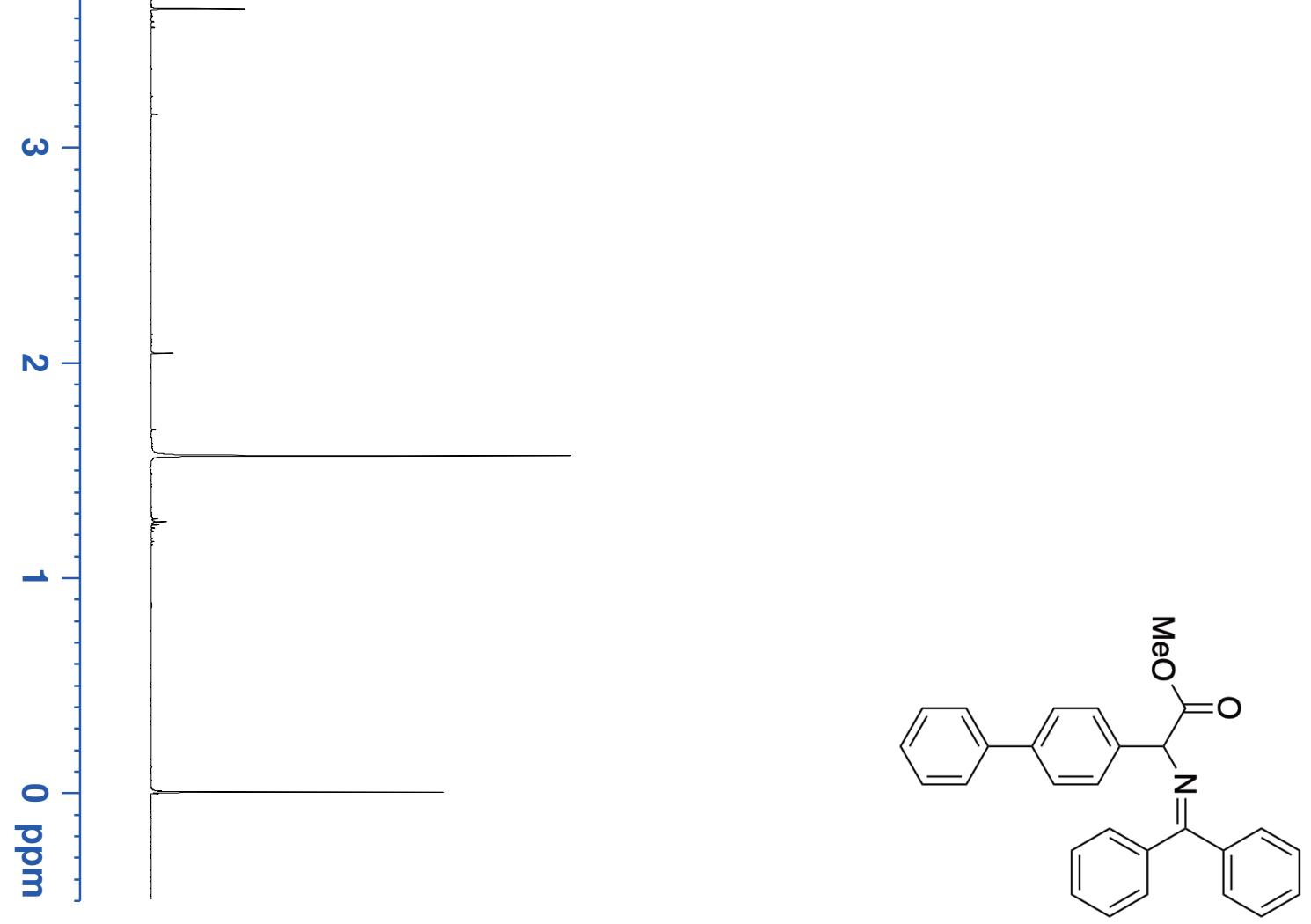

$\left[\begin{array}{l}7.440 \\ 7.436\end{array}\right.$

7.433

7.425

7.419

7.416

7.412
7.409
7.409

7.404

7.400

7.390

7.387

7.385

7.370

$-7.366$

7.364

7.360

$-7.357$

$-7.353$

7.347
-7.344

$-7.336$

7.332

7.332
-7.328

-7.328
-7.317
-7.315

7.320
-7.315

$-7.124$

$-7.119$

7.112

7.105

5.208 


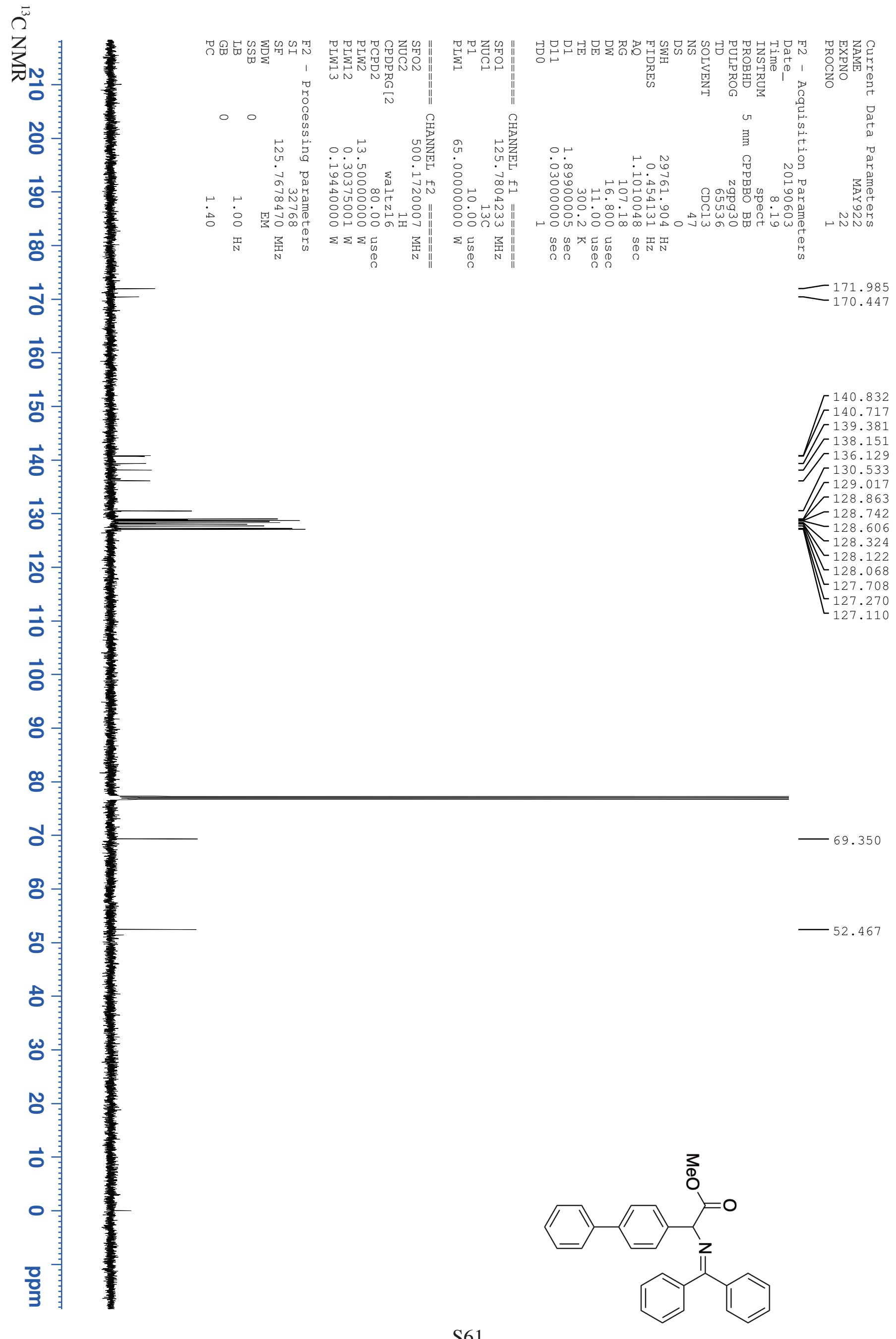




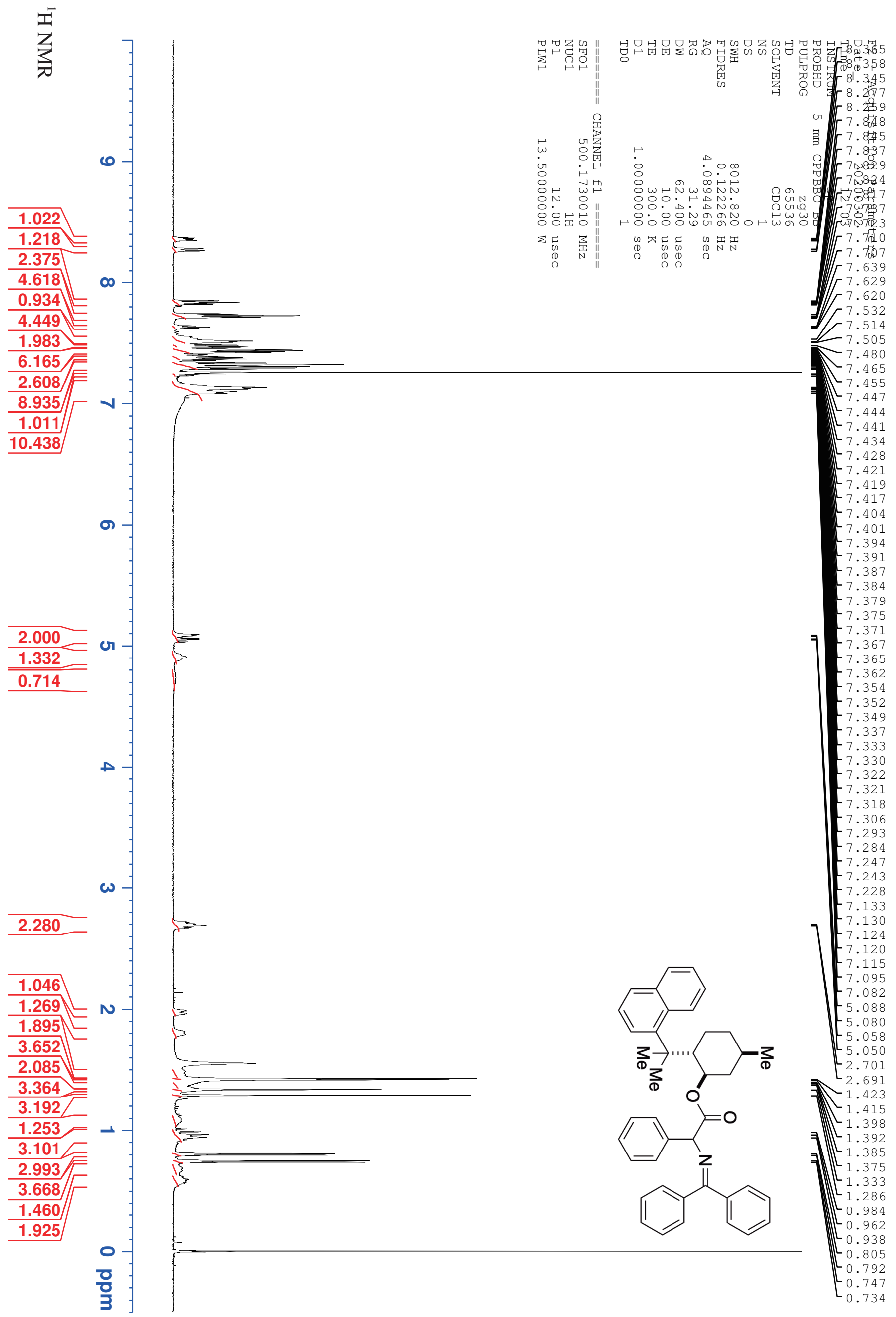




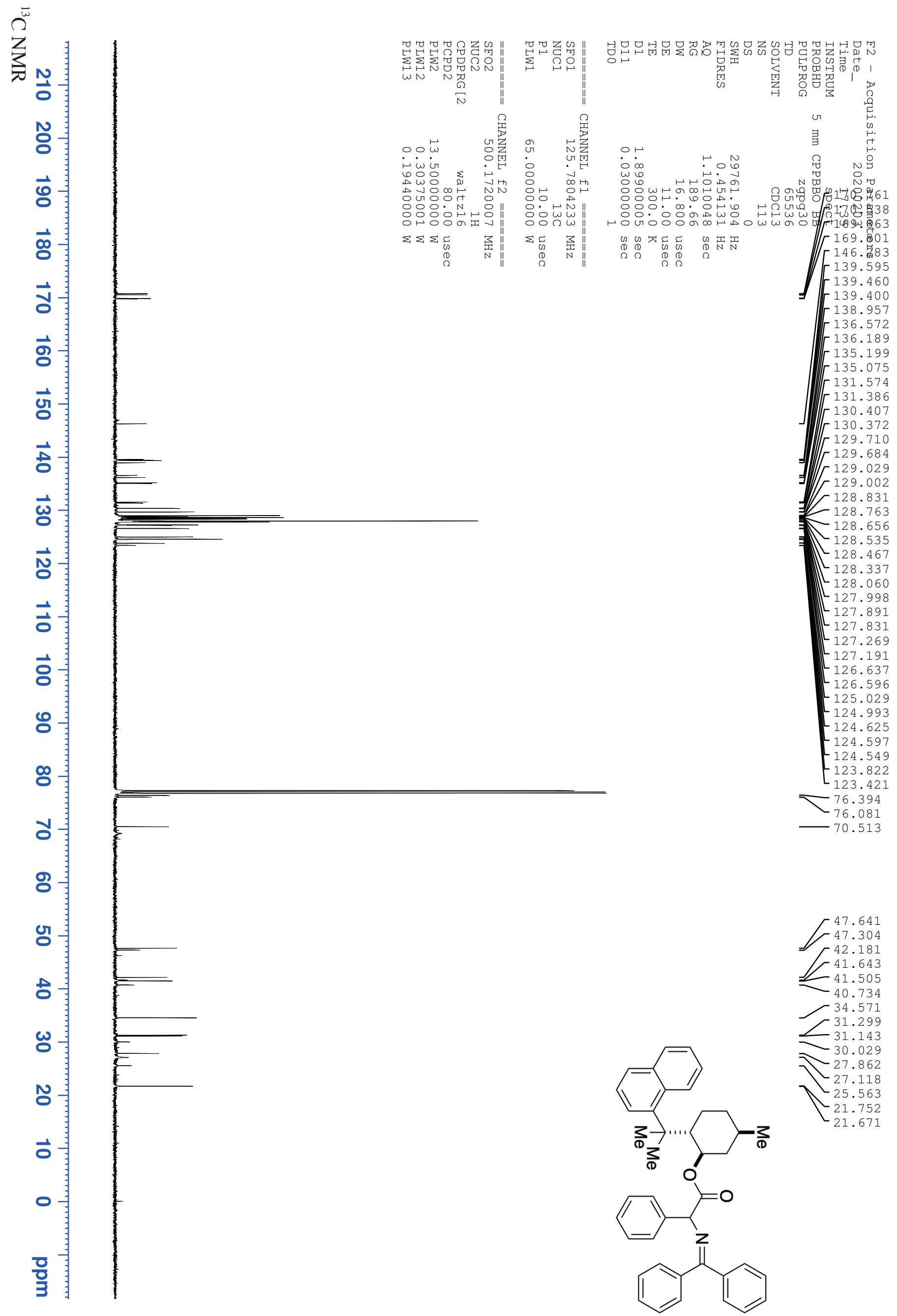


艺

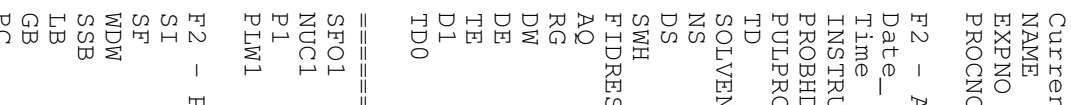

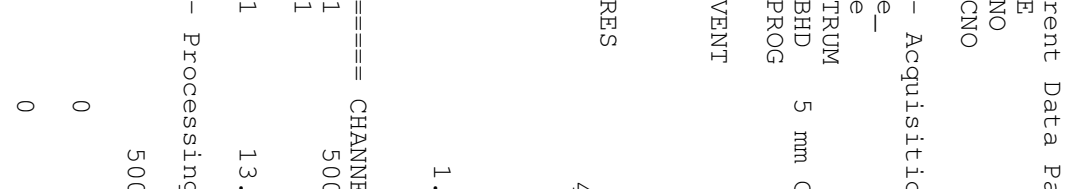

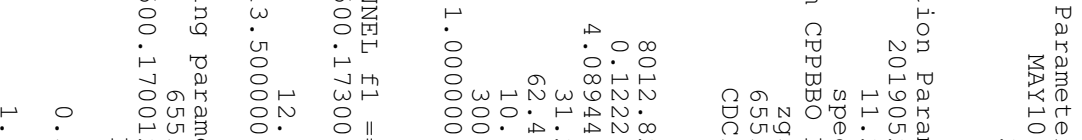

7.649
7.635
7.457

7.649
-7.635
-7.457

7.450

7.446

7.439

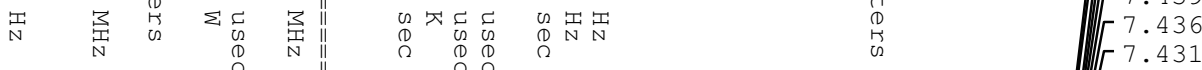

$\infty$

$\frac{1.996}{2.960}\llcorner$
$\frac{2.076}{2.036}$
1.959

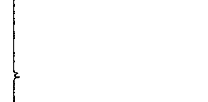

$\sigma$

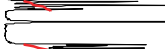

7.402

7.399

$-7.387$

7.383

7.375

[7.372

7.370

7.341

7.325
7.311

7.325
7.165

$-7.161$

7.150
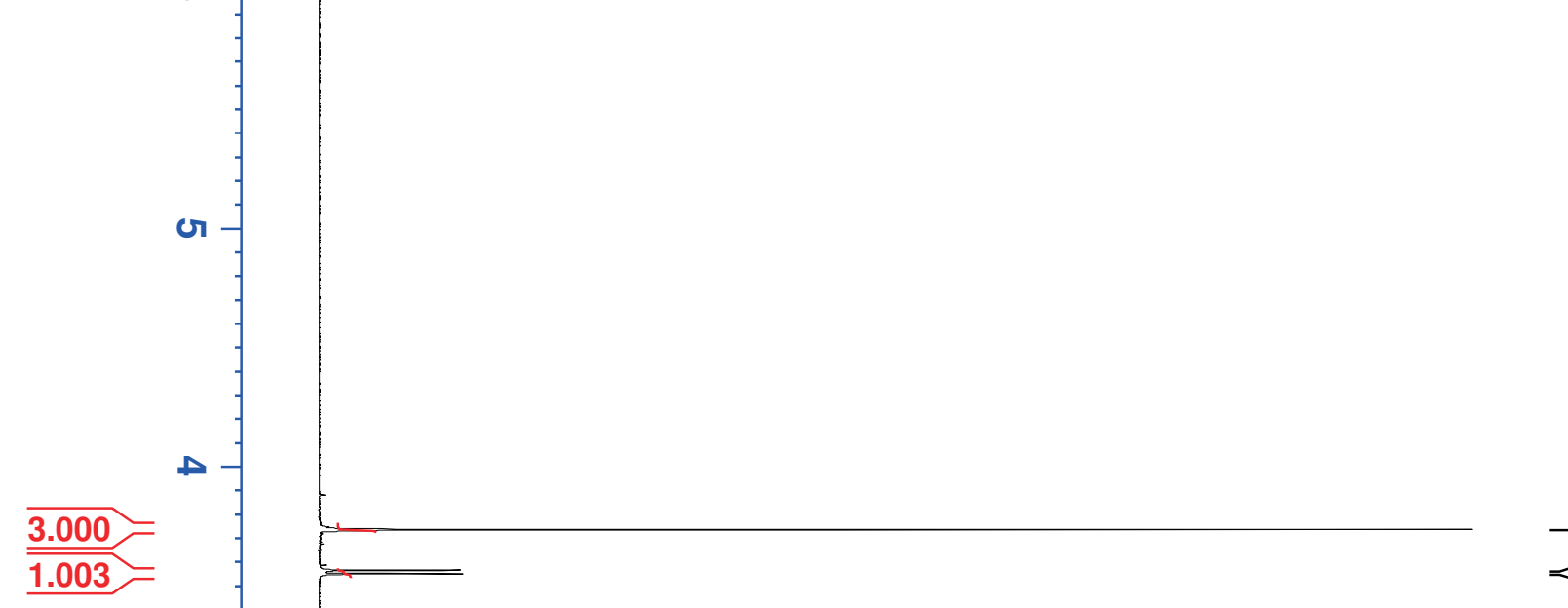

$-3.733$

3.562

3.547
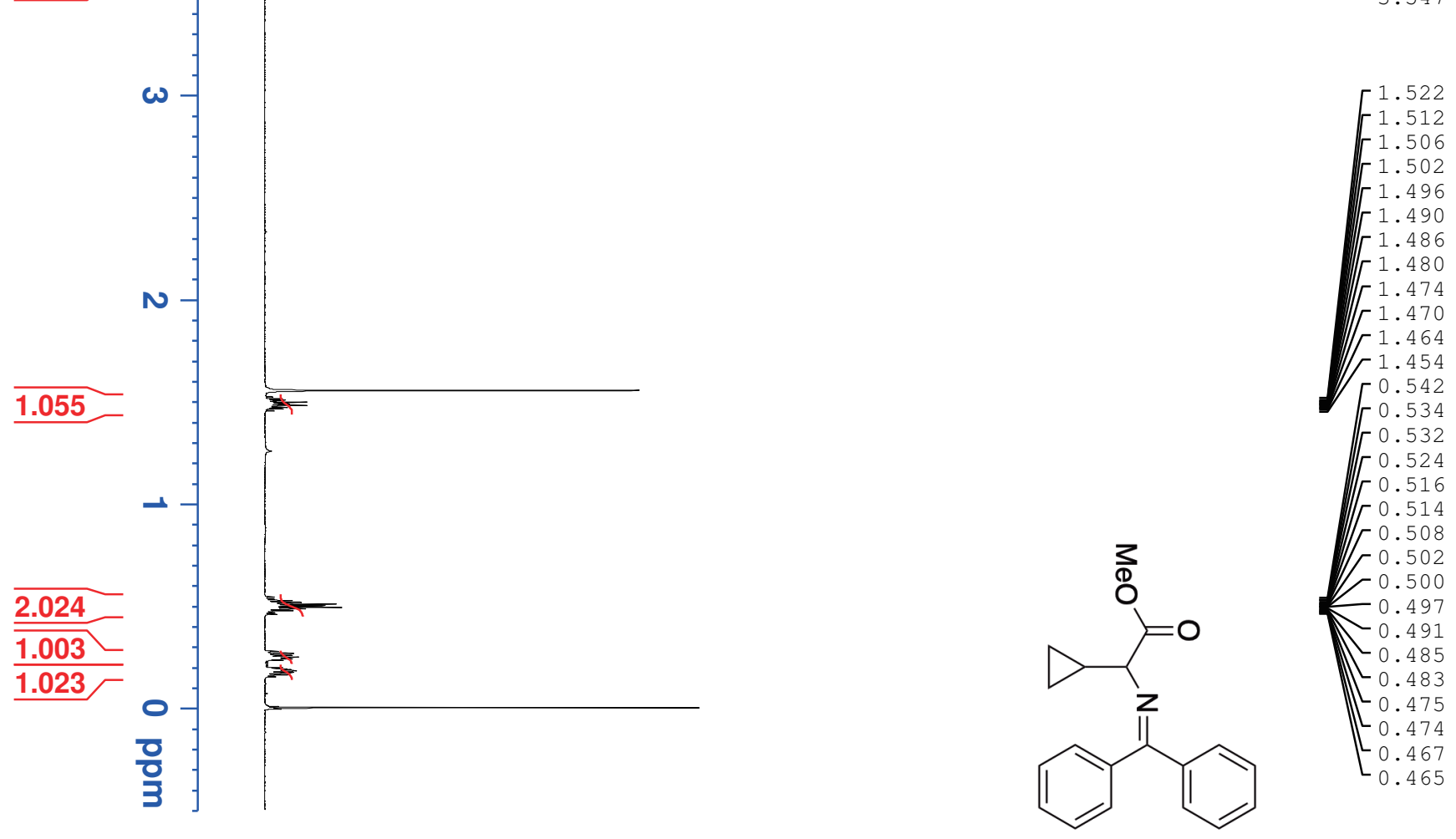


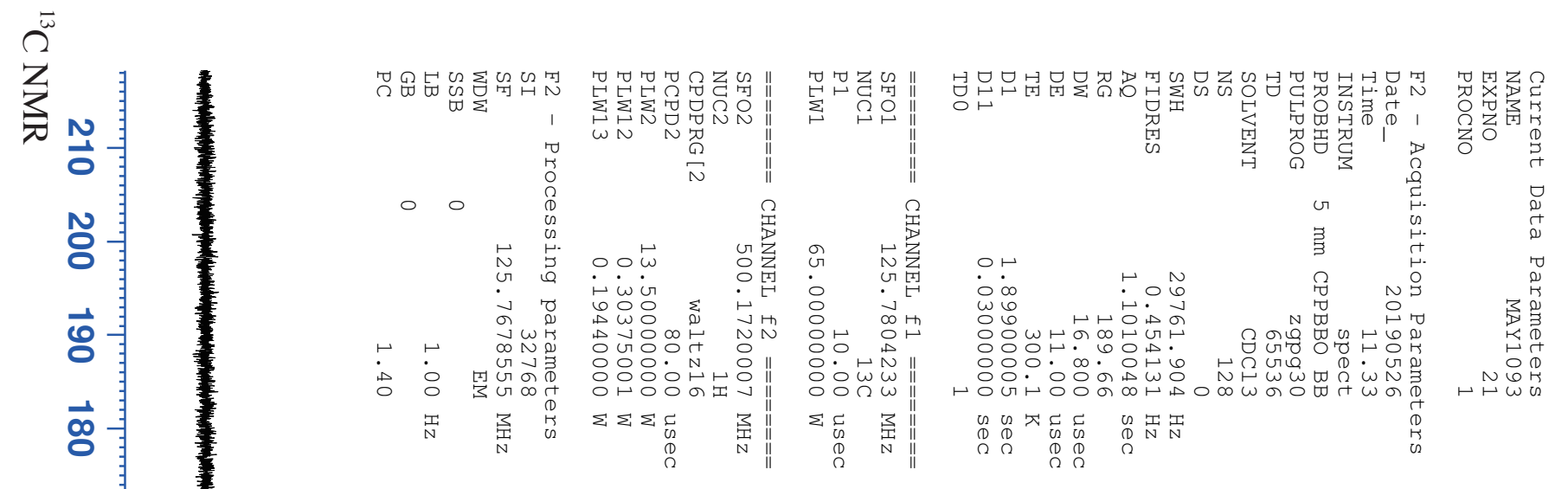

$\overrightarrow{0}$

$\vec{\circ}$

ज्ञ

$\overrightarrow{0}$

$\vec{\omega}$

$\overrightarrow{\mathrm{N}}$

$\overrightarrow{\overrightarrow{0}}$

용

c)

\&

フ

g

당

o

$\omega$

응

$\overrightarrow{0}$

o

응 毫毫

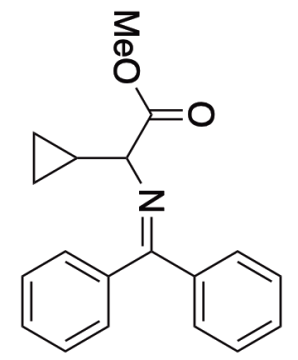

139.497
-136.250

$-136.250$

130.258

128.820
128.566

$-128.413$

127.974
127.880 


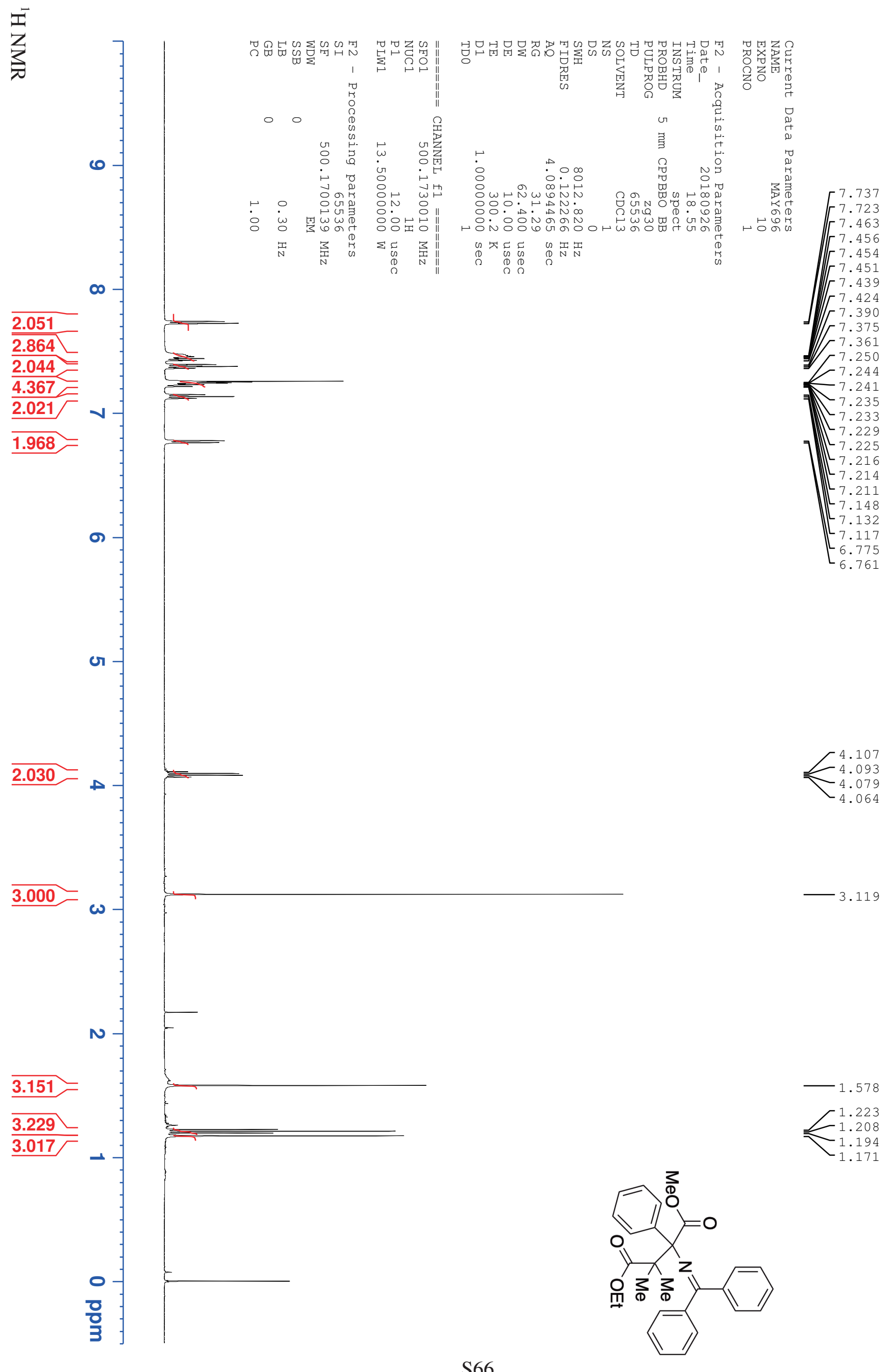




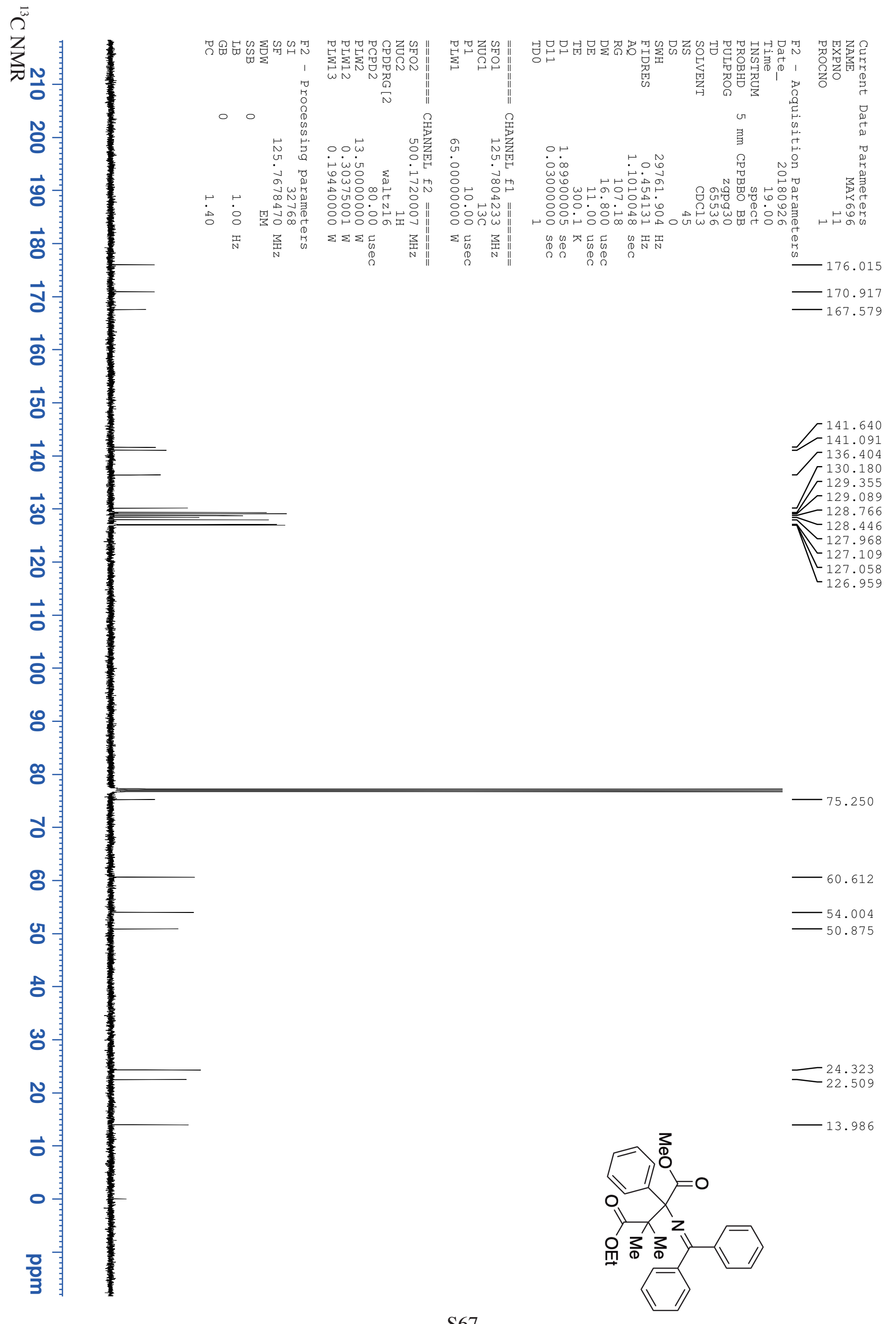




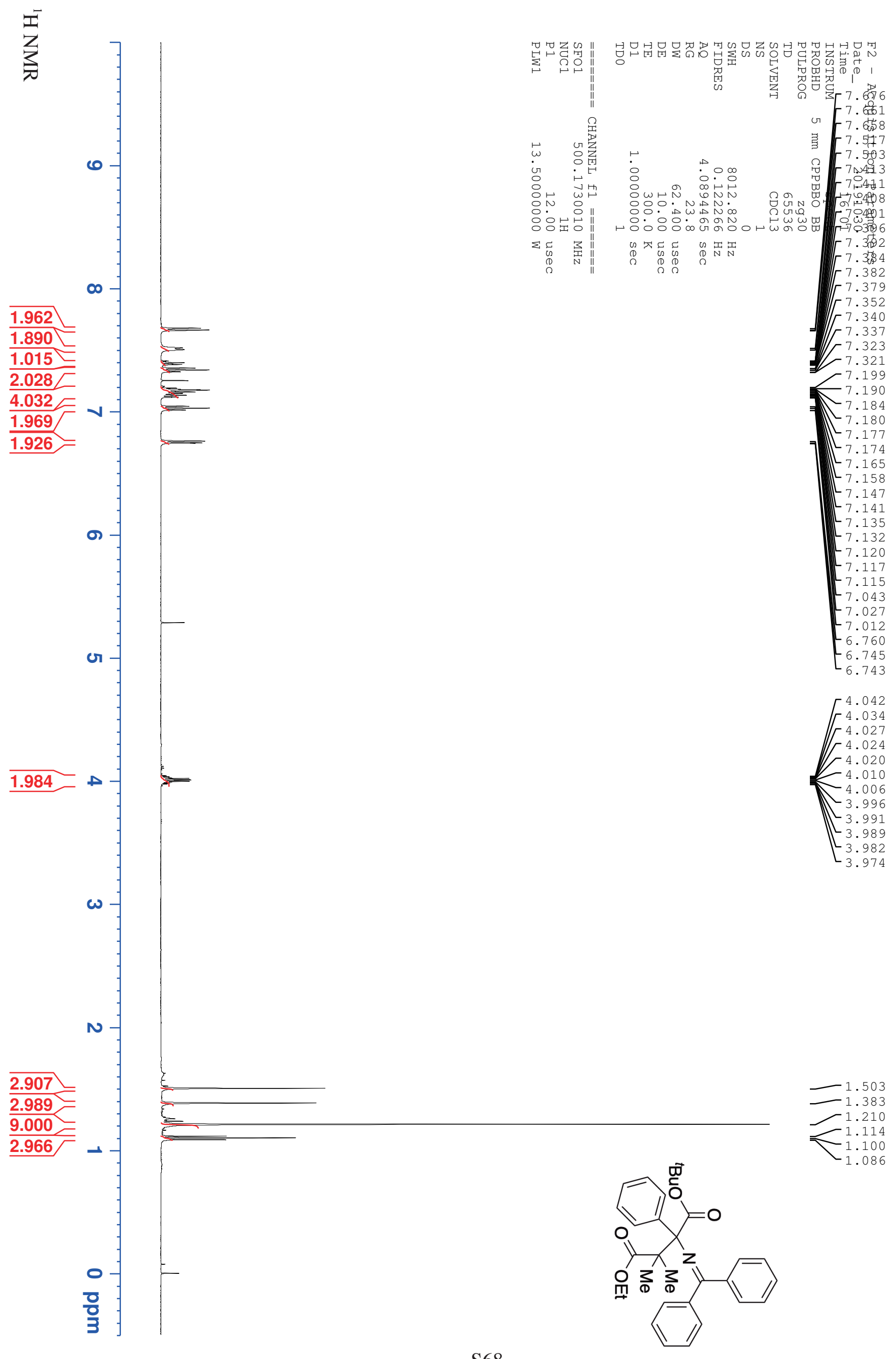




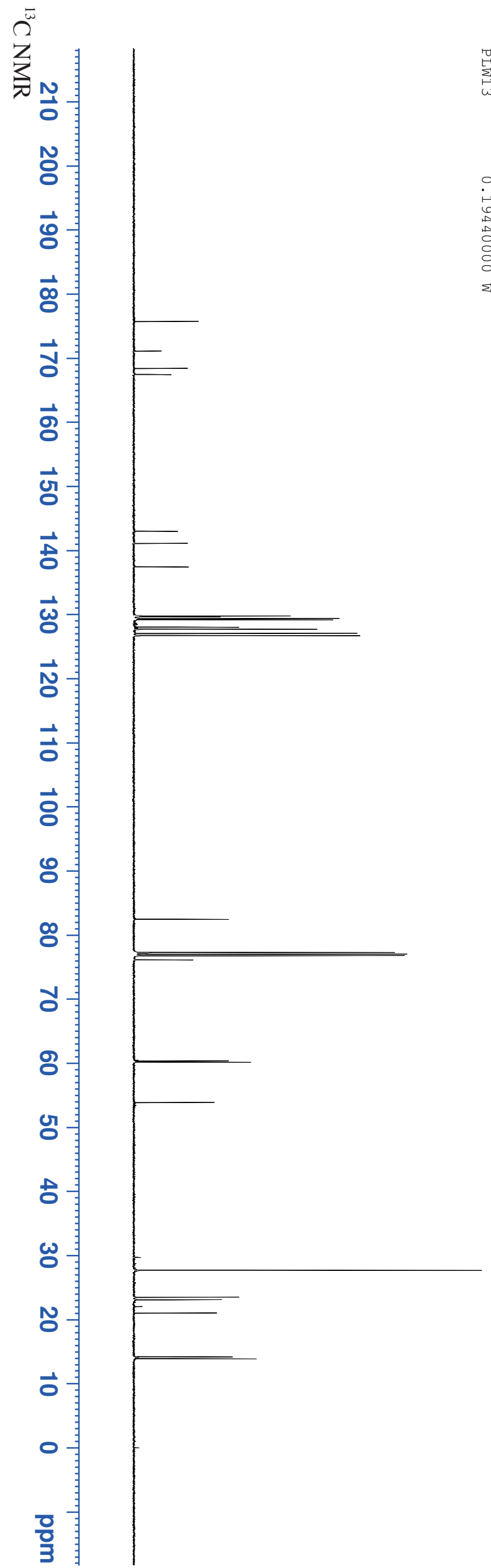

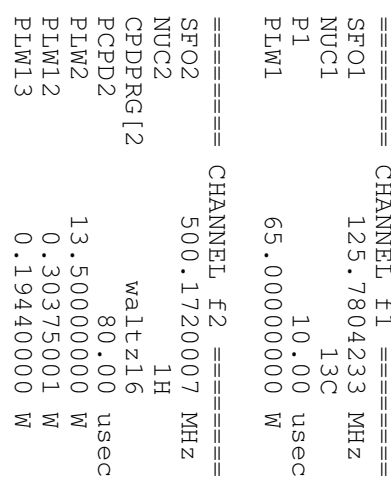

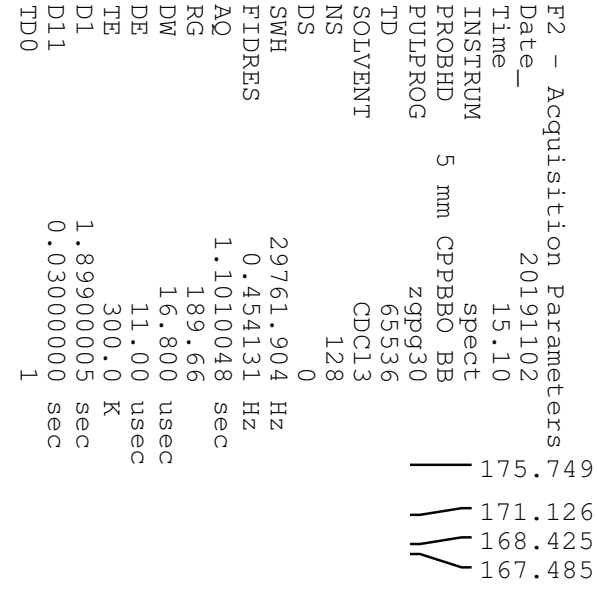

$\Gamma^{143.024}$

${ }^{141.140}$

Fin.

$\left[\begin{array}{r}129.802 \\ 129.684\end{array}\right.$

129.399

129.218

128.677
128.468

128.468

$-128.245$

$-128.070$

$-127.916$

127.726

127.081

$\left[\begin{array}{r}126.741 \\ 126.717\end{array}\right.$

$-82.488$

$-76.131$

$-60.382$

$-60.179$

$-53.891$
$-27.716$

$-23.508$

23.098

$-21.037$

$-14.200$

13.909

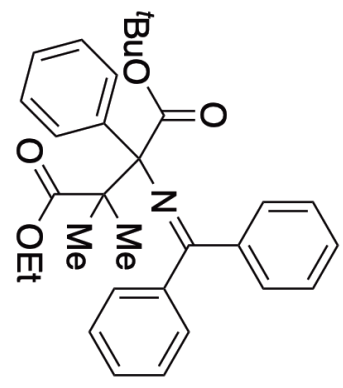




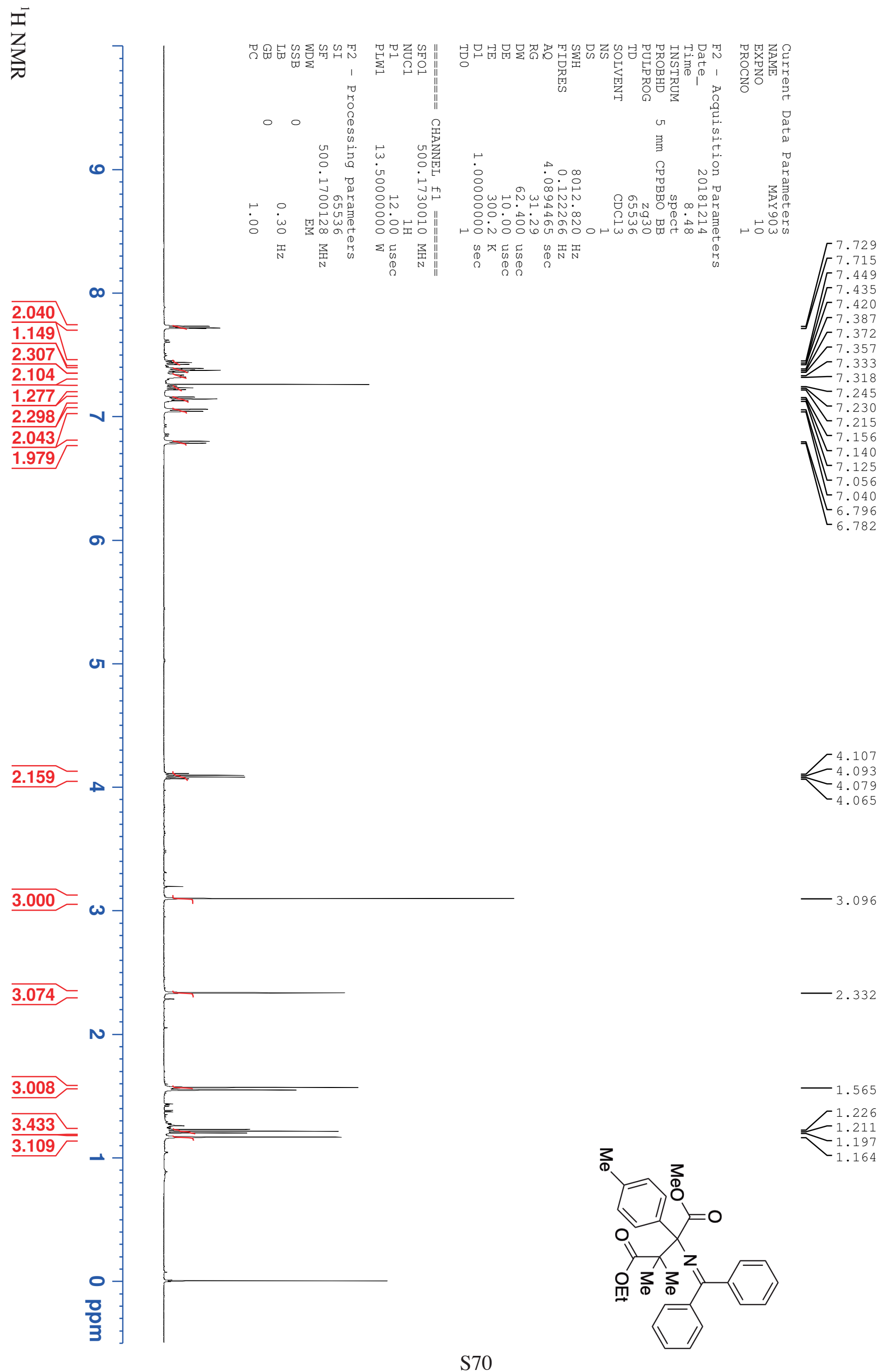




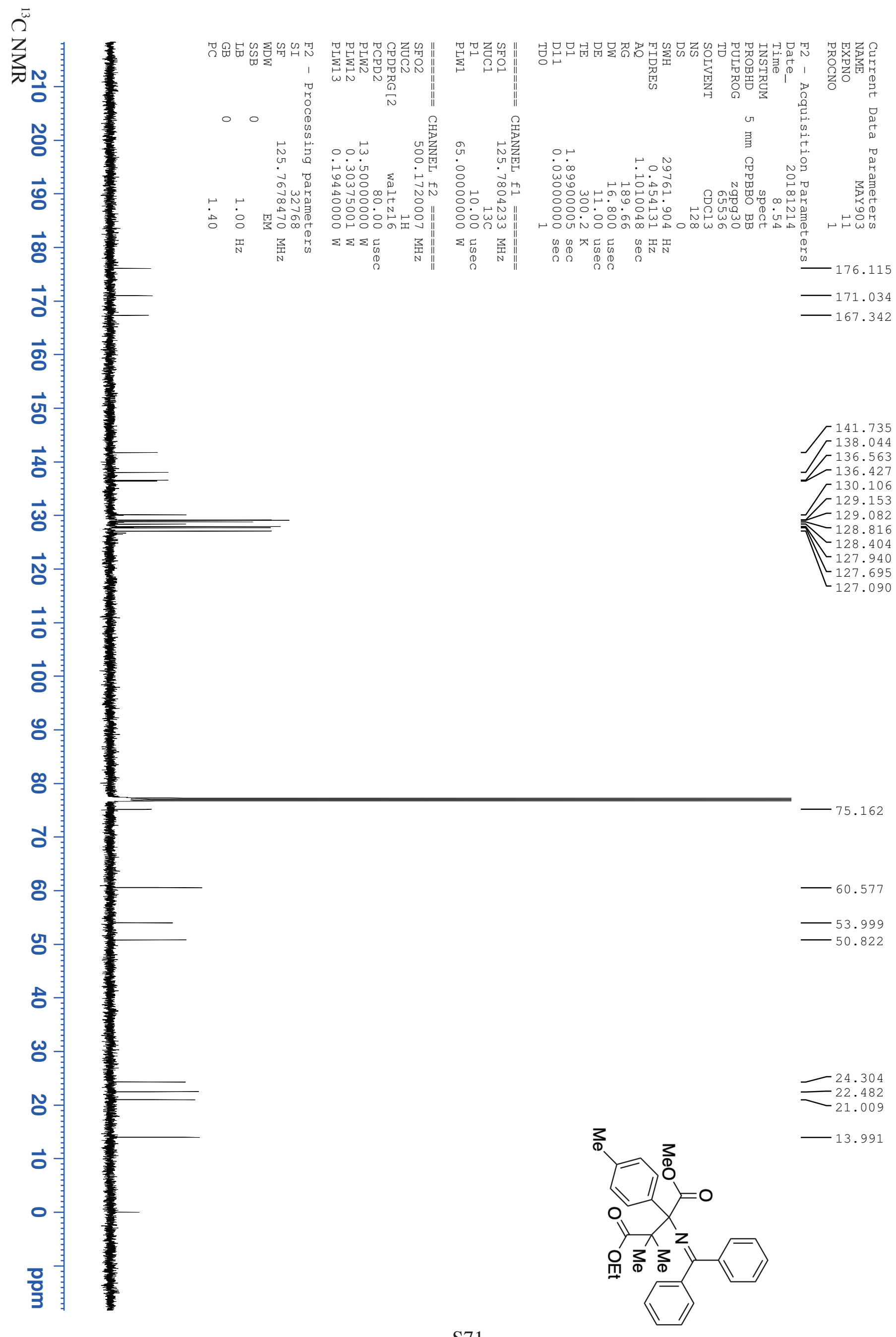



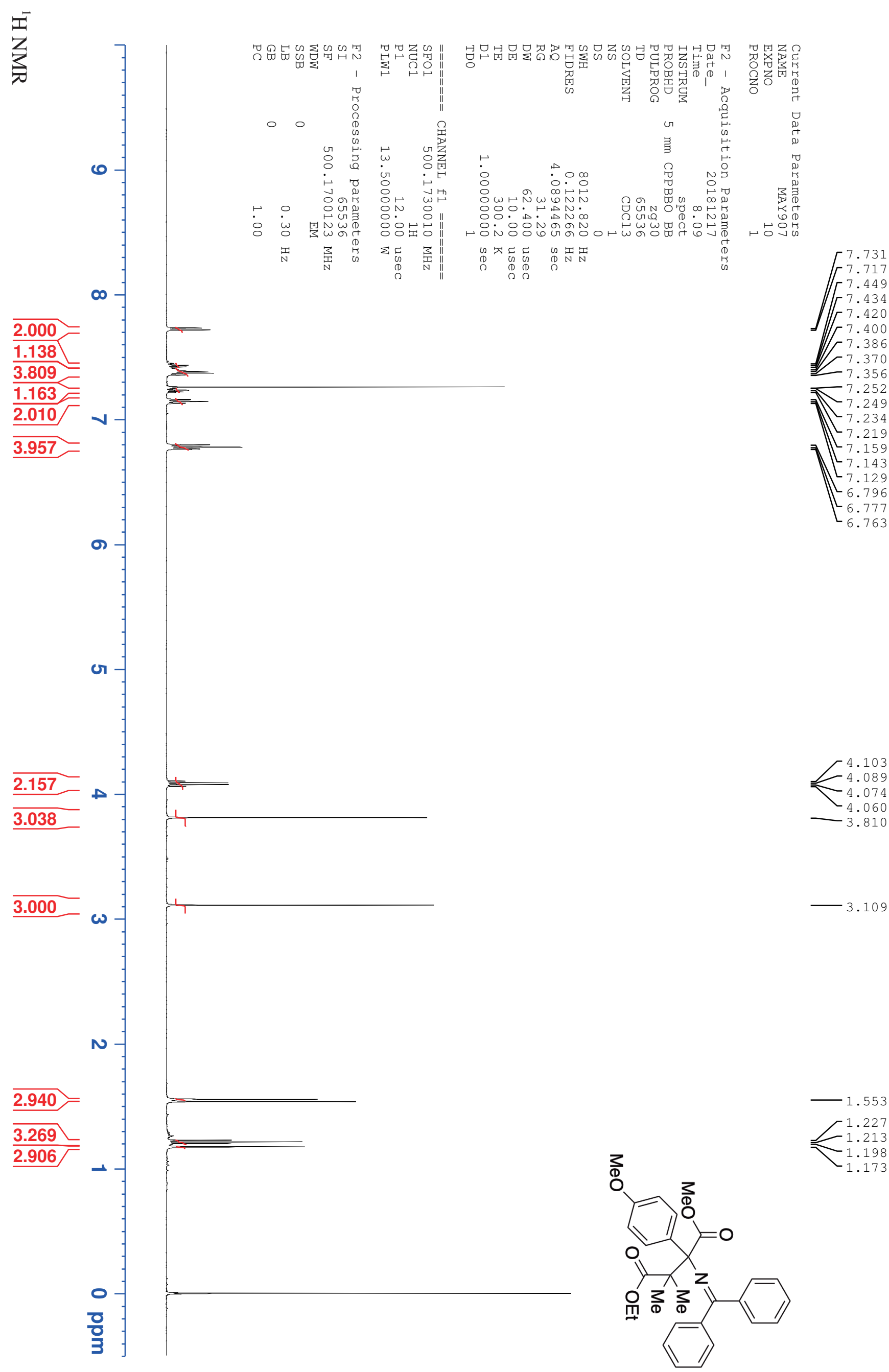


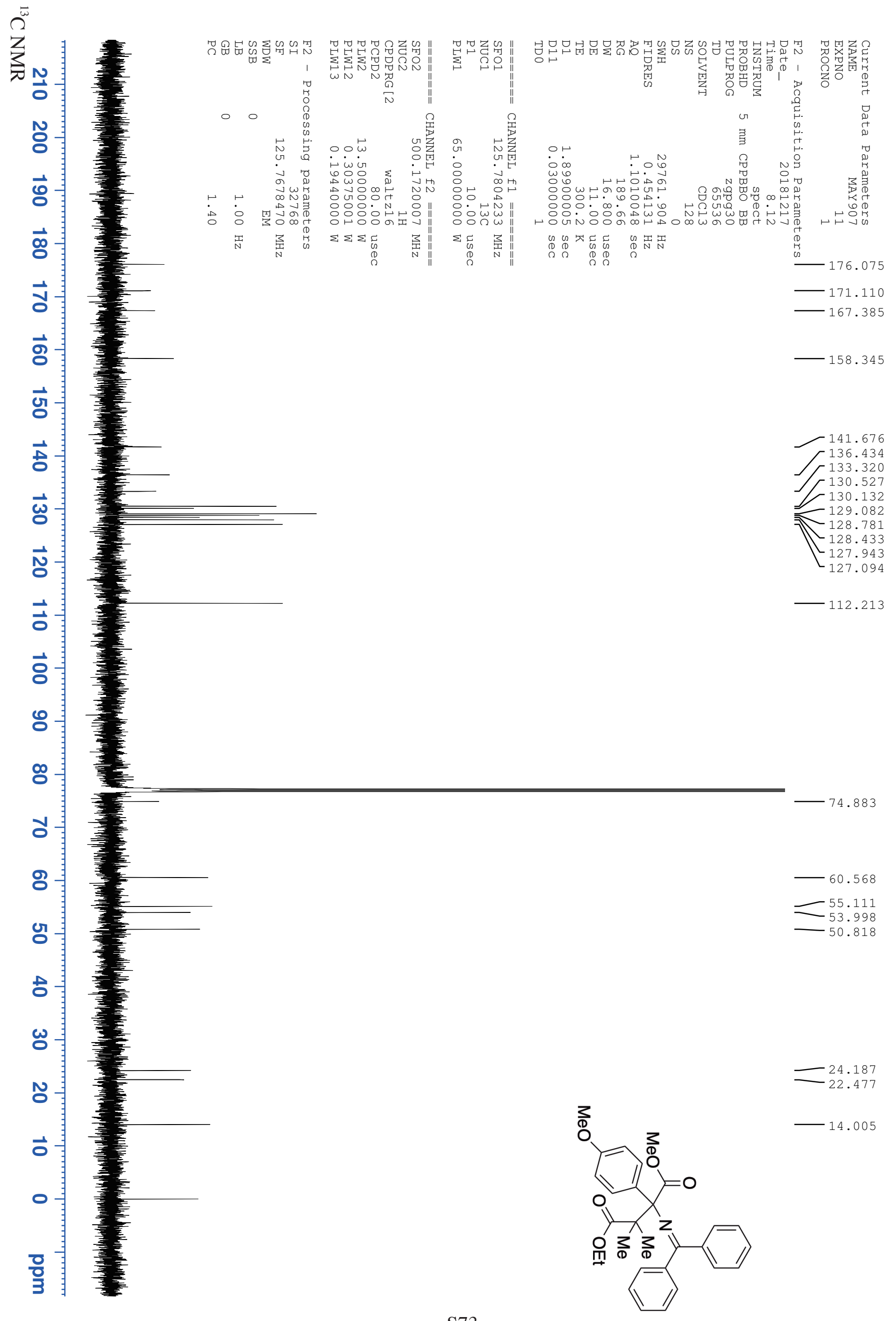



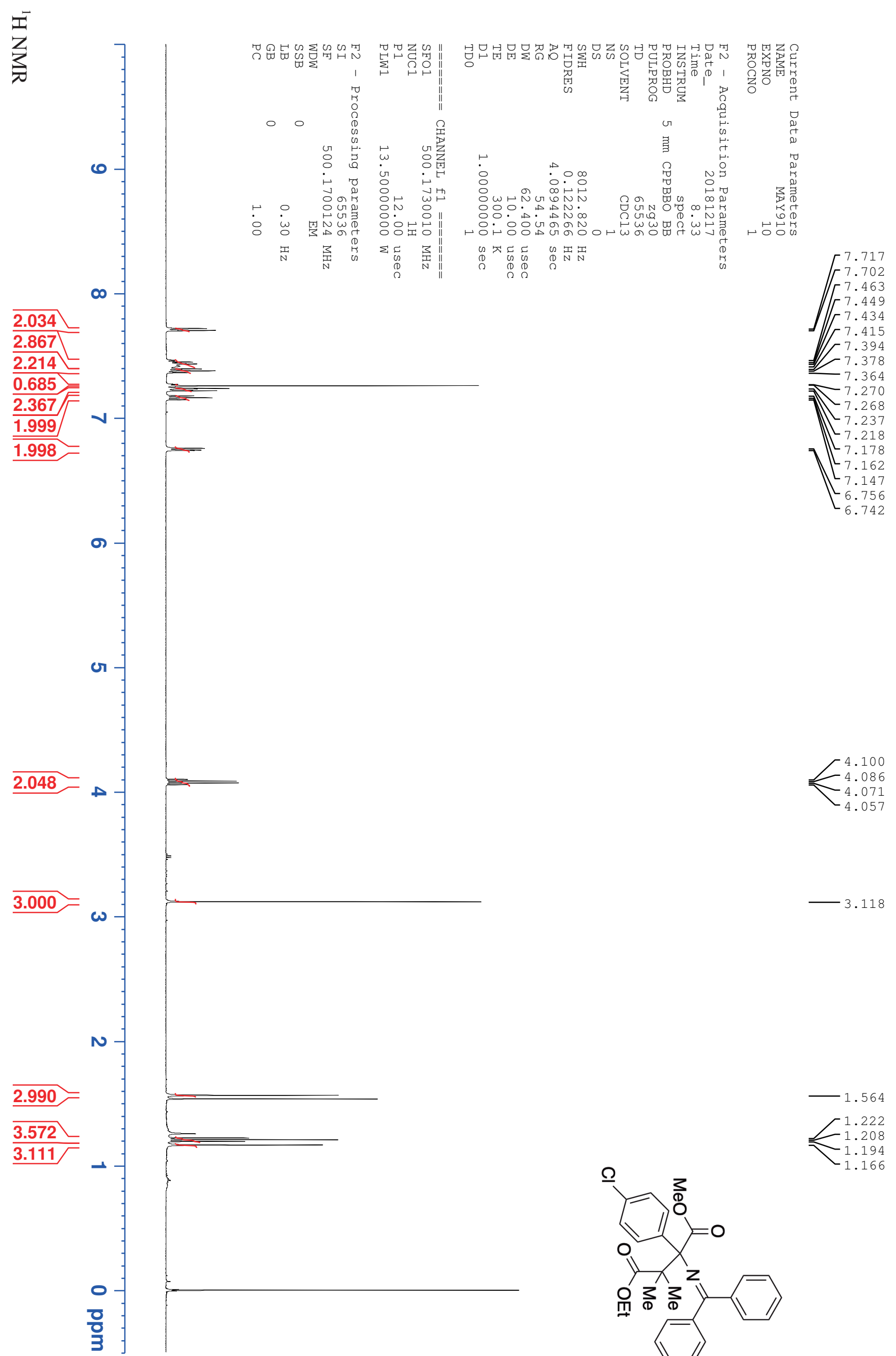

$-3.118$

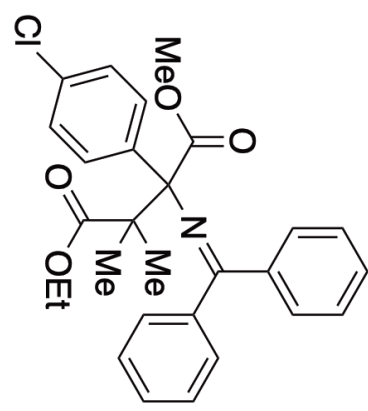

4.100

4.086
4.071

4.057

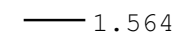

1.222

1.208

1.166 


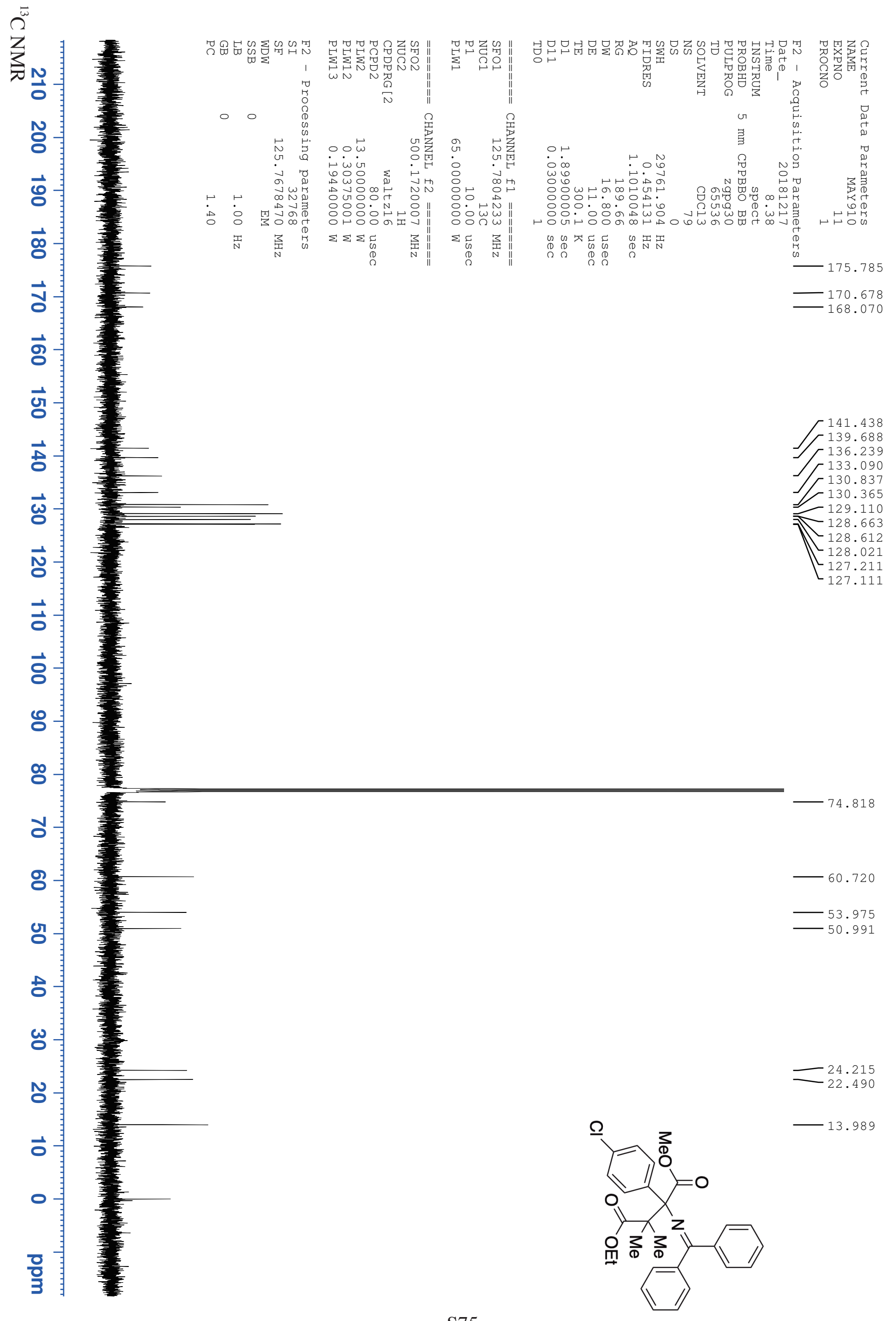



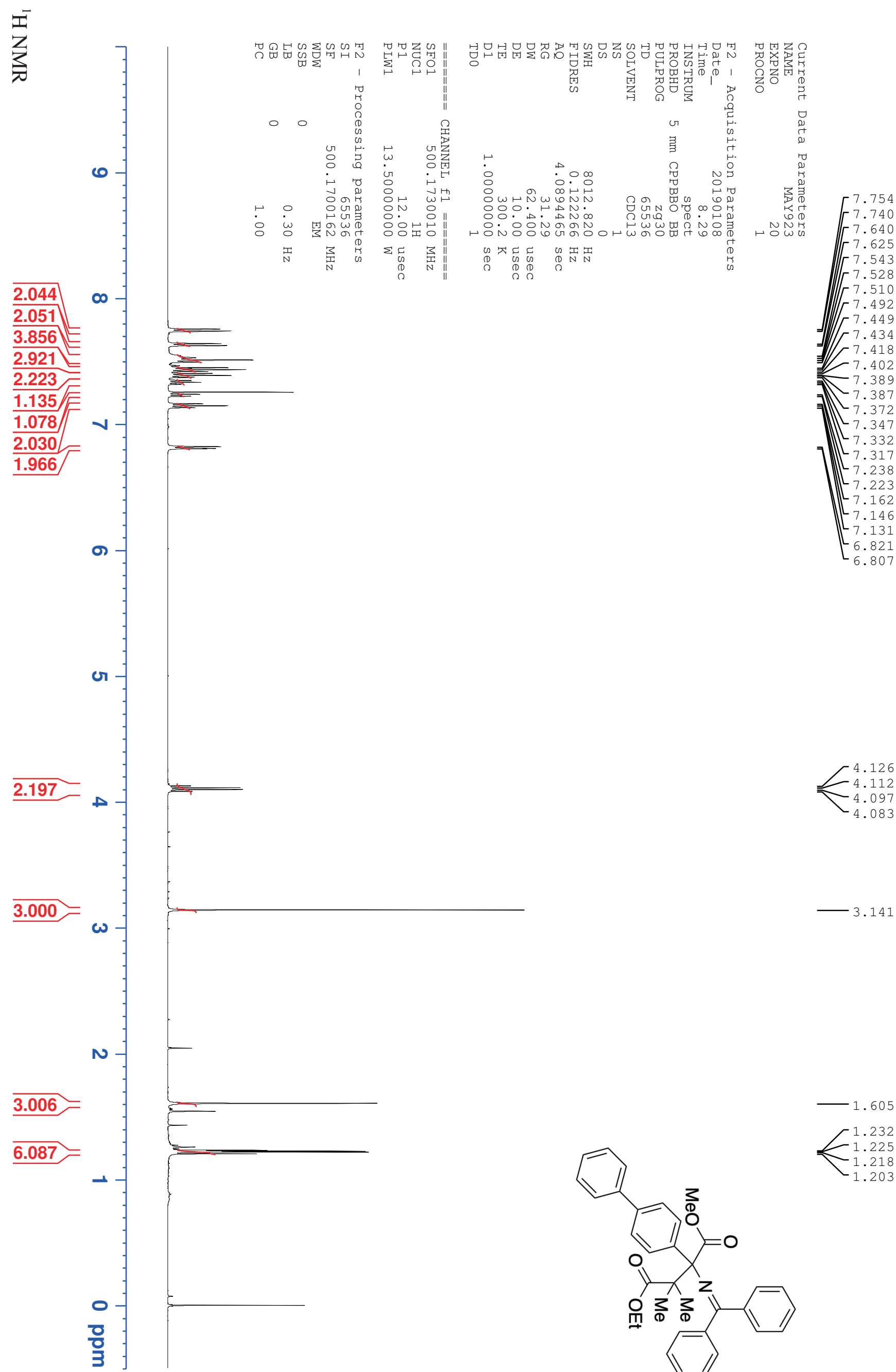

4.126

4.112

4.097
4.083
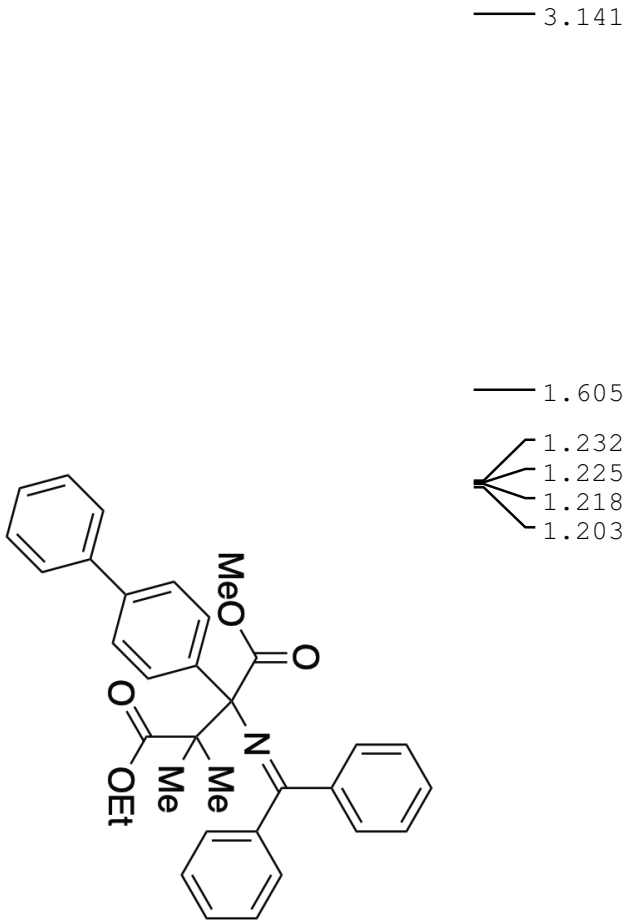

1.232

$\mathcal{K}^{1.225}$

$-1.203$ 


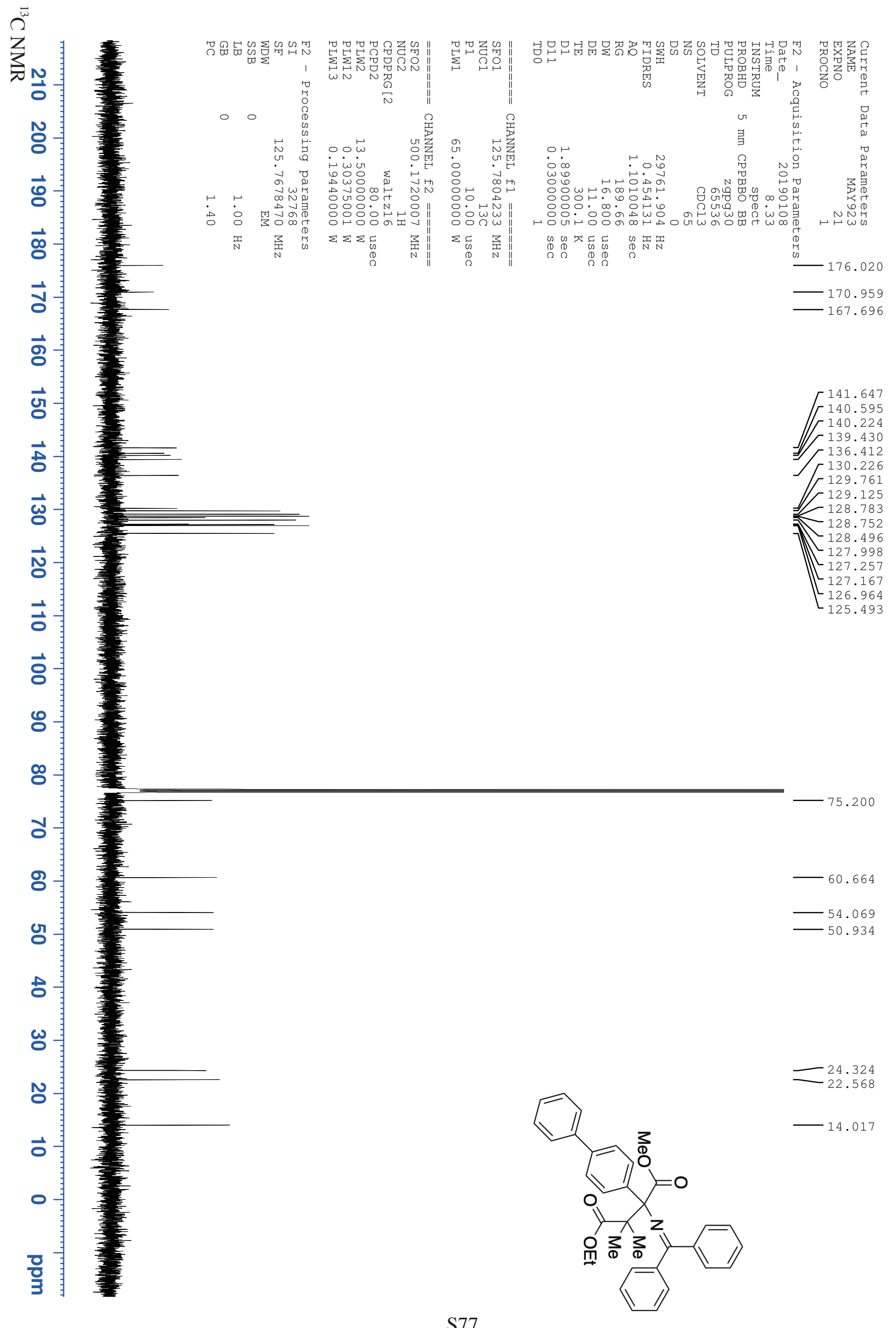




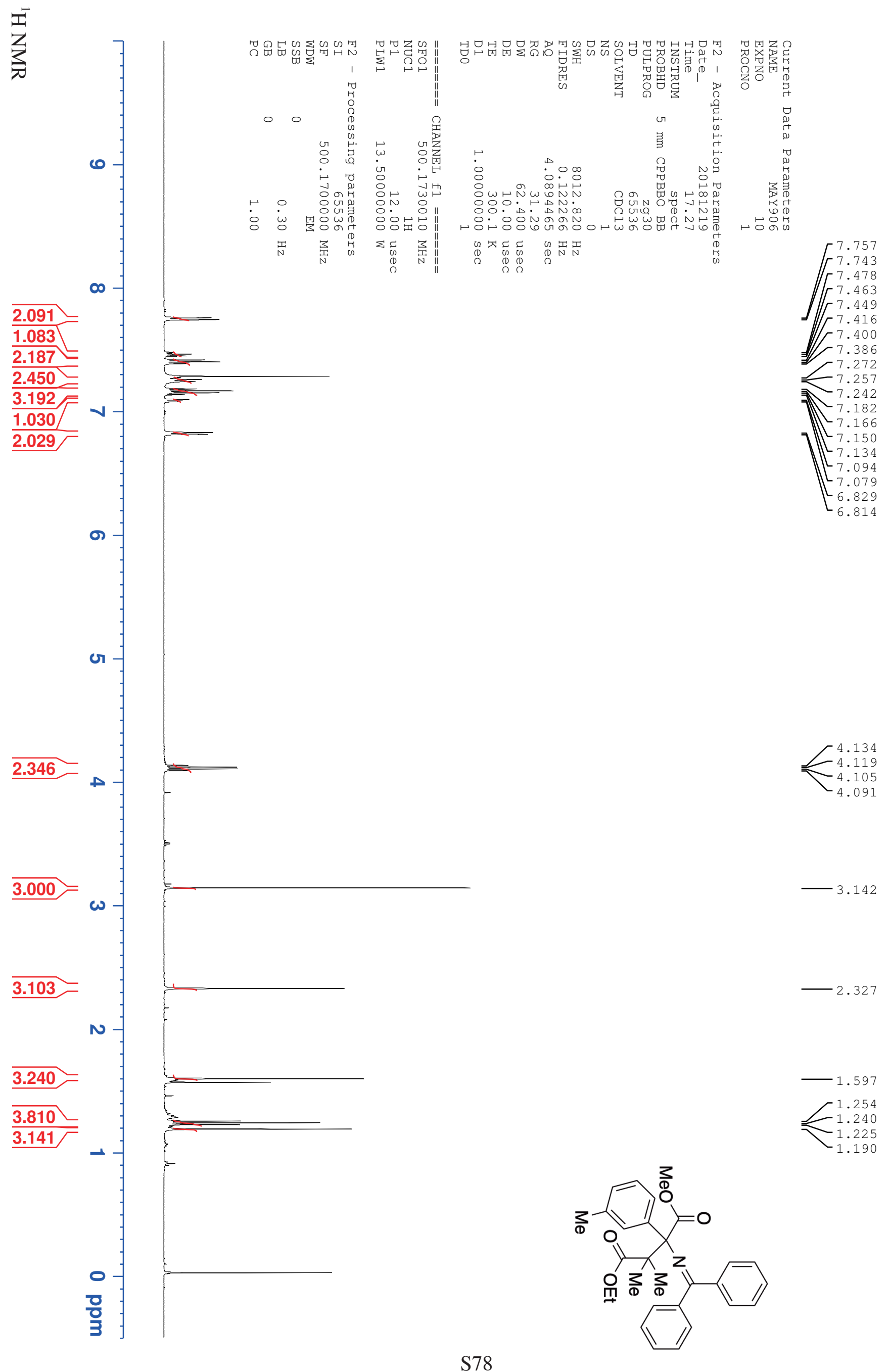




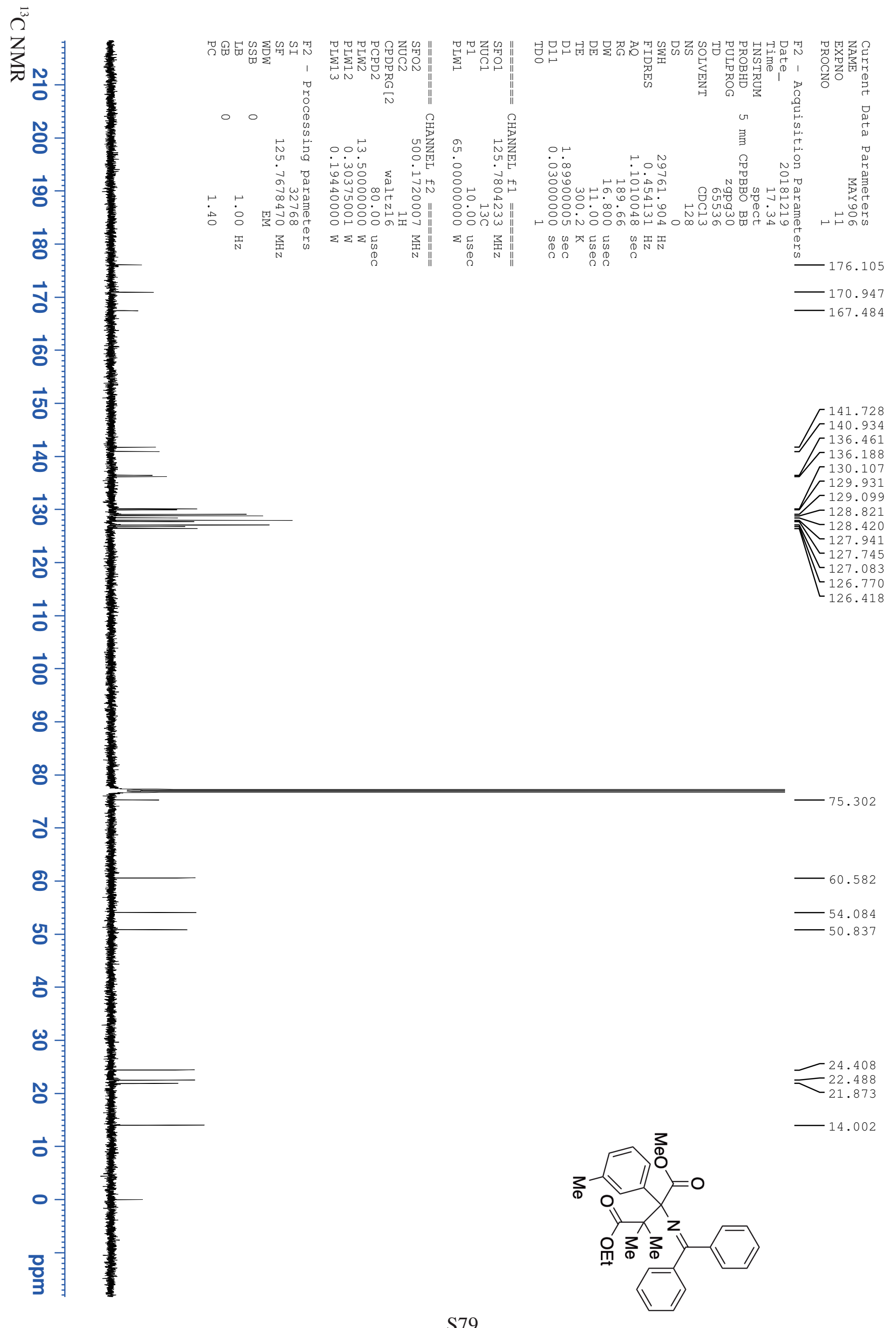




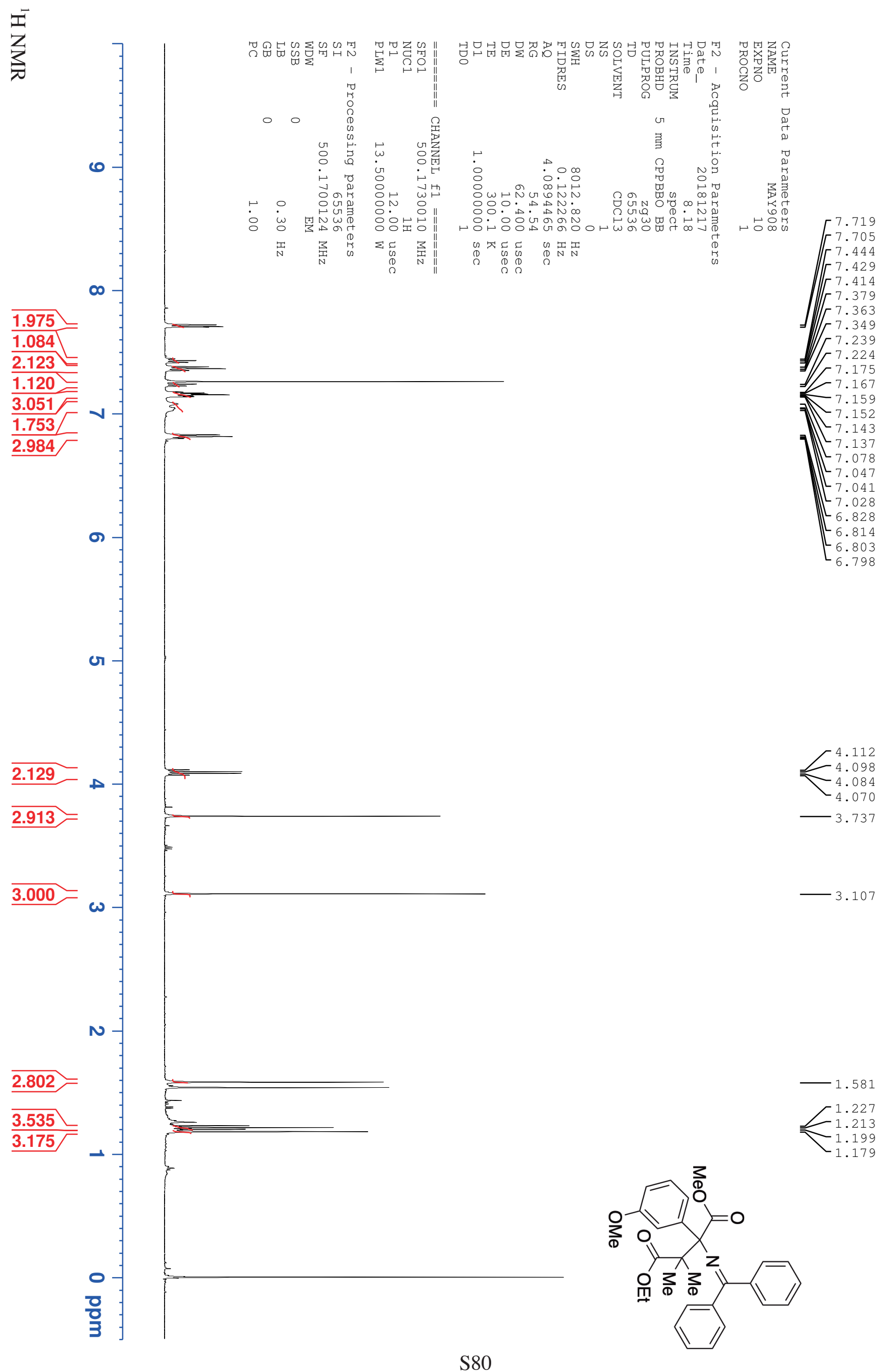




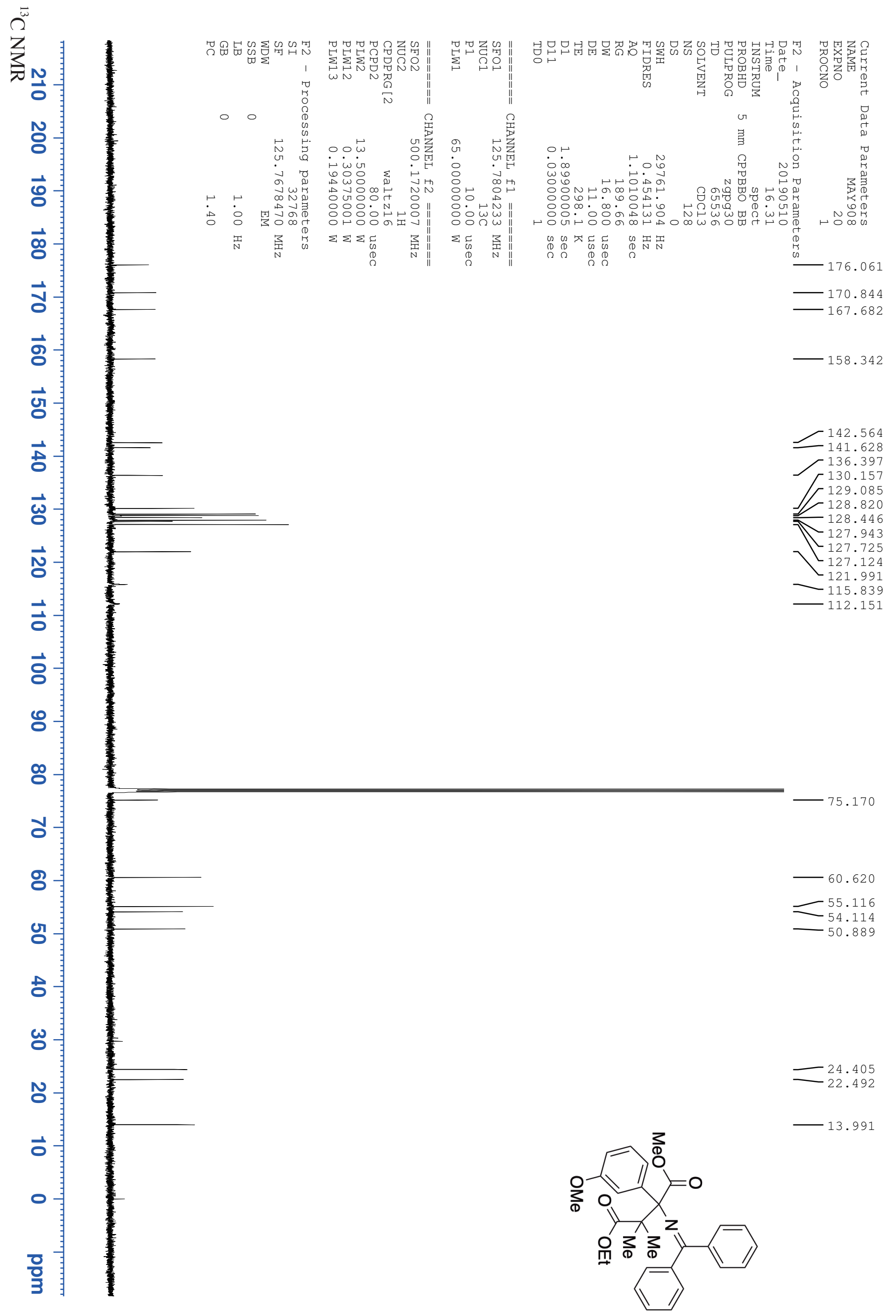




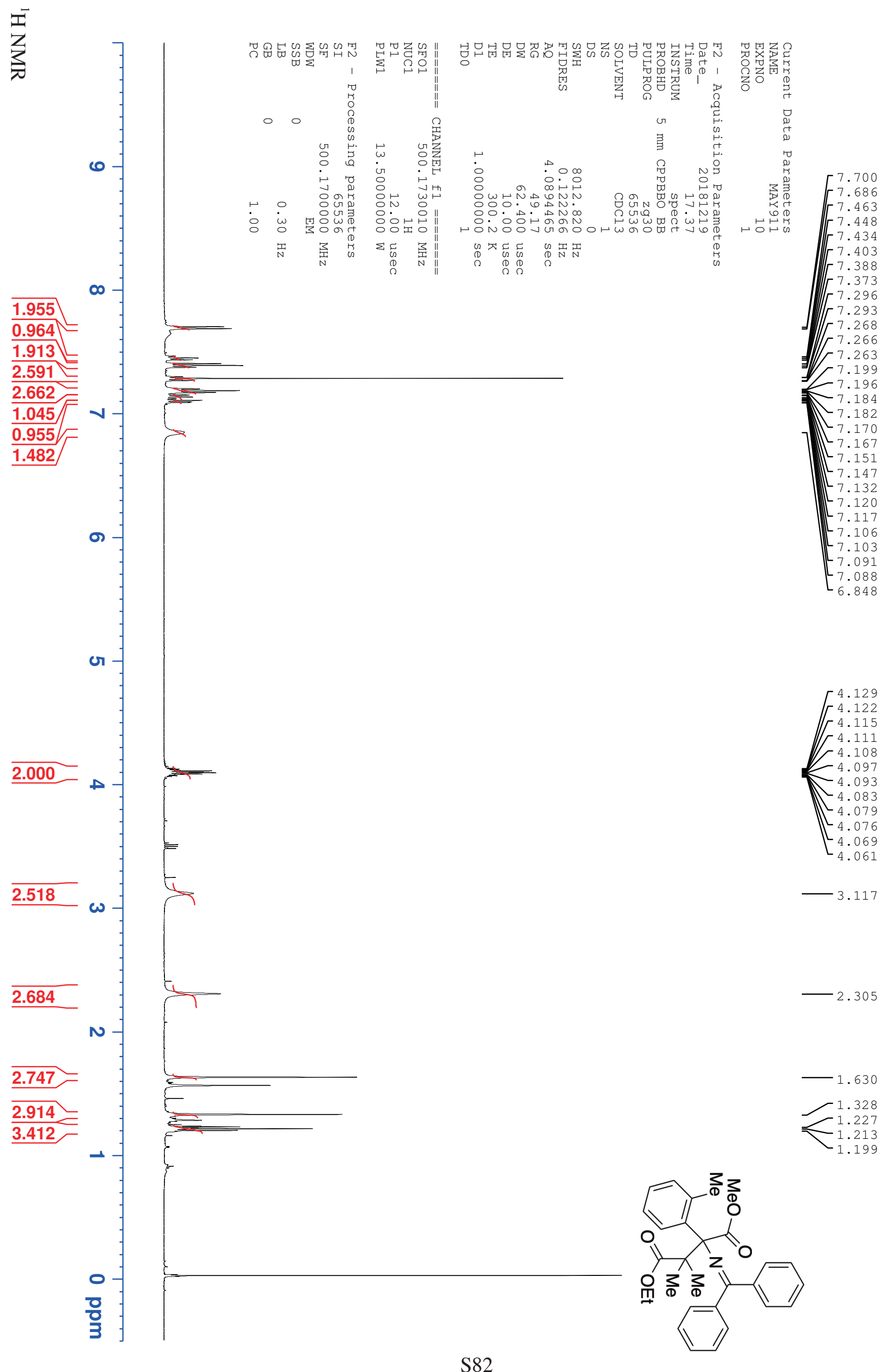




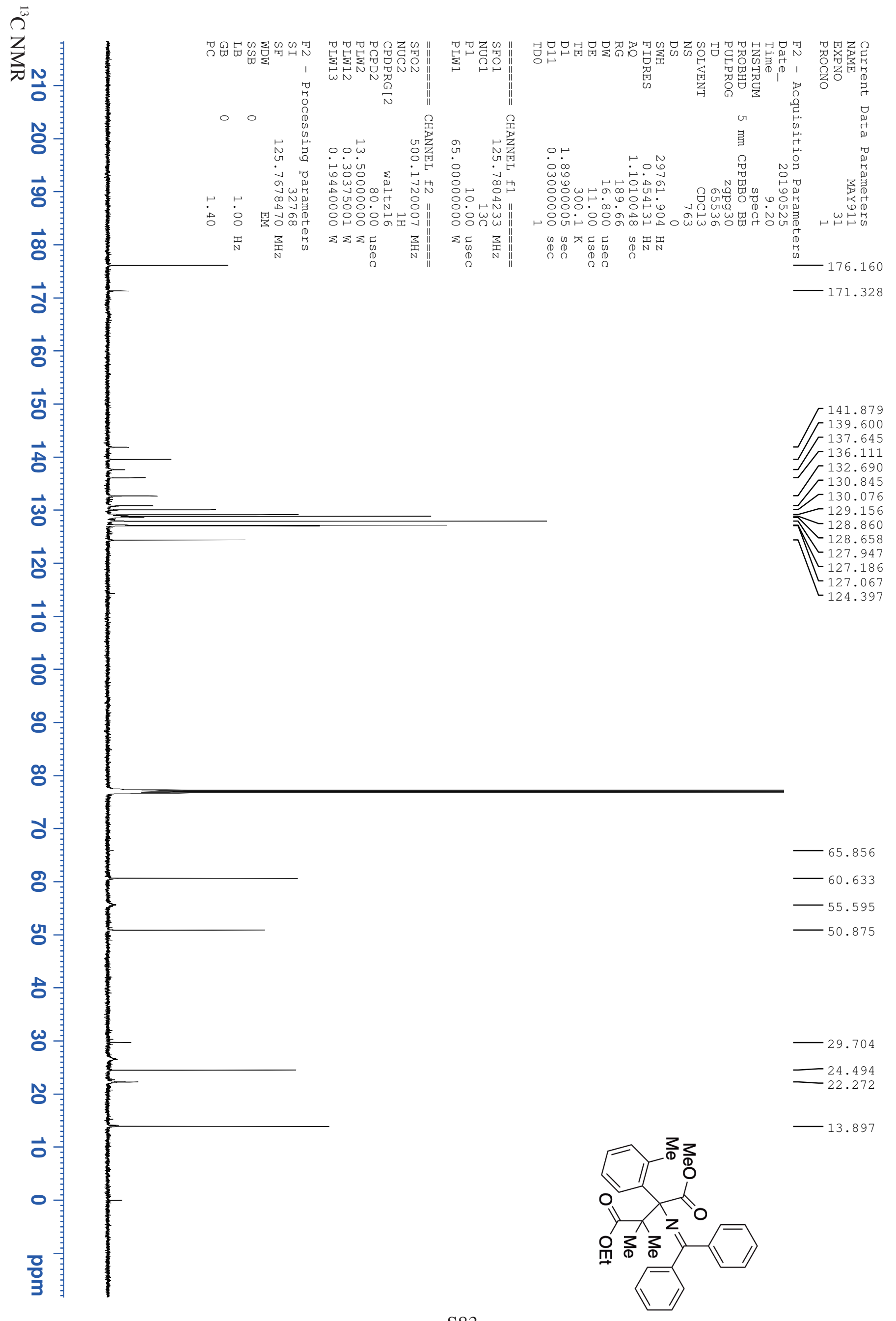




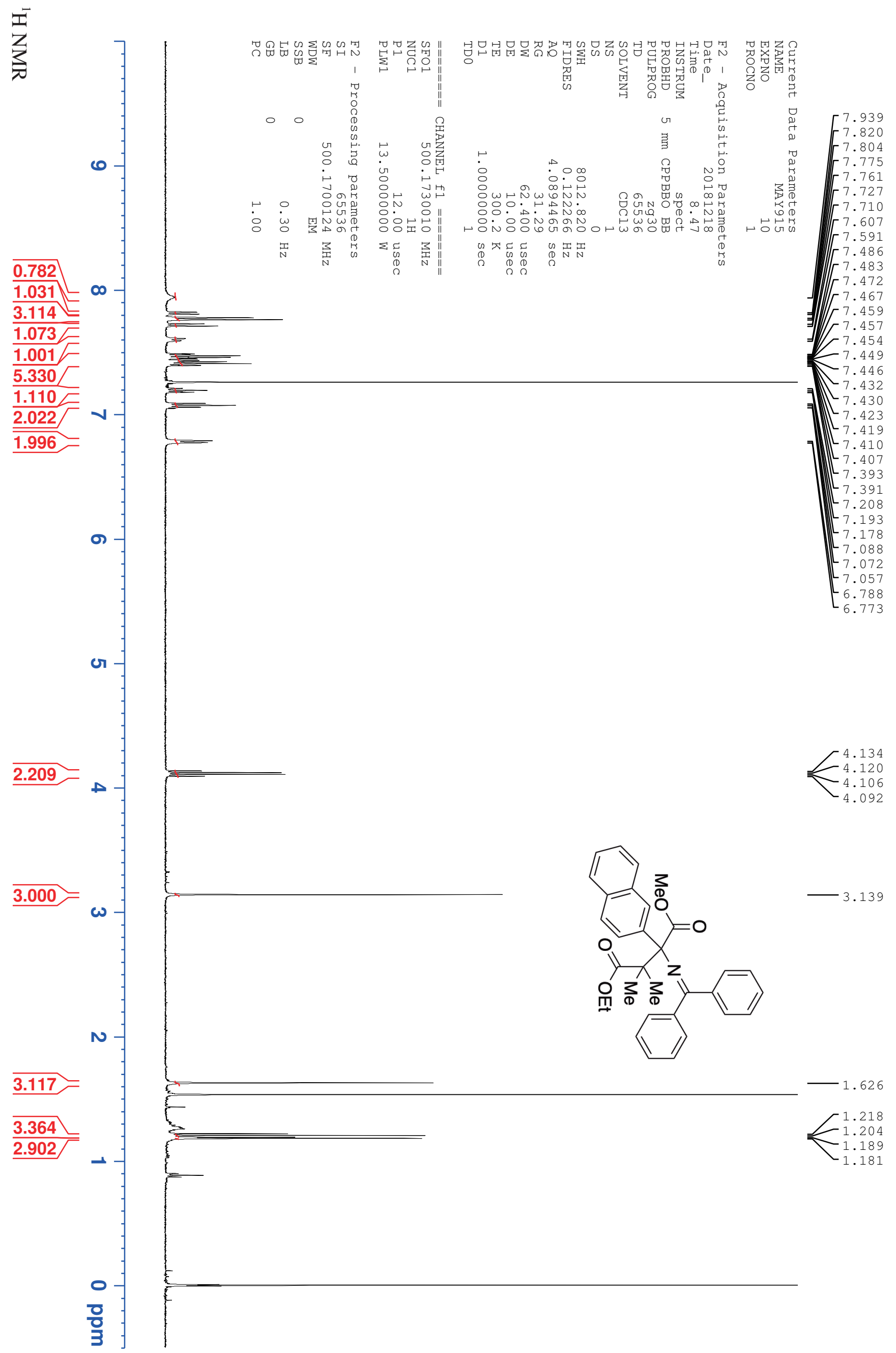




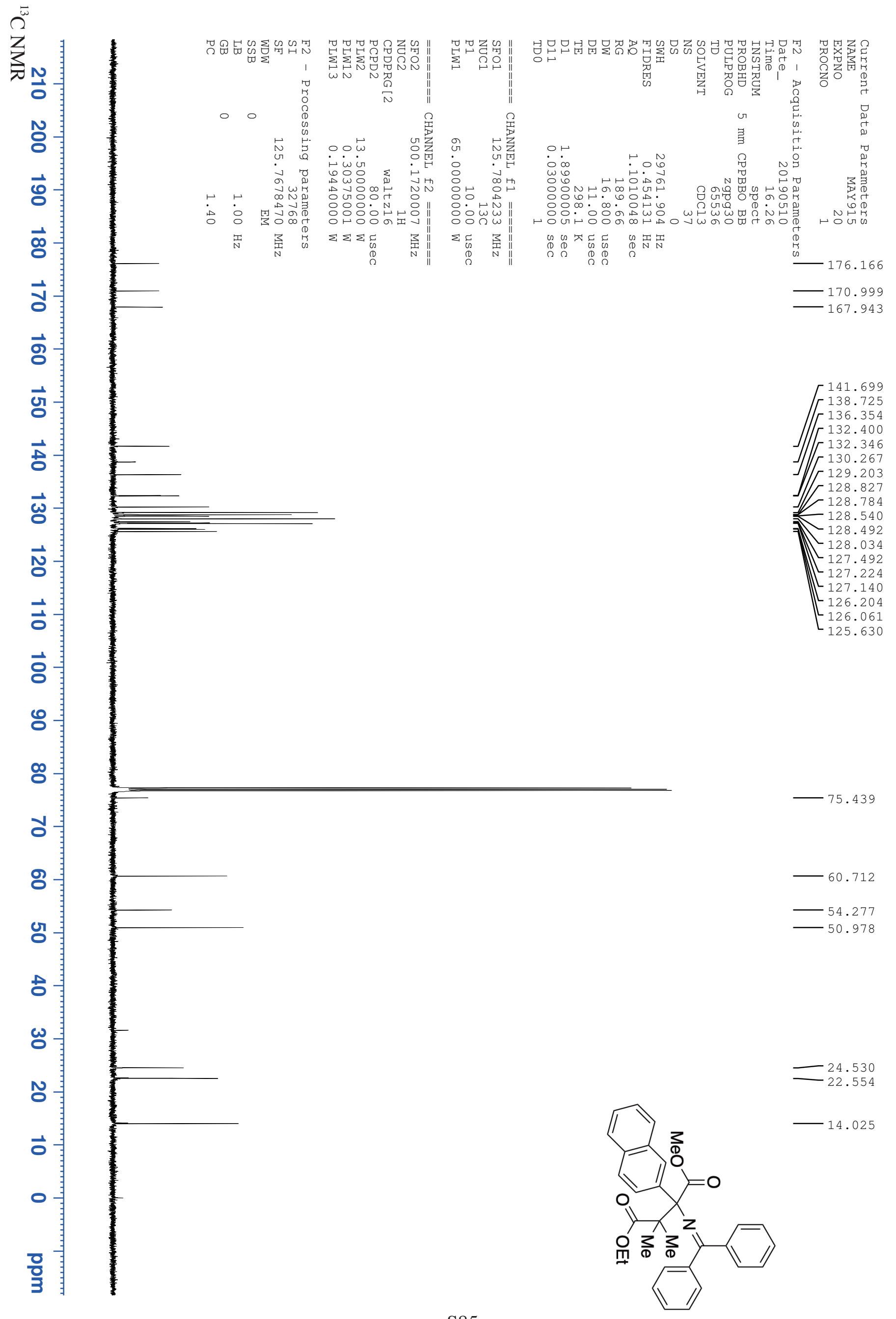




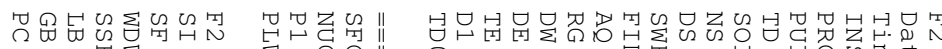

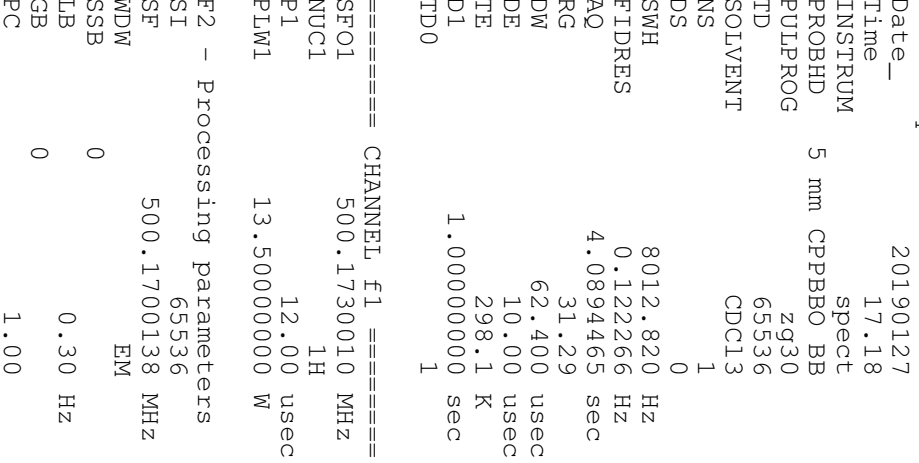

或离菌
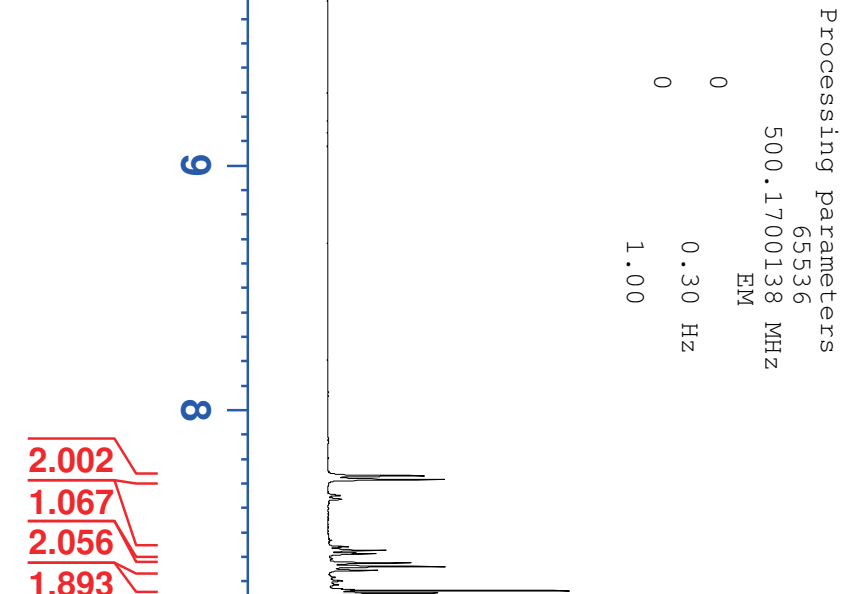

7.236

7.233

7.231

$-7.171$

7.157

7.092

7.090

7.085

7.083

6.943

6.940

6.933

$\left[\begin{array}{c}6.728 \\ 6.714\end{array}\right.$
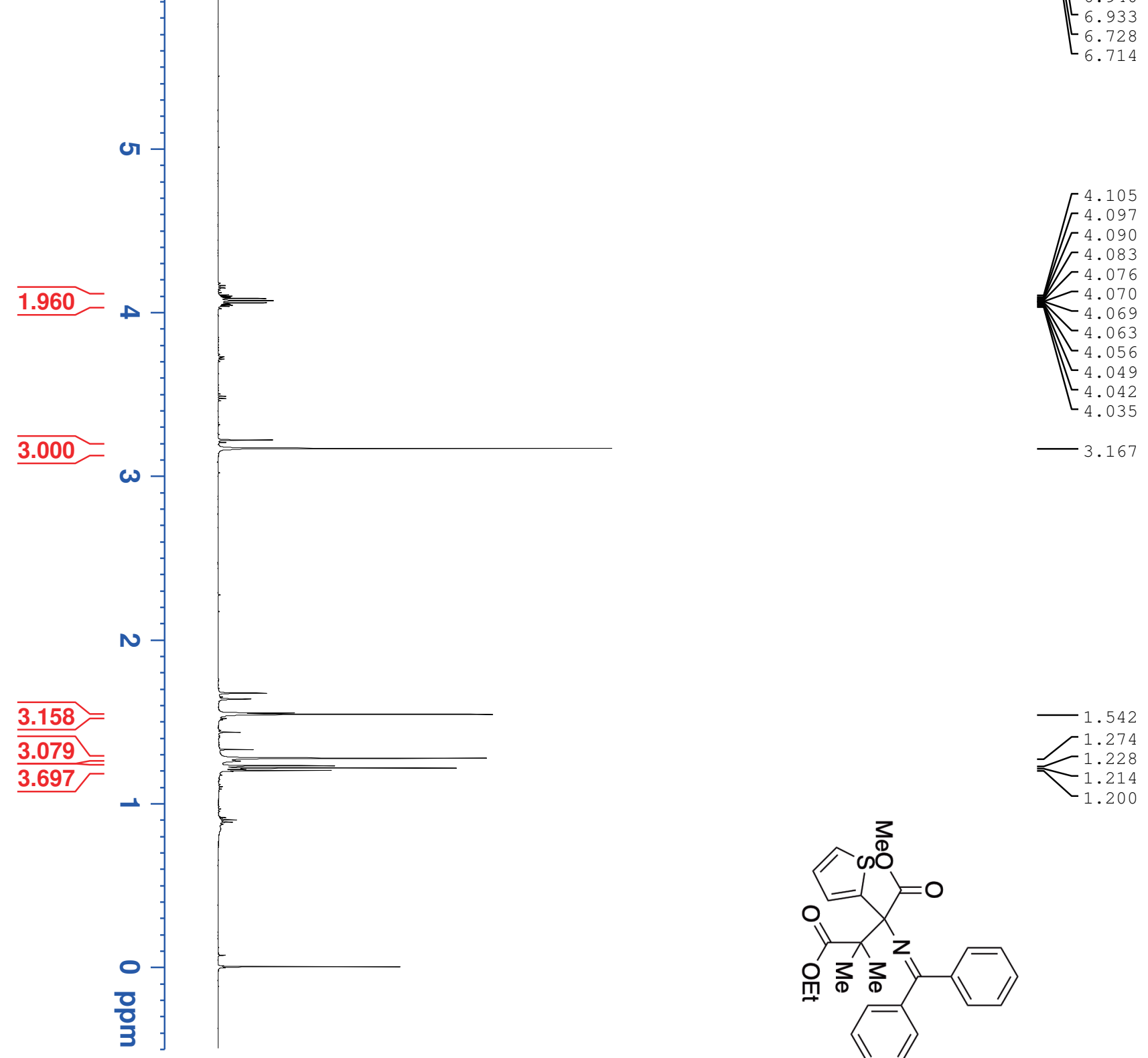

1.542

1.274

1.228

1.214

1.200

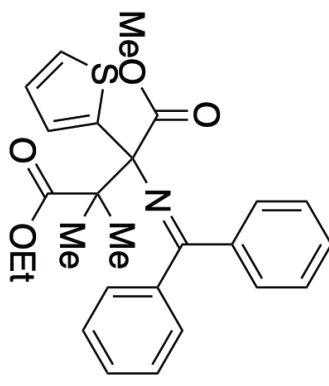




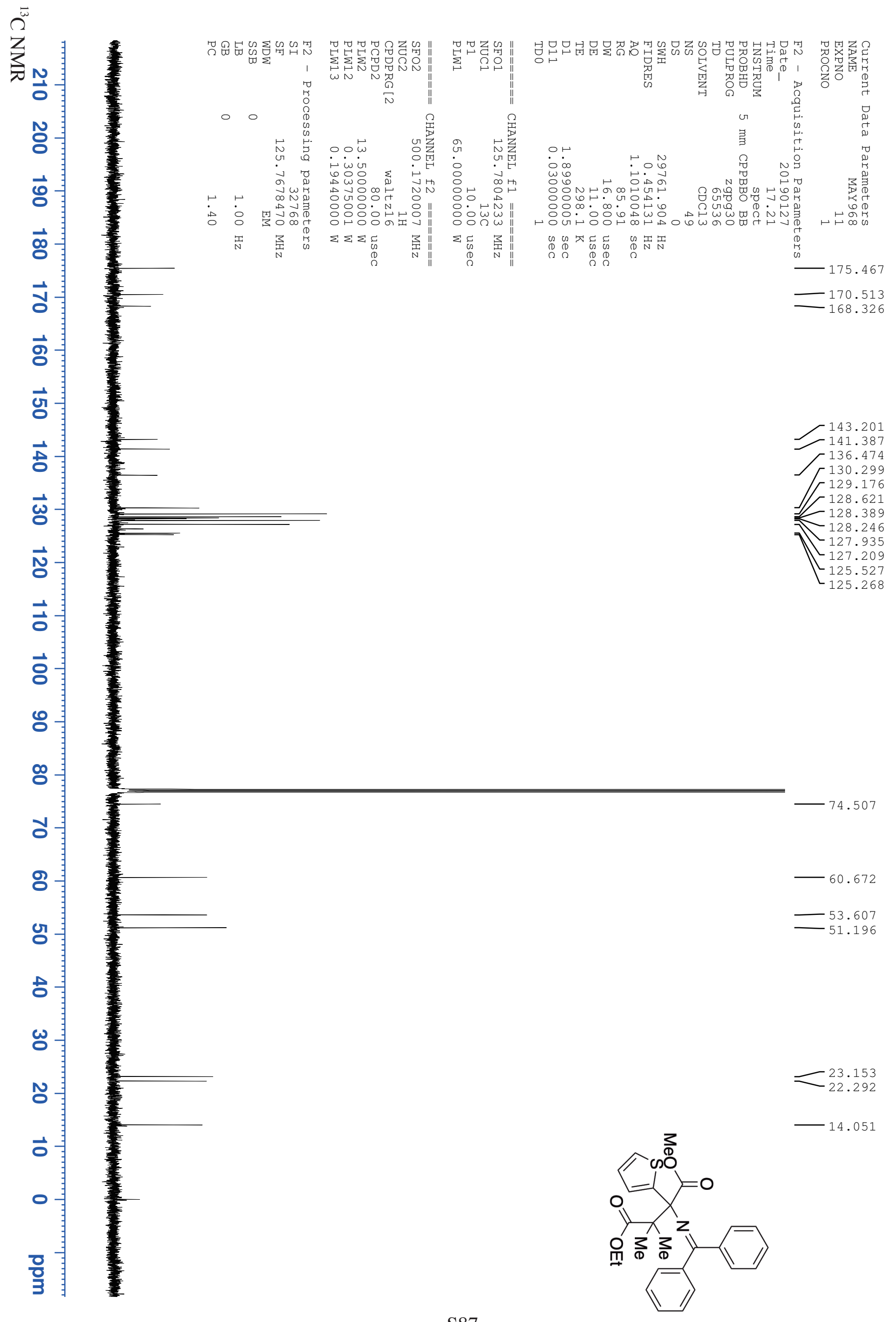



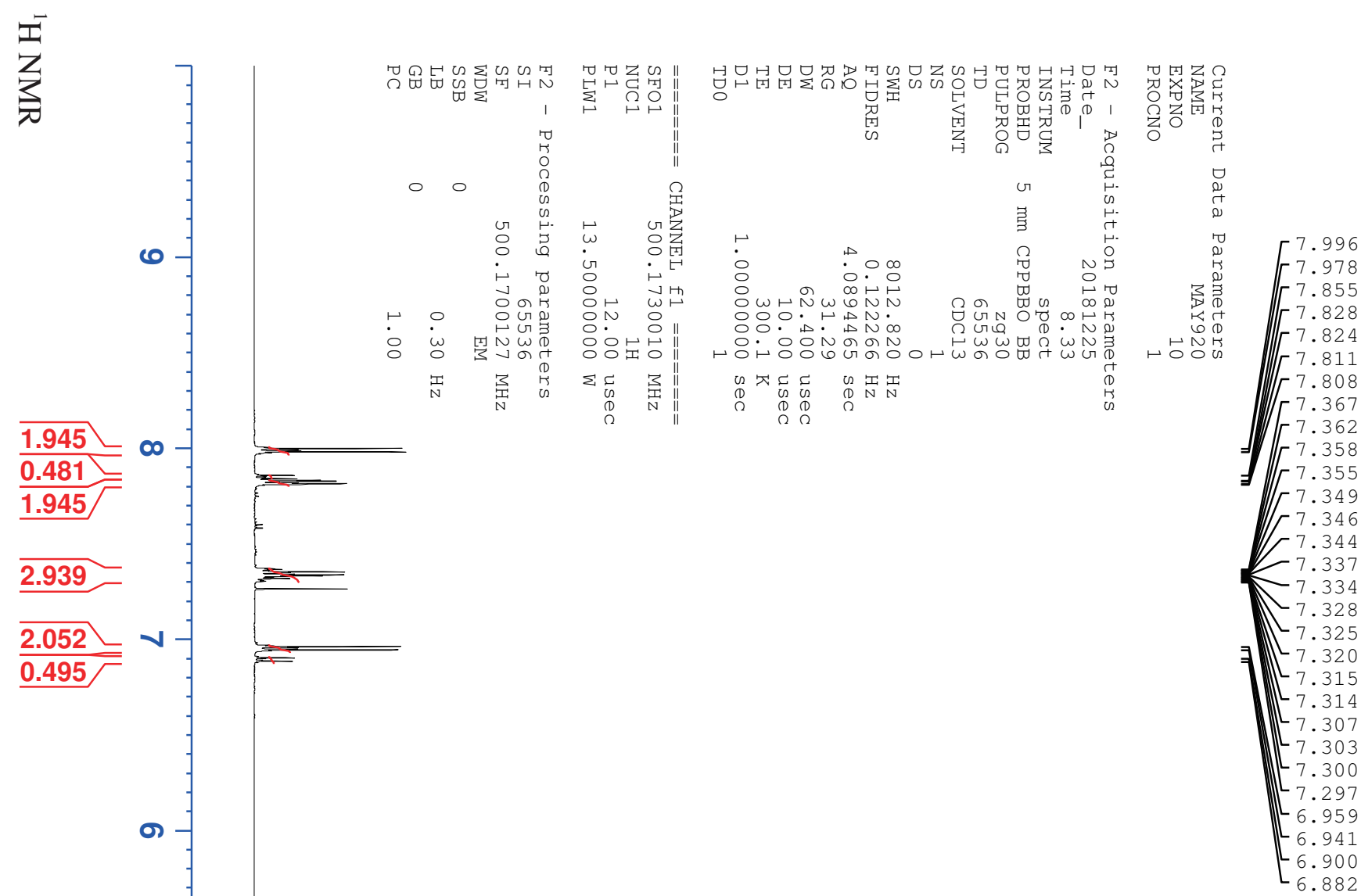

2.939

2.052 0.495
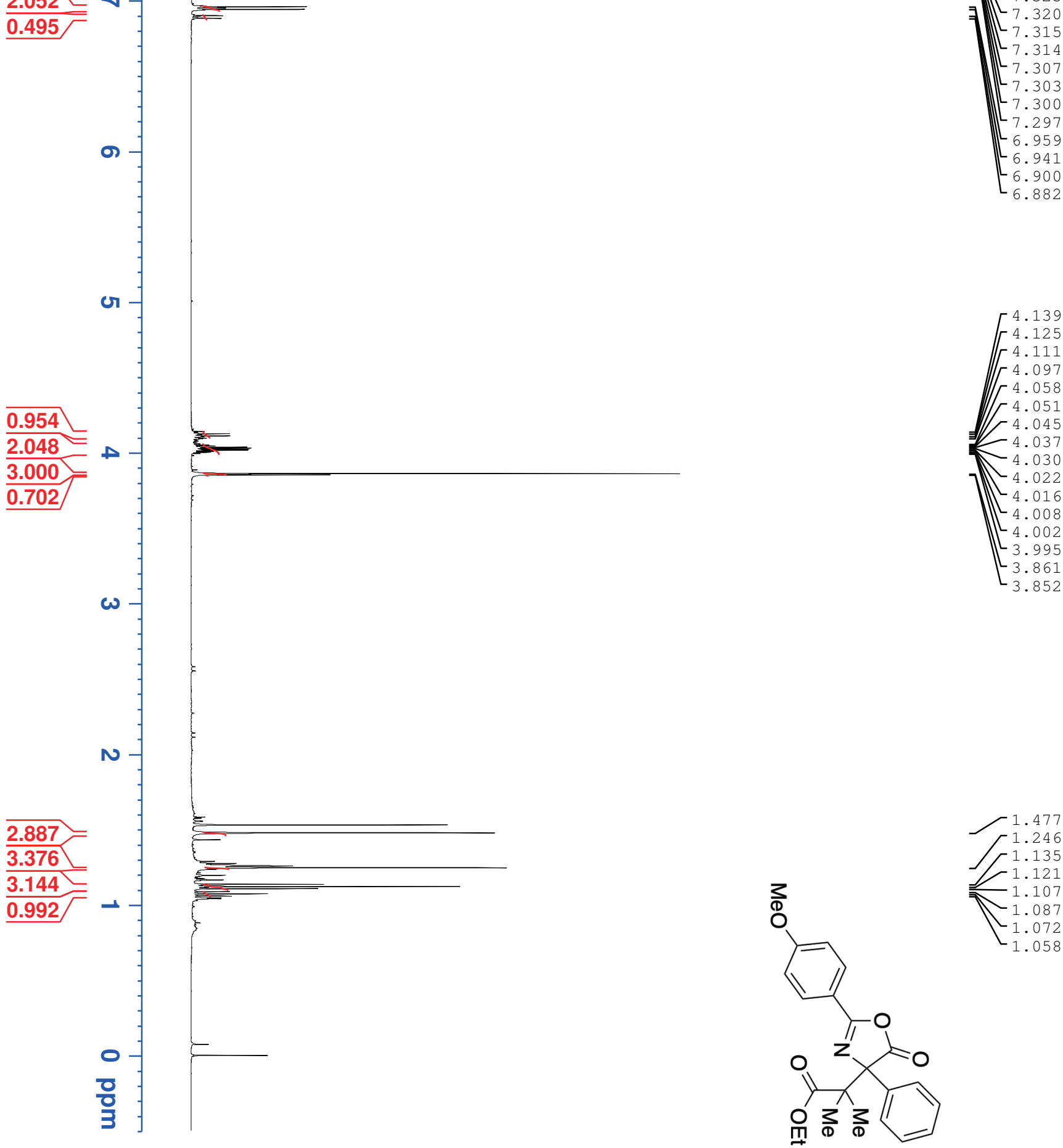


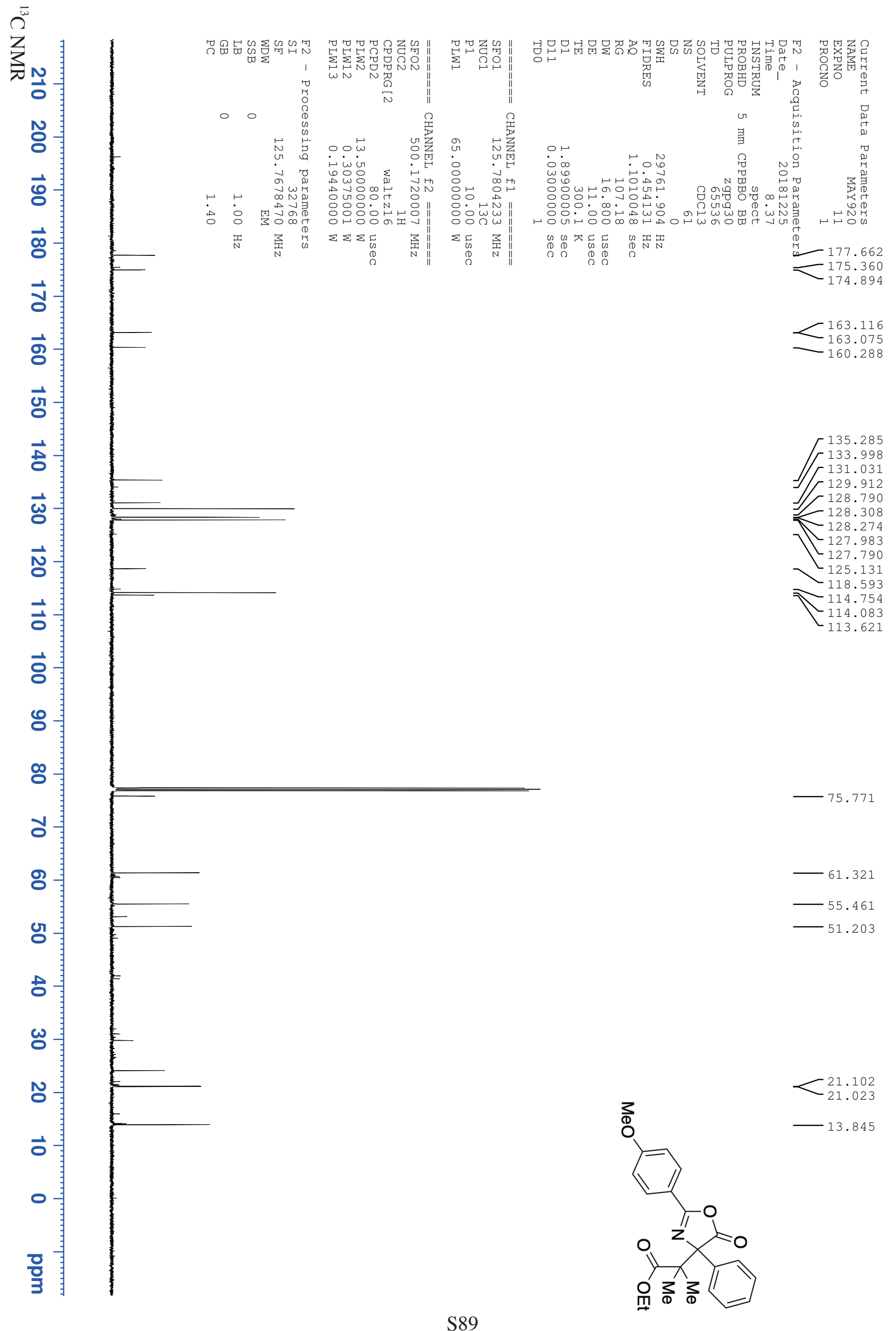




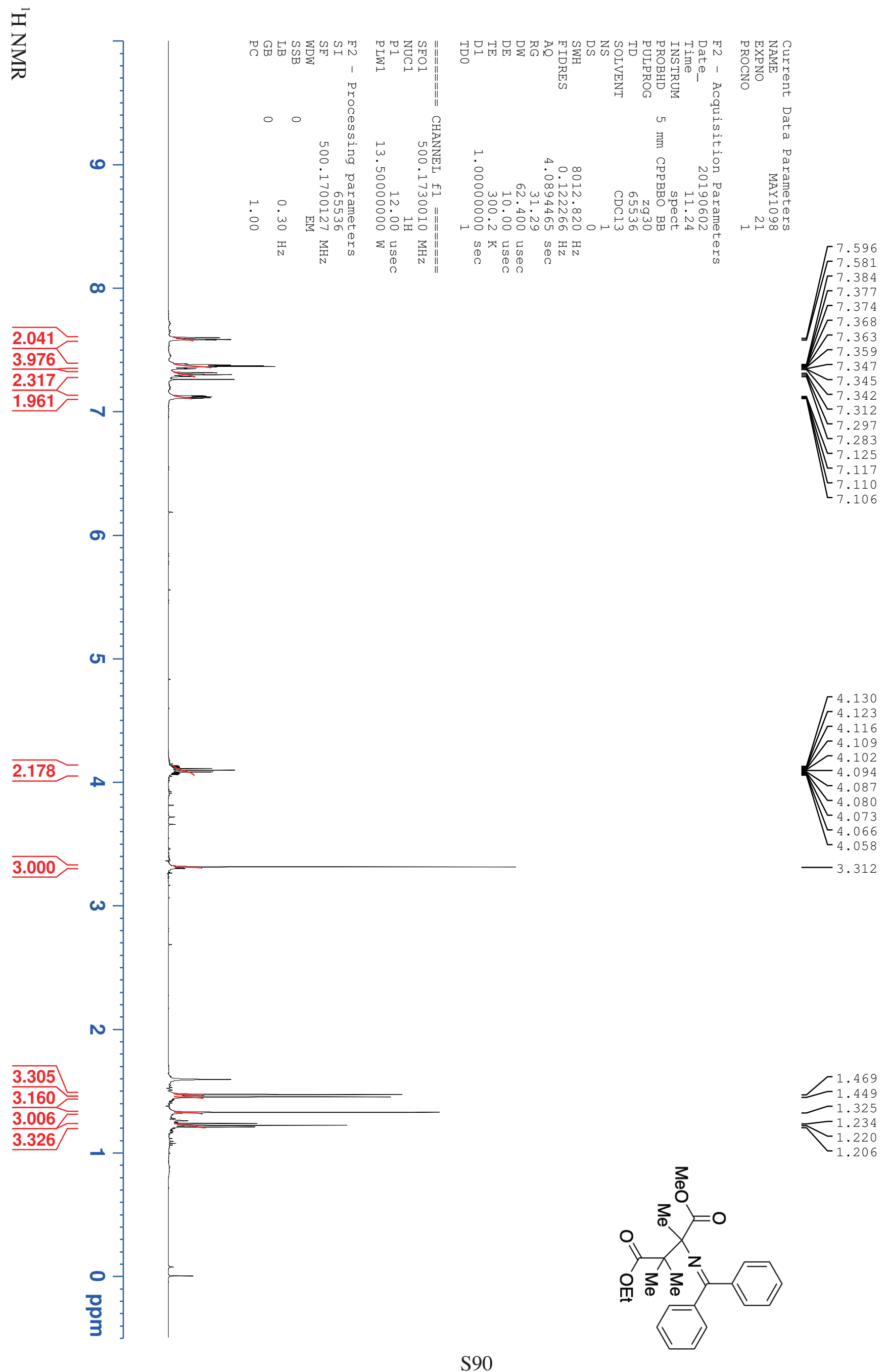




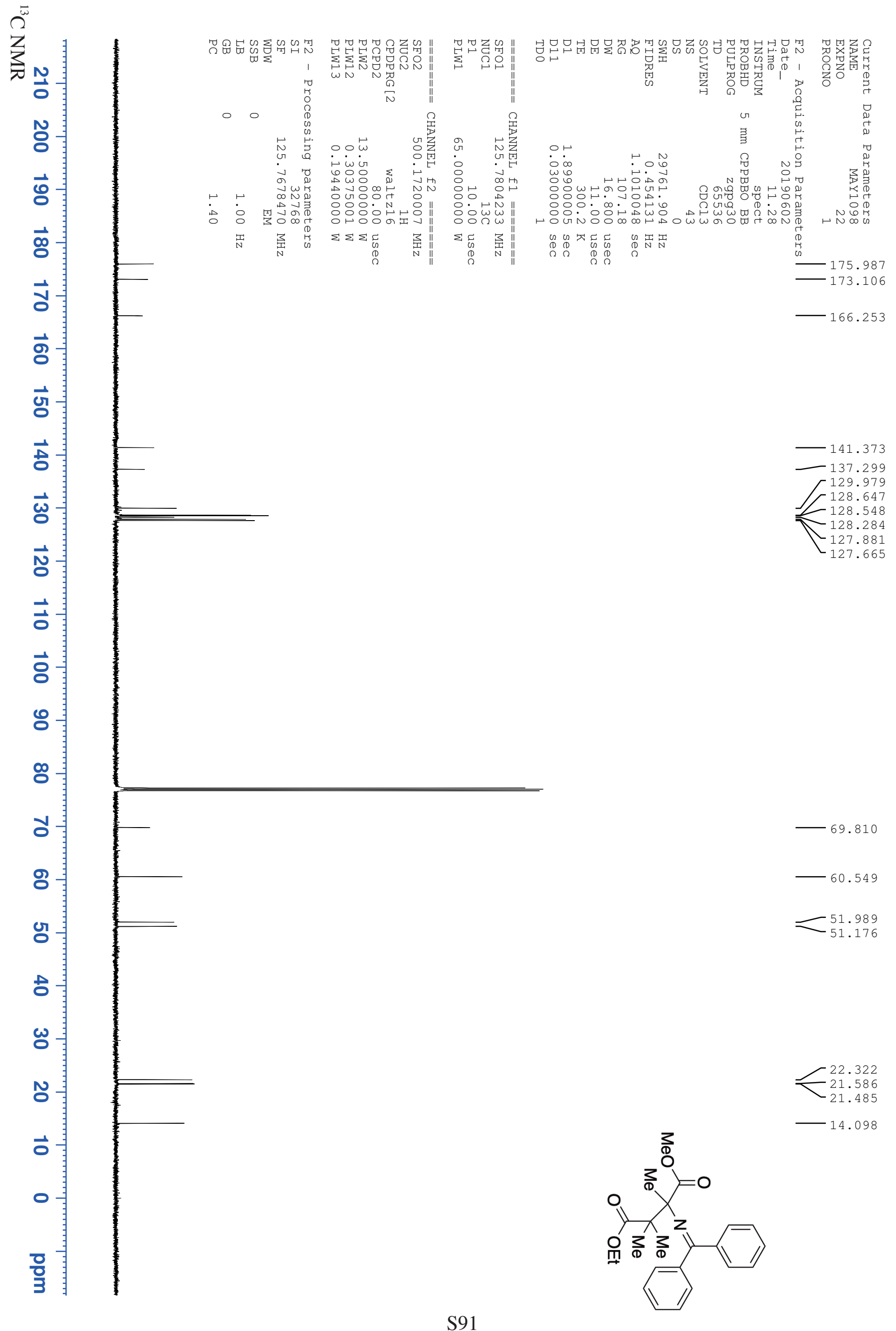




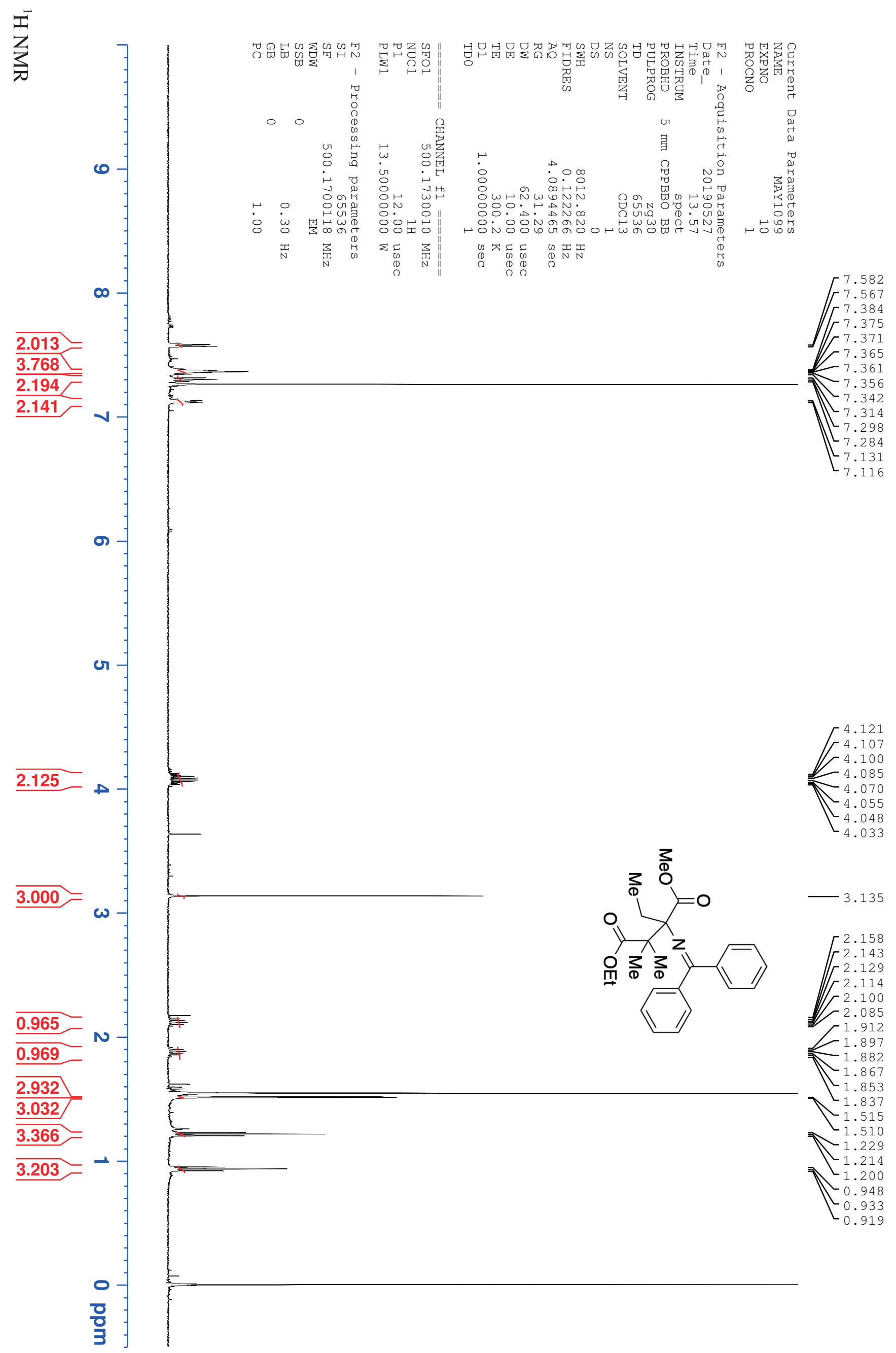




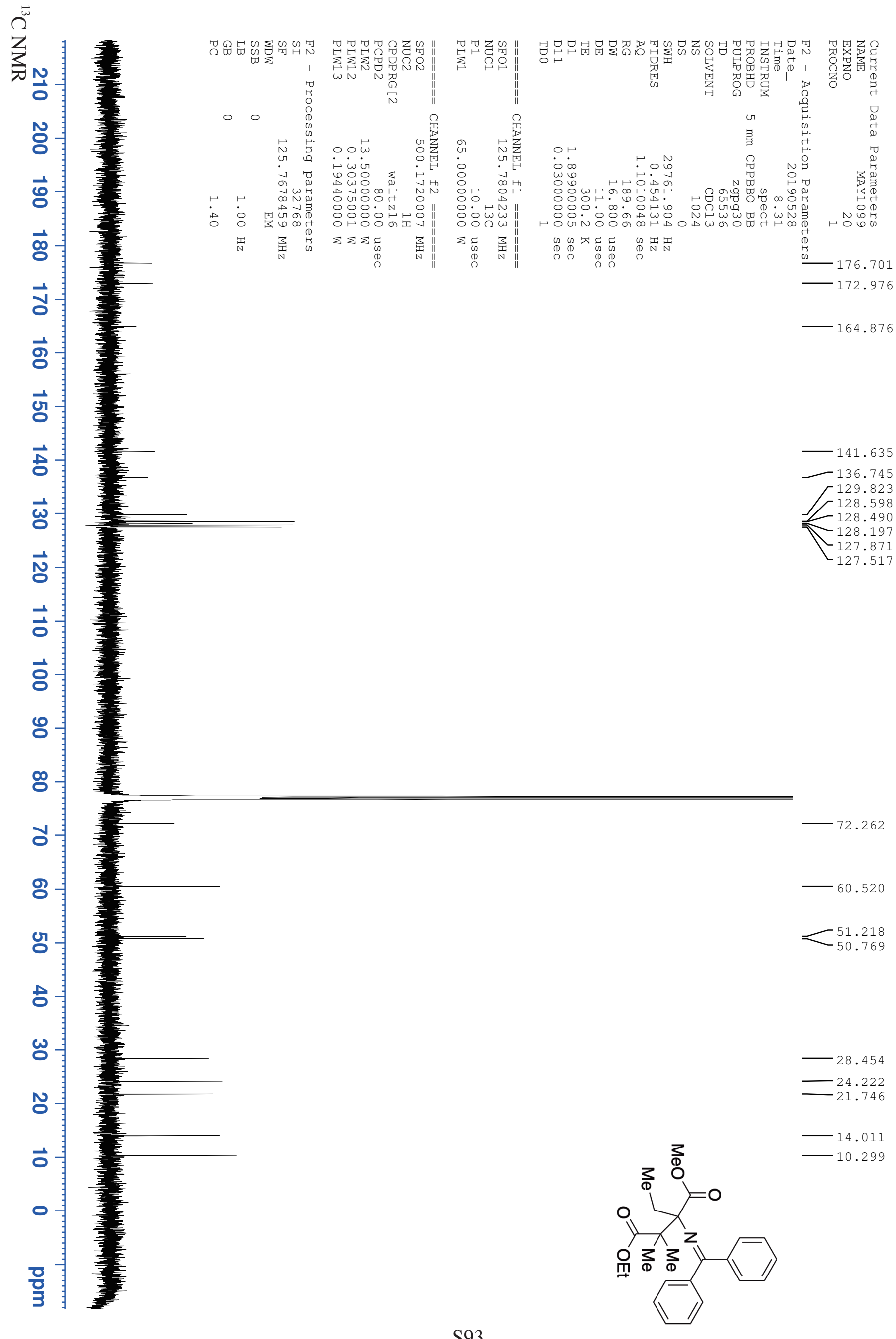




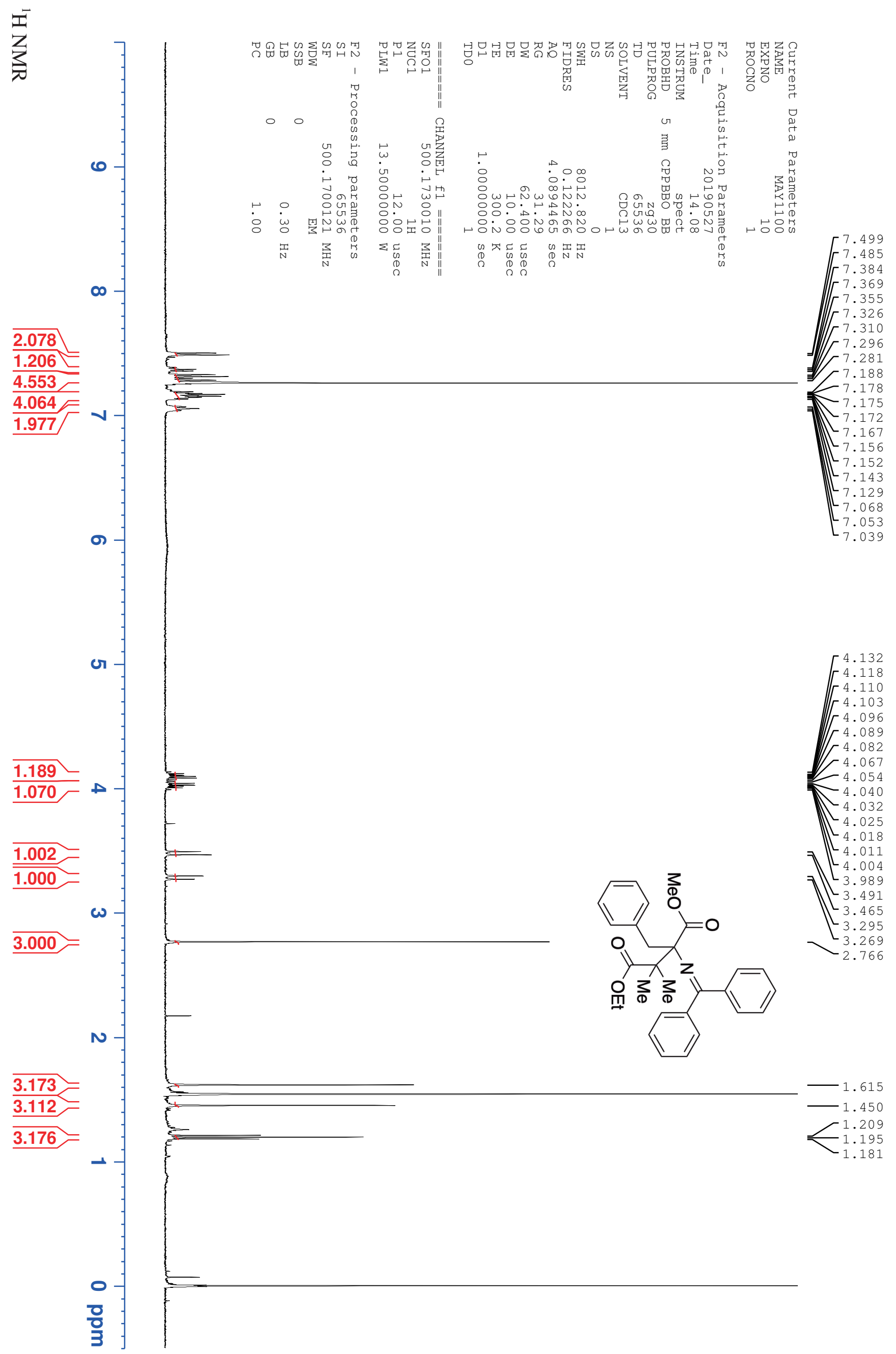




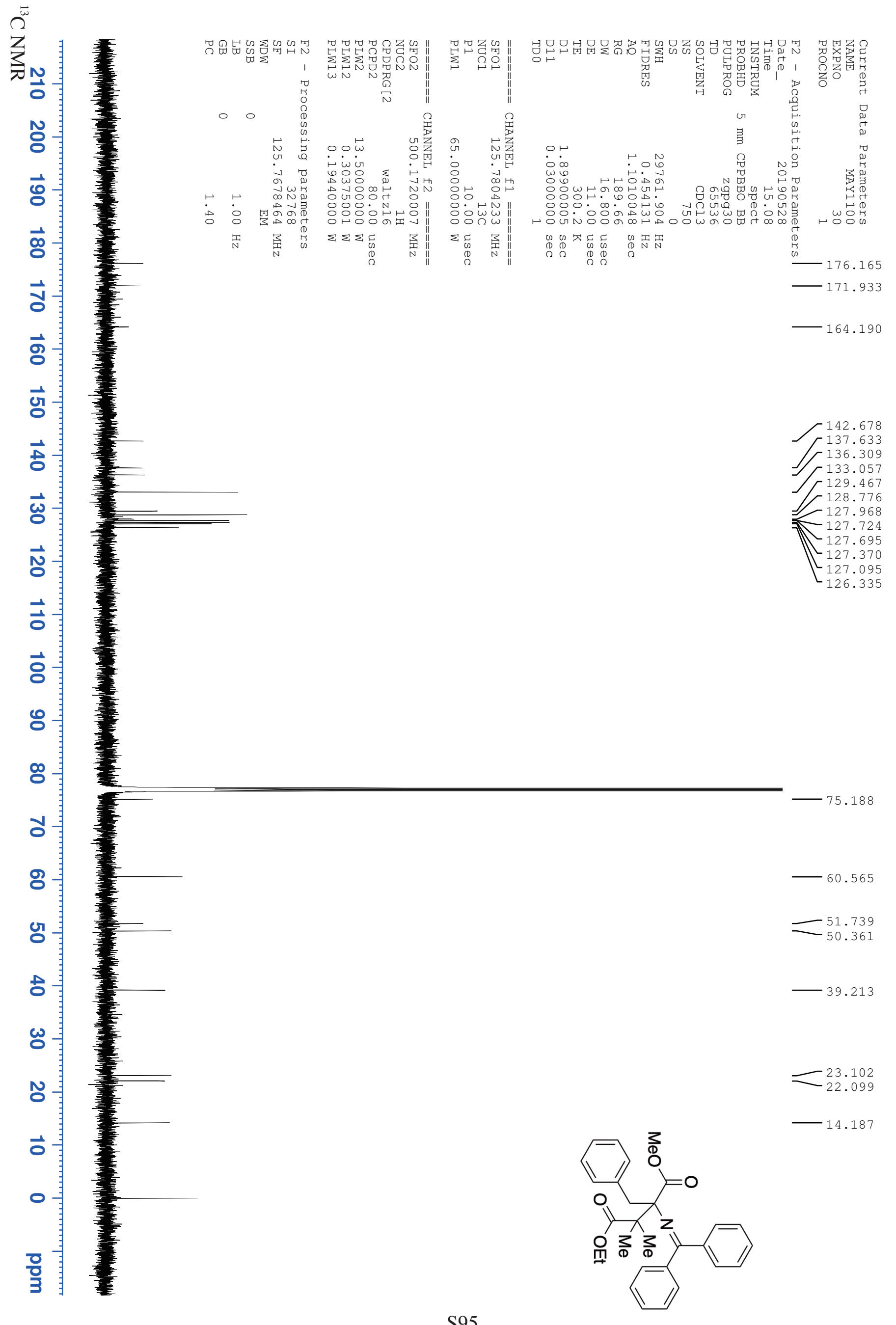




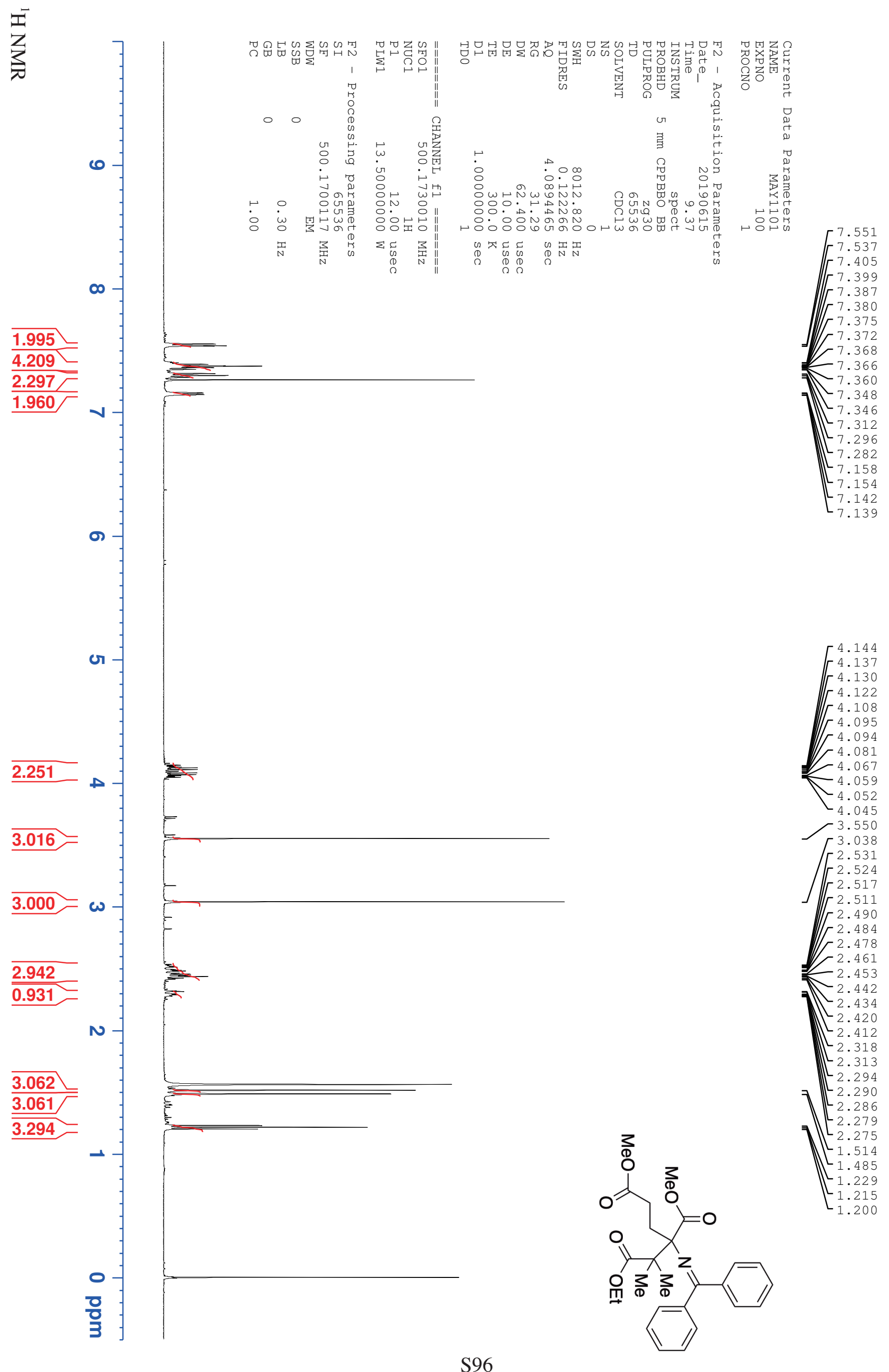




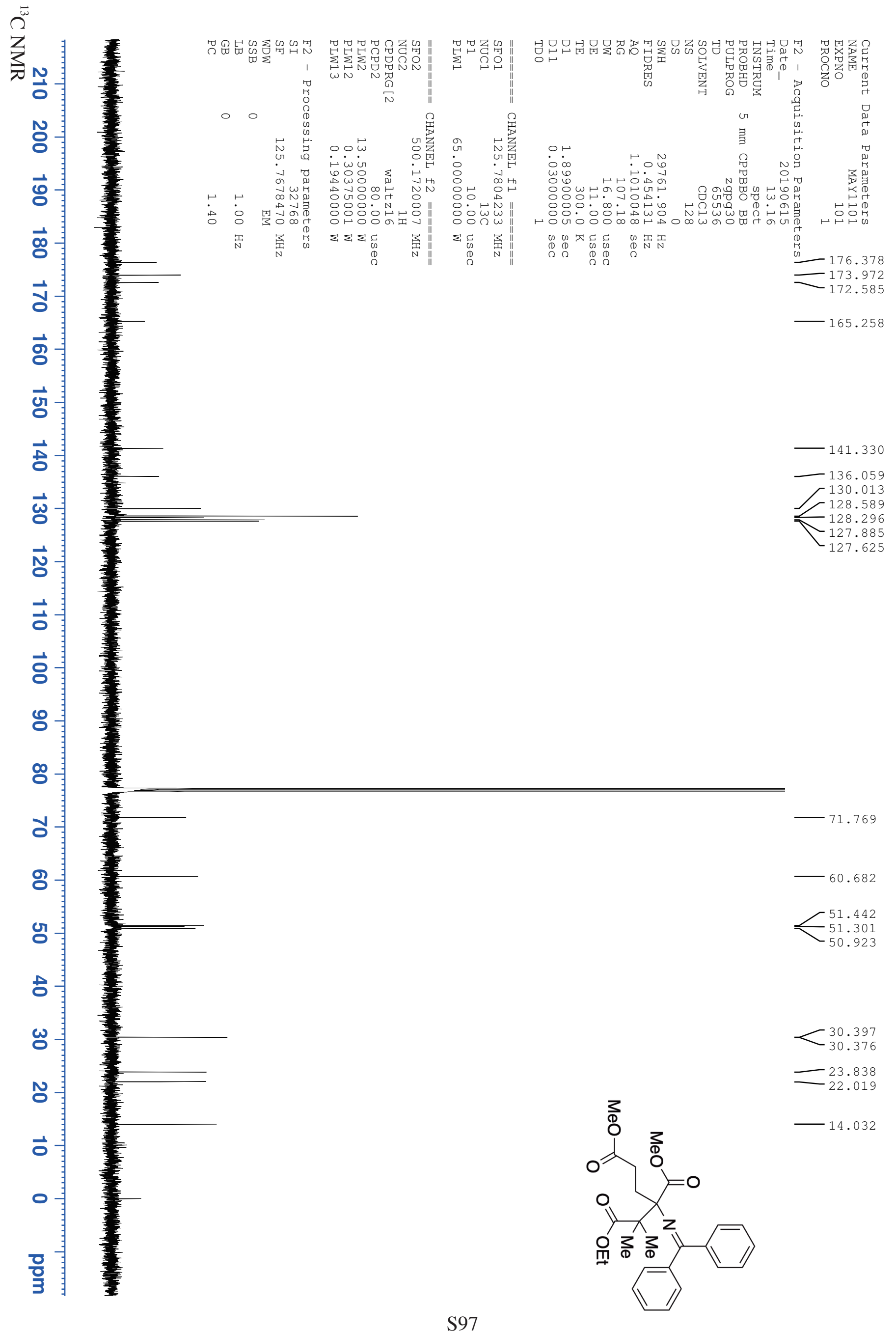



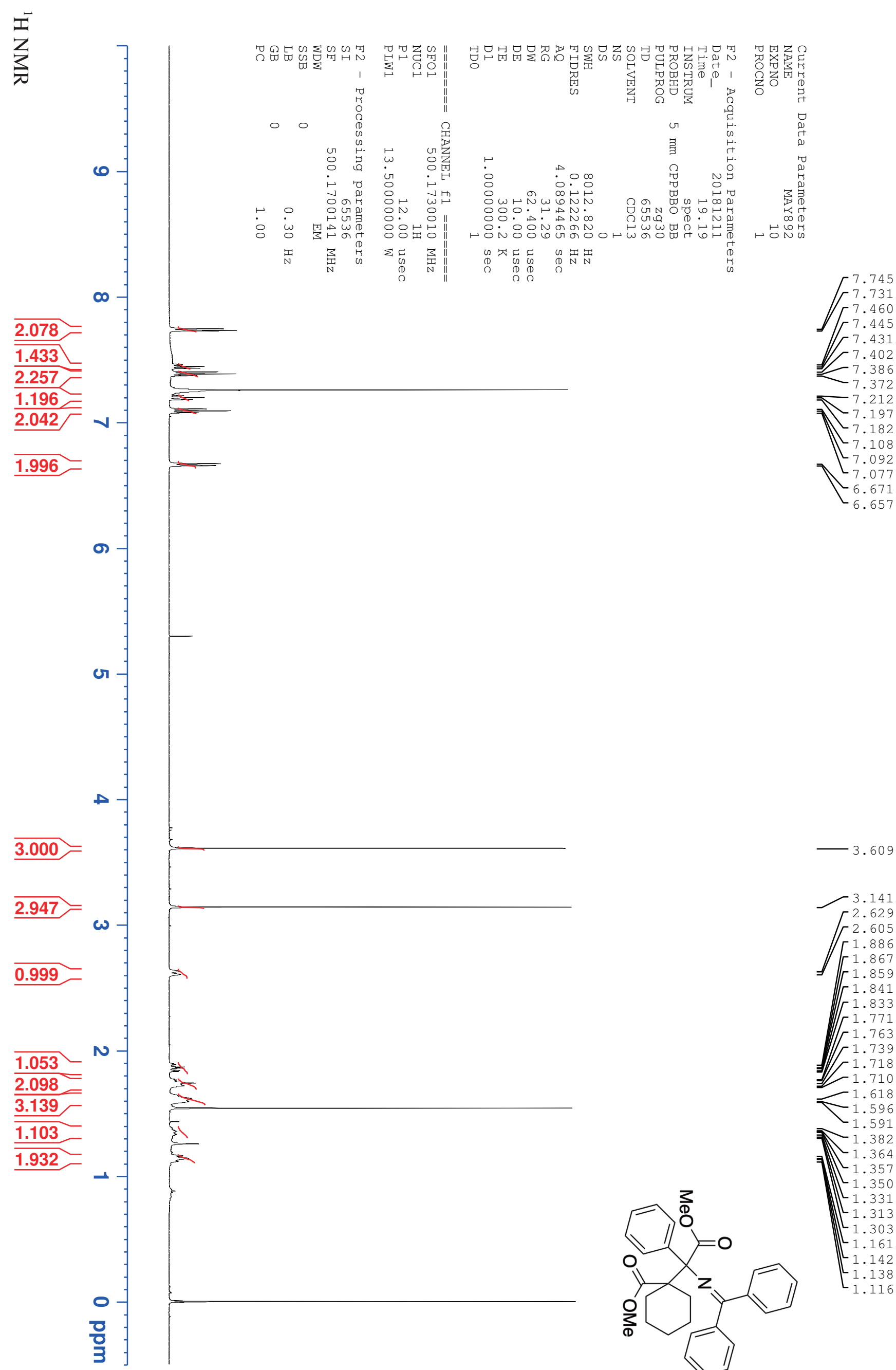

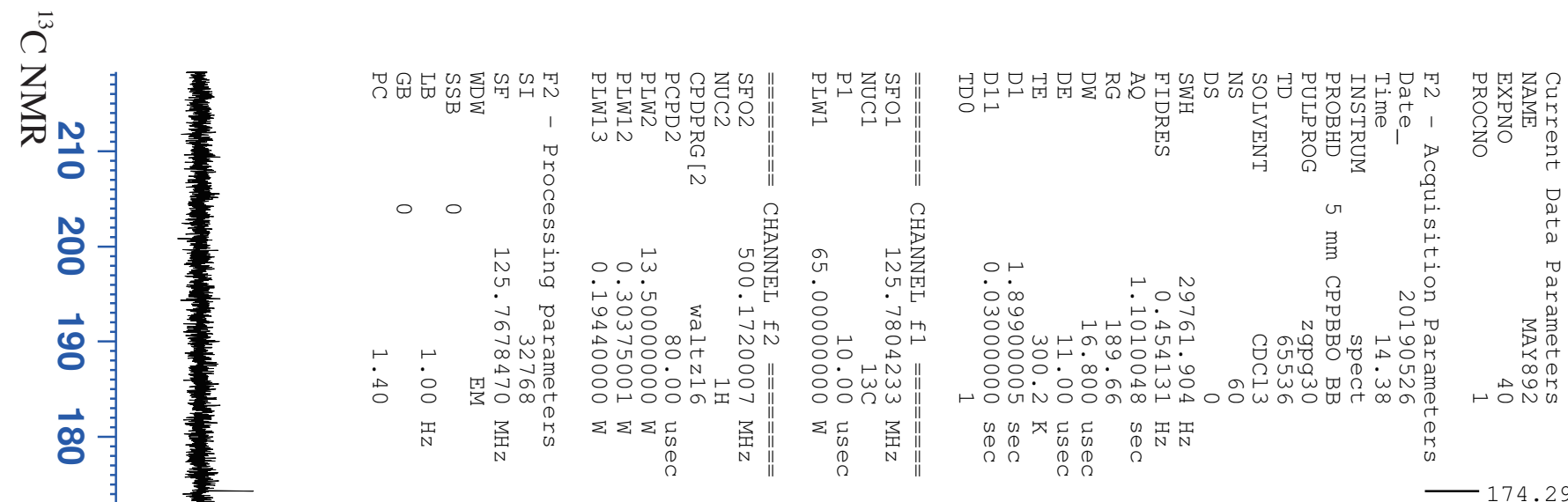

- 174.294

170.454

ป

용

जु

$\vec{f}$

డ్ర

ज̃

룽

홍

$\varnothing$

\&

ป

8

용

응

$\omega$

ก

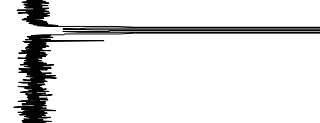

141.702

140.694

136.739

130.168

129.780

129.096

128.770
128.367

$-128.367$

$-127.999$

127.081

127.045
126.699

8

$8=$

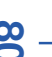

-

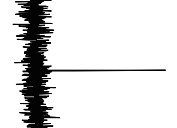

59.118

$\Upsilon 51.375$

0.819

促

$\overrightarrow{0}$

$\circ$

总

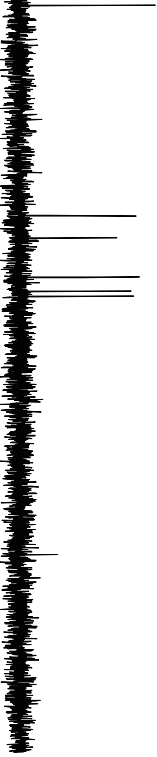

$-31.330$

29.287

25.64

24.365

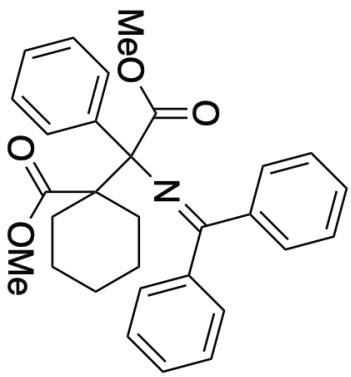




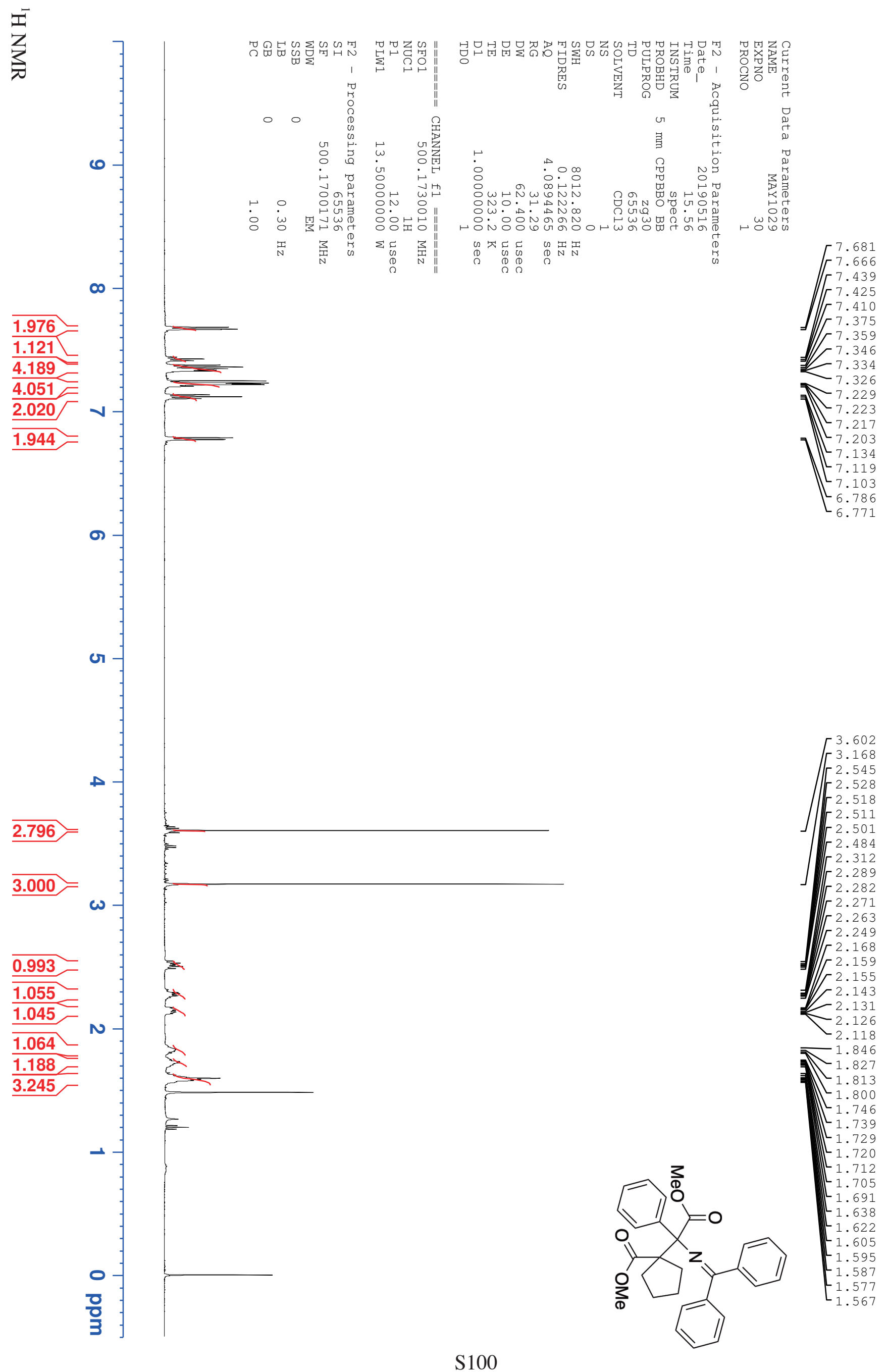




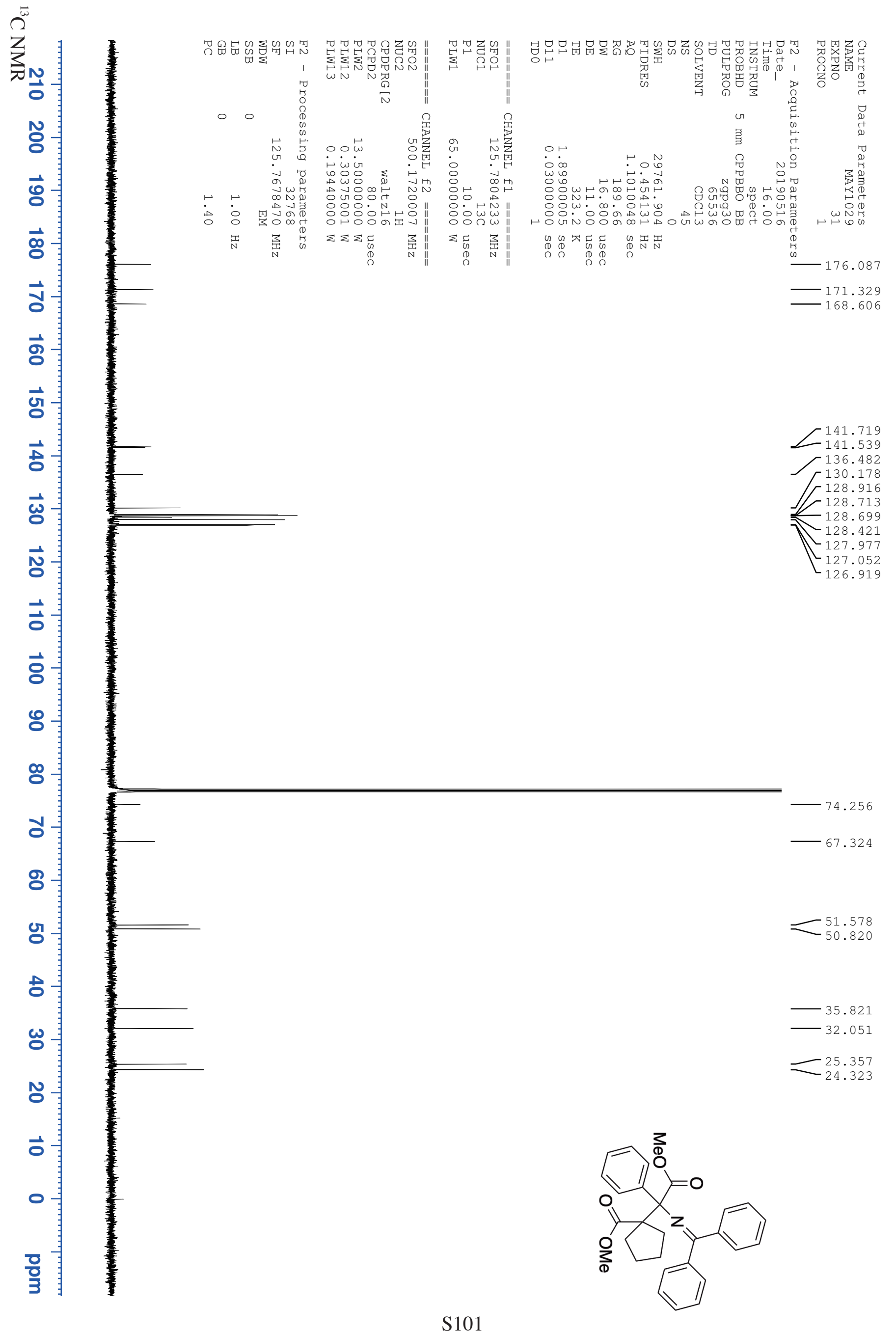




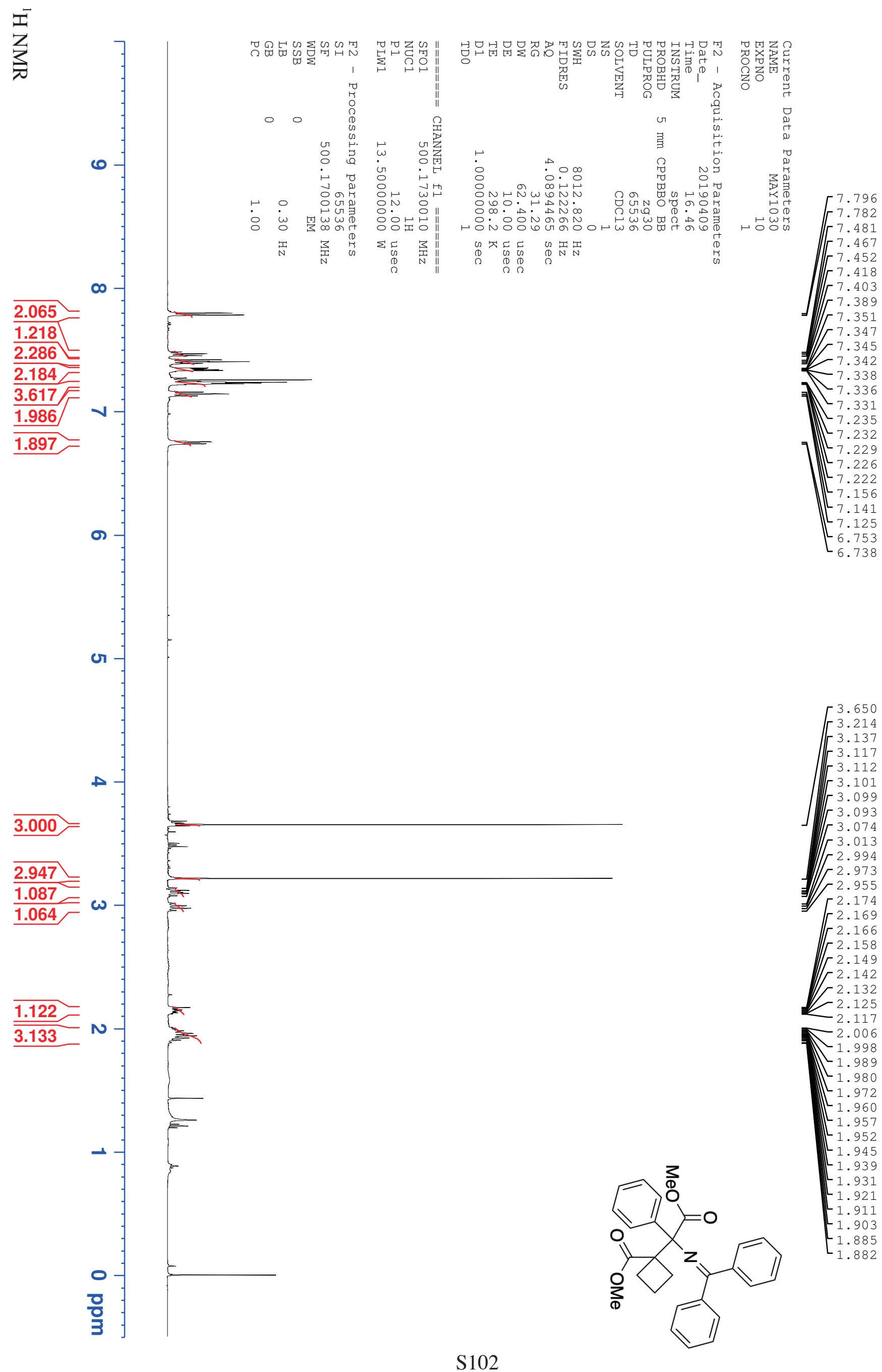




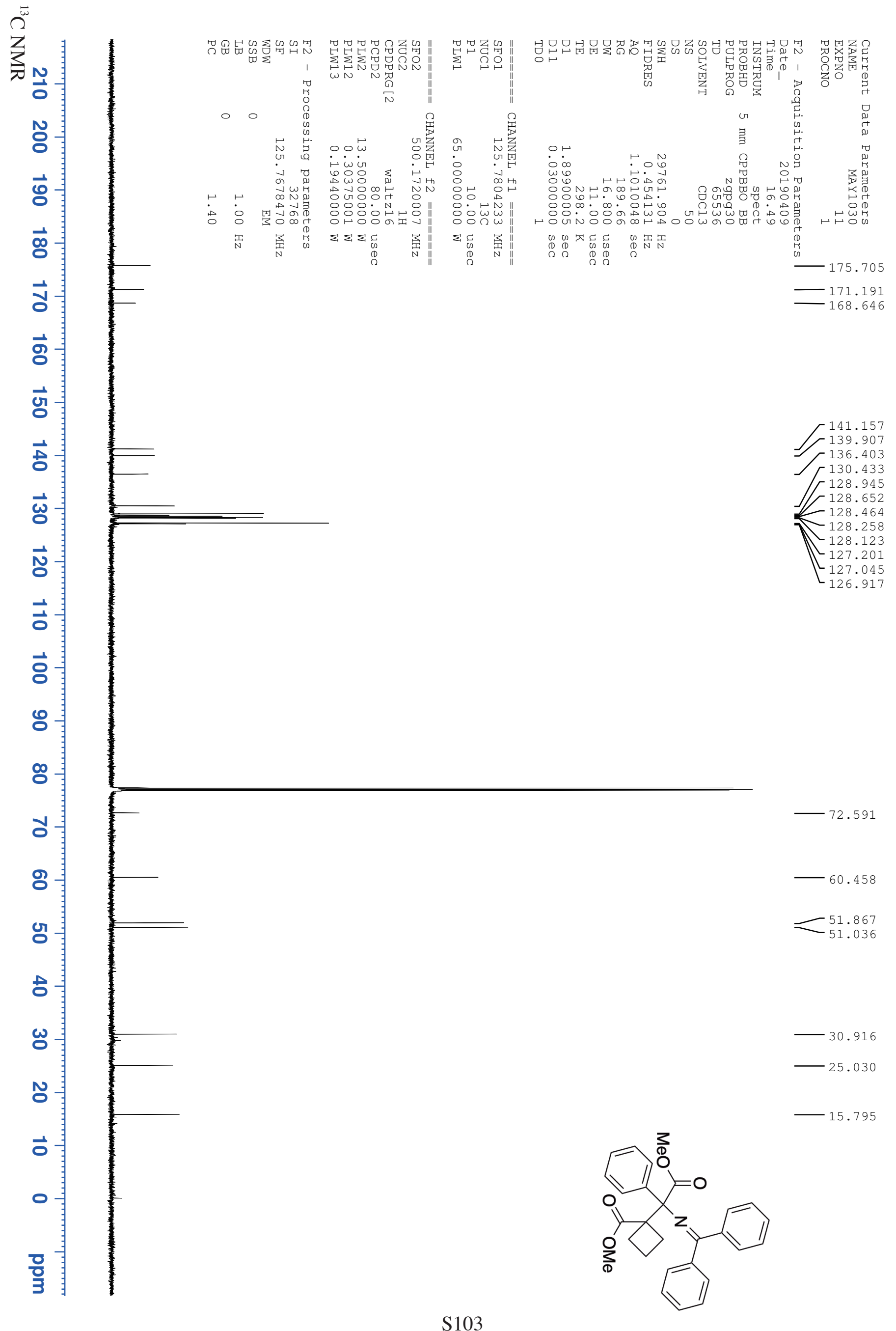




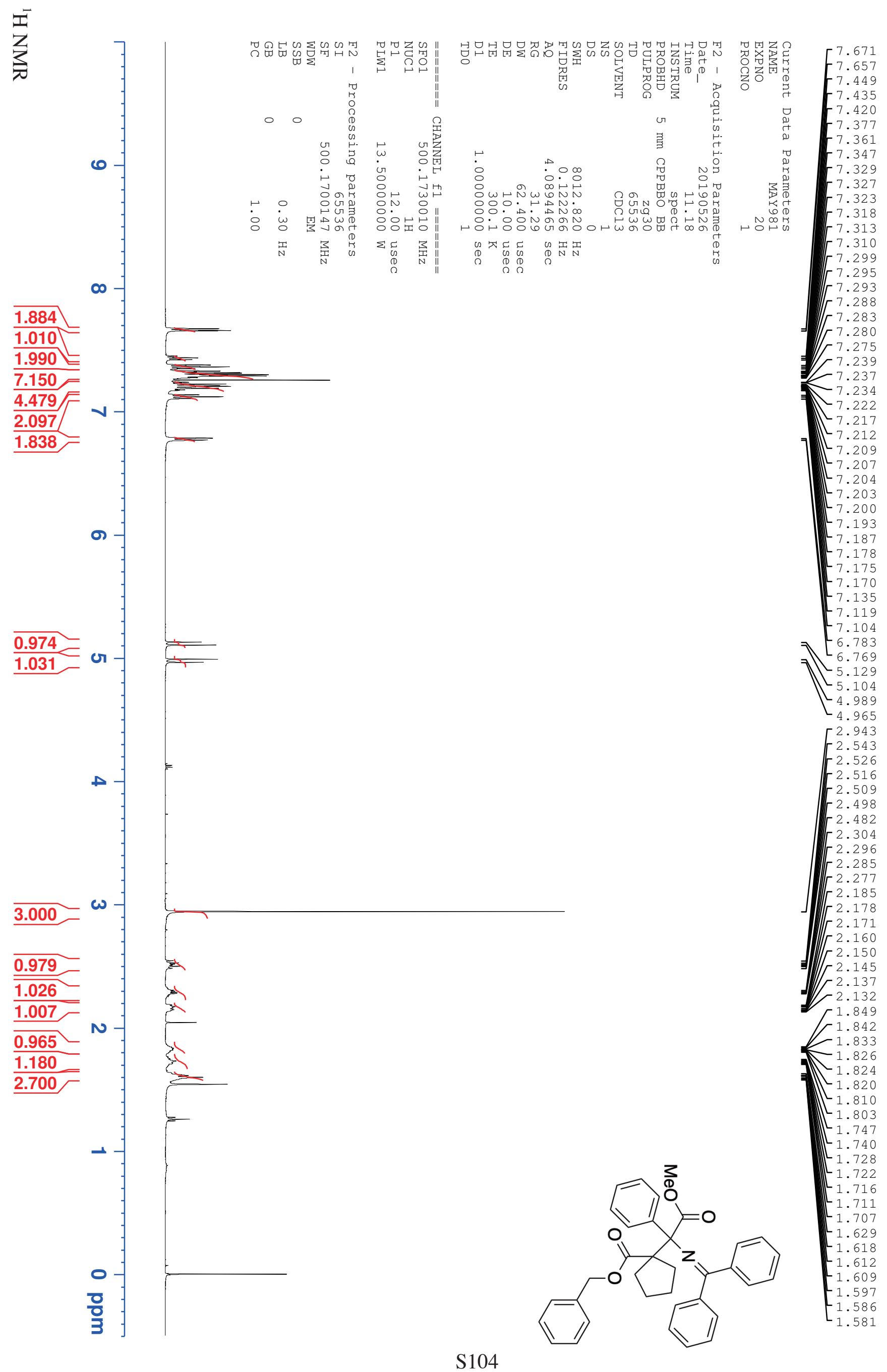




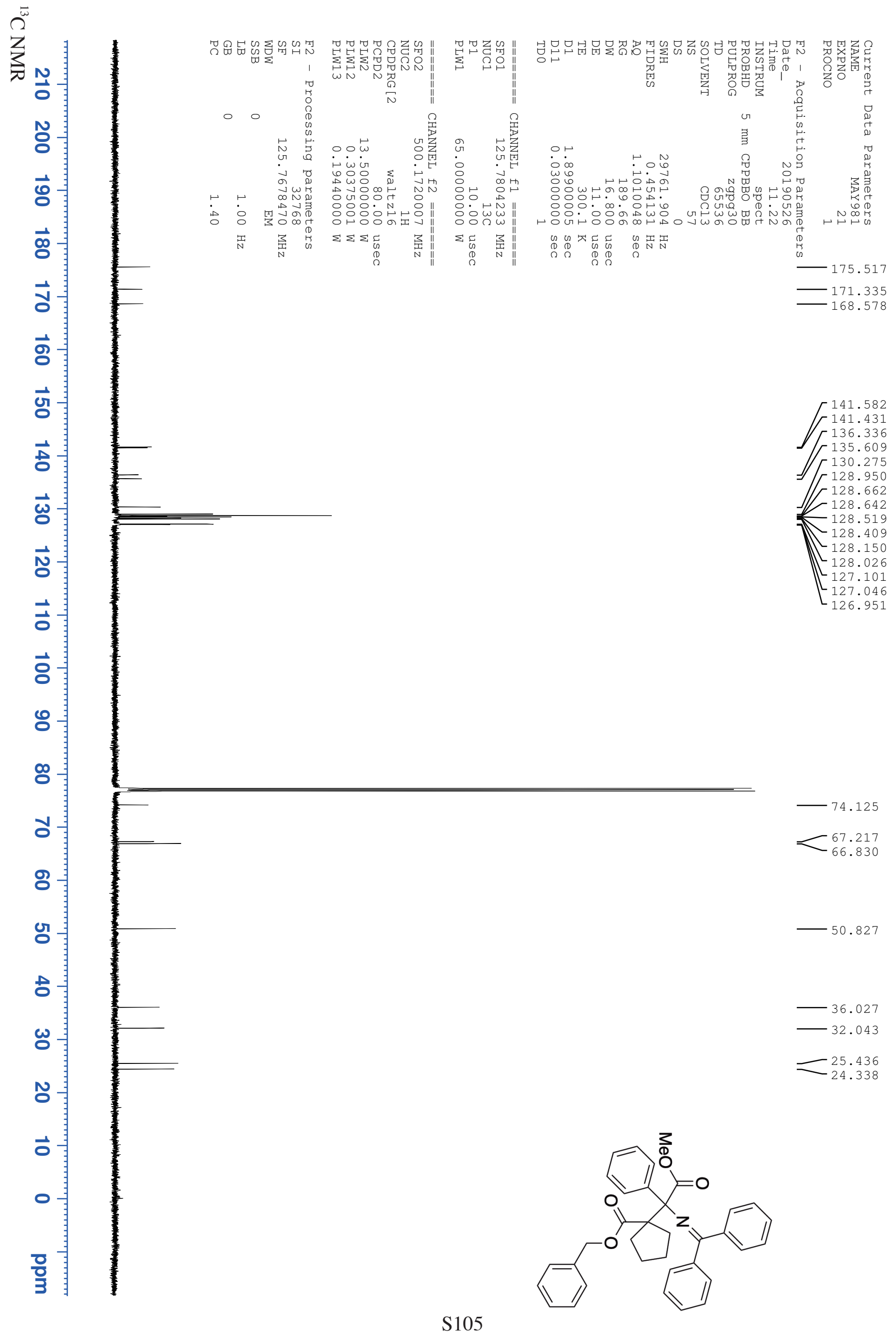




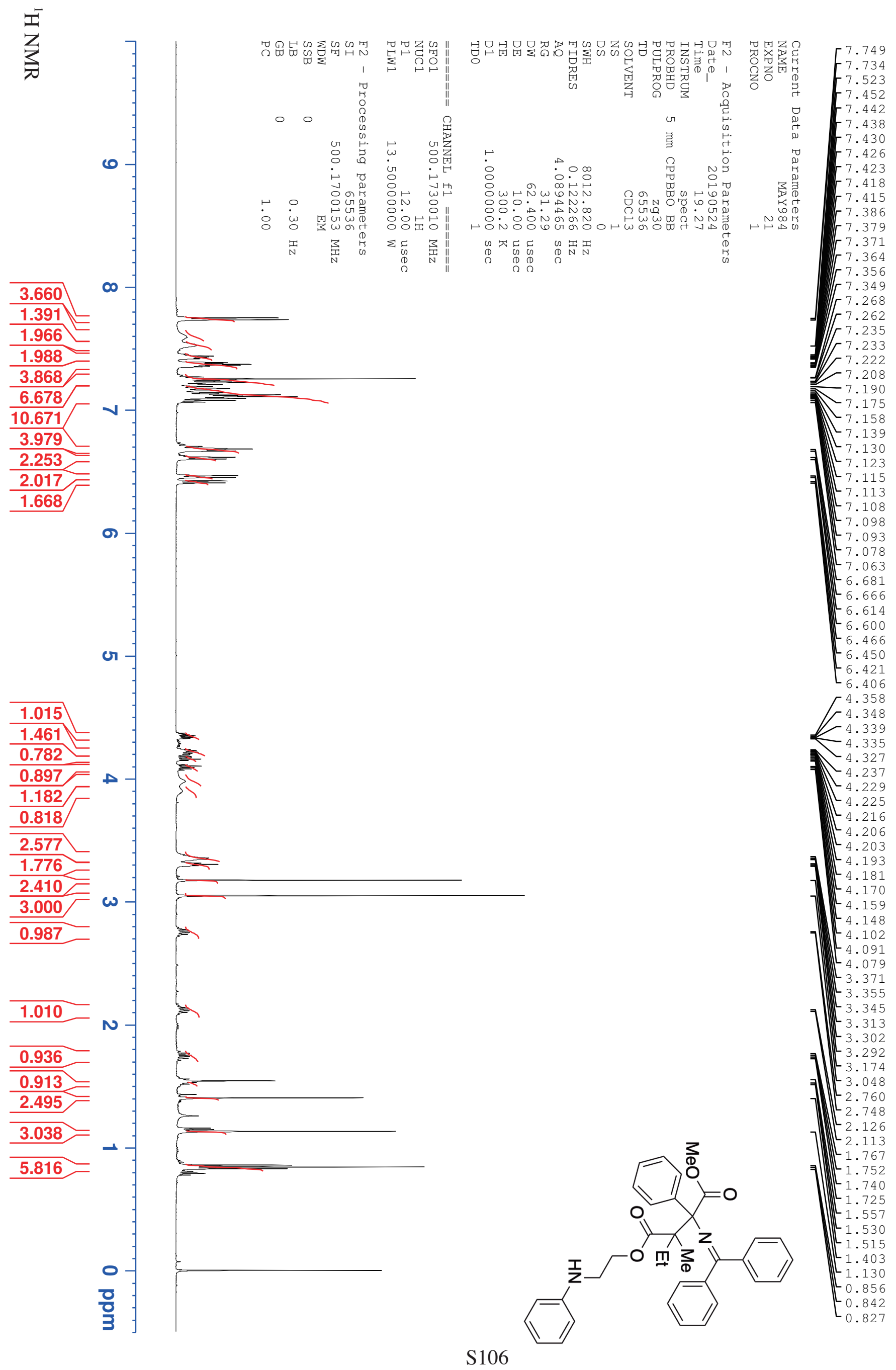




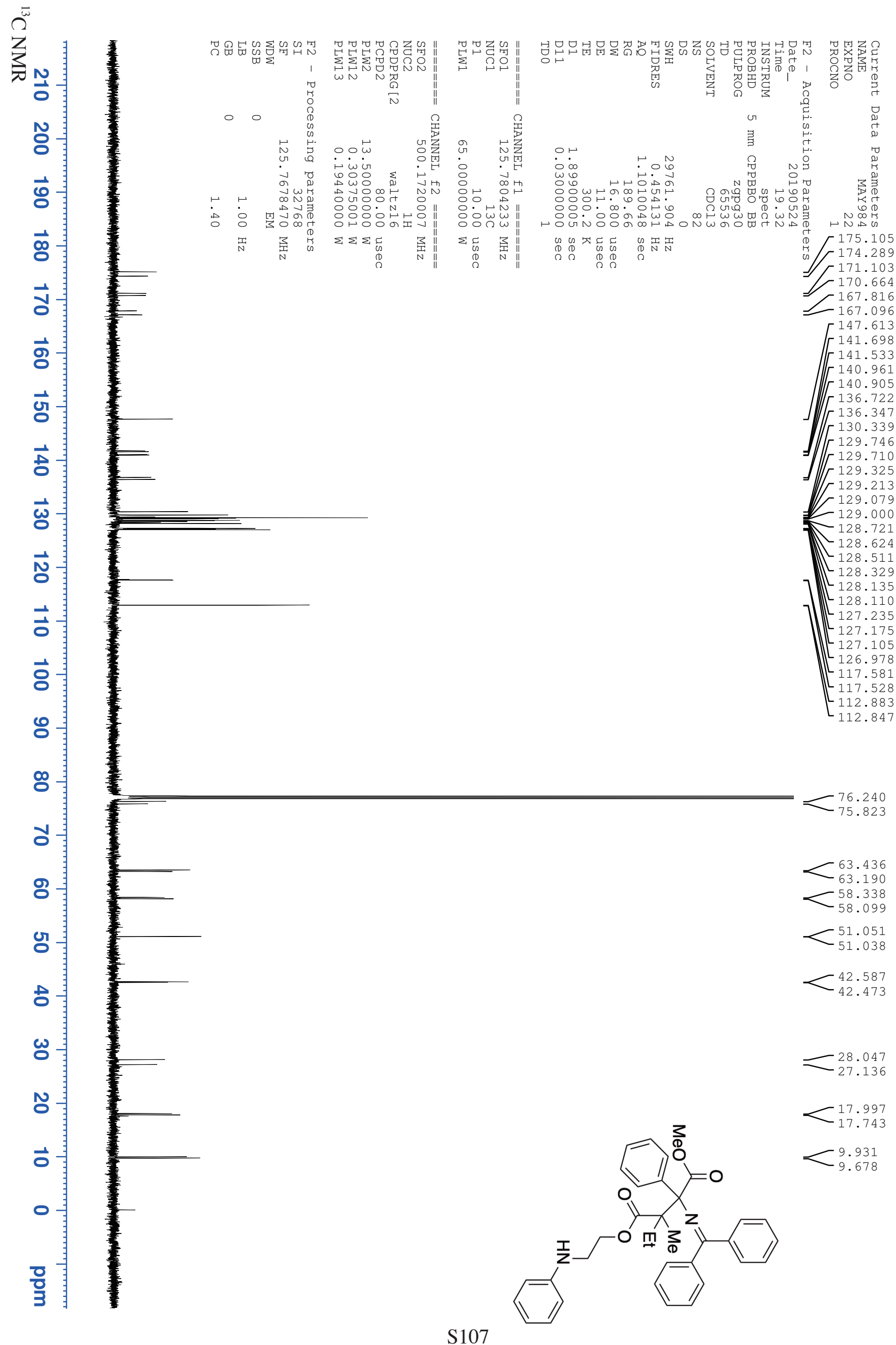




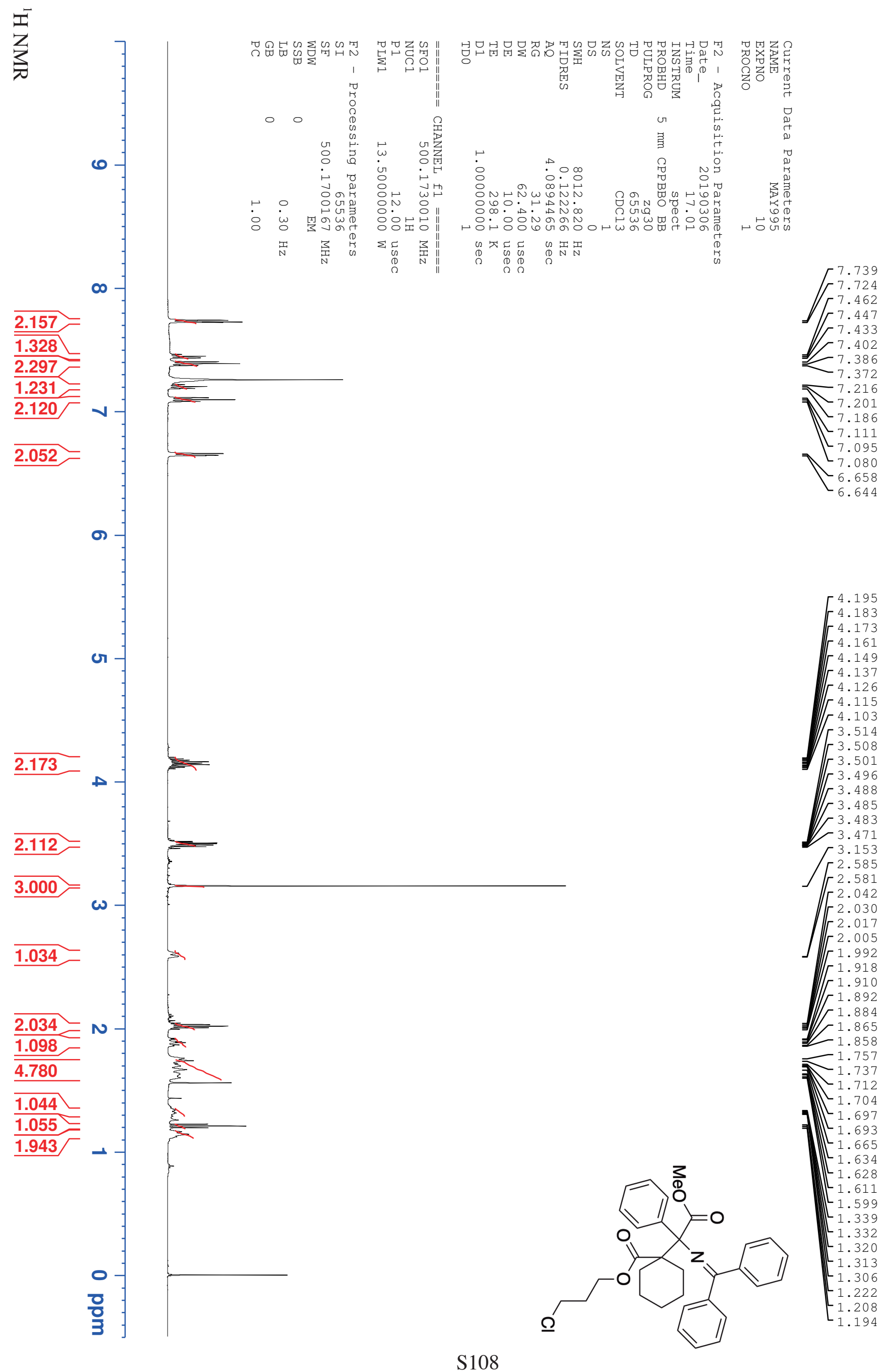




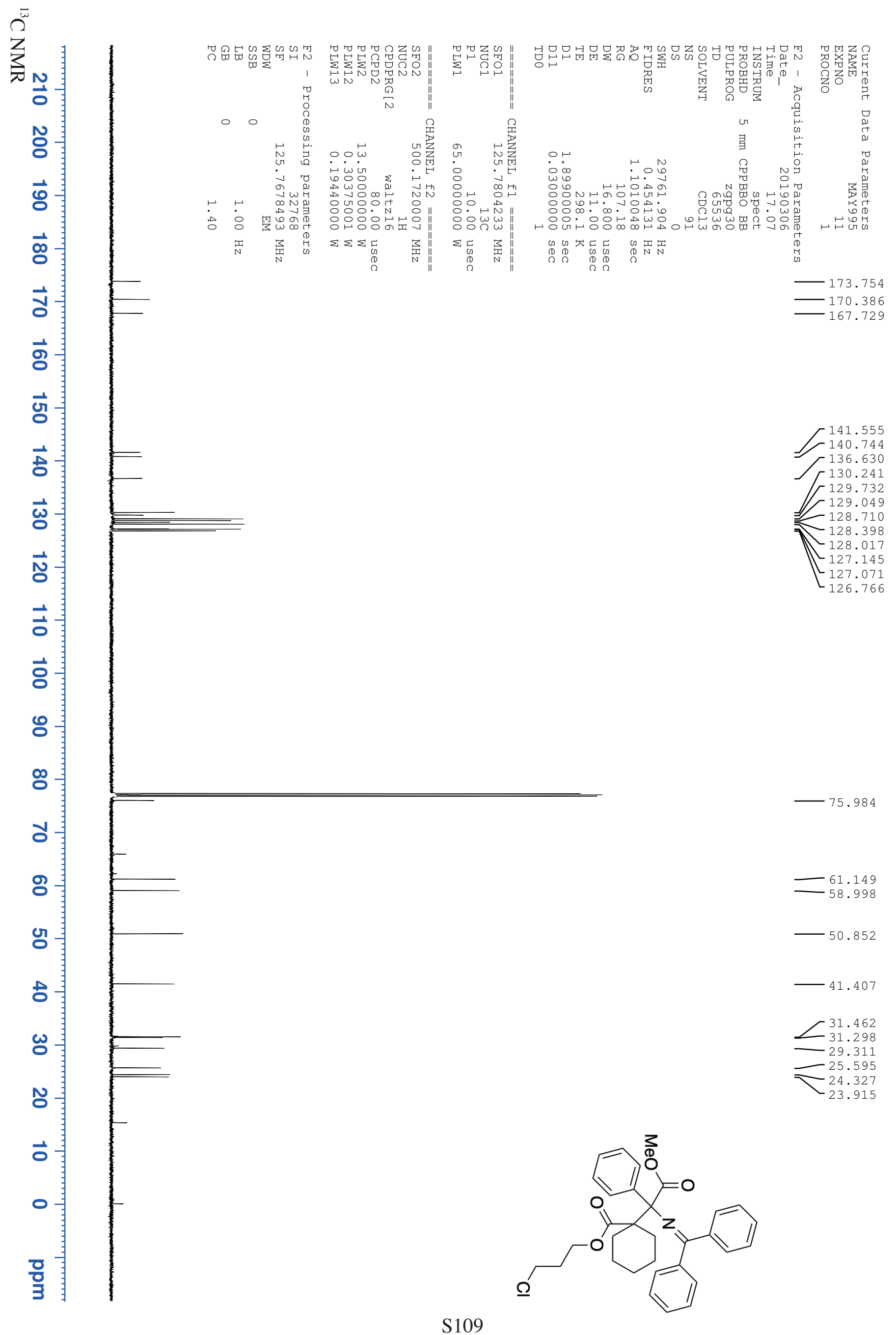




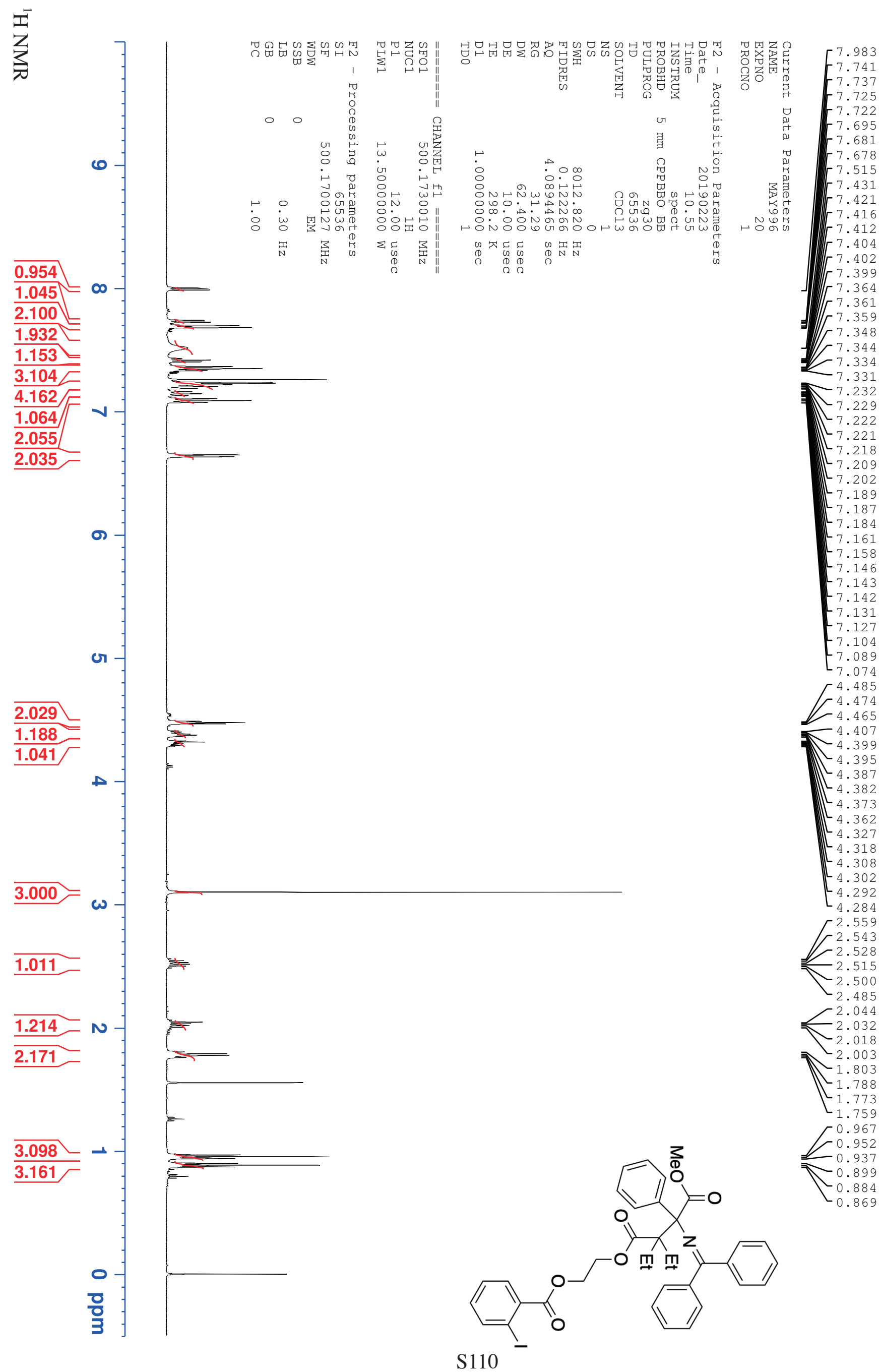




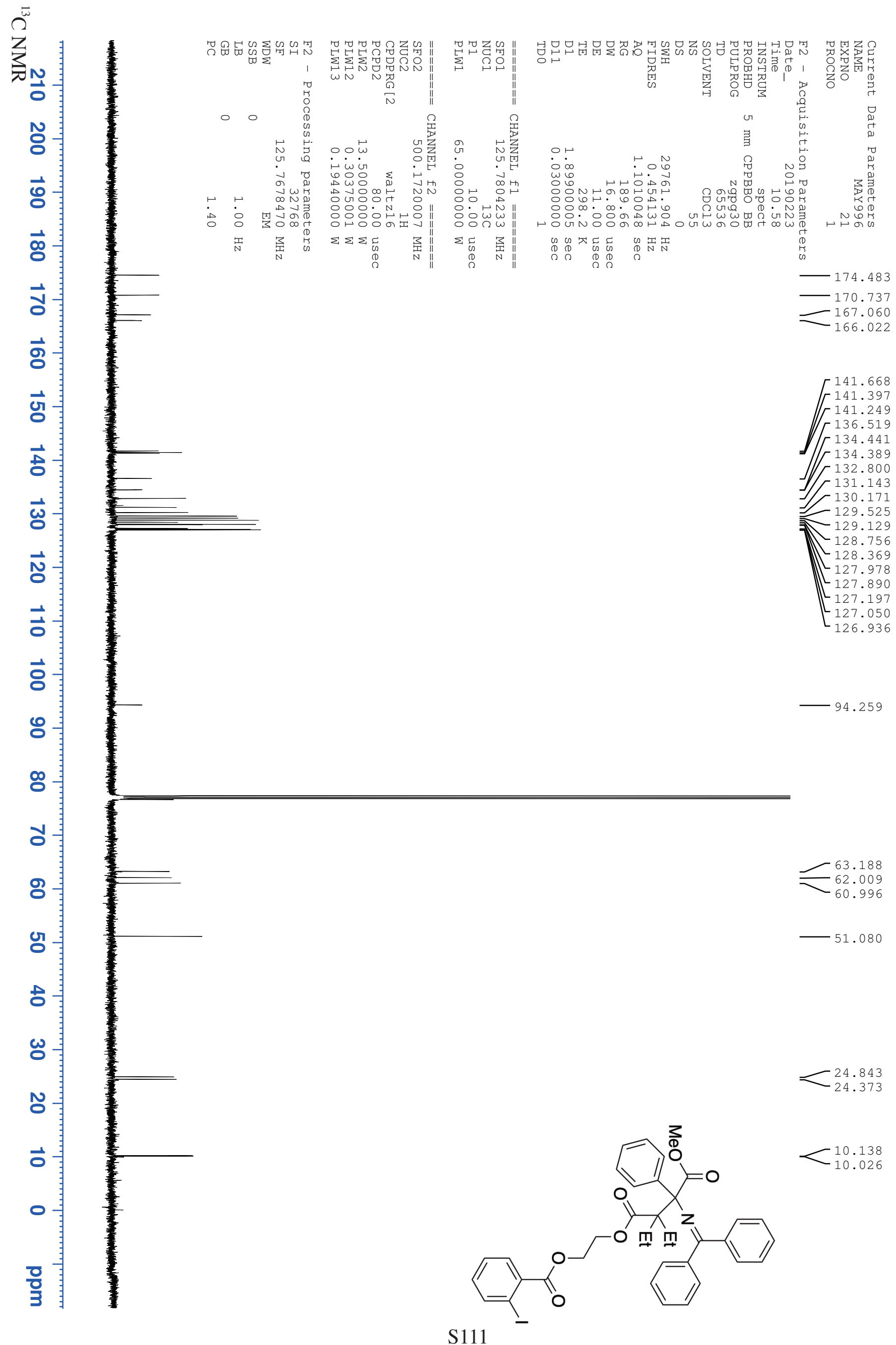



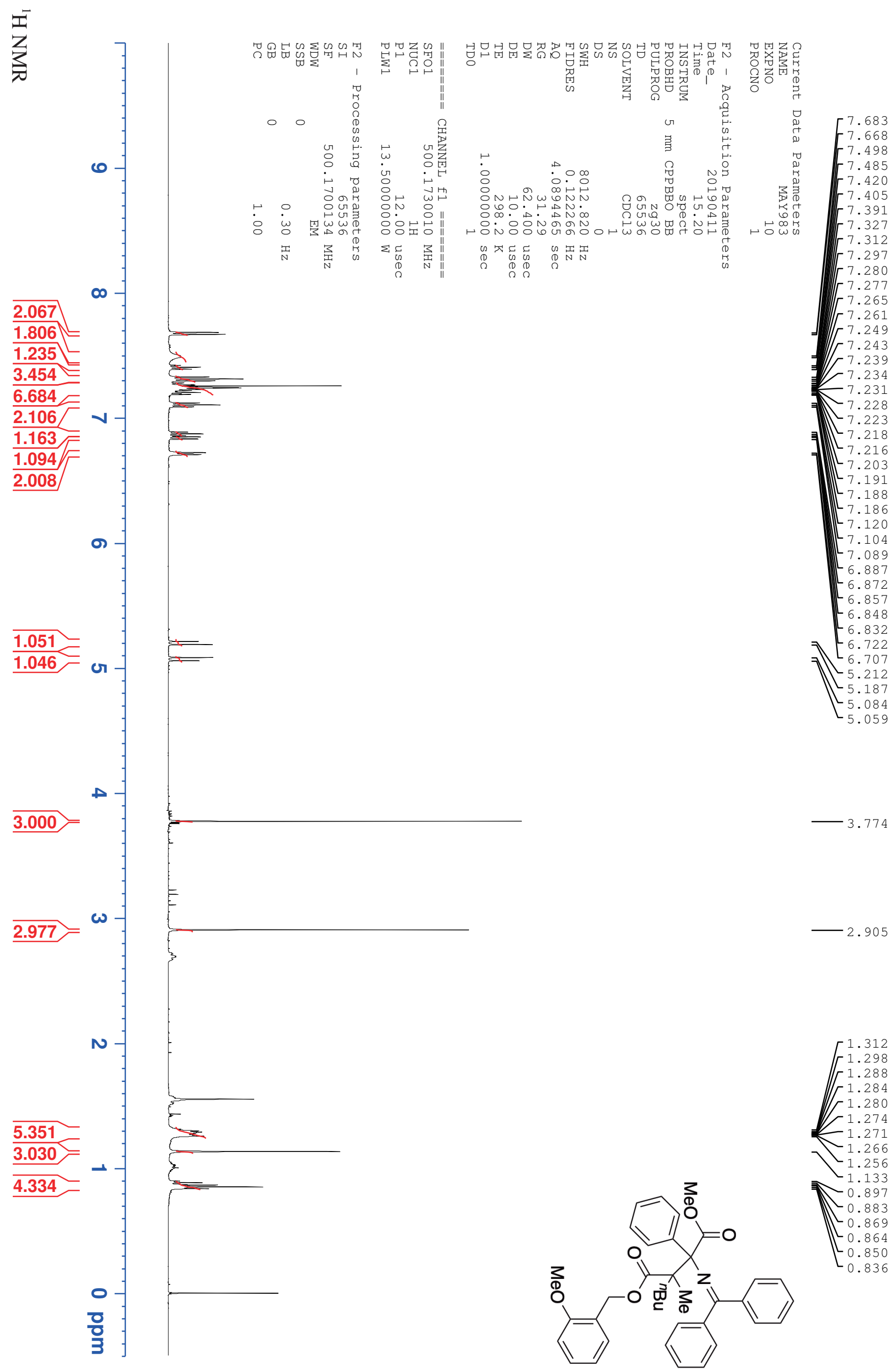


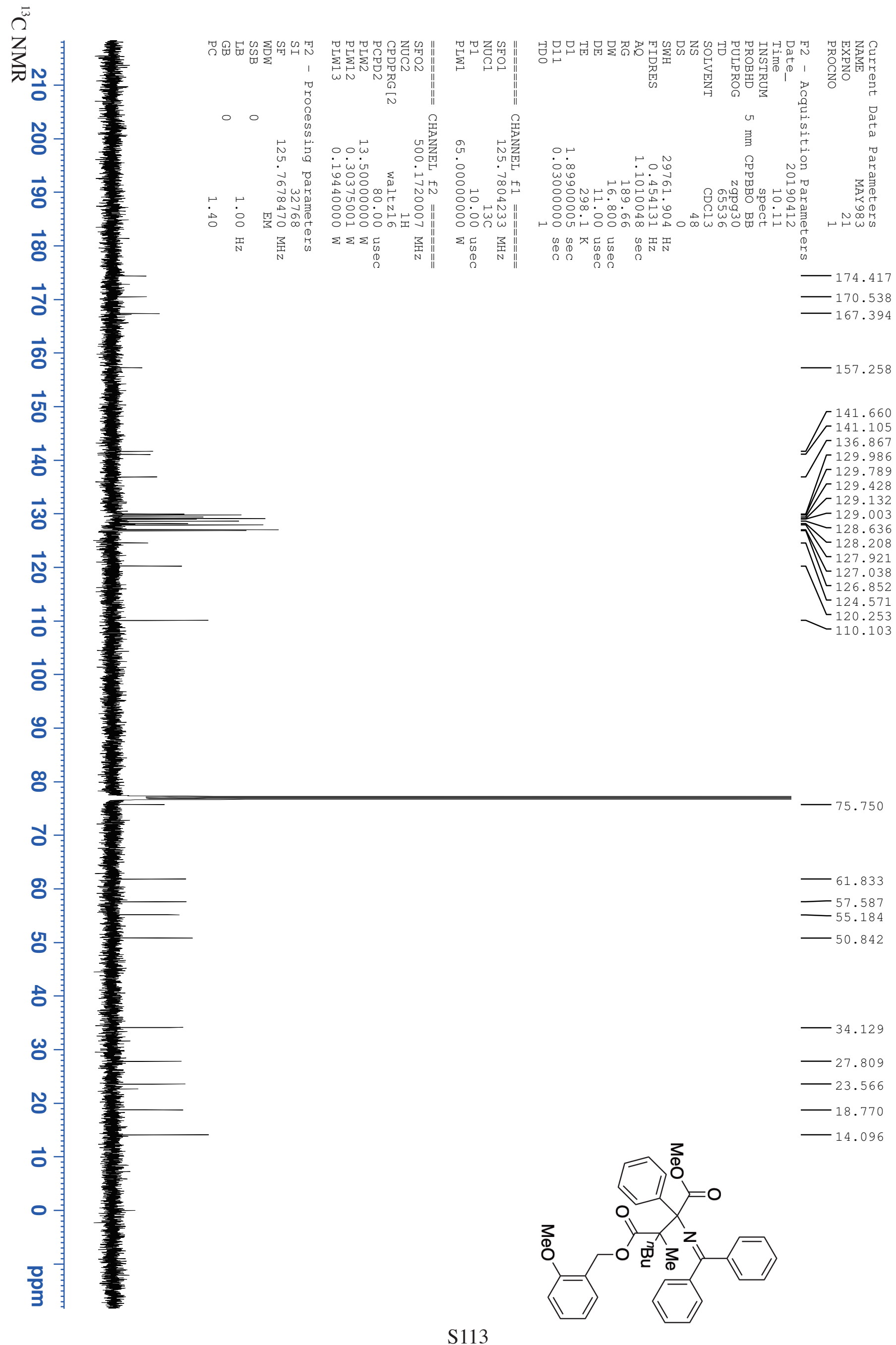




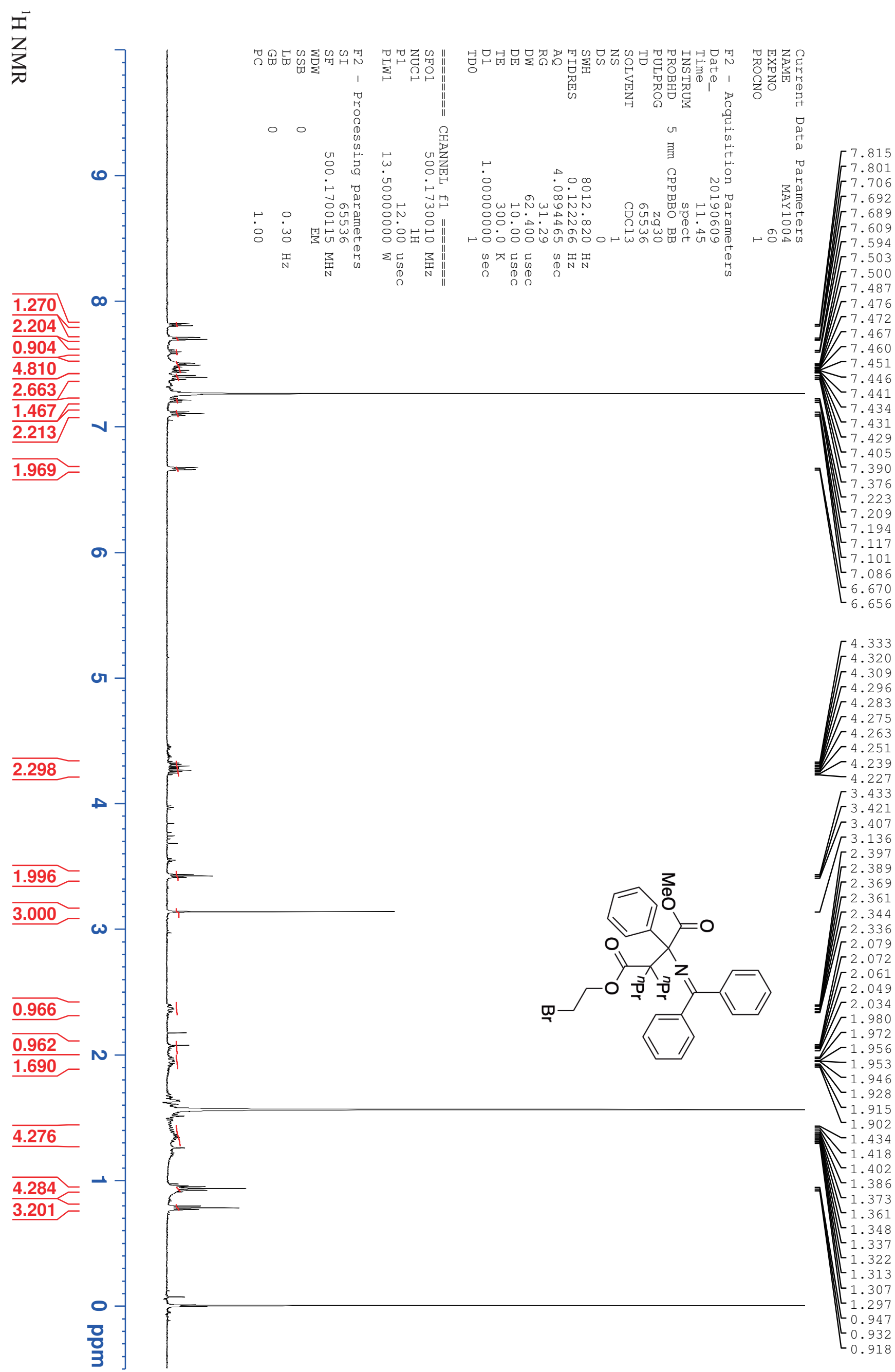




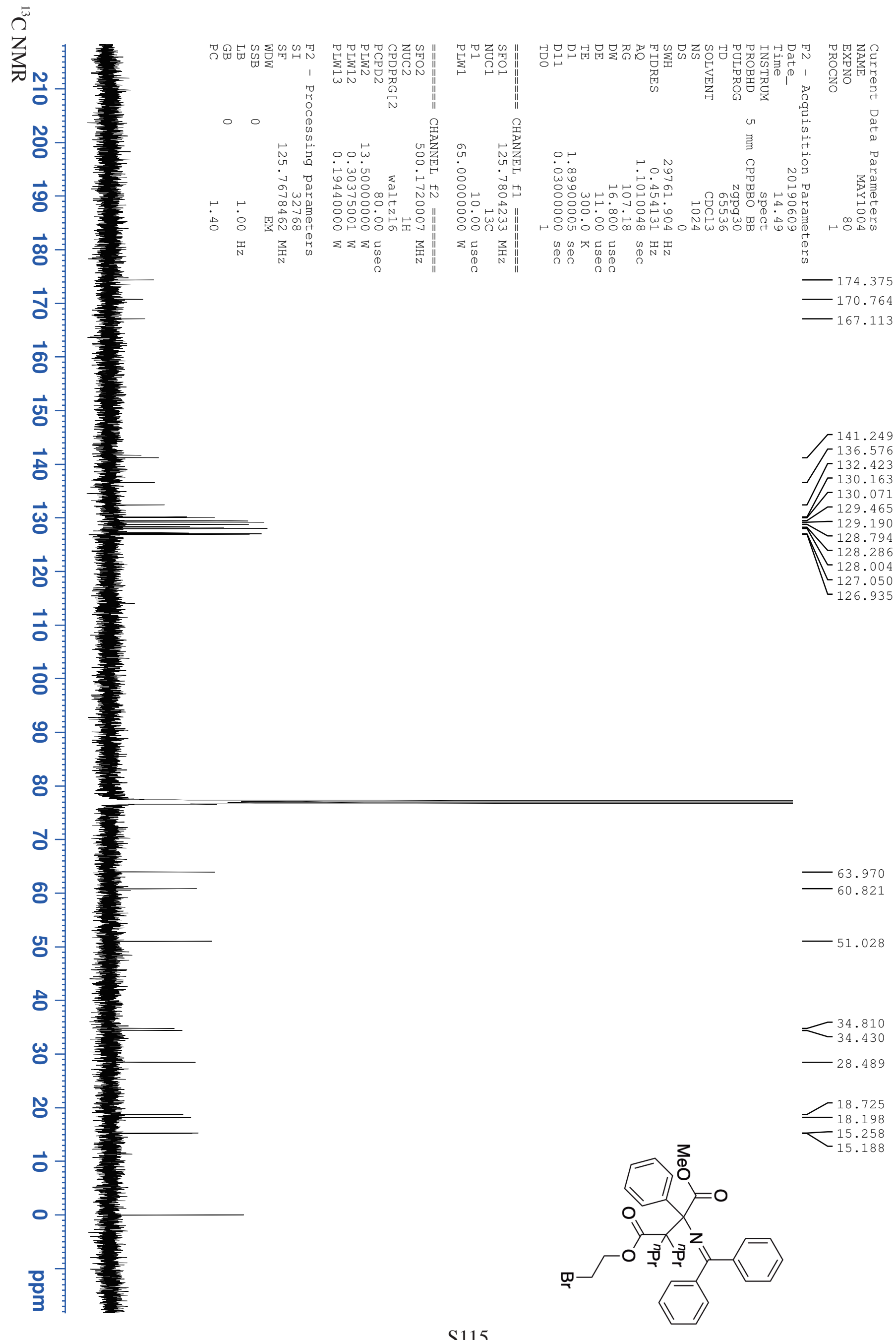




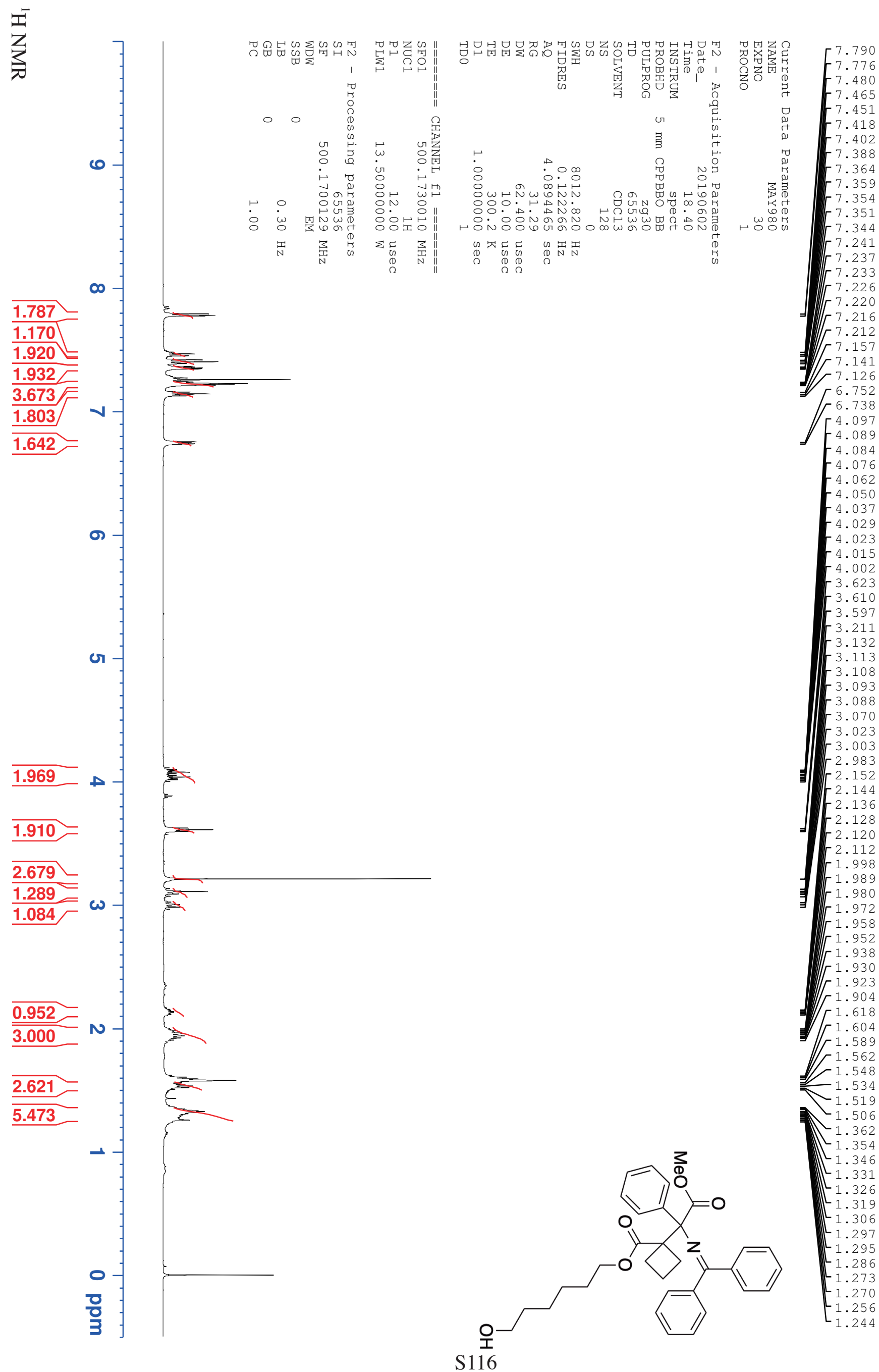




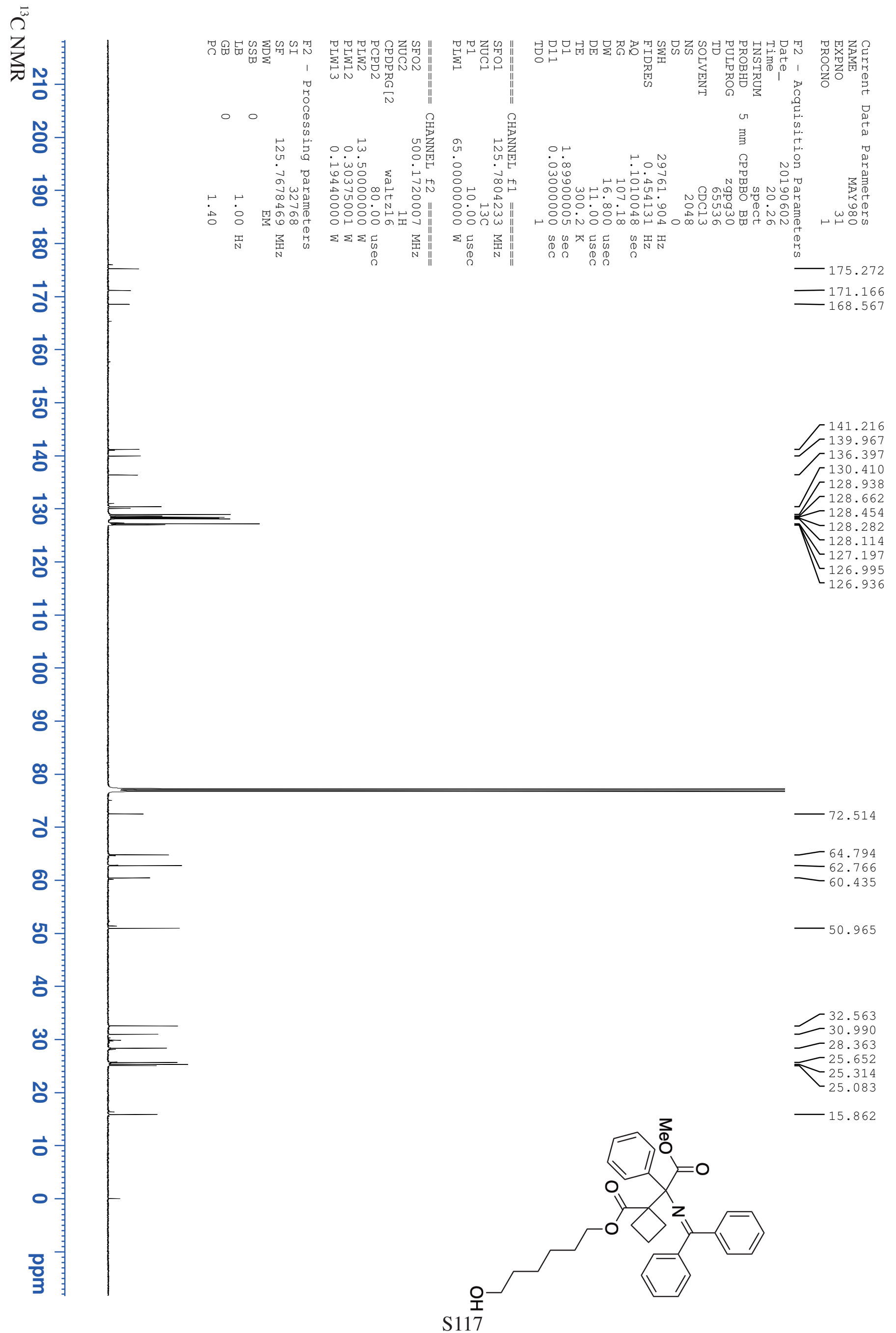




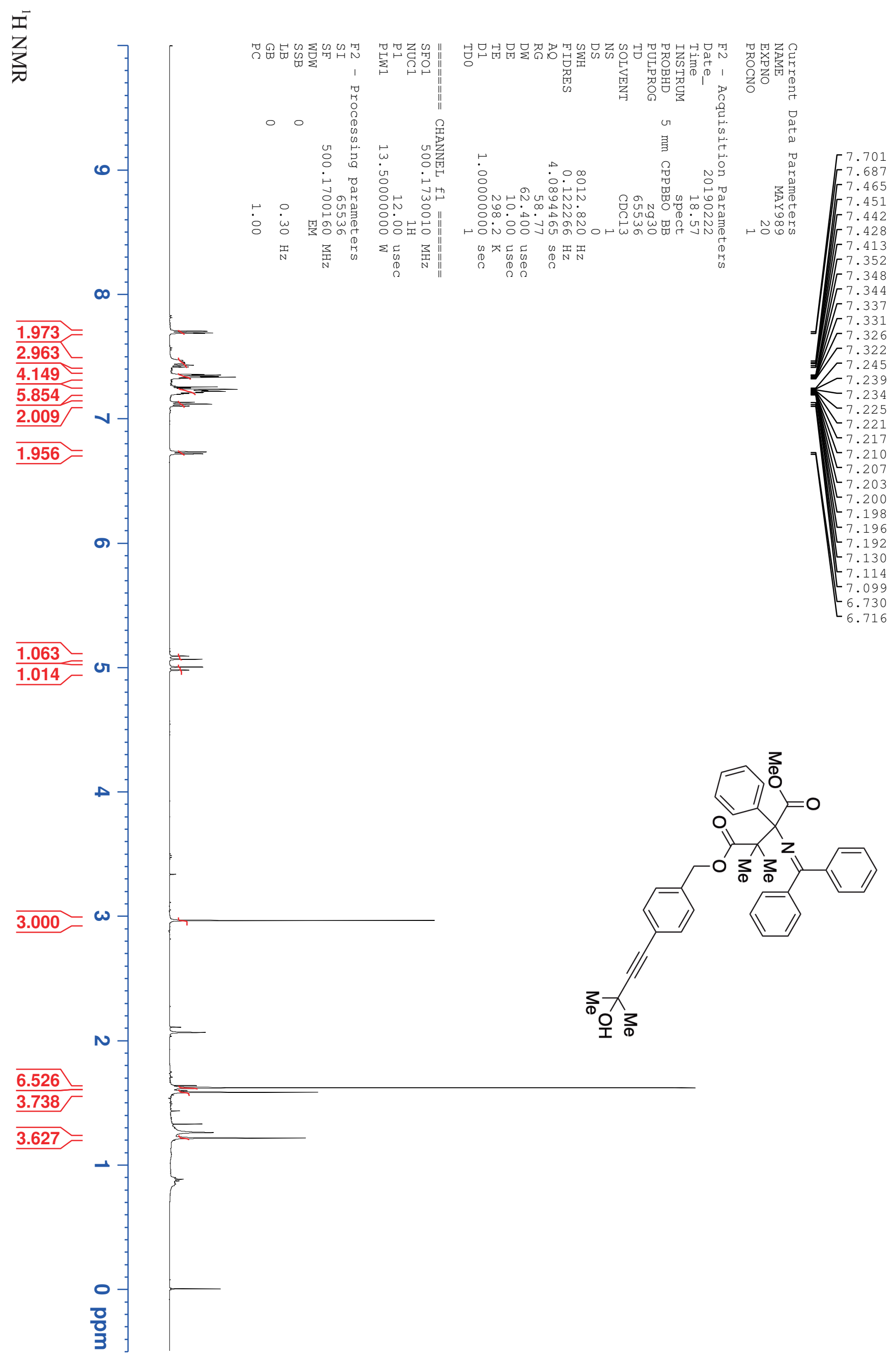




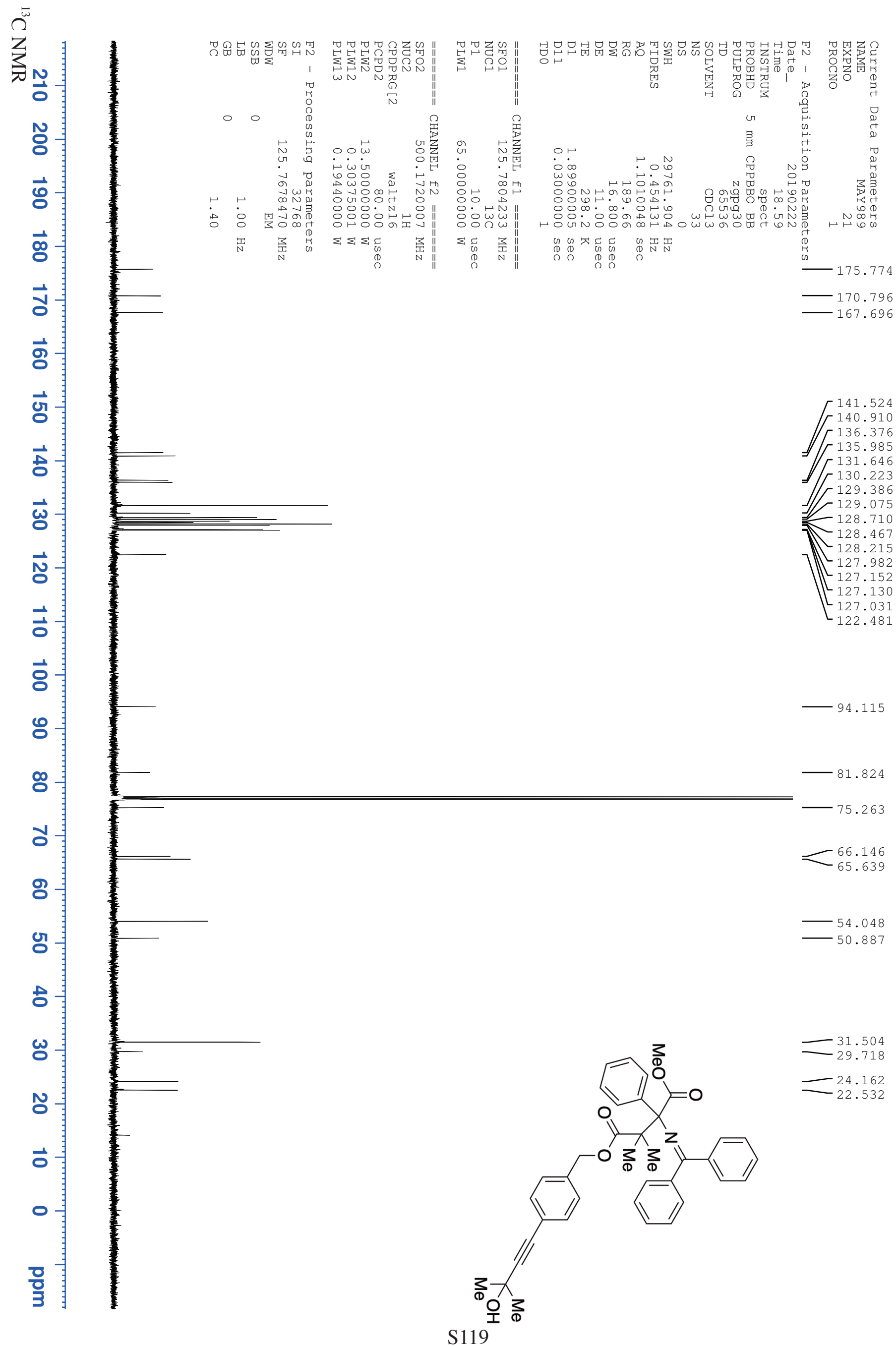




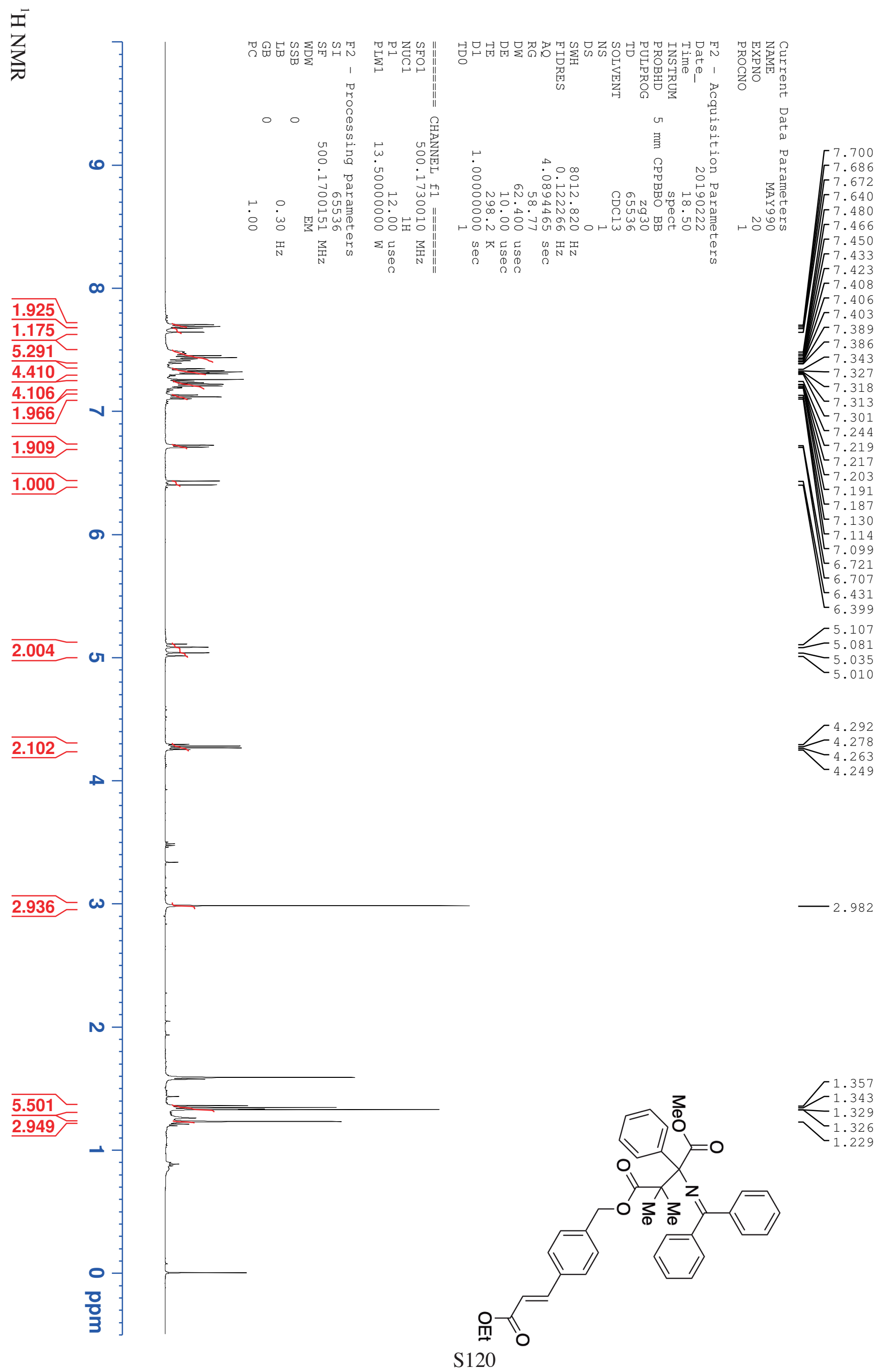




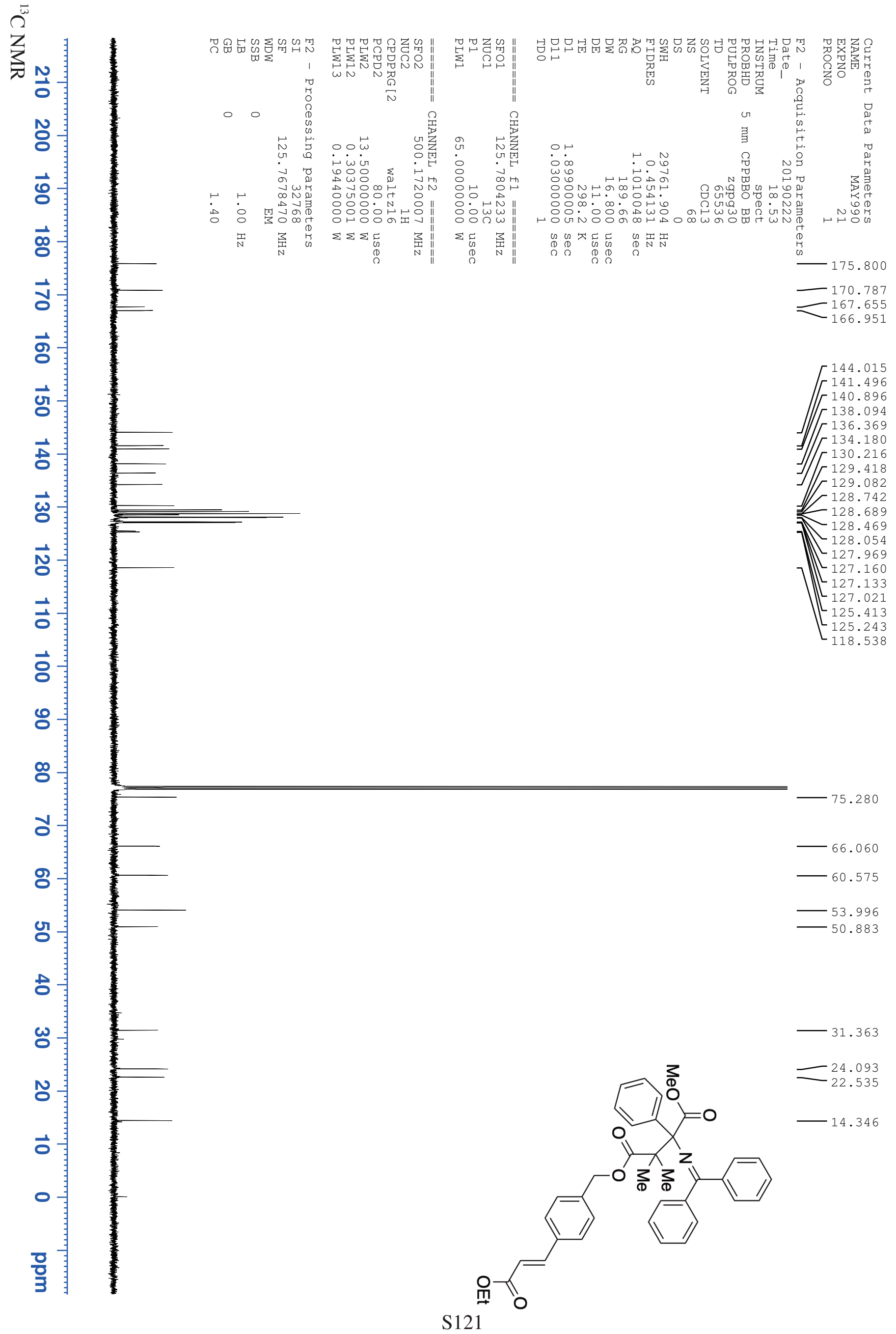




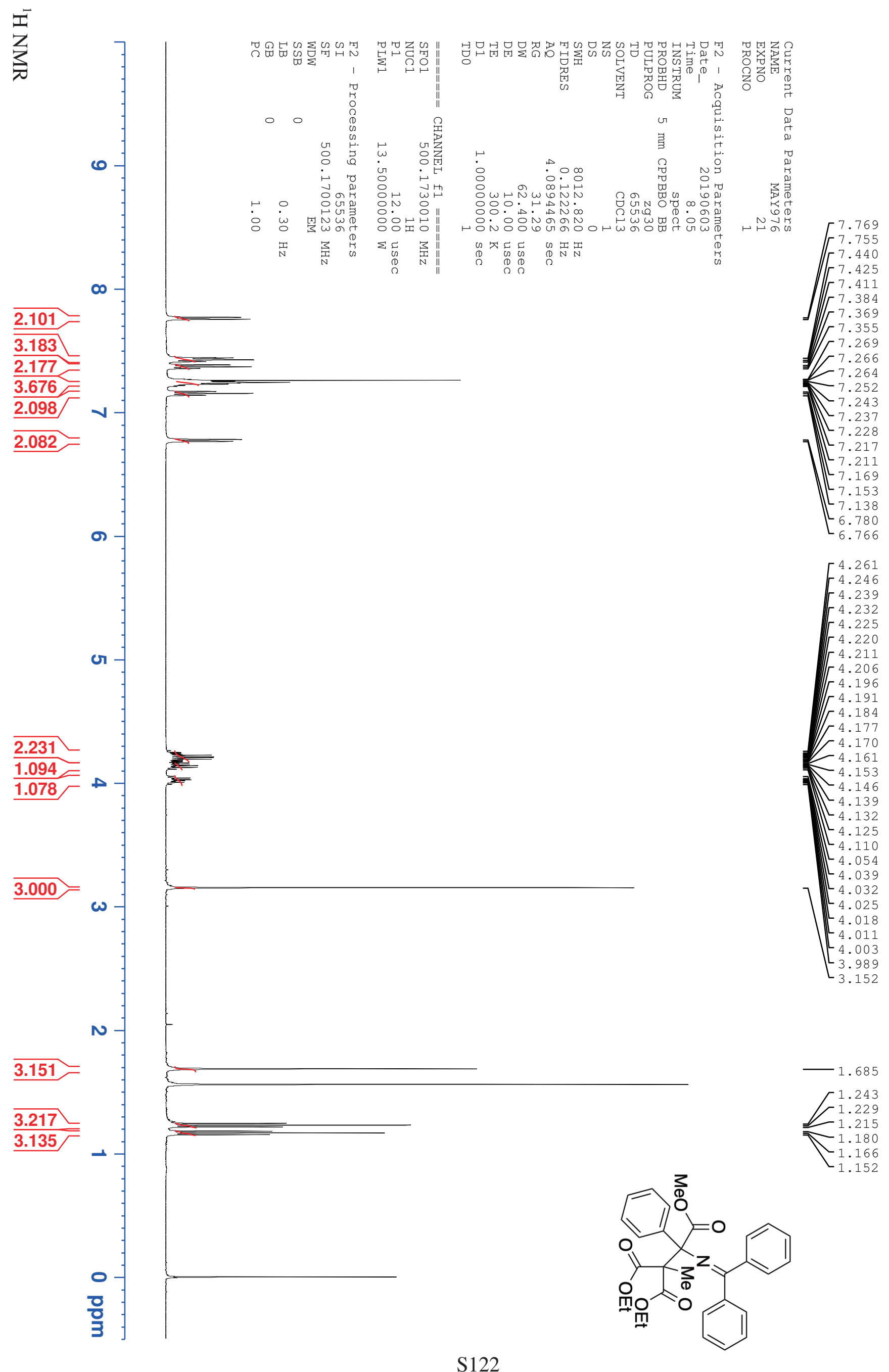




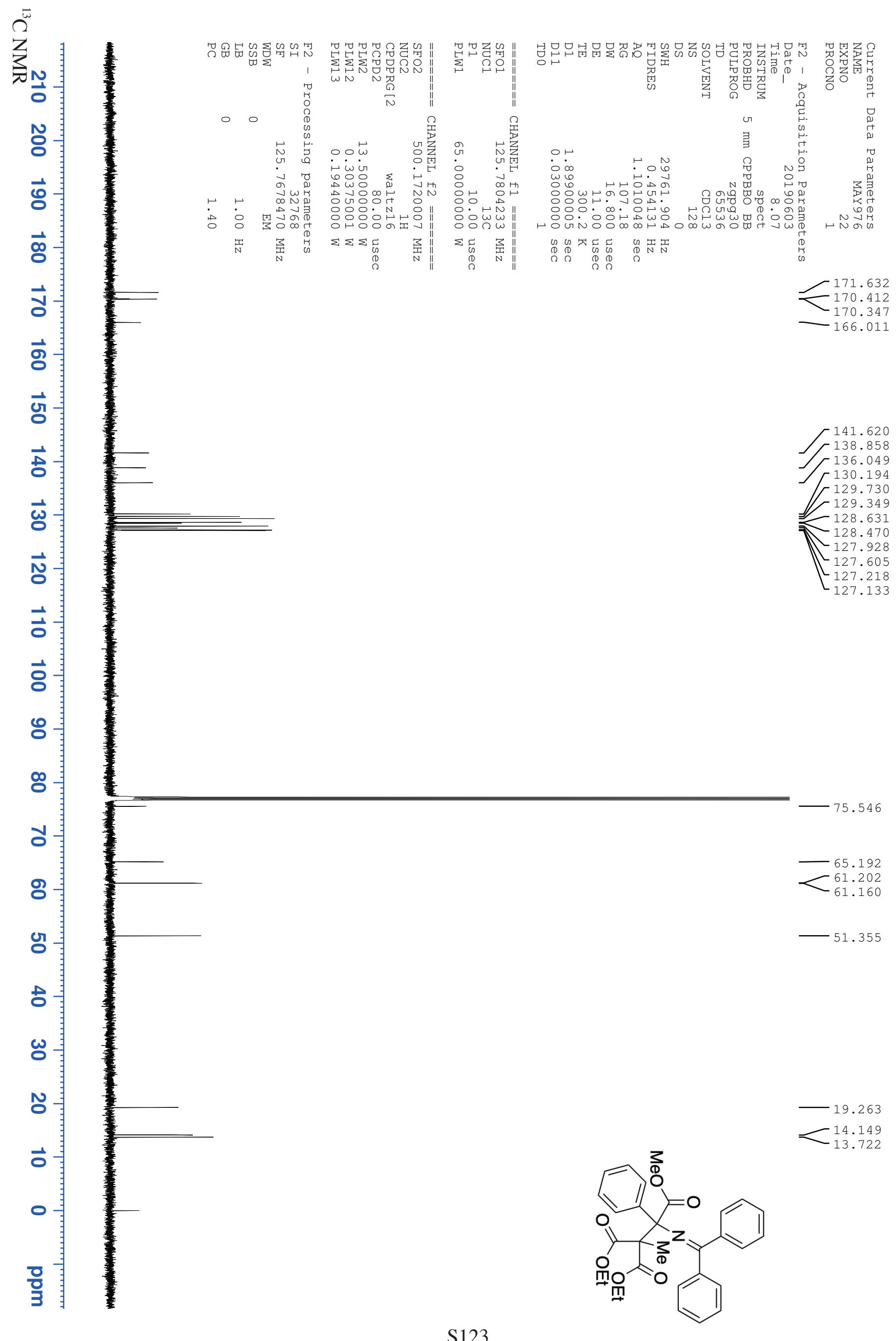




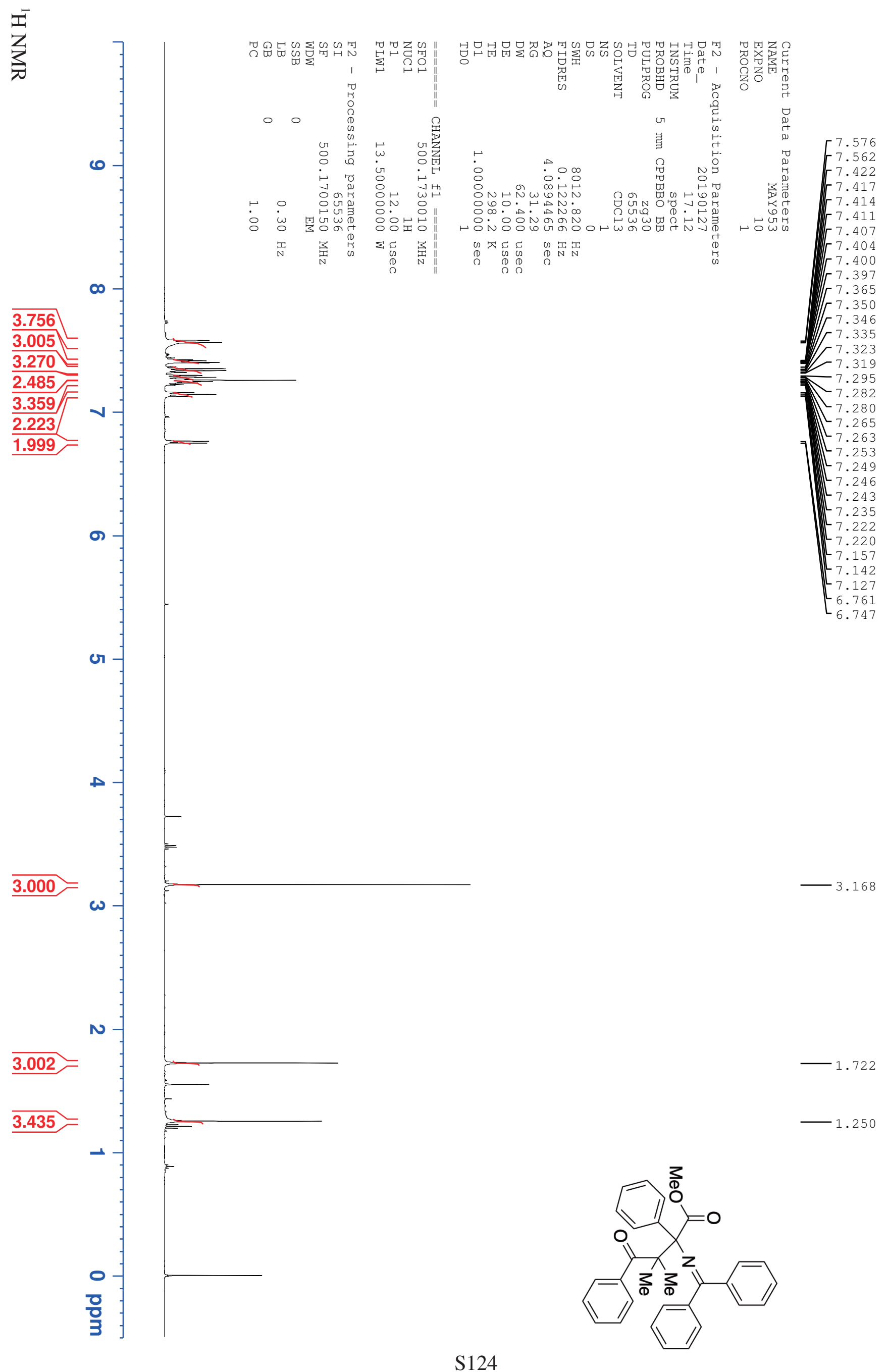




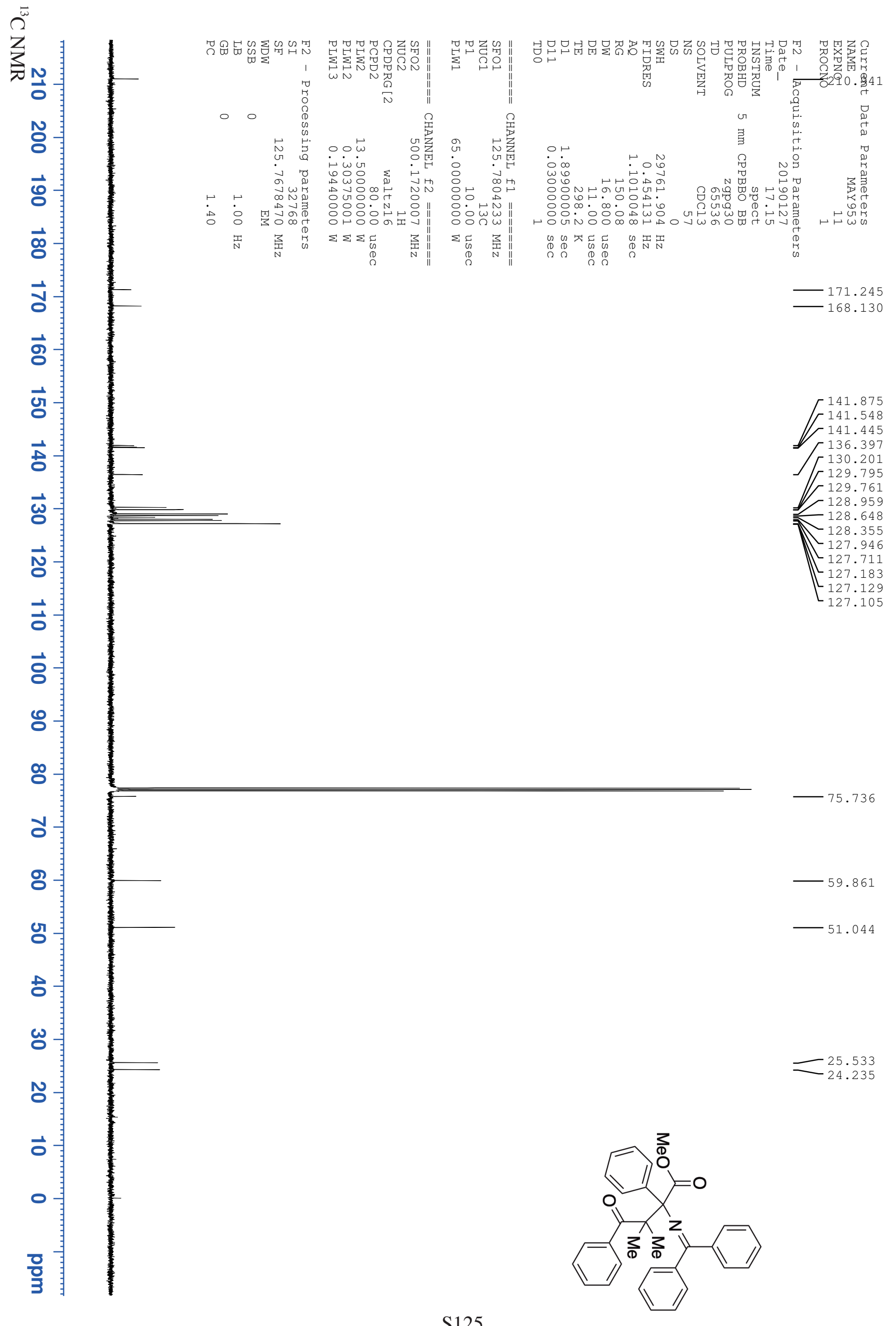




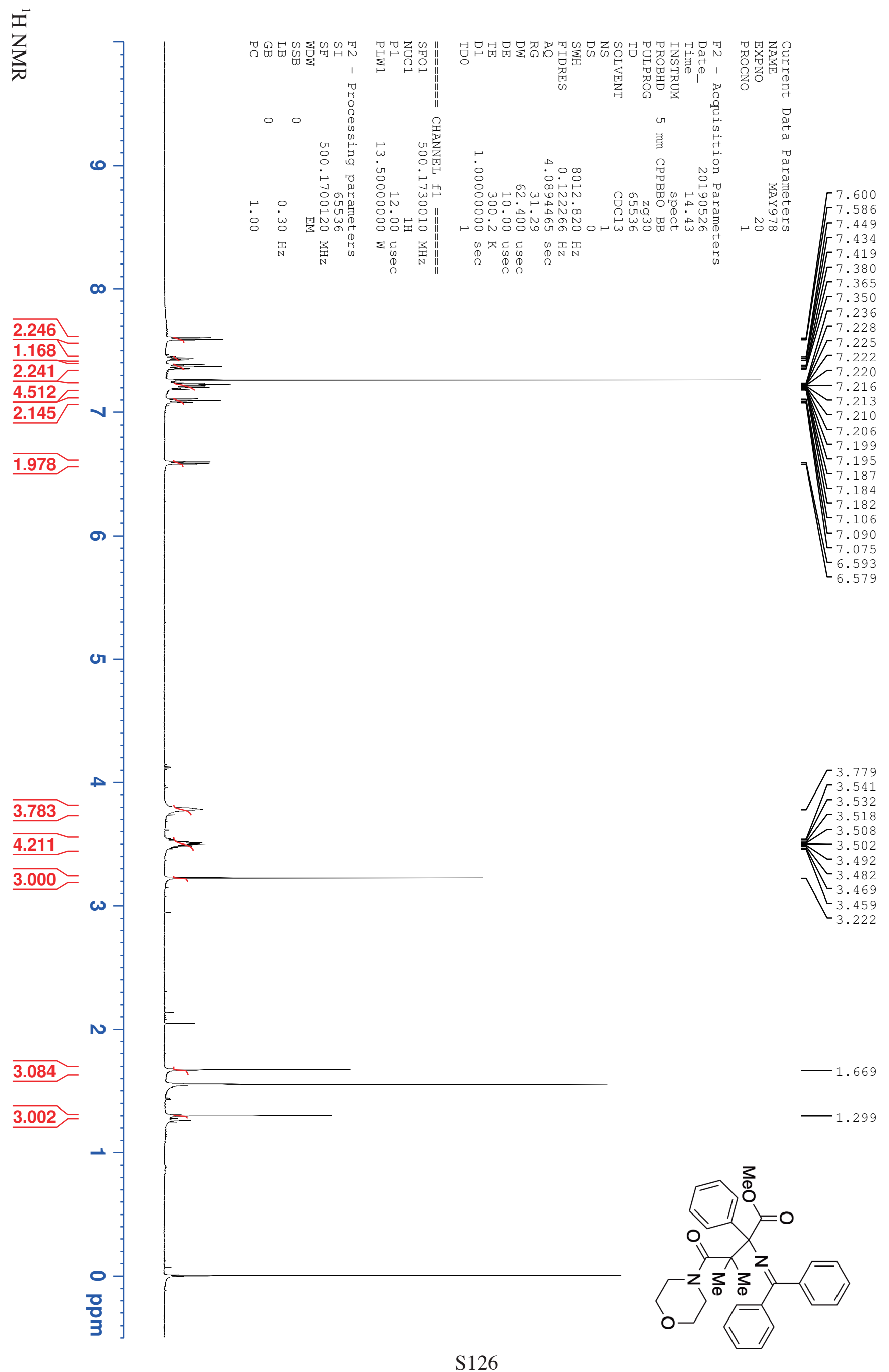




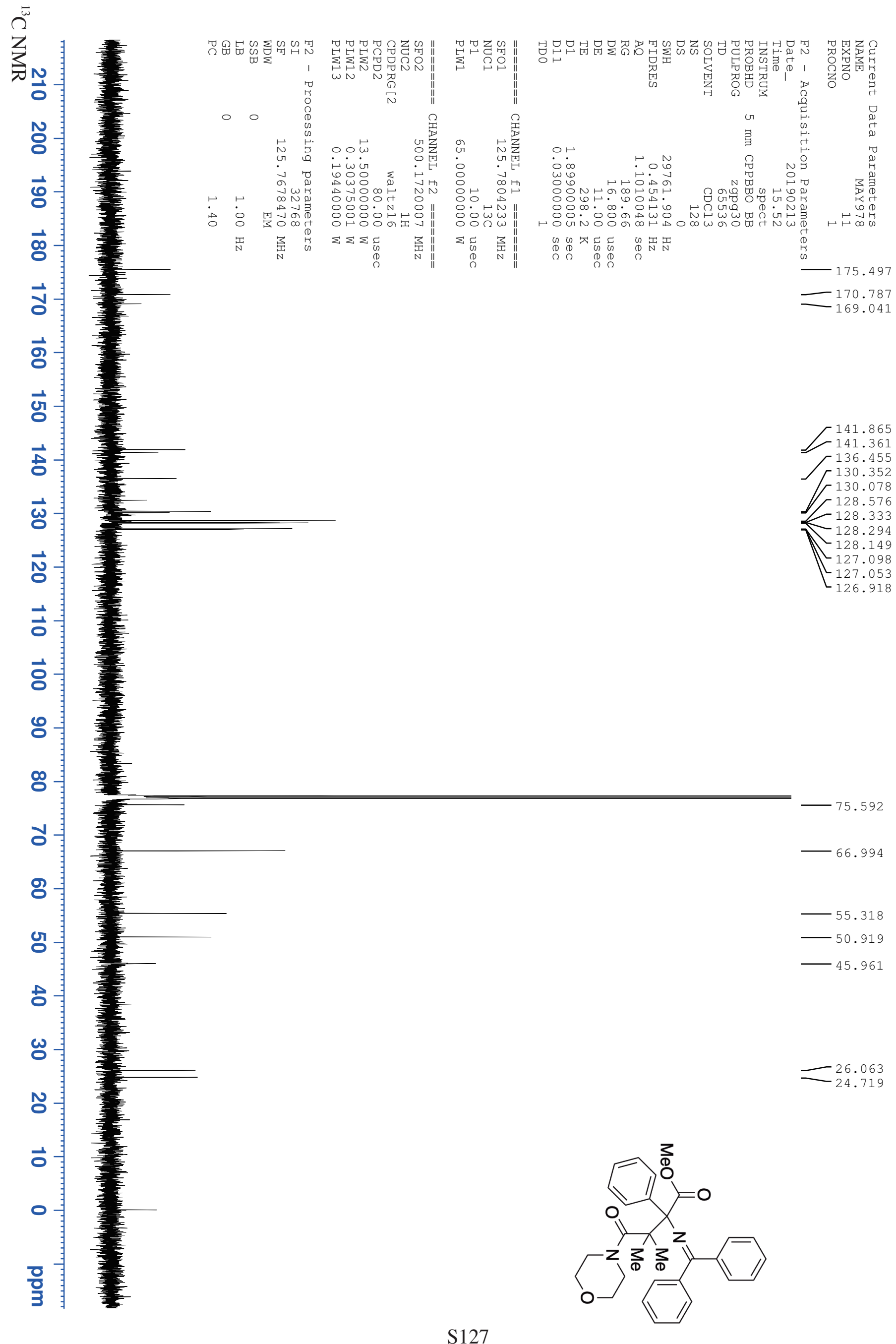




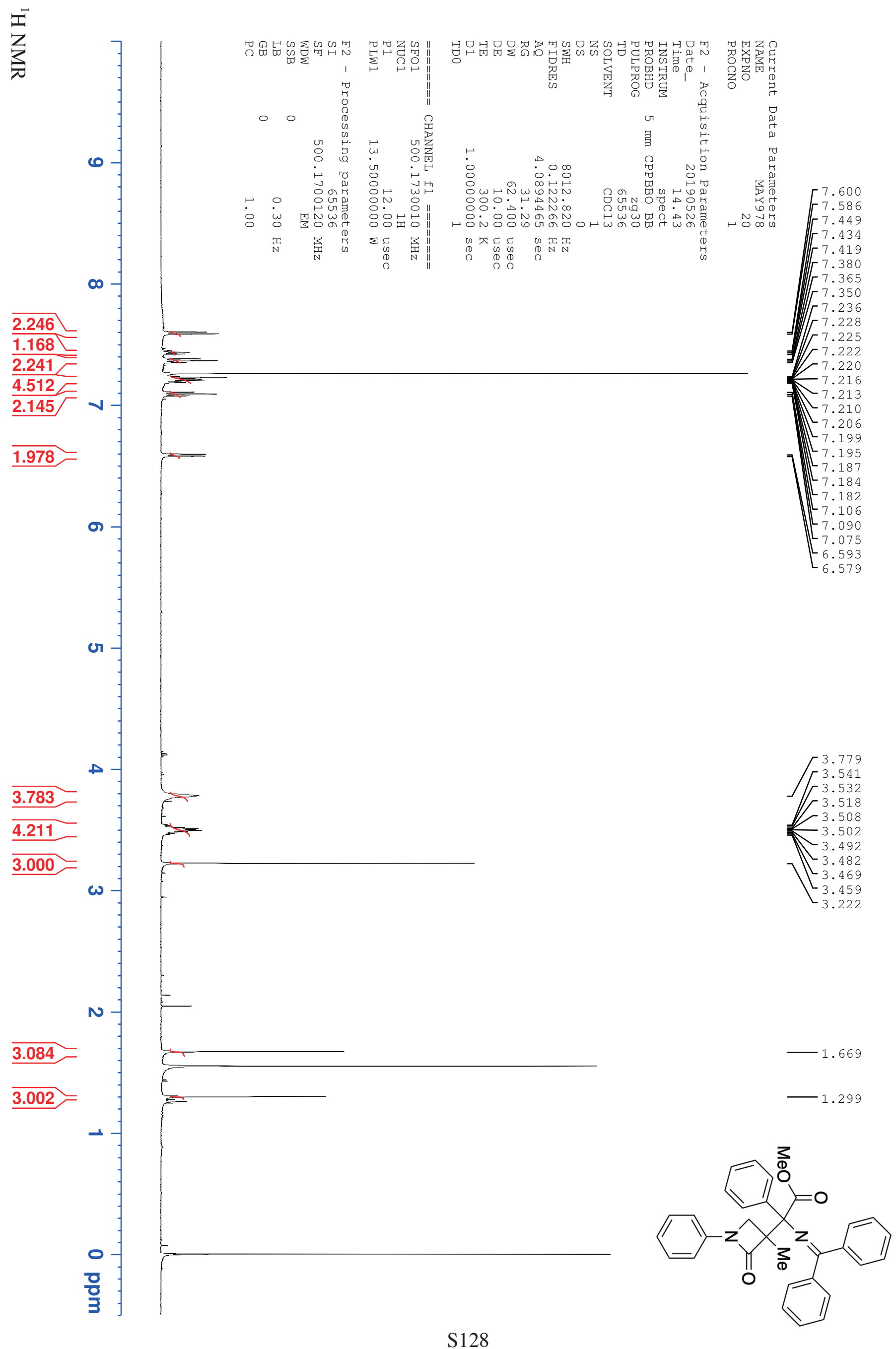




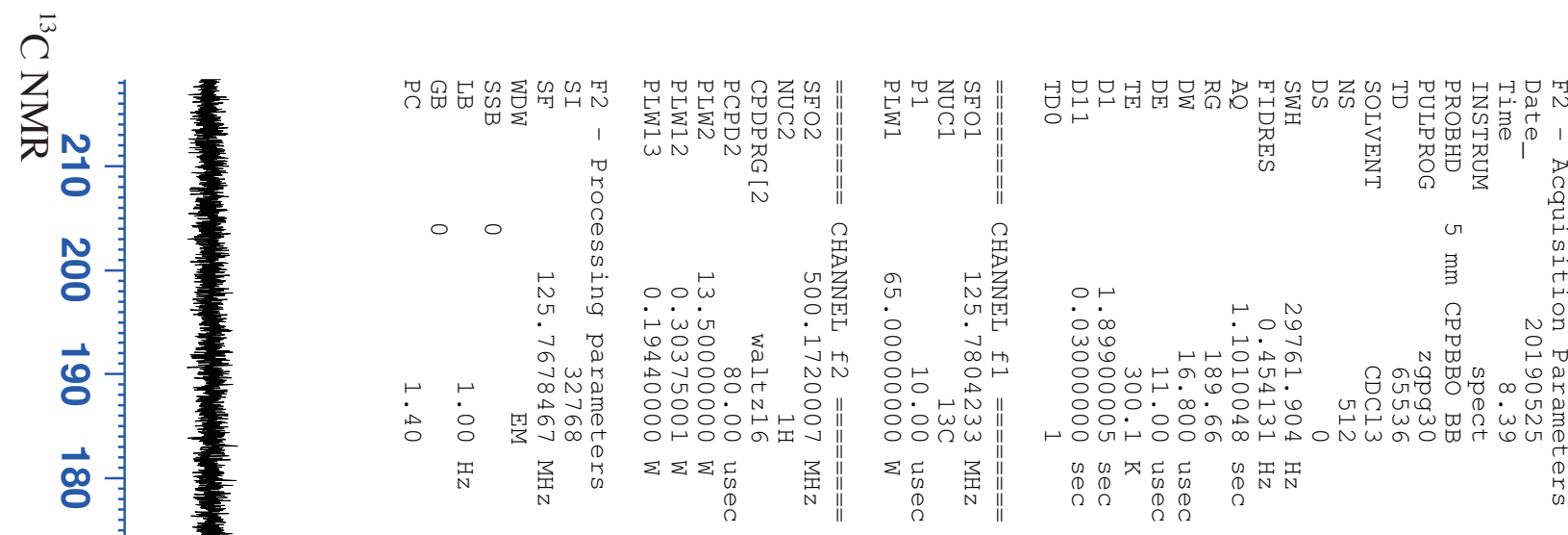

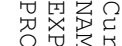

$\overrightarrow{0}$

홍

o

\&

ปั

8

o

웅

ఱ

ㅇ

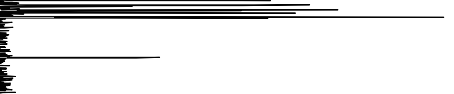

官

$=$

$=$

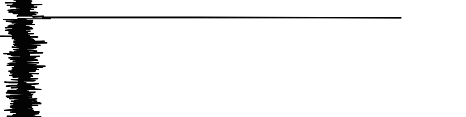

138.711

138.634

138.501

136.502

136.409

$F^{130.327}$

130.274

$\left[\left[\begin{array}{c}129.540 \\ 129.127\end{array}\right.\right.$

129.100

129.077

129.040
-129.0402

$-128.842$

128.404

$-128.372$

128.332

128.245

127.930

127.860

127.656

127.331
127.285

127.285
127.222

123.568

123.525

$\left[\begin{array}{r}116.425 \\ 116.402\end{array}\right.$

年稁

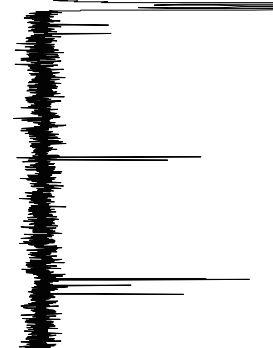

$\Upsilon_{74.300}$

$-63.077$

51.812

$-51.718$

51.200
-50.391

$\overrightarrow{0}$

○

몹

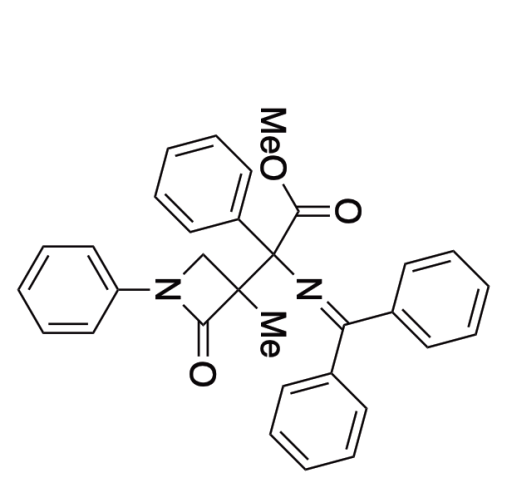

${ }^{18.489}$ 


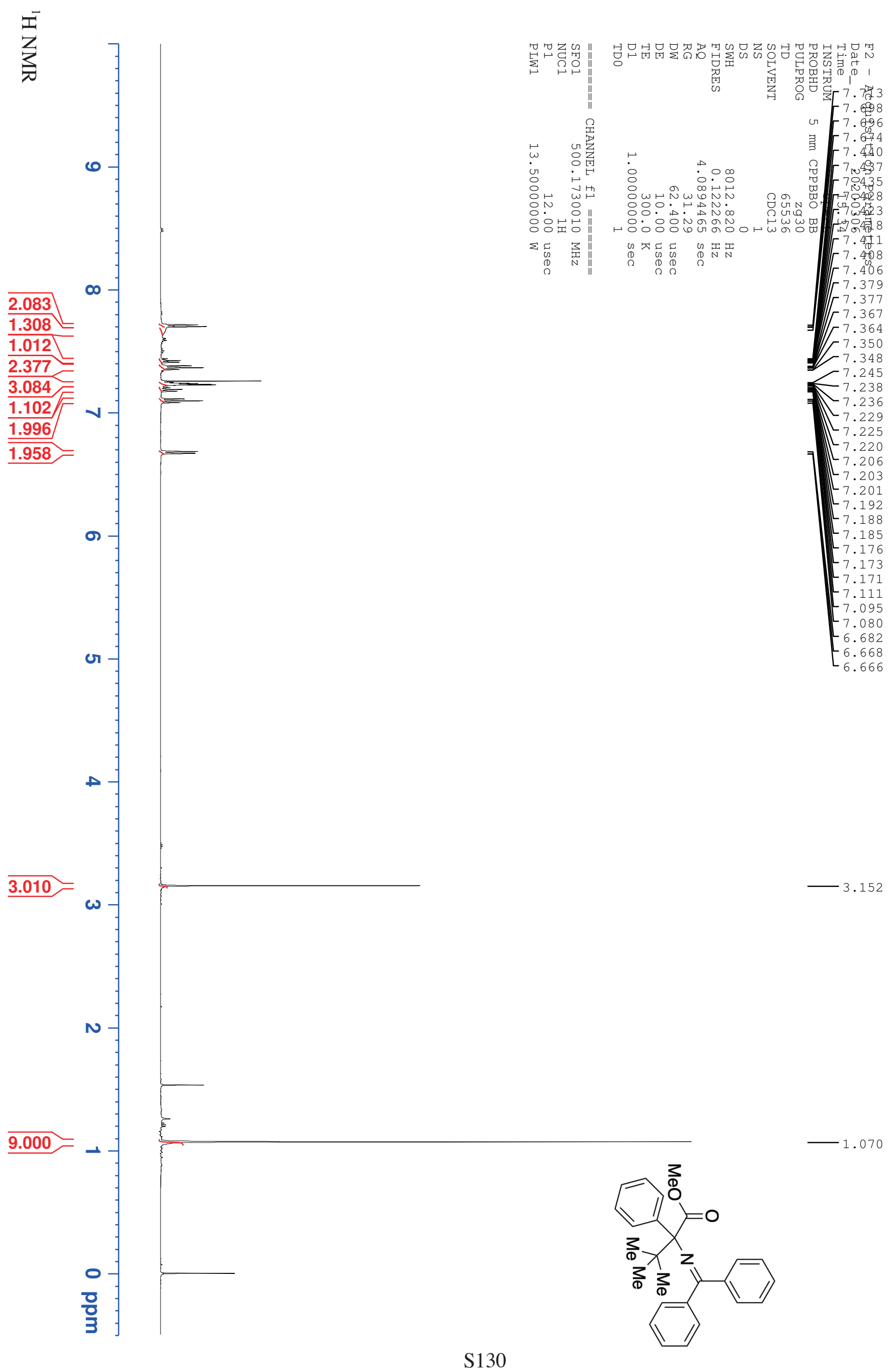




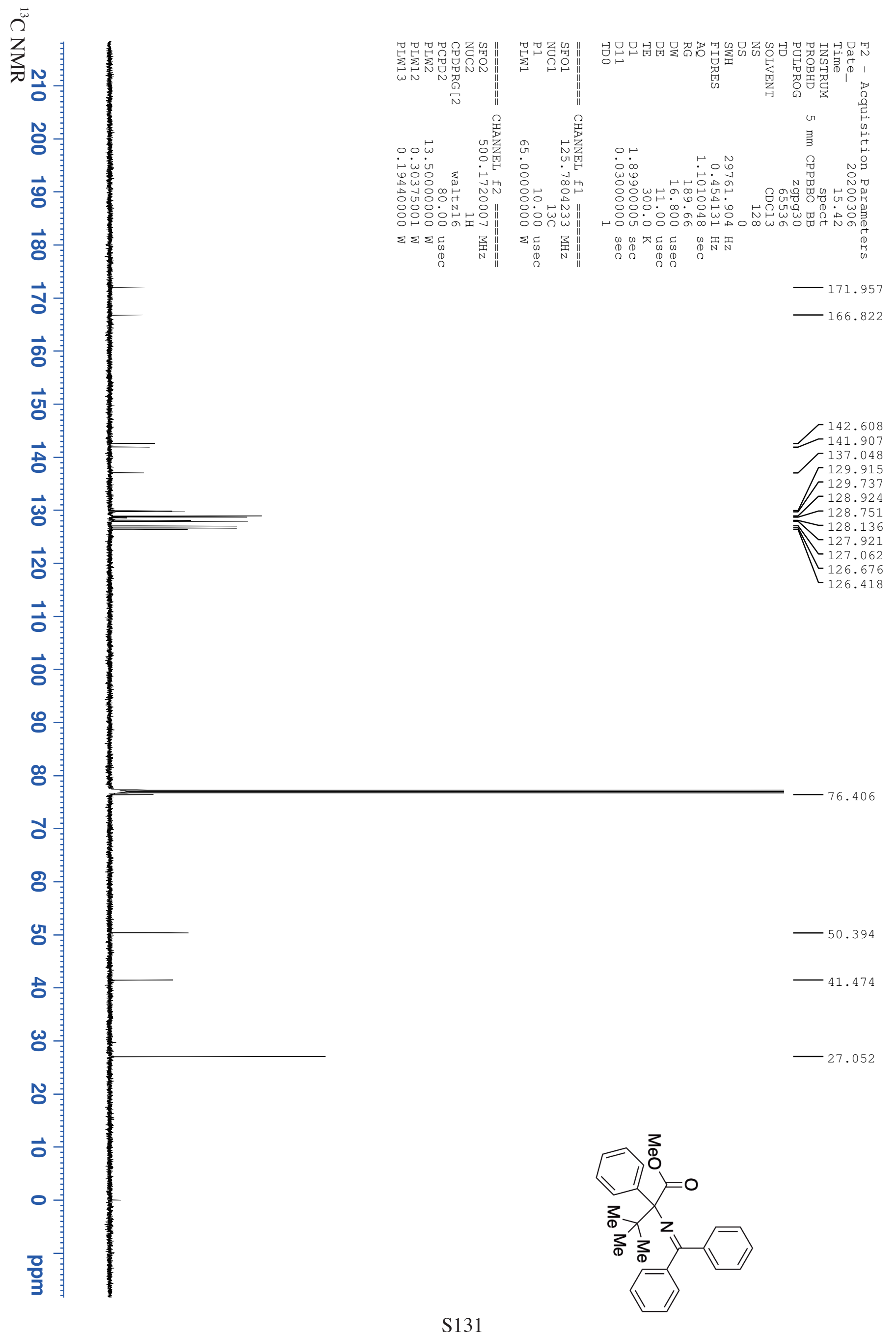




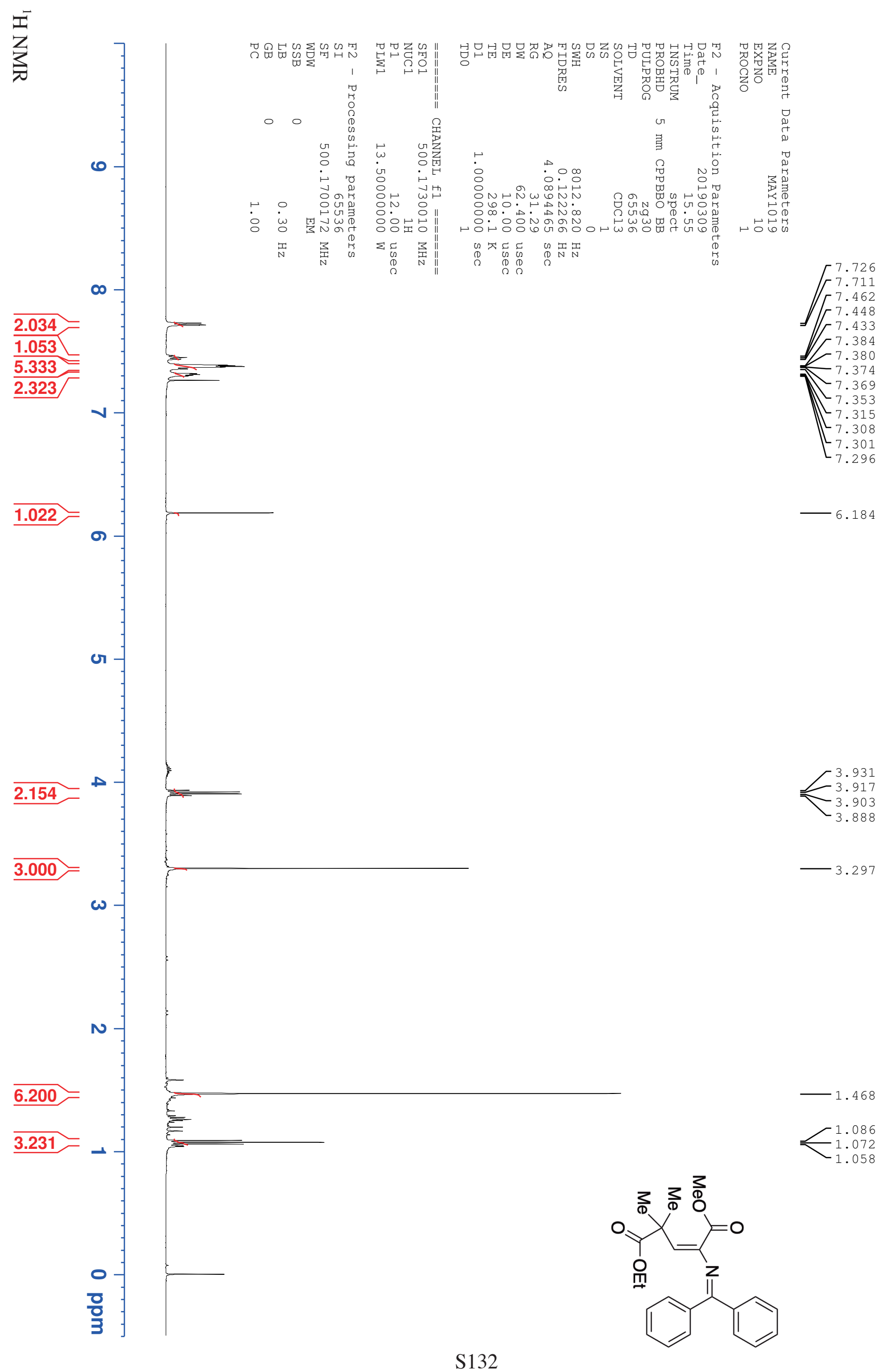




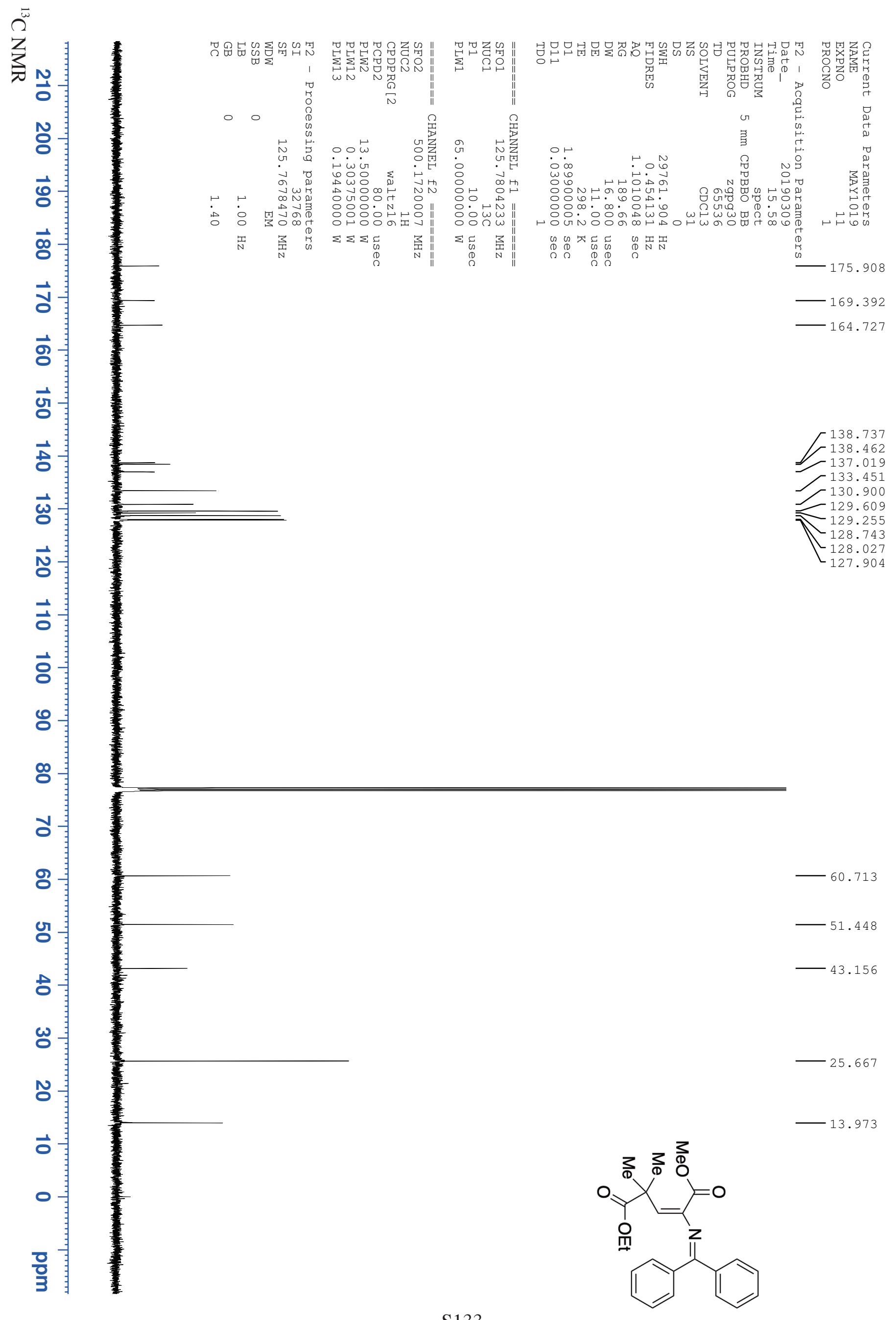



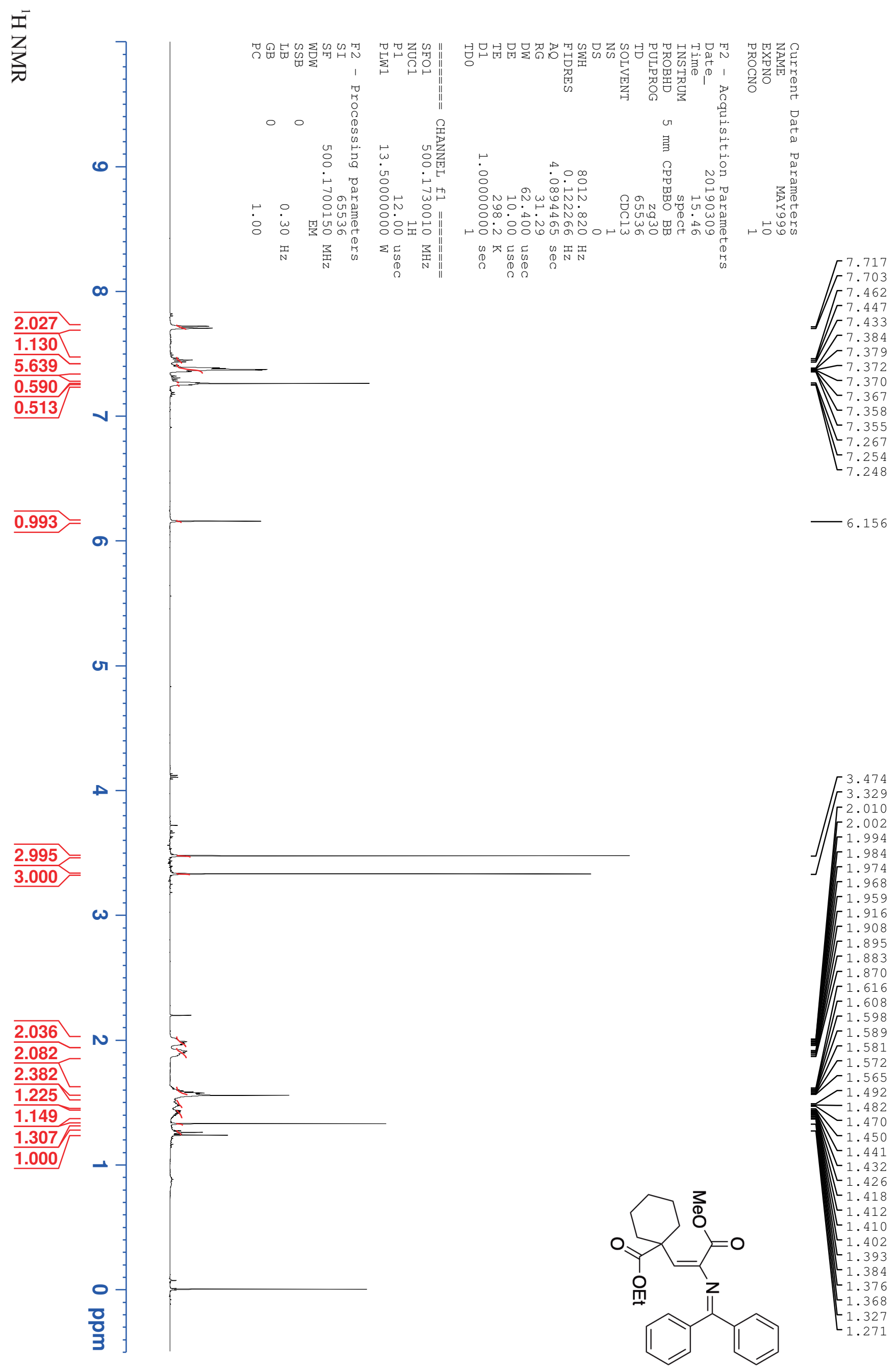


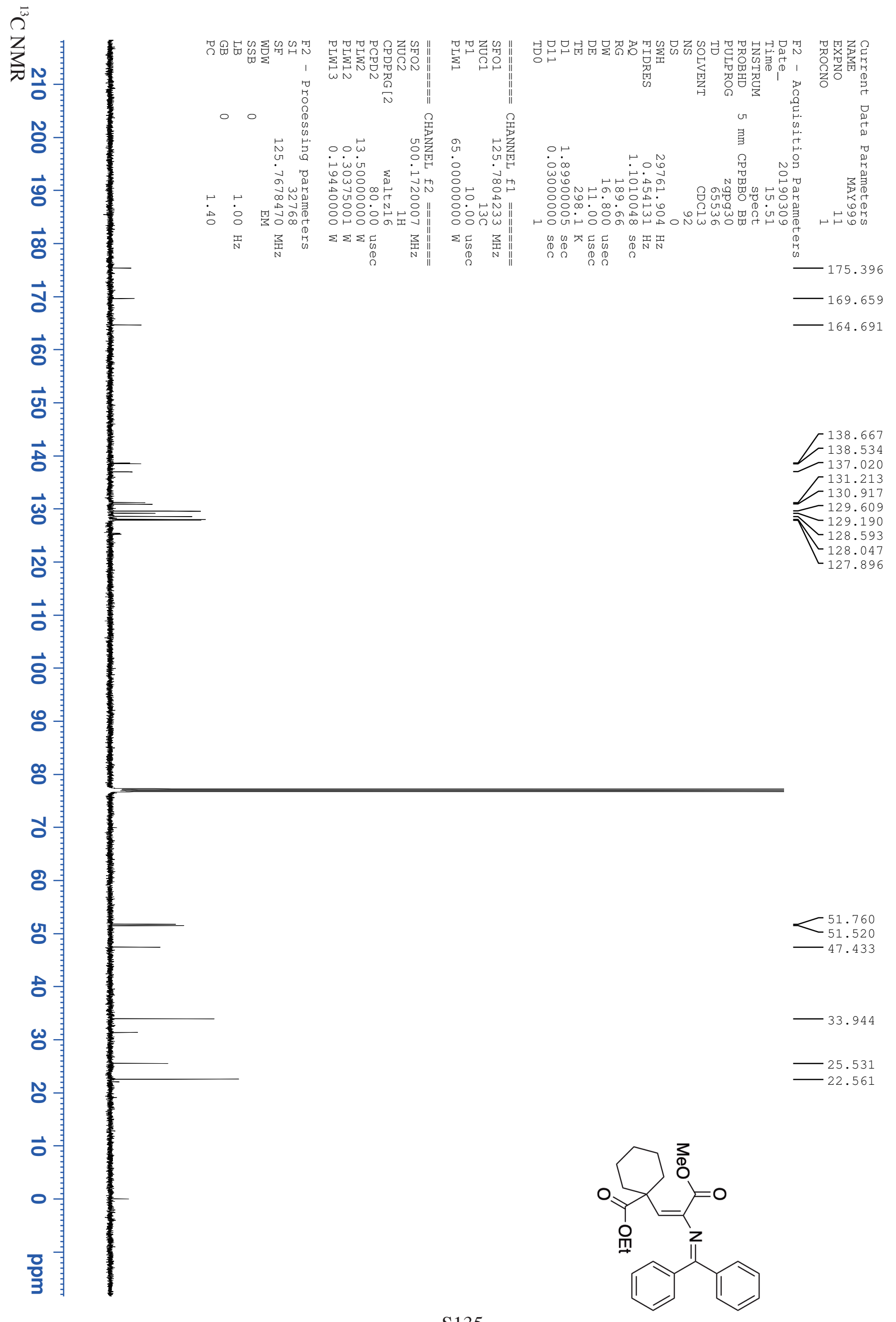




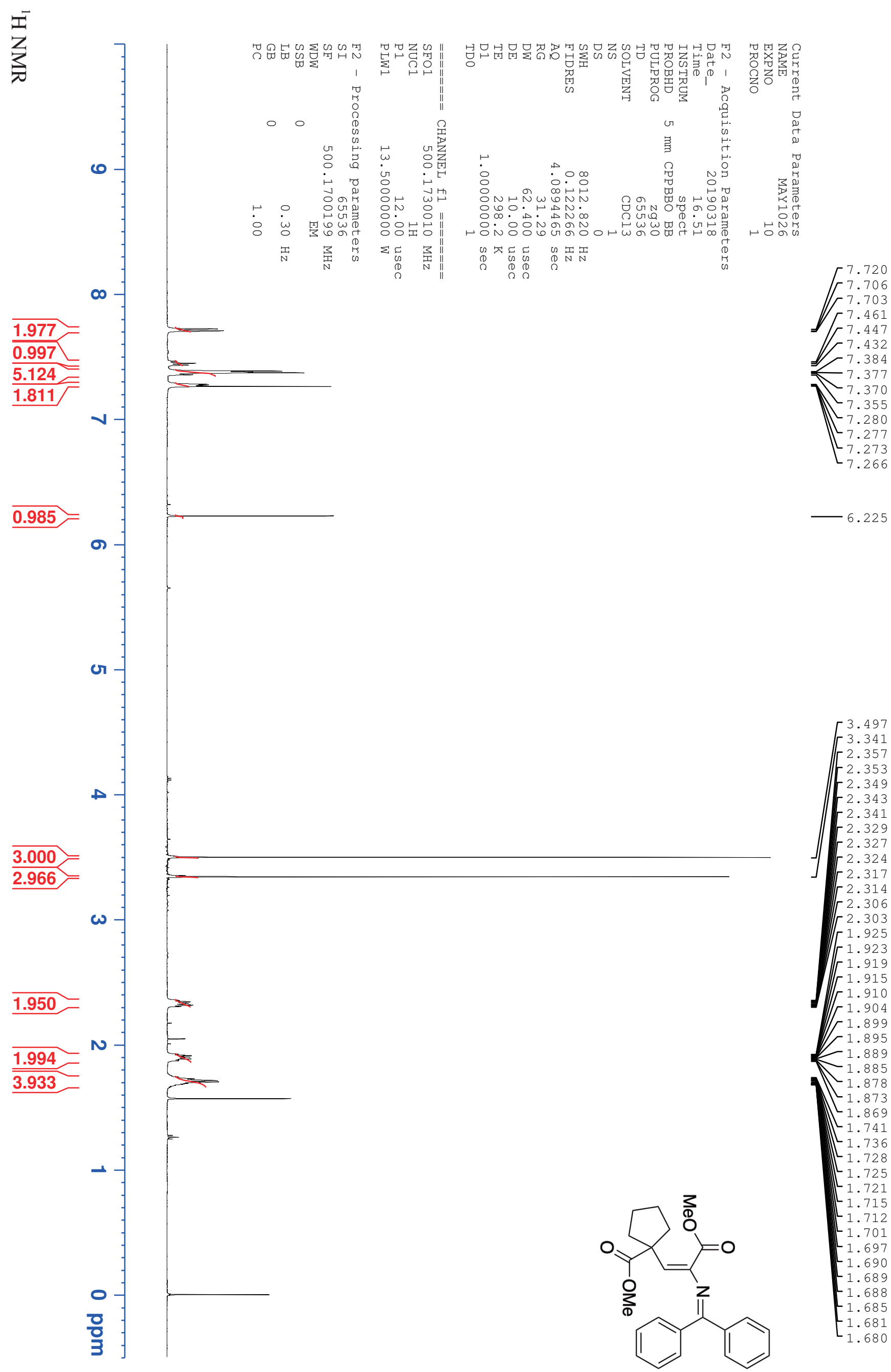




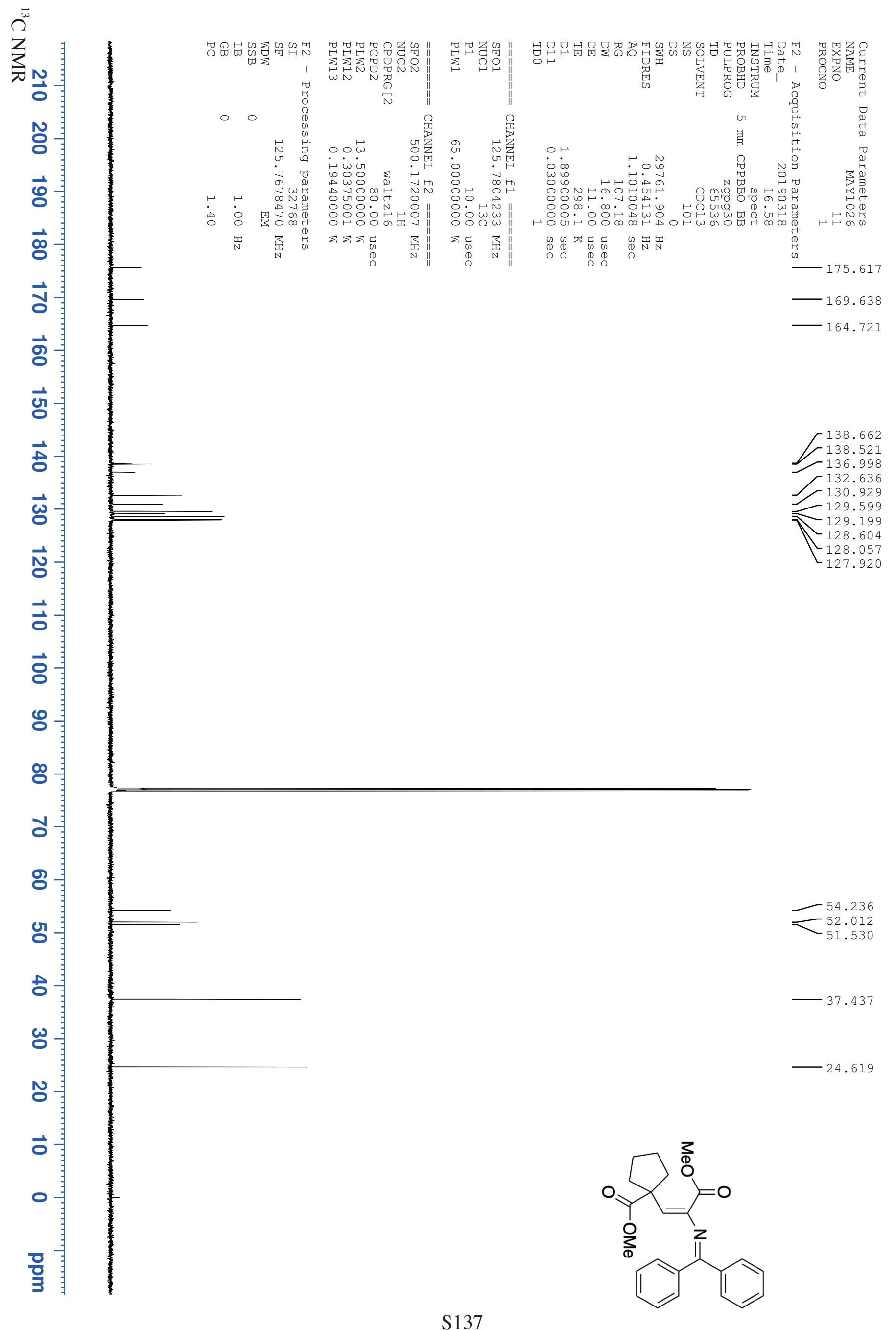




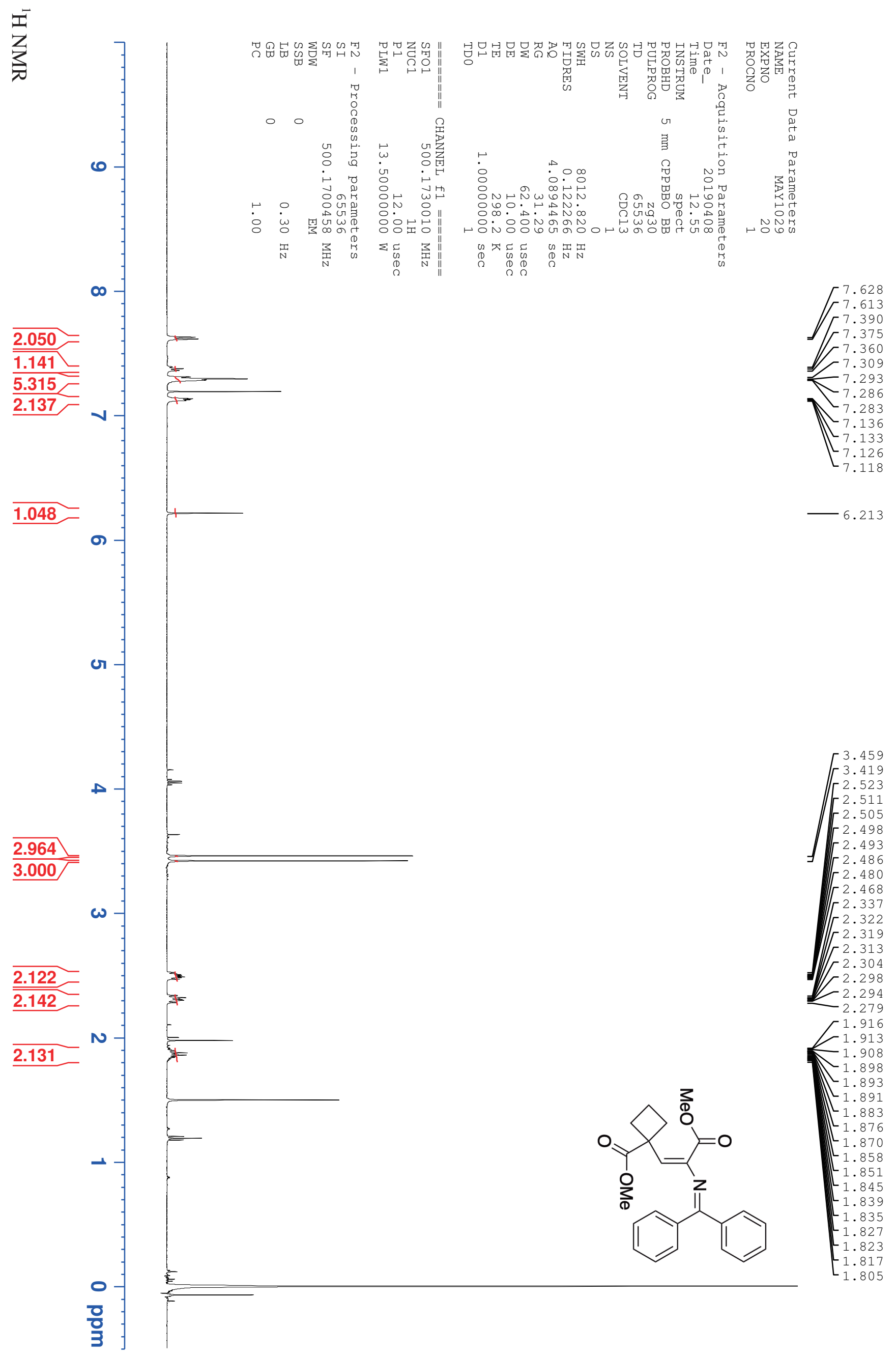




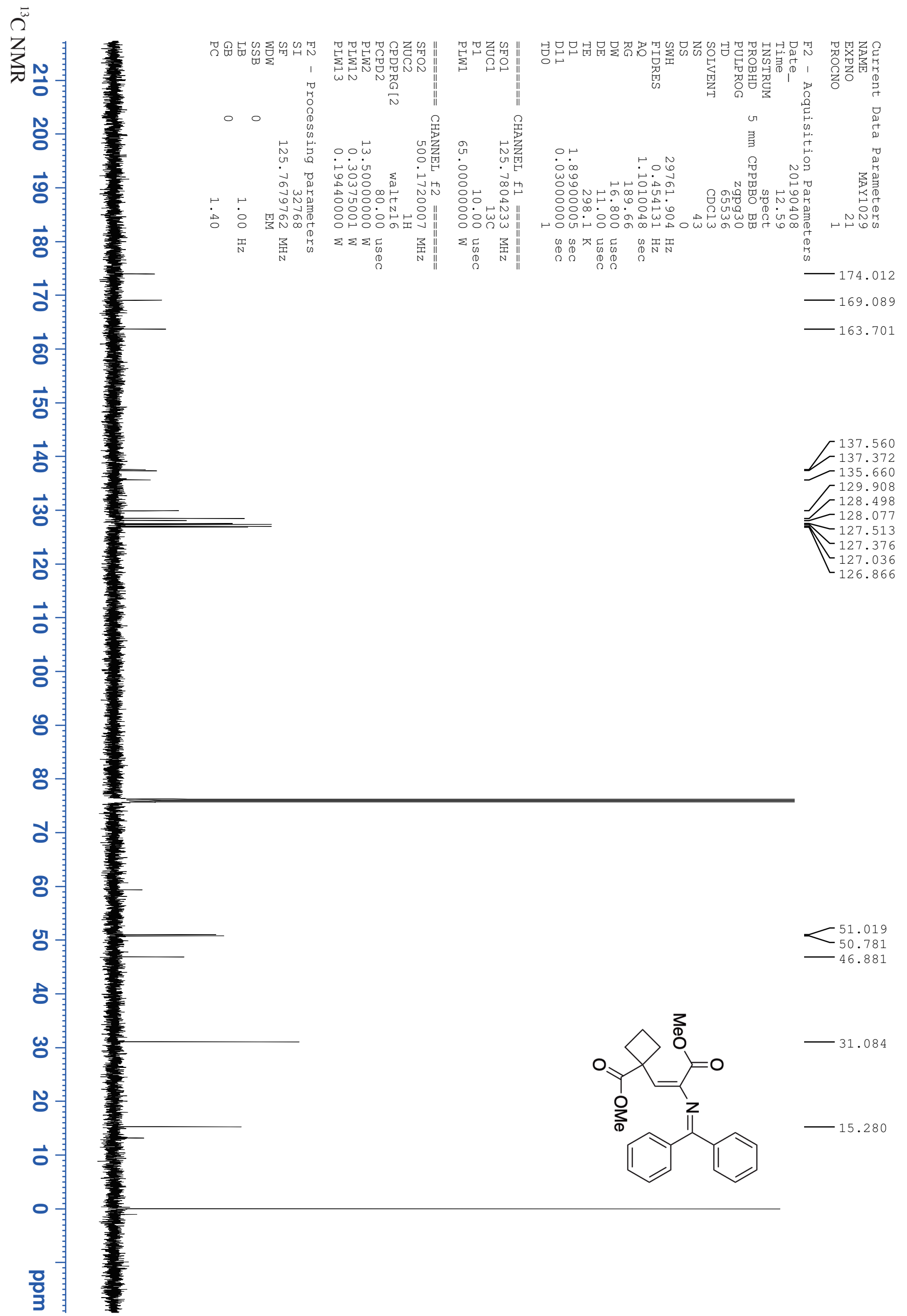




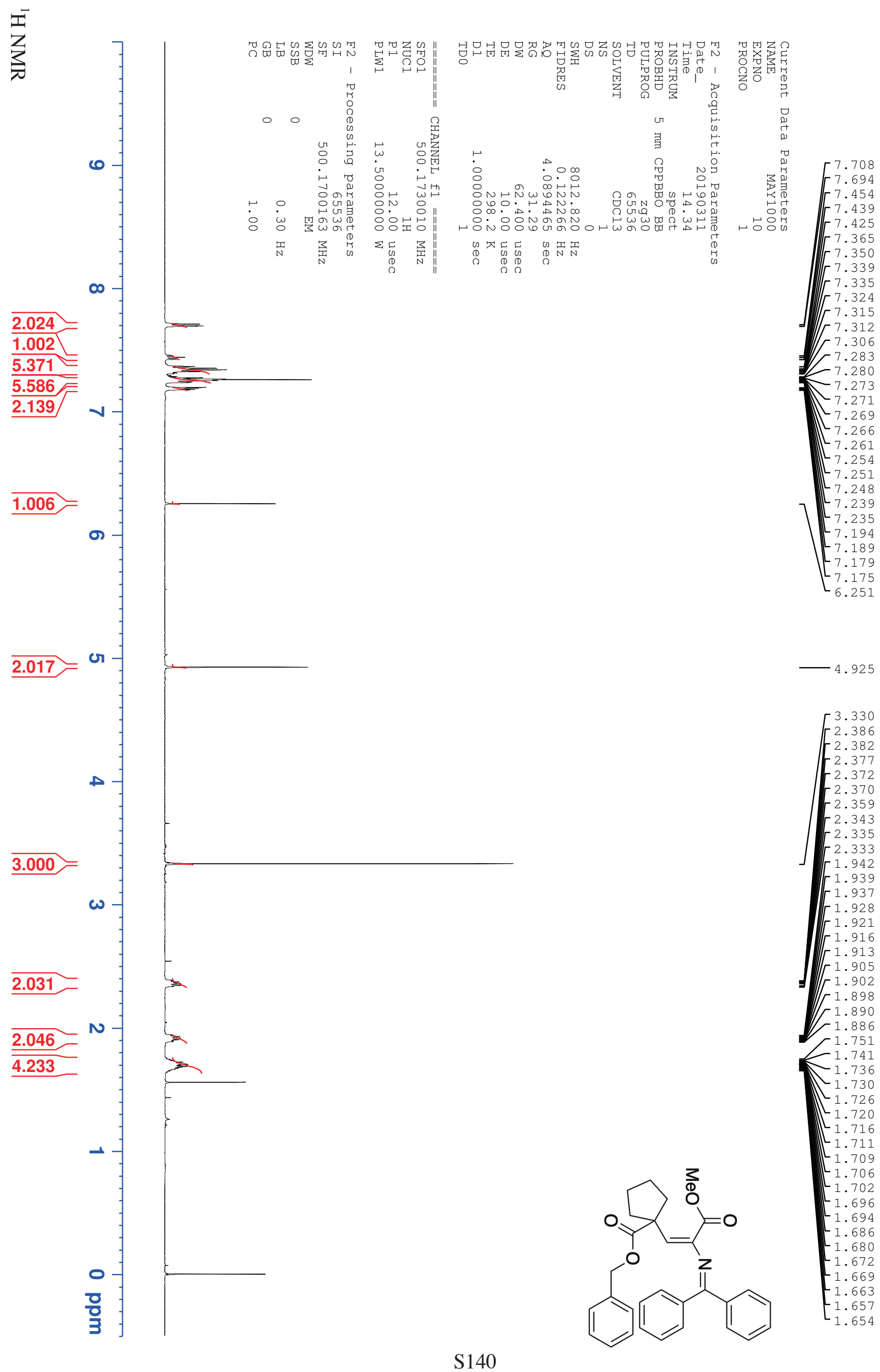




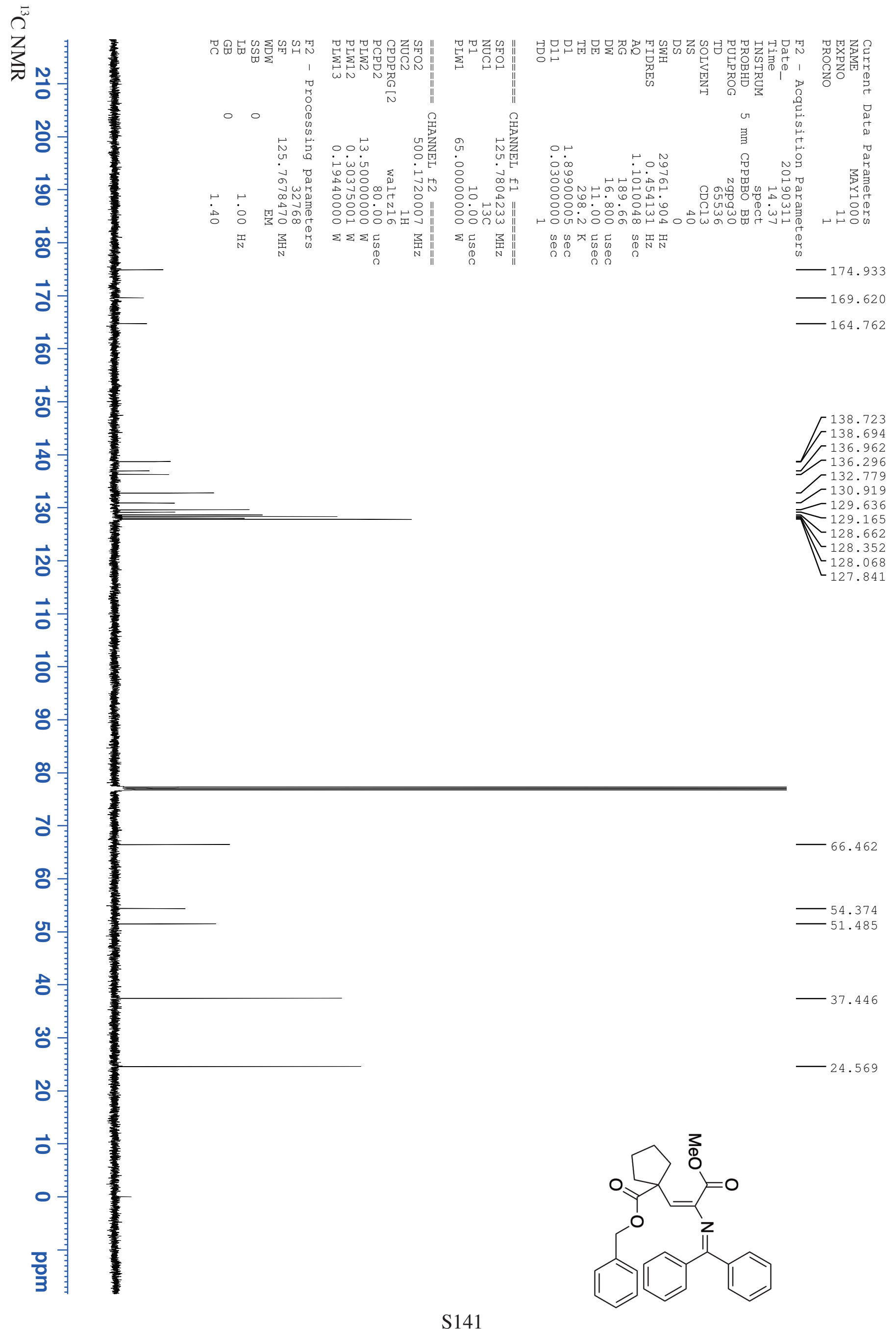




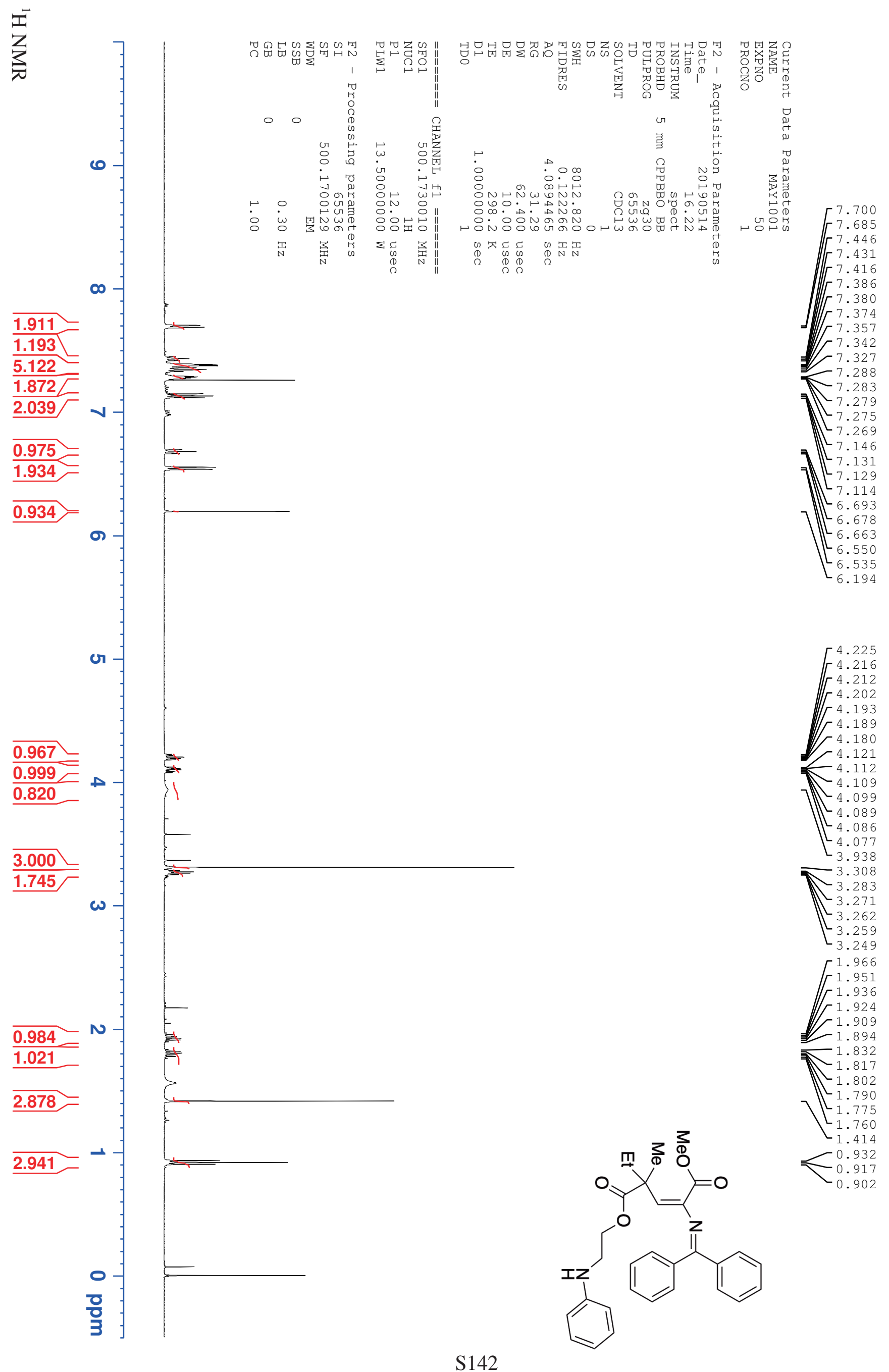




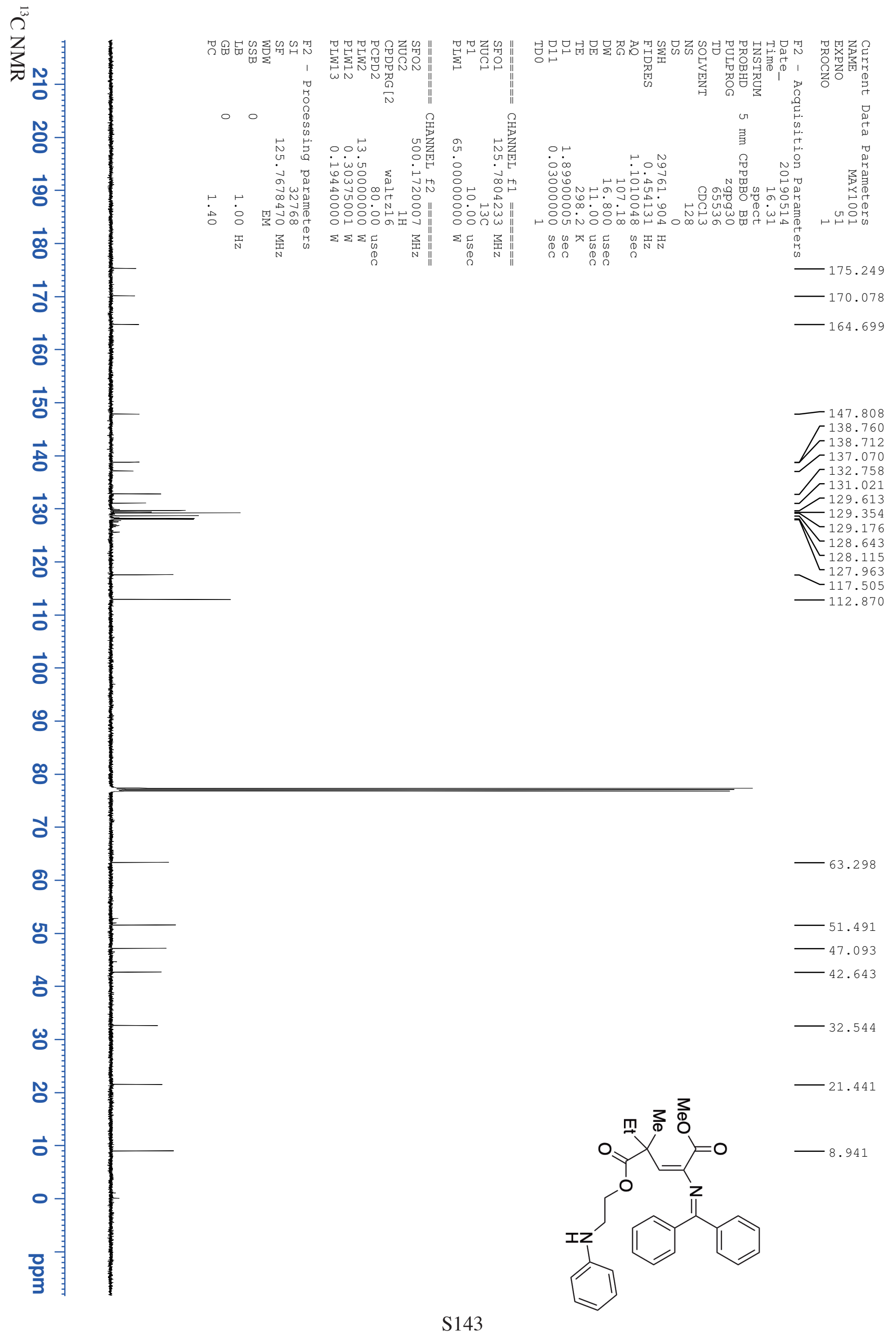




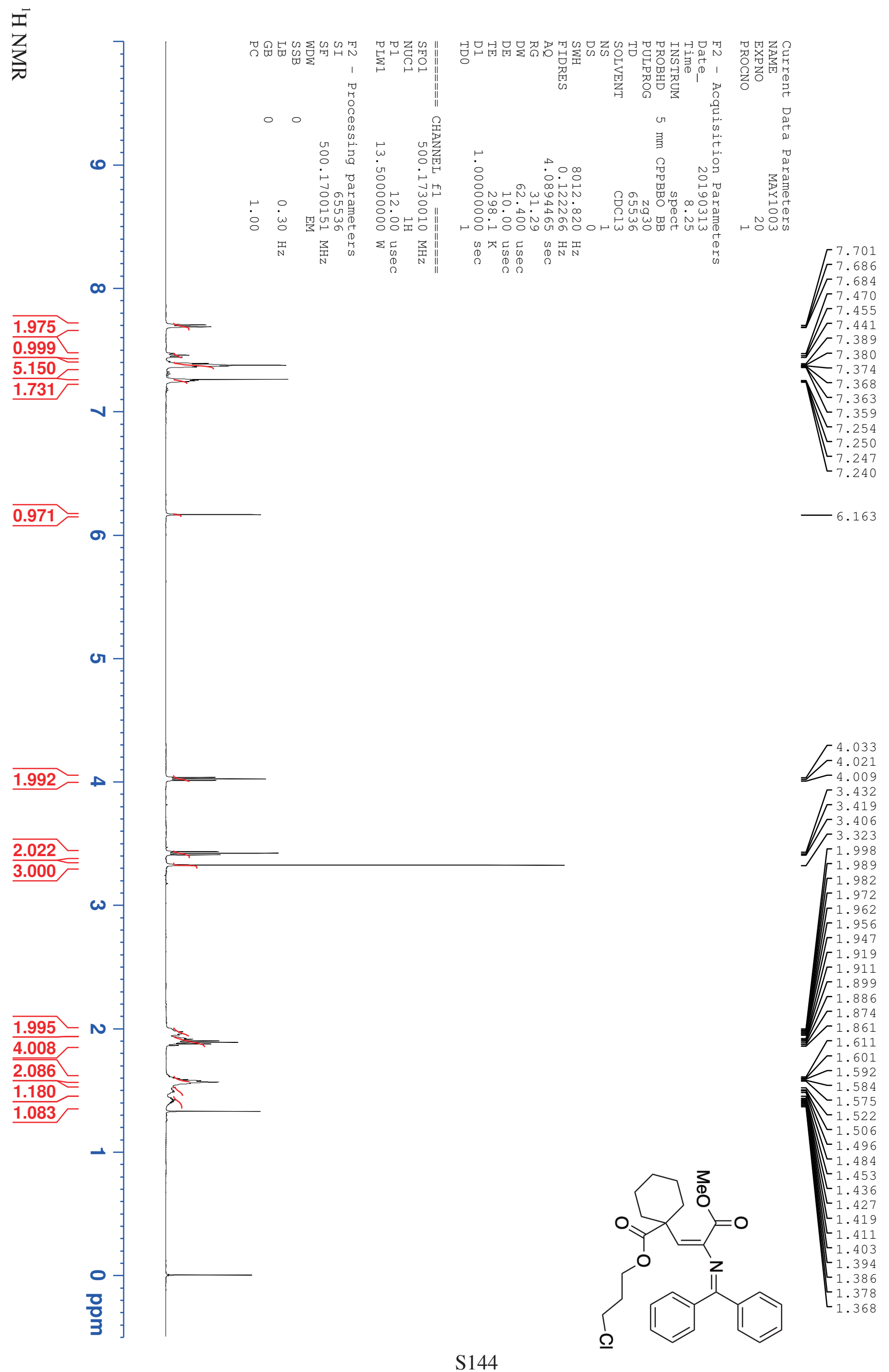




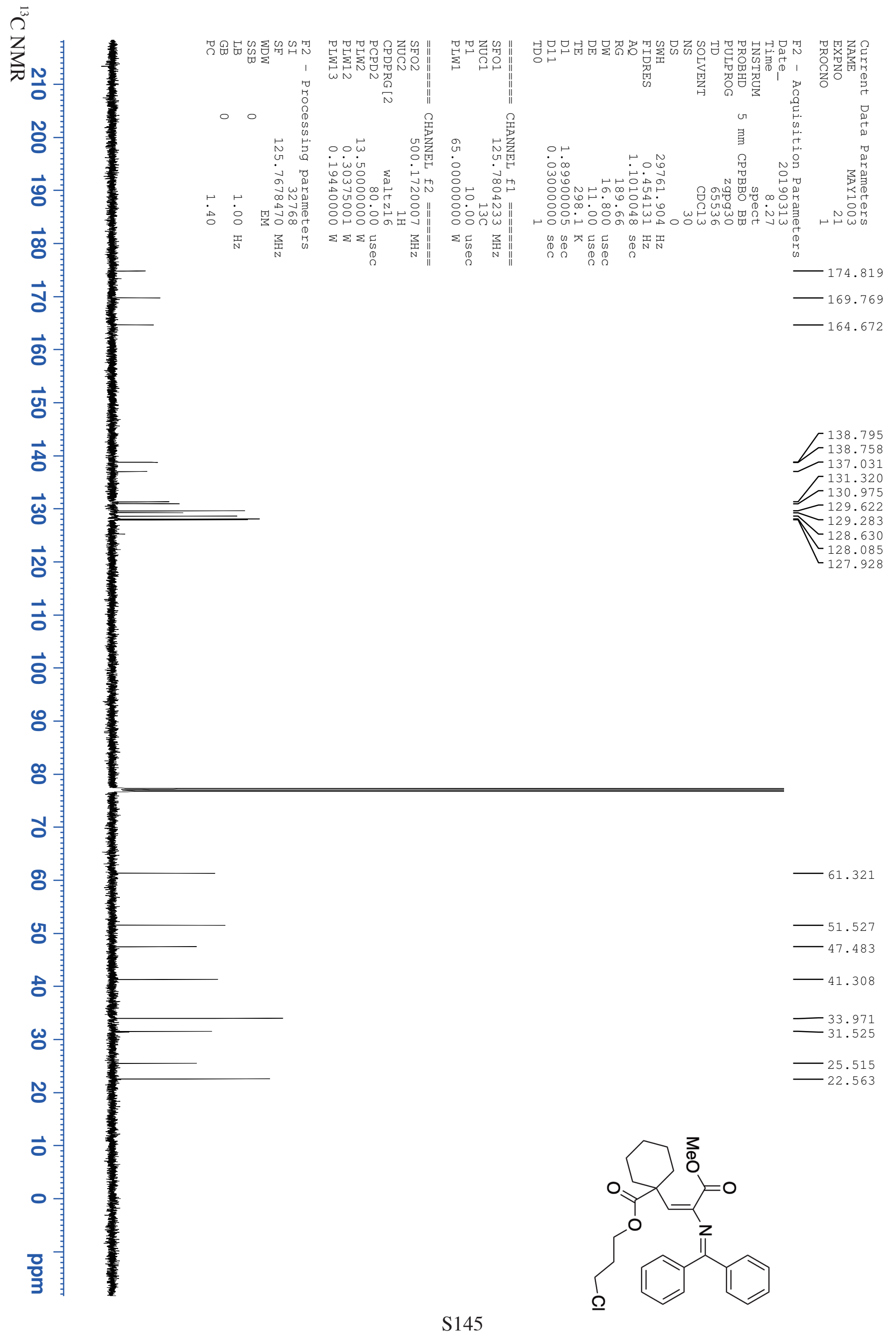




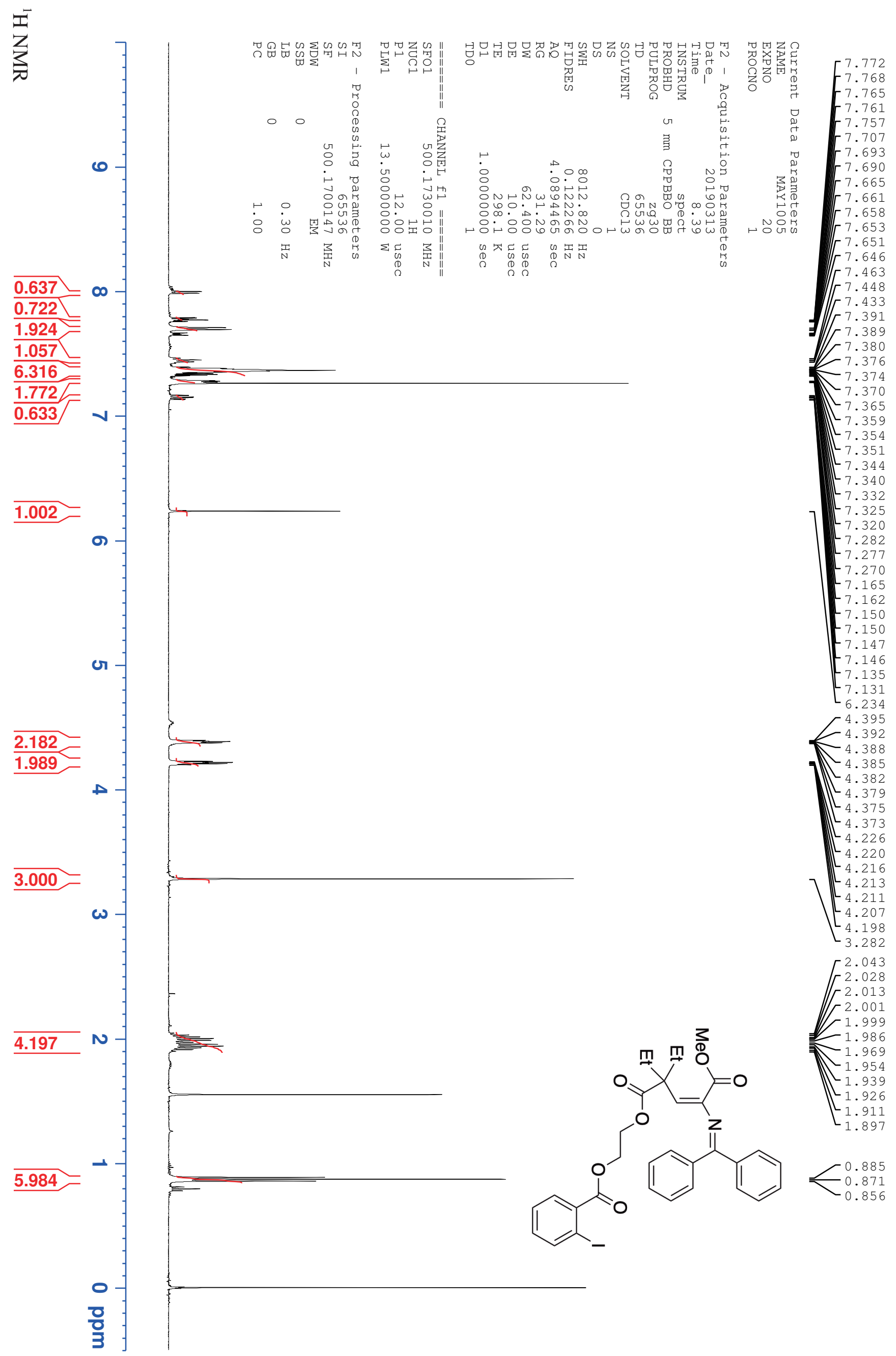




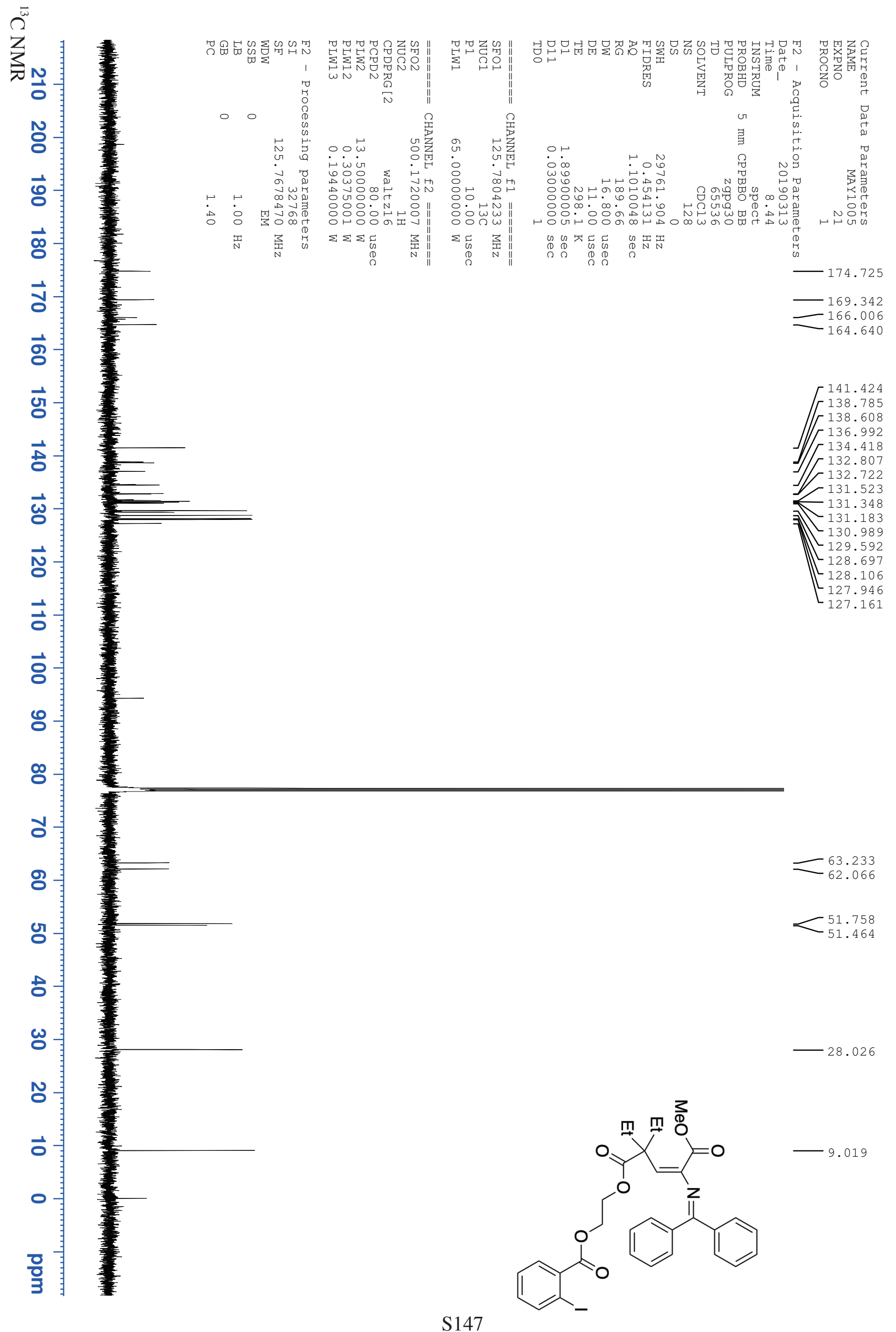




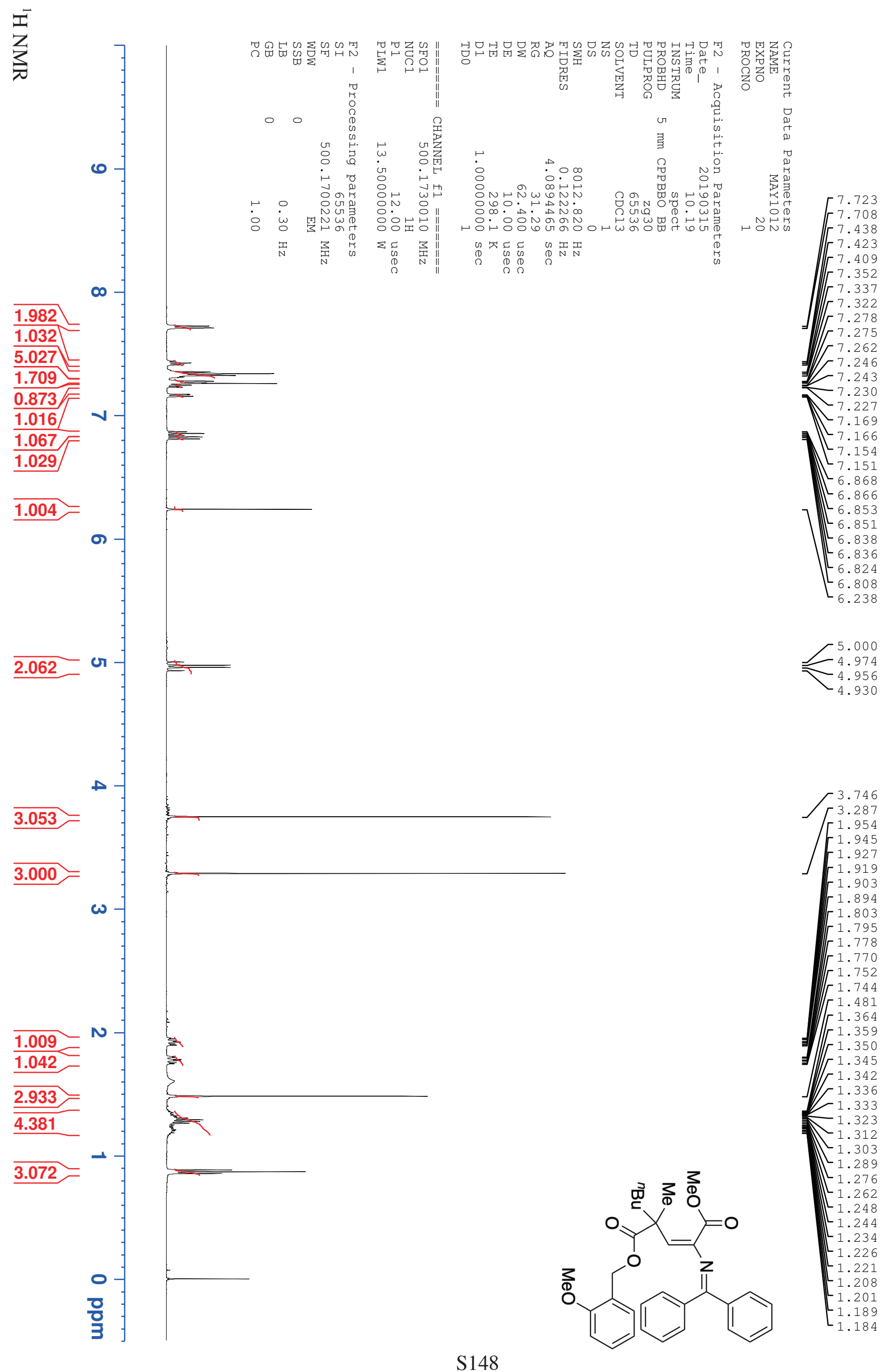




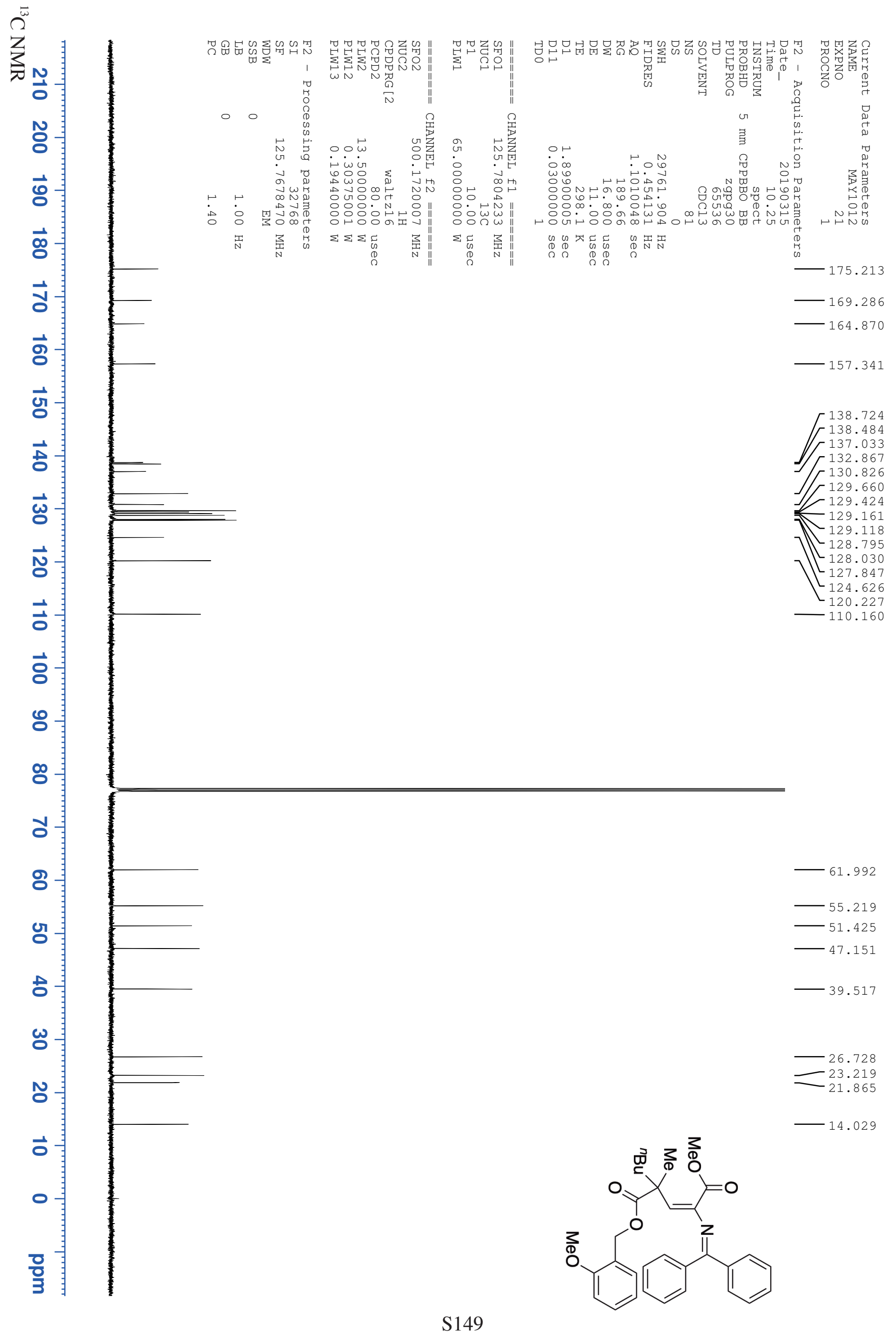




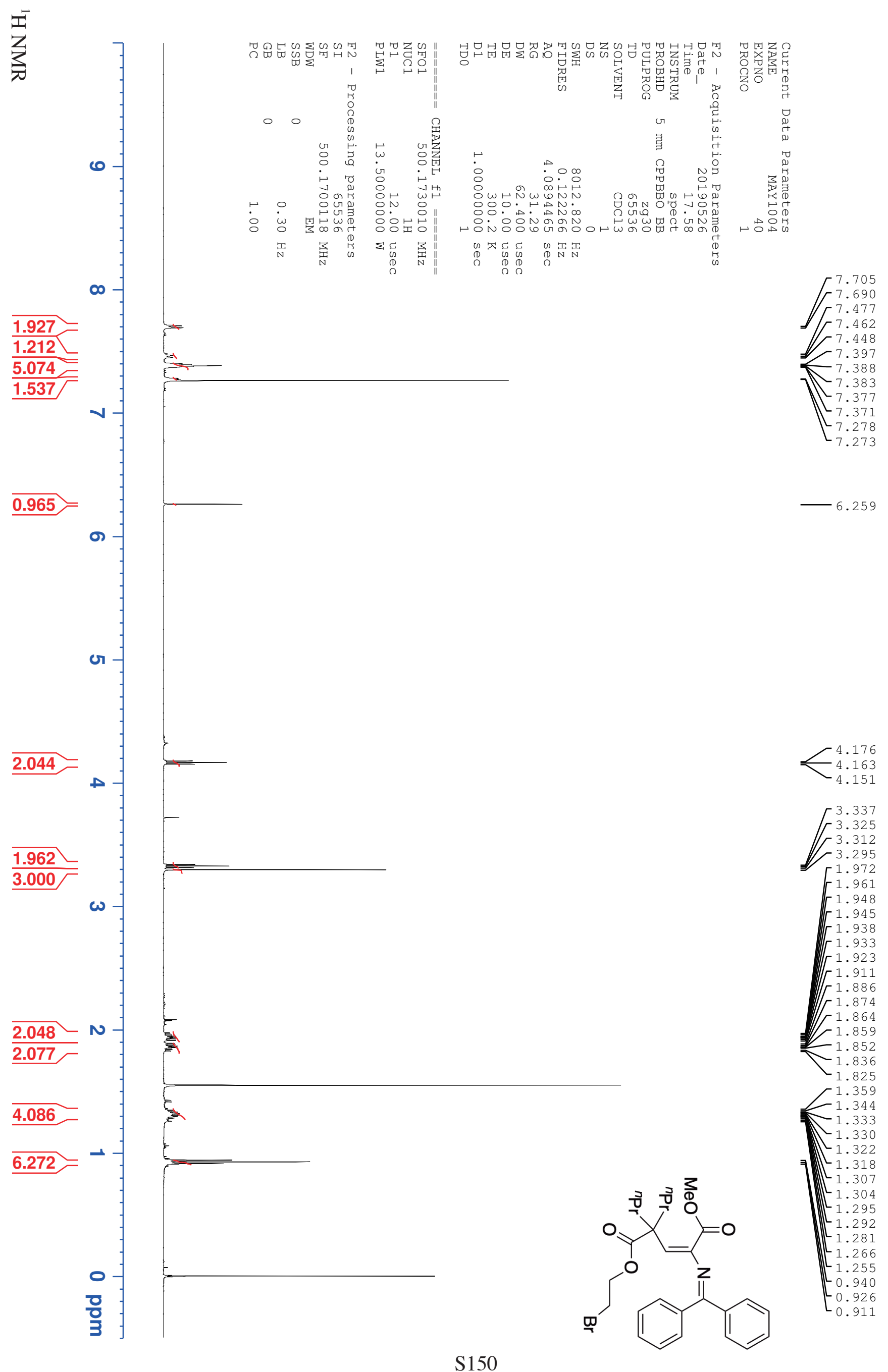




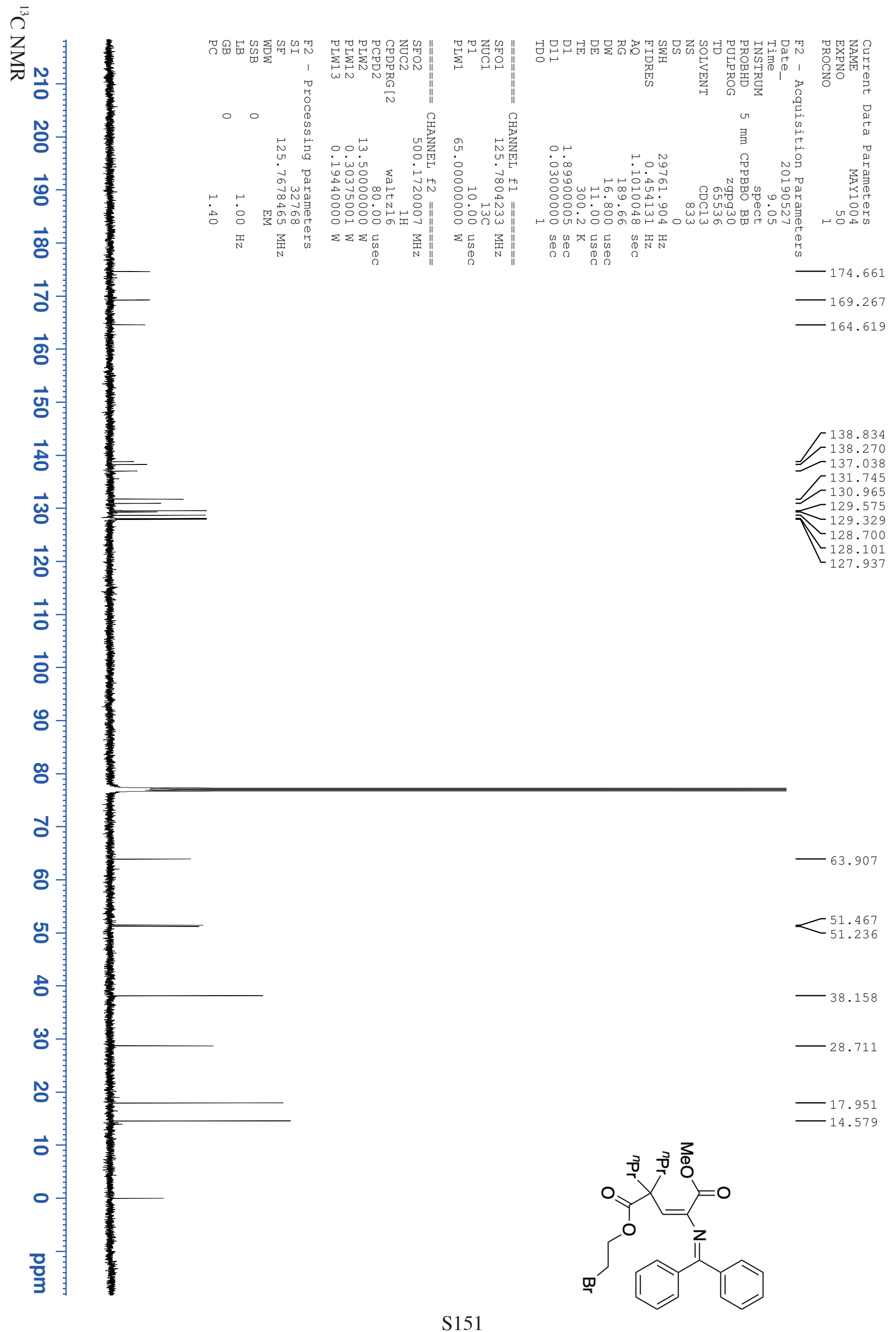




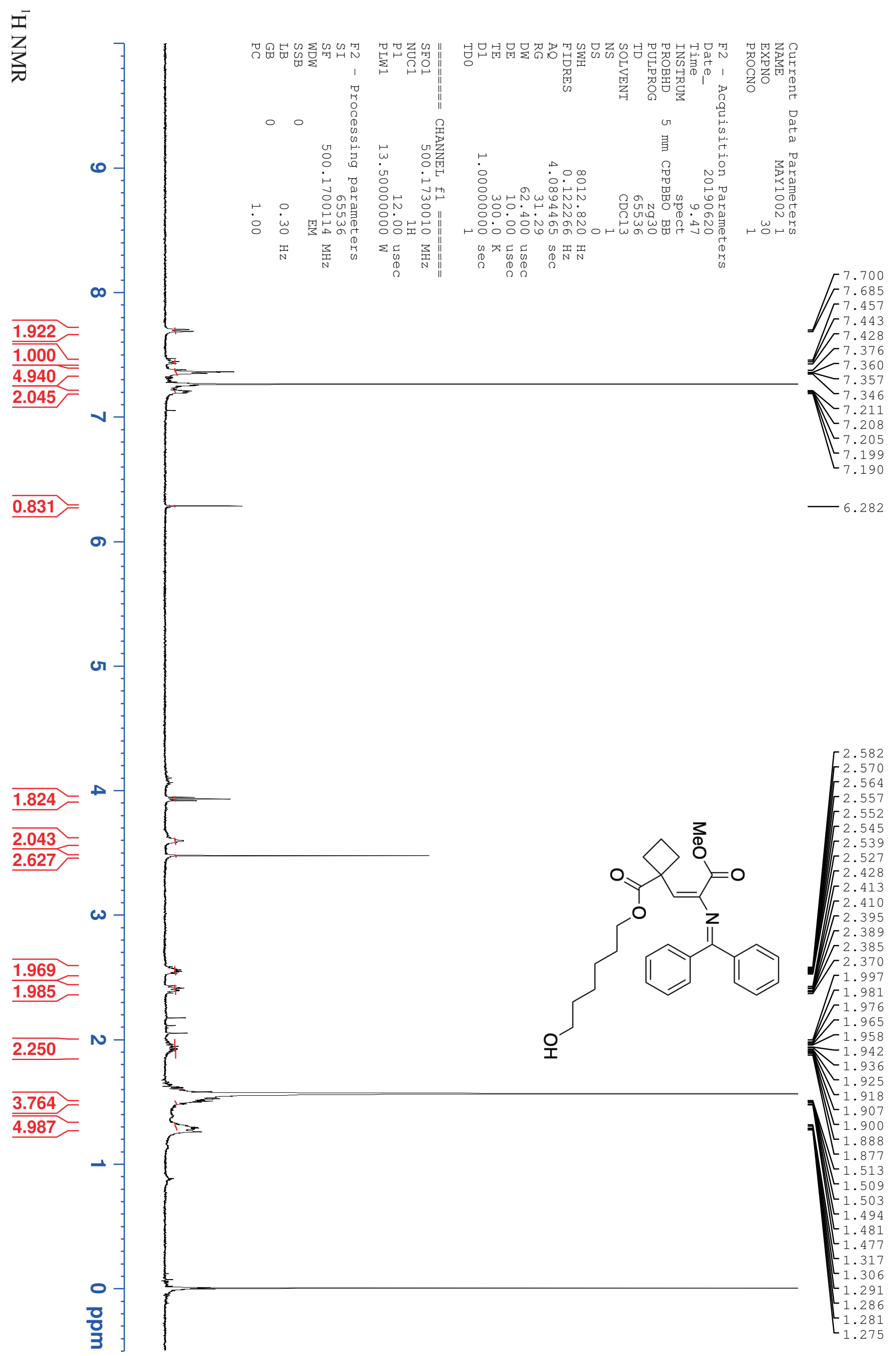




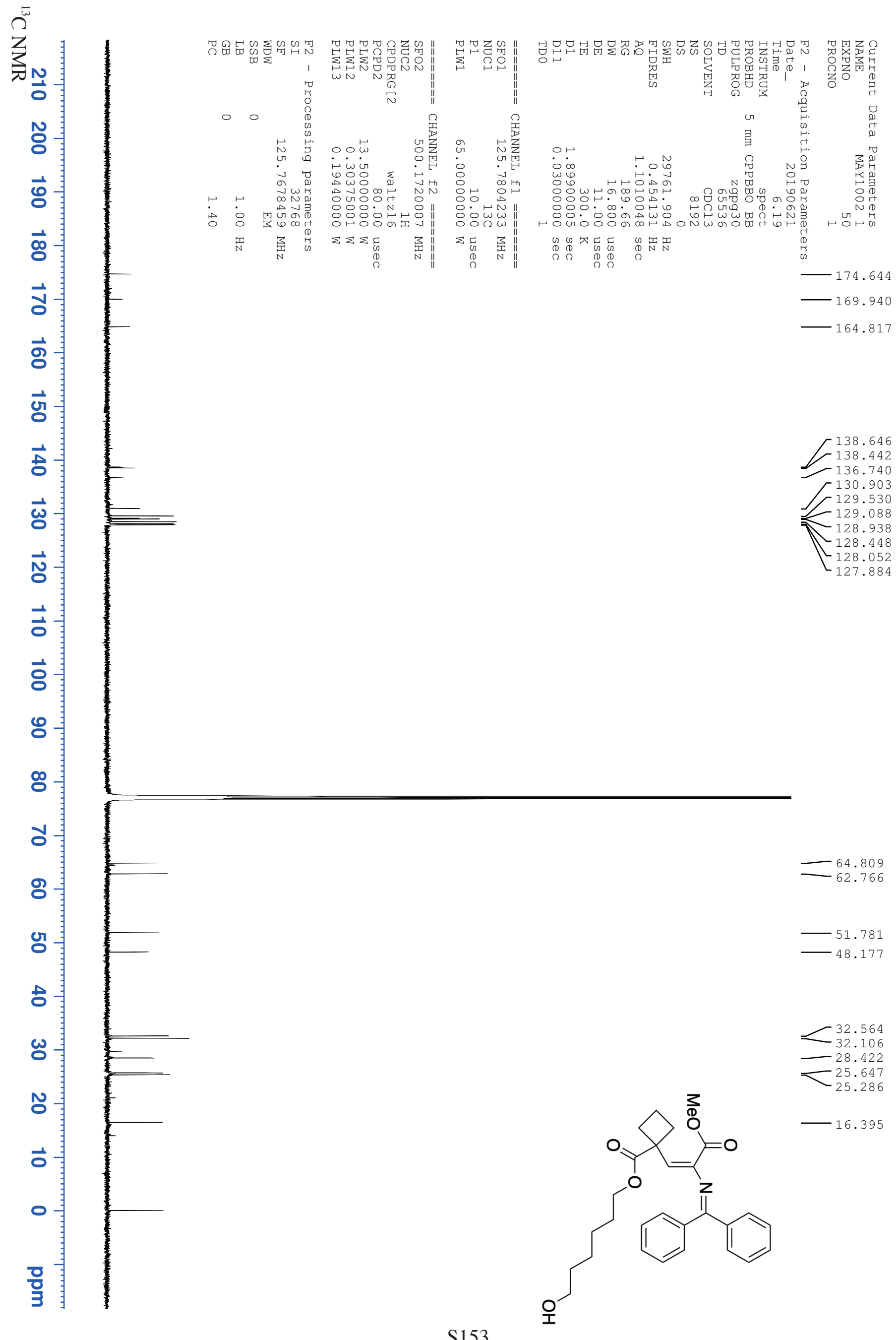




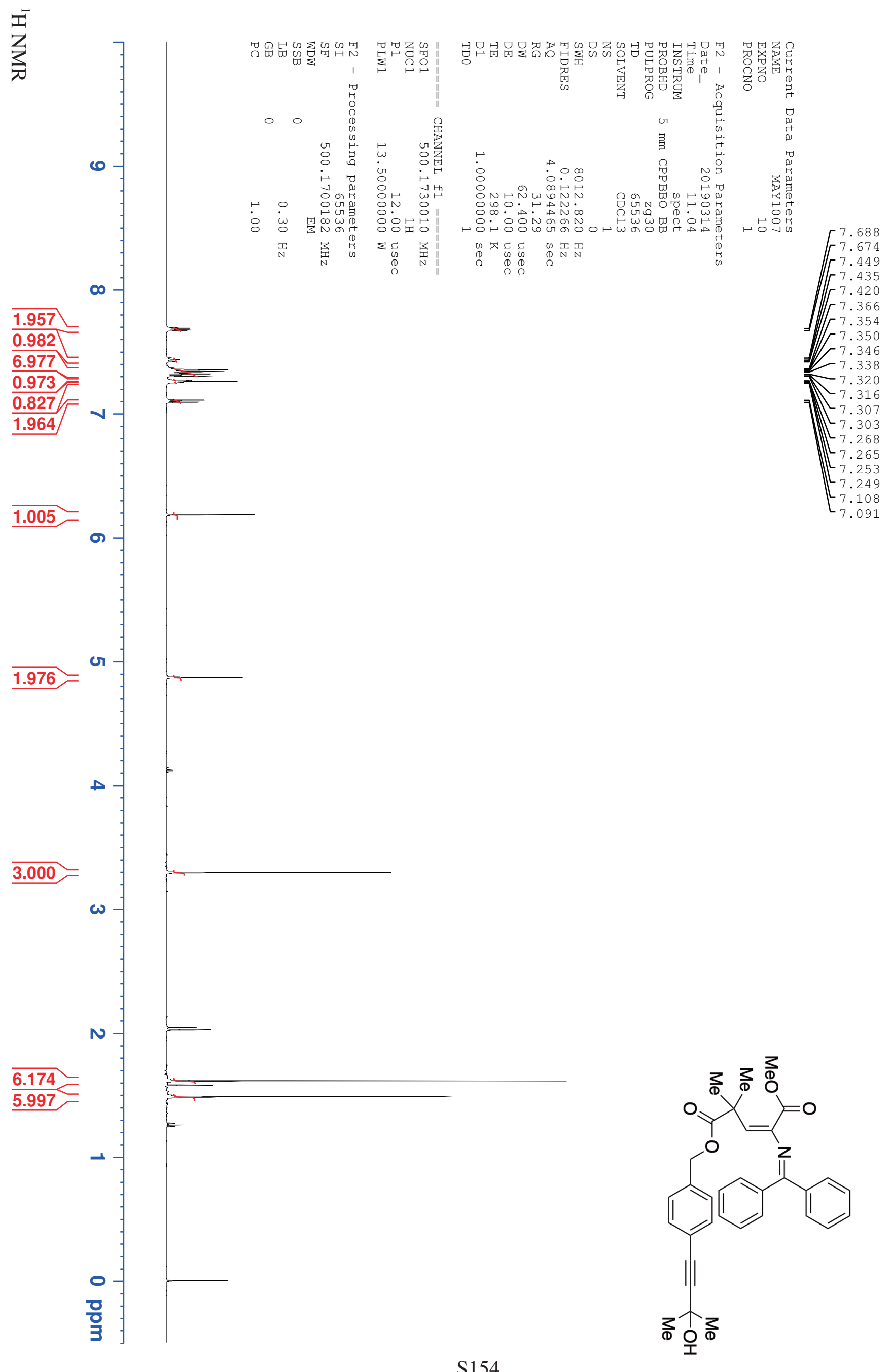




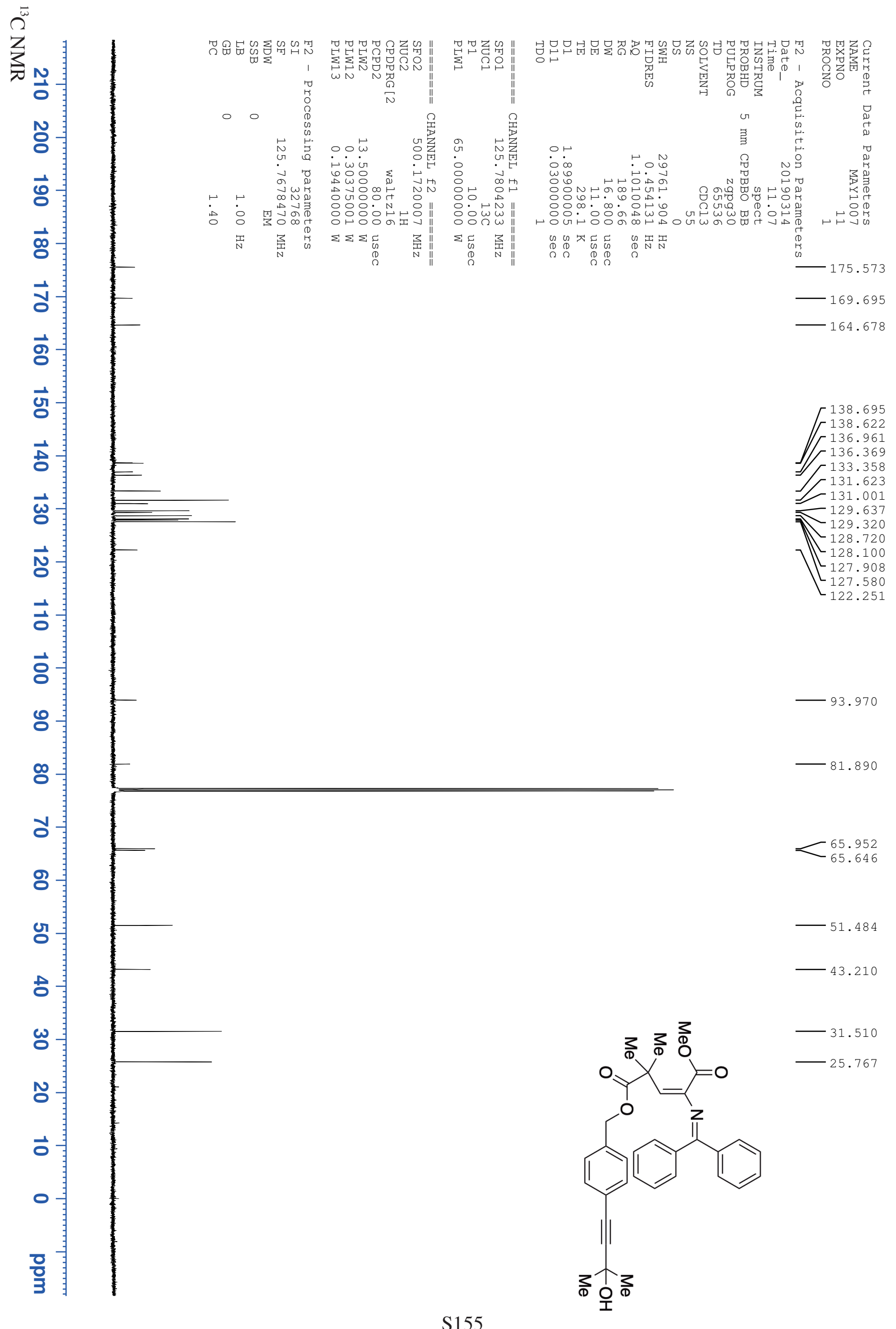




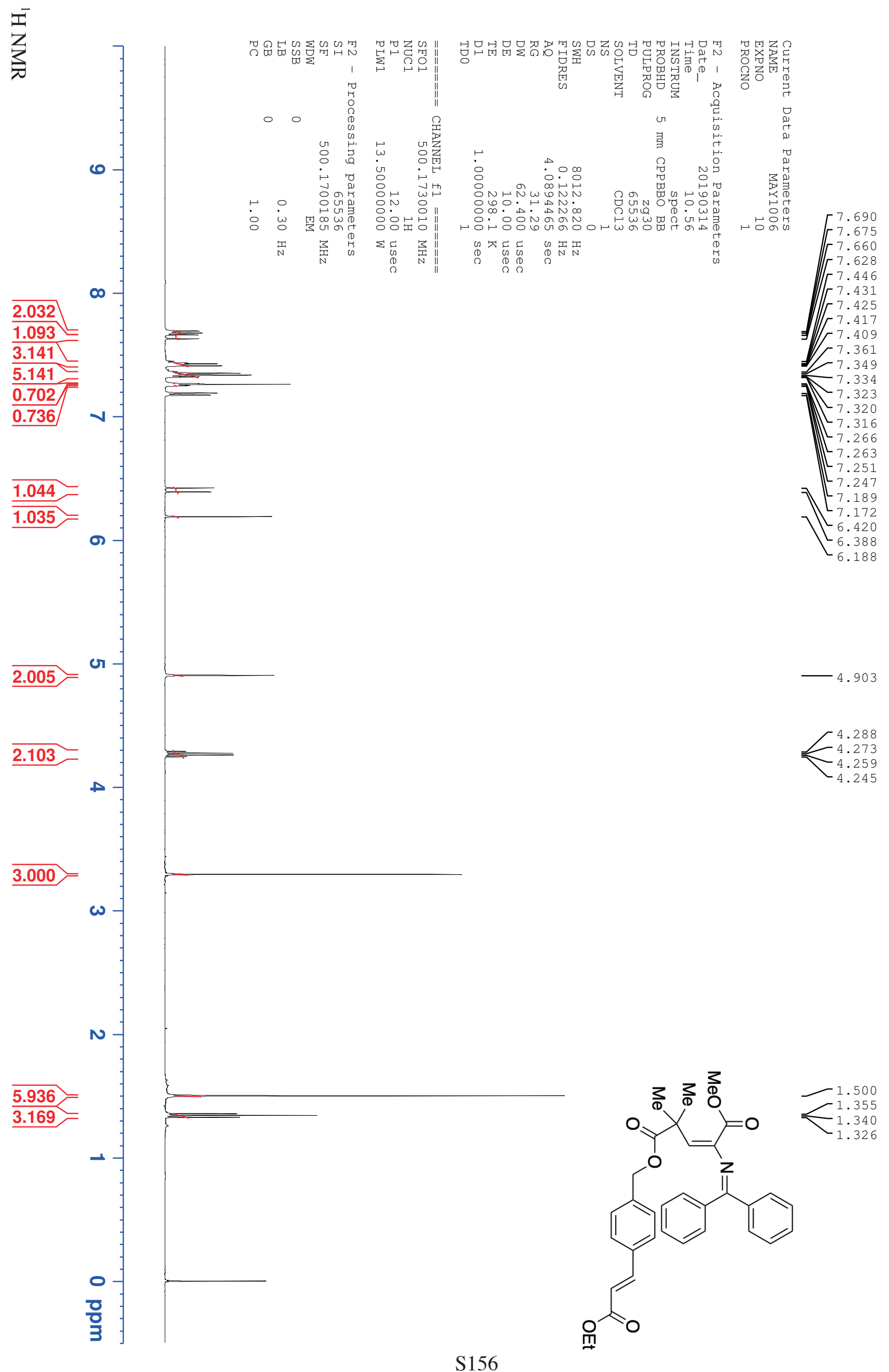




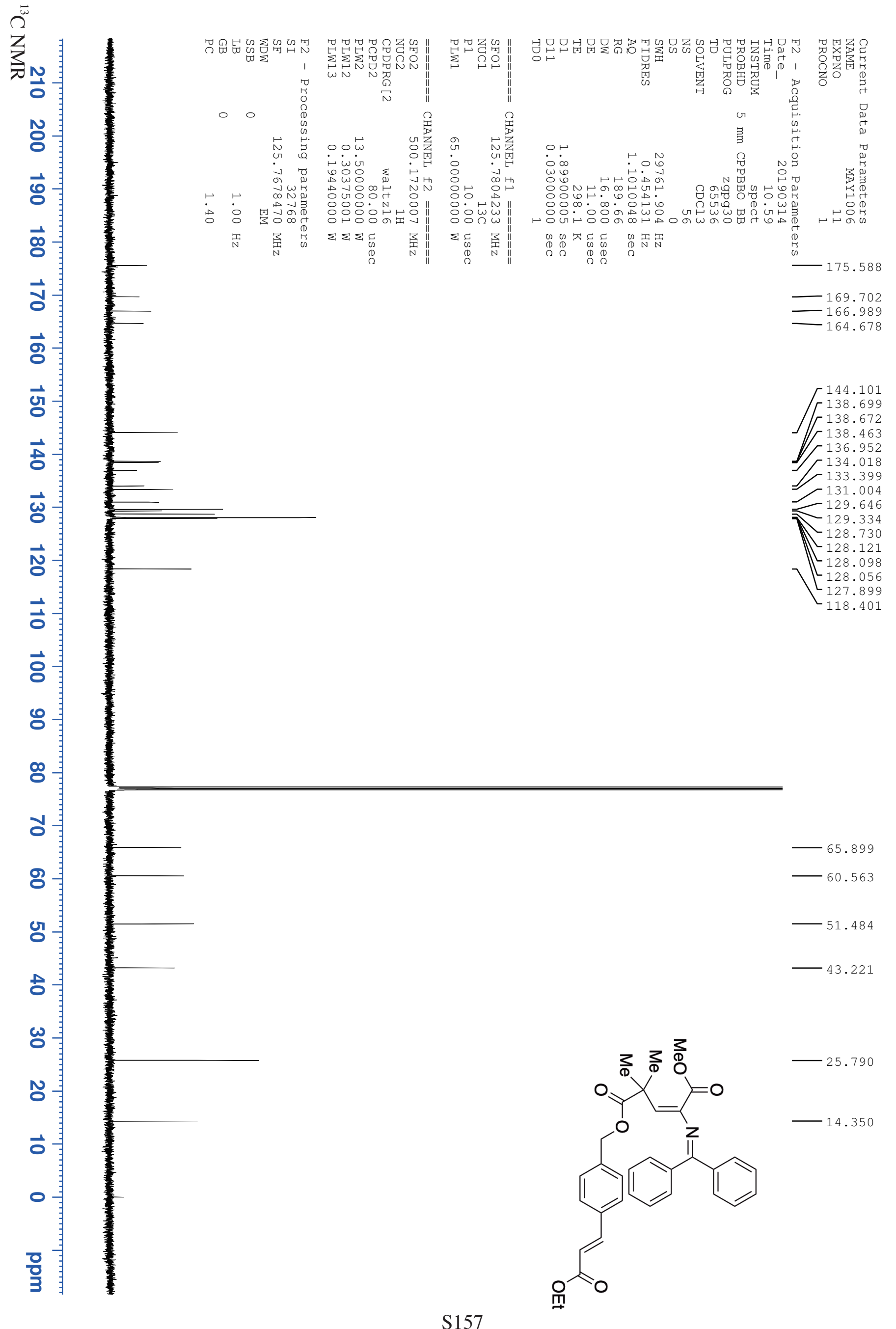




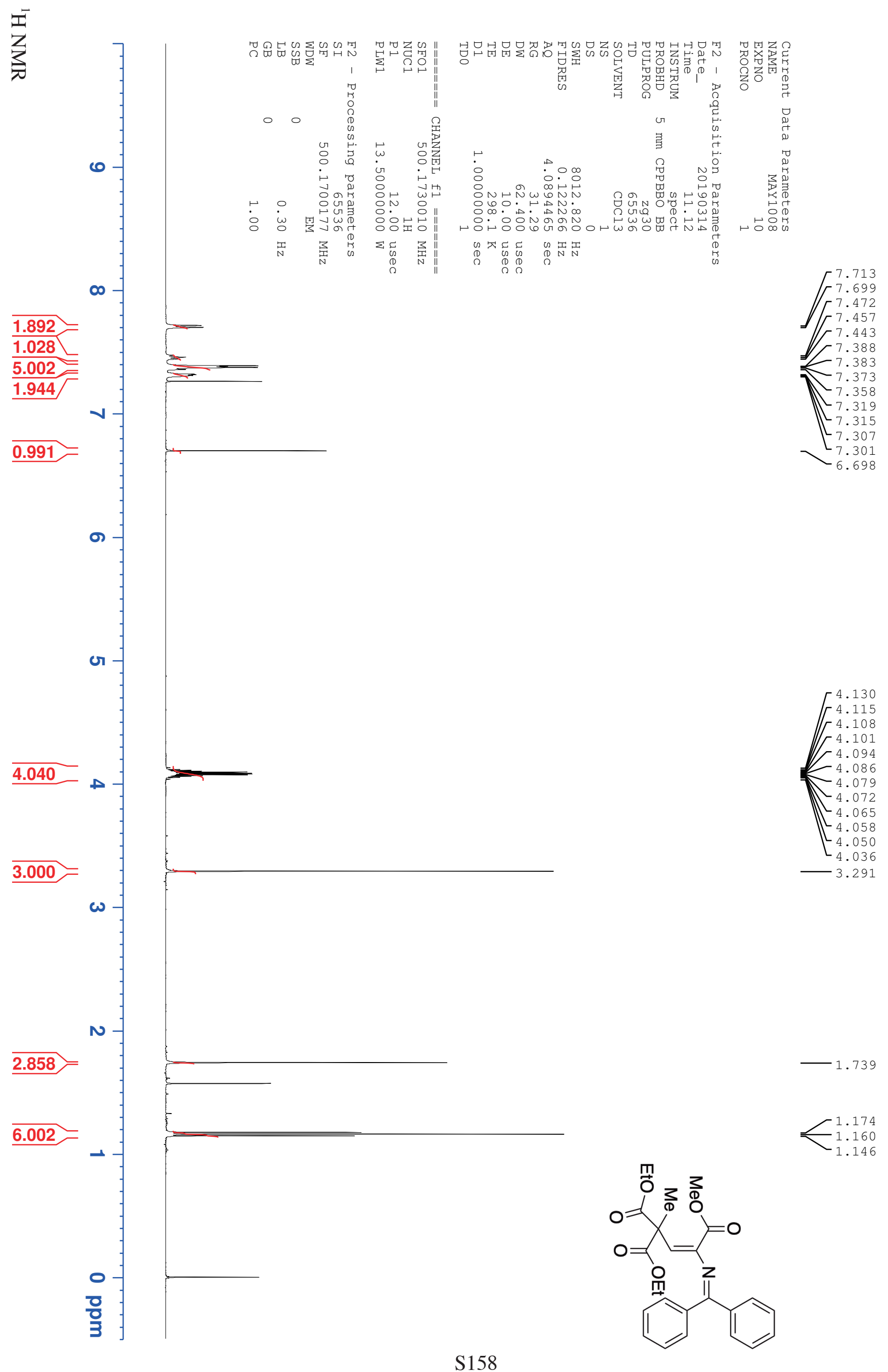




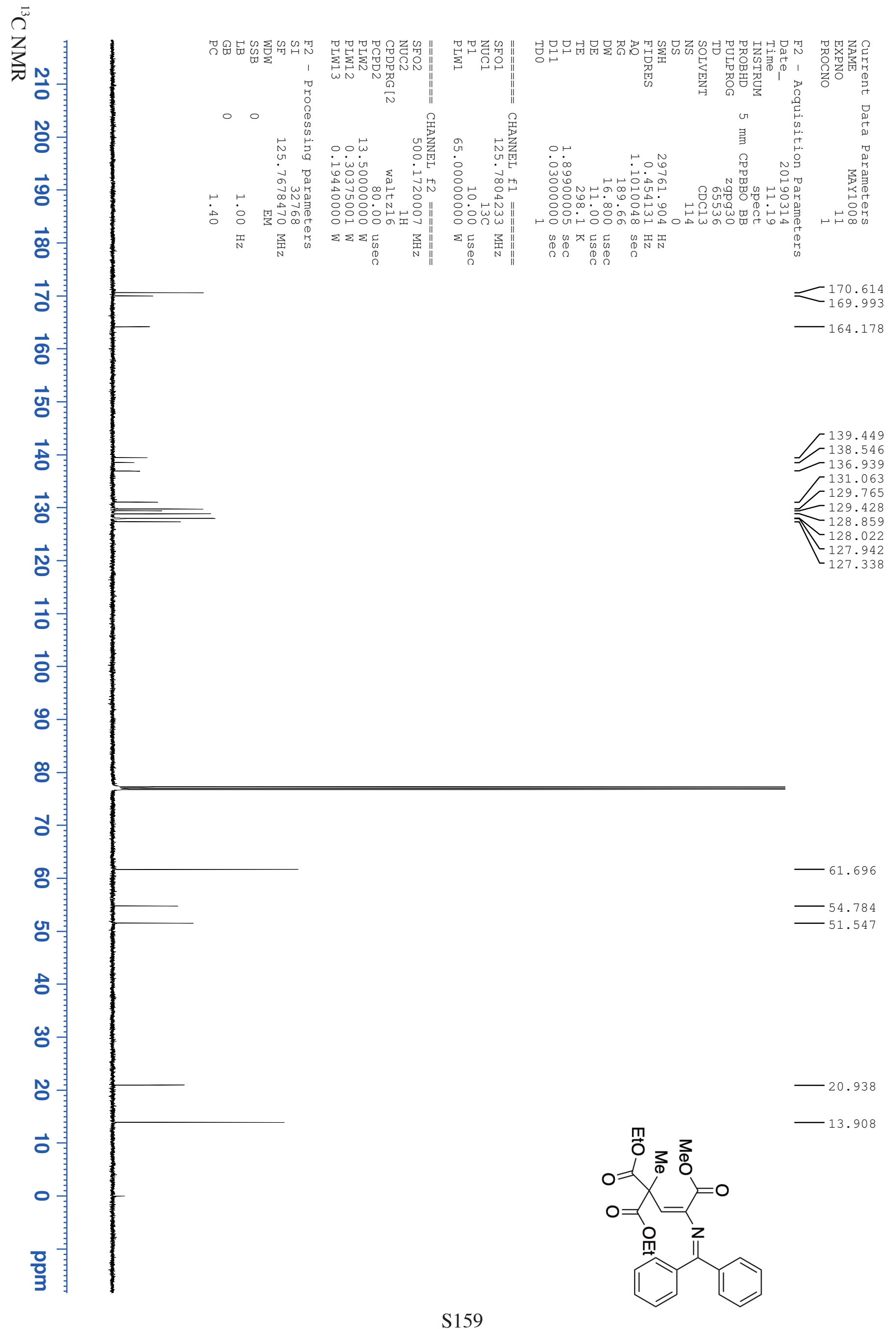



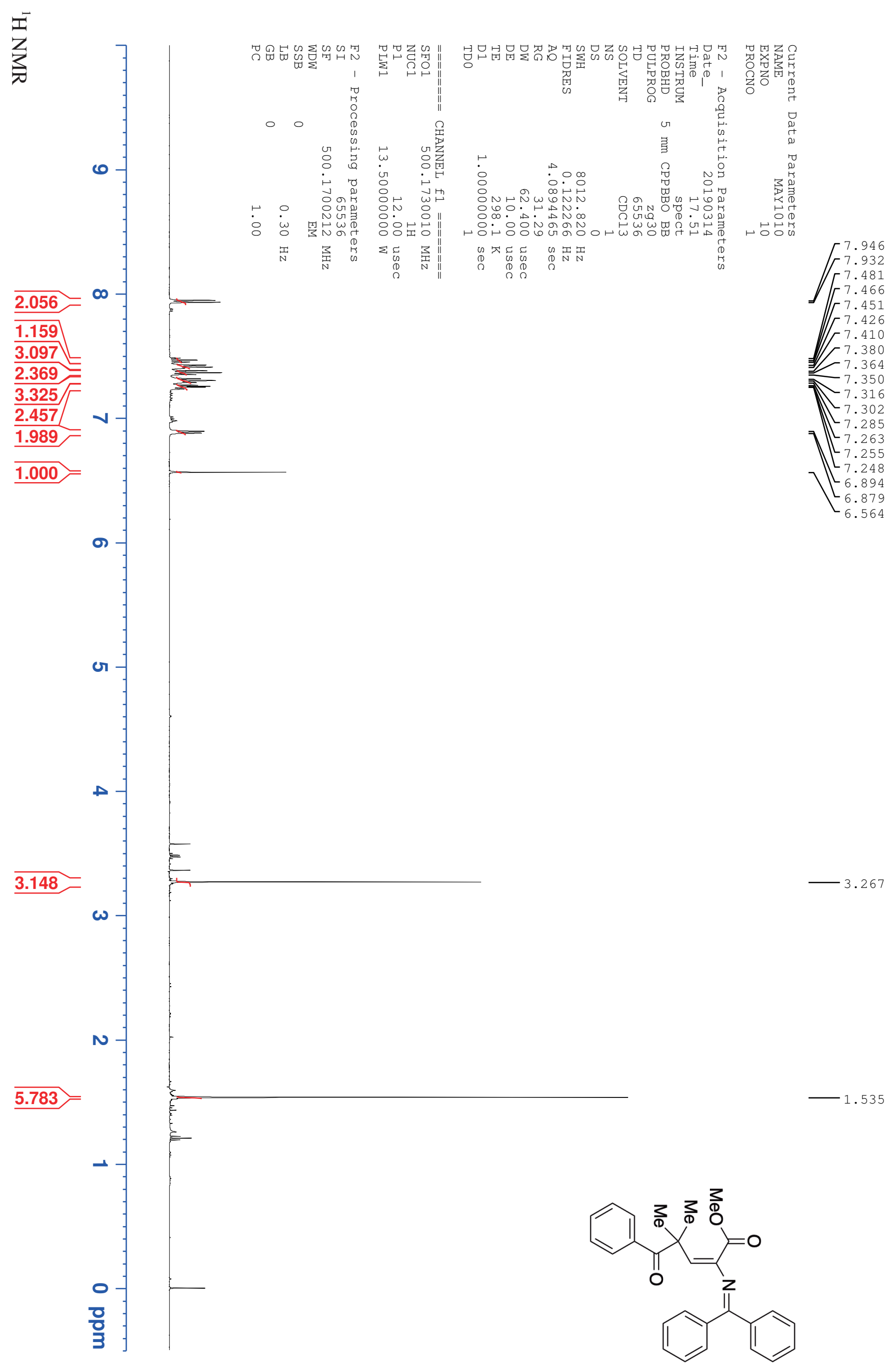


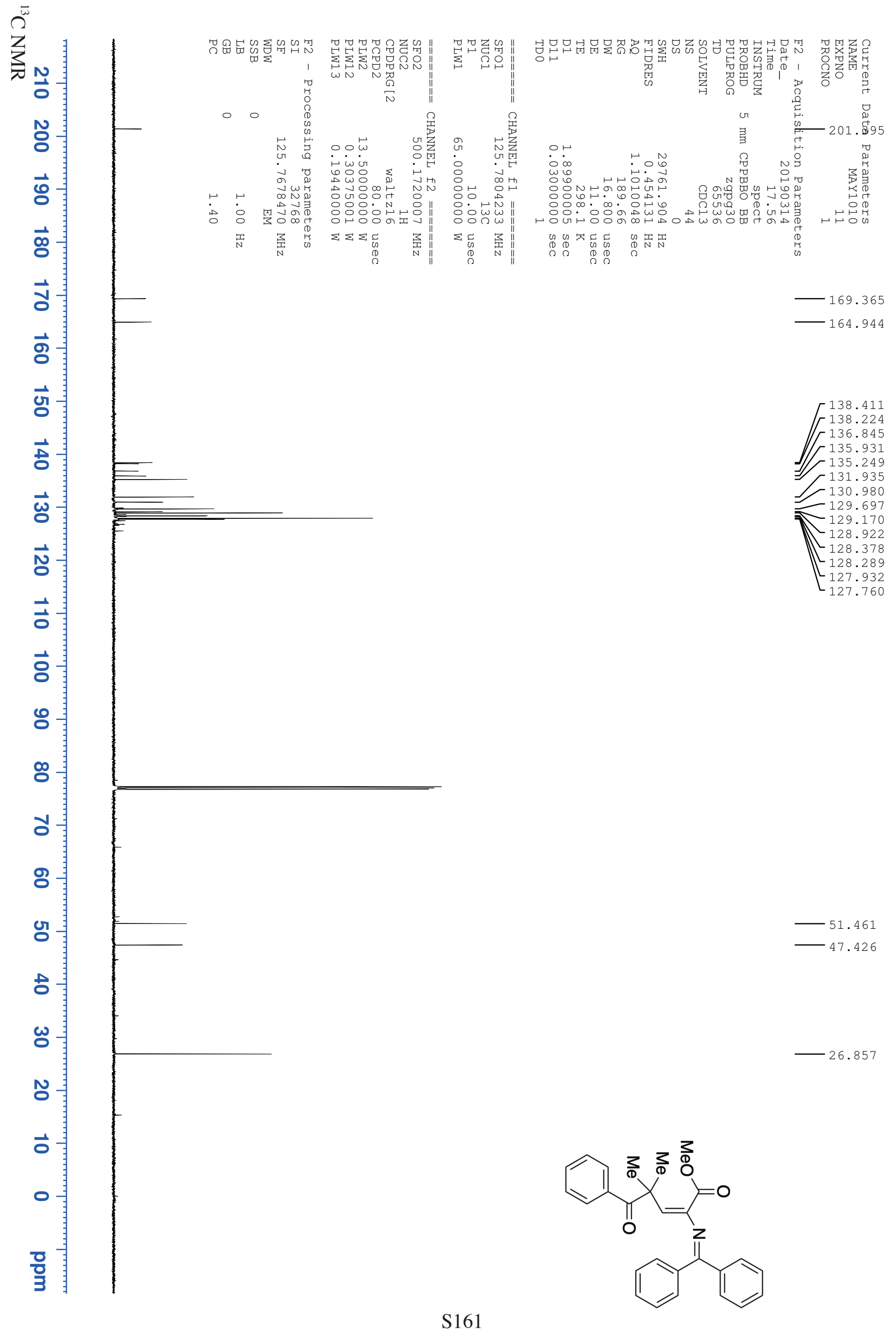




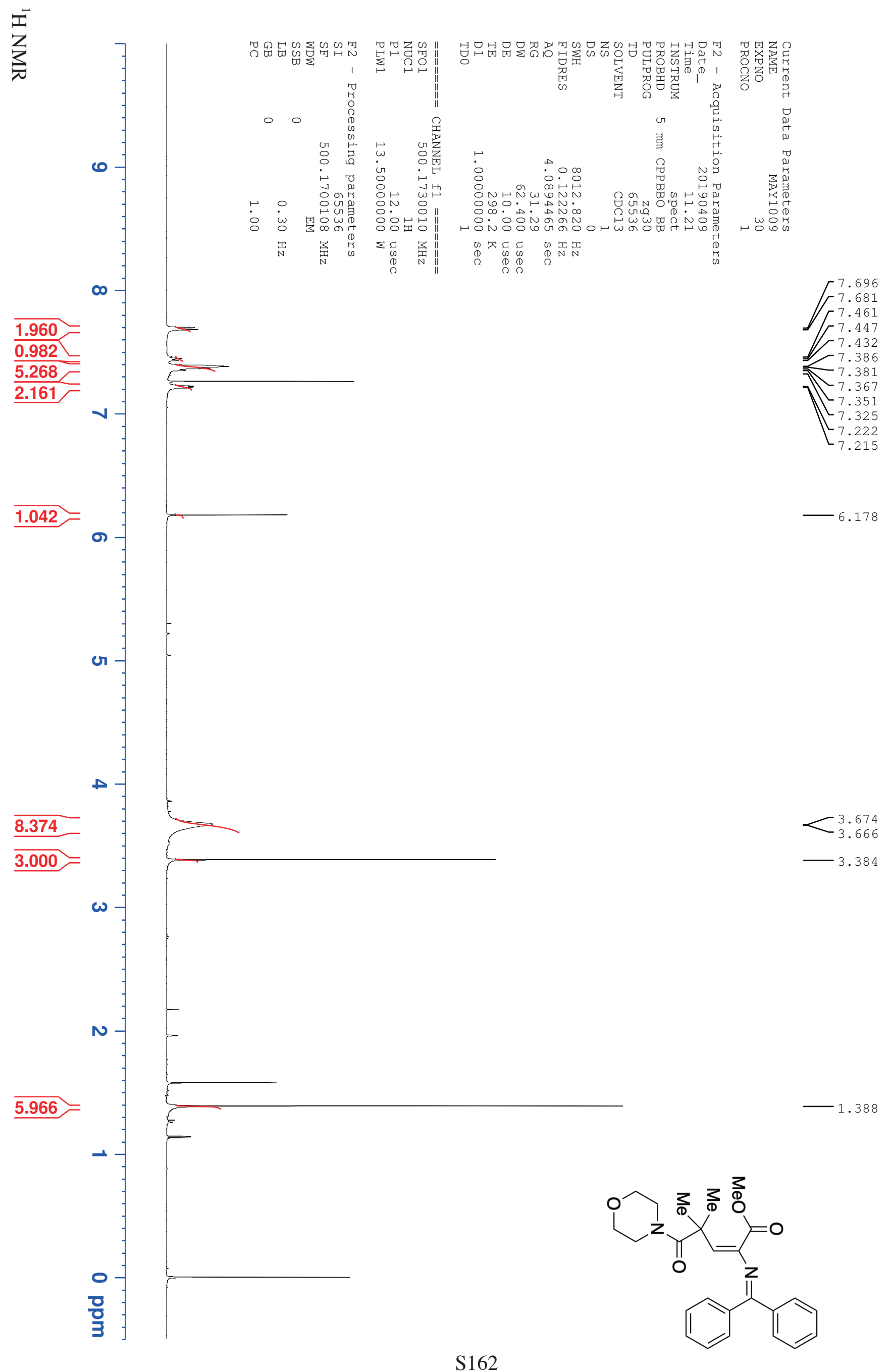




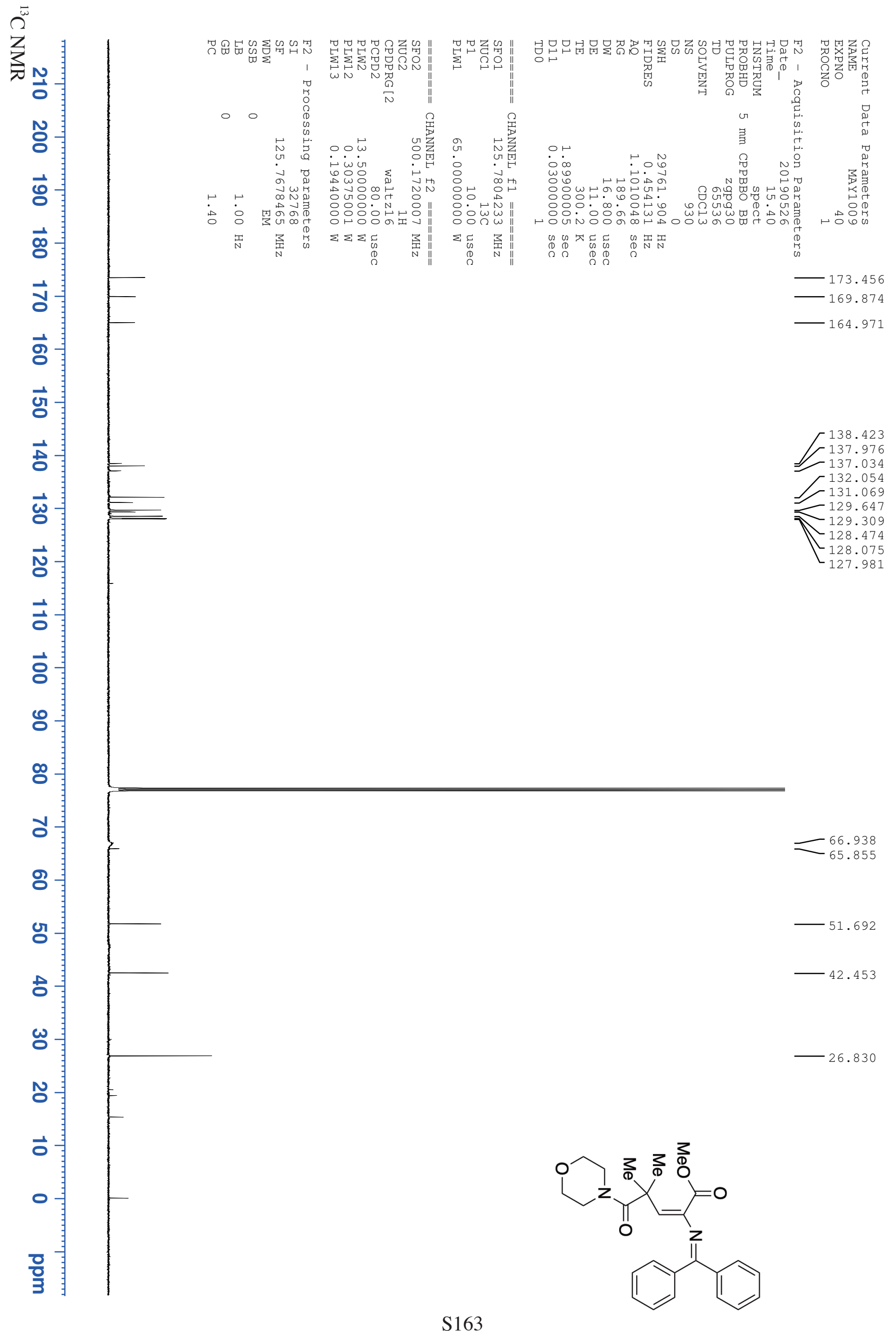




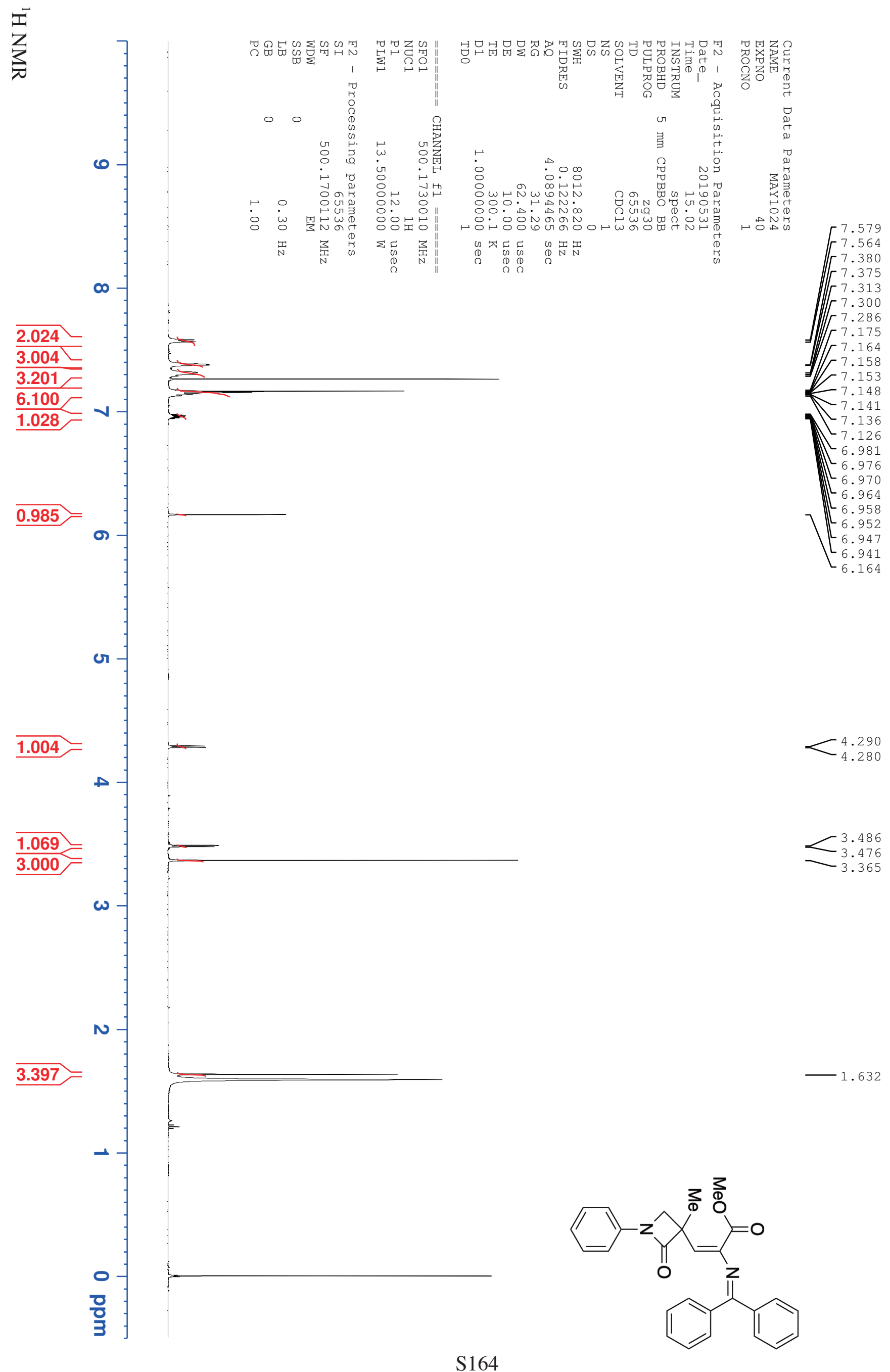



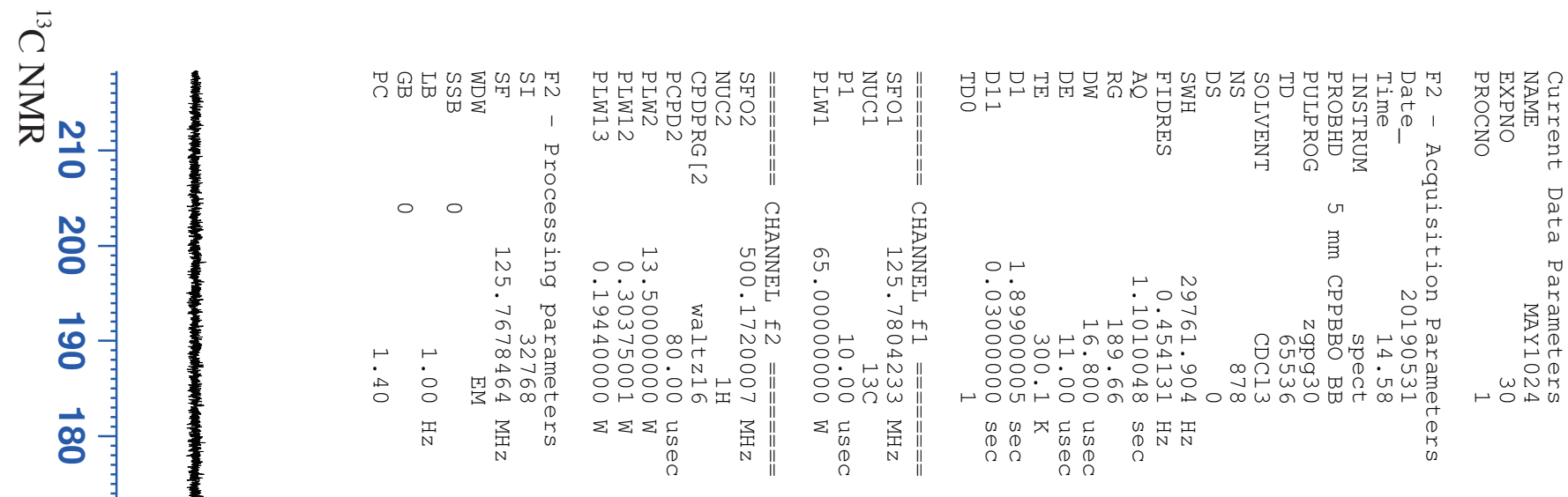

a

a

믐

咅

㟧

종

孛

å

8

๓

ปั

8

gr

\&

$\omega$

ก

o

$\circ$

흡

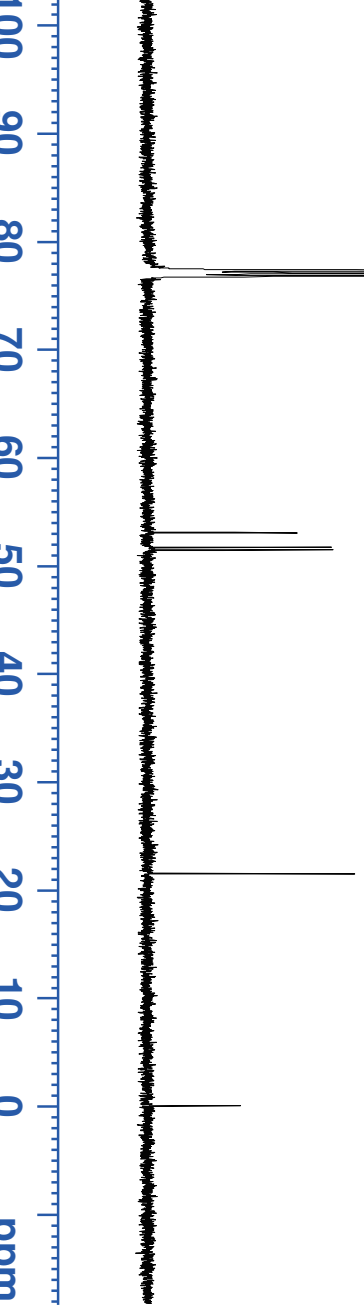

$\Gamma^{140.923}$

138.489

138.215
-136.782

130.914

130.914
12969

$-129.310$

128.841

$\sqrt{127.935}$

$-127.816$

$-125.268$

123.495
116.204

$\overrightarrow{0}$

$-170.516$

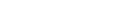




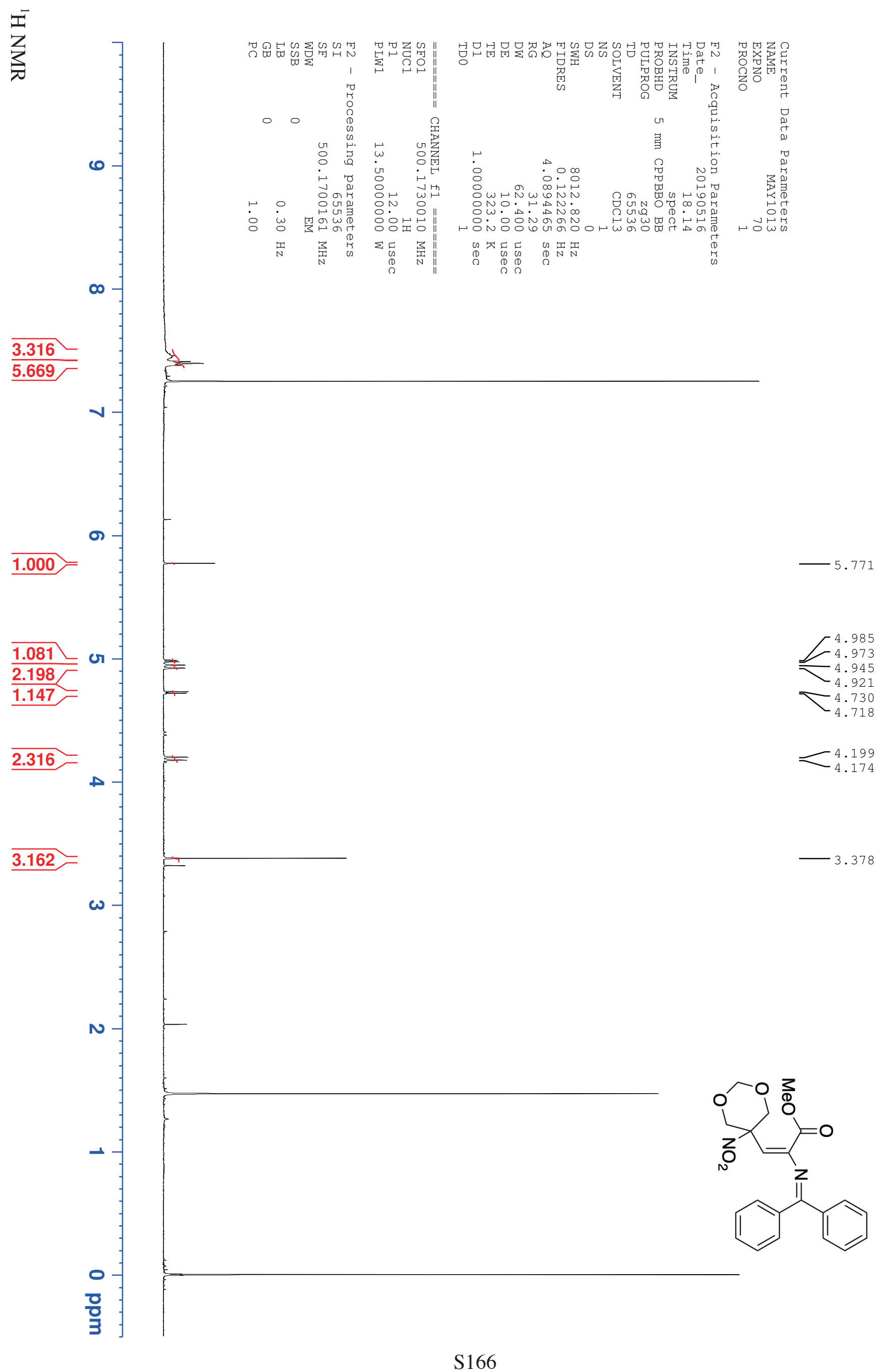




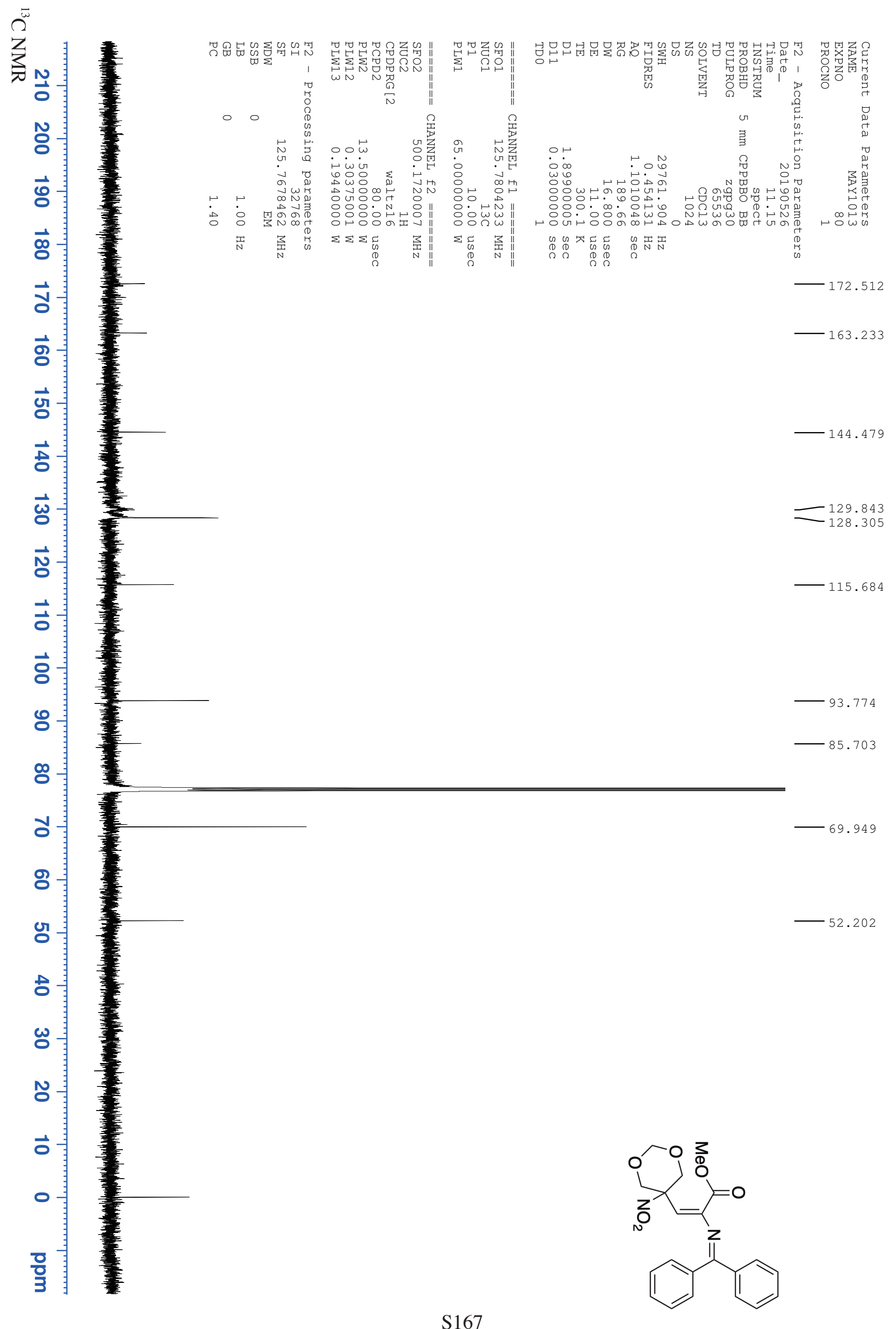




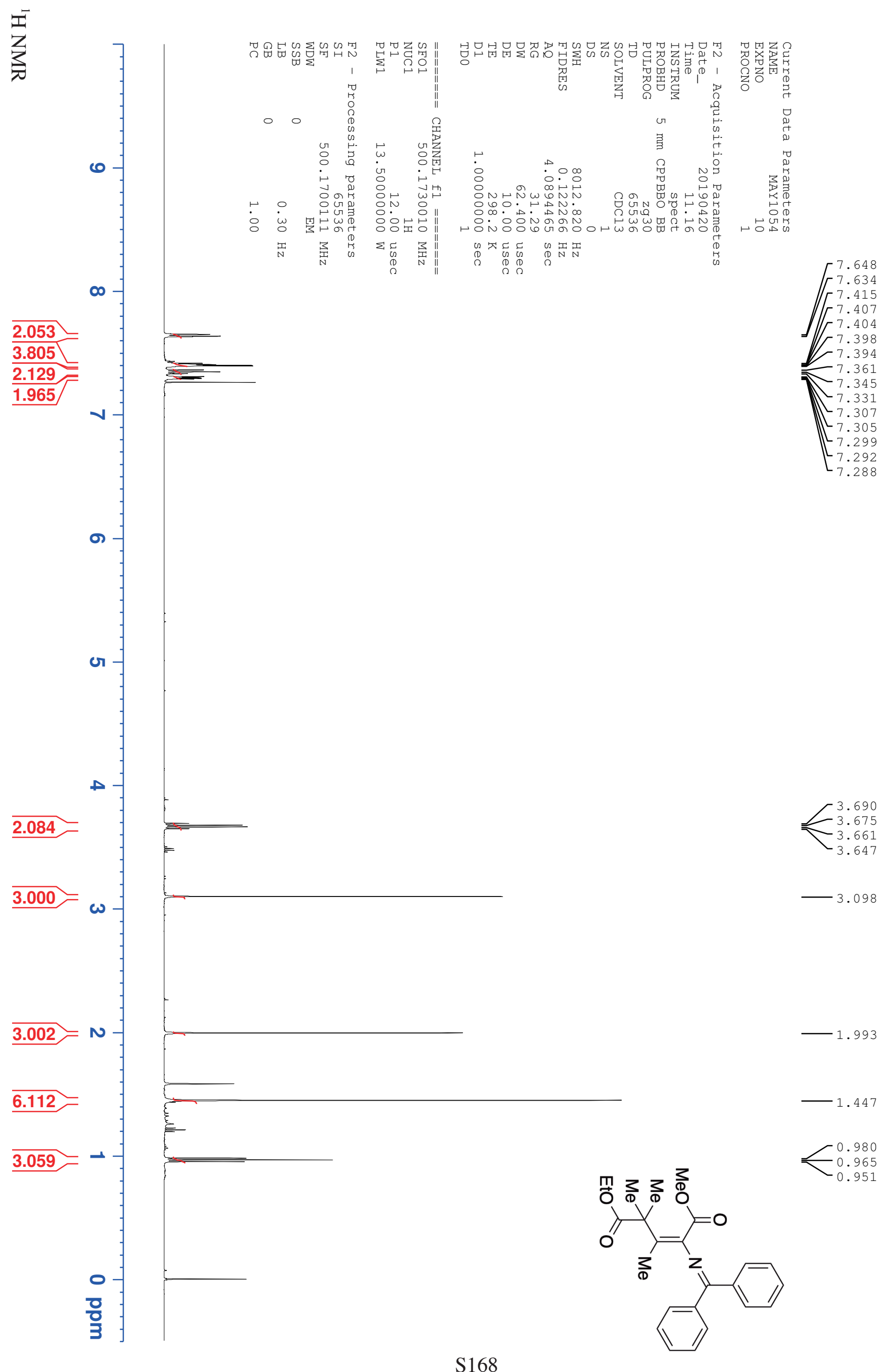




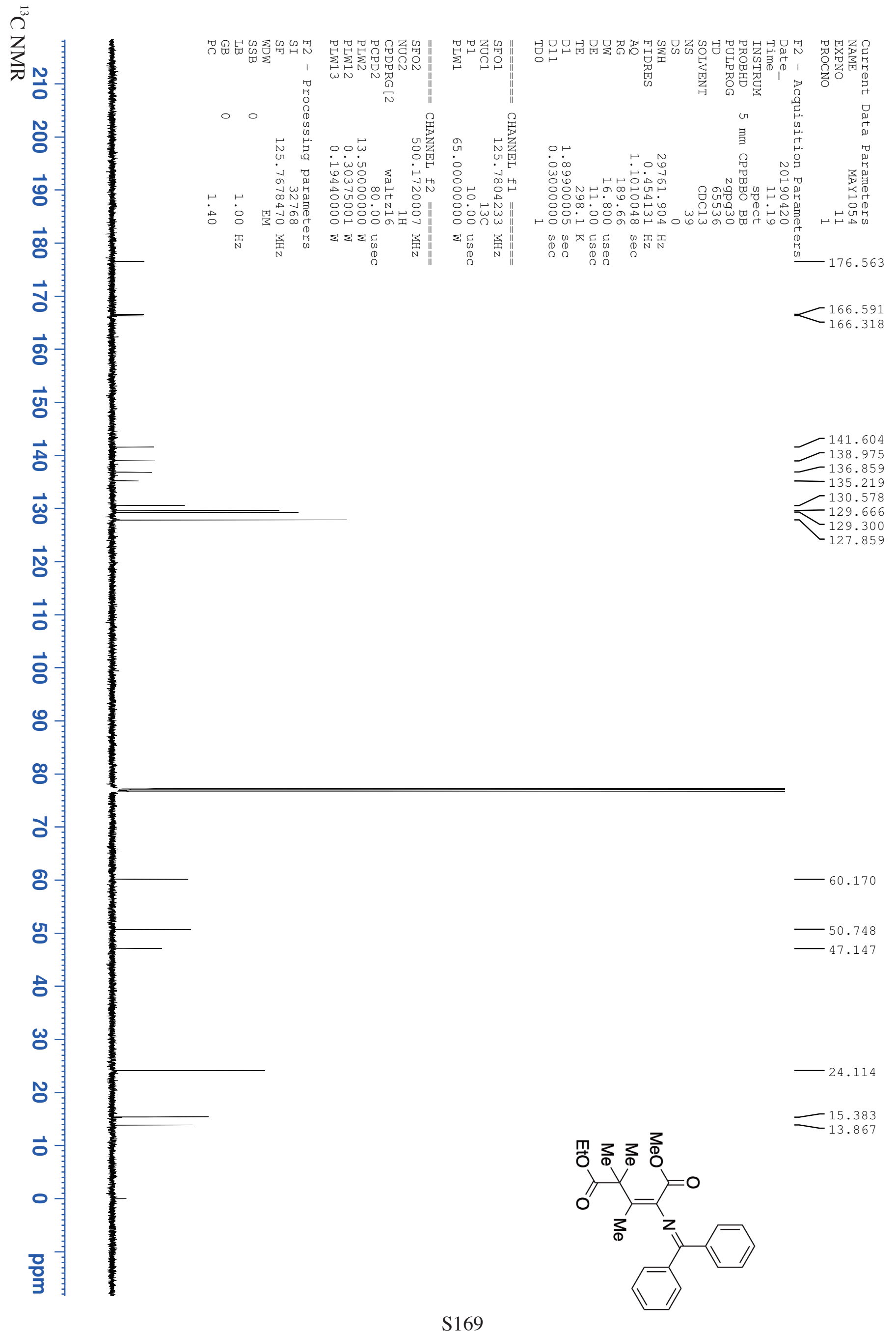




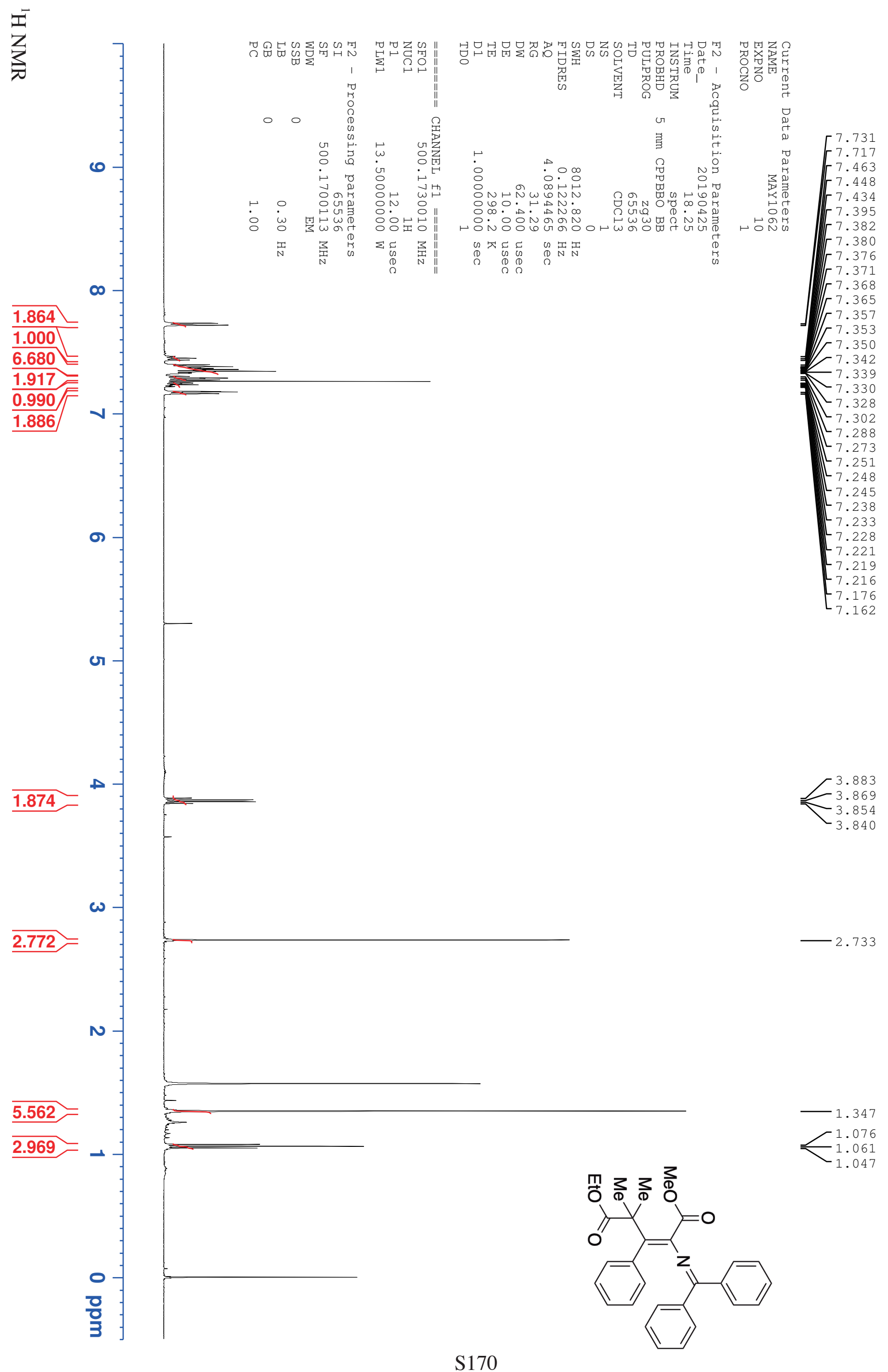




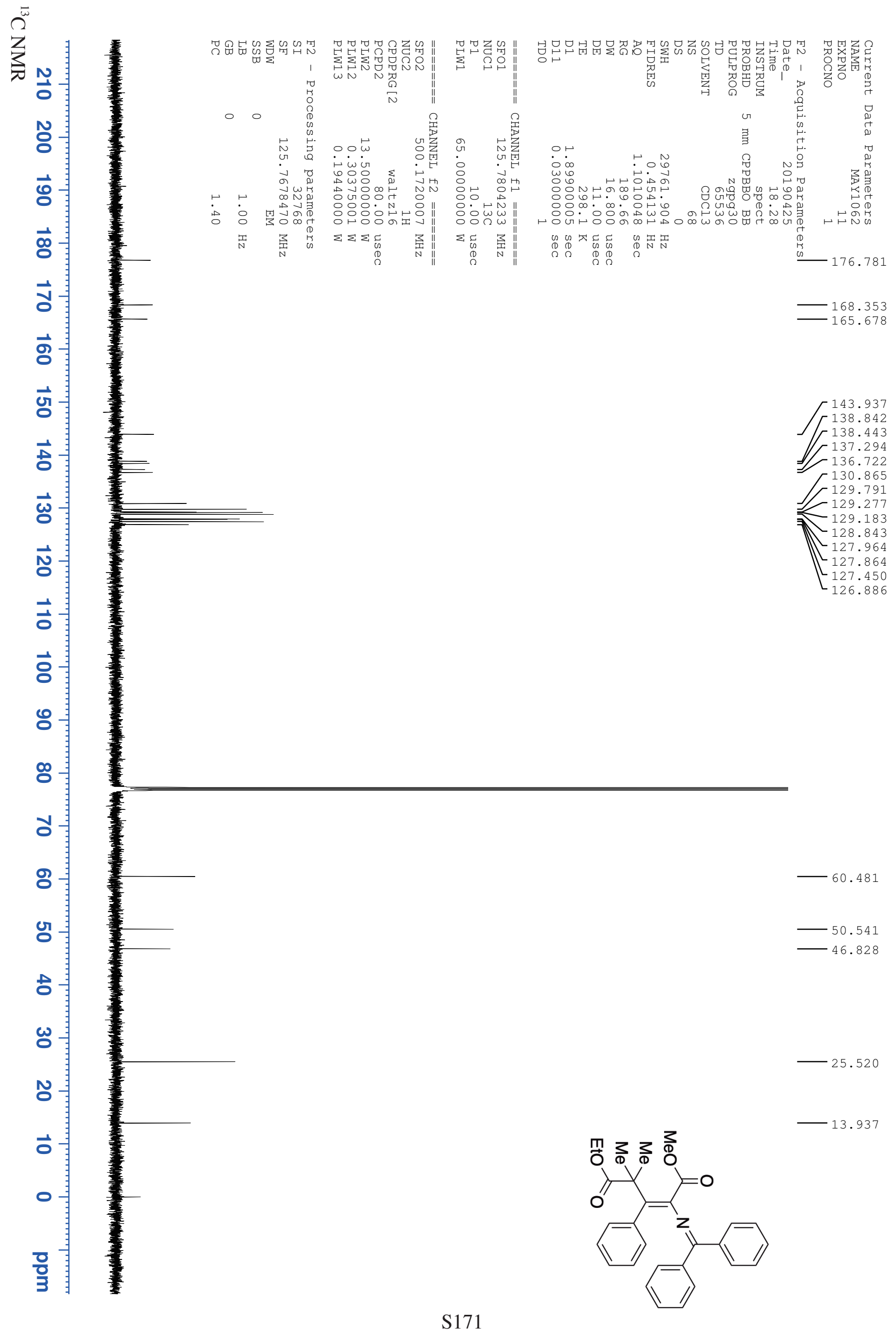



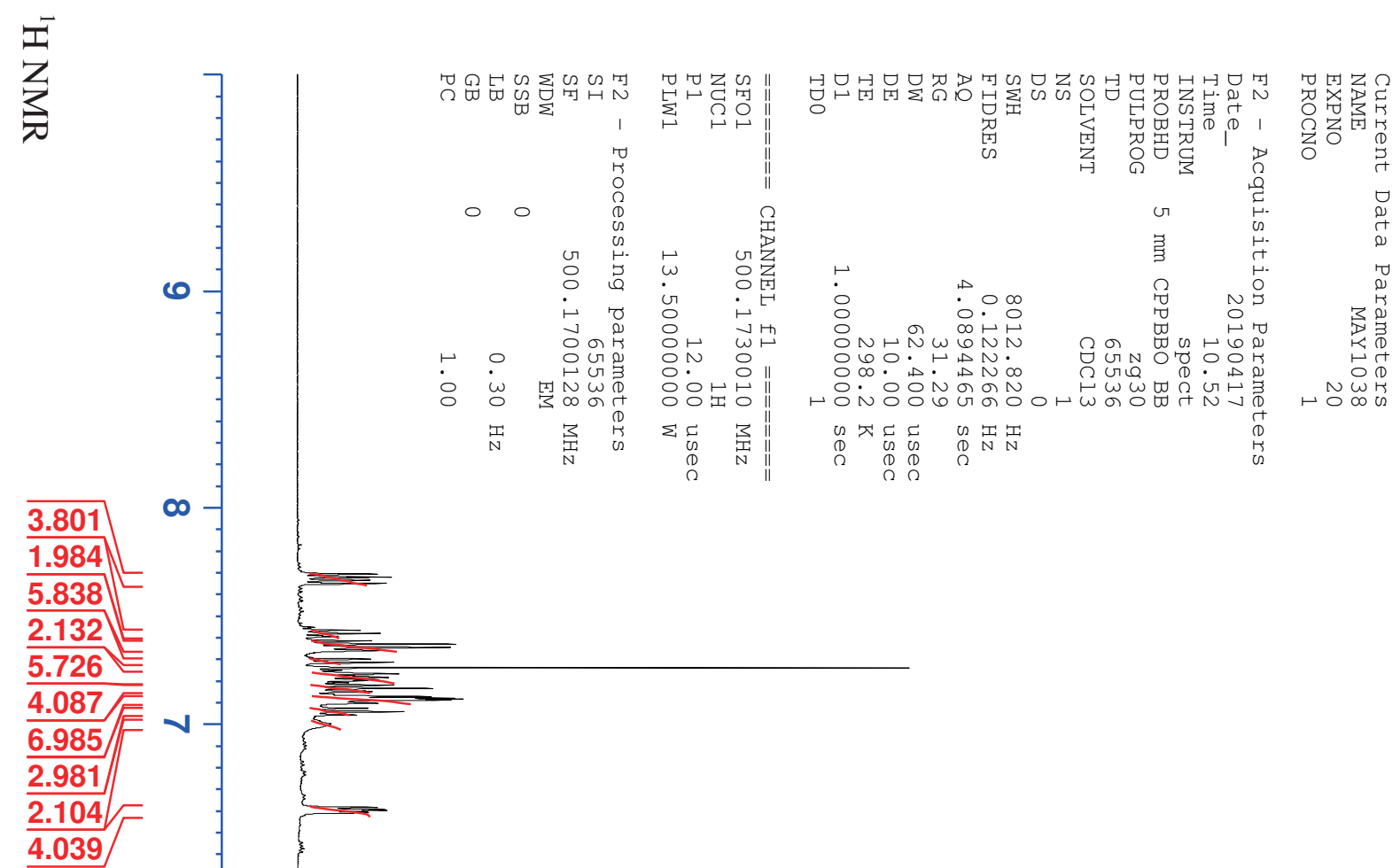

4.039
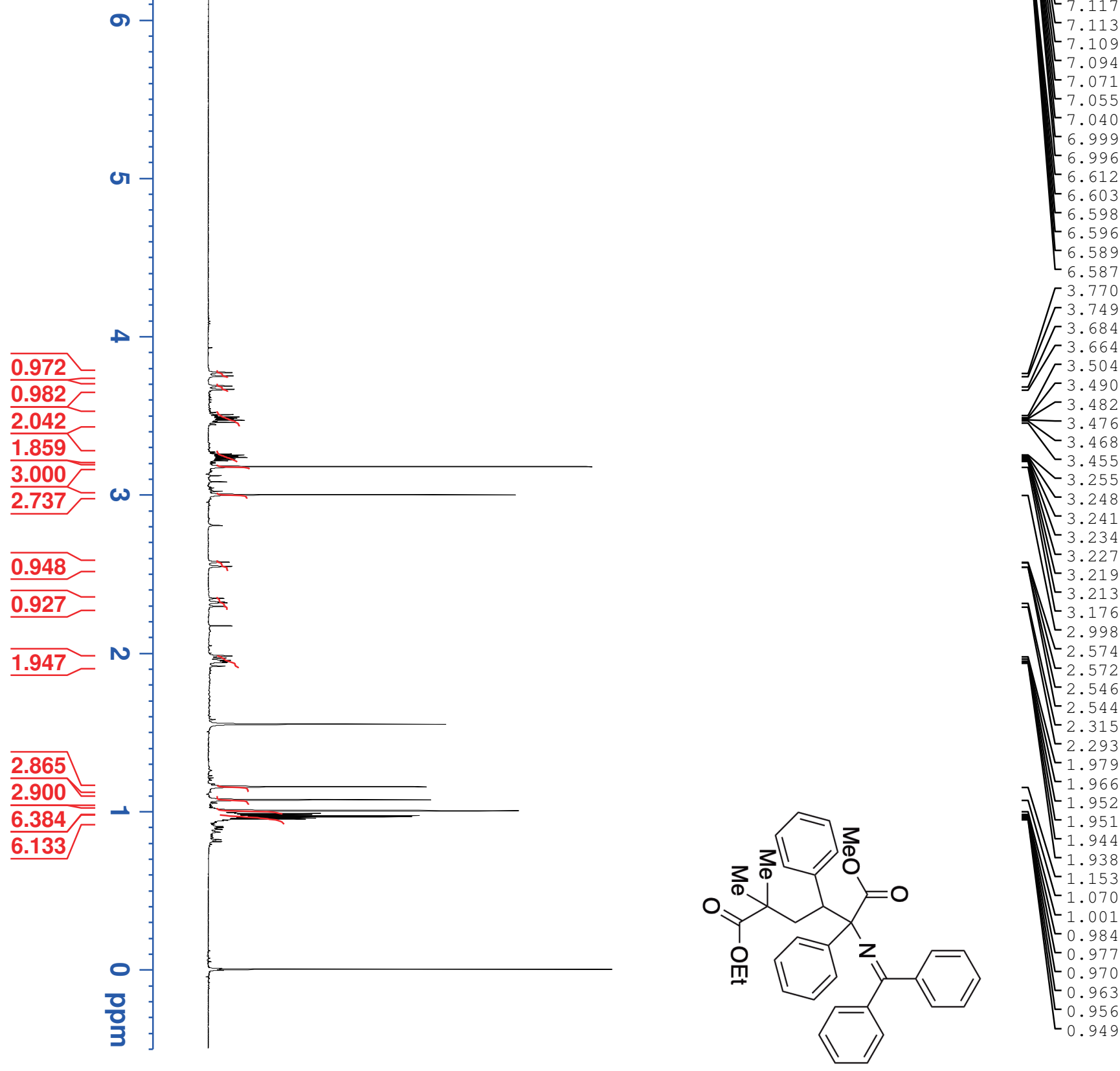


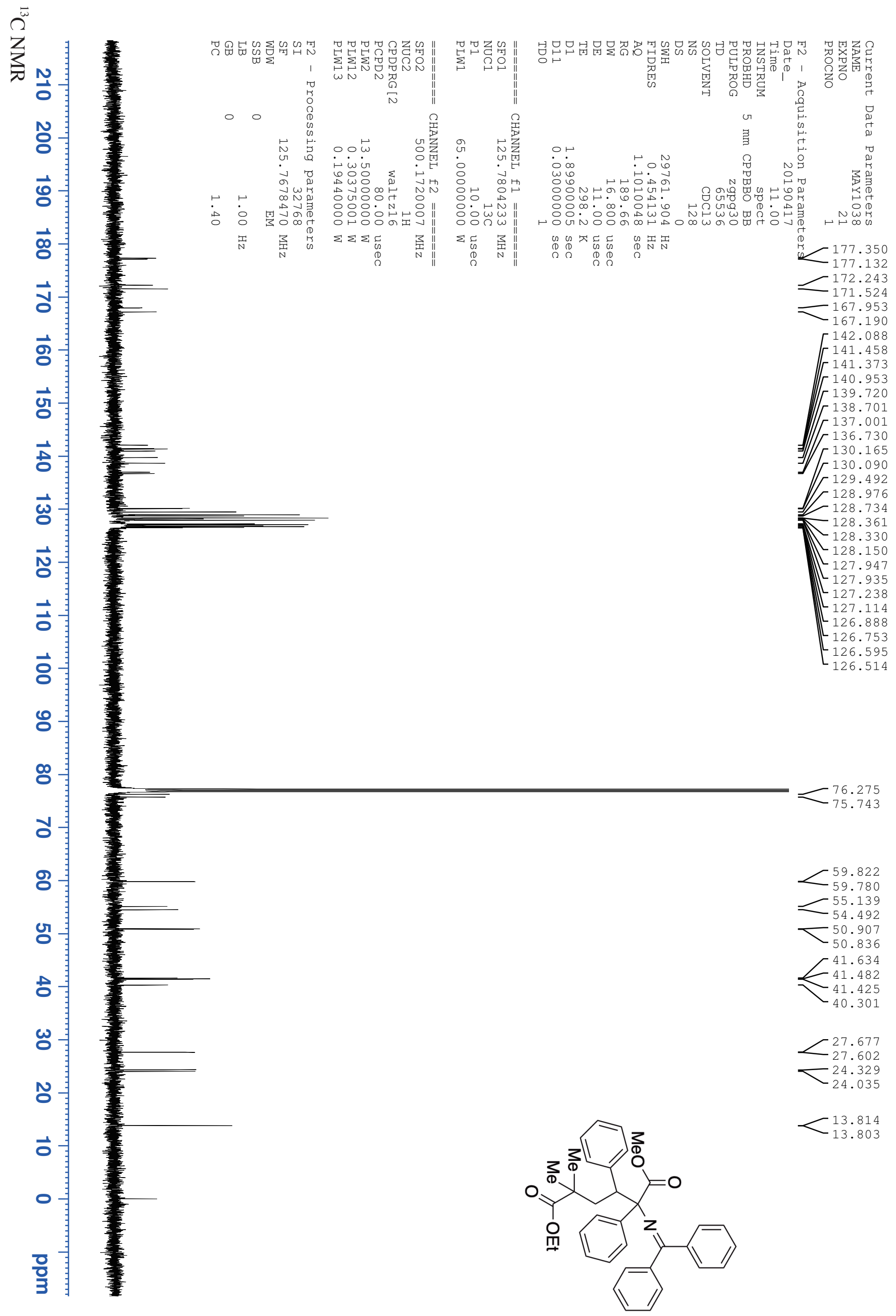




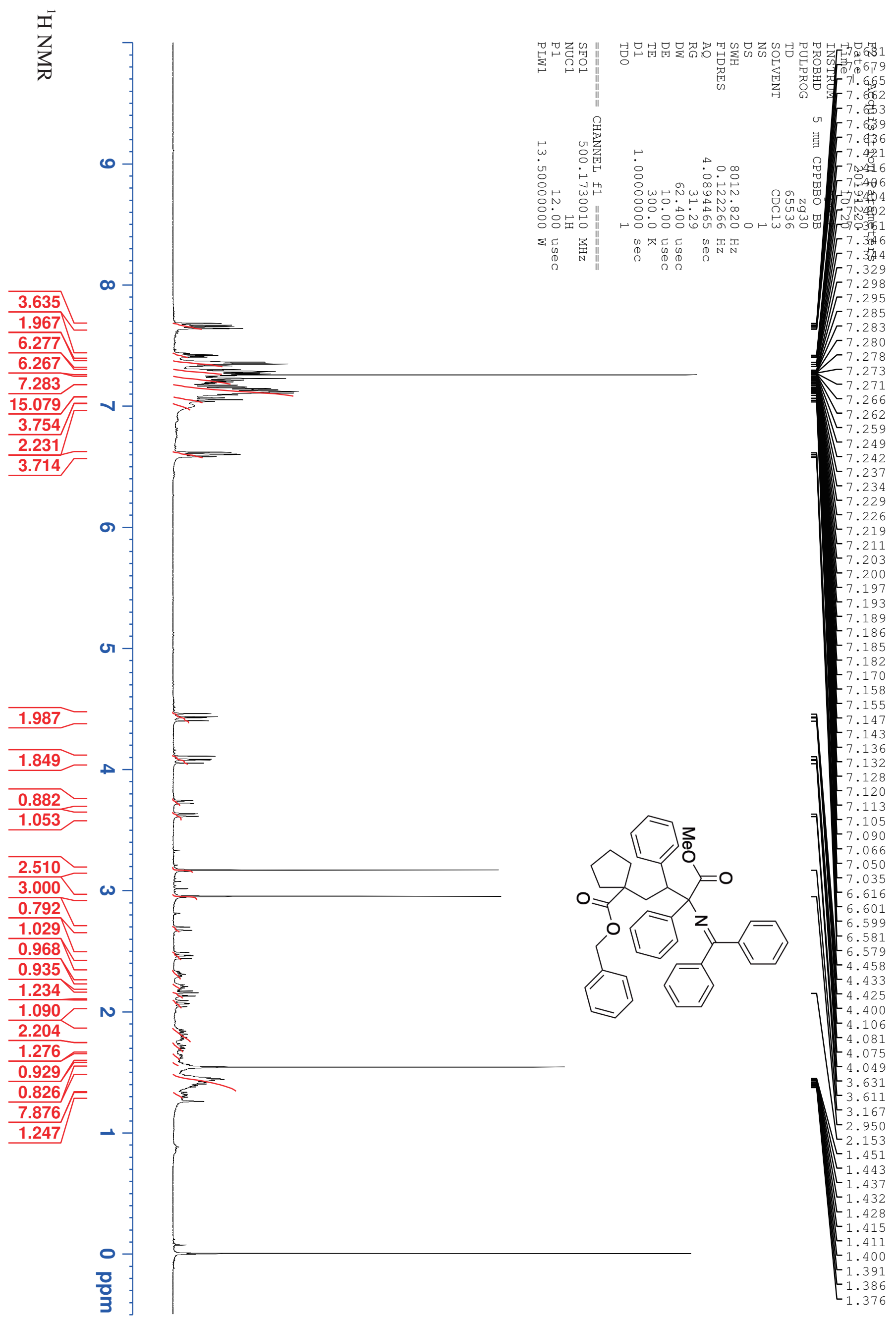




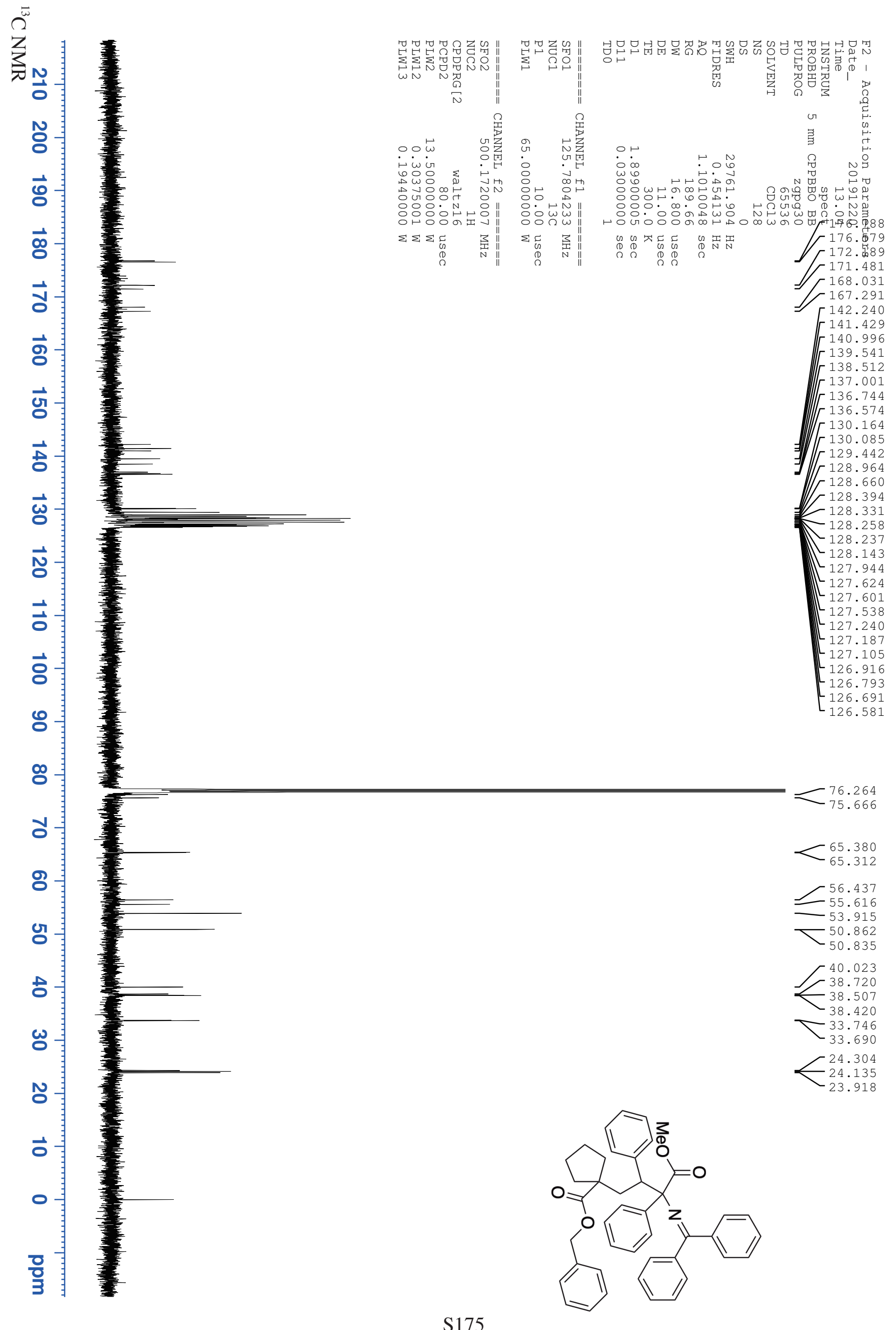




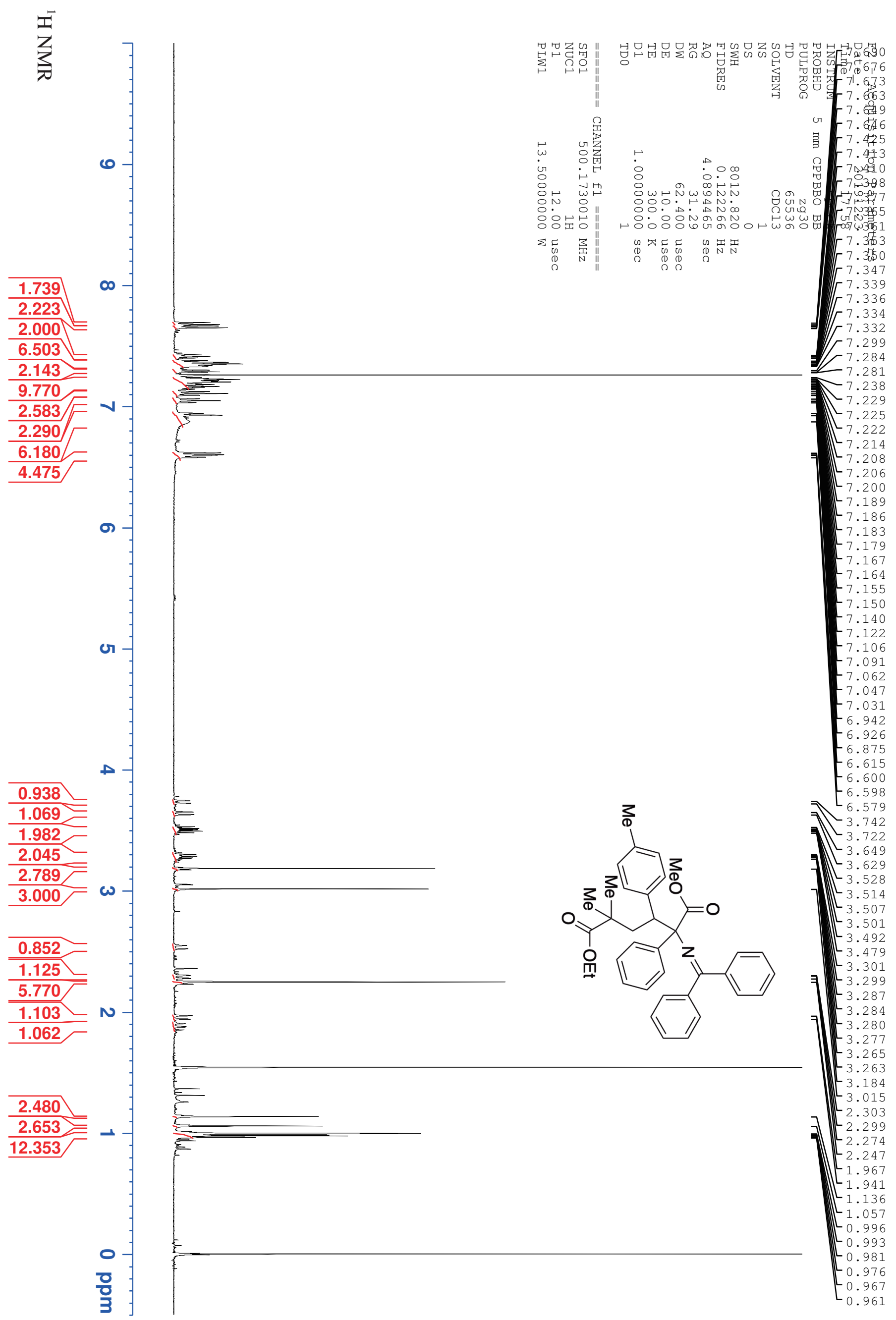




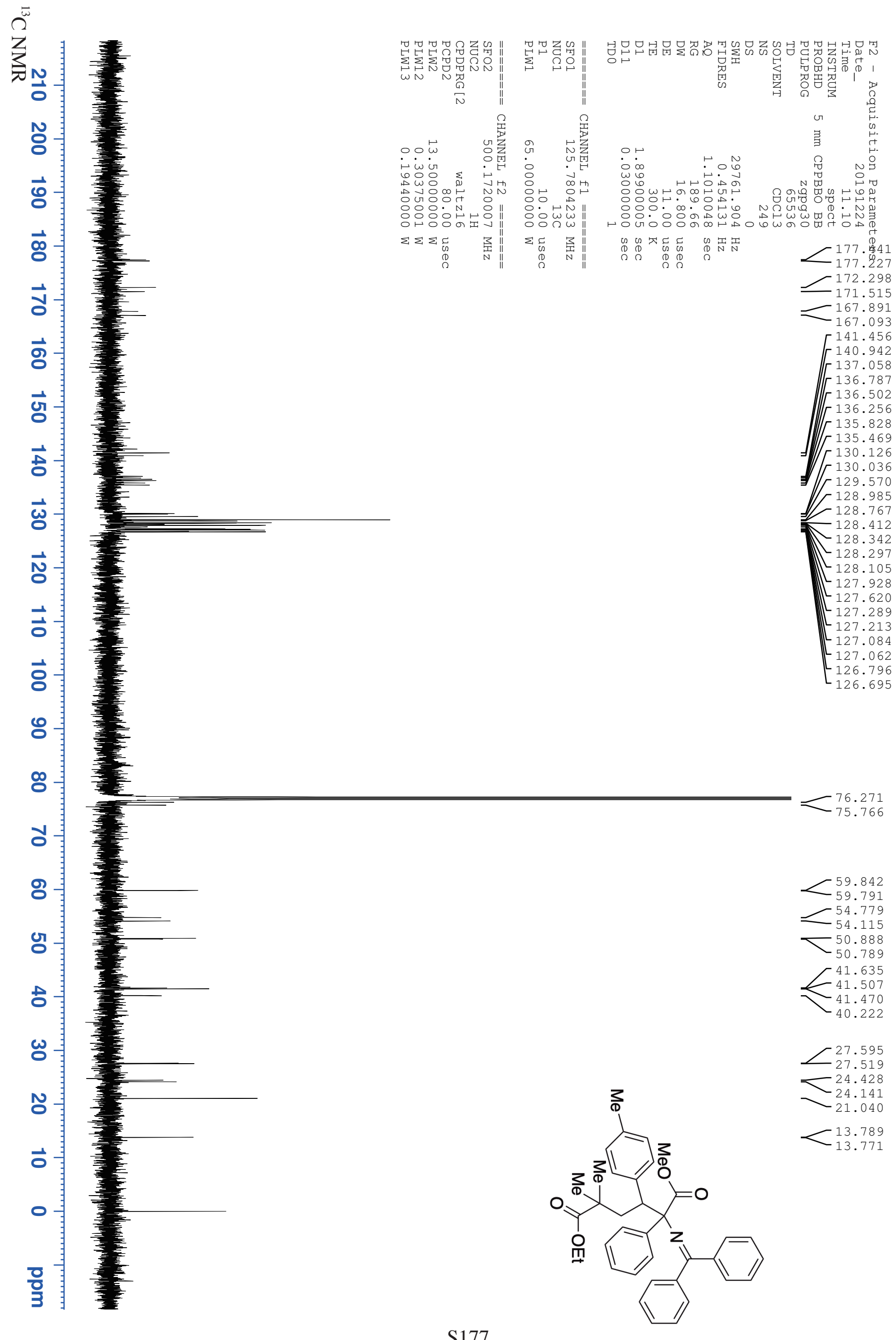




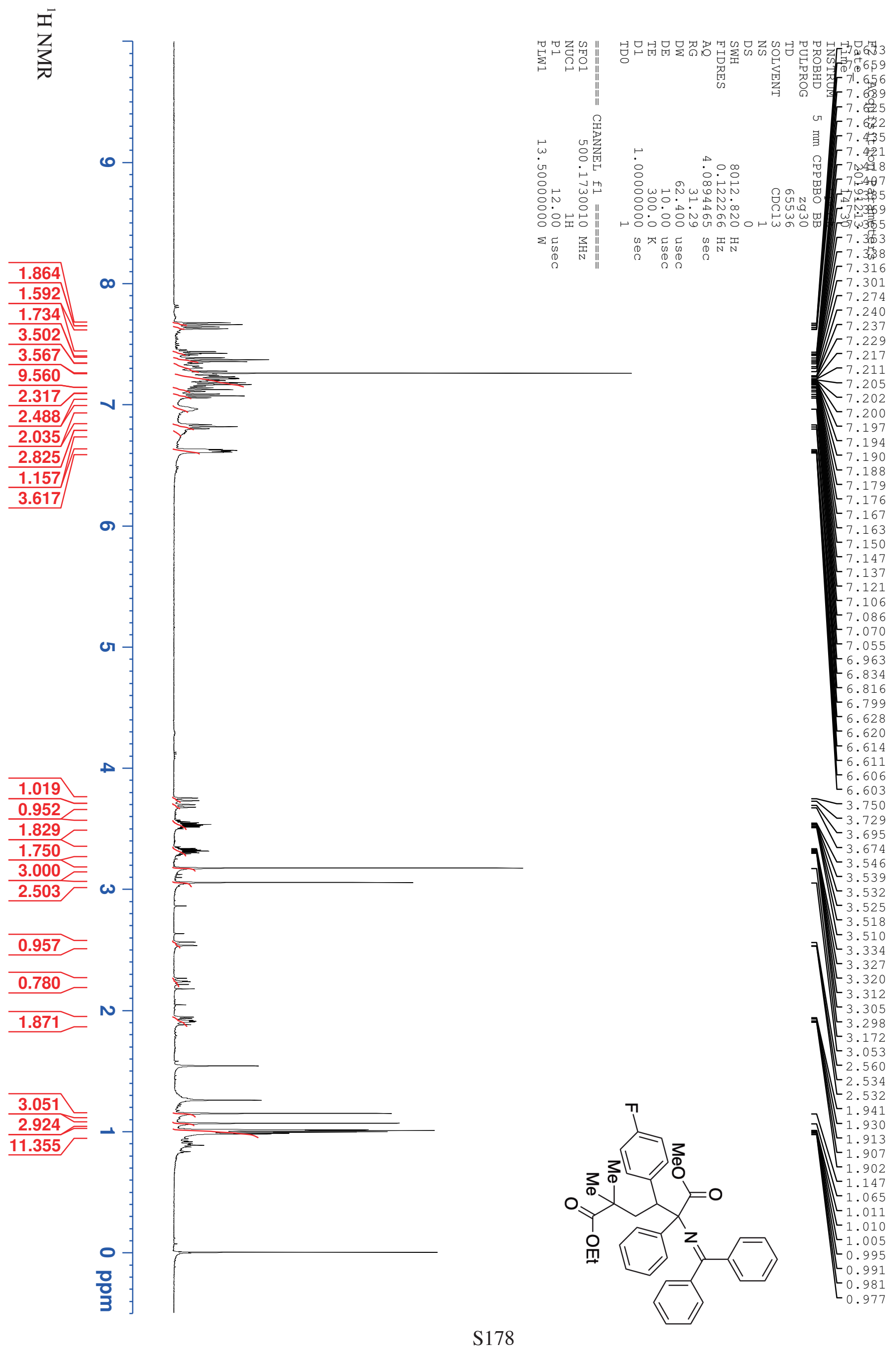




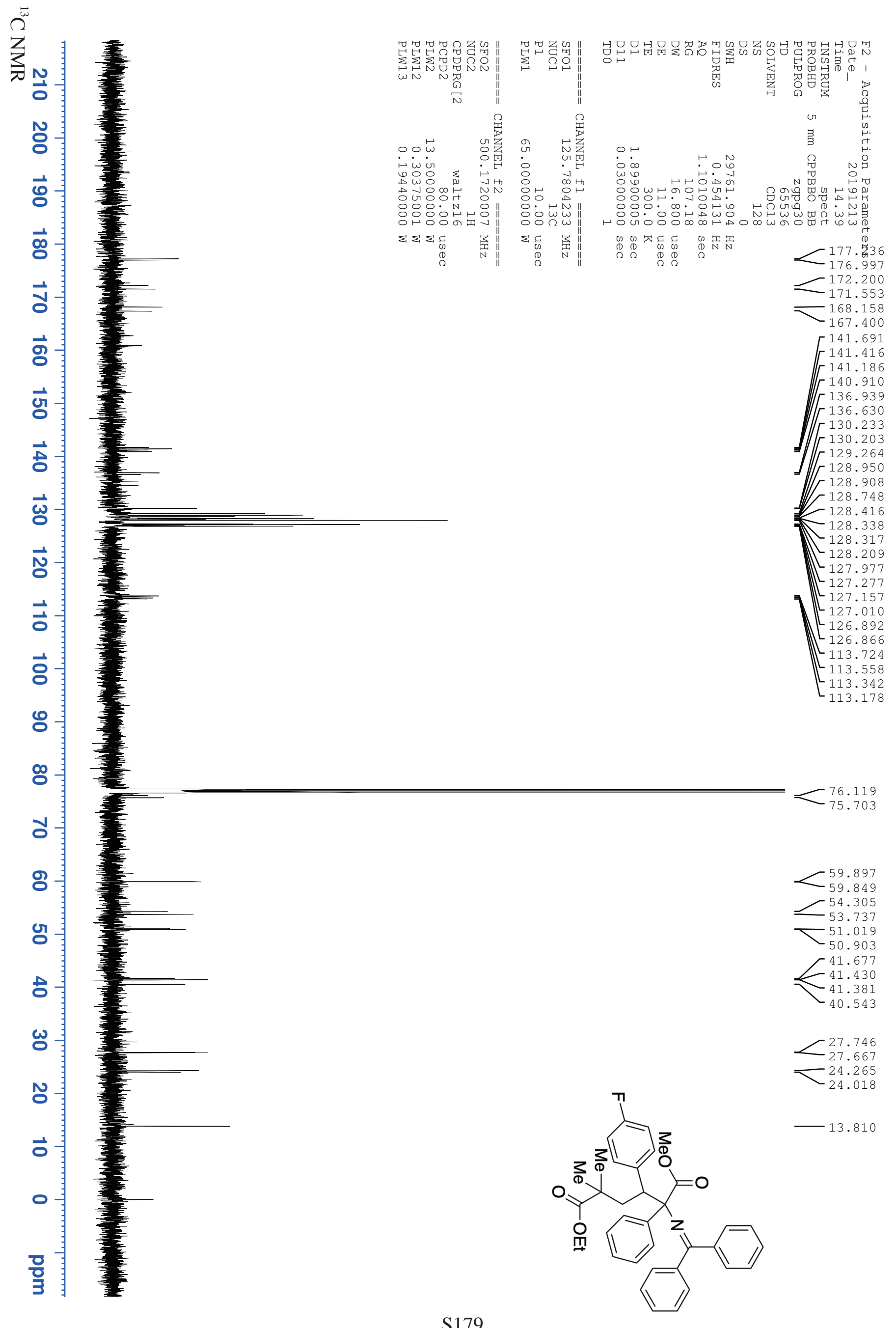




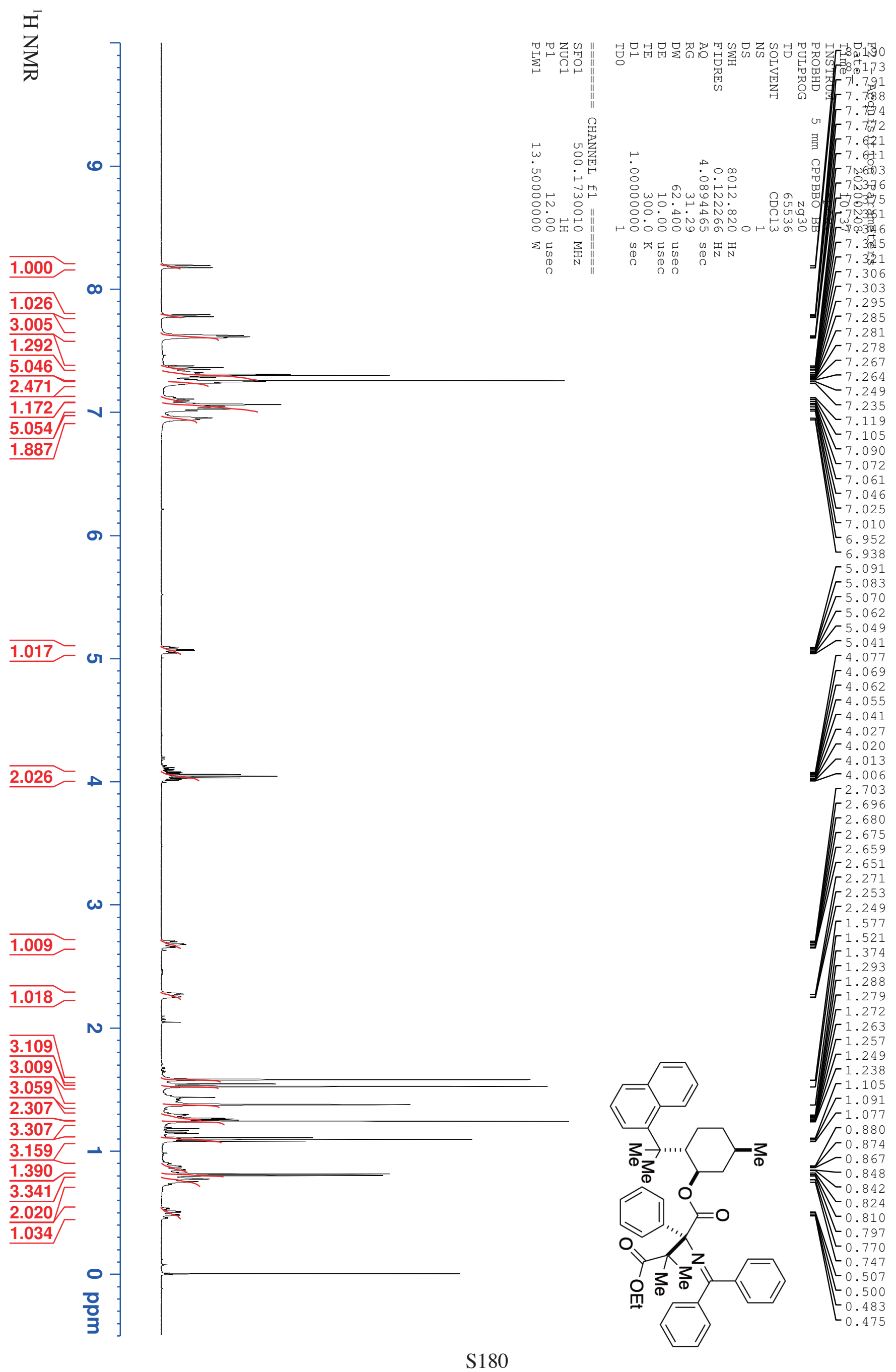




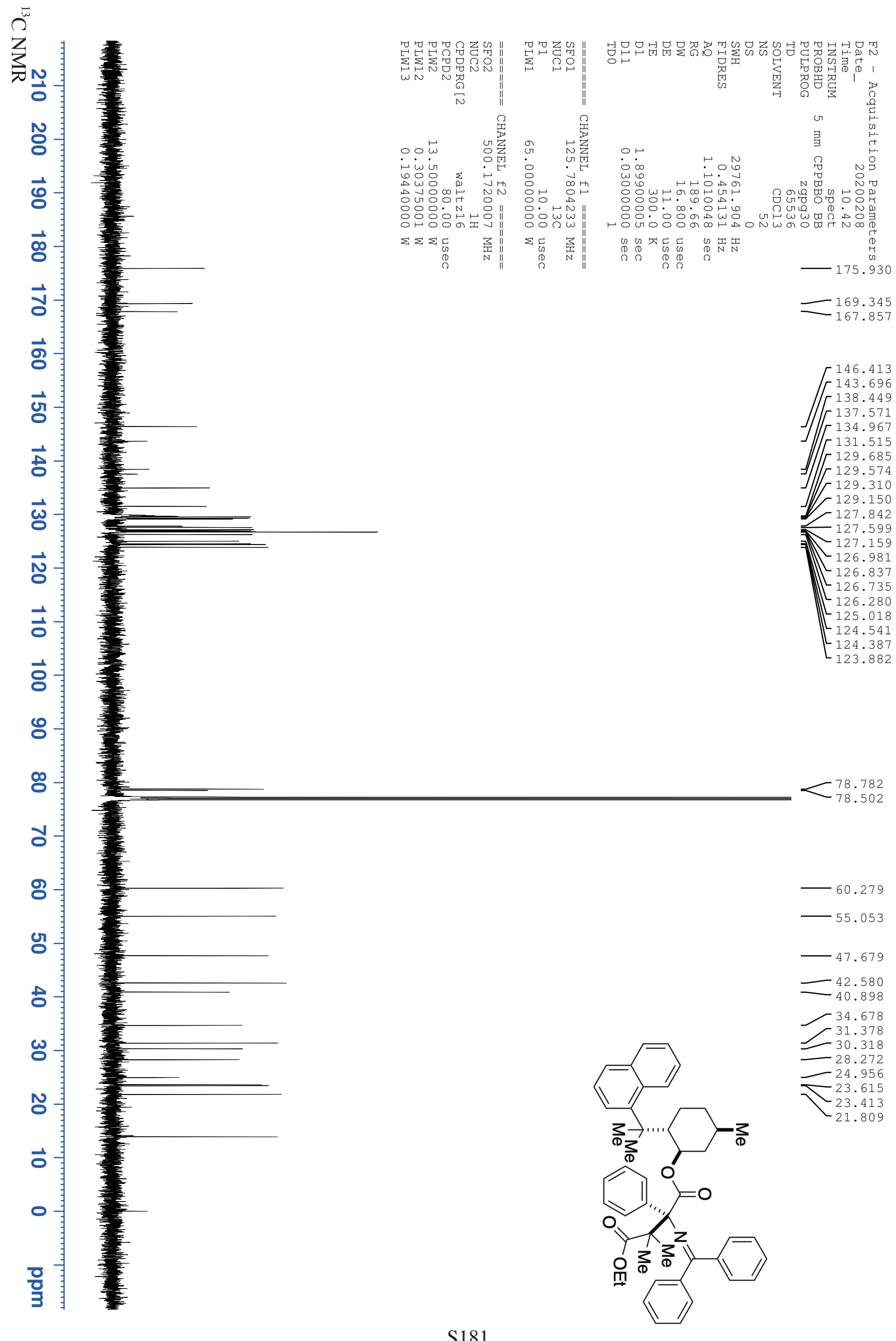




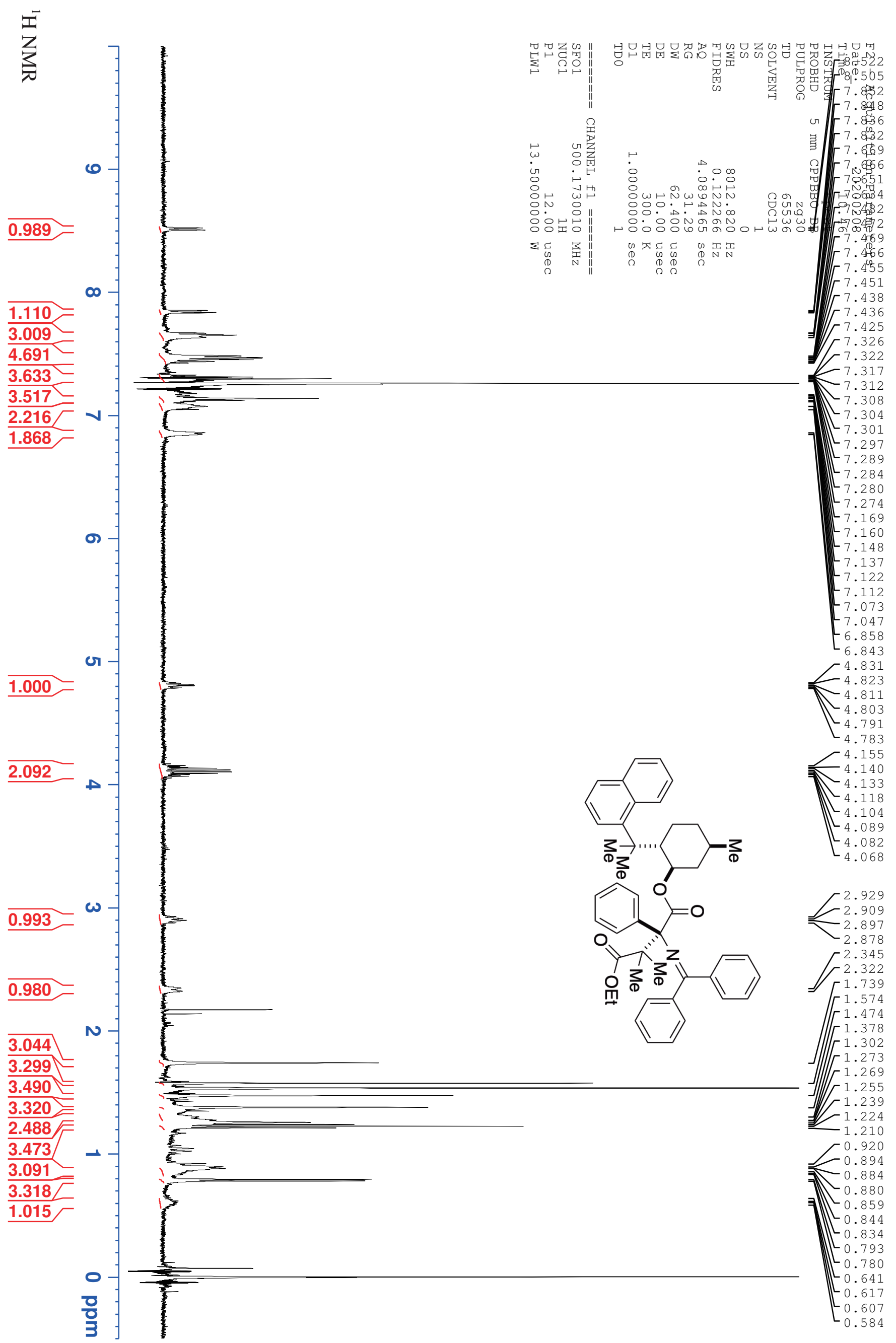




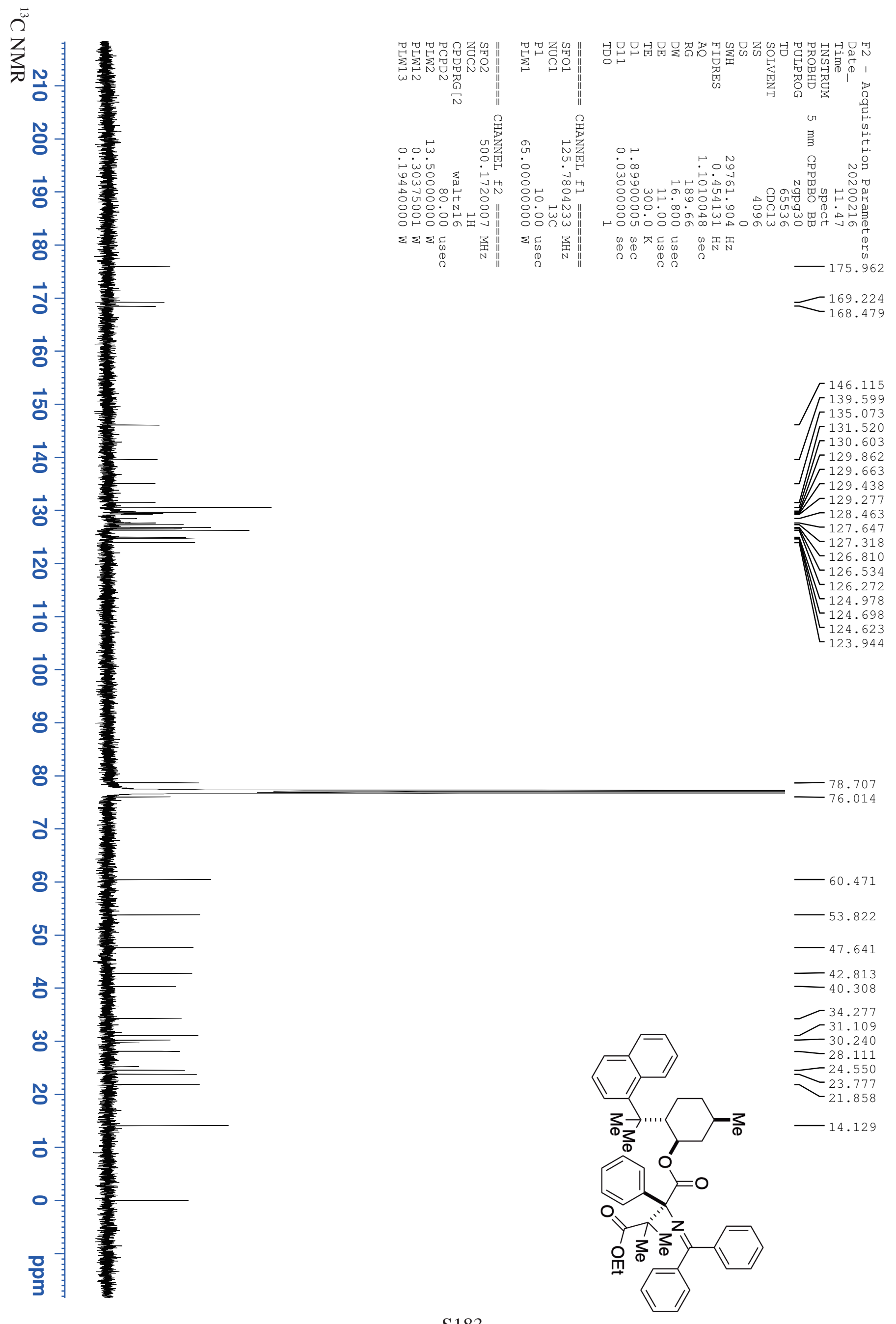




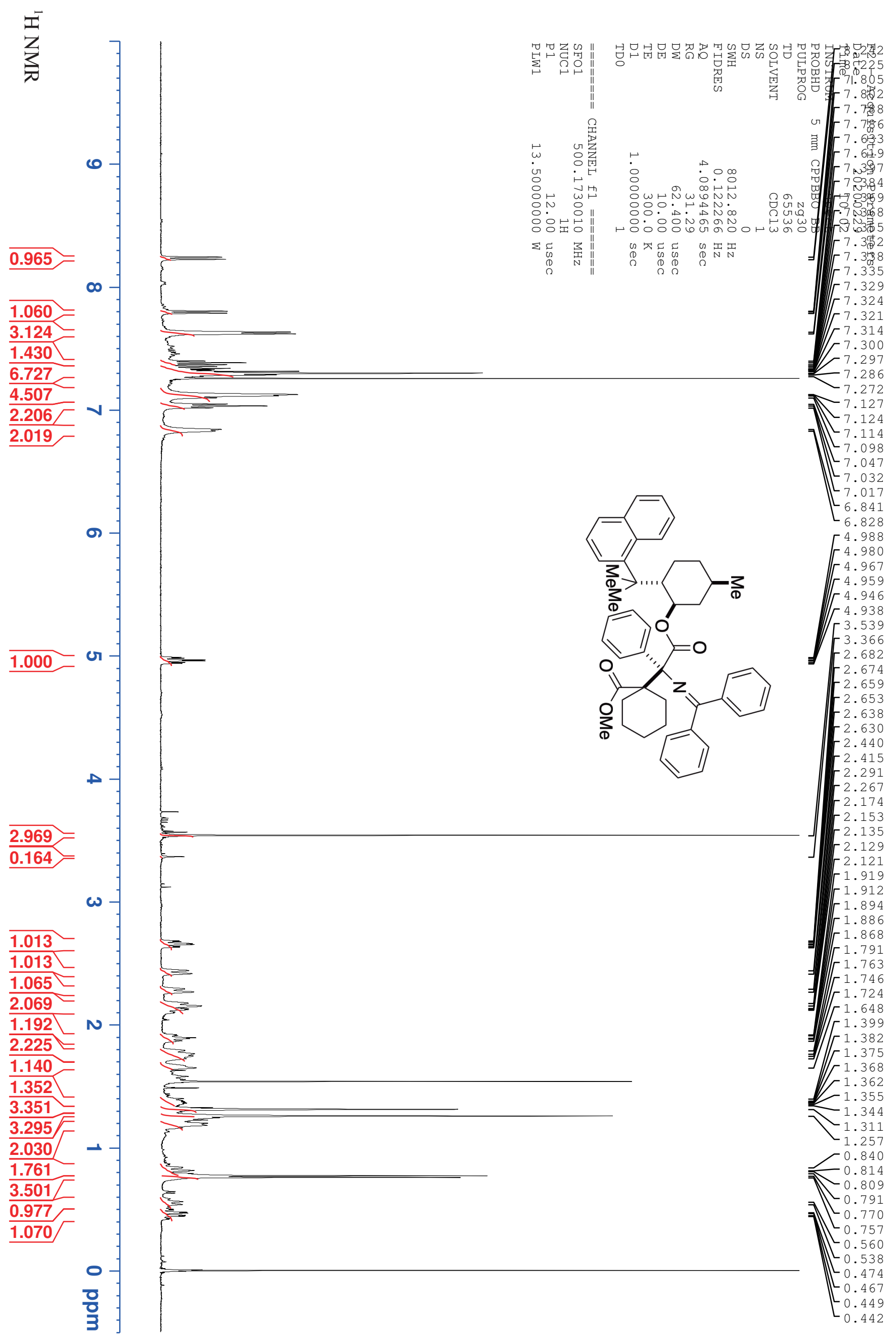




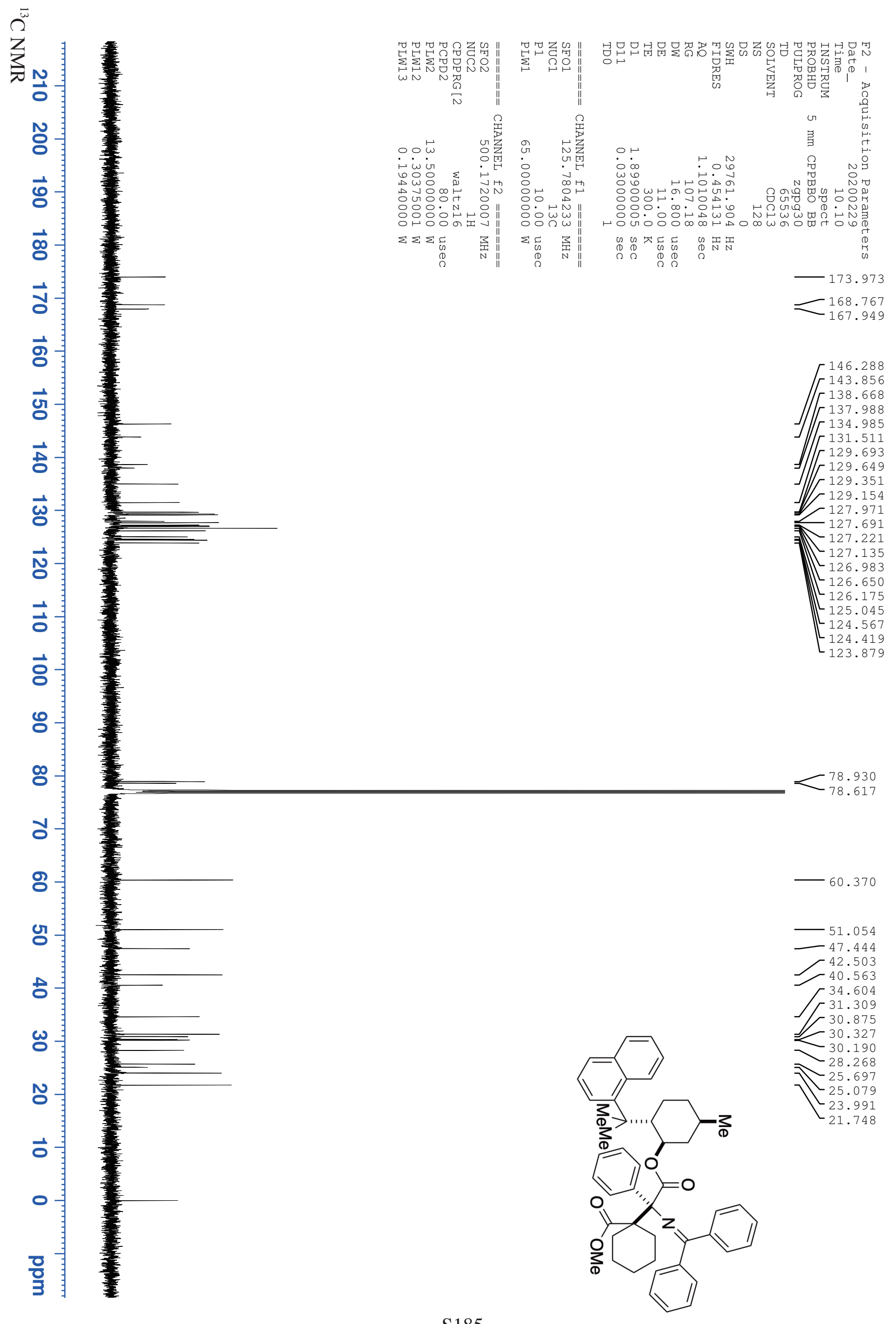




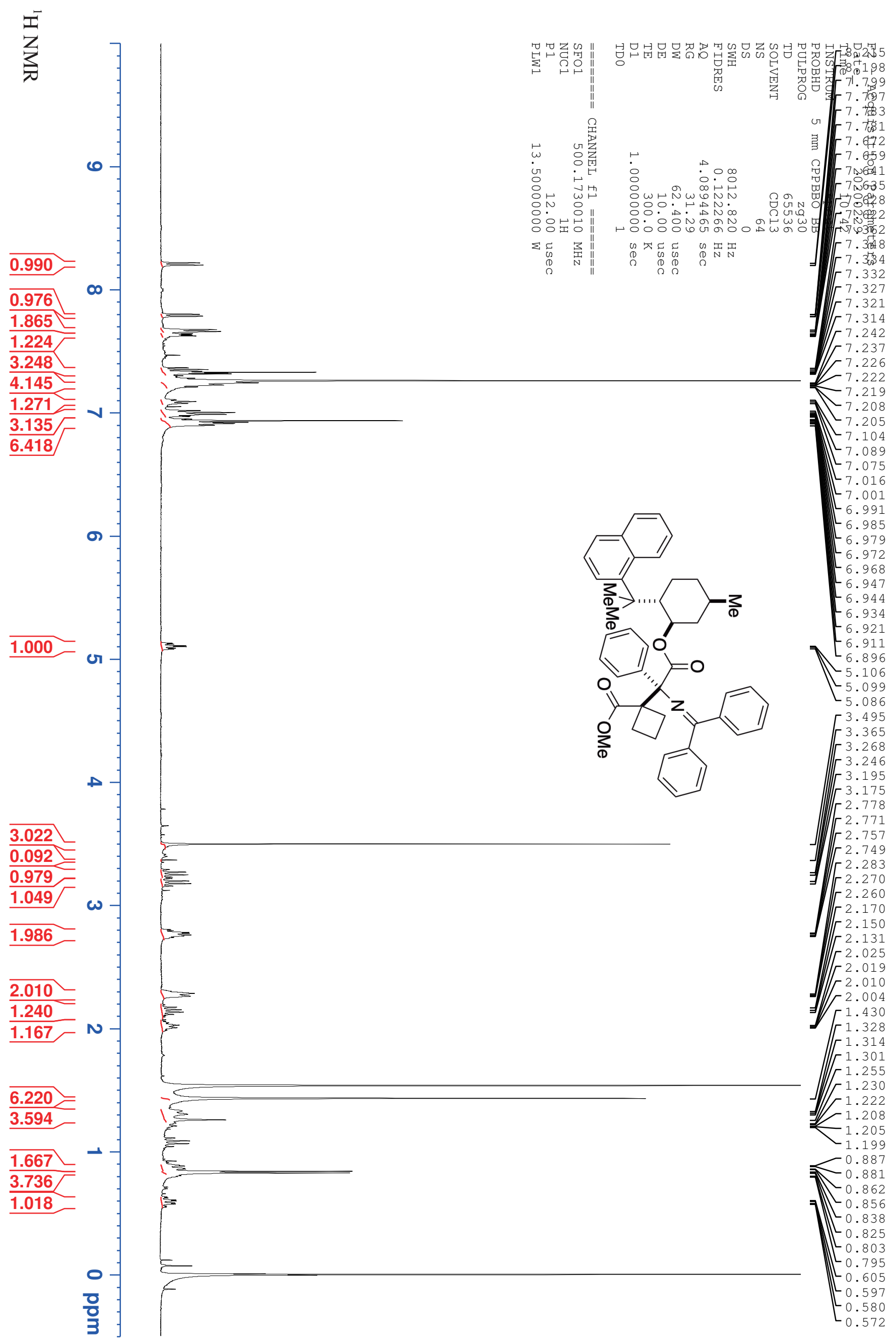




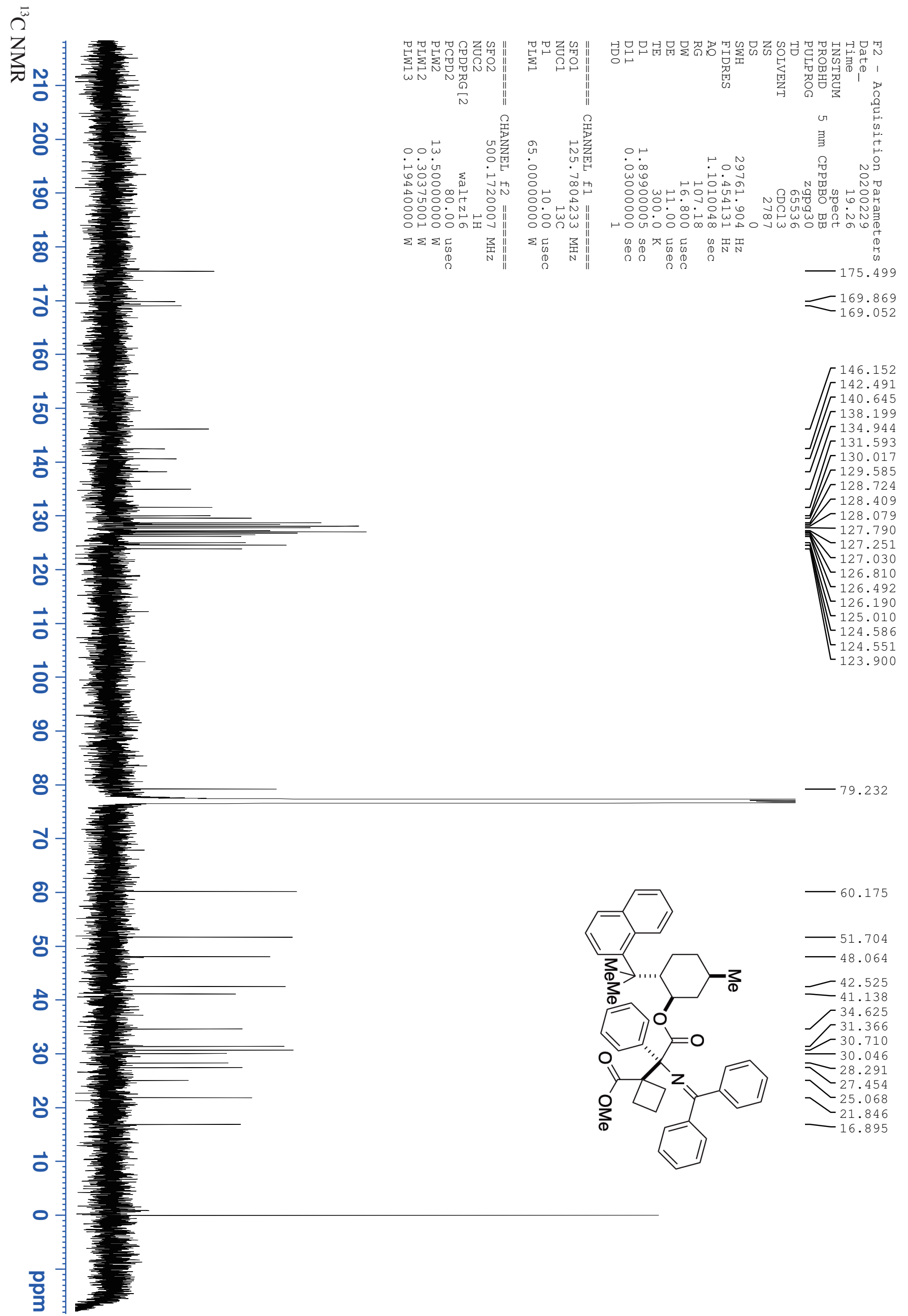




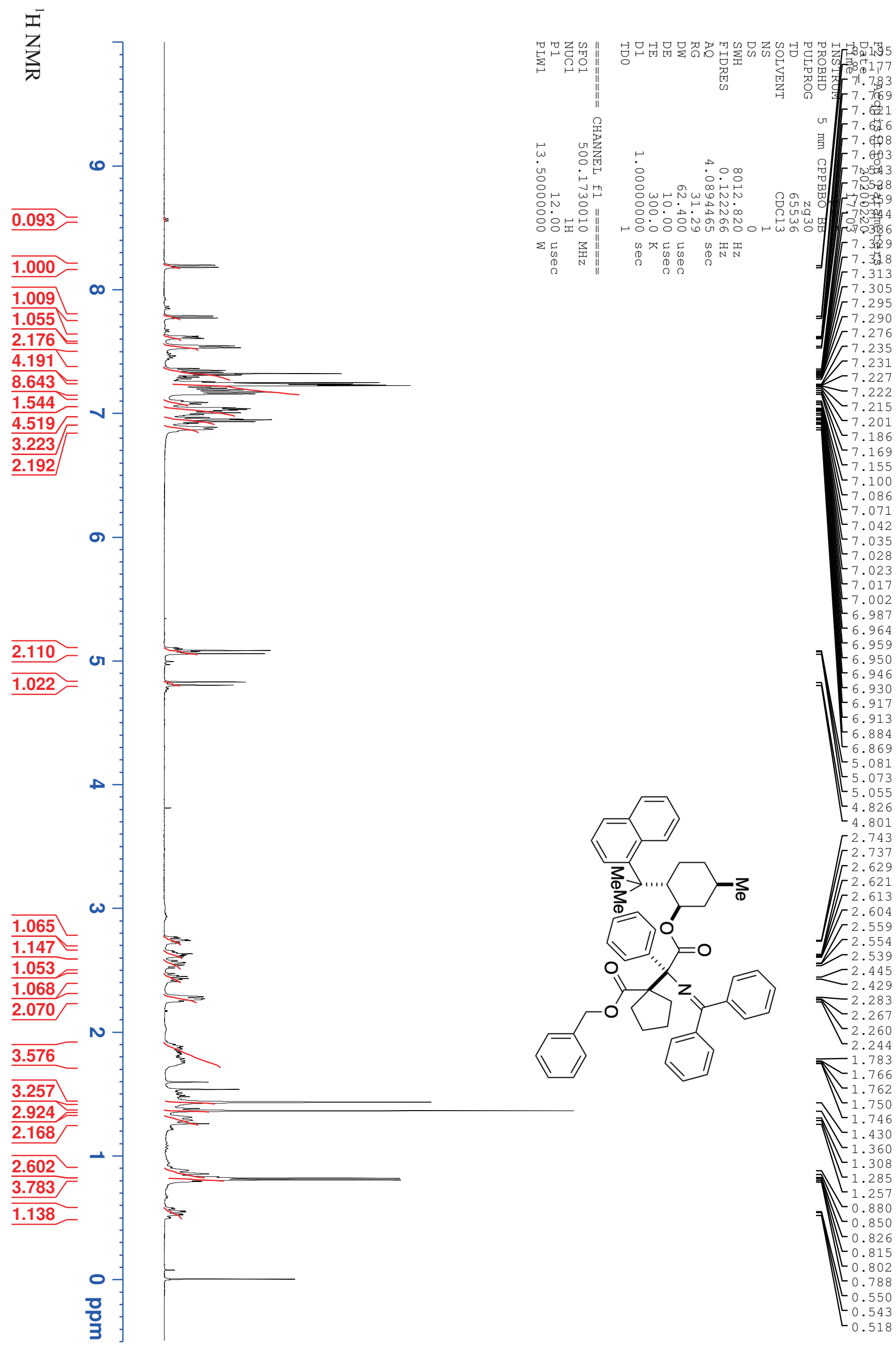




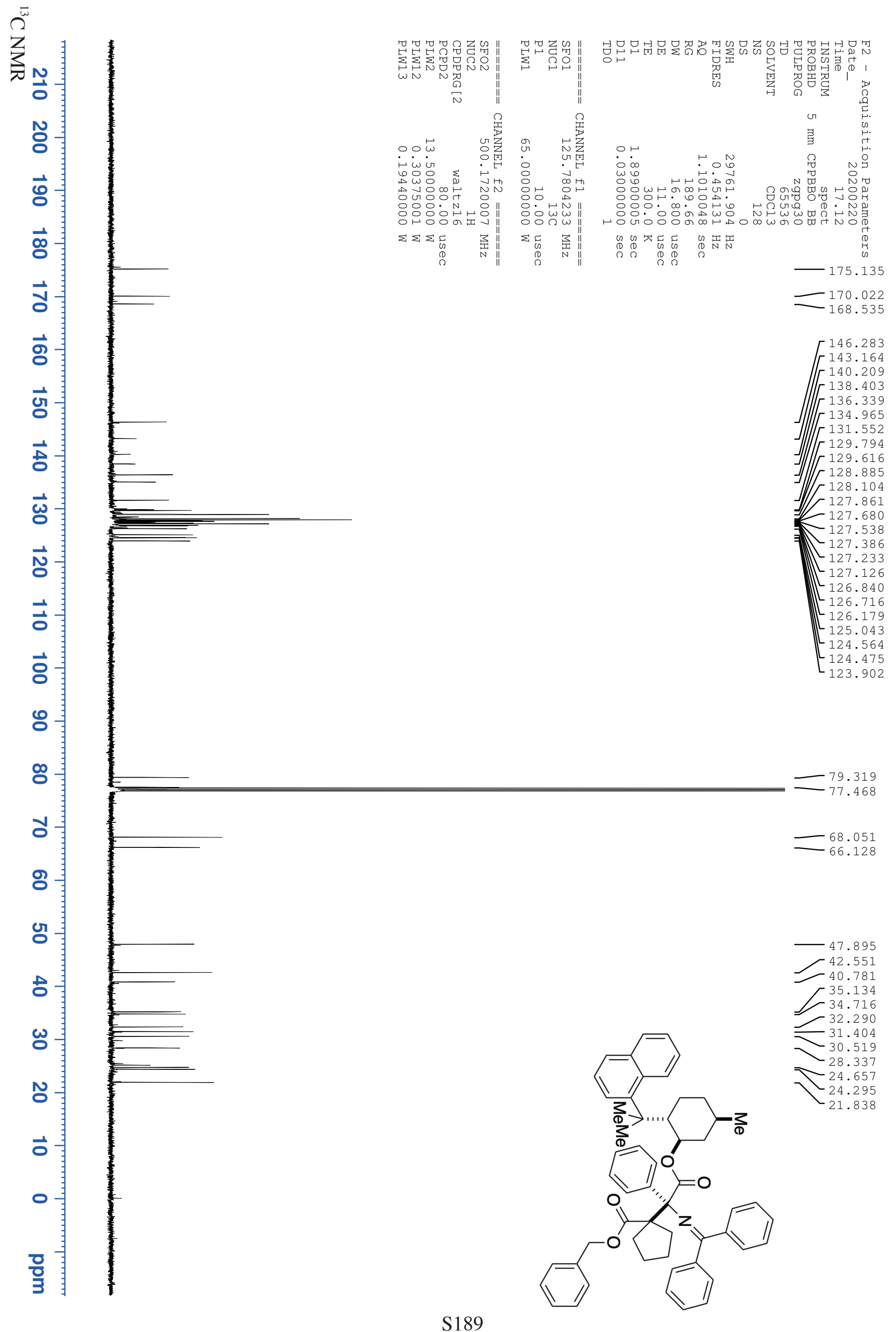




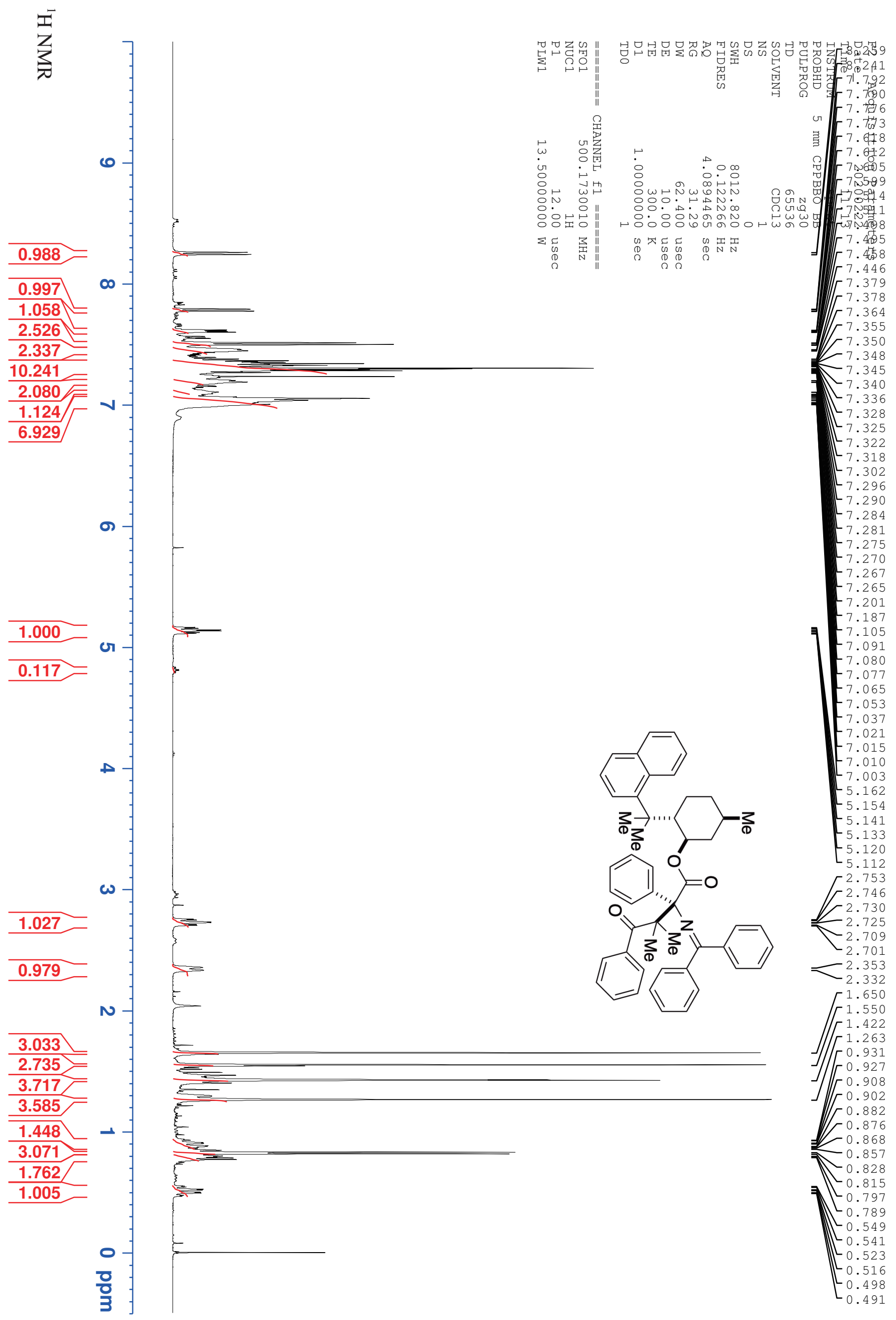




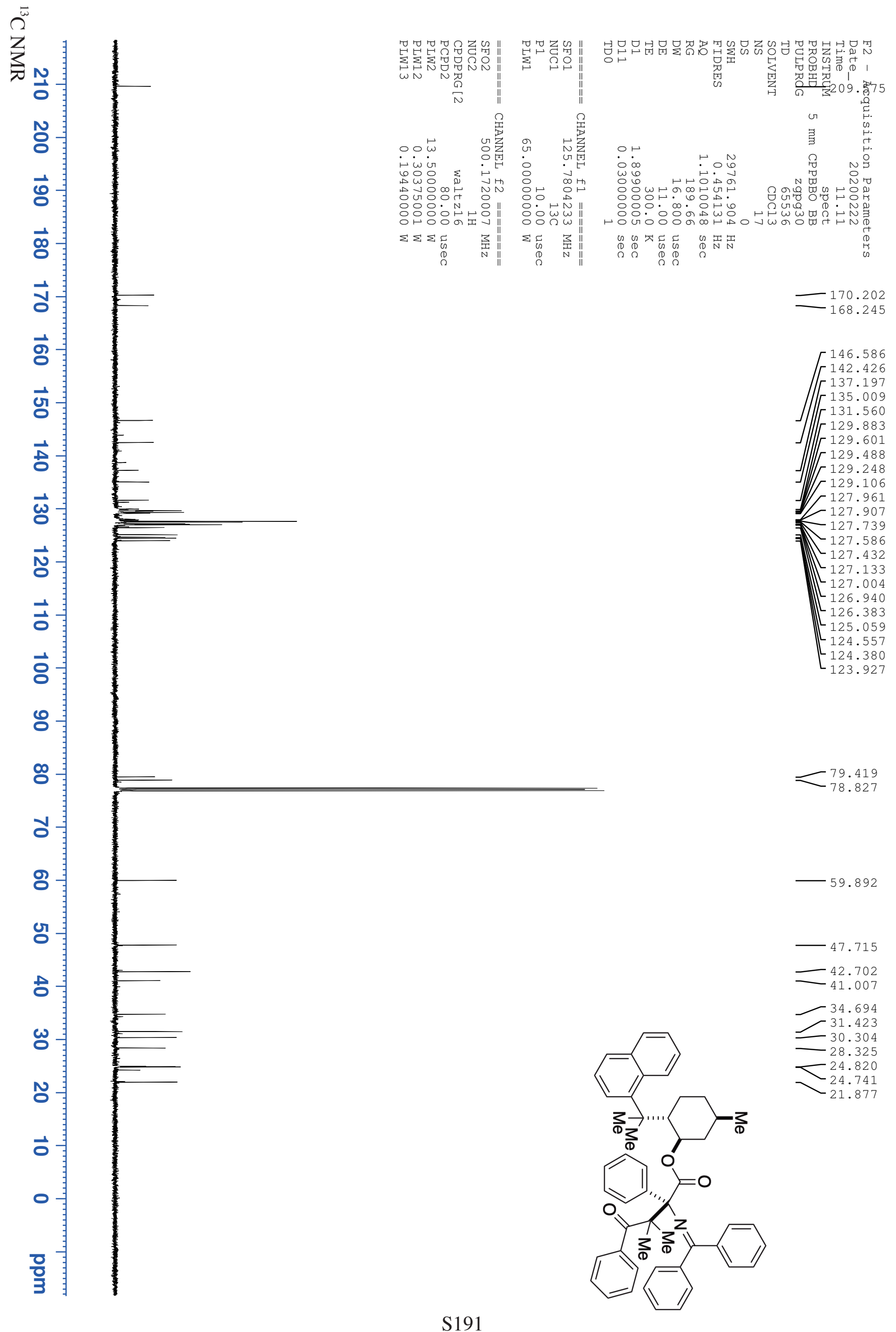




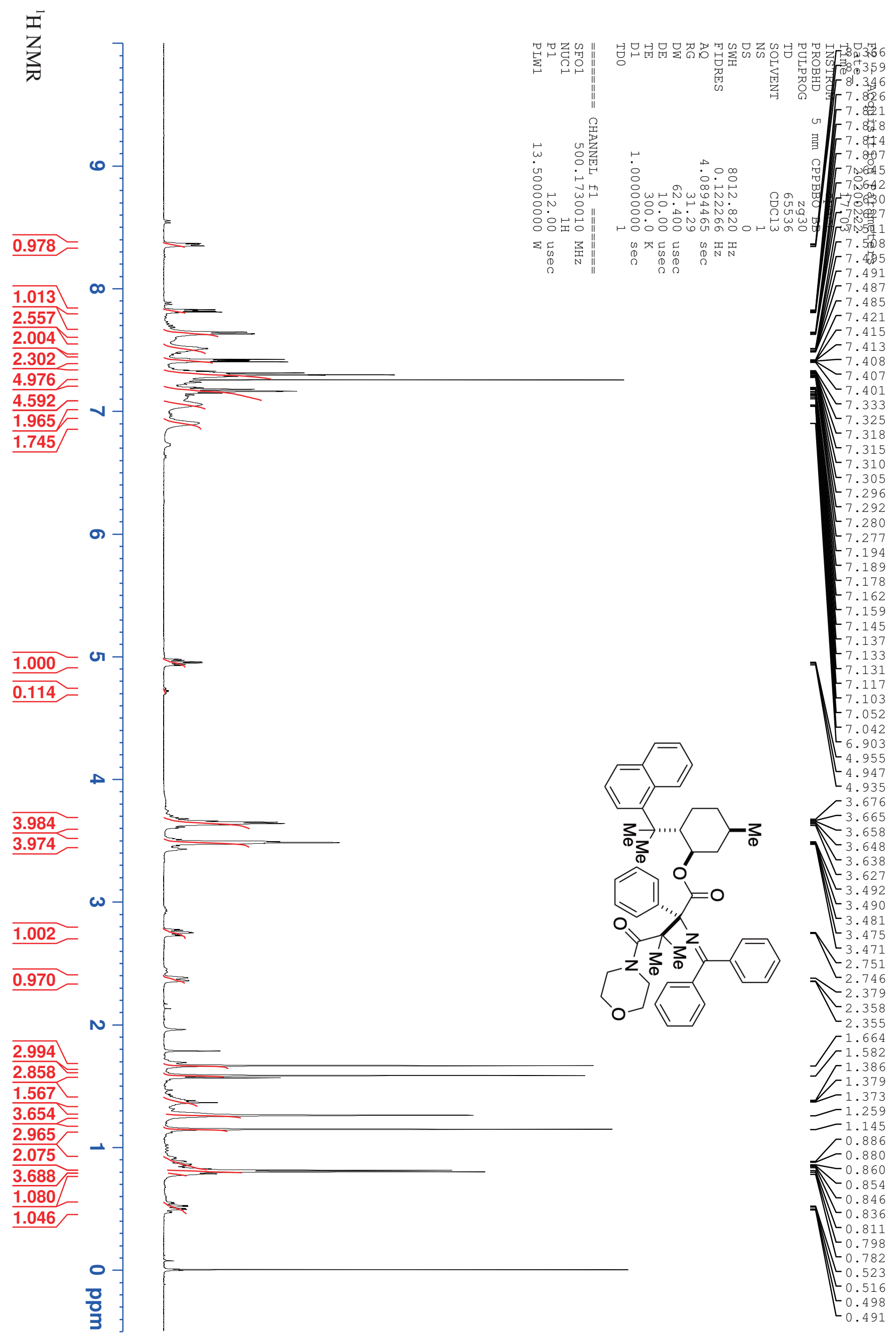



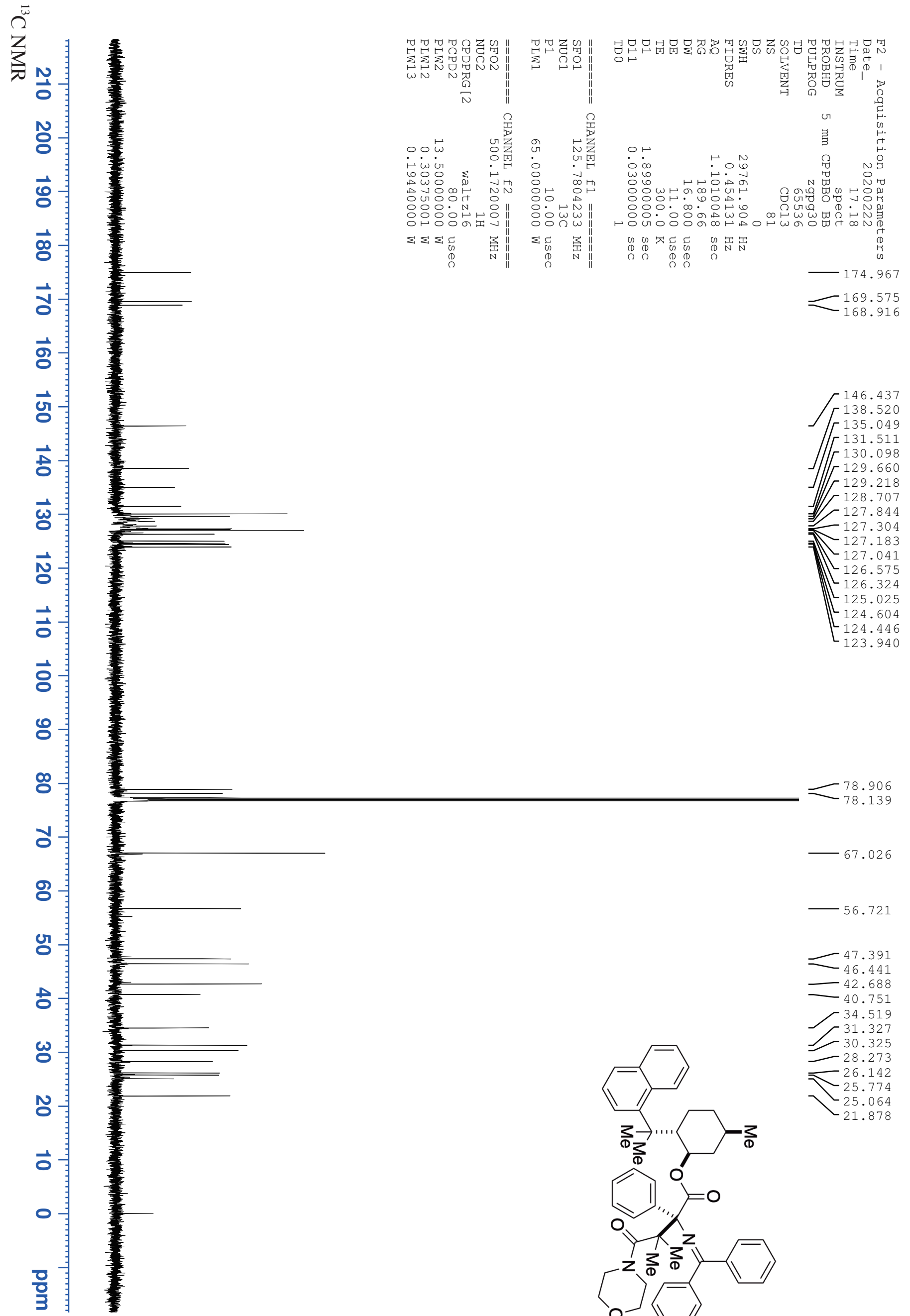

$-47.391$

- 46.441

$-42.688$

$-40.751$

- 34.519

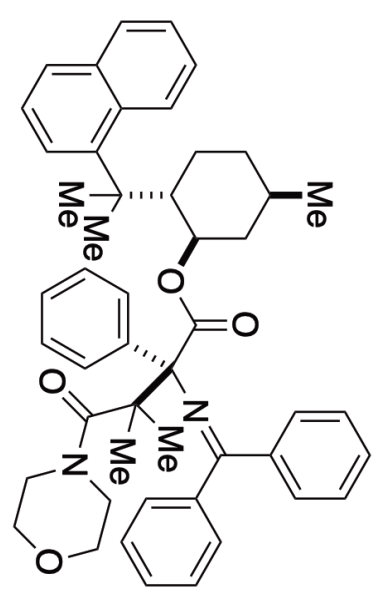

$\begin{array}{r}31.327 \\ -30.325 \\ \hline\end{array}$

$-28.273$

26.142

$-25.774$

$-25.064$

21.878 


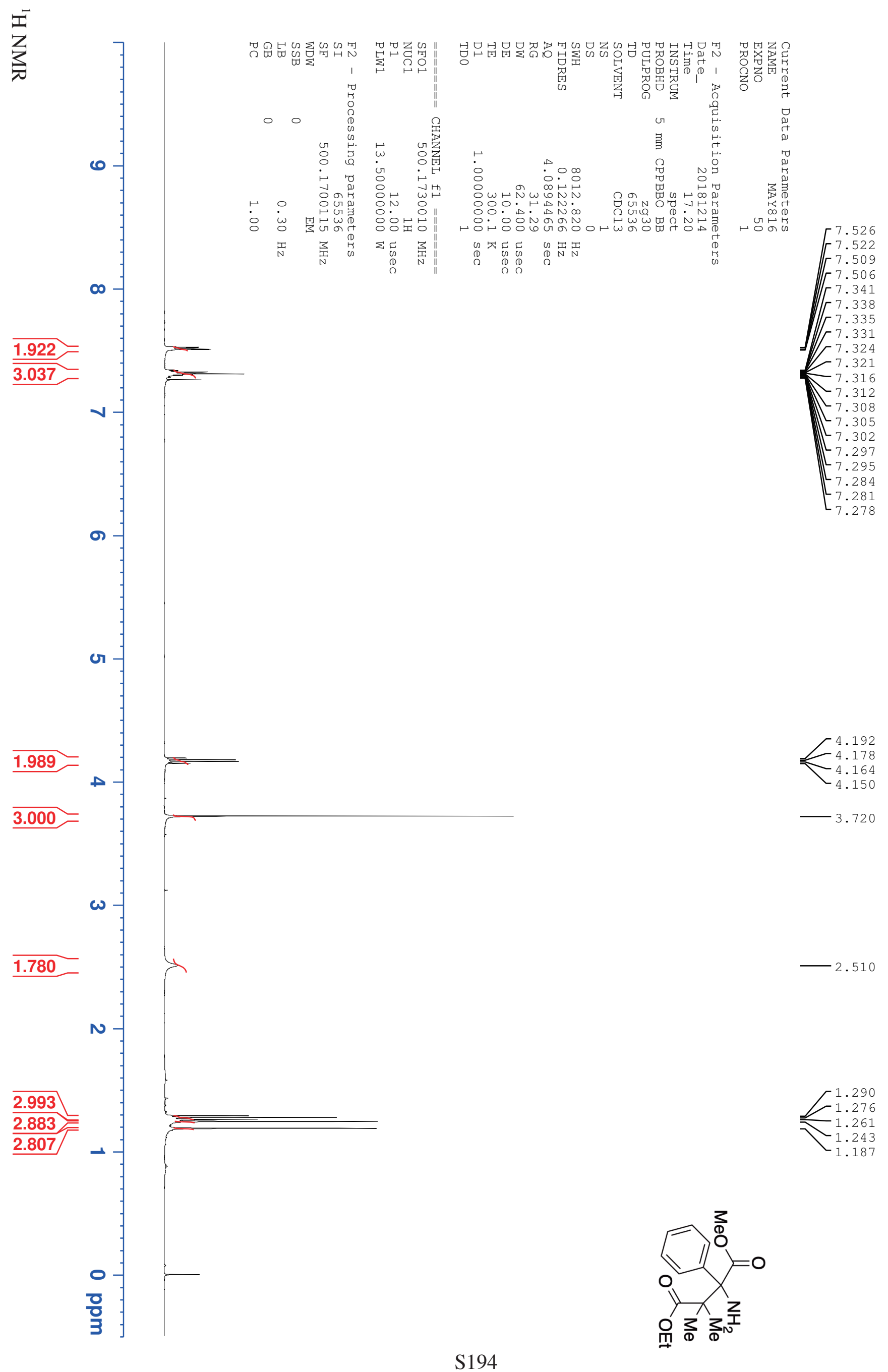




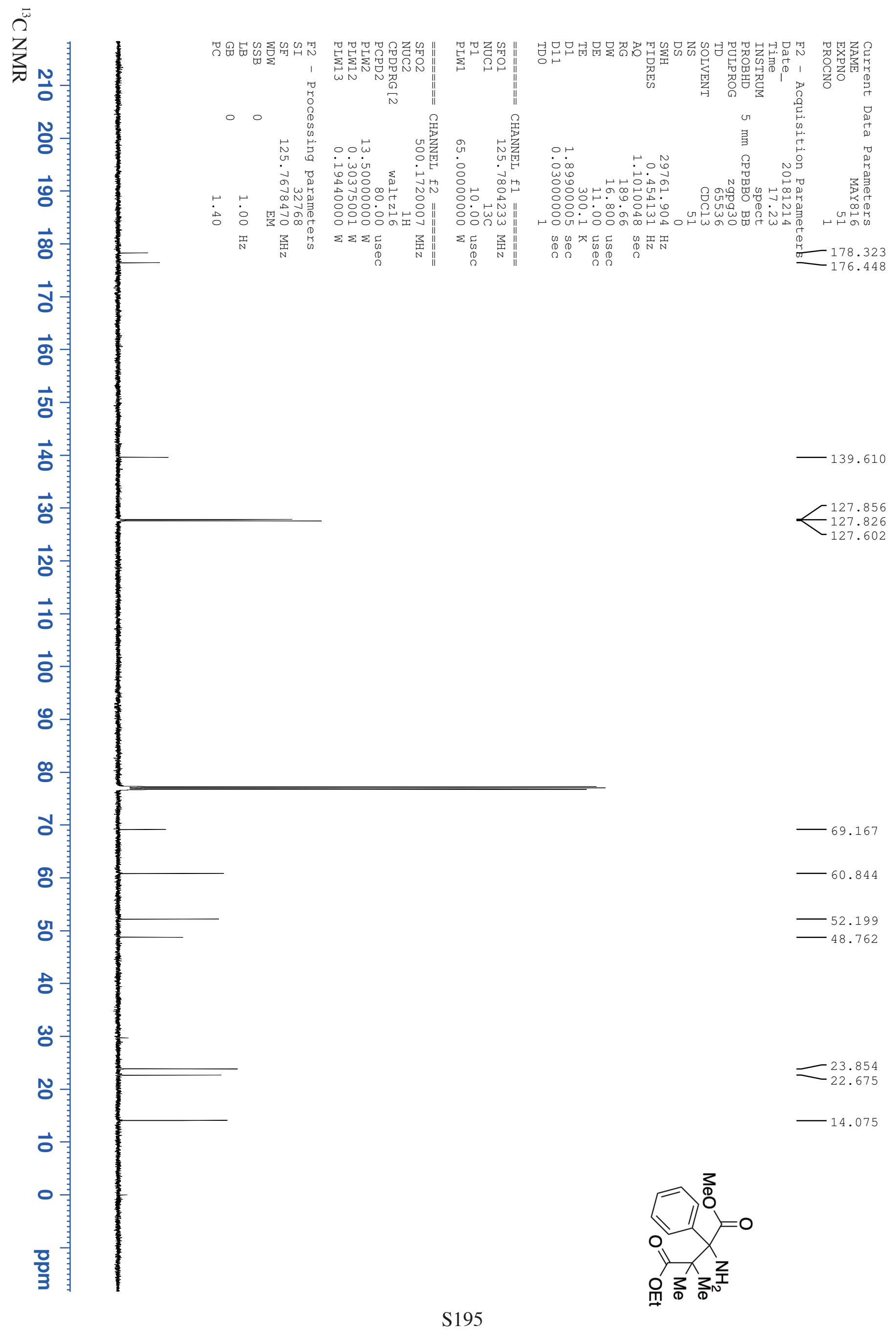




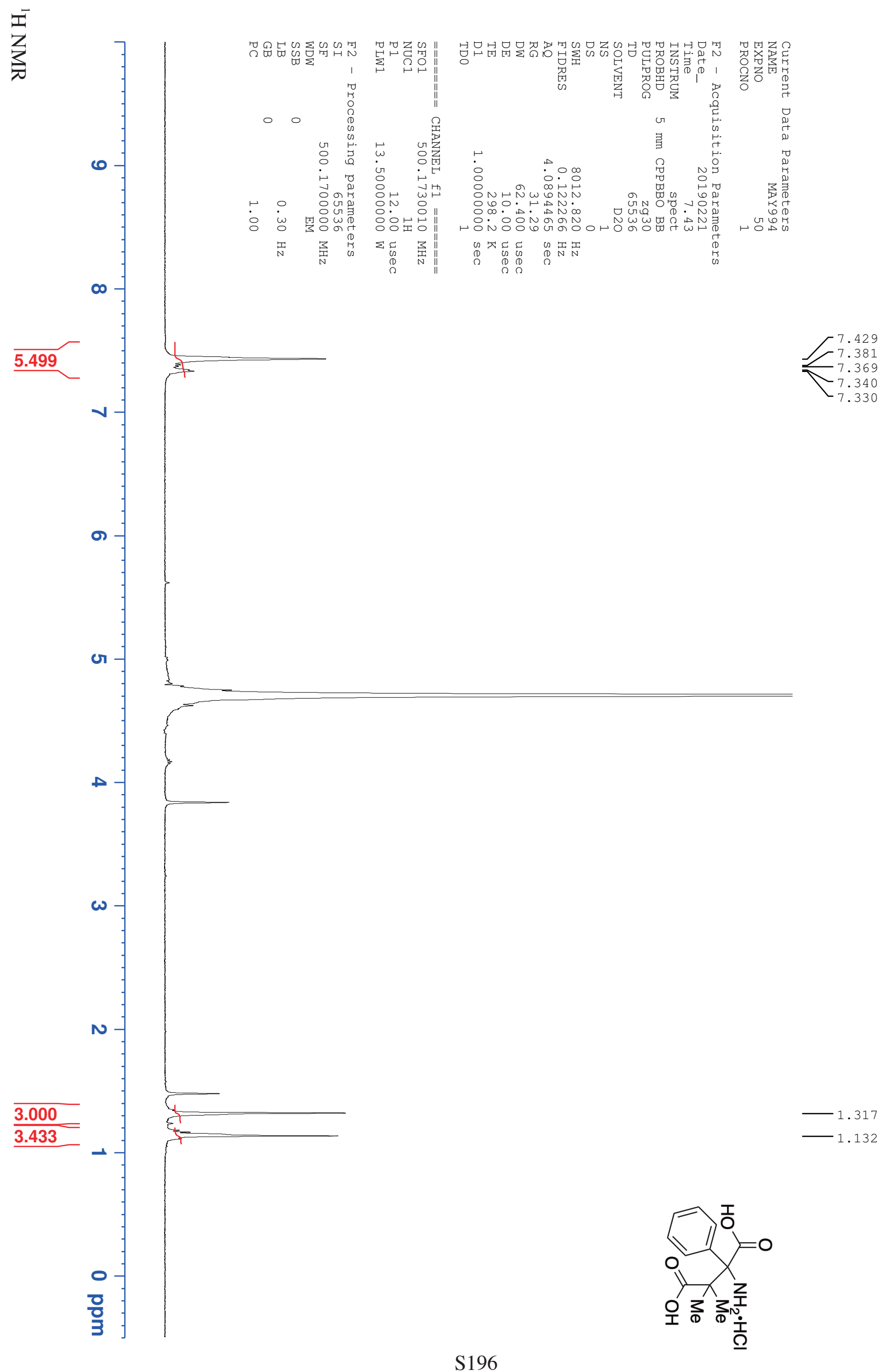




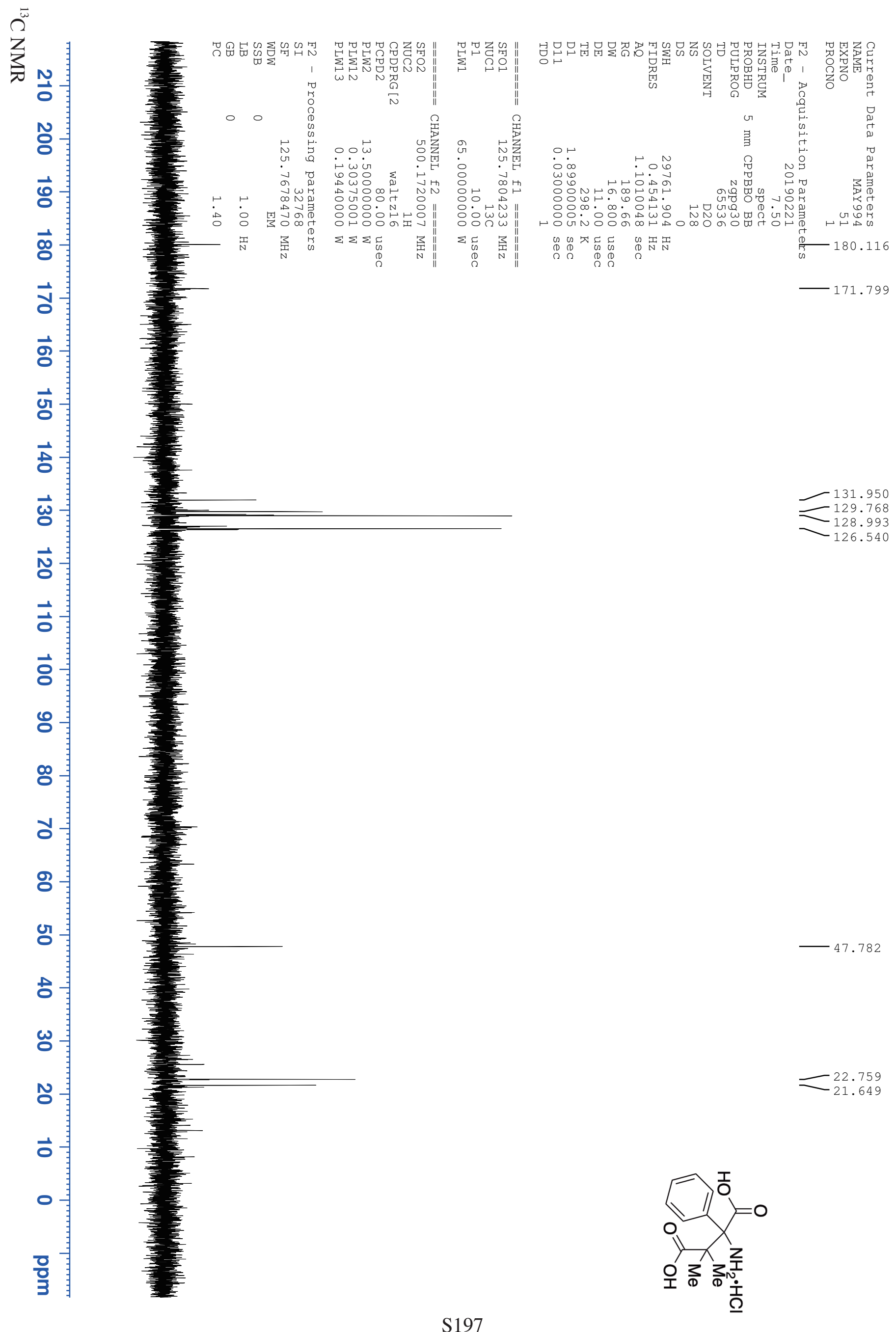


$a$ 4.2714
4.2572

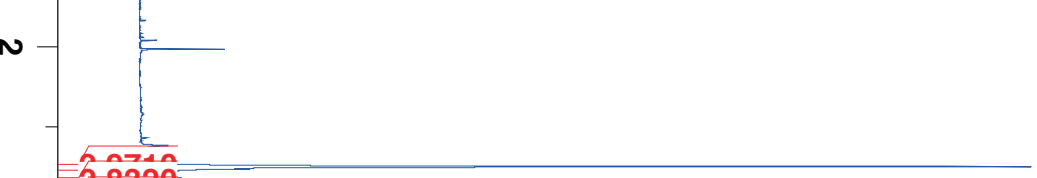

2 onn

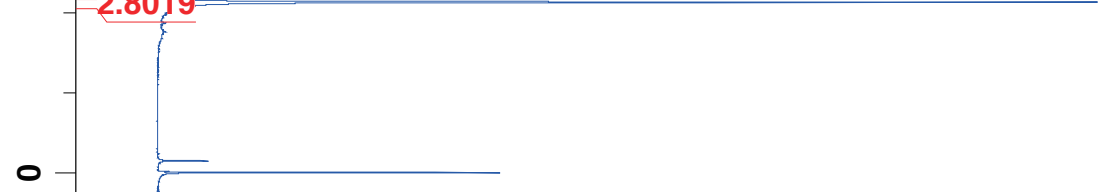

0

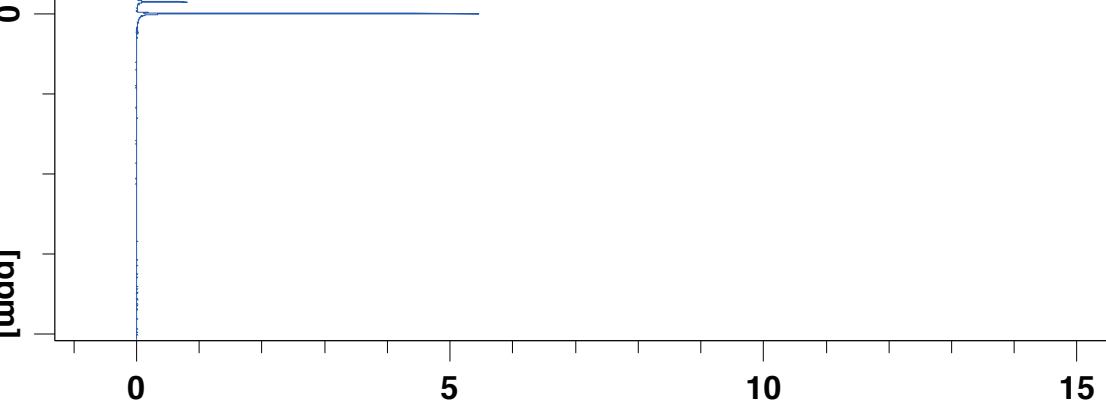

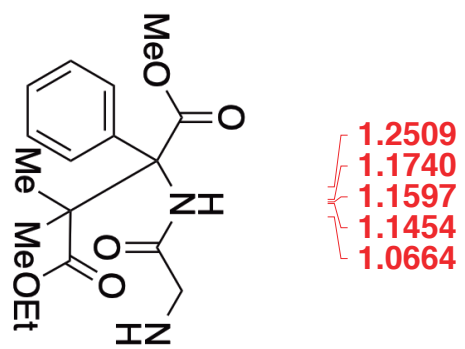

1597

1.1454

1.0664

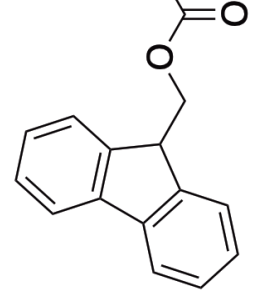




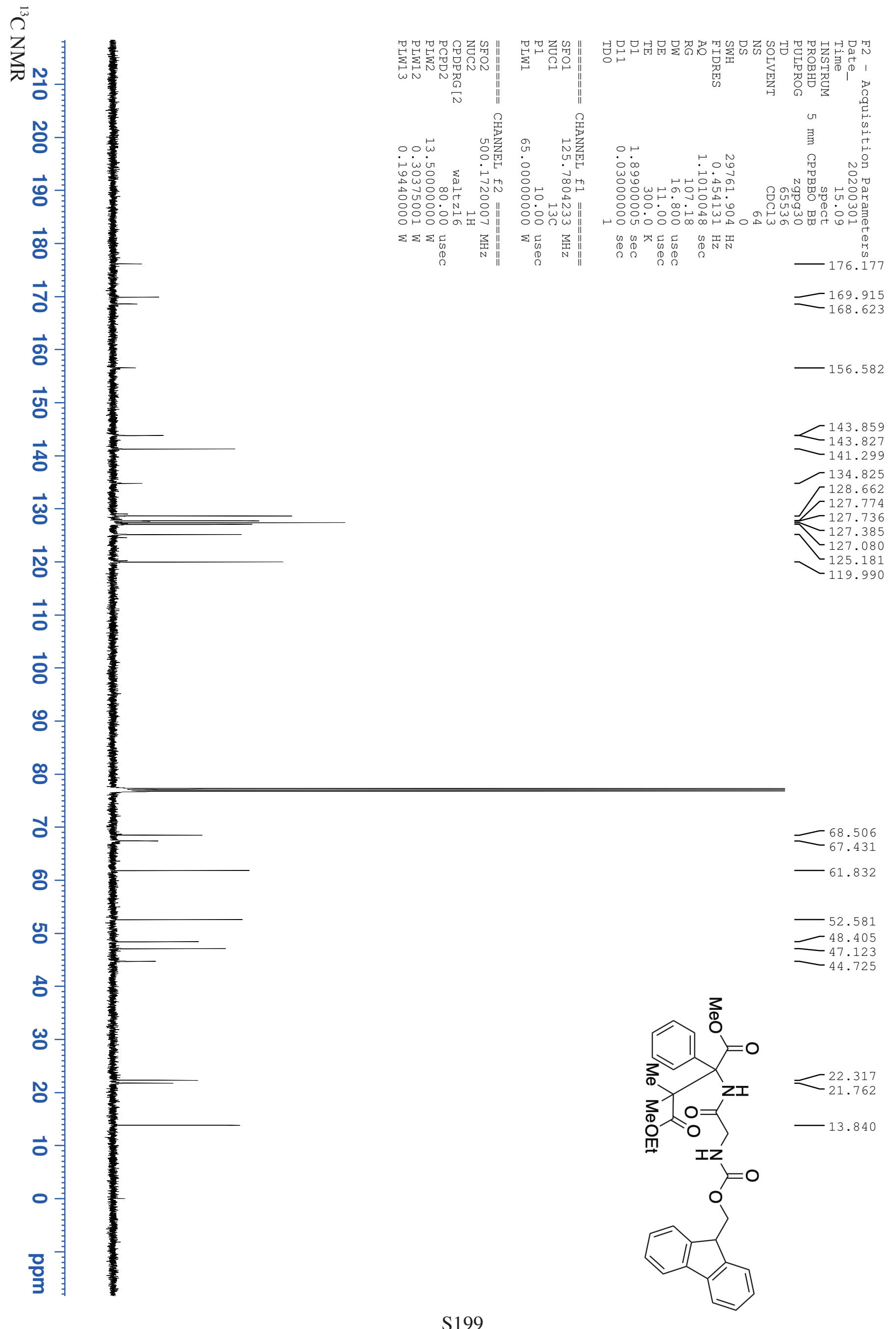




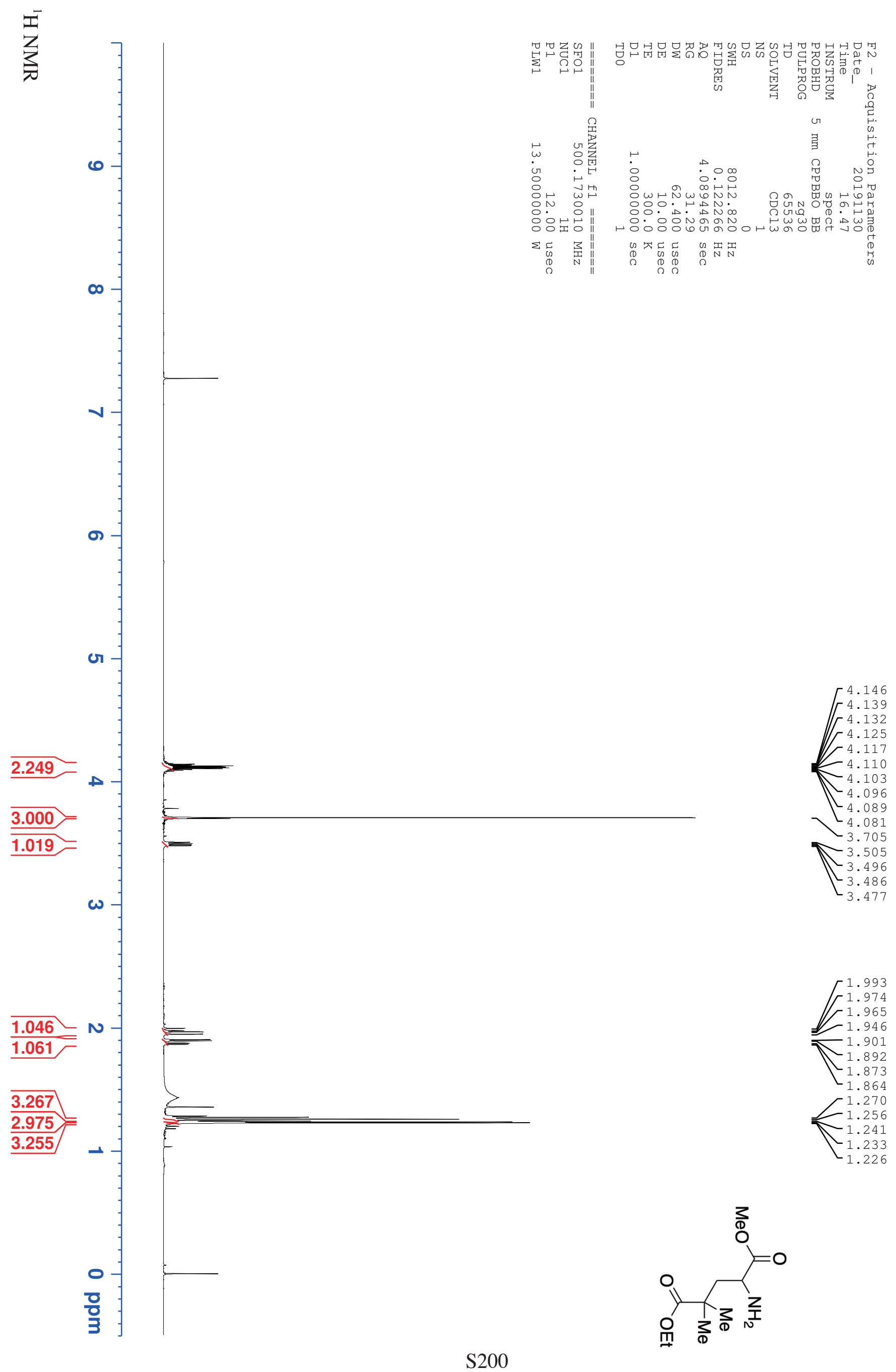



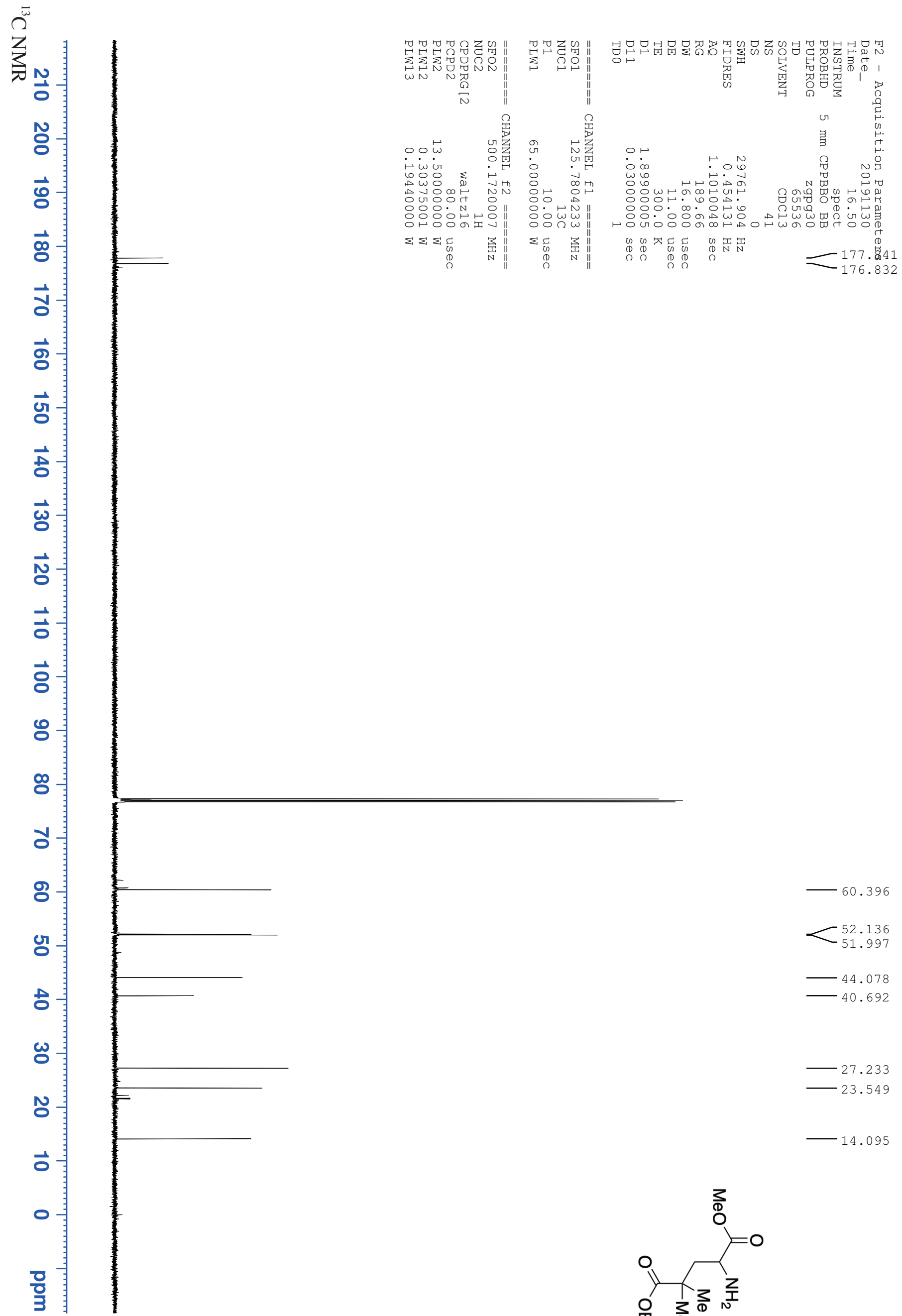

52.136

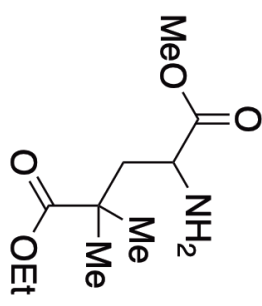




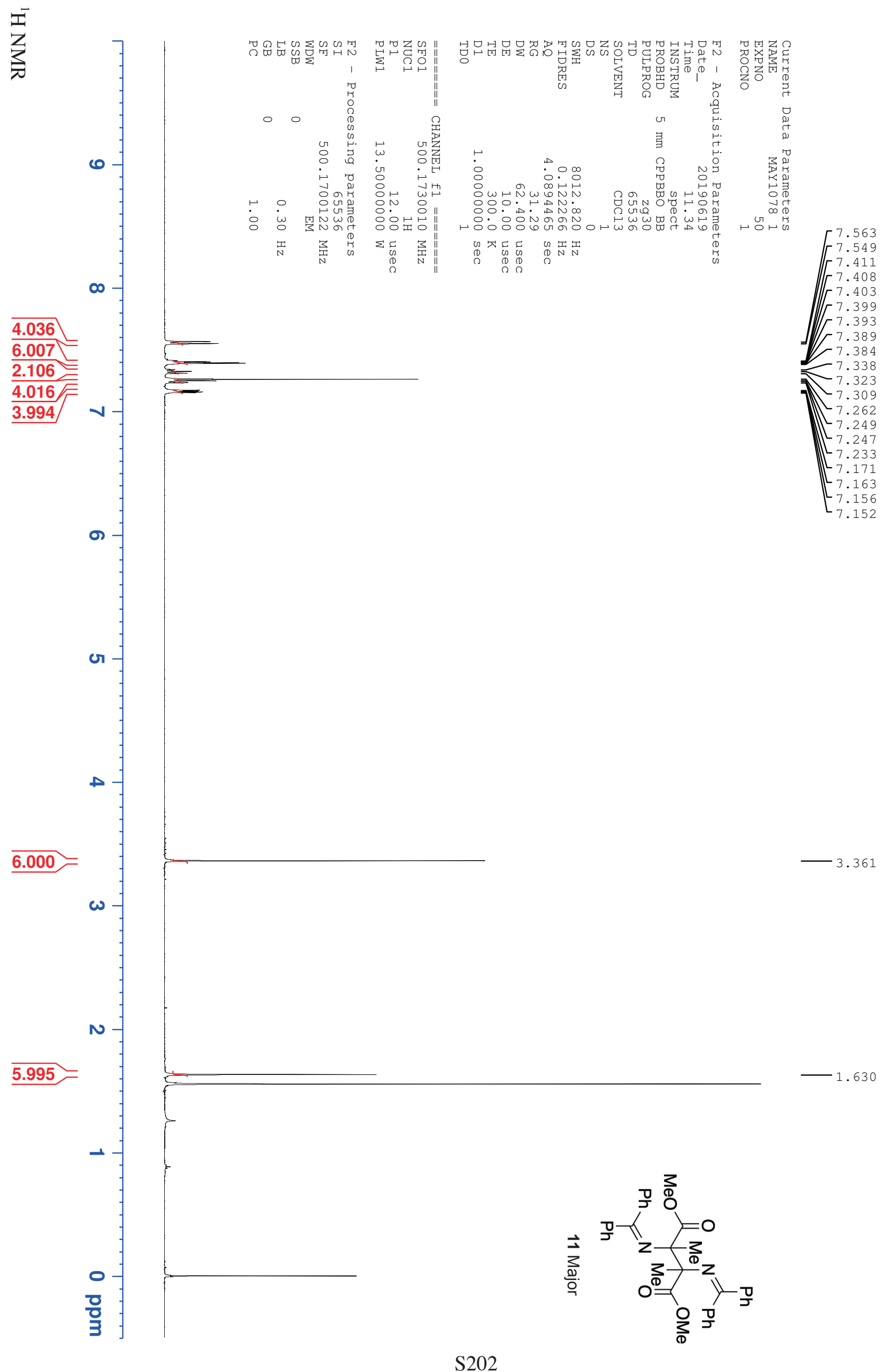




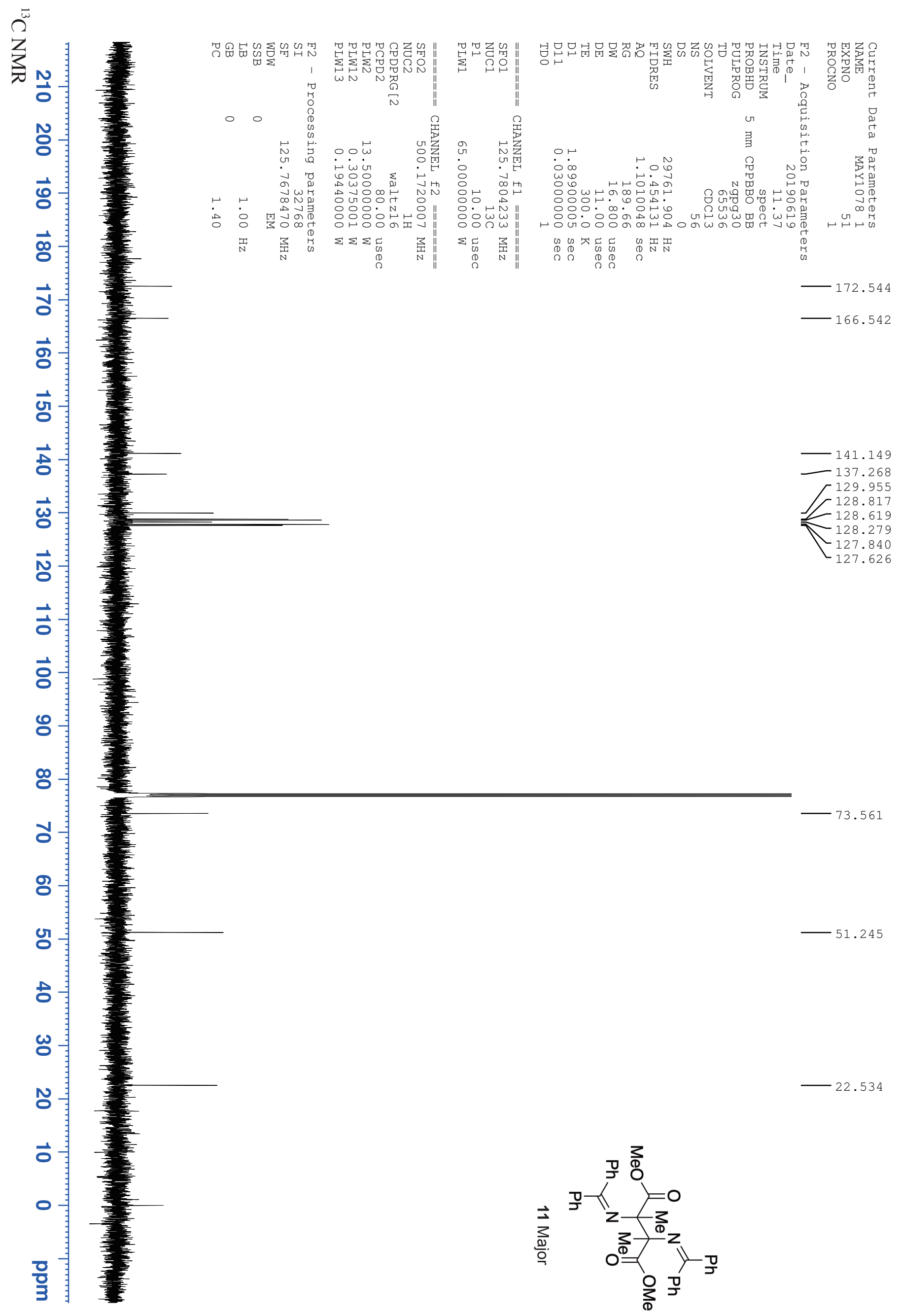




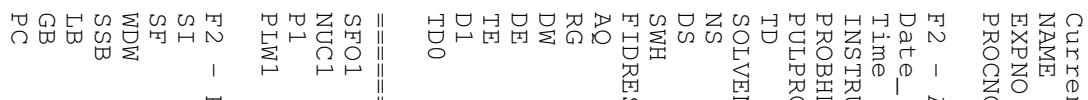
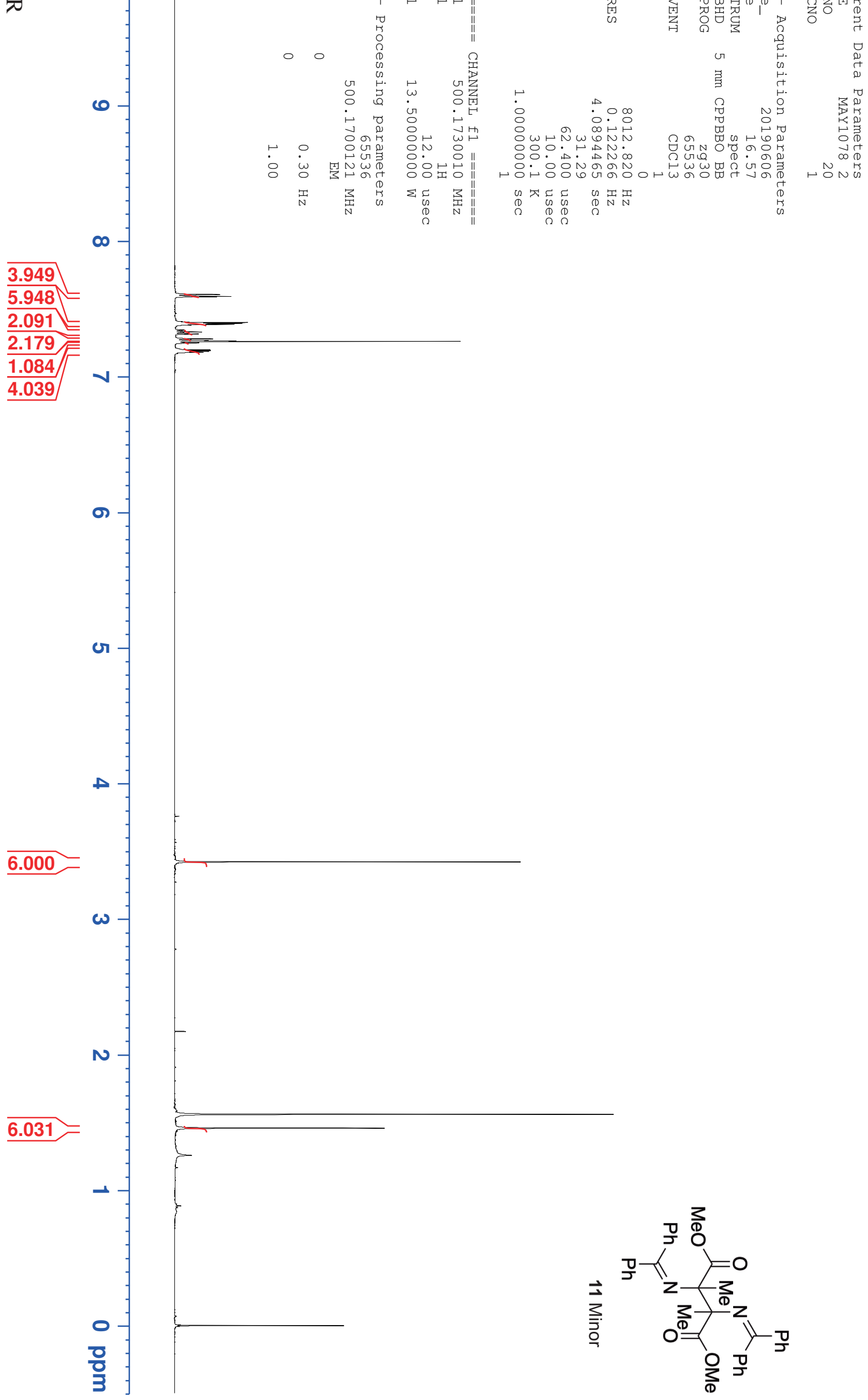


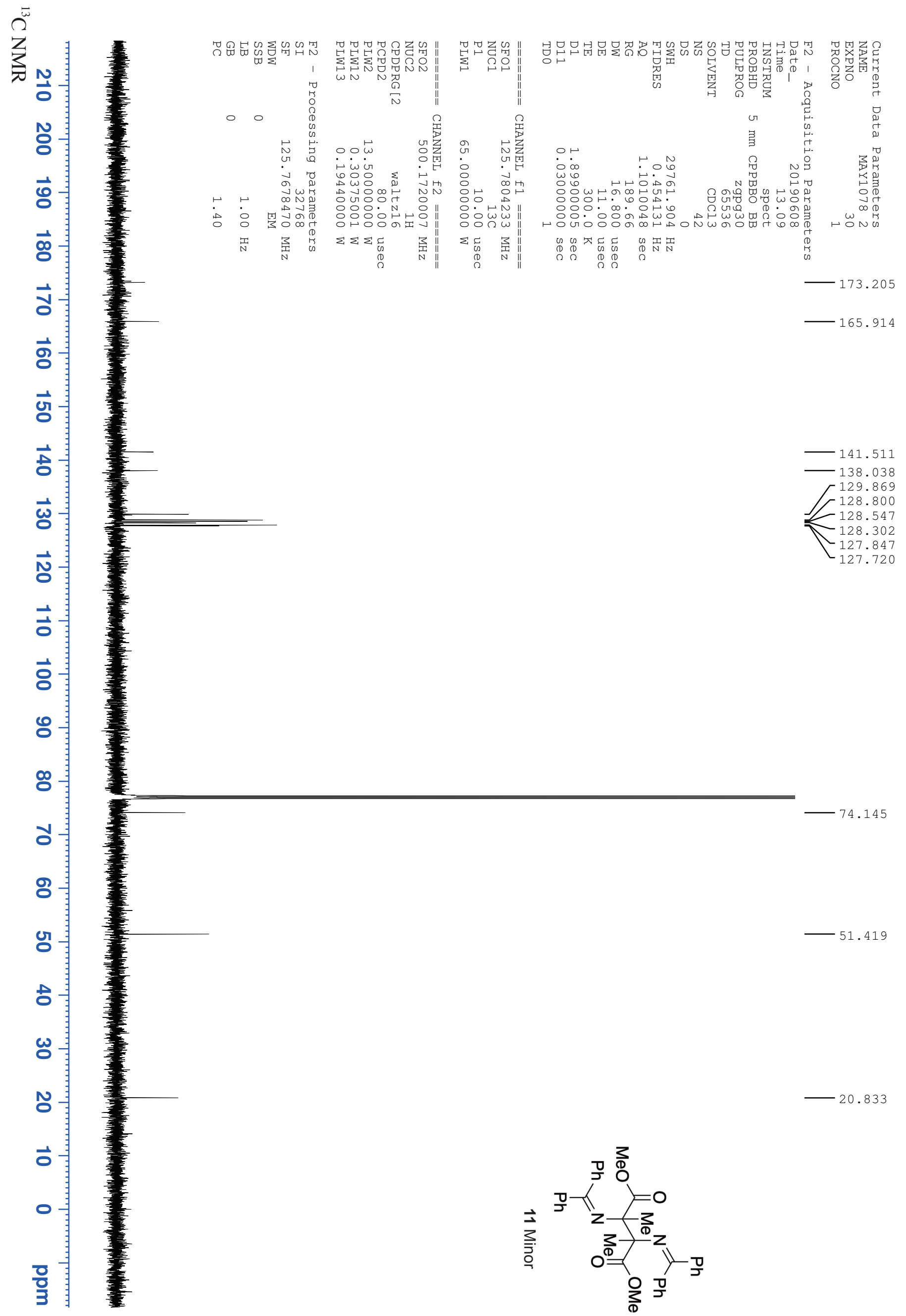




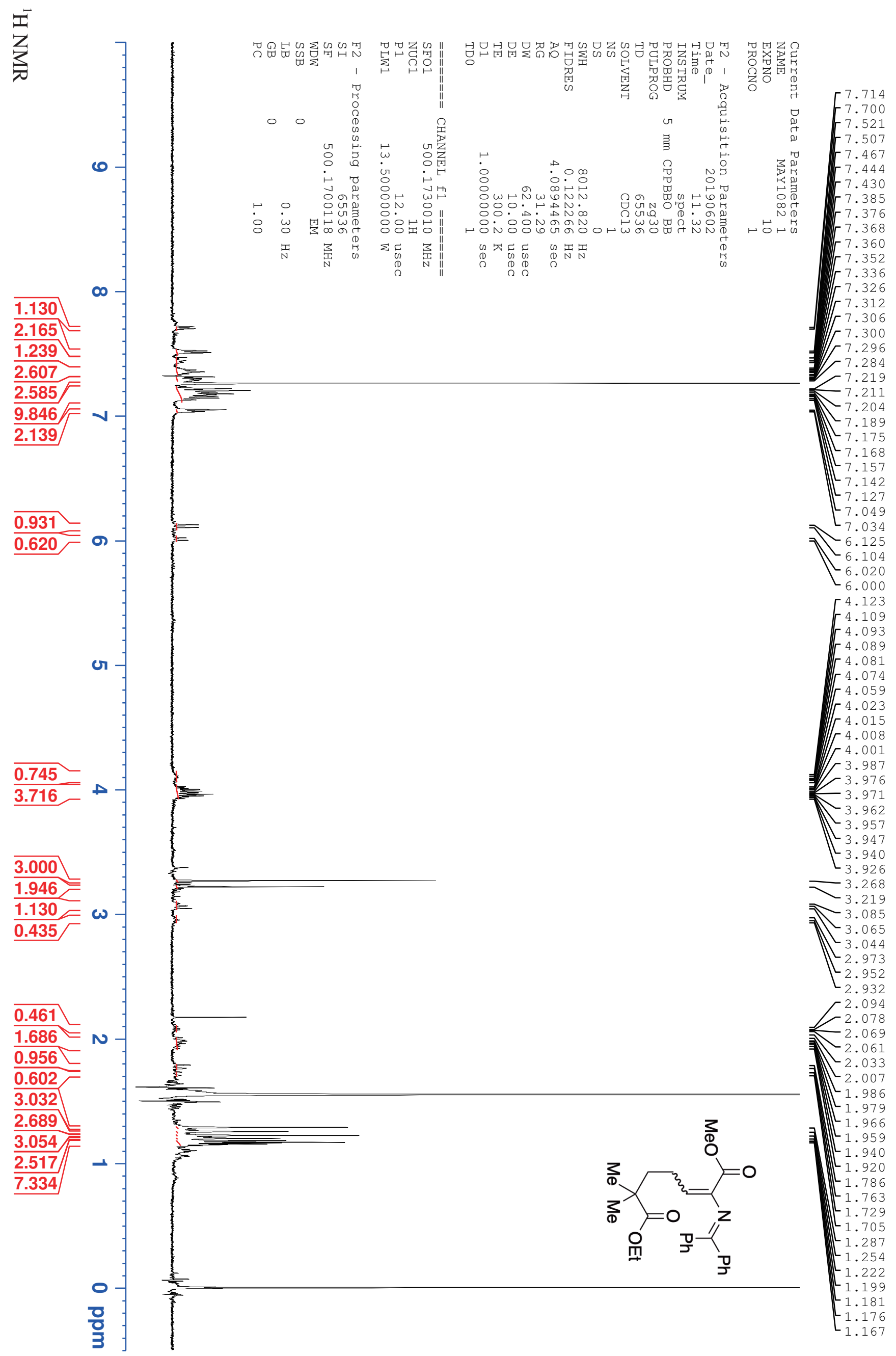




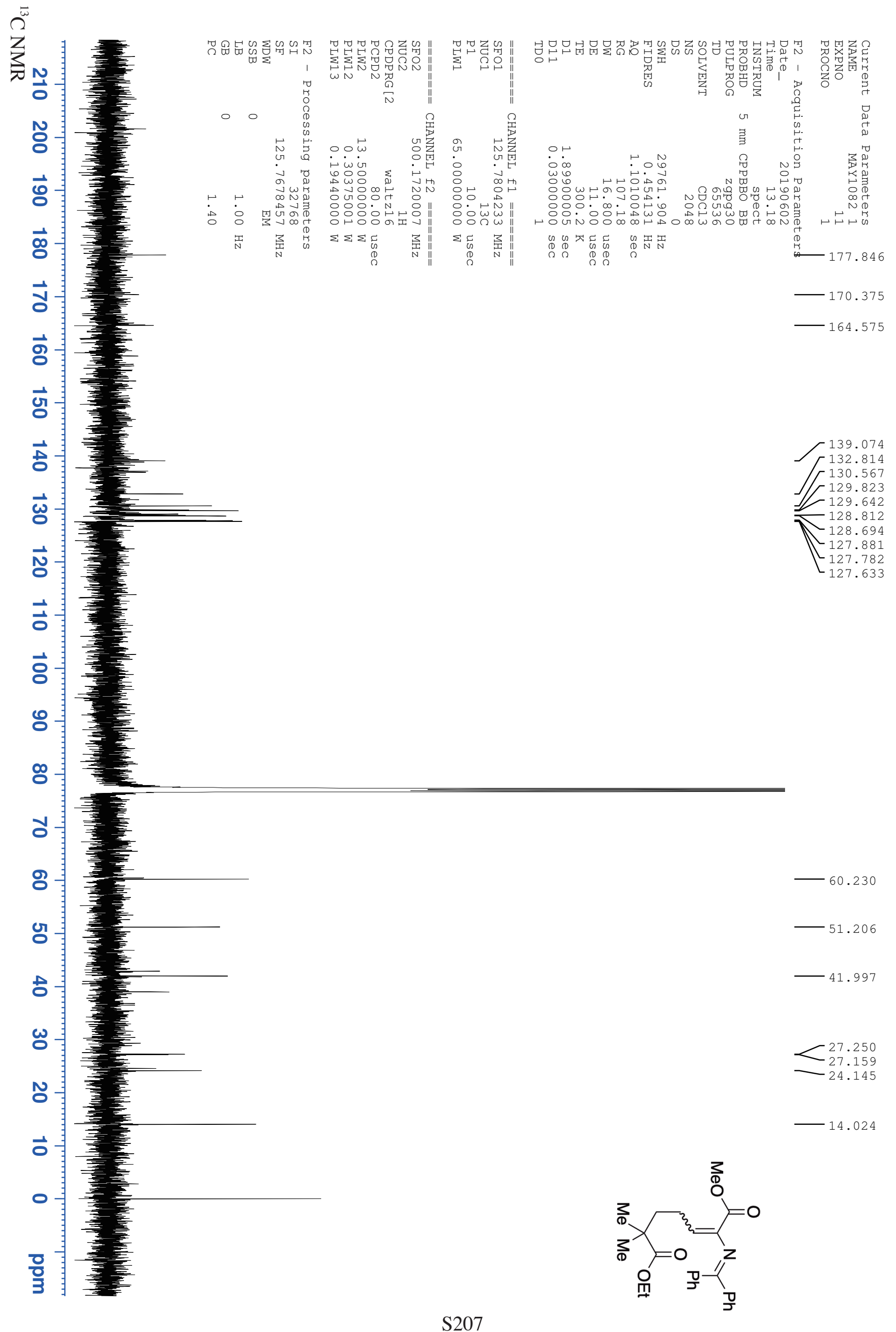




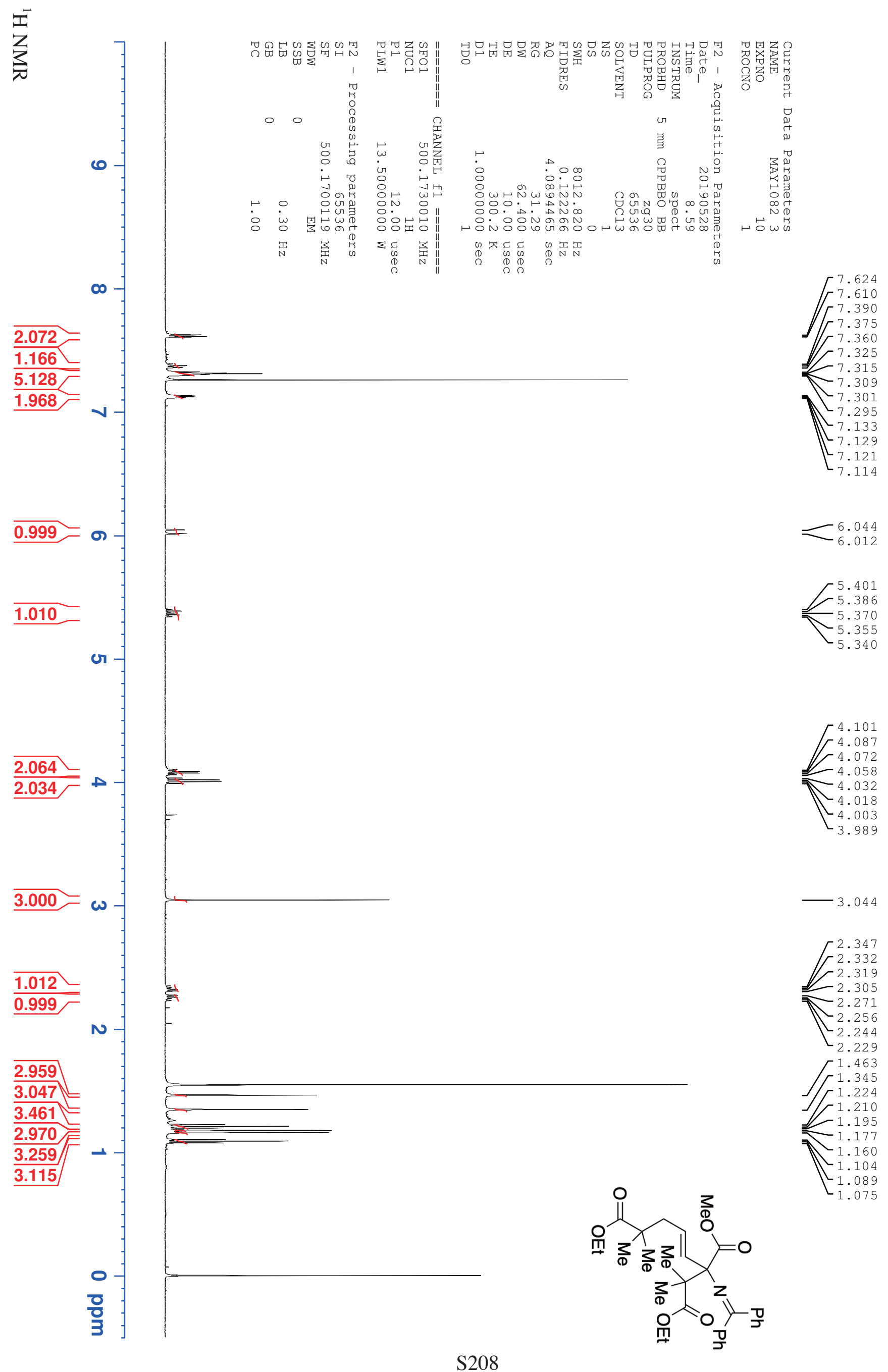




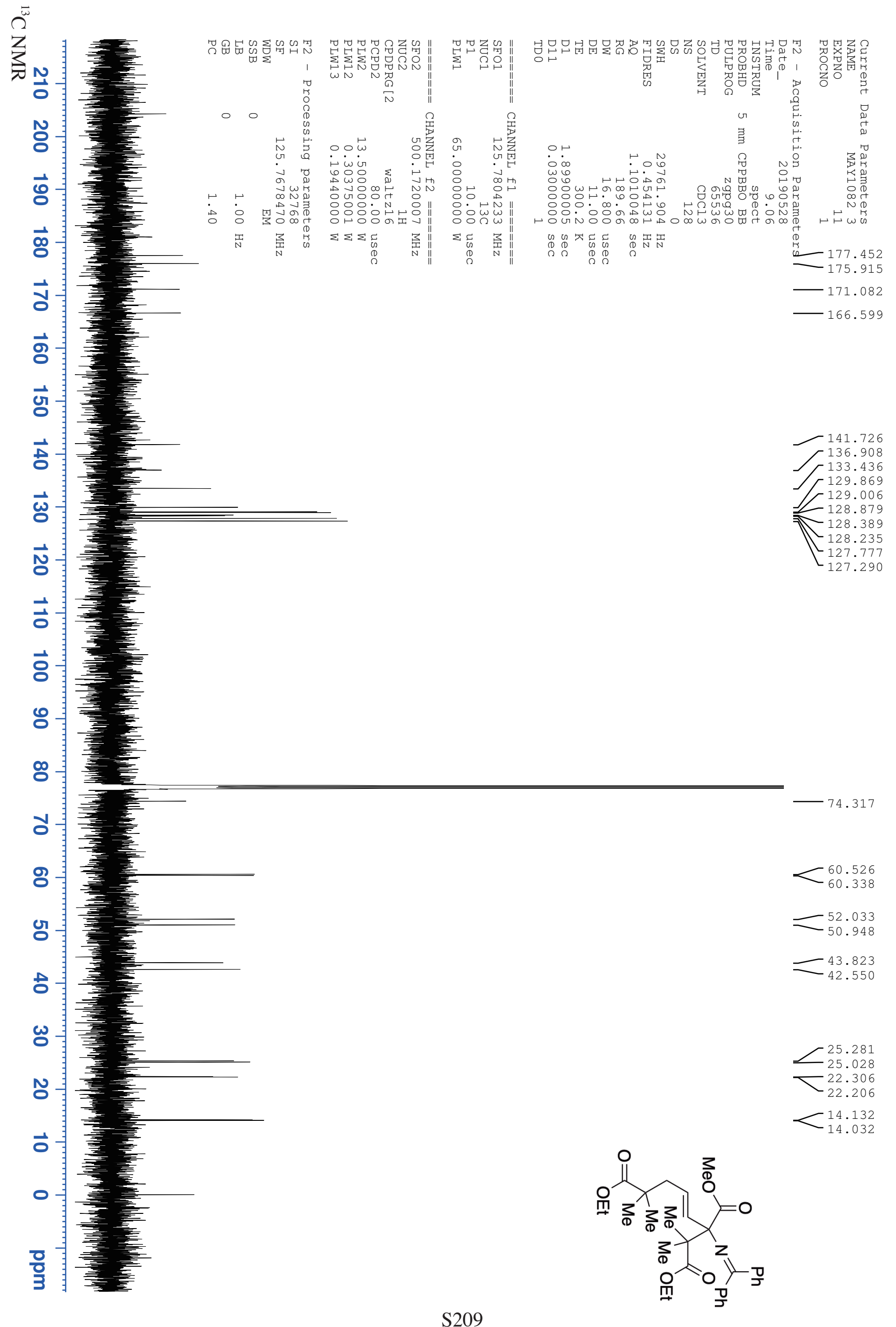

